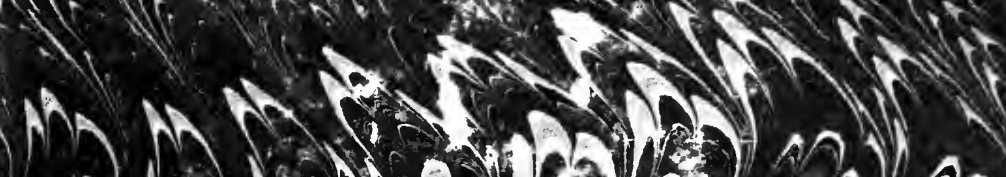
A

(2)

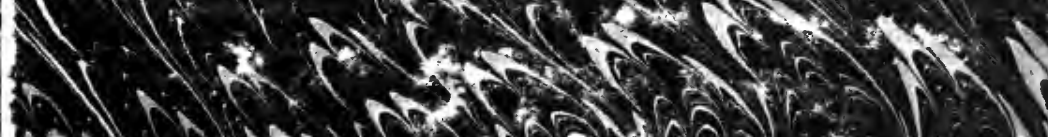
5.) COR 1 C.V.

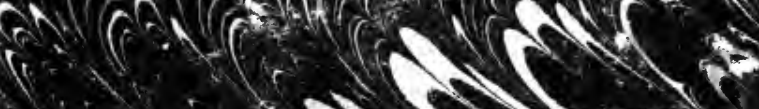

AR Â.

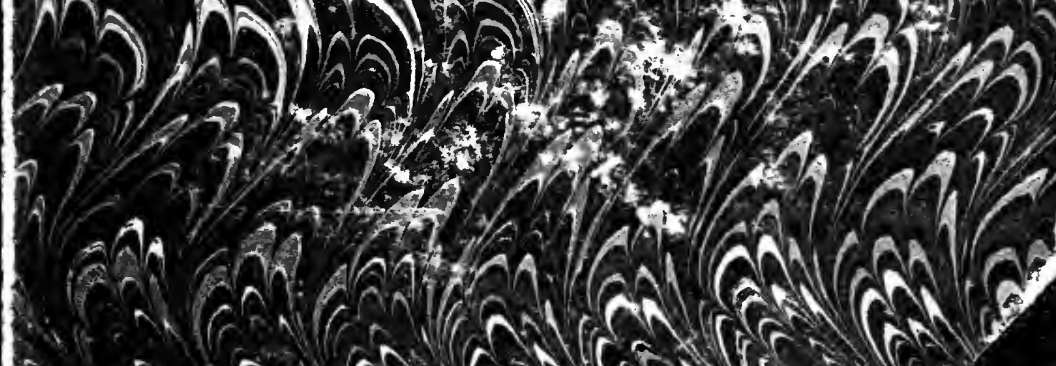

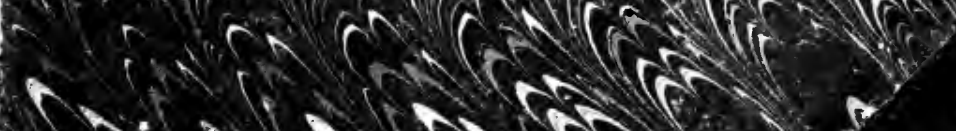




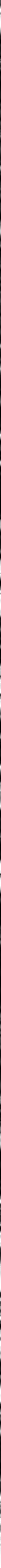


Digitized by the Internet Archive in 2007 with funding from Microsoft Corporation 




\section{THE MODERN APPLICATIONS OF}

\section{E L E C T R I C I T Y}



TLE

$H$

\section{THE MODERN APPLICATIONS}

OF

\section{ELECTRICITY}

BY

E. HOSPITALIER

TRANSLATED AND ENLARGED BY

JULIUS MAIER, Рн.D.

science master, cheltenhaM college

SECOND EDITION, REVISED, WITH MANY ADDITIONS

IN TWO VOLUMES

VOI. I.

ELECTRIC GENERATORS: ELECTRIC LIGHT

WITH NUMEROUS ILLUSTRATIONS

\section{O N O N}

KEGAN PAUL, TRENCH \& CO., 1, PATERNOSTER SQUARE 


$$
\begin{aligned}
& \frac{5774}{301900} \text { bot } \\
& b
\end{aligned}
$$




\section{PREFACE TO THE SECOND EDITION.}

Is the present wholly revised edition, a large number of important alterations and additions have been made, with the view of giving an account of the numerous inventions which have during the last two years been made in practical electricity.

The necessity of a second edition twelve months after the publication of the first clearly shows the general interest taken in the sulject which is treated in this book; and, on the other hand, the necessity of dividing the book into two volumes must be ascribed to the enormous progress electrical appliances have lately made, and are making daily and hourly.

As a practical illustration of this progress, I may mention that this preface has been set up in type by a composing machine which is worked by electricity.

In the first volume a considerable number of electric generators and are lamps have been added to those given originally, and a better account of the incandescent lamps will, I trust, be likewise acceptable. The descriptions and illustrations of these additions are mostly taken from that 
excellent weekly publication, La Lumière Électrique; ${ }^{1}$ for the account of the Edison system I am indebted to Mr. Dredge's book on Electric Illumination.

Chapter IV. of the first volume, containing the applications of electric light, is entirely new, and is also for the most part taken from La Lumière Électrique.

Part II. of the second volume, which treats of the "Various Applications of Electricity," has been added nearly in its entirety; as also the third part, which gives an account of Marcel Deprez's interesting researches and successful experiments on electrical transmission of energy.

I have to thank Messrs. Crompton, Lane Fox, Lever, McEvoy, Swan, the Brush Company, and the Pilsen and Joël Company, for their great kindness in supplying me with information and illustrations of their respective apparatus; and also the editors of the Engineer and the Electrician for the loan of blocks illustrating the Edison machine, Brown and Saunder's' telephone system, and the Ferranti machine. I am also under great obligation to $\mathrm{my}$ friend, Mr. J. E. Julian, of the Inner Temple, for looking over the proofs.

\section{Chaltexham,}

$$
\text { July, } 1883 .
$$

1 In addition to the Figures described in the text as having been taken from La Lumière Electrique, the following are also from the same source:Figs. 6,54 to $62,70,71,73,74,76$ to $80,86,93$ to 96 . 


\section{CONTENTS.}

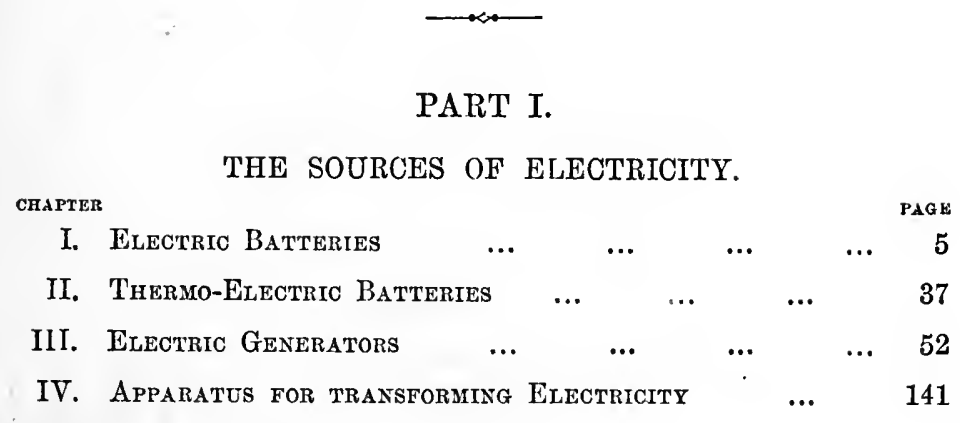

\section{PART II.}

ELECTRIC LIGHTING.
I. ARó LaMrs
$\begin{array}{llllllllll}\cdots & & \cdots & & \ldots & & \ldots & & \ldots & 185 \\ & & & \ldots & & \ldots & & \ldots & & 284\end{array}$

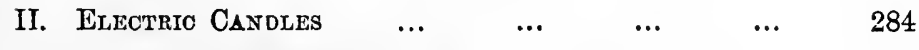

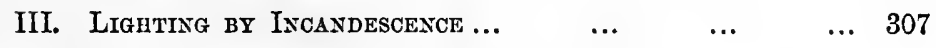
IV. The Applications of lilectrio Lighting $\quad \ldots \quad 345$

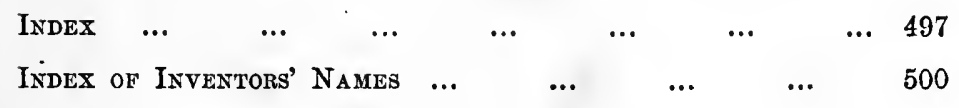





\section{THE MODERN APPLICATIONS}

\section{oF \\ ELECTRICITY.}

\section{PART I.}

\section{THE SOURCES OF EJECTRICITY.}

Tне first electric phenomenon is mentioned by Thales of Miletus, who observed, 600 B.C., that amber, after being rubbed, acquired the property of attracting light bodies; but Otto de Guéricke, towards the middle of the seventeenth century, was the first to observe the electric spark.

The identity of lightning and electricity was not demonstrated until a century later, in 1752 , by the illustrious Franklin; and fifty years after that, Volta inaugurated, by the construction of the electric pile, a new era in the history of electricity, the field of which has been so immensely widened during the last few years.

The whole of the eighteenth century is full of studies and discoveries in statical electricity, and it was reserved for Volta to lay the foundation of the numerous discoveries in dynamical electricity. This distinction has almost disappeared, and we shall see in the following chapters how the so-called statical electricity is changed into dynamical electricity, and vice versâ.

From the point of view of practical application-the only VOI. I. 
part of electricity which interests us in this work-the electrical machines (friction machines, etc.) have given no results, and the high-tension currents which they give have only been used for amusing experiments, for lecture purposes, and for purely scientific research.

We shall, therefore, not consider them, but refer our readers to the treatises on elementary physics, where they will find these phenomena described at length. We are solely concerned with the different modes for producing so-called dynamical electricity, and with its principal applications.

Before proceeding to this, our special task, it will not be out of place to say a few words concerning the modern views on the nature of electricity itself.

Whatever may be the exact nature of electricity, it is now regarded chiefly as a medium for the transmission of force; or, more properly, of kinetic energy.

Some physicists regard electricity as a peculiar vibration of what corresponds to the luminiferous ether (a highly attenuated fluid pervading all matter). It is probable that electrical induction is due to some such vibration. The late Professor Clerk Maxwell has investigated the subject very fully, his enquiries going far to prove that if electrical induction is due to a vibration of the luminiferous ether itself, the vibration is such, that the waves undulate in a plane at right angles to those of light and heat.

An electric current is probably nothing more than the actual transference of this "ether" through a conducting material-that is to say, a material through which it can be made to pass without meeting with any great resistance. The difference between electricity and an electric current may be likened to that between air at rest and air in motion. We 
very commonly talk of there being no air when the air is only very still; but of course we know there is every bit as much air, when it is still, as when it is in violent motion in the form of a gale of wind. In the latter case, however, the effects are very different, the air when in motion being capable of exercising a very considerable pressure upon whatever opposes itself to its motion. So it is with electricity. When at rest, it escapes our observation; when in motion, it produces phenomena which are comparable in magnitude and visibility to those produced by wind. Electricity, indeed, may very well be conceived of as a subtle fluid or gas pervading all matter, and made apparent to us only in its effects, when transferred from one thing to another, and accumulated or set in motion.

This "fluid theory" of an electric current satisfies every known phenomenon, as does also the undulatory theory of electric induction.

Classification of the Sources of Electricity.-Motion of electricity, or the electric current as it is called, can be produced in various ways. All the apparatus used till now for the production of the current can be divided into three large, perfectly distinct classes, characterized by the nature of the action which comes into play.

1. Apparatus in which chemical action is utilized, and which directly transform chemical affinity into electricity. These are galvanic piles, or galvanic batteries.

2. Apparatus which directly transform heat into electricity. These are thermo-electric batteries.

3. Apparatus which directly transform work into electricity. These are electric generators; and they are subdivided into magneto-electric and dynamo-electric machines. 
'THE SOURCES OF ELEC'TRICITY.

We shall deal separately with these three distinct classes of apparatus ; in a special chapter we shall consider those electrical apparatus which are not, properly speaking, generators of electricity, but which impart to the electric current particular properties by changing its relative qualities. 


\section{CHAPTER I.}

ELECTRIC BATTERIES.

WE can form a fairly accurate idea of a battery by comparing it to a source of heat; for instance, the furnace of a boiler. This source produces, by the combustion or chemical combination of coal with the oxygen of the atmosphere, heat, which raises a certain volume of the products of combustion to a certain temperature.

The amount of heat produced by the combustion will partly serve to produce a certain volume of steam at a certain pressure.

The furnace of our boiler is nothing but the battery itself; zinc is the fuel, dilute acid is the agent producing combustion. By this combustion an electric current is generated, having a certain tension or electro-motive force, and a certain intensity; as vapour has a perfectly distinct pressure and volume.

This crude comparison is on the whole fairly accurate; it enables us to understand the often complicated phenomena connected with the battery, and renders, as it were visible, facts whose theoretical explanation often presents great obscurity.

The simplest form that can be given to a battery is that of a battery with a single liquid. An element consists in that case of-

1. A vessel containing the acting materials; 
2. A material to be acted upon (zinc is almost exclusively used), forming the negative pole of the battery: the fuel;

3. A liquid, for instance dilute sulphuric acid: the agent producing combustion ;

4. A plate not attacked by the acid-copper, carbon, or platinum-which forms the positive pole on receiving the electricity from the liquid by conduction.

Fig. 1 represents a simple element thus constituted:

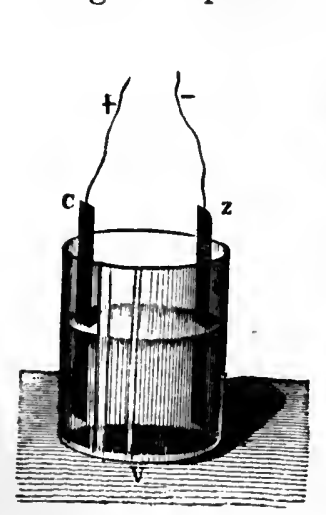

Fig. 1.-Simple element. a glass vessel $\mathrm{V}$; a zinc plate $\mathrm{Z}$, forming the negative pole of the battery; a liquid, water with $\frac{1}{10}$ of its weight of sulphuric acid; and a copper plate $\mathrm{C}$, receiving by conduction the polarity of the liquid in order to form the positive pole. On connecting, by a conducting wire, the two plates $\mathrm{Z}$ and $\mathrm{C}$, an electric current will circulate through this wire.

This electric current can be simply represented as a circulation of continuous electricity, going from the negative to the positive pole in the interior of the battery, and from the positive to the negative pole on the exterior by means of the connecting wires.

Let us for a moment put aside the secondary phenomena produced in the interior of the simple element which we have described, and examine the current circulating in the outside circuit.

Tension, Electro-motive Force, Intensity of the Electric Current, Resistance of the Conductor.-When a zinc plate comes in contact with the acid, a difference of electric 
tension is produced between them, constituting the electromotive force of the element.

The tension of the current corresponds to the pressure which causes water to circulate through a conducting tube. The analogy is complete, for the intensity of the current in the circuit is analogous to the volume of water delivered by the conducting pipe. The electric conductor forming the outside circuit is nothing else but the conducting tube; this conductor offers a resistance to the elcctric current as the tube offers a resistance to the running of the water. There are therefore, with regard to a given electric circulation, three distinct characteristic elements-

1. The difference of potential (sometimes called tension), the cause by which the electric current is established, and of which the electro-motive force is the effect. This latter term is frequently used for difference of potential; this is, of course, not strictly correct, it is a substitution of the effect for the cause.

2. The volume of the current, its intensity, or the quantity of electricity traversing any section of the circuit in unit of time.

3. The resistance of the circuit, or the resistance which the conductor opposes to the circulation of the current, taking into consideration its dimensions and its nature.

Ohm's Law.-The law which connects the resistance of the conductor, the electro-motive force, and the intensity of the current in an electric circuit, has been established mathematically by $\mathrm{Ohm}$, a celebrated German savant, in 1827, and experimentally by the French physicist Pouillet.

This law can be expressed very simply by the following formula :-

$$
\mathbf{I}=\frac{\mathbf{E}}{\mathbf{R}}
$$


where I represents the intensity of the current, $\mathrm{E}$ the electromotive force or difference of potential at the two extremities of the circuit, $\mathrm{R}$ the electric resistance of the circuit.

This formula expresses: The intensity of a current in an electric circuit is in direct proportion to the electro-motive force, and in inverse proportion to the resistance.

We shall have to refer to this law on many occasions in examining the sources of electricity and its applications.

Electrical Units.-We know that a current which traverses an electric circuit of a certain resistance possesses a certain electro-motive force, and a fixed intensity. To have an exact idea of this current, we must measure its elements : difference of potential, intensity, and resistance. The measuring of electric elements is a very delicate and difficult task, and is beyond our province, but we must say a few words about the units which have been fixed upon after continued research, and have now been universally adopted.

In 1863, the British Association appointed a committee, composed of the most eminent electricians of Great Britain, to fix upon a standard for electric measurement. After eight years of work, the committee published a very detailed report, and fixed upon the electrical units, guided by considerations upon which we cannot enter here.

The units adopted by the English Commission were founded on the centimetre, the gramme, and the second. They are all derived from one another by definitions which are sometimes of a very complicated nature, but which present the advantage of clearly establishing these units, and allow of their being fixed anew, in case it should be necessary.

But these units possess a still greater advantage over the empirical units which were formerly in use. They require no other coefficient of reduction but multiples of 
ten. The introduction of these coefficients of reduction arises from the fact that the original units, to which the committee has given the name of absolute units, were rather too small for practice, which necessitated the use of large numbers in calculation; decimal multiples of these units have been substituted for them, and these multiples have been named after men celebrated in electric science.

The unit of resistance adopted by the committee is the $0 \mathrm{hm}$. It corresponds to the resistance of an iron wire of four millimetres diameter and about a hundred metres in length. In France, it was the custom-a custom which is gradually disappearing-to count the electrical resistance in kilometres of telegraph wire. The kilometre of wire is equivalent to about ten ohms. In Germany, the Siemens unit is used, represented by the resistance of a column of purified mercury, one metre long and one millimetre square in section: it is nearly equal to the ohm.

The unit of electro-motive force takes the name of Volt. It corresponds very nearly to the electro-motive force generated by one Daniell element whose exact value in volts is 1.079. The thermo-electric unit of Gaugain is occasionally used, whose electro-motive force is $\frac{1}{18 \frac{1}{2}} \cdot \frac{6}{6}$ a volt, and $\frac{1}{19} \frac{1}{7}$ of the Daniell element.

This is the electro-motive force developed by a thermoelectric couple of copper and bismuth, one of the solderings of which is kept at $0^{\circ}$ and the other at $100^{\circ} \mathrm{C}$.

This unit, now almost obsolete, has the advantage, from a scientific point of view, of being very constant, and of allowing at all times of a practical determination without any difficulty. The use of the volt, however, although not representing a real standard, is preferred on account of the simplicity which it introduces into calculation. 
Unit of intensity. It is defined by Ohm's formula-

$$
\mathbf{I}=\frac{\mathbf{E}}{\overline{\mathbf{R}}}
$$

and takes the name of Ampere. It used formerly to be called Weber, and is the intensity of current which traverses a conductor, whose resistance is one ohm, and whose electromotive force is one volt.

Unit of quantity. It takes the name of Coulomb, and represents the quantity of electricity which traverses, during one second, a conductor of one ohm resistance and a difference of potential of one volt.

This enables us at once to establish the relation between the intensity of a current and the quantity of electricity. A current with an intensity of one ampère yields per second a quantity of electricity equal to one coulomb. A current with an intensity of $\frac{1}{100}$ ampere will yield a quantity of electricity equal to one coulomb in 100 seconds, etc.

The currents employed in telegraphy vary between five and ten milliampères; those which serve for the electric light vary between one and fifty ampères; finally, in certain electro-chemical operations, the intensity of the current reaches 1000 ampères. It is most essential to employ very powerful currents for these operations, because the quantity of metal deposited is proportional to the intensity of the current, that is to say, to the quantity of electricity which passes during unit of time.

Joule's Law.-The law discovered by the celebrated physicist Joule is expressed by the formula-

$$
\mathrm{W}=\mathrm{I}^{2} \mathrm{R} t .
$$

The quantity of heat or of work W developed by an electric circuit is proportional to the square of the intensity 
of the current $I$, to the resistance of the circuit $R$, and to the time $t$.

This formula gives, on replacing $\mathrm{R}$ by its value taken from Ohm's formula-

$$
\text { W = IE. }
$$

The work is proportional to the intensity of the current and to the electro-motive force. We find here again the analogy with the flow of water, where the work is proportional to the volume and to the pressure or height of the fall.

Unit of capacity. It takes the name of Farad, and represents the capacity of a condenser which contains one coulomb of electricity when charged to the potential of one volt. The quantity of electricity contained in a condenser is proportional to the electro-motive force, as in a reservoir of compressed air the weight of the enclosed air is proportional to the pressure, always supposing that the air follows Mariotte's law.

In practice, the farad is too large a quantity, and the unit adopted is the microfarad, which equals 1000000 farad.

We have, therefore, five electrical quantities and five corresponding units: (1) electro-motive force, expressed in volts; (2) resistance of the conductors, in ohms; (3) intensity of the current, in ampères; (4) quantity of electricity, in coulombs ; (5) capacity of a condenser, in microfarads.

We can now enter upon an account of the electric batteries, confining ourselves to the newest systems which are most extensively used.

Classification of Batteries.-The batteries can be divided into several perfectly distinct groups, although there exists no absolutely rational and methodical classification- 
1. From the nature of the soluble electrode; in practice, however, this character has no importance, for in almost all batteries zinc is used as the soluble electrode.

2. From the nature of the acting agent, which is sometimes an acid, generally sulphuric acid; acetic acid in Pulvermacher's battery; sometimes a salt like common salt, sal ammoniac, mercuric sulphate, lead sulphate, etc.

3. From the nature of the depolarizing agent, which can be copper sulphate, nitric acid, peroxide of lead, peroxide of manganese; in some cases the depolarizing and the acting agent form one single solution in the same liquid, as for instance in. Poggendorf's battery, where zinc and carbon are immersed in a solution of potassic bichromate and sulphuric acid.

We shall therefore examine these batteries in groups of like nature, following the excellent classification of $\mathbf{M}$. Alfred Niaudet, in his Traité Élémentaire de la Pile Électrique.

\section{Single-Liquid BatTeries.}

Since Volta's piles, a great number of single-liquid batteries have been constructed: the crown of cups or couronne de tasses, Cruikshank's battery, Wollaston's battery, the spiral battery, Muncke's battery, are only modifications of the fertile principle discovered by the Italian physicist.

These apparatus are very little used now in practice, on account of the rapid polarization of the elements. They. are described in almost all treatises on physics.

In certain single-liquid batteries copper has been replaced by electrodes of other substances. 
In 18t9, Mr. Walker used carbon electrodes; others used iron as the unattacked electrode.

In 1840, Smee recommended platinized platinum to facilitate the generation of hydrogen bubbles. Walker replaced it, in 1857 , by platinized carbon; in this form the single-liquid battery is still very much used for the railway service.

The negative electrode, or positive pole, in the singleliquid batteries is nowadays coated with substances which facilitate the generation of hydrogen or its combination with oxygen to form water.

In other single-liquid batteries the nature of the liquid has been varied. Hydrochloric acid has been used, but without success.

Nitric acid has also been used, but this is too expensive, and, like hydrochloric acid, gives off noxious vapours. Pulvermacher has used acetic acid to excite his galvanic chains.

Independently of acid liquids, common salt has been used ; zinc-carbon batteries and common salt are extensively used in Switzerland for electric bells.

In his electric buoy Duchemin uses sea-water; in Bagration's battery sal ammoniac is used, and in Stöhrer's battery a solution of alum.

Whatever may be the chosen liquid and electrodeand the number of these combinations is, so to speak unlimited-it is very doubtful whether we shall ever again return to them, having at our command batteries with depolarizing mixtures, some of which we are going to describe.

Polarization of Batteries, Batteries with Two Liquids. - Let us take again our simple element, Fig. 1, which consists of 
a zinc plate, a copper plate, and water with sulphuric acid. If we close the current of this battery on a galvanometer, we produce a deflection of the needle which will gradually decrease till it becomes almost imperceptible under certain conditions. This decrease of intensity of the current, whatever may be the causes which produce it, is called polarization.

The polarization of the battery depends upon the following principal causes:-The impurity of the zinc, by creating small secondary couples in the midst of the zinc itself, is a cause which tends to diminish the electromotive force of the couple. Mr. Kemp proposed in 1839 the use of amalgamated zinc to counteract this cause; and at the same time Mir. Grove read a paper before the Academy of Sciences to explain the importance of amalgamated zinc in batteries. Nowadays the zinc of all batteries is amalgamated. The weakening of the liquid by chemical action tends also to weaken the current, partly by the weakening of the chemical action, partly by the increase of the resistance which the liquid opposes to the passage of the current.

The deposition of zinc on the copper by the current also tends to diminish its intensity, because the copper, coated with zinc, and acted on by the acid of the element, produces a new current in an inverse sense to the original one.

But the most powerful cause of polarization is the deposition of hydrogen on the copper.

The oxidation of the zinc and its transformation into zinc sulphate produce a certain quantity of hydrogen, which is deposited on the electro-negative plate (copper, platinum, or carbon). This deposition of gas on the copper plate opposes a material obstacle to the transmission of the 
current by the bubbles of gas. On the other hand, this hydrogen forms, with the oxygen given off at the other electrode, a sort of gas battery, and in consequence an electric flow in an inverse sense to the former. A theoretically perfect battery, in which we could do away with all these causes of polarization, would yield a constant current, and would really deserve the name of constant battery.

In practice this name is given to all those voltaic combinations which have for their aim the prevention or diminution of the deposition of hydrogen on the electronegative plate. We shall see, on reviewing the batteries actually in use, the different ways in which this desideratum is more or less realized.

Theoretical Conditions of a Perfect Battery.-The following are, according to Mr. Fleming Jenkin, the essential conditions which such a battery must fulfil :-

1. It must possess a great electro-motive force.

2. It must have a feeble and constant interior resistance.

3. Its electro-motive force must be constant, whatever the intensity of the current produced may be.

4. The materials used must be inexpensive.

5. The battery must not consume anything when it produces no current, that is, when the circuit is not closed.

6. It must be so arranged that we can easily verify its condition and working, and add fresh materials when required.

No known battery realizes all these conditions in the highest degree. The special exigencies in each case must determine the choice of a suitable element for the intended purpose.

Two-Liquid Battery with Copper Sulphate.-The use of copper sulphate or nitrate as a depolarizing agent was first 
recommended by Becquerel in 1829 after long and numerous experiments. The results obtained were very satisfactory, and he has always used these solutions.

It was not until 1836 that Daniell invented the battery which is called after him. Becquerel's and Daniell's batteries only differed in the nature of the porous cell, which was of gold-beater's skin in Daniell's, and of kaolin in Becquerel's, for which have been substituted porous cells of pipe-clay.

The arrangement of the Daniell element is well known; we need not refer to it again. Its electro-motive force is, as we have seen, 1.079 volt, when the sulphuric acid is diluted with four times its weight of water; and its interior resistance varies, according to the size of the elements, their condition, etc., between one and twenty ohms. Some medical batteries, however, which, for the convenience of transport, must be of small dimension, have an interior resistance of 100 or $200 \mathrm{ohms}$.

The Daniell battery is constructed in many different styles; we know it in the form of flasks, as batteries with copper outside, and as trough batteries. Daniell's trough battery, constructed by Mr. Muirhead, is very much used, and the Post Office authorities have more than twenty thousand in use for telegraph service.

M. Carré has constructed a Daniell battery in which the porous cell was replaced by a cell of porous parchment paper, which considerably diminished the interior resistance of the battery, sixty elements of which were sufficient for the electric light. This is the first instance of the Daniell battery being employed for such a purpose, where a current of a certain intensity is required.

M. Minotto of Venice has invented a sand battery, 
which is very much used in Italy and in India. The separation of the two liquids arises from their different densities; zinc and copper are formed into two flat horizontal dises, the copper underneath. The copper sulphate is separated from the sand by a sheet of blotting paper or a piece of cloth. This battery offers a great interior resistance, but renders excellent service in telegraphy.

Trouvé's Moist Battery.-This is a Daniell battery, possessing the great advantage of acting without a liquid; the liquid used for the moistening of the elements can be poured out, or runs out of its own accord.

The elements of this battery are constructed in the following manner:-A round zinc disc $Z$ (Fig. 2), and a copper disc C, are placed parallel to one another, and are separated by a pile of paper discs of a somewhat smaller diameter.

This mass of paper can absorb much water, and remains moist for a very long time, especially under conditions which we shall mention below.

The lower half of the paper dises is soaked in a solution of copper sulphate, the other half in a solution of zinc sulphate.

It will be seen that we have here all the constituents of an ordinary Daniell battery, in which the two liquids remain much more effectually separated than in porous cells.

The copper disc is supported in the centre by a rod insulated from the paper and zine dises; it reaches beyond the slate cover fixed at the top of the glass or ebonite vessel in which the element is placed to shelter it from currents of air, and from dust. The border of this vessel is rough, and the slate well adjusted; the element is thus con-

voL. $\mathrm{I}$. 
tained in a hermetically closed space, and consequently preserved from evaporation.

Thus constructed the element acts for more than twelve months without requiring any looking after.

After a longer or shorter time, according to the amount of work done by the battery, the copper sulphate is reduced, and the battery ceases to act; it must then be recharged, which is an easy operation, only requiring a little care. It consists in dipping the lower part of

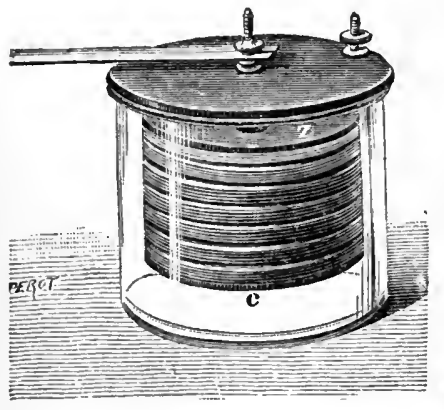

Fig. 2.-Trouvé's moist battery.

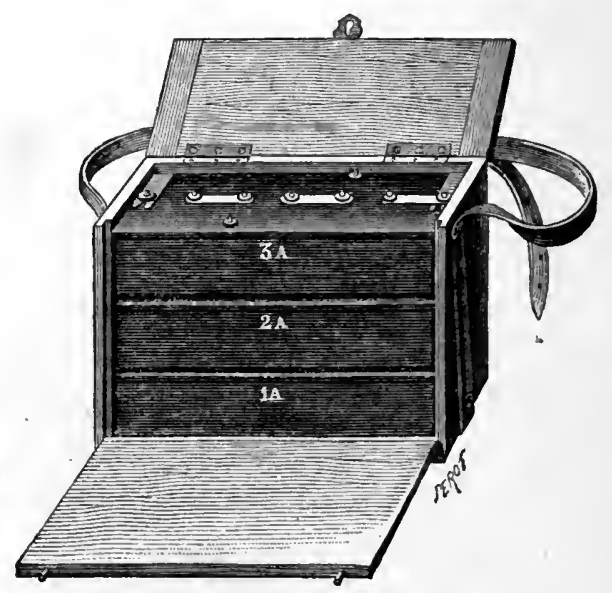

Fig. 3.-Trouvés battery for military telegraphy.

the element into a hot solution saturated with copper sulphate. This solution is prepared in a copper basin made for the purpose; it rises up to a certain level, and the cover of the element fits on to the edge of the basin, so that the paper is impregnated up to the desired height. As regards the zinc sulphate, it is constantly formed by the action of the battery, and therefore never needs to be replaced. The zinc itself is dissolved, and must be replaced after a certain interval; the paper is renewed at the same 
time. The copper on the contrary, when freed from the copper deposited on it in form of a powder by the action of the current, serves for an indefinite time, like the other parts of the battery.

The principal advantage of this new arrangement, invented by $M$. Trouvé, is the almost complete suppression of the interior work of the battery when the circuit is open. We may say of a Daniell battery which gives no current that it is a horse in the stable-which means, it consumes without producing; this is the only, but very grave objection tu an ordinary Daniell battery.

This inconvenience does not exist, so to say, in Trouvé's moist battery, because there the liquids cannot easily mix, owing to their different densities.

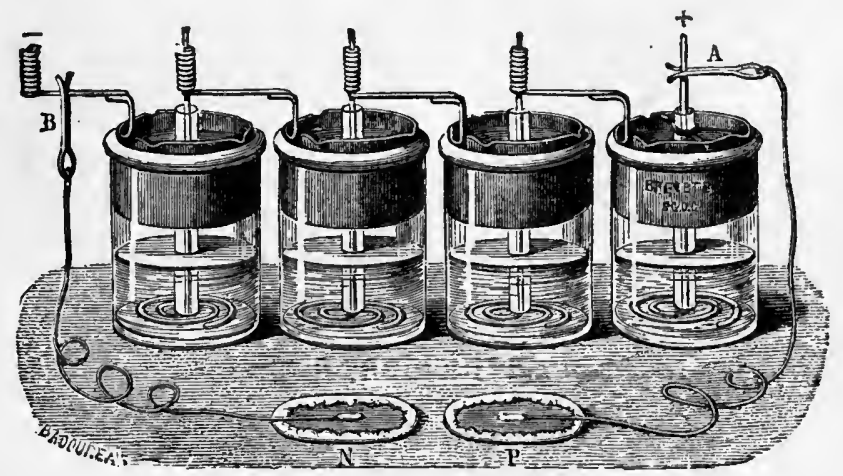

Fig. 4.-Callaud's battery, after Trouvé's model.

Callaud's Battery.-The method for the separation of the two liquids of the Daniell battery, by means of their different densities, was found simultaneously by Callaud in France, and Meidinger in Germany.

The Callaud battery has received several modifications in practice. We will describe here one of these modifications now very much in use. 
Callaud's Battery, after Trouvé's Model.-This battery is very simple and inexpensive. The glass vessel is only twelve centimetres high, and seven centimetres wide. The zinc rests on the border of the vessel by means of three spouts, or folds, made with a pair of pincers (Fig. 4). A flat spiral of copper wire ends in a vertical piece rising in the centre of the vessel, and protected by a small glass tube. The connection of the elements is effected by means of a small coil which terminates the copper wire soldered to the zinc, and into which fits the copper wire, forming the electrode-pole of the following element.

Four Callaud-Trouvé elements, arranged in a little box, are sufficient to charge and keep charged the secondary
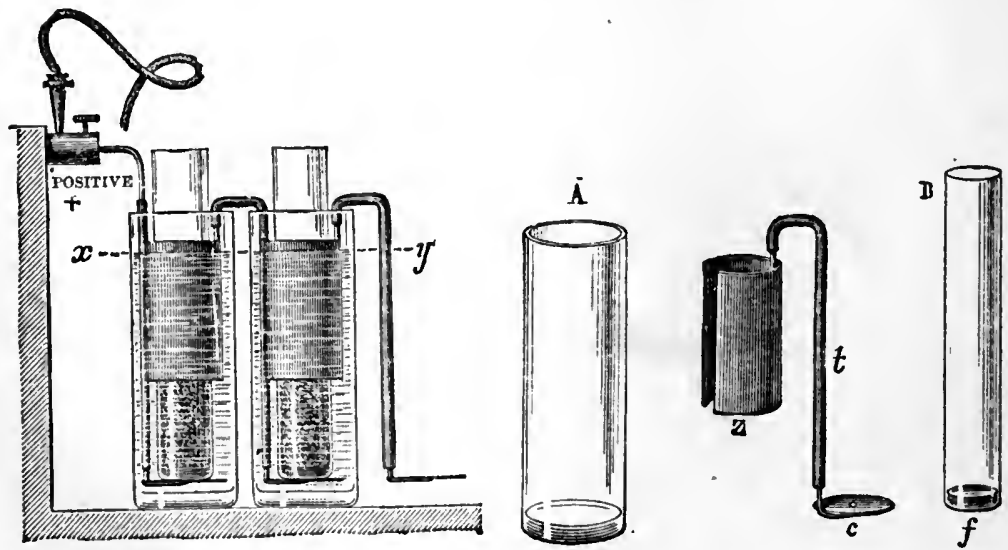

Fig. 5.-Dr. Onimus' battery : details of an element.

batteries of M. Gaston Planté, of which we shall speak in Chapter IV. .

Onimus' Battery.-Dr. Onimus' battery is another Daniell battery more especially arranged for medical application of continuous currents. It consists of an exterior glass vessel $\mathbf{A}$, enclosing a small zinc cylinder $\mathbf{Z}$ 
(Fig. 5), and a copper rod $t$ terminating in a copper plate $c$. A glass tube B, open at both ends, is placed in the centre of the vessel, and closed at its lower extremity $f$ by gunwadding, calibre 24 , which can easily be replaced if necessary. The tube B contains crystals of copper sulphate. The wadding acts the part of a porous cell. The battery is charged by pouring water into the vessel and the tube up to the height $x y$. A current is established after a few hours, and the battery works regularly for several months, and only requires a few drops of water and some sulphate crystals to be added at rare intervals.

The advantages of this battery for medical purposes are

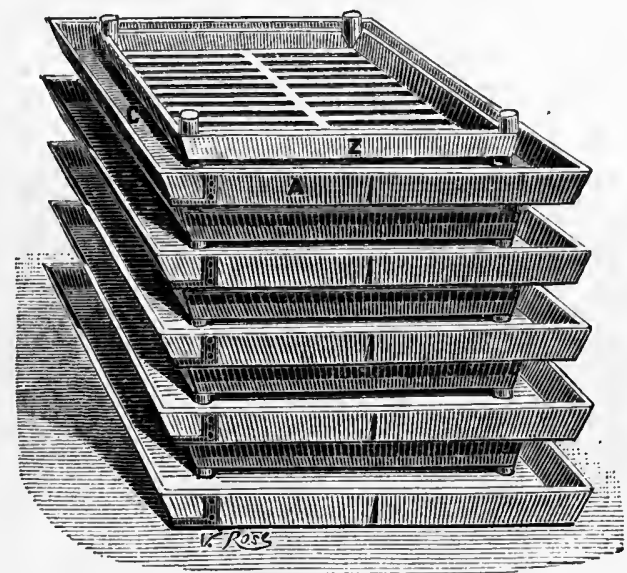

Fig. 6._Sir William_Thomson's battery.

its great constancy and feeble chemical action, for the solution of copper sulphate penetrates but little through the wadding. No mixing of the liquids takes place during transport, and it suffices to remove the small tubes containing the crystals of copper sulphate, in order to arrest the action of the battery, for then the liquid sinks to a lower level, and 
the zincs are out of contact. On replacing the tubes in the vessels the battery acts as before.

Sir William Thomson's Battery.-This battery, shown in Fig. 6, has a very large surface, and is composed of wooden troughs A lined inside with lead. The copper plate $\mathrm{C}$ is at the bottom; the zinc, in the form of a grating $\mathrm{Z}$, rests on wooden brackets; the zinc and copper plates are thus close together. By placing seven or eight of these elements one above the other a powerful battery is obtained, applied by Sir William Thomson to the siphon recorder.

\section{Sulphate Batteries.}

Batteries have been constructed in which the copper sulphate and the copper plate are replaced by the plate of another metal and the corresponding sulphate; but these

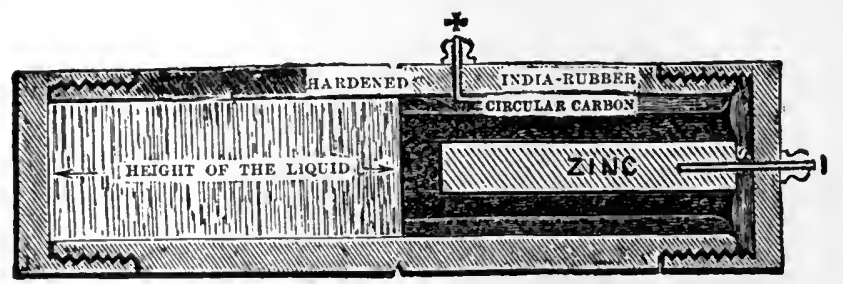

Fig. 7.-Trouvé's hermetical battery.

apparatus have on the whole no practical interest. The most important is Marié-Davy's mercuric sulphate battery. The copper sulphate is replaced by mercuric sulphate, and the copper plate by a piece of carbon. The chemical action is analogous to that of the Daniell, except that the mercuric sulphate is nearly insoluble. Mercuric sulphate batteries have also been used without porous cells, and in Trouve's reversible battery it is the solution of the mercury which forms at the same time the depolarizing and the acting agent. 
Trouvé's Hermetical Mercuric Bisulphate Battery.-The battery, represented in natural size (Fig. 7), consists of a zinc-carbon couple, placed in a hermetically closed ebonite case. The zinc and the carbon only occupy the upper half of the case; the other half is occupied by the exciting fluid, which is a solution of mercurous sulphate.

As long as the case is kept vertical, with the zinc at the top, the couple will not plunge into the liquid, and no electricity is generated. On reversing the case the zinc and the carbon are immersed; a current is produced immediately, which ceases at once when the case is replaced in its former position. Hence the name of reversible battery. Trouvé has applied it to several induction apparatus, to his electric explorer, and to his little electric trinkets. (See Tissandier, Récréations Scientifiques, Bibliothèque de La Nature.)

Messrs. Gaiffe and Ruhmkorff also use mercuric sulphate batteries with a single liquid to incite the induction coils of their small medical pocket apparatus.

Lead Sulphate Batteries.-Lead sulphate was used for the first time by Becquerel, then in 1860 by Marie-Davy, who gave to the element the form of a Daniell battery without Sir William Thomson's porous cell. Becquerel restored afterwards the ordinary Daniell form.

These batteries are no longer used now on account of the enormous interior resistance of the elements.

\section{ACID Batteries.}

The first battery with nitric acid as depolarizer dates from 1839, and was invented by the eminent physicist Grove. In Grove's battery the negative electrode, or positive pole, consists of platinum foil immersed in a 
porous cell, which contains concentrated nitric acid. Grove had proposed to replace the platinum by wood charcoal or graphitoidal charcoal deposited in gas retorts; but he did not follow up this idea, which was taken up again in 1843 by Bunsen, of Heidelberg.

All the Bunsen batteries constructed till 1849 were made with hollow carbon cylinders, placed with the acid in the exterior cylinder, and the zinc placed in the interior of the porous cell.

In 1849, Archereau first used prismatic carbon, which he placed in the porous cell, while he placed rolled zinc in the outside vessel. The battery which is universally known under the name of Bunsen battery, is therefore really the invention of Archereau.

The Bunsen battery has since undergone a number of modifications, which however have not succeeded in supplanting Archereau's original invention. Ruhmkorff however has constructed a Bunsen battery with flat elements, possessing two advantages-a small interior resistance, owing to the close neighbourhood and the large surface of the electrodes, and a small volume of nitric acid on account of the narrowness of the porous cell, where the carbon, in form of a thick prismatic piece, occupies about one-third of the space.

Tommasi has also modified Bunsen's battery with the idea of doing away with the nitrous vapours, and thus rendering the apparatus fit for domestic lighting. We are still waiting for the fulfilment of these handsome promises.

Chloric acid has also been tried as a depolarizer, as well as chromic and hydrochloric acids and aqua regia, etc.; but these batteries have not answered in practice, and have not reached beyond the state of laboratory experiments. 


\section{Oxide Batteries.}

The depolarization in a battery being effected by the oxidation of hydrogen, this result can be obtained by using oxides which are easily decomposed.

De la Rive employed peroxide of lead and peroxide of manganese more than thirty years ago, but his batteries were never used in practice, and had been forgotten, when Leclanché invented the battery which bears his name, and is now extensively used.

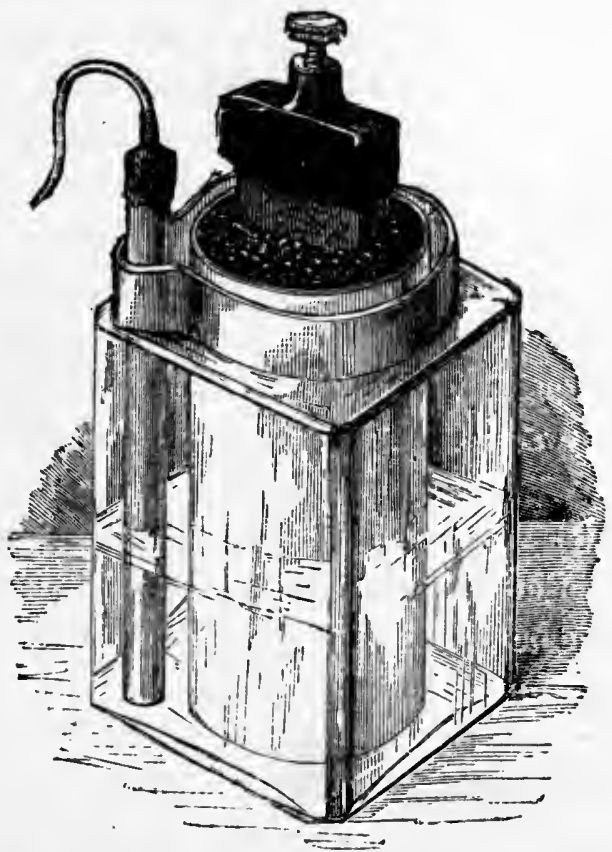

Fig. 8.-Leclanchê's manganese dioxide battery.

Leclanché's Manganese Dioxide Battery.-An element of this battery is represented in Fig. 8. The exterior glass vessel is square: this form has the advantage over the round form of saving space when enclosed in a box; in 
other words, a less cumbrous battery is the result. This vessel is provided with a neck of nearly the same diameter as the porous cell; so that the vessel is almost closed, and the possible evaporation of the liquid considerably diminished. This neck has a spout for the introduction or removal of the zinc, and is also convenient for the emptying of the liquid contained in the vessel.

The soluble electrode consists of a simple cylindrical rod of zinc, one centimetre in diameter; in the upper part of this zinc rod is a small central hole, into which is soldered a galvanized iron wire. This electrode-pole is at the same time flexible and solid; it can be formed into a spiral cylindrical coil, and is thereby rendered elastic, which is often desirable.

The porous cell has, as we have said, nearly the same diameter as the neck of the exterior vessel; it contains about equal quantities of manganese dioxide and gas carbon. In the centre of this mass is a carbon plate, with a piece of lead at the top; a brass button is screwed into the lead, and is connected with the negative electrode-pole of the following element.

The outer vessel is half filled with a solution of ammonium chloride in water. The liquid penetrates after some time into the porous cell and the substances it contains.

The mixture of manganese dioxide and carbon is covered over with wax or melted resin to protect it in transport. A hole is made in this coating, through which the air can escape, when the water penetrates into the porous cell.

There is no chemical action in Leclanche's battery till the circuit is closed. Its electro-motive force varies between 1.4 and 1.5 volt. In the model of fourteen centimetres height the resistance varies between five and six ohms. 
It contains no poisonous substances; gives off no acid vapours, no appreciable smell; the substances used are cheap, and resist the most intense cold. This battery is very much used in telegraphy, for electric bells, and is now in general domestic use. The depolarization obtained by the manganese dioxide is not complete, and with a short circuit the electro-motive force decreases rapidly. But it depolarizes very rapidly with the open circuit. In the case of intermittent service, as for instance electric bells and telegraphs, the polarization is imperceptible.

Leclanché has constructed a new battery, in which the porous cell is replaced by a mixture of forty parts of manganese dioxide, fifty-five of carbon, and five of gumlac exposed to a pressure of three hundred atmospheres at $100^{\circ} \mathrm{C}$. The contact of the zinc rod and the cylinder is prevented by placing two very prominent indiarubber rings on the zinc rod.

For electric bells, Leclanche's batteries can be used four or five years without any other trouble than to add from time to time a little water in the outer vessel, to replace the water lost by evaporation.

Clamond and Gaiffe's Sesquioxide of Iron and Sal Ammoniac Battery.-This battery differs from Leclanchés only by the substitution of sesquioxide of iron for manganese dioxide. When the circuit is closed, the ammonium chloride attacks the zinc, and forms with it a double chloride of ammonium and zinc; the liberated ammonium acts upon the sesquioxide of iron, decomposes it, takes up part of its oxygen, and is given off as free ammonia.

This element, represented in Fig. 9, consists of a glass vessel, a porous carbon prism $C$, containing sesquioxide of iron in its pores, and a ring of amalgamated zinc Z. A 
cork, made of mastic, closes the vessel, and prevents the evaporation of the ammonium chloride solution.

The electro-motive force of the couple is 1.2 volt; it has the advantage of not being used up when the circuit is

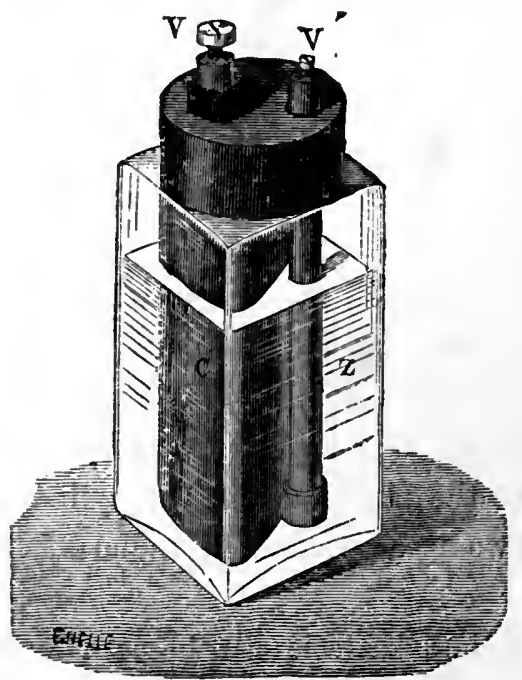

Fig. 9.-Clamond and Gaiffe's battery. open. The depolarizing body can, during the time of rest, take up the oxygen which it has lost during the time of action. Its action is unlimited, on condition that it is not overworked, and that the zinc and ammonium chloridearereplaced from time to time. It can be used for medical batteries with a continuous current, for electric bells, and for house telegraphy, but from its enormous interior resistance it is but a feeble source of electricity. With larger dimensions it can be used for railway telegraphs and medical induction apparatus.

Chloride Batteries.

In these batteries the depolarization is effected by chlorine instead of oxygen.

We only mention Daniell's platinum chloride battery, which, of great value from a theoretical point of view, would be too expensive in practice.

Warren de la Rue's Silver Chloride Battery.-Marié-Davy was the first to use silver chloride as a depolarizing agent 
in 1860 ; but Warren de la Rue has used it in his battery since 1868, and the improvements effected by him are the result of his investigations on high-tension currents.

Fig. 10 represents the whole of a group of ten elements, mounted ready for action, and Fig. 11 the different parts constituting each element.

The outer vessel is a cylinder, thirteen centimetres long and three centimetres in diameter; the soluble electrode is a

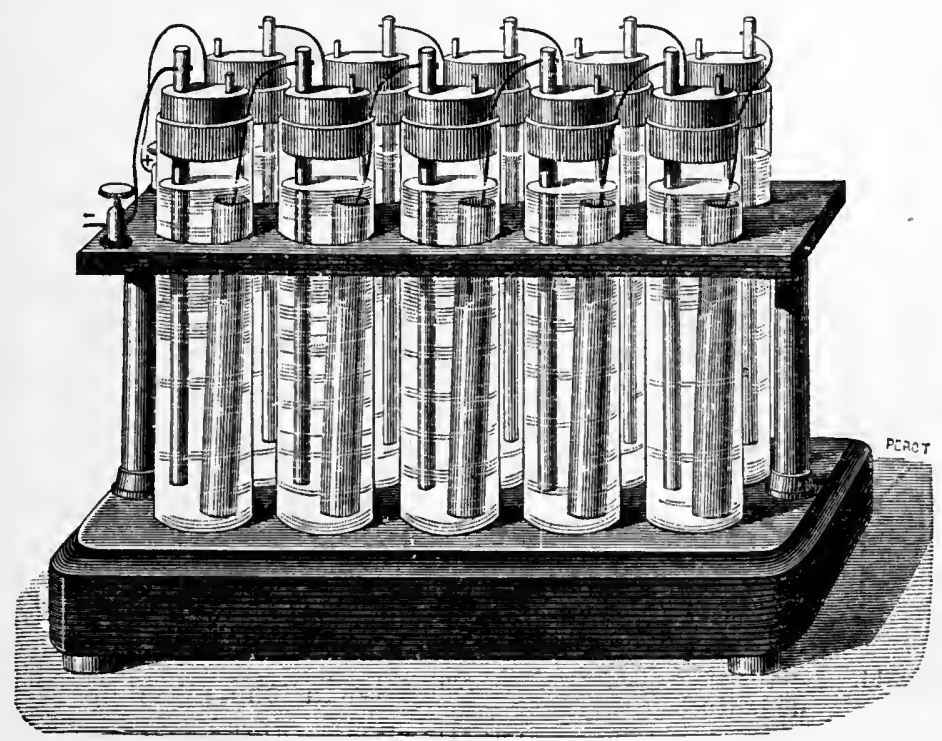

Fig. 10.-Warren de la Rue's battery.

zinc rod not amalgamated (a rare exception from the general use of amalgamated zinc). Warren de la Rue employs, in preference, zinc from Vieille-Montagne, on account of its superior quality. A small silver strip, which forms the positive pole of the following element, fits into a hole made in the upper part of the zinc rod; the contact is assured by a small brass pin $\mathrm{C}$, of slightly conical shape. 
The second electrode is formed by a silver ribbon surrounded by a cylinder of silver chloride. This silver chloride-stick is placed in a little cylinder of parchment paper A, to prevent accidental contacts.

The liquid is a solution of twenty-three grammes of ammonium chloride in one litre of water. The outer vessel is closed by a paraffin cork, one of the best insulators known, and which, in virtue of its anti-hygrometric properties,

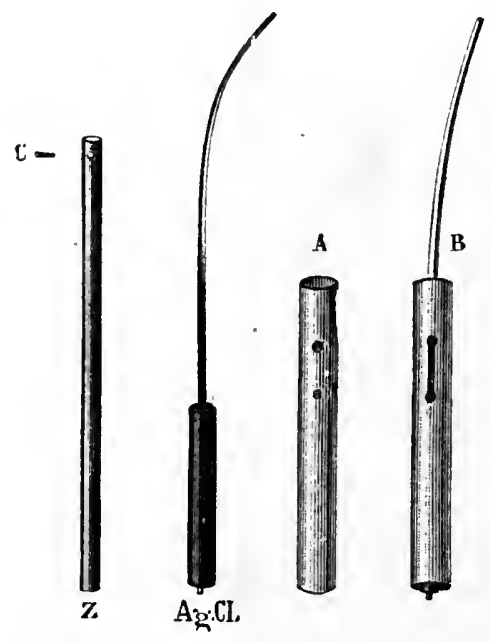

Fig. 11.-Details of un element of Warren de la Rue's battery. prevents the water which might be spilled on its surface, from spreading, and thus causing a deviation of the current.

The action is very simple: The zinc dissolves, and replaces the silver in the chloride; the silver resulting from the decomposition is deposited in the form of a porous substance, first on the surface, and then gradually in the mass of the silver chloride. This battery gives rise to no local action, as long as the exterior circuit is not closed. This is very important in experiments of short duration, repeated at long intervals. Although this is a single-liquid battery, it must be remarked that the acting agent, chloride of silver, is a solid body, which is not dissolved in the liquid in contact with it, but supplies the chlorine necessary for the solution of the zinc.

The electro-motive force varies between 1 and 1.07 volt; 
its interior resistance depends upon the time of action and the dimensions of the element. In the model which we have just described, the resistance varies from $2 \cdot 7$ to $4 \cdot 3$ ohms. Warren de la Rue has 11,000 elements of this battery, which enable him to make the most interesting experiments on the electric spark, the description of which, however, does not come within our scope.

Gaiffe also uses the silver chloride battery for continuous currents and for medical induction apparatus. His elements are of very small dimensions, and are kept in hermetically closed ebonite boxes, with covers to screw on.

Duchemin proposed, in 1866, the use of iron perchloride as depolarizer, but there is only an incomplete depolarization effected in the element, and the zinc is soon covered with non-conducting deposits.

Marié-Davy has tried lead chloride, but the electromotive force of this element is feeble, and lead chloride is rather expensive; the battery has, therefore, no special advantage.

Niaudet's Battery with Bleaching Powder.-In Niaudet's battery, represented in Fig. 12, the zinc is immersed in a solution of sodium chloride, and the carbon in a solution of bleaching powder, which acts here the part of depolarizer. The bleaching powder of commerce, used as bleaching agent and disinfectant, is a mixture of lime, calcium hypochlorite, and calcium chloride. The hypochlorous acid which it contains is composed of oxygen and chlorine, which can both unite with hydrogen to form water and hydrochloric acid. This latter acts upon the lime, and produces calcium chloride. The substances formed being all soluble, the liquid remains clear. When the circuit is open, the zinc is not acted on; there is, therefore, no waste. 
The electro-motive force of this element is 1.6 volt. The bleaching powder possessing a very disagreeable smell, the vessel containing the battery must be closed by means of a cork coated with wax. The zinc, with its bent endpiece, is kept at a regular, very short distance from the porous cell by means of small pieces of wood, squeezed

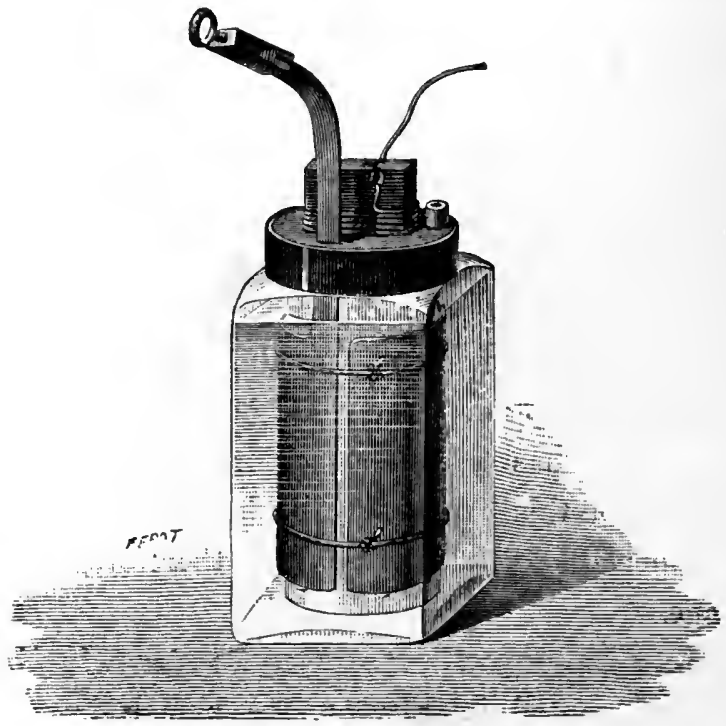

Fig. 12.-Niaudet's bleaching powder battery.

between the zinc and the cell by two springs. By this arrangement the zinc does not descend to the bottom of the liquid, and the formation of local couples, which would result in mere waste, is avoided.

\section{Batteries with Depolarizing Mixtures.}

We have examined in the preceding pages batteries in which the electro-negative electrode (copper, platinum, carbon, or silver) is surrounded by substances easily giving off oxygen or chlorine, which combine with the generated 
hydrogen, and produce partial or total depolarization. Another method consists in surrounding this electrode by two substances which, by their reaction upon one another, produce either oxygen or chlorine.

The batteries of this kind which are most extensively used are potassic bichromate batteries.

Poggendorff was the first to use a mixture of potassic bichromate and sulphuric acid for depolarizing the conducting electrode.

Since then the bichromate battery has been improved in various ways, and has become very convenient and very practical.

The electro-motive force of the elements reaches two volts; it is greater than that of the Bunsen and Grove elements, but, in spite of the mixture, a rapid polarization is produced, especially when the external circuit has little resistance. Complicated reactions ensue in these elements, tending to the formation of chrome alum, which, by its formation, produces a current in the opposite direction of the element; the result is an energetic polarization and a rapid exhaustion of the liquid.

Grenet's Battery.-In 185̆6, Grenet had established a system of ventilation in the interior of the liquid which prevented the deposition of chrome alum, and destroyed, by the action of the air on the nascent hydrogen, the polarization which might have ensued.

In practice nothing could be expected of so complicated a system, and the battery which now bears the name of Grenet is nothing else but the well-known flask or bottle battery, which renders such good service for experiments of short duration.

Potassic Bichromate Battery, with Windlass-Stand.-This VOL. I. 
battery gives a powerful current, without smell, provided it is only used for a short time, and that the zincs are taken out of the liquid after each experiment.
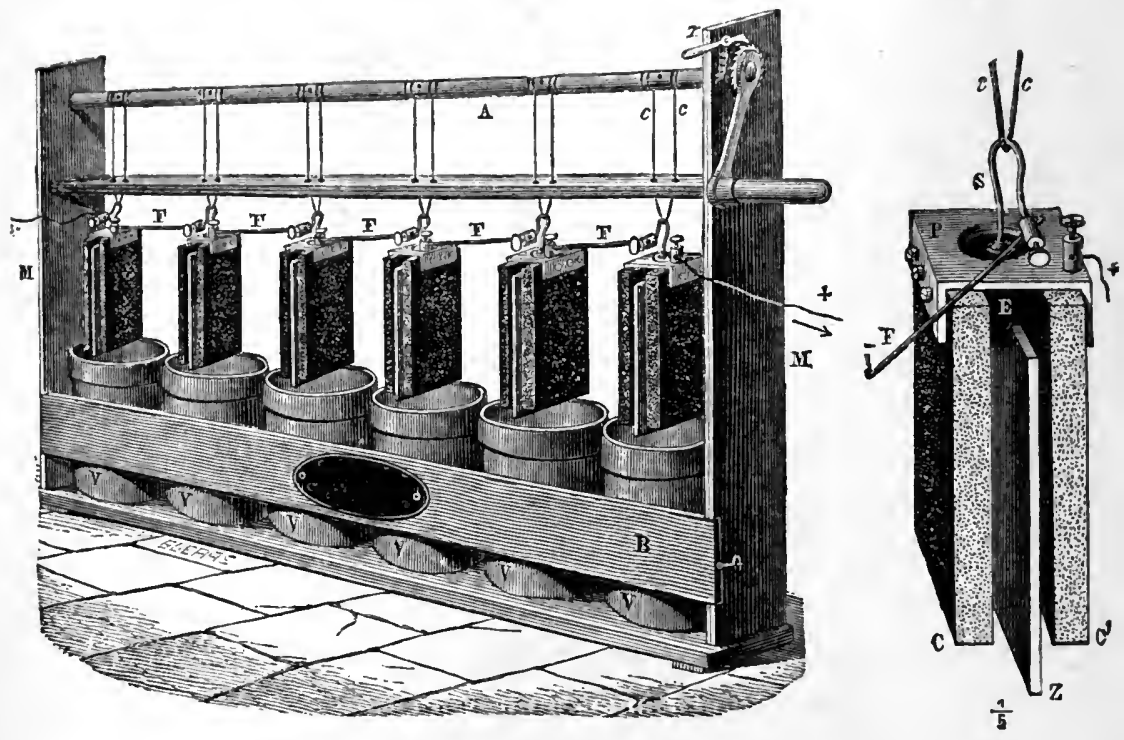

Fig. 13. - Bichromate battery, with windlass-stand.

Ducretet has constructed a model composed of bichromate elements, suspended by cords, wound up by a ratchetwheel A $r$ (Fig. 13). Each element consists of a large amalgamated zinc plate $\mathrm{Z}$, placed between two carbons $\mathrm{C} \mathrm{C}^{\prime}$, and insulated by a block of ebonite $\mathrm{E}$; a brass hook $\mathrm{S}$ fixes this plate, and serves at the same time as conductor, and as hook for the cord of the stand. The carbons are screwed on a metallic square $P$, a metal post with binding-screw being fixed on this square for the conducting wire. The best solution consists of -

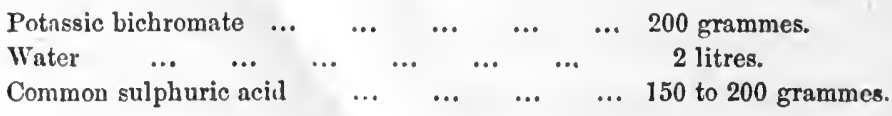


It is advisable to add five grammes of mercuric bisulphate to keep the zincs well amalgamated.

Gaiffe has constructed a similar battery, with stand, in which the carbons and zincs are permanently fixed to a cross-bar, which prevents the elements from swaying to and fro when they are raised, and also the acid liquid from being thrown out.

Trouvé has constructed another model, in which the elements can easily be taken to pieces and cleaned, the zincs amalgamated anew, and replaced if necessary.

Camacho arranges the elements one above the other, and causes the depolarizing mixture to circulate; this produces a certain motion favourable to depolarization. The carbon electrode is placed in a porous vessel filled with fragments of gas carbon; an enormous surface is thereby given to the electrode, which renders polarization very slow.

Cloris Baudet has also constructed a battery to which he gave the somewhat pretentious name of "Impolarisable" (not capable of polarization), and in which the porous vessel, containing the zinc, has two small vessels soldered to it sideways: one of these small vessels contains sulphuric acid, the other, which is pierced with holes, contains potassic bichromate. Thus a stock of materials is provided which fairly maintains the solution in a state of sufficient concentration, but this does not explain how polarization is prevented.

Fuller has invented a bichromate battery, used in America and in England for telegraphy, where the zinc, placed in the centre of the porous vessel, plunges into mercury, which preserves its amalgamation.

Use of Batteries in Electric Appliances. Available Maximum Work.-In all the batteries which we have described, 
we invariably find zinc as the fuel. The available work in the external circuit of a battery depends therefore on the number of calories produced by the combination of zinc with the acting agent. Looking at it from this point of view, the battery which renders valuable service in telegraphy, for electric bells, medical apparatus, laboratory experiments, etc., is absolutely unable to supply large quantities of electricity. Zinc is an expensive fuel; an equal weight of zinc costs fifteen times as much as coal, and generates five times less heat. This fact alone sufficiently explains why batteries are seldom, if ever, used for electric lighting, and why we prefer the use of machines for this purpose, as well as for galvano-plastic and many other appliances, in spite of their being more complicated.

We reserve the battery for work where electricity is required, more for the rapidity and delicacy of its action, than for its efficiency; its application to telegraphy and telephony are instances of this. For electric lighting and for electro-motors, where calories and work are required, the batteries labour under the disadvantage of burning a too expensive fuel-zinc, and also of burning it too slowly; which necessitates the use of a considerable number of elements, without yielding the corresponding available maximum work.

Unless a complete revolution in the production of electricity by direct chemical action takes place, the principles with which we are acquainted now do not permit of the construction of a battery, at the same time powerful and economical, for applications which require these two qualities united to a very high degree. 


\section{CHAPTER II.}

\section{THERMO-ELECTRIC BATTERIFS.}

TherMo-ELECTRIC batteries are apparatus which transform heat into electricity. The discovery of this highly important fact was made in 1821, by Professor Seebeck, of Berlin. On soldering to a plate of bismuth the ends of a copper plate,

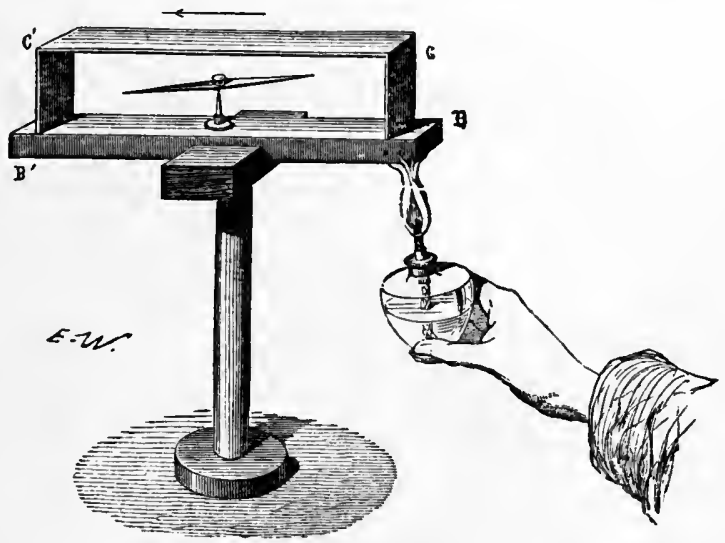

Fig. 14.-Seebeck's experiment.

bent in such a manner as to leave an empty space between the two plates, Seebeck found that on heating one of the solderings of this metallic couple, a magnetic needle, placed between the two plates, was deflected, the current passing always from the hot to the cold soldering of the copper, and from the cold to the hot soldering of the bismuth. 
Becquerel attributes the production of the current to the unequal propagation of heat in different parts of the heterogeneous circuit caused by the two different metals.

Whatever may be the metals entering into the composition of the thermo-electric couples, they are easily distinguished from hydro-electric couples by the nature of the currents. In a battery, in fact, each couple or element possesses a strong tension, varying from one to two volts, and a rather considerable interior resistance (varying from 0.2 to 15 and $20 \mathrm{ohms}$ ); in the thermo-electric couple, on the contrary, for a difference of temperature of $100^{\circ}$ between the solderings, the electro-motive force, very variable with the nature of the constituting elements and the mean temperature of the two solderings, varies between $\frac{1}{20}$ and $\frac{1}{300}$ volt, whilst the interior resistance is very weak. We express this fact by saying that thermo-electric batteries give quantity, but little tension; a large number of them must therefore be joined to obtain a certain electro-motive force. We shall give some figures with regard to the different elements which we are going to describe.

Becquerel, by making experiments between $0^{\circ}$ and $20^{\circ}$ with different.metals, has been able to classify them in such order that each of them is negative with regard to the preceding ones, and positive with regard to those which follow.

The following is the order of the metals experimented on by Becquerel :-

Bismuth, platinum, silver, tin, lead, copper, gold, zinc, iron, antimony. It would seem, $a$ priori, that on taking the most remote elements of the series for the formation of a couple, the largest electro-motive force would be obtained for a given difference of temperature. Other considerations 
of a theoretical and practical nature show that such is not the case.

Practice suggests the choice of metals, of alloys, or substances in which the difference of temperature of the solderings can be maintained at the highest possible degree, without altering the battery. The nature of the metals used naturally influences the cost of the battery.

The law, stating that the electro-motive force of thermoelectric currents is proportional to the difference of temperature, is only correct with special reservations as to the mean temperature of the two solderings, and to the neutral point which varies with the nature of the metals employed. An example will show better than any theoretical explanation, what we mean by the neutral point of a thermoelectric couple. Let us take, for example, a thermo-electric couple formed of the two metals copper and iron. Let us suppose the cold soldering of the couple to be at $70^{\circ}$, and the hot soldering at $450^{\circ}$. No current will pass through the couple thus constituted, although the difference of temperature is $380^{\circ}$. The reason is that the neutral point of iron with regard to copper is $210^{\circ}$, that is to say, that at $210^{\circ}$, the thermo-electric current formed by the two metals changes its direction, and as the mean temperature between the two solderings is precisely equal to $210^{\circ}$, two currents of equal tension, but different directions, are produced which destroy each other.

While maintaining, therefore, a great difference of temperature between the two solderings of a thermo-electric couple, we must take care that the temperatures are not, the one higher, and the other lower, than the neutral point of the two metals forming the couple, for we should then obtain only a differential current, liable to become null 
when the mean temperature of the two solderings corresponds to the neutral point. ${ }^{1}$

If the difference of temperature in a thermo-electric couple is constant, the current itself is constant, for no polarization or variation is produced in the resistance of the couple. The electro-motive force of a thermo-electric battery is proportional to the number of elements constituting the same, exactly as is the case in hydro-electric batteries.

These principles being established, we will pass in review the different thermo-electric couples used in practice.

Ersted and Fourier constructed the first thermoelectric battery. It was composed of small bars of bismuth and antimony soldered together in regular order, and in a straight line. The bars of bismuth terminated in a soldered piece which plunged into ice at zero, whilst the other solderings were heated from $200^{\circ}$ to $800^{\circ}$ by small lamps.

Nobili has simplified Fourier's battery by bending the bars in zigzag; he has thereby enclosed fifty elements in a cube, the sides of which were two centimetres long. Melloni has used this cube in his thermo-multiplier.

Marcus' Battery.-In this battery alloys are used instead of simple metals. One of these alloys consists of nickel, copper, and zinc, known as German silver; the other of antimony, zinc, and bismuth. The solderings are heated by a small Bunsen burner. Wheatstone and Ladd have each constructed a battery with the alloys recommended by Marcus; the former obtained sparks with this battery, as well as the incandescence of a platinum wire, and the decomposition of water in a voltameter; the latter set in action a Ruhmkorff coil which gave a spark of thirty-eight millimetres.

' See, for further details, Electricity and Magnetism, by Fleming Jenkin ; and Philosophical Transactions, 1856, p. 708. 
Farmer's Battery.-Farmer, of Boston, constructed in 1868 a similar battery to that of Marcus; its positive plates consisted of ten parts of copper, six of zinc, and six of nickel; and the negative plates of twelve parts of antimony, five of zinc, and one of bismuth; the two metallic plates were screwed into each other, the lower joints heated by a gas flame, and the upper joints cooled by a stream of water.

Becquerel's Battery.-In 1865, Becquerel, in his researches on the thermo-electric force of artificial copper sulphide, found that this substance, heated to $200^{\circ}$ or $300^{\circ}$, is strongly positive; its point of fusion is above $1000^{\circ}$, and it can therefore be used at very high temperatures. The metal which Becquerel associated with it was German silver (ninety of copper and ten of nickel); the battery is heated with gas, and cooled by a stream of water. ${ }^{1}$

Noë's Thermo-Electric Battery.-This battery is extensively used in Austria and Germany. In the model represented in Fig. 15, only two Bunsen burners are required to set in action forty elements, three for sixty, and so forth, one for each group of twenty, according to the intended application.

The elements in each group are placed horizontally, like the radii of a circle, the hot solderings in the centre, the cold solderings in the circumference.

The two metals used are German silver and an alloy of antimony and zinc; they are soldered together without the use of an intermediate metal. The extremities of the German silver wires reach into a brass capsule, which forms the bottom of the mould in which the other metal is cast. Fig. 16 represents two separate elements in natural

'See the description and diagrams in Ganot's Physics, translated by Atkinson, 3rd edit., p. 787. 
size; the small capsule $c$ is attached to the element, and forms part of the apparatus. Into this same capsule reaches a small copper rod $r$, whose extremity is likewise held fast by the molten metal, and which conducts the heat to the hot soldering. The extremities of these copper rods form a small circle, fastened between two mica plates, and

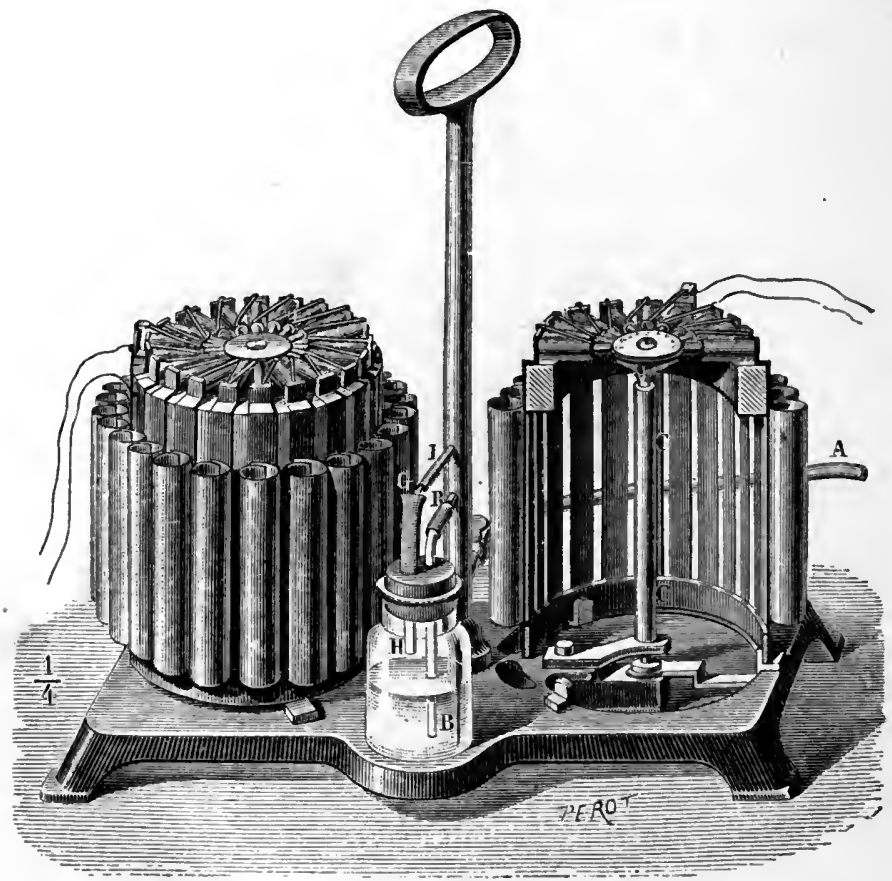

Fig. 15.-Noë's thermo-electric battery.

are all heated simultaneously by a single flame, generally a Bunsen burner; a spirit lamp is sometimes used.

By heating the solderings by conduction, they are protected against overheating, which would fuse the metal and render the battery useless. The loss of heat by radiation is 
prevented by covering the rods with small insulating tubes $t$ (Fig. 16).

For the cold soldering the fusible metal is soldered to a copper plate, to which are also soldered the "maillechort" (an alloy of copper, nickel, and zinc) wires of the next element; the copper plate, of cylindrical form, presents a large surface for radiation favourable to its cooling, which is besides accelerated by the circulation of air inside the copper tubes.

Under the most favourable conditions, that is, by heating the solderings to nearly their point of fusion, the Noë elements, according to Waltenhofen's experiments, have an electro-motive force between $\frac{1}{9}$ and $\frac{1}{10}$

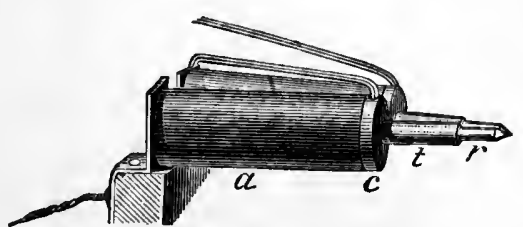

Fig. 16.-Details of an element of Noë's thermo-electric battery. volt; whilst the Marcus elements have only an electromotive force of $\frac{1}{18}$ volt.

In practice, however, these figures are not reached, for all the elements cannot be equally heated, and we can only reckon upon $\frac{1}{16}$ volt per element; under these conditions the interior resistance is $\frac{1}{40} \mathrm{ohm}$.

Each group of twenty elements has, therefore, an electromotive force of 1.25 volt, and an interior resistance of $0.5 \mathrm{ohm}$.

We have to mention here a very practical regulator for the pressure of the gas, which prevents the overheating of the battery, and the accidents which might arise from it.

This safety apparatus consists of a glass bottle (Fig. 15), containing water, and closed by a cork. Two tubes pass through this cork : the one $\mathrm{B} R$ is a branch tube of the gas- 
supplying tube, and reaches to the bottom of the bottle; the other, $\mathrm{H}$, only reaches to the level of the water-it

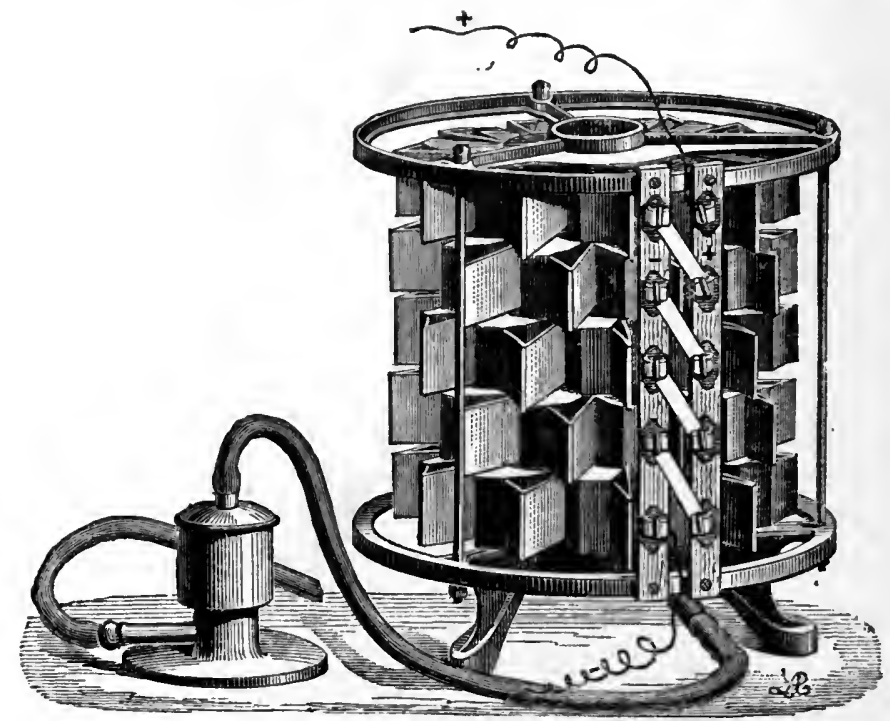

Fig. 17.-Clamond's thermo-electric battery, heated with gas.
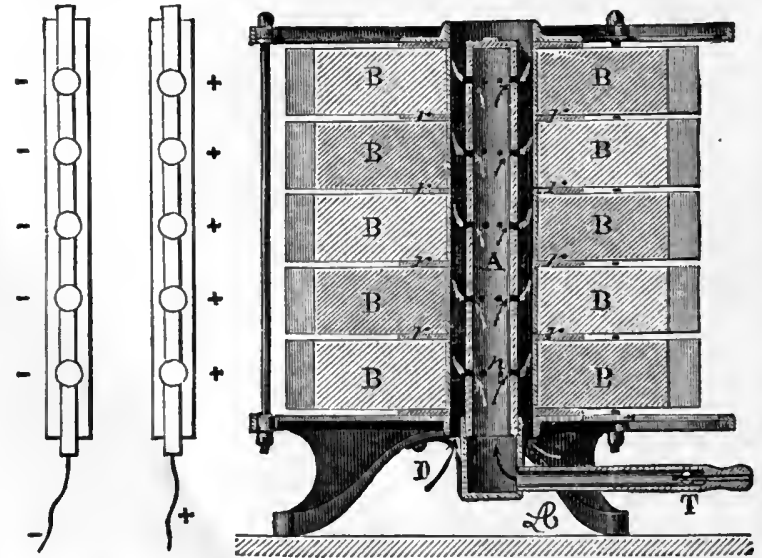

Fig. 18.-Clamond's thermo-electric battery (vertical section). T, gas supply tube; $\mathbf{A}$, perforated earthenware tube, where the gas is mixed with air, the mixture passing into the outside cylinder, where it is burned; B B, thermo-electric bars; $\mathrm{D}$, entrince of air for combustion; $r r$, discs of asbestos for the insulation of the elements. 
carries off the gas which may be collected in the bottle, and leads it before another branch tube, terminating in a burner $\mathrm{I}$, which is kept constantly burning. In case of an excess of pressure, the gas rises in bubbles through the water, escapes at G, and is burned, without producing any accident or any bad smell.

The bottle acts thus like a real safety-valve. Twenty elements suffice for the decomposition of water, and forty to charge Gaston Planté's secondary battery, and to set an induction coil in action.

Two minutes are sufficient to generate a current, and the action can be stopped at any moment.

This battery suffers no alteration in the course of time like the other batteries described before, and in which continuous heating would produce a considerable increase of interior resistance, and, in consequence, a corresponding weakening of the outer current.

Mure and Clamond's Battery.-The first battery constructed by Clamond, in co-operation with Mure, was shown to the Institute by Becquerel, on the 31st of May, 1869. It consisted of galena and leaden plates.

The intensity of this battery is diminished by use, for the interior resistance increases, in consequence of the oxidation of the crystallised bar at the points of contact, and of the splitting of the bar perpendicular to its length.

Clamond's Battery.-On the 20th of April, 1874, Jamin exhibited before the Académie des Sciences a new battery by Clamond, heated with gas, in which these drawbacks are avoided. Clamond has adopted, for the construction of his couples, Marcus' alloy of zinc and antimony, which is a good conductor of electricity, and which can easily be 
manufactured by easting. The armature consists of iron plates, in preference to copper and German silver, because the iron resists very well, whilst the two metals named are acted upon and dissolved, and soon become useless.

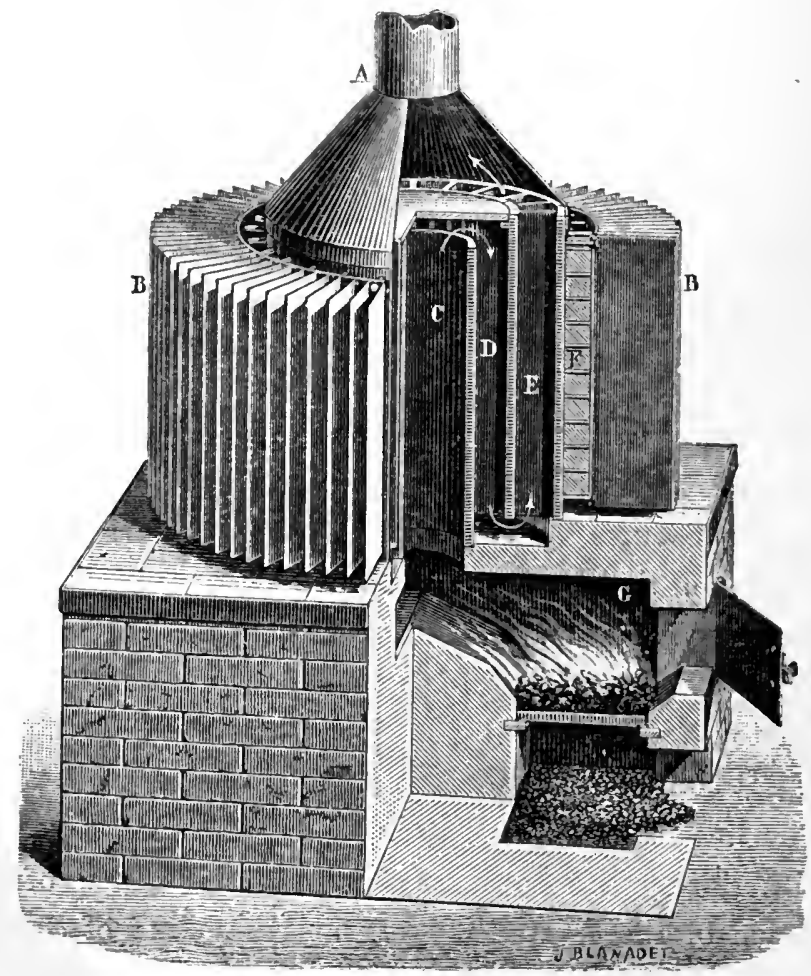

Fig. 19.-Clamond's thermo-electric battery, heated with coke.

The bars are arranged in form of a star, and coupled for tension.

These stars placed one above another, of ten elements each, are separated by asbestos discs. The whole apparatus forms a cylinder, lined inside with asbestos (Fig. 17), and heated with gas by means of a perforated earthen- 
ware tube. The gas, mixed with air, passes through this tube, and burns in the annular space between the tube and the bars. The extremities of these stars fit into copper clamps, fixed on two small planks, and this arrangement permits the coupling together of the stars of ten elements, either for tension or for quantity.

The gas supply is regulated by Giroud's rheometer. Figs. 17, 18, and 20 represent the exterior view, the vertical and horizontal sections of Clamond's battery. The explanations accompanying the sections will suffice to explain the arrangement of the battery. This apparatus of fifty elements consumes five centimes' worth of gas per hour, and can deposit twenty grammes of copper per hour. It is used in the printing department of the

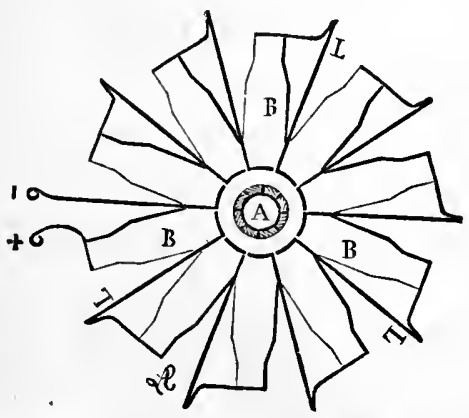
Fig. 20.-Clamond's thermo-electric battery. Horizontal section of the bars and their armatures; $\mathrm{B} B$, thermoelectric bars; $\mathrm{L}, \mathrm{L}$, plates forming the armatures.

Bank of - France, and in

the photographic studios of Messrs. Goupil and Co. at Asnières.

Clamond's Battery heated with Coke.-Since 1874, Clamond has continued his researches on thermo-electric batteries, and on the 5th of May, 1879, Count du Moncel submitted to the Académie des Sciences a new battery, much more powerful than the former ones, which can be used for electric lighting.

Fig. 19 (p. 46) represents a view of Clamond's new battery; Figs. 21, 22, and 23 give the principal details.

The apparatus consists of three parts- 
1. The furnace and the collector, for heating the interior solderings of the battery.

2. The battery itself, arranged in form of a star.

3. The diffusor, for cooling the exterior solderings.

Furnace and Collector.-The battery is heated with coal or coke. The gases, produced by combustion, pass from the furnace $\mathrm{G}$ (Fig. 19), through the cylindrical cast-iron pipe $\mathrm{C}$, downwards through a series of compartments $\mathbf{D}$ arranged in form of a star, then upwards again at $E$, through a second series of compartments, and finally escape through the chimney A. The heating of the elements, therefore, is not direct; there is even no contact between the elements and the hot gases, but these gases heat the mass of cast iron through which they circulate; this mass acts as a collector of heat, which it gives off to the ele-

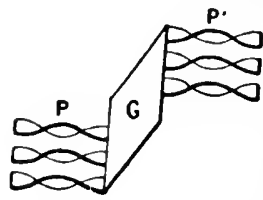

Fig. 21.-Armature of an element of Cla. mond's battery. ments in a more uniform manner.

Battery.-The battery consists of a series of chains arranged in form of a crown around the collector, and heated on the inside. The battery of three thousand couples consists of sixty chains of fifty couples each.

Each couple consists of a thermo-electric bar, three centimetres long and wide, and two centimetres thick. These little prisms are formed of an alloy of antimony, and joined together by iron armatures (Fig. 21). The armatures are cut into thin sheets, twisted like corkscrews at their extremities $P$ and $\mathrm{P}^{\prime}$. To make a chain, these armatures are placed in a special mould, after having surrounded the flat part $G$ with a coating of asbestos, and the alloy is cast in the mould. The fifty elements are thus made at once, the parts $\mathbf{P}$ and $\mathbf{P}^{\prime}$ of each armature 
are joined to two successive couples by a soldering made of the same material as the armature. The chains are arranged vertically and in form of a star, between the collector $\mathrm{A}$ and the diffusor B (Fig. 23), by placing between them and the chains thin laminæ of mica, which insulate them, while they allow the circulation of heat. In a battery of 6000 elements, two groups, of 3000 elements each, are arranged for tension. For a temperature of $360^{\circ}$ in the heated, and of $80^{\circ}$ in the cooled solderings, 3000 elements represent, according to experiments made by G. Cabanellas, an electro-motive force of 109 volts, and an interior resistance of $15.5 \mathrm{ohms}$.

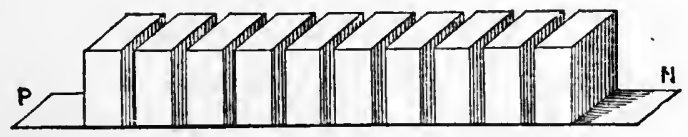

Fig. 22.-Thermo-electric chain of ten elements.

Diffusor.-The diffusor maintains the exterior solderings of the chains at the lowest possible temperature, which, in practice, does not exceed $80^{\circ}$. It consists of a series of copper plates, placed all round the battery, which increase the surface of cooling. These plates are represented at B (Fig. 23). Clamond's coke battery is therefore, in fact, an electrical heat-engine, and can be used in all cases as such. An apparatus of 6000 elements consumes from nine to ten kilogrammes of coke per hour. With an arrangement of two circuits of 3000 elements each, two Serrin lamps can be fed, giving a light of from thirty to fifty gas-burners each (equal to 450-750 candles).

We have here two remarkable results, worthy to be followed up, and we regret that the experiments, commenced VOL. I. 
in 1879, have not been continued. Although hitherto the efficiency of thermo-electric batteries-that is, the proportion of heat consumed to the heat changed into electricity-has been very slight (it does

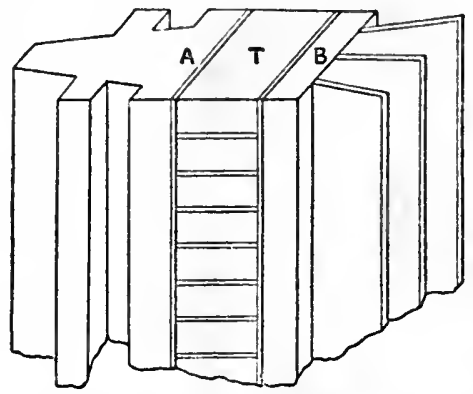

Fig. 23.-Clamond's battery. A, collector; T, thermo-electric ehain; $B$, diffusor.

not exceed four to five per cent.), these apparatus are interesting, for they transform, in a direct manner, heat into electricity; whilst in the case of electric generators, which we are now going to describe, heat has first of all to be converted into work by a steam or gas engine, and the energy thus generated has to be transmuted into electricity. It is, therefore, always easier to keep up an electrical heat-engine, or to feed a gas battery, than to pass through the more complicated intermediate stages by the employment of an electric generator.

Gaugain's Thermo-Electric Battery.-Gaugain uses bismuth-copper couples, arranged in horse-shoe furm alongside of each other on a cross-beam of wood, which allows of their being raised or lowered at once into two liquids, one of which is distilled water at $0^{\circ}$, and the other heated paraffin at $100^{\circ}$. This battery has a constant electromotive force, and is therefore an excellent standard battery. Its electro-motive force is $\frac{1}{1^{\frac{1}{2}}}$ volt ; by arranging therefore, 182 elements for tension, and keeping the solderings exactly at $0^{\circ}$ and $100^{\circ}$, we have a battery with an electro-motive force of exactly one volt.

Contrary to all expectation, no progress has been made, 
within the last two years, in the practical application of thermo-electric apparatus, and, with the exception of some applications to galvano-plastic, made by the Bank of France and by Messrs. Goupil, of Paris, these apparatus only present a scientific interest. 


\section{CHAPTER III.}

\section{ELECTRIC GENERATORS.}

WE are fully aware that the term "electric generators" includes galvanic and thermo-electric batteries, as well as magneto- and dynamo-electric machines; we use it simply as a shorter designation for the two latter kinds of machines when speaking of them collectively.

The transformation of work into electricity is the most complicated means for producing an electric current, and yet it is, up to the present, the most economical and the most extensively used in all applications which require powerful currents. This extensive employment of machines is of comparatively recent date, although the discovery of the fundamental principle of all machines was made by Faraday in $\mathbf{1 8 3 0 .}$

The way to this discovery had been prepared by CErsted's experiments, made as early as 1820, on the action of a current on the magnetic needle, and by Arago's discovery, made shortly afterwards, of the magnetization produced by a current. The action of electric currents on magnets, and their magnetizing action on soft iron, being known since 1820 , it seems but natural to draw an $a$ priori conclusion that an inverse action must take place when a permanent magnet acts on a closed spiral circuit; but ten 
years elapsed before Faraday observed this phenomenon and investigated its different bearings.

We need not enter here upon the theory of induction currents produced by the approach or withdrawal of a magnet or current; the laws of these currents, as stated by Lenz and Matteucci, are to be found in any text-book on physics.

All electric generators are founded on Faraday's discovery, and the laws of Lenz and Matteucci, applied to practical methods for the most effective collection of induction currents.

Classification of Electric Generators. - Every electric generator consists of two fundamental, necessary, indispensable parts : the inductor, or field magnet, and the armature. By moving the inductor before the armature or, as a general rule, the armature before the inductor, electric currents are produced in the wire of the armature, whose nature, intensity, and sense are defined by Lenz's laws. These laws show that, in order to obtain powerful currents, the armature must be moved with great speed before powerful inductors.

Any mechanical arrangement which enables us to obtain this result is an electric generator.

The function of the inductor is to create a special condition of movement of the surrounding ether, and this is called the magnetic field. This magnetic field can be produced by two very different methods, and, according to these methods, electric generators can be divided into two classes.

If the magnetic field of the inductor in which the armature moves is produced by a fixed and permanent magnet, the machine is called magneto-electric.

If the magnetic field is formed by an electro-magnet, 
whereby its intensity is increased-electro-magnets being, for equal weight, much more powerful than permanent magnets-the machine is called dynamo-electric.

The above division is based on the nature of the inductor; there is a second one, more important still, based on the nature of the currents collected outside the machine on the external circuit. According to Lenz's laws, when an element of the spiral wire of an armature traverses a magnetic field, the current engendered in this element circulates first in one sense and then in another;-this is an alternating current.

When the machine directly collects the alternating currents thus produced, without reversing them, that is to say, if the currents pass into the outer circuit without changing their sense, the machine is an alternating-current machine.

If, on the contrary, the machine reverses the alternating currents, which then circulate always in the same sense in the external circuit, it is a continuous-current machine.

We shall only describe those types of machines which are the most extensively used-

$$
\begin{aligned}
& \text { A.-Machines with Contincoos Corrents ... }\left\{\begin{array}{l}
\text { a.-Magneto-electric. } \\
\text { b.-Dynamo-electric. }
\end{array}\right. \\
& \text { B.--Machines witu Alternativg Currests... }\left\{\begin{array}{l}
a .- \text { Magneto-electric. } \\
b .- \text { Dymumo-electric. }
\end{array}\right.
\end{aligned}
$$

\section{A.-Machines with Continuous Currents.}

$$
\text { a.-Magneto-Electric Machines. }
$$

Although the discovery of induction was made by Faraday, in 1830, the electric spark by induction was only obtained on the 31st of January, 1832, by Nobili, in 
Florence; but even in his case there was no current in the proper sense of the word.

The first machine which gave a current was constructed by Hippolyte Pixii, of Paris, and was brought before the Académie des Sciences on the 3rd of September, 1832.

Pixii's Machine. - We only mention this historical machine to point out a rather curious peculiarity which is not to be found in any other magneto-electric machine. The well-known apparatus consists of two coils and a horse-shoe magnet, whose poles are placed to face the coils, and turn in such a manner as to present their two poles successively before the extremities of the coil.

Instead of moving the inductor in the magnetic field, the magnetic field is moved before the inductor; but this arrangement does not in any way affect the principle. The currents thus developed are alternating, but by means of a commutator invented by Clarke, the currents were reversed, and Pixii's machine was the first with continuous currents. The first improvement in this machine was effected by Saxton.

Saxton's Machine.-This machine is arranged like Pixii's, that is to say, the magnet and the coils are placed end to end, but the magnets are fixed, and the coils are movable. Consequently, as there is a smaller mass to be moved, the machine need not be so heavy for equal power.

Clarke's Wachine.-In 1834, Clarke constructed a machine in which the magnet was vertical and the coils horizontal; they presented themselves, therefore, to the inductor, no longer by their ends, but by the flat sides of the horse-shoe magnet. In this form, magneto-electric machines have been introduced into all physical laboratories, and are still so used in medicine. 
Clarke's Machine after Gaiffe's Model-_A, B, B' (Fig. 24), is an electro-magnet; $\mathrm{H}$, one of the coils of the soft-iron armature turning before the arms of the magnet; $R$ is the toothed wheel, and $M$ the winch-handle, which communicate the rotatury motion to the coils. The whole apparatus, with the hand-pieces, the crank, and all accessories, can be enclosed in a small box.

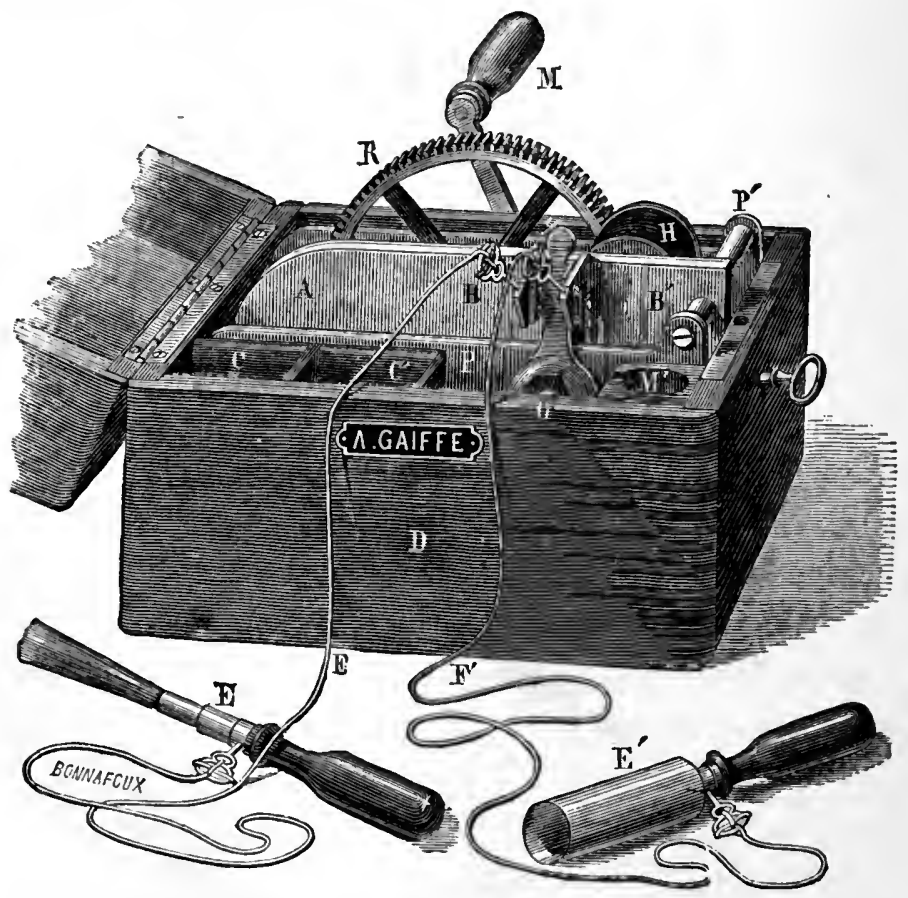

Fig. 24.-Clarke's machine after Gaiffe's model.

The power of the currents can be varied by increasing the speed of rotation. By arming the magnet a less powerful current is generated.

Page's Machine.-In 1835, Page, an American, constructed a machine, in which the fixed coils surrounded the magnet, which was fixed likewise. The currents, produced by the 
rapid rotation of a soft-iron armature before the extremities of the magnet, were alternating, in consequence of the successive overcharging and weakening of the magnetic field; these currents were afterwards reversed in the ordinary manner by a Clarke's commutator.

All these machines and several others based on the same principle, were only apparatus of feeble power, and found but a very limited application. In 1849, Nollet, Professor of Physics at the École Militaire, Brussels, constructed a Clarke's machine on a large scale, and that machine is now called the Alliance machine.

Nollet's apparatus gave no favourable results until Masson, Professor at the Ecole Centrale, suggested to Van Malderen, the engineer of the Compagnie l'Alliance, to transform the continuous-current machine into an alternating-current machine. We shall refer to this again when speaking of these machines.

Siemens' Coil.--In 1854, Siemens and Halske, of Berlin, advanced the question a good deal by inventing the coil which bears their name, and which we shall find again in a great number of apparatu', such as Wilde's, Ladd's, Marcel Deprez's, etc.

Siemens' coil is a soft-iron cylinder with two longitudinal grooves parallel to its axis, which give it, in transversal section, the form of a double $T$. An insulated copper spiral fits into the grooves, and the extremities of the spiral are fixed to the two halves of a commutator for the reversion of currents, as in Clarke's machines. By turning the coil rapidly between the poles of a magnet, induction currents are produced in the wire, owing to the alternately inverse polarities of the two enlarged poles of the coil; these currents are subsequently reversed and 
collected in the usual manner. In 1879, Marcel Deprez modified Siemens' machine by placing the axis of the coil parallel to the arms of the magnet.

We shall refer to this modification again in connection with electric motors; for Siemens' machine, and electric generators with continuous currents generally, are reversible, that is, if they develop electricity by expending work, they can also jroduce work by expending electricity.

Finally, Gramme's machine, laboratory size, ought to find a place here, but its description must be reserved for the next chapter.

Between Siemens' coil of 1854 and Gramme's ring, which dates from 1870, a whole series of new principles must be considered, before we can speak of an invention which has given such a powerful impetus to the mechanical production of electricity.

\section{b.-Dynamo-Electric Machines with Continuous Currents.}

We know that the electric current is produced in electrodynamic machines by a rapid motion of the induced wire of the coils in a powerful magnetic field formed by the inductor. The power of the resulting currents depends to a great extent on the power of the magnetic field.

Wilde's Machine.-As an electro-magnet has at least twenty-five times as much power as a steel magnet for equal weight, Wilde thought that, by using electromagnets instead of common magnets as inductors, the intensity of the magnetic field, and consequently the intensity of the resulting current, would be considerably increased.

Wilde's machine, which figured at the French Exhibition of 1867, was really composed of two machines placed one 
above the other. The first was a Siemens' rnagneto-electric machine, which transmitted the current to two large electro-magnets; these latter served as inductors to a second Siemens' coil, which formed the external circuit. Wilde's small machine feeds and excites the inductors of the second coil ; it is, therefore, called an exciting machine. We shall find it again in a great number of other electric generators, both with continuous and with alternating currents. It is also sometimes called the inducing or priming machine.

One of the drawbacks of this machine is the great speed which must be imparted to the coils-2000 to 2500 revolutions per minute. This produces a very high temperature, which has to be counteracted by circulating cold water between the polar pieces of the electro-magnet serving as inductor to the coil of the external circuit.

Ladd's Machine.-Ladd's machine is the first application of a new principle, discovered simultaneously by Wheatstone and Siemens, and which was brought before the Royal Society on the same day, the 14th of February, 1867. This principle consists in the gradual increase of intensity of an electro-magnetic system under the influence of the induction currents which it develops. A mere trace of magnetism arising either from the momentary action of a battery, from terrestrial magnetism, or from remanent magnetism, is sufficient to produce magnetizing action and increased intensity of magnetism up to a certain maximum, which will depend on the speed of rotation of the coil, the resistances of the circuit, and the point of magnetic saturation of its inductors.

In Ladd's machine, which is constructed on this principle, we find again the two Siemens' coils employed by :Wilde, but the permanent maguet is suppressed. 
The electro-magnet consists of two large iron plates surrounded with wire, thus forming two straight electromagnets, whose visible poles are of different sense; the two Siemens' coils are placed transversely to the two extremities of these electro-magnets. The smaller of the two transmits its current to the electro-magnets to preserve their magnetism, the second is utilized for feeding the external circuit. A trace of remanent magnetism in the plate of the electro-magnets is sufficient to produce, in consequence of successive overcharges, a very intense current,

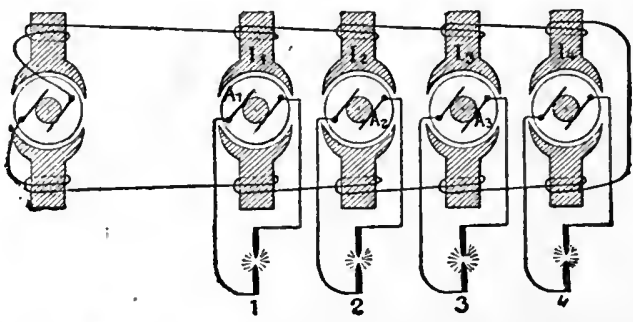

Fig. 25.-Machines excited by a separate exciting machine.

which imparts great power to the electro-magnets, and thus develops a magnetic field of very great intensity. This power of the magnetic field can, moreover, be regulated by modifying the speed of rotation.

The great speed of the coils, however, produced considerable heat in the machine, owing to the rapid changes of magnetism in the soft iron. The use of two machines, on the other hand, complicated the transmission of motion, entailing the employment of two wheels and two belts.

Mode of Charging the Inductors of Dynamo-Electric Machines.-Whatever may be the shape of the part which receives the current, whether Clarke's or Siemens' coil, 
Gramme's ring, etc., dynamo-electric machincs can be divided into three classes, distinguished by the manner in which the field-magnets are charged.

Fir'st Class.-In Ladd's machine, which is the type of this class, the inductors are charged by a distinct and perfectly separate circuit fed by a special machine, which frequently feeds not only one but several machines. Fig. 25 shows an arrangement of a self-exciting machine feeding four machines, each of which supplies an arc lamp.

Second Class.-The electro-magnets forming the magnetic field are arranged in the same circuit as the external armature and circuit. This is the arrangement invented by Wheatstone and Siemens, in 1867, and which is now applied to nearly all dynamo-electric machines. Fig. 26 shows this special arrangement. The continuous current produced by the armature A tra-

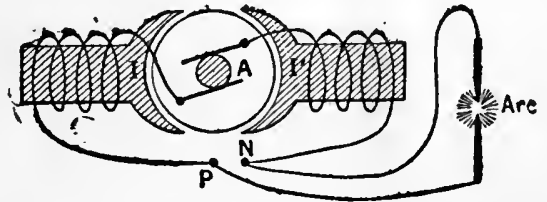

Fig. 26.-Diagram of the ordinary mounting of a dyuamo feeding a voltaic arc.

verses the two inductors $I$ and $I^{\prime}$ at the same time as the arc which it feeds. The magnetic field, therefore, depends on the strength of the traversing general current.

Third Class.-The arrangement which characterizes the machines of this class was also invented by Wheatstone, in 1867 , but was only taken up again in 1880 by Siemens. This arrangement consists in placing the inductors in a shunted, and not in the same circuit, and for this purpose these inductors must be provided with a long wire of considerable resistance. The working conditions of the machines are hereby rendered much more favourable for 
the regularity of the current. ${ }^{1}$ Edison's machine, of which we shall speak further on, offers an instance of this.

Principle of Gramme's Machine.-The most important progress in the mechanical production of electricity, since Siemens' and Wheatstone's discovery, is due to the invention of a former workman of the Compagnie l'Alliance, named Gramme. Thanks to his machine, the applications of electricity to lighting, galvano-plastic, and transmission of motive force, have made very rapid strides within the last few years.

Gramme's movable armature presents the appearance of a ring, turning round its centre and in its own plane; it can be considered as formed of a straight electro-magnet which had subsequently received a circular form, and been soldered at the end in such a manner that core was joined to core, and wire to wire.

The idea of employing a ring as a movable armature is not new; as carly as 1852 , Page constructed a motor founded on this principle. In 1861, Pacinotti also constructed a motor which, not only in the employment of a ring armature, but also in the application of the segmental collector or commutator was the prototype of the Gramme machine. Pacinotti's machine figured at the Exhibition of 1881, and gained for its inventor an honourable mention.

Pacinotti's Machine.-It will be interesting to give a description of this historical machine in Pacinotti's own words, from an account published in 1864 in "Il Nuovo Cimento."

"I took a cast-iron ring, provided with sixteen equal

- See, for further details, La Lumiere Electrique, of the 15th of April, 1880 , p. 151. 
teeth; this ring is supported by four arms or vertical pieces of brass B B (Fig. 27) which connect it to the shaft of the machine. Between the teeth small triangular wooden prisms are placed, and into the hollow spaces between the prisms and the teeth coils of silk-covered copper wire are fitted. This arrangement aims at obtaining between the iron teeth of the wheel a perfeet insulation of the bobbins thus formed. In all the bobbins the wire is coiled in the same sense, and each of them is formed of nine spirals. Two consecutive bobbins are separated from one another by an iron tooth of the wheel, and by the small triangular wooden prism. On leaving one bobbin to construct the following one, I fix the end of the copper wire to the piece of wood which separates the two bobbins.

"On the axle which carries the wheel thus constructed I have grouped all the wires. One extremity of these wires forms the end of one bobbin, and the other the commencement of the following bobbin, and this is effected by causing them to pass through holes made for this purpose in a wooden collar centred on the same axis, and then attaching them to the commutator which is likewise mounted on the axis.

"This commutator consists of a small wooden cylinder having on its circumference two rows of sockets, into which fit sixteen brass pieces, eight into the upper, and eight into the lower sockets, all concentric with the wooden cylinder, over which they slightly protrude, and whose thickness separates one row from the other.

"Each of these brass pieces is soldered to the two ends of wire which correspond to two consecutive bobbins, so that all the bobbins communicate with one another, each of them being connected to the following one by a con- 
64 THE SOURCES OF ELECTRICITY.

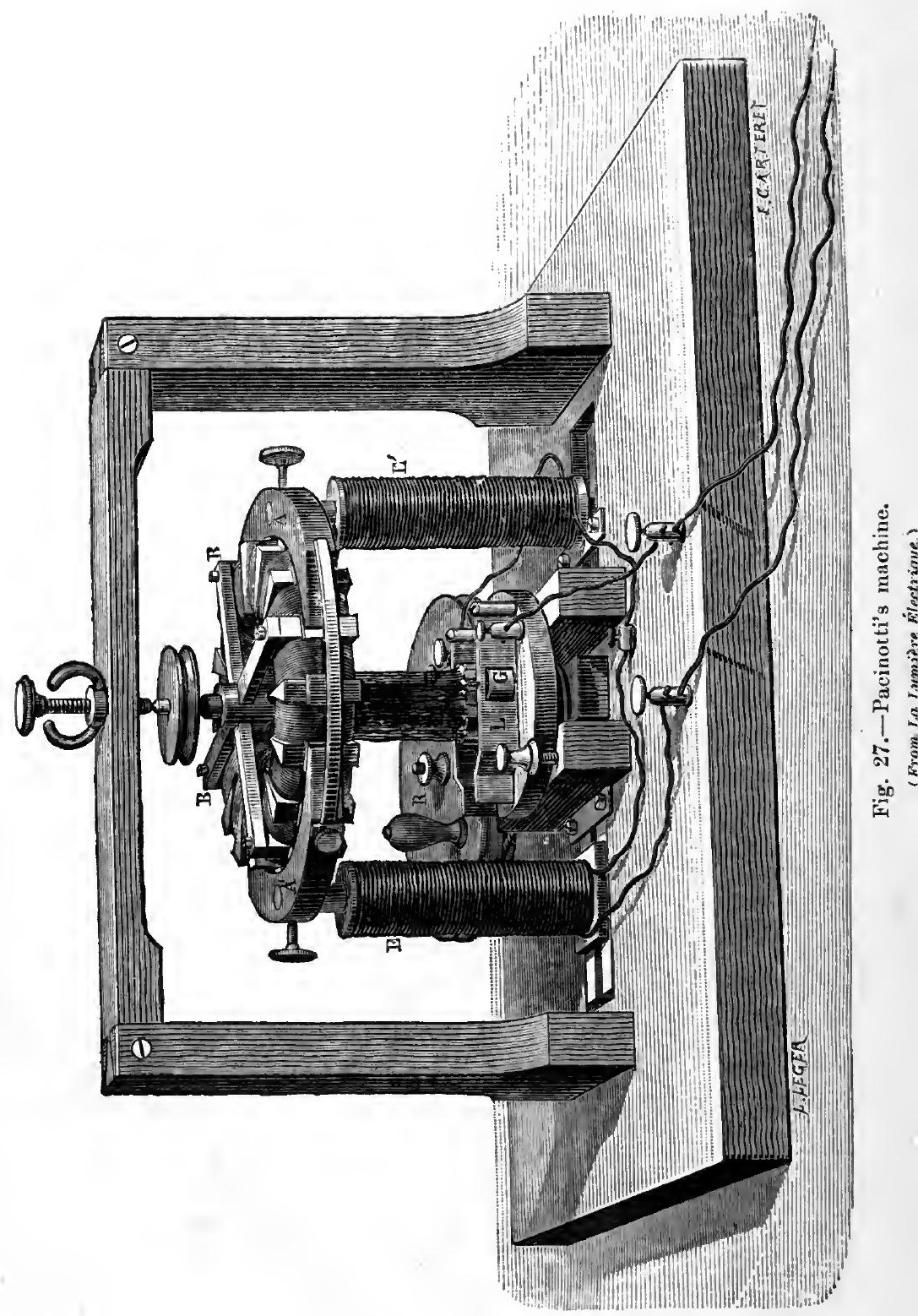


ductor, part of which is formed by one of the brass pieces of the commutator. If, therefore, two of these brass pieces are placed in communication with a battery by means of two metallic rollers $\mathrm{G}$, the current on dividing will traverse the helix on either side of the point from whence start the ends of the wires attached to the brass pieces which communicate with the rollers, and the magnetic poles will appear in the iron of the circle on the diameter perpendicular to A A'. On these poles act the poles of a fixed electro-magnet, which determine the rotation of the transversal electro-magnet round its axis, since in the transversal electro-magnet, when it is in motion, the poles are always reproduced in the fixed positions which correspond to the communications with the battery."

Pacinotti's claims to priority are no longer a matter of dispute, and it is to be regretted that he did not fully realize the importance of his discovery, leaving to Gramme the practical and industrial development of his principle.

Gramme's machine, however, in spite of the priority claimed by Pacinotti, has a real and absolutely personal value, justified, moreover, by its many successful applications which we shall describe in this volume.

To understand the action of Gramme's machine, let us recapitulate the simplest experiment of induction. Let us suppose a magnetized bar, one metre long, and a spiral conducting wire in reciprocal motion; if the spiral is brought near the bar, an induction current is developed in the former. Let us suppose that the bar penetrates into the spiral by a series of successive, uniform movements. It will be observed that an induction current corresponds to each of these movements. All these currents are of the same sense, until the VOL. I. 
spiral arrives at the neutral line; they alter their sense if the movement continues in the same direction beyond the neutral point, the iron ring is magnetized, and the magnetism is distributed in it in the following way:-

$\mathrm{A}$ and $\mathrm{B}$ (Fig. 28) are the poles, $\mathrm{M}$ and $\mathbf{M}^{\prime}$ the neutral points.

By turning the ring, this distribution of magnetism is not altered; the poles are fixed in space, although the ring is displaced, because the coercive force of the soft iron of which it is made is zero, or can be neglected. The effect is,

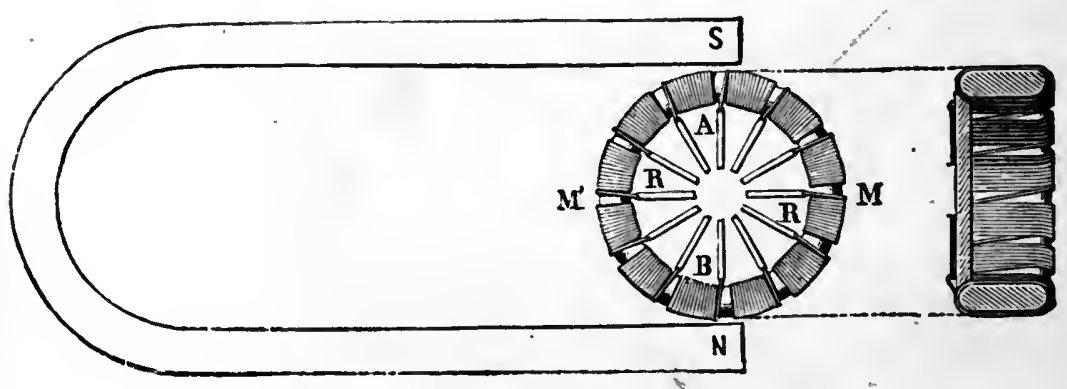

Fig. 28.-Priviciple of Gramme's ring.

therefore, the same as if the iron were immovable, the wire spirals alone moving over a magnetized bar.

In each of the elementary coils, therefore, a current is developed which, starting from one of the poles $A$, remains direct up to the neutral line $M$, takes the inverse sense from $\mathrm{M}$ to $\mathrm{B}$, keeps that sense from $\mathrm{B}$ to the neutral line $\mathrm{M}^{\prime}$, and becomes direct again from $\mathbf{M}^{\prime}$ to the pole $A$. The current, therefore, remains of the same sense from one neutral point to the other.

All the coils which, at a given moment, are in the upper semicircle, are all at the same time traversed by currents of direct sense, which are joined for tension; the coils of the lower semicircle are traversed by currents of inverse 
sense, also associated for tension. A state of equilibrium is thus established, and the system can be compared to two series of batteries, equal in number and in power, acting in opposition. By putting the two extremities of an external circuit in communication with the opposite common poles of the two batteries, these batteries are associated for quantity; in the same way, by establishing

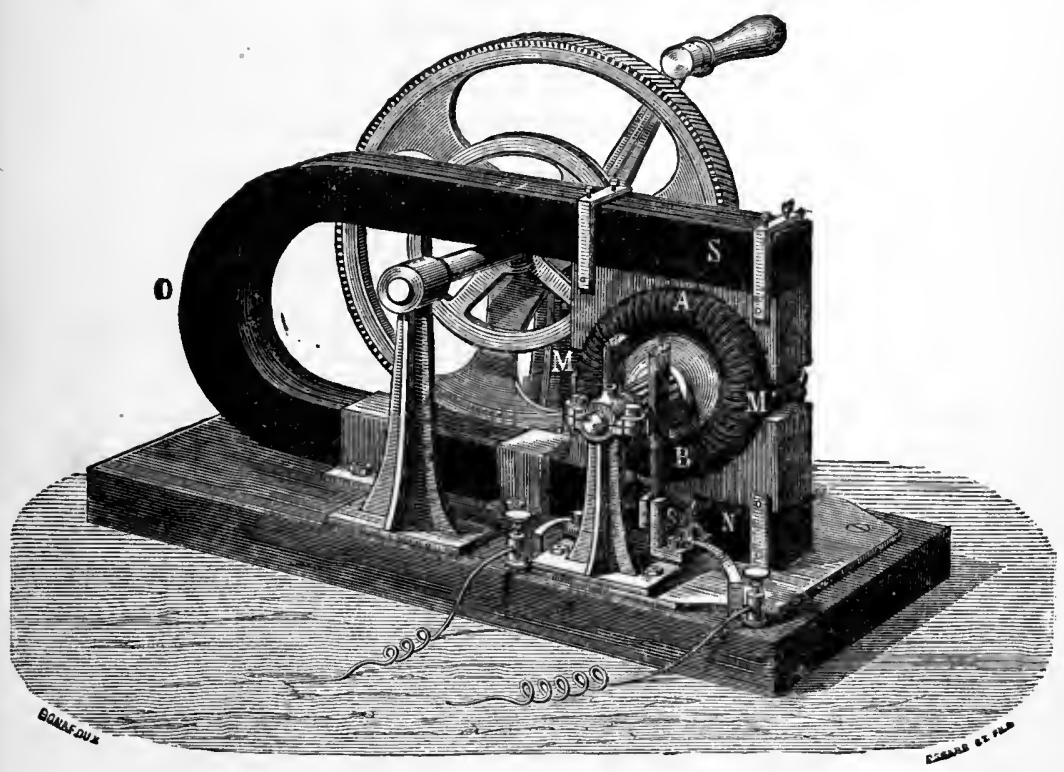

Fig. 29.-Gramme's laboratory machine, with ordinary magnets.

collectors on the neutral points of the ring, the currents developed in the ring can be collected. These collectors are formed of copper brushes, which rub against a series of radiating pieces $R$ (Fig. 28) insulated from one another, and each connected with the end-piece of one coil and the beginning piece of the next. These currents are therefore, by the action of the collectors, transferred from the soldering of the two coils to the piece $R$. 
In Fig. 29, which represents the machine in its practical form, the pieces $R$ are joined to form a cylinder of small diameter, but always insulated from one another.

Between certain limits, the electro-motive force increases in proportion to the speed; the sense of the current changes with the sense of rotation. The current is continuous, for the circuit is never broken, and the rubbers or brushes begin to touch one of the radii before having left the preceding one. On account of their flexibility and the large number of copper wires of which they are composed, one part of each brush, if not the whole, is always in contact.

By changing the thickness of wire of the coils, tension or quantity can be produced at will. Fine wire increases the interior resistance and the tension of the current; thick wire diminishes this resistance, and increases quantity at the expense of tension. Tension and quantity are, therefore, the two elements of an electric circulation, just as pressure and volume supplied are the elements of the circulation of fluids in hydrostatics. The energy expended in each case is the product of two factors, and this product is constant, whilst the factors vary ad infinitum.

Gramme's Machine with Jamin's Magnets. - Gramme has constructed a considerable number of different machines founded upon the principle of the ring and collector. The one we have taken as a type to explain the production of the currents and the way of collecting them, is magnetoelectric.

Fig. 30 represents the model of a magneto-electric machine to which Gramme has successfully applied Jamin's laminated magnets. Numerous and accurate experiments have proved that laminated magnets, for equal weight, are much more powerful than ordinary magnets, provided their 
extremities (Figs. 30 and 31) carry pieces of soft iron which share their magnetism with the magnet.

Before the adoption of the soft-iron armatures, only five or six sheets could be superposed with any effect; with the soft-iron armature, as many as twenty can be used. Jamin's magnets have the further advantage of possessing a much larger magnetic surface-for magnetism is especially power-

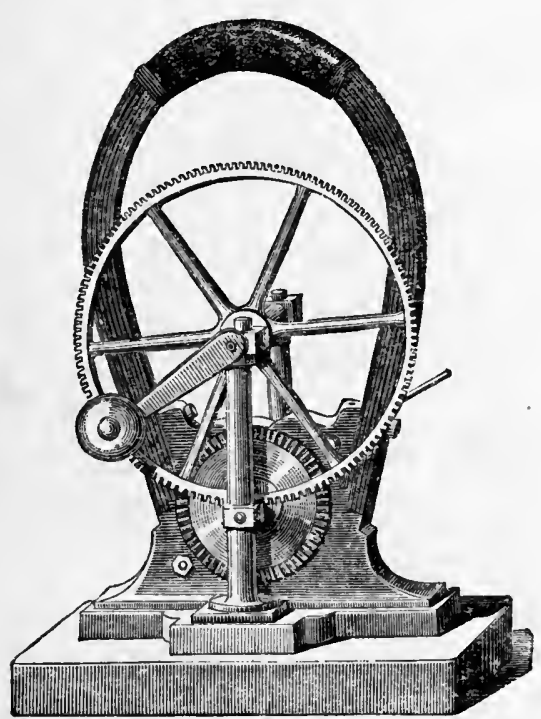

Fig. 30.-Gramme's machine with Jamin's magnet (small laboratory model).

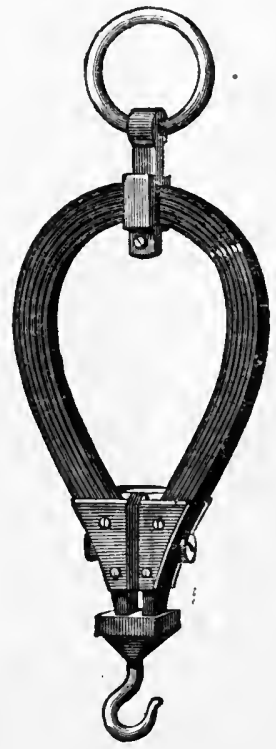

Fig. 31.-Jamin's magnet.

ful on the surface-and a greater facility of mounting, dismounting, and replacing the sheets.

The magneto-electric machine with Jamin's magnets is now very much used in the laboratory. It is very easily set in motion by a pedal or a winch-handle, and it renders very good service for experiments of short duration, charging of secondary batteries, etc. 
Gramme's Dynamo-Electric Machines.-However great may be the power of the magnetic field developed by a

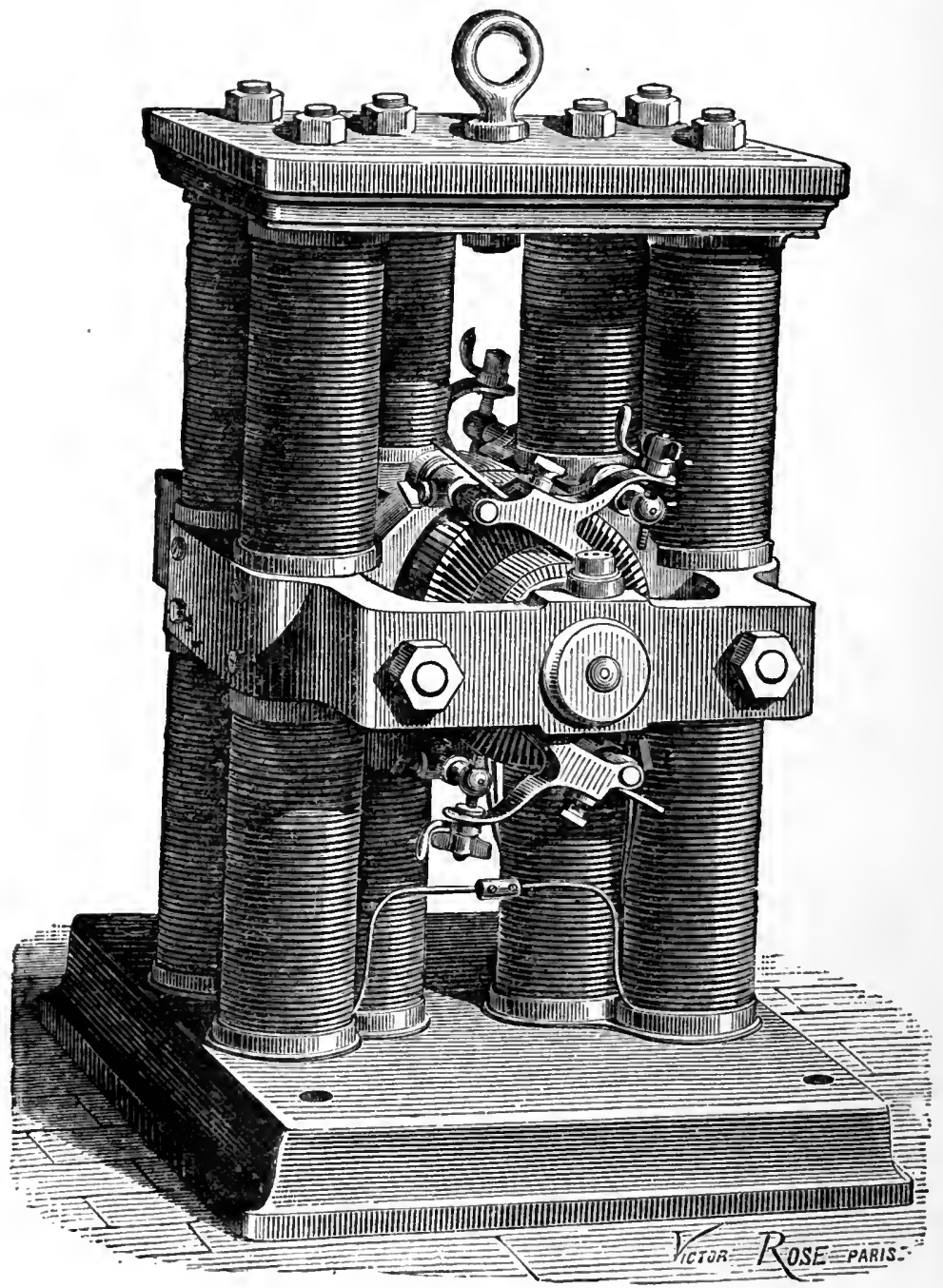

Fig. 32.-Gramme's machine, with two rings for electric lighting.

Jamin's magnet, it is far from being equivalent to that of 
an electro-magnet, and although, theoretically, magnetoelectric machines may have a much greater efficiency than dynamo-electric ones, these latter are preferred, because, for equal weight, they transform into electricity a much larger amount of work than the former.

For these reasons, Gramme, after having constructed the machines we have described, which are all of rather feeble power, applied to his ring the principle discovered by Wheatstone and Siemens. The first dynamo-electric machine constructed by this inventor dates from 1872; it was used for galvano-plastic in Messrs. Christofle and Co.'s works. It consisted of four electro-magnetic bars and two coils. The first Gramme machine intended for electric lighting had six electro-magnetic bars and three coils.

We find in these two machines the principle applied by Ladd to his electric generator. One of the rings served to charge the inductors, the two others supplied the external current, and could be associated, according to the requirements, either for tension or for quantity.

Fig. 32 represents a smaller model, in which there are only two rings: one of them produces the magnetic field, and the other feeds the external circuit.

In another model, there is only one single ring formed of 120 partial coils, which are connected with two collectors placed on each side of the ring, so that all the even coils are in connection with the collector on the right, and all the odd coils with the collector on the left; the machine, therefore, works as if there were two distinct rings, which can afterwards be associated for tension or for quantity.

In this machine, the inductors are arranged in the same circuit as the coils, as indicated by Wheatstone and Siemens, in 1867; but according to Fontaine, Gramme's patent 
concerning this arrangement is prior to the communication of these two savants. By simplifying his invention more

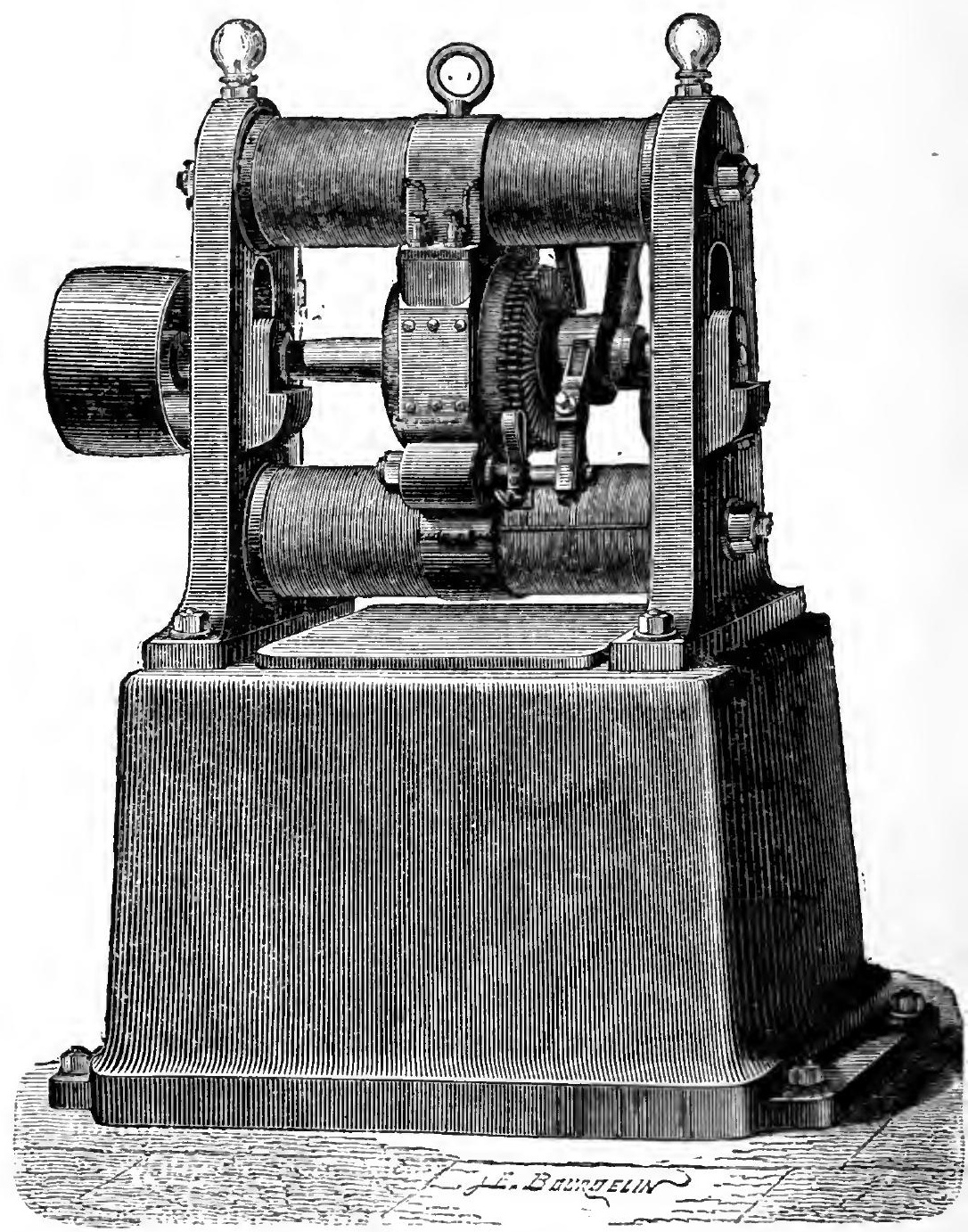

Fig. 33.-Gramme's dynamo-electric machine (workshop type). and more, Gramme has finally succeeded in constructing 
a machine with two electro-magnetic bars and one single central ring.

This machine is called "type d'atelier" (workshop type), or type $\mathrm{A}$; it is represented in Fig. 33. We find in it again the coil and the collectors of the magneto-electric machine; the inductors consist of two soft-iron rods fixed on the frame, and carrying in their centre, where the poles are situated, two armatures, which surround threefourths of the circumference of the ring, and thereby effectually divide the magnetic field and increase the effects of induction.

In machines for galvano-plastic, where quantity and but little tension is required, the armature of the electro-magnet inductors is formed of a single band of thin copper, occupying the whole width of half a bar of the electro-magnet; the coil itself is formed of very thick flat wire, strong enough to resist the effects of centrifugal force.

Gramme's machine, in its present form, realizes all the conditions of a good electric generator; its only drawback is the difficulty of coiling the ring, and this drawback is not a serious one, thanks to the improved methods of construction.

Gramme's machine transforms, under favourable conditions, eighty-five to ninety per cent. of the work expended into electricity; these figures clearly show that the efficiency of these machines leaves nothing to be desired.

The only improvements of which they are capable would be greater facility of construction and repair, and lowering of weight and price.

We are now going to describe a certain number of other machines which, although for the most part founded on the principles that have been explained, present certain interesting peculiarities on different accounts. 
Schuckert's Machine.-This machine, which is extensively used on the Continent and also in this country, is shown in Fig. 34. It consists of two vertical frames between which are fixed the cores of the electro-magnets; the cylindrical cores have a groove in the centre to receive

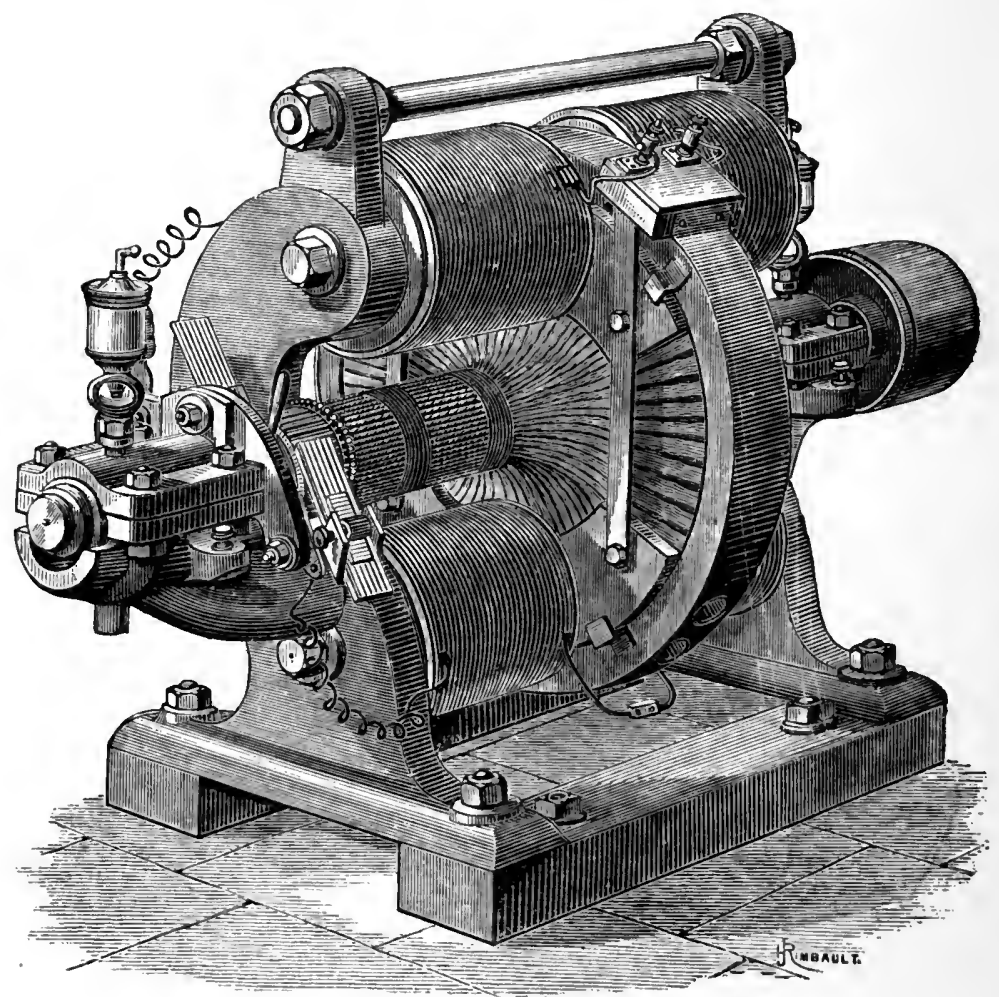

Fig. 34.-Schuckert's machine.

the revolving armature. The latter consists of a ring built up of a number of thin discs, insulated from eacl other and covered with coils. This ring is nearly enclosed by the semicircular pole-pieces already mentioned, and which approach each other within a short distance, near 
the horizontal axis of the ring. The object of this special arrangement is, of course, to bring as large a number of the convolutions of the armature coils as possible within the influence of the magnetic field. The commutator of this machine consists of a number of metal segments insulated from each other, these segments being connected to the sections of the armature coil. The number of these sections varies from ten to one hundred, according to whether the machine is intended for low or high tension; and the end of each section is connected to its corresponding segment by a screw, so as to be easily removed for repair or renewal.

This generator has probably the highest electro-motive force of any in practical use, and is, therefore, especially applicable to installations in which the driving station is at a considerable distance from the are to be illuminated, but it of course requires proportionately increased care in handling and in the manipulation and fixing of the conductors.

Niaudet's Machine.-In 1872, Alfred Niaudet constructed a machine of Clarke's type with Gramme's collector. It is magneto-electric (Fig. 35), but its description, without a previous account of Gramme's ring, would have entailed useless repetitions. To understand the working of this apparatus, each coil must be considered as one of the partial coils of Gramme's ring, for Clarke's coils are all connected with one another; the beginning piece of each being connected with the end-piece of the following coil. The currents are collected by means of brushes which touch the connecting points of the different coils.

The continuity of the current results from the fact that the collecting springs, before leaving one of the radial pieces, already begin to touch the next. 
Gramme's machines supply, for equal magnetic power and length of wire employed, a more powerful electric current, while expending less motive force; this constitutes a superiority in their favour, which Niaudet is far from contesting.

He thinks, however, that for telegraphy without batteries, with continuous-current machines, the extreme facility of construction of eylindrical fine wire coils, with

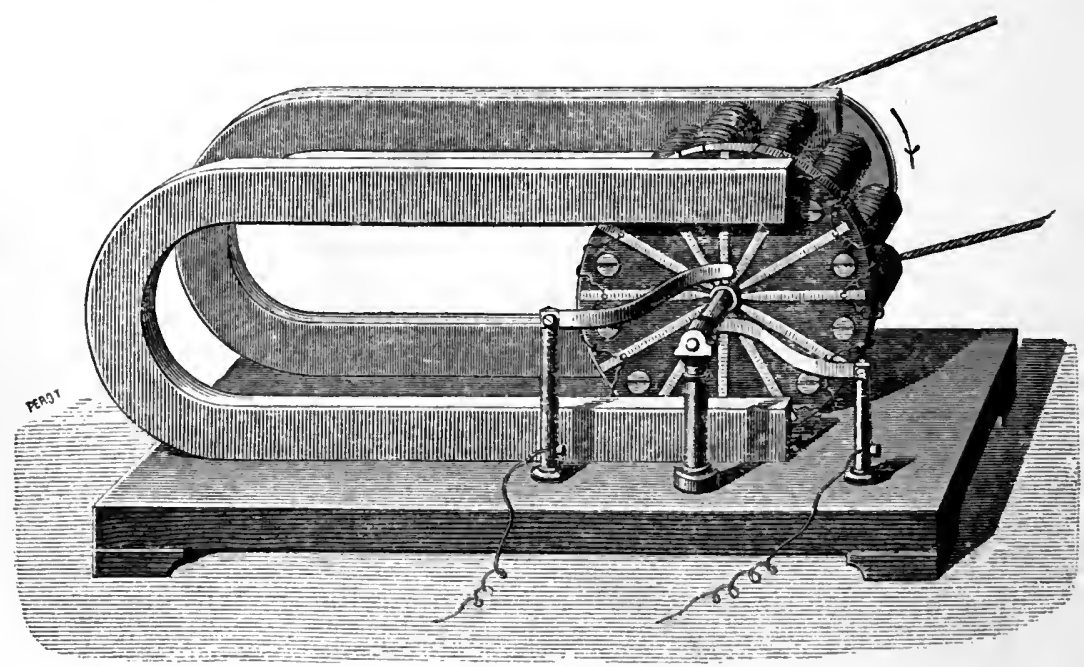

Fig. 35.-Niaudet's continuous-current machine.

the object of obtaining currents of great tension and small quantity, will justify the employment of this apparatus, the simplicity of construction of which will compensate for its inferior efficiency-an efficiency which, in this particular case, is altogether of secondary importance.

Lontin's Machine.-Lontin's machine, patented in 1874, is a dynamo-electric machine with continuous currents, the field-magnets being in the same circuit as the armature 
and the externa] circuit. It differs from Gramme's machine by the form of the armature, which is composed of forty small coils D D (Fig. 36), with their axes converging into one point, like the radii of a circle. To this arrangement the armature owes its name of magnetic pinion.

The forty coils, divided into ten rows of four coils each,

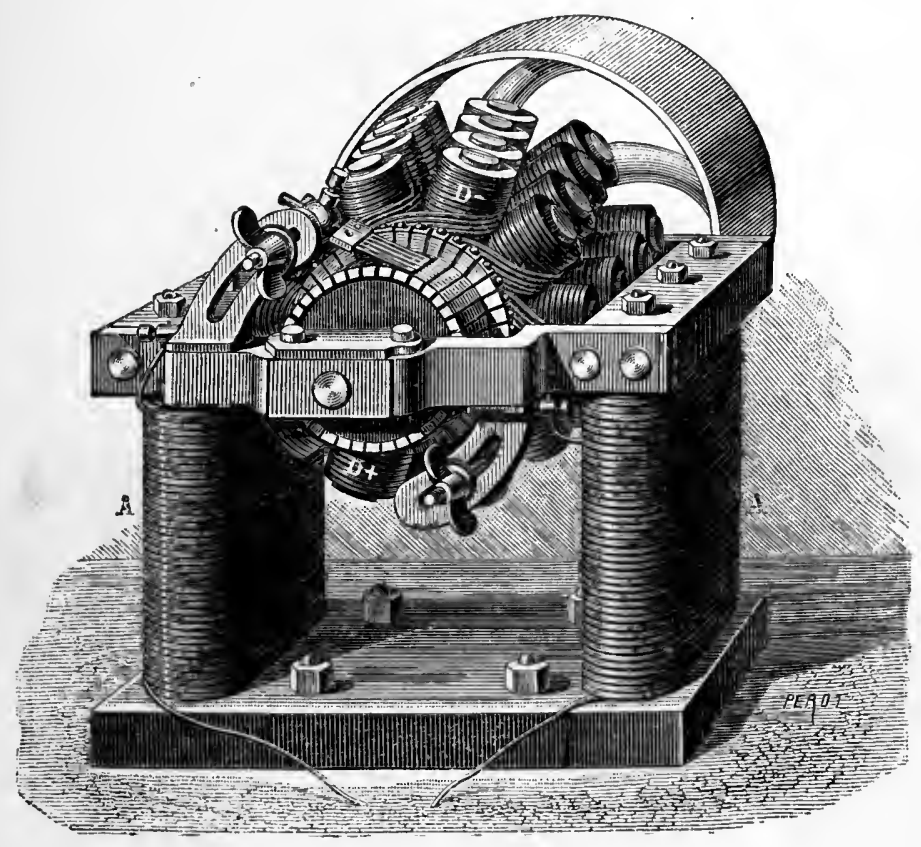

Fig. 36.-Lontin's continuous-current machine.

are placed after the manner of screws on each of these rows, and are provided with an iron core. They thus constitute a series of electro-magnets which, by turning between the poles of the inductor $\mathbf{A}$, assume polarities of different sense at each semi-revolution.

The currents thus produced are collected on a Gramme collector by brushes formed of very thin copper-foil. 
Both principle and theory of this machine are identical with those of Gramme's : the only point in which it differs from this latter is the special form of its armature.

Lontin uses this machine especially for the charging of the inductors of his alternating-current machine, which will be described on p. 116.

Siemens' Machine.-By this name are generally designated the machines invented by Hefner Alteneck, engineer in the employ of Messrs. Siemens, of Berlin. The machine

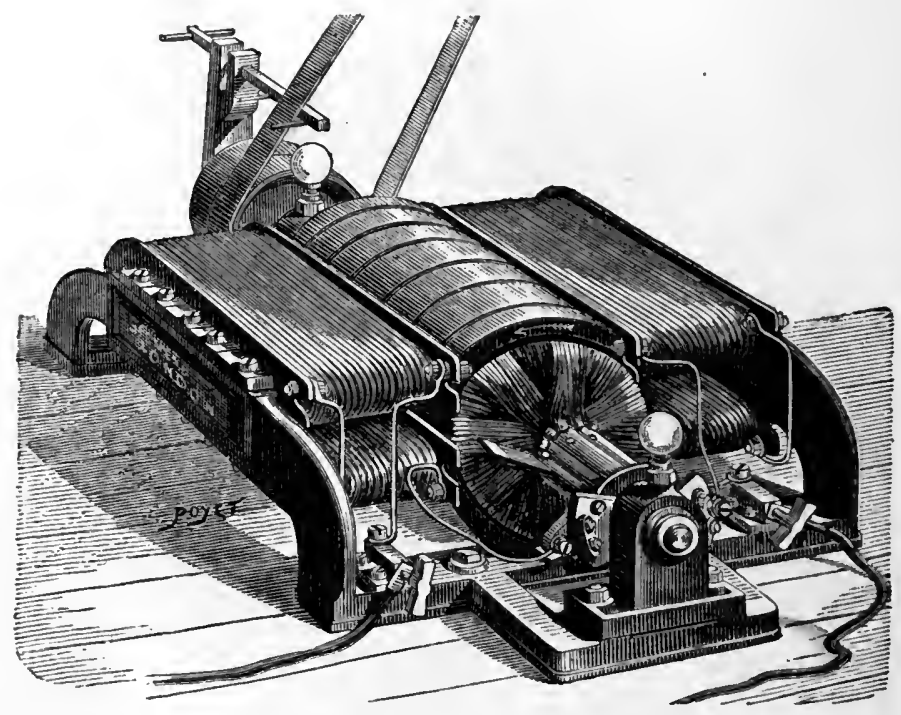

Fig. 37.-Siemens' continuous-current machine (middle size).

(Fig. 37) has a cylindrical armature. The induced coil, formed of sections connected with one another and with a Gramme collector, is of considerable length and coiled in a special manner; the wire only covering the external part of the supporting iron frame. The induction is, therefore, only produced by the exterior face which is presented 
to the inductors; and the thin iron frame, which replaces Gramme's wrought-iron ring, plays a very subordinate part.

The coiling of the wire is thus rendered easier, and the coil can be fixed and centred on its axis of rotation in a much simpler manner.

The inductors, formed of a series of iron plates, are slightly arched close to the coil; this" arrangement allows

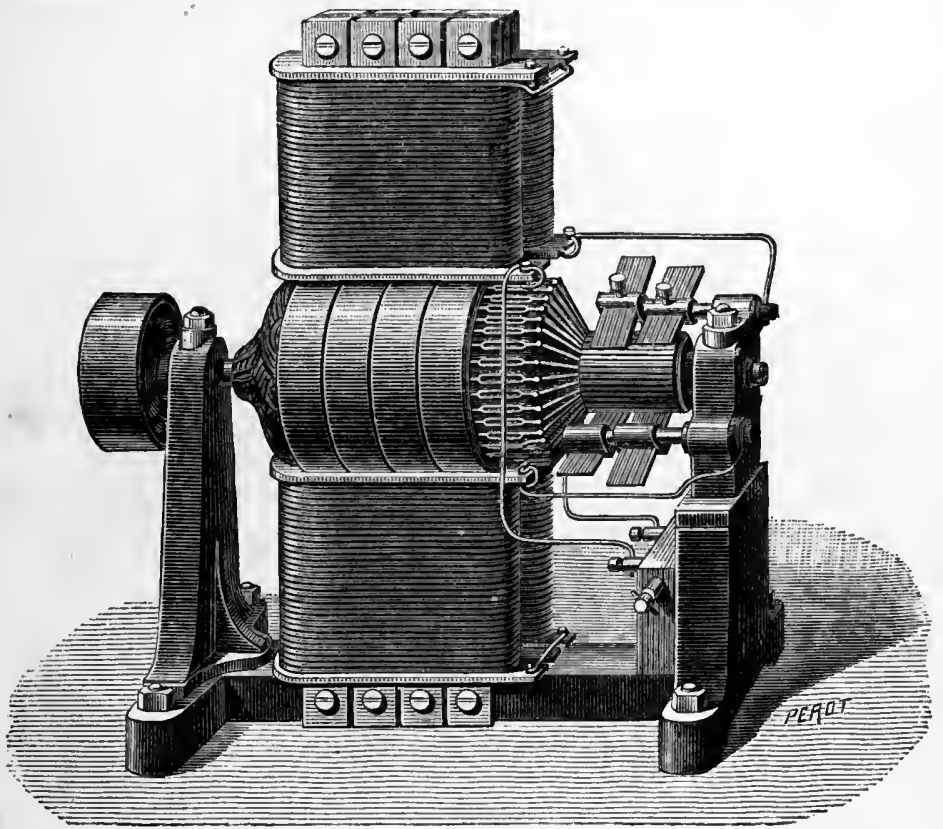

Fig. 38.-Siemens' continuons-current machine (small size).

of a thorough division of the magnetic field, imparting to this latter its maximum density at the very point where it ought to act with the greatest energy. The general form of the machine is also very favourable to its stability. The collector and the brushes are absolutely identical with those of Gramme's machine. 
In this form, and by simply varying the relative dimensions of the different parts and of the inducing and induced wires, the machine can be employed for galvano-plastic, electric lighting by arc or incandescent lamps, transmission of motive force, etc., like Gramme's machine.

The machine of smaller size (Fig. 38) is more especially used for charging the inductors of alternating-current machines, of which we shall speak hereafter, and differs from the larger size by the vertical position of its inductors,

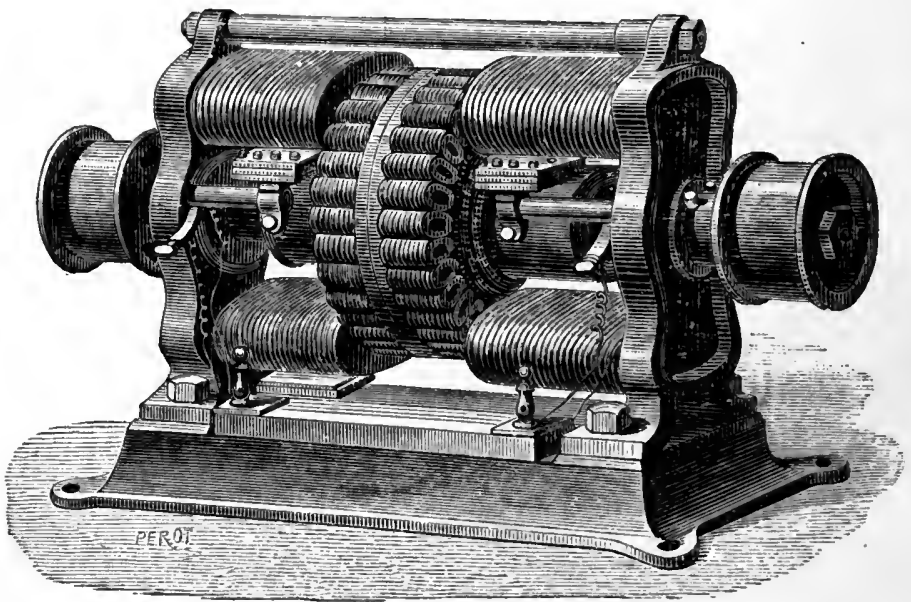

Fig. 39.-Wallace-Farmer's machine.

by which the induced coil is kept further away from the ground. The collecting brushes are double for each pole. By this arrangement, one metallic part of the collector is always in contact with one of the brushes when the other brush meets an insulated part, and the current is thereby rendered more regular.

Wallace-Farmer's Machine.-This machine, which owes its reputation to Edison, who recommended it before he 
invented his own machine, was invented by Moses Farmer, of Boston, in 1875, and subsequently improved by William Wallace, of Ansonia (Connecticut). It consists (Fig. 39) of a disc carrying two series of flat coils in order to utilize the surface of the discs more thoroughly; each of these coils is connected with the following, and with a Gramme's collector, as in Niaudet's machine (p.75). The inductors are

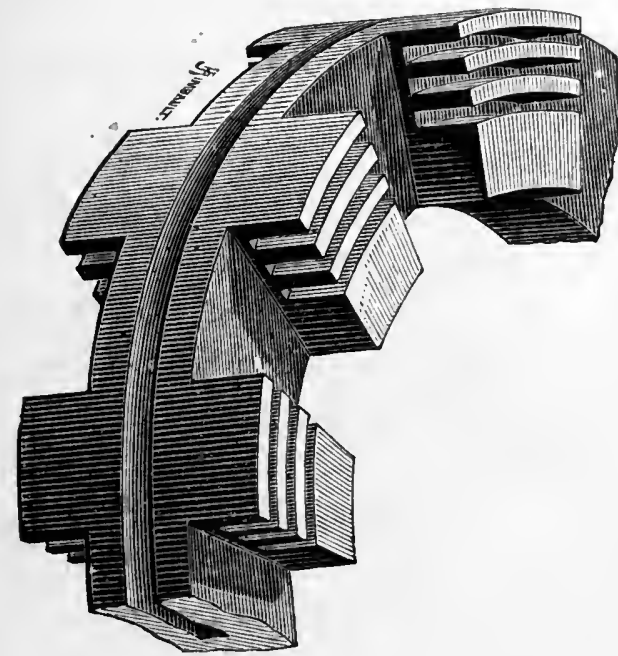

Fig. 40.-Portion of the armature ring, without coils.

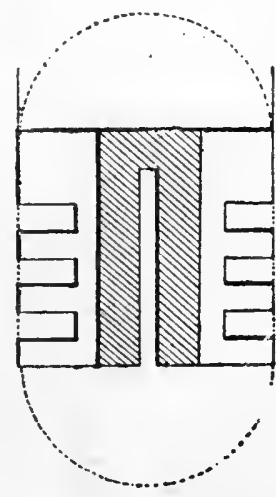

Fig. 41.-Cross-section of the armature ring.

arranged for tension in the external circuit. The machine is double, and each of its halves can act independently, or the two can be joined together, either for quantity or for tension.

The Brush Machine.-The most characteristic feature of the Brush machine lies in the form and construction of its armature, which consists of a cast-iron ring, the cross-section of which is generally rectangular, but in the direction of its circumference it is alternately wide and narrow, as shown in Fig. 40, which represents a portion voL. I. 
of the armature ring. On reference to Fig. 40, it will be seen that the ring is divided into as many sectors as there are bobbins to be wound, by a number of rectangular depressions or grooves; in these the coils of insulated copper wire are wound until the groove is filled up and the flat converging recesses become flush with the face of the intermediate thicker portions or pole-pieces by which they are separated from one another.

Fig. 41 is a cross-section of one side of the ring taken through one of these portions, and it will be observed that the intermediate thicker portions of the ring are grooved out by a series of deep concentric grooves, the object of which is partly to reduce the mass and lessen the weight of the revolving armature, partly for the purpose of ventilating the ring and thus carrying away a portion of the heat generated by the working of the machine, but chiefly for the localization and isolation of local currents generated by induction in the iron, and which would tend not only to reduce the efficiency of the machine by diminishing the magnetic capacity of the armature, but also to produce a heating of the ring, and therefore of the coils, whereby a portion of the current would be lost through their resistance being increased. For a similar reason, the periphery of the ring is grooved out deeply so as almost to sever the ring; by this means all cross currents are effectually cut off, and induction currents are compelled to flow in directions which are not detrimental to the efficiency of the machine. This again increases the area of radiating or cooling surface, and consequently helps to prevent the armature becoming overheated.

Fig. 42 is a sketch of the armature ring with all its coils wound; the two sides of each groove, and therefore of 
each coil of wire, are parallel to the centre line or radial plane of the coil, and by the adoption of that form of bobbin, one of the practical difficulties in the winding of annular armatures of the ordinary form is avoided. All the

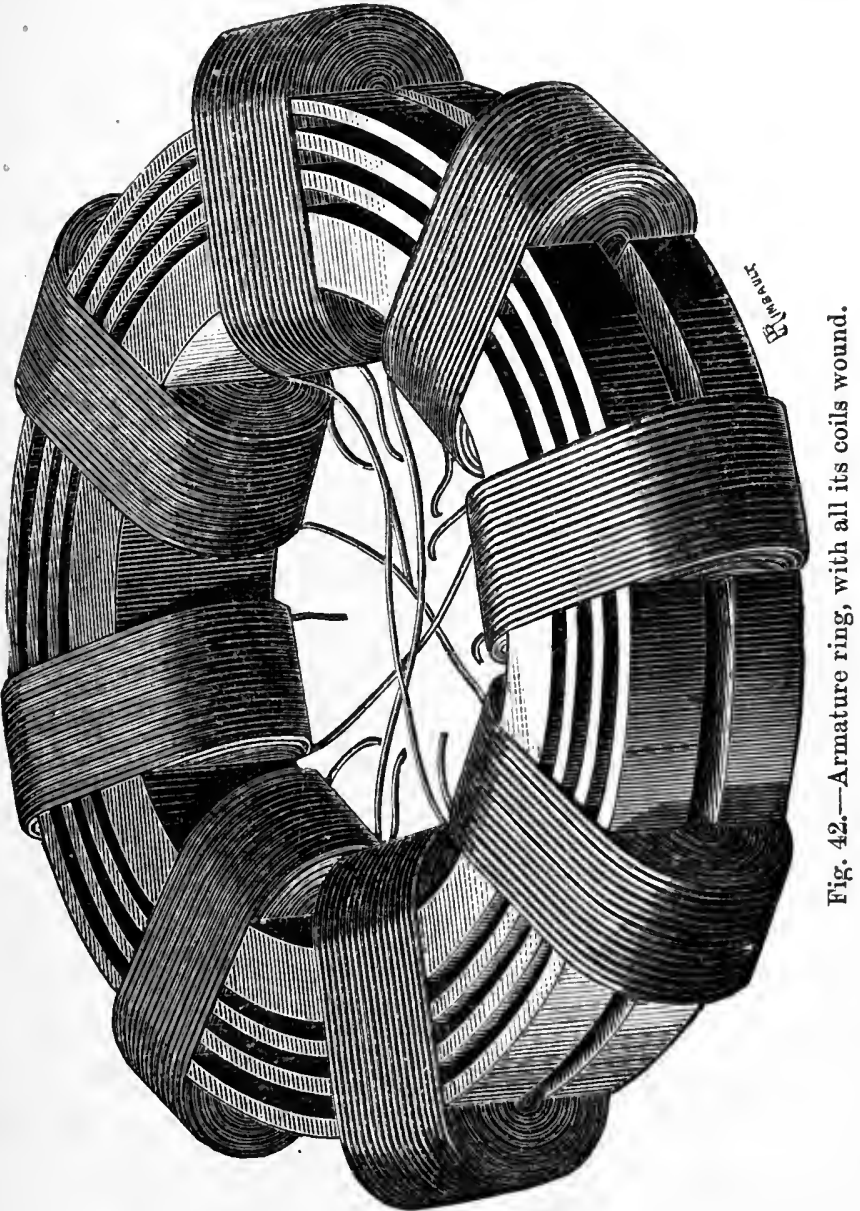

coils are, like those in the Gramme machine, wound in the same direction.

Fig. 43 shows the connections of the coils and the action of the commutator. The coils are connected in pairs, each 
to that diametrically opposite it, and carcfully insulated from those adjacent to them. For each pair of coils there is a separate commutator, so that for the ordinary ring of eight coils there are four distinct commutators side by side upon the axis. The brushes are arranged so as to touch at the same time the commutators of two pairs of coils, but never of two adjacent pairs, the adjacent commutators being always connected to two pairs

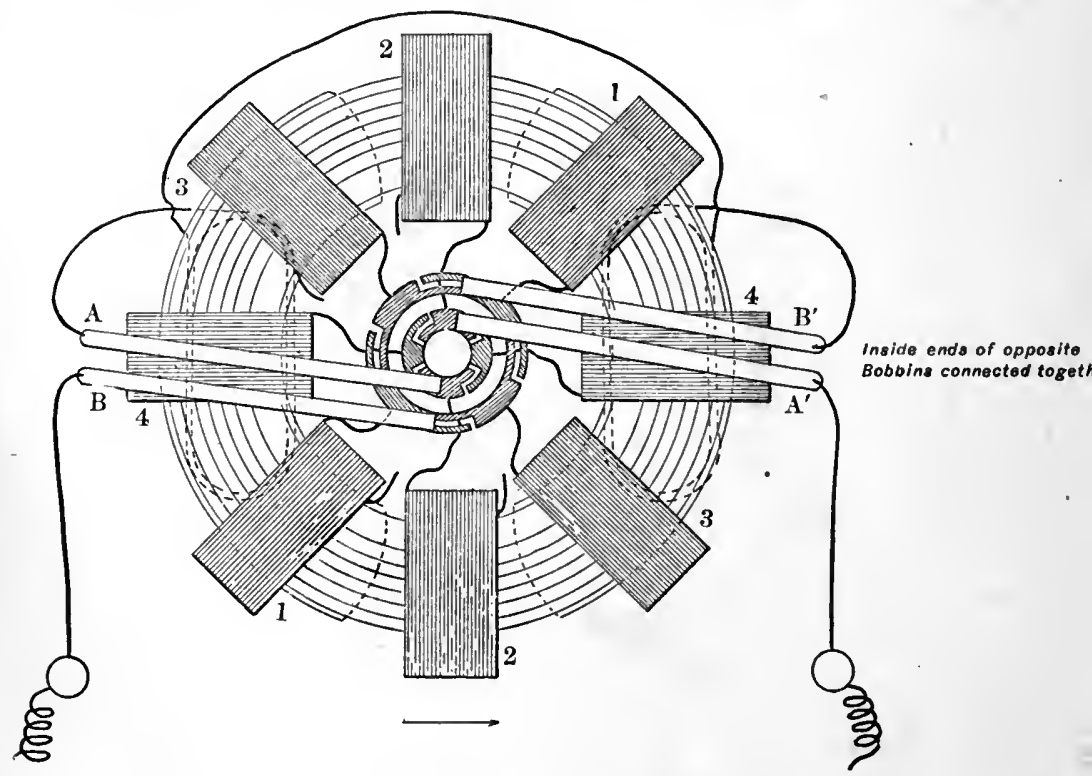

Fig. 43.-Connections of coils and commutator.

of coils that lie at right angles to one another in the ring. The arrangement is shown in Fig. 43, where each pair of coils has its own commutator, to which pass the outer ends of the wire of each coil, the inner ends of the two coils being united across to each other. In the machine, each pair of coils, as it passes through the position of least action (i.e. when its plane is at right angles to the direction of the 
lines of force ${ }^{1}$ in the field, and when the number of lines of force passing through it is a maximum, and the rate of change of these lines of force is a minimum), is cut out of connection. This is accomplished by causing the two halves of the commutator to be separated from one another by about one-eighth of the circumference at each side. In the figure it will be seen that the coils marked 22 are "cut out." Neither of the two halves of the commutator touches the brushes. In this position, however, the coils 4 , at right angles to 22 , are in the position of best action, and the current powerfully induced in them flows out of the brush marked $\mathbf{A}$ (which is, therefore, the negative brush) into that marked $A^{1}$. This brush is connected across to the brush marked B, where the current re-enters the armature. Now, the coils 11 have just left the position of best action, and the coils 33 are beginning to approach that position. Through both these pairs of coils, therefore, there will be a partial induction going on. Accordingly, it is arranged that the current, on passing into $B$, splits, part going through coils 11 , and part through 33 , and reuniting at the brush $\mathrm{B}^{\mathrm{l}}$, whence the current flows round the coils of the fieldmagnets to excite them, and then round the external circuit, and back to the brush A. (In some machines it is arranged that the current shall go round the field-magnets after leaving brush $\mathrm{A}^{\mathbf{1}}$, and before entering brush $\mathrm{B}$; in which case the action of the machine is sometimes, though not correctly, described as causing its coils, as they rotate, to feed the field-magnets and the external circuit alternately.)

1 We call a magnetic field a region subject to magnetic action; the intensity of a magnetic field at a given point can be measured by the deflection of a magnetic needle placed at this point. The position taken up by this needle indicates the direction of the resultant of the magnetic actions at this point, and the lines along which the needle sets arc called lines of force. 
The rotation of the armature will then bring coils 11 into the position of least action, when they will be cut out, and the same action is renewed with only a slight change in the order of operation. From this it follows that, whichever pair of coils is in the position of best action, is delivering its current direct into the circuit; whilst the two pairs of coils which occupy the secondary positions are always joined in parallel, the same pair of brushes touching the respective commutators of both.

The four pairs of coils of the Brush machine really constitute four separate machines, each delivering alternate currents to a commutator, which commutes them to intermittent uni-directional currents in the brushes; and these independent machines are ingeniously united by the device of letting one pair of brushes press against the commutators of two pairs of coils; these paired machines are then connected in series, by bringing a connection round from brush A to brush B.

The commutator, which is attached to and rotates with

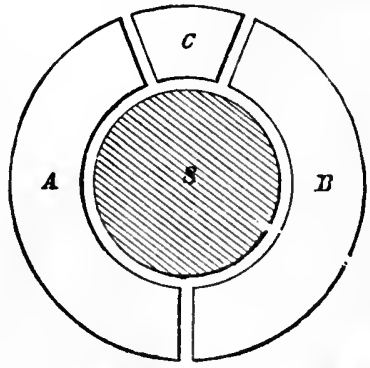

Fig. 44.-Section of a copper ring of the commutator. the driving shaft of the machine, consists of a set of copper rings or flat cylinders, of which there are as many on the shaft as there are pairs of coils on the armature, and each of these cylinders consists of two segments insulated from one another on ons side of the shaft by a small air space about oneeighth of an inch wide, and on the other by a piece of copper separated from the segments by two smaller air spaces. The arrangement is shown in Fig. 44, in which $A$ and $B$ are the two segments connected respectively to corresponding coils 
on opposite sides of the armature, and attached by an insulating material to the shaft $\mathrm{S}$; $\mathrm{C}$ is the copper insulating piece, the object of which is to separate either of the flat copper brushes or collectors, which press upon the periphery of the commutator, from either of the segments during the interval occupied by one pair of coils passing the vertical, or in other words, through the neutral portion of the magnetic field; this occurs twice in each revolution of the armature, and therefore of the commutator.

Fig. 45 is a diagram illustrating the connection between the armature bobbins and the magnet coils at the time

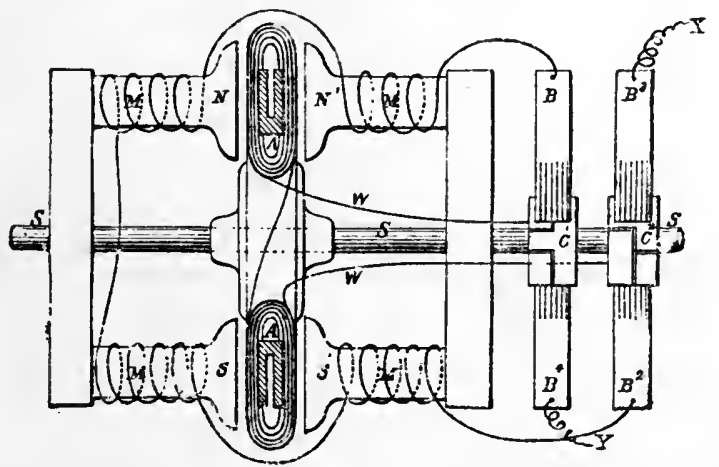

Fig. 45.-Connection between armature bobbins and magnet coils.

when the commutators are placing them in the same circuit. Referring to this diagram, $M$ M and $M$ M are the two magnets having their similar poles presented towards one another on opposite sides of the armature coils $A A^{1}$. Thus the coil $\mathrm{A}$ is under the influence of a magnetic field produced by the two north poles $\mathrm{N} \mathrm{N}^{1}$, while at the same time its corresponding bobbin $\mathrm{A}^{\mathrm{x}}$ is under the influence of the two south poles $\mathrm{S} \mathrm{S}^{1}$. A current is therefore induced in the pair of bobbins $\mathrm{A} \mathrm{A}^{\mathrm{l}}$, which is transmitted by wires passing through the shaft $\mathrm{S}$ to the commutator $\mathrm{C}^{1} \mathrm{C}^{2}$, whence it is 


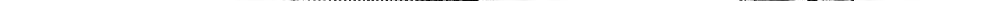


collected by the brushes $\mathrm{B}^{1}$ and $\mathrm{B}^{2}$, and by them transmitted to the magnet coils, which are all connected together in series, and at the same time, the other portions of the commutators (which are in connection with the other working armature bobbins) are in contact with the brushes $\mathrm{B}^{3}$ and $\mathrm{B}^{4}$, by whieh they are placed into the external circuit of the machine.

The commutators are a special feature of the Brush system; and the arrangement by which the position of the collecting brushes can be adjusted, so as to place them in contact with the commutators in any desired angular direction with respect to the position in the magnetic field occupied by the several bobbins to which the commutators are connected, is exceedingly simple and ingenious.

The commutating apparatus is represented on the extreme right of the general view of the machine (Fig. 46), and consists of two pairs of rings, of the form shown in Fig. 44, attached to and revolving with the main shaft, and, therefore, their position is fixed with respect to the revolving armature of the machine. On to the cylindrical circumferences of these rings are pressed two pairs of copper collecting brushes, which rub tangentially against the commutator rings, one pair pressing above and the other pair pressing below; a line forming the points of contact being a diameter of the ring. The copper "brushes," as they are called, are flat strips of elastic copper, about two inches wide, cut at the ends which press against the rings, into eight tongues, so as somewhat to resemble a grainer's comb, and each comb or brush is wide enough to cover or be in contact with two armature rings, and in this way, although two of the coils are insulated twice in each revolution, the main circuit is never interrupted.

For the purpose of adjusting the brushes so as to make 
contact with the commutators at the most effective angular position with respect to the magnetic field, they are mounted to the two opposite ends of two rocking levers, which are capable of oscillating on the driving shaft, and can be fixed in any desired position by means of a setserew which clamps a stout wire issuing from the base of the machine. The currents are conveyed from the brushes by wide strips of thin sheet copper, shown in the general view, and in order to allow for the variable distances of the free ends of the brushes from the base of the machine, they are made undulating or wavy, doubling up as the distance is shortened, and stretching out when it is increased.

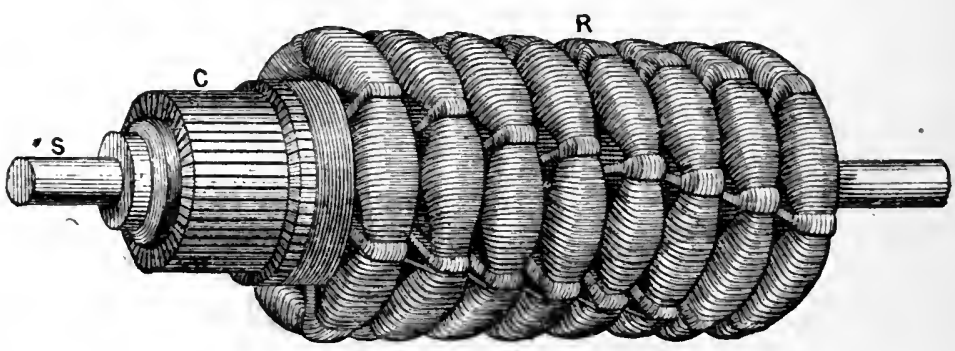

Fig. 47.-Armature of Bürgin's machine.

Bürgin's Machine.-In 1877, Bürgin, of Bâle, in order to bring the mass of the ring closer to the inductor, constructed a dynamo-electric machine, in which the induction is effected on eight distinct rings $R$, mounted alongside one another on the same shaft (Fig. 47). The magnetic core of each ring is a square frame of iron wire, round which are wound the spindle-shaped serews forming the armature, thicker in the middle, so that the cormers of the square frame are nearly in the same circumference as the exterior spirals of the spindle-shaped screws, and thus pass very close by the poles of the inductors. The cight rings are 
mounted in somewhat slanting direction, so that the coils present themselves in suecession before the inductors, as in Lontin's magnetic pinion. The forty-eight coils are connected with one another, and also with a eollector $\mathrm{C}$, like the coils of Gramme's machine, and the working of the two is identical.

The inductors are of cast iron, and mounted for tension on the general circuit. This machine has the advantage of being simple, of easy construction, and allows of a precise adjustment of the rings on the axis of rotation.

Crompton's Compound Machine.-The want of some sort of automatic apparatus which would regulate the current to

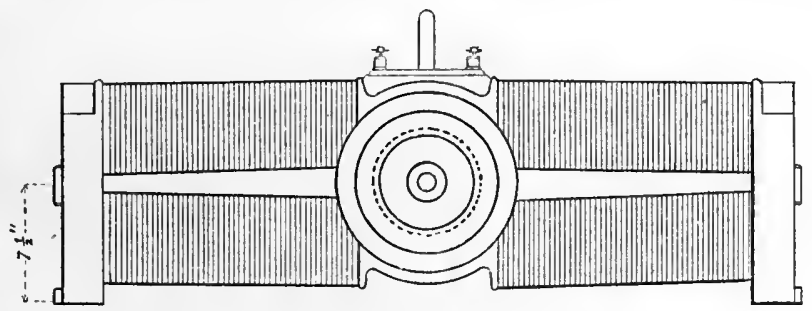

Fig. 48.-Crompton machine (side elevation).

the number of lamps in eireuit has long been felt, and several electro-mechanical devices have been invented and added to ordinary machines, with a view of making them suitable for this class of work. But if it be remembered that any kind of exterior regulator can only begin to act after the disturbanee which it is intended to avoid has already commenced to take place, and that even the most delicate meehanieal contrivance must necessarily be clumsy and sluggish in comparison with the lightning speed with which electrical charges are propagated, there seems little hope that regulation can ever be obtained by such means. Yet the electro-mechanical governor ought not to be altogether disearded; its proper 
place should be to assist the centrifugal governor of the engine in keeping the right speed. The regulation of Crompton's compound machine, although perfectly sufficient to protect the lamps from injury, and giving them a current which is constant enough for most cases, is not mathematically perfect over the whole range for which the machine is designed, and therefore it is desirable to have an apparatus which will vary the speed of the engine, so as to make the regulation mathematically perfect.

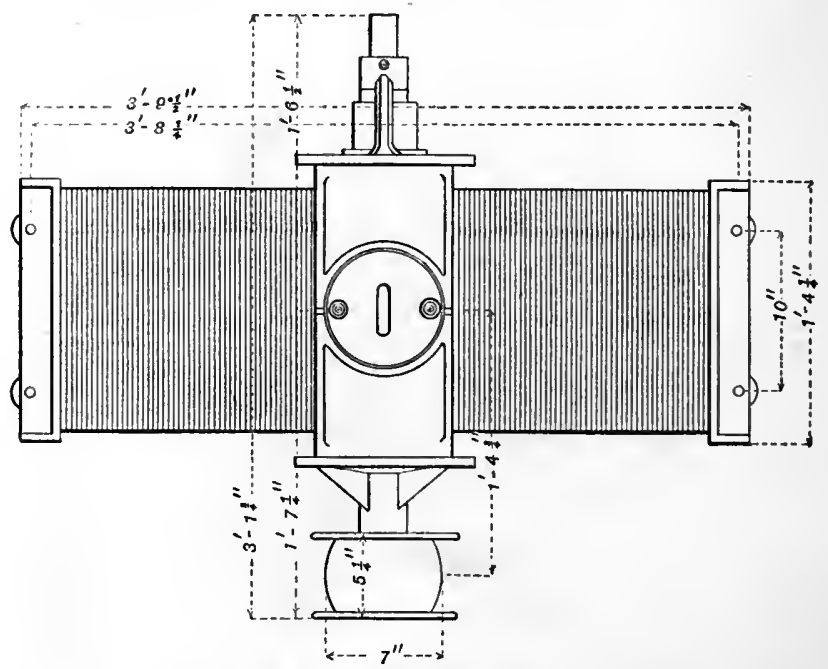

Fig. 49.-Crompton machíne (plan).

The object of maintaining a constant electro-motive force in the external circuit is obtained by a combined system of shunt and main coils. The machine shown in Figs. 48, 49 50 , is, in general appearance, like an ordinary CromptonBürgin dynamo, but with the arms of the field-magnets sixteen inches long instead of eight, and the pole-pieces heavier than usual. The magnets are excited, partly by the main current, which is split up into four exciting coils 
wound round the four arms of the field-magnets, and reunited after having traversed them; and partly by a shunt current, which flows in series round the fine-wire coils, which are laid on the top of the main coils. The terminals of the shunt coils are coupled direct to the two brushes.

The object of splitting up the main current into four parts is to reduce the resistance of the main exciting coils, without unduly increasing the size of wire, which would be a practical difficulty for even winding.

It may be mentioned that the electro-motive force of the machine has been increased through inclining the arms of the field-magnets, whereby the length of yoke is diminished.

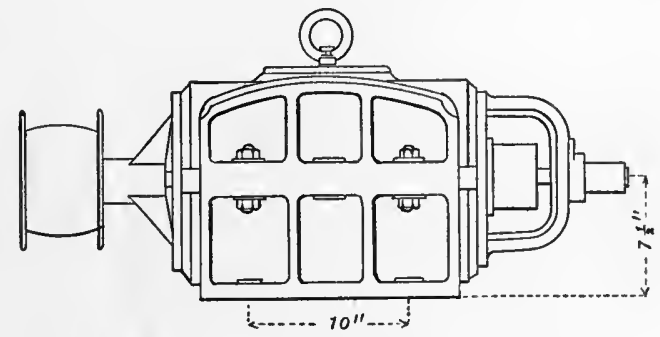

Fig. 50.-Crompton machine (end elevation).

The speed of this machine is from 1400 to 1800 revolutions, according to the electro-motive force required.

Edison's Machine.-This machine consists of twelve castiron field-magnets connected in two series of six, the series having a resistance of $12 \cdot 23$ and $12 \cdot 22 \mathrm{ohms}$. The two series are connected in multiple are, the aggregate resistance of the field-magnets being thus reduced to $6.11 \mathrm{ohms}$. The magnets are wound with No. 10 wire; their poles are massive pieces of cast iron. These enormous magnets have excited considerable attention from time to time, and a good deal of adverse criticism has been made upon them; but Mr. Edison strongly maintains the wisdom of a large invest- 
ment in iron, which is cheap, and which allows him a large dividend in the utilization of energy. The armature consists in the first place of a steel shaft, over which is fitted a wooden cylinder. On the central portion of this compound cylinder a large number of thin discs of sheet iron are slipped, the discs being separated from each other by sheets of tissue paper of similar diameter. There are, however, eight intermittent iron dises of a quarter of an inch in thickness,

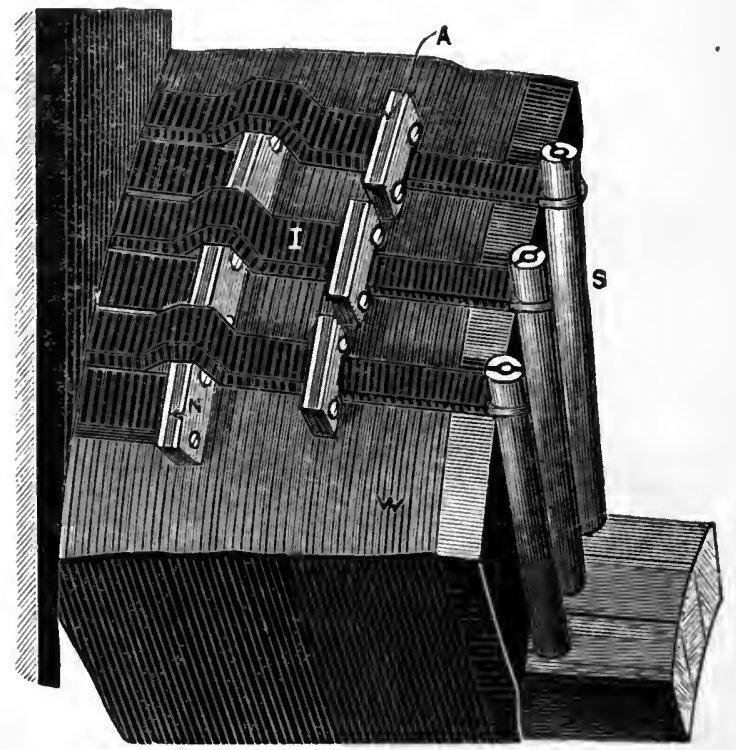

Fig. 51.-Armature of the Edison machine (conuections made).

to give the necessary strength, and the whole is bolted together by eight bolts. These iron dises form the core of the armature. Copper dises of a somewhat larger diameter than the iron discs are placed at each end of the built-up core. There are fifty-three of these discs wound with $0 \cdot 102$ wire, with alternate discs of prepared paper for insulation. These copper dises have each two lugs on their circumference, to which the armature rods are connected. In the details 
shown in Fig. $51, \mathrm{~W}$ indicates the copper disc, A the lug, I the armature rod, $\mathrm{S}$ the connection to the commutator. The arrangement is shown both when the connections are made (Fig. 51) and when disconnected (Fig. 52).

The special feature of the machine is the use of copper rods I to take the place of the usual windings of wire; they are 106 in number, narrowing slightly to the inside face, with rounded edges. These rods are wrapped by nachinery with prepared paper, the layers of paper being each coated with a special insulating material. The

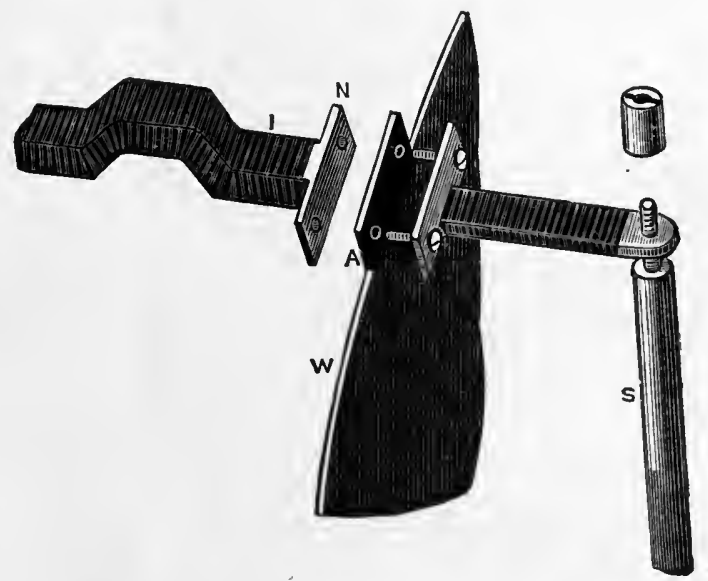

Fig. 52.-Details of armature (disconnected).

rods are separated from each other at every foot of their length by pieces of vulcanite fibre encased in mica and also by an air space. Every alternate rod is connected through a radial copper rod with the commutator. The blocks of the commutator K (Fig. 53) are fifty-three in number, and taper in a similar manner to the armature bars; they are bevelled at the ends and separated by strips of mica. At the back of the radial bars, and acting as a facing to the copper discs, is a mahogany disc. The ccmplete armature is 
wound with steel wire, with double mica insulation underneath ; this wire is intended to counteract any centrifugal action. The bar upon which the brush-holders are fastened is shown at $\mathrm{H}$ (Fig. 53); the brushes are connected with the main conductors $\mathrm{C}$, through levers as shown, and can be changed as regards position in the commutator as required. The drawing shows clearly how this is done. So careful has the inventor been to consider everything connected with the machine, that, to prevent oxidation, the threads of the radial bars, the lugs on the armature, bars, discs, serews, and other contacts are lightly plated with gold. This perfection of contacts, together with the large sectional area of the armature rods, ensures low resistance.

The engine is forty horse-power nominal, wo:king up to about 125 horse-power indicated. The engine is connected direct to the shaft of the armature, which revolves at the rate of 350 revolutions per minute. This machine is intended to supply the current for 1000 Edison lamps of sixteen candle-power each.

In the engraving $\mathbf{A} \mathbf{A}$ shows the channels by which air is driven to circulate around and keep the commutator cool.

The Weston Machine.-The machine represented in Fig. 54 has, by the arrangement of the field magnets, a eertain resemblance to one of the forms of the Gramme machine. These inductors consist of six cylindrical horizontal electro-magnets, whose poles are joined to two polar pieces placed one above the other below the armature. The coils of these electro-magnets are coupled for series and traversed by the current of the machine. The armature performs about 900 revolutions per minute. The polar pieces which surround this latter,instead of being continuous, are cut up into grooves T, T, T, T', T', T' (Fig. 55). The polar pieces 
$\therefore$ is. 


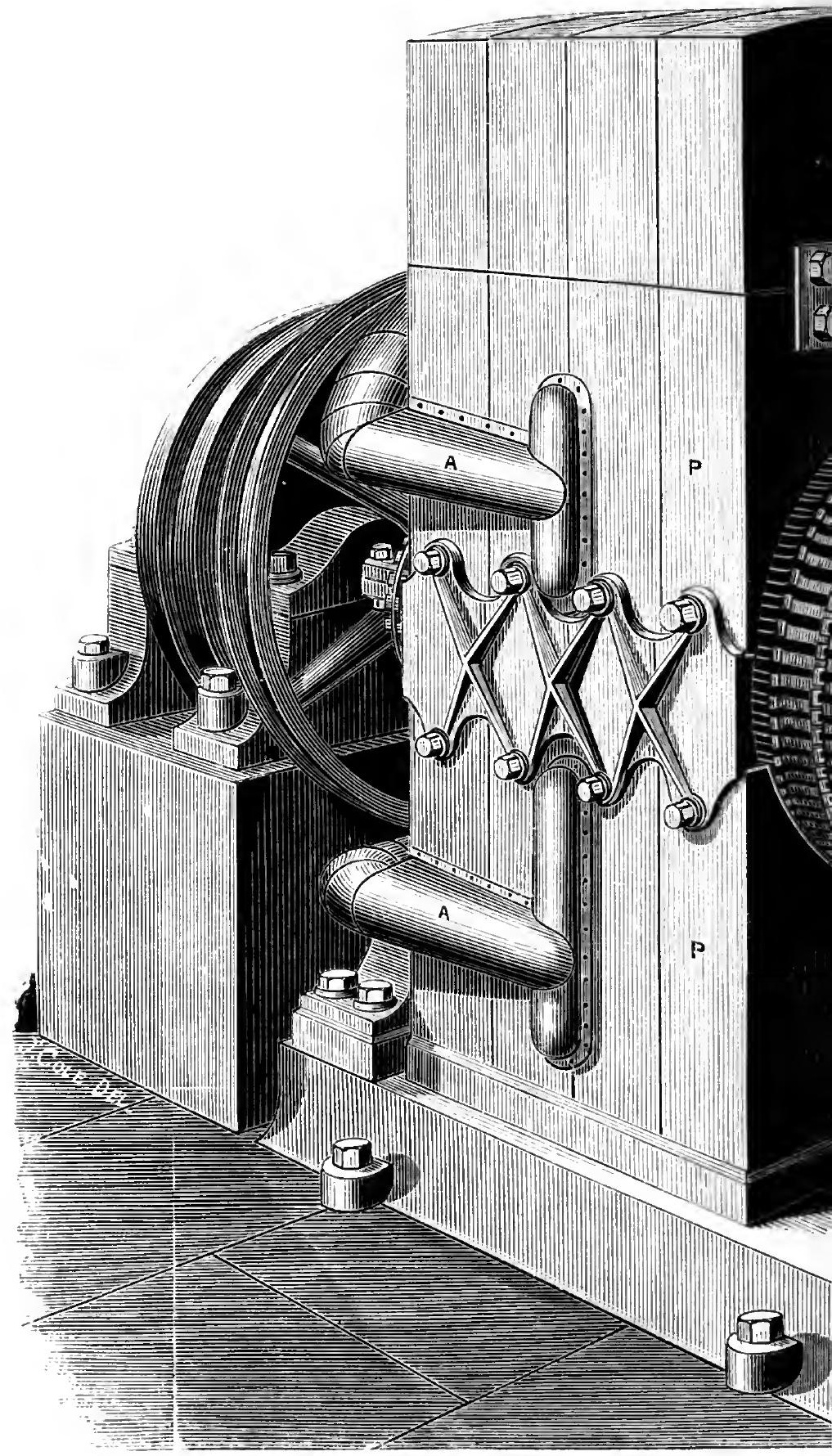

Fig. 53. 


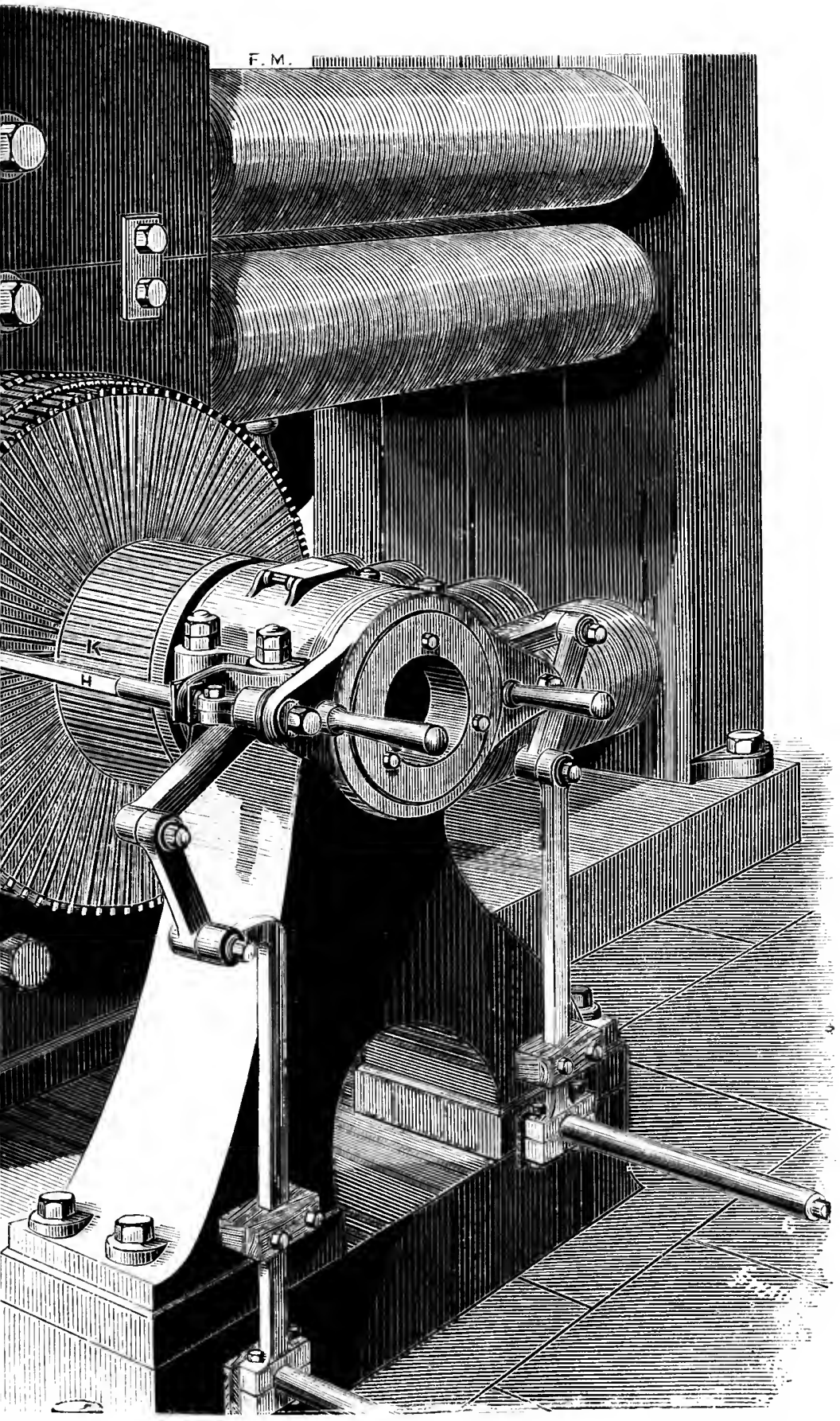



ELECTRIC GENERATORS.

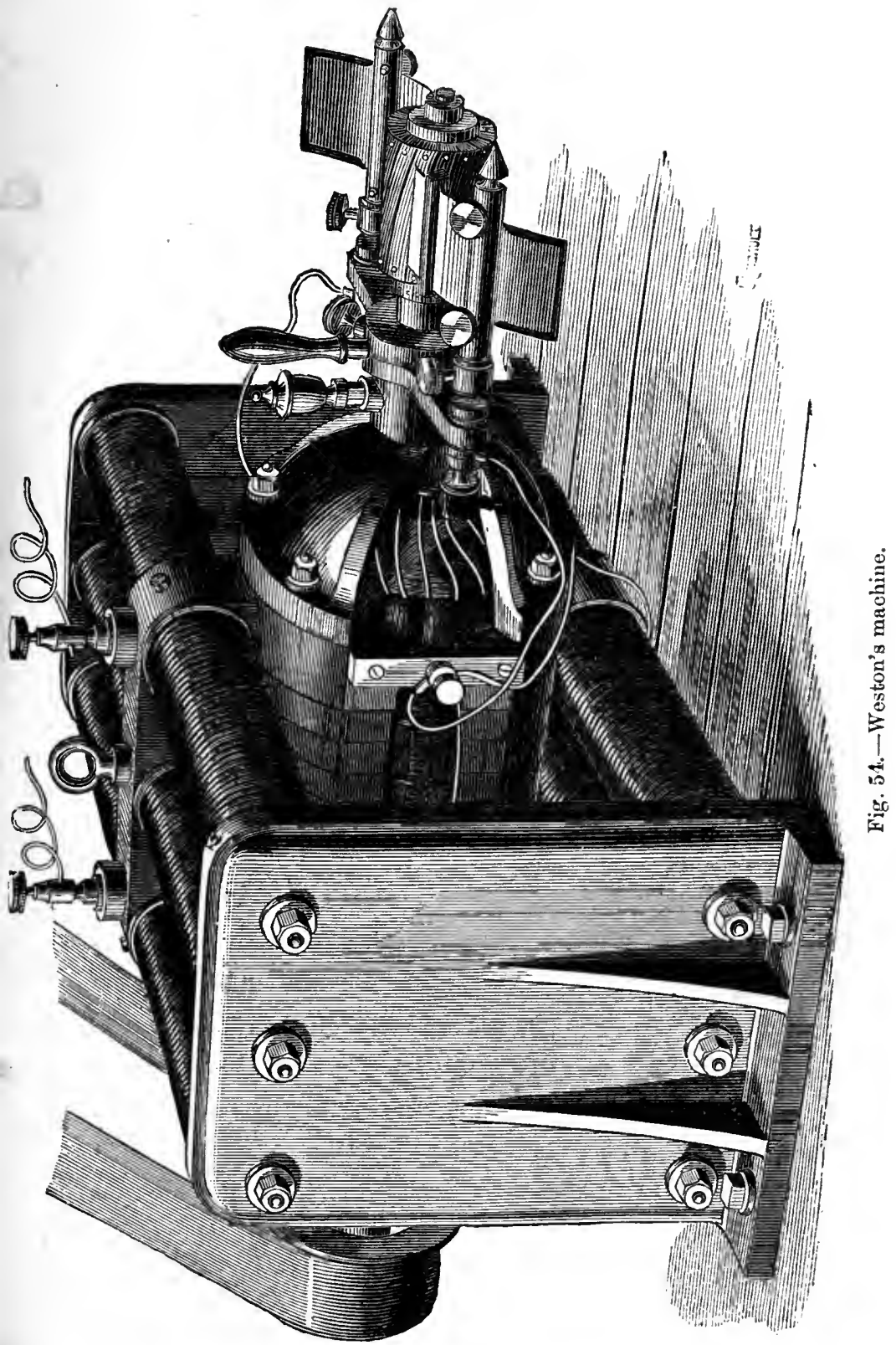

VOL. I. 
in this figure are represented as they would be seen from one of the ends of the machine after the plates at the end

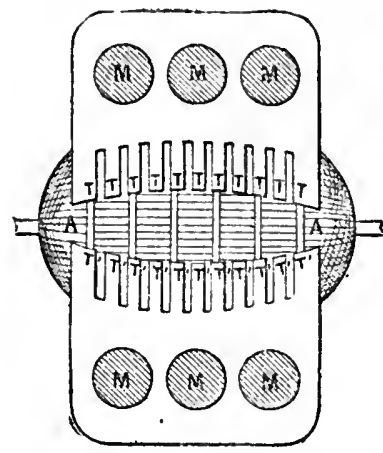

Fig. 55.-Polar pieces of the armature. and the coils had been removed. It will also be seen that the space between the two polar pieces is larger in the centre than towards the extremities of the armature, the length of the grooves diminishing towards the centre. According to Weston, a greater regularity of current is ensured by this arrangement, as the induction of the armature coils is effected from the centre towards the extremities, and vice versa, instead of taking place simultaneously along the entire length of one of the armature clements. The grooves in the polar pieces are meant to prevent the production, in the iron mass, of Foucault's currents, and further to facilitate the cooling, by ventilation, of the polar pieces and of the armature.

This latter has quite a particular construction. The cylinder on which the wire is wound, instead of being of

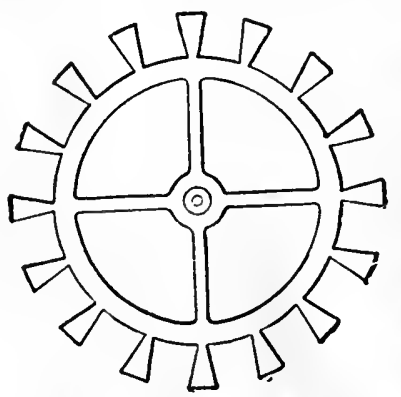

Fig. 56.-Armature-wheel. same diameter as the discs are fastened to the shaft to keep the toothed wheels and dises in their places. The armature has now the form of a 
cylinder, with hemispherical extremities and sixteen longitudinal grooves. These grooves contain the wire coils, each group of wire being placed in a special groove. Perfect ventilation is thus ensured, the armature being not only hollow but having $16 \times 36$, or 576 , openings. The hemispherical extremities are also provided with windows, and a continuous circulation of air is thus established in the apparatus.

The collector shown in Fig. 54 is similar in principle to the Gramme collector, but the plates have a slightly curved shape, and are only separated by air instead of a solid insulator. The object in giving the plates that peculiar shape, is to obtain a continuous contact of the brushes with the two plates, and thus to ensure greater regularity of current. The brushes are formed of a dozen separate picces of thin sheet copper, the edges of which press against the curved plates. The brushes are besides mounted on a movable support, which allows of their being placed in a convenient position.

Fein's Machine.-In this machine the ring is fastened to the shaft in such a manner that nearly the whole length of wire is energized by the inductors. Fig. 57 represents the section and Fig. 58 the perspective of the apparatus.

The ring $\mathrm{R} R$ is fastened by means of screws and screwnuts to an indented piece of brass $\mathrm{S} \mathrm{S}$, which is bolted on the shaft $a c$, and, together with the shaft, driven by the flywheel J. The extremities of the wires of each group pass either between the branches of each indented piece or through holes pierced in these branches and lined with some insulating material, and are all finally connected to the commutator $\mathrm{C}$. This latter, for the sake of convenience, is placed outside the mounting of the machine. 
The current is, as usual, collected by two brushes B and B. In order to avoid the formation of Foucault's currents, and the rise of temperature consequent upon it, the ring is formed of a series of very thin iron plates insulated from one another.

The cores of the field-magnets $\mathrm{E}$ E are connected to the armatures $\mathrm{M} \mathrm{M}^{\prime}$, which enclose the ring, and to these armatures are serewed the funnel-shaped polar pieces $\mathbf{A}$ A,

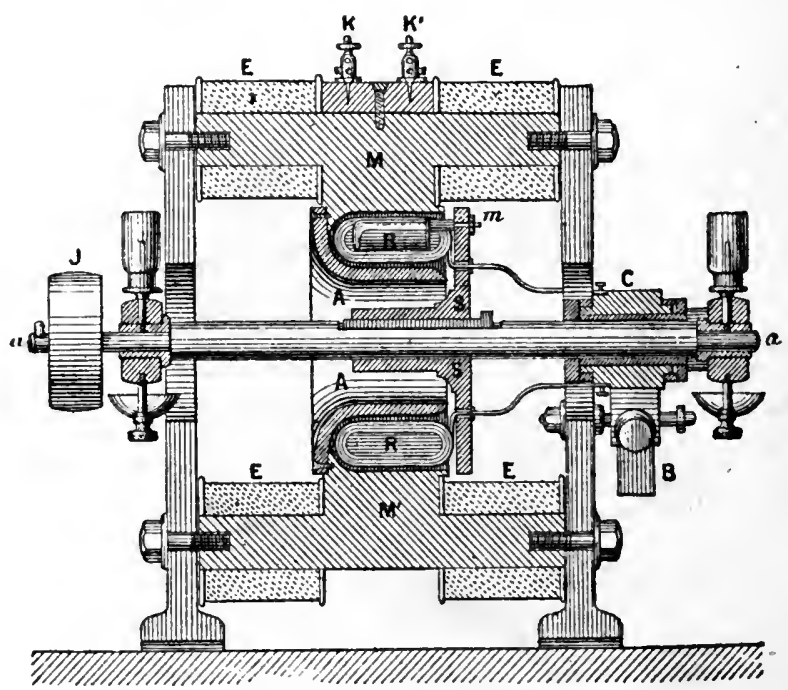

Fig. 57.-Fein's machine (section).

which surround the interior and one of the sides of the ring, as shown in Fig. 57.

By this arrangement nearly the whole length of wire is acted on by the inductors, and the only part which does not contribute to the production of electro-motive force is the part fixed to the indented piece.

It is very easy to ascertain that this arrangement incrcases the efficiency of the machine. After having worked 
it in its normal condition, we have only to take out the interior polar pieces and work the machine like an ordinary Gramme; the intensity of the current will now scarcely be one-half of what it was originally.

The advantages of this mode of construction may therefore be summed up as follows:-

1. The wires of the ring are, almost in their entire length, exposed to the action of the inductors, and the

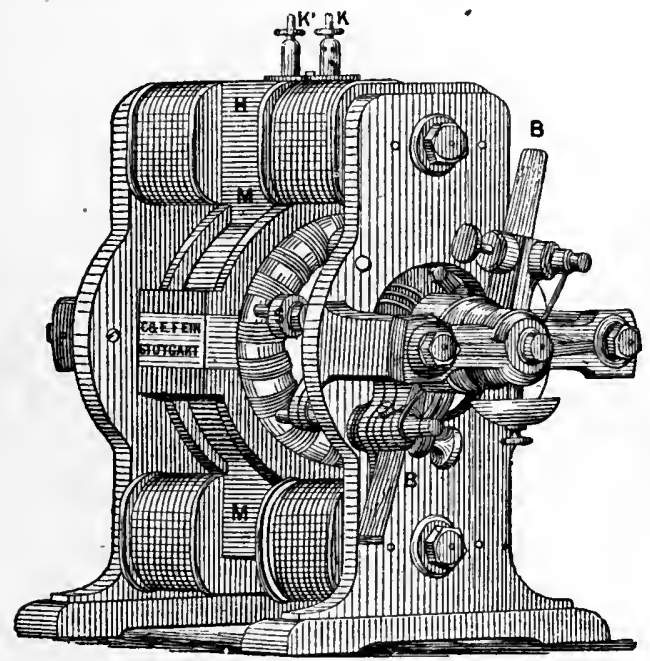

Fig. 58.-Fein's machine (perspective).

resistance in those parts of the wire which do not produce any electro-motive force is very feeble.

2. The arrangement of the ring is such that the formation of Foucault's currents is nearly eliminated; the loss of work through overheating is therefore very small.

3. The ring, being of moderate size, rotates very easily, and the machine, in proportion to the effects which it produces, expends but a small amount of work.

The Jürgensen Machine.-This machine, as will be seen 
from Figs. 59 and 60, is a modification of the Gramme machine.

The inductors $a_{1} a_{3}$, placed on the foundation plate $a_{1}$ form a large horseshoe electro-magnet, with polar enlargements. To prevent the deleterious vibrations of the two arms, their poles are joined at the top by an insulated plate, and sustained below by two insulated supports $l l$. The coiling of the wire of these field-magnets presents this peculiarity, that the number of coils increases in proportion as they approach the polar enlargements: this

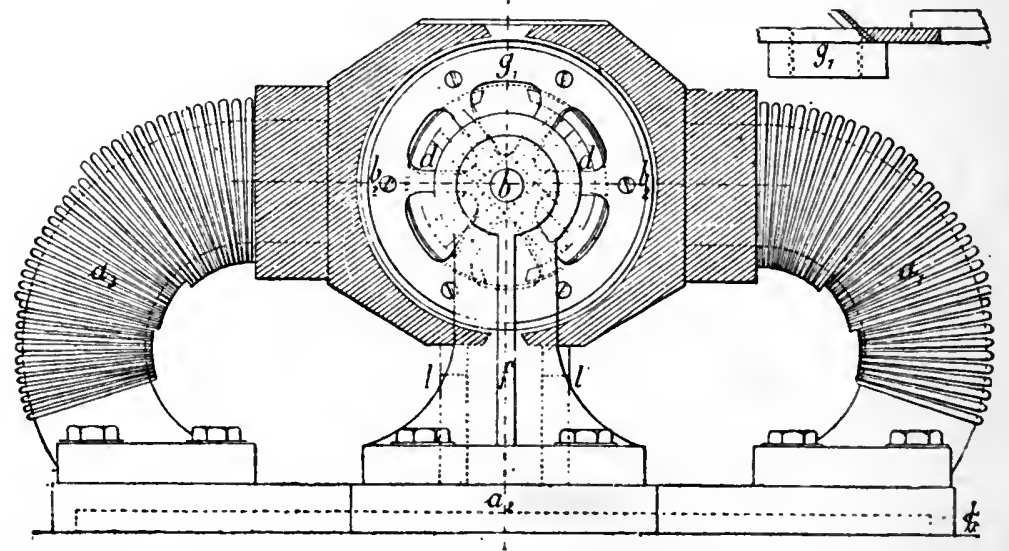

Fig. 59.-The Jürgensen machine (elevation).

arrangement is intended to concentrate as much as possible the magnetic intensity towards the poles. There is also a certain amount of empty space left between the individual wire coils, in order to facilitate the circulation of air and to diminish the heating of the wires. In addition to these field-magnets, the machine contains yet another system of electro-magnets placed inside the revolving armature. These electro-magnets are flat, slightly shorter than the armature, and arranged in the form of a cross. 'The poles of the same name are placed alongside one another, and are joined by a 
common piece of soft iron. Two interior poles are thus obtained facing the external poles of the same name, and a very powerful magnetization of the armature ensues.

This latter is composed of a certair number of iron rings $b_{1}$ of the same size, insulated from each other, and joined by transverse pins along the whole length of the armature. At each of its extremities the iron cylinder of the armature carries three spindles $b_{3}$, on which are fixed the copper discs $g_{1}$ and $g_{2}$. One of them $g_{1}$ carries a steel ring, turning freely round the spindle $e$ whose projecting part

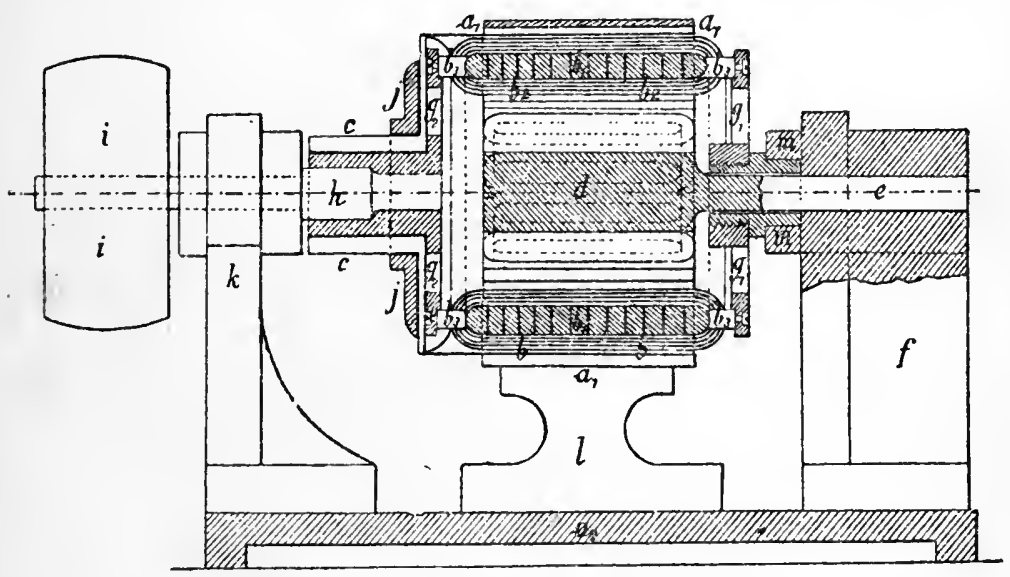

Fig. 60.-The Jürgensen machine (section).

revolves in the bearings $m$ of the main standard $f$. The other disc $g_{2}$ is attached to the driving shaft $h$, which carries the pulley $i$. The dises $g_{1}$ and $g_{2}$ are each pierced by six openings, the edges of which are bevelled off or provided with fans, as will be seen from the small sketch annexed to Fig. 59; in this way the cylinder, in turning, acts as a ventilator, and sends a strong current of air into the interior of the machine, which prevents the overheating of the armature. The massive plates $g_{1}$ and $g_{2}$ also contribute, by their conductivity, to the cooling of the iron cylinder. 
The armature wires are coiled as in Gramme's machine, and the collector is also the same, only Jürgensen, instead of employing two brushes, uses four. They are carried by the upright piece $k$, can be rotated round the axis of the machine, and are thus placed in the most convenient position.

When the two principal brushes alone are employed, the current traverses first of all the field-magnets, and then the external circuit, unless a special exciting machine is used. The auxiliary brushes are intended for taking a shunted current from the collector, by means of which the field magnets are excited.

The original parts of Jiirgensen's machine are, first of all, the electro-magnet inside the armature; secondly, the special coiling of the wires of the field-magnets; and thirdly, the effective ventilation produced by the rotation of the armature. This latter arrangement is advantageous because it lessens the heating of the machine.

Hefner Alteneck's Machine.-By an ingenious arrangement of commutators and collectors, Hefner Alteneck succeeded in transforming Siemens' alternating-current machine into a continuous-current machine. The result is interesting, in so far as it shows the prevailing tendency of inventors to create continuous-current machines, which are capable of a more general application than machines with alternating currents.

The Arago Machine.-Machines in which a circular series of coils or of electro-magnets successively traverses alternate magnetic fields are for the most part alternating-current machines. Such are, for instance, the Alliance, Lontin's, De Méritens', Siemens', etc., machines, which will be found on p. 109 and following. There has been a tendency, however, to construct, with the same arrangement, continuous- 
current machines, and the Arago machine belongs to this kind of apparatus, as well as Hefner Alteneck's and Hopkinson Muirhead's machines.

The Arago machine, constructed by the American Ball, consists of two electro-magnets, with two coils E E (Fig 61) fastened to the mounting of the machine. These electro-

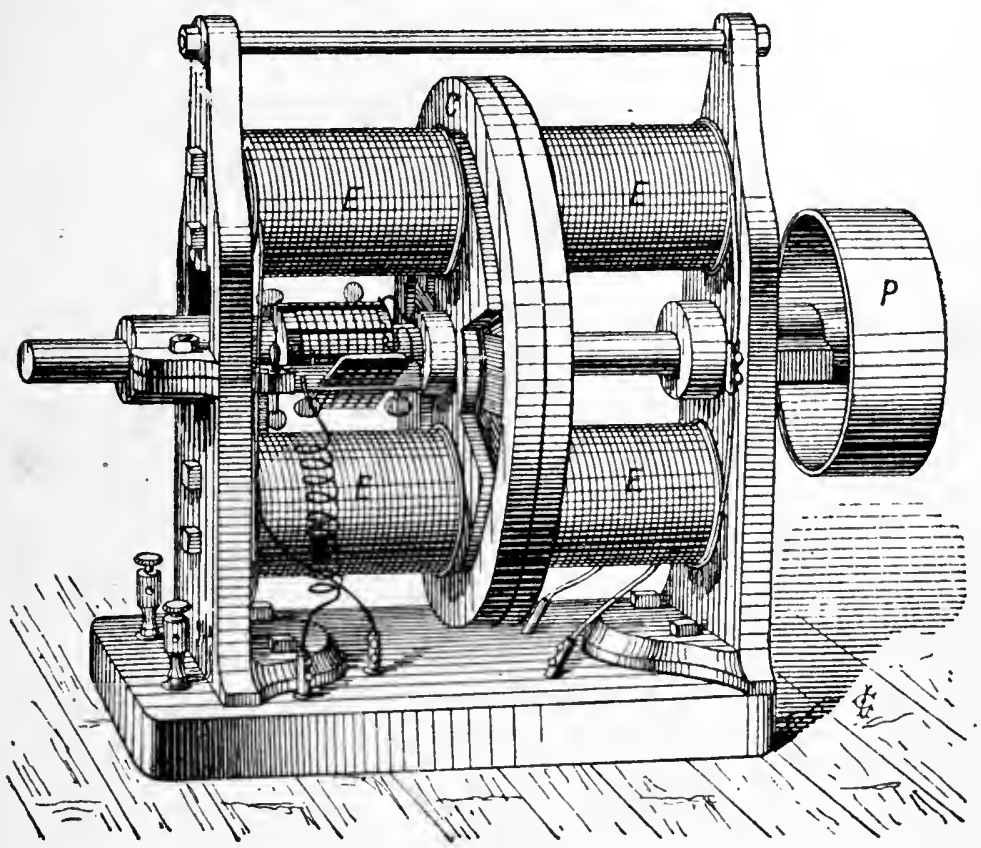

Fig. 61.-The Arago machine (with two electro-magnets).

magnets carry enlargements in the form of sectors, which enclose on each side a great part of the armature ring. This latter is formed of six coils without iron; the wire is wound round a piece of wood, and the coils formed in this way are held between two discs fastened on the shaft, and a double copper ring which forms the circumference. The six coils are joined together in series, and from the 
point where the wire of each is connected to the following coil, they are, by means of a conducting wire, placed in communication with a Gramme collector. Thus we have an armature of similar construction to that of Siemens' alternating machine, but with a Gramme collector. The brushes are arranged as in most of the machines having a collector, and in certain cases the plates of the collector are so arranged as to short-circuit that pair of opposite coils which happens to be in the neutral line; thus the pair of inactive coils does not introduce any injurious resistance into the working. It will be remembered that a similar result is also obtained in the Brush machine.

A second type, represented in Fig. 62, has altogether twelve electro-magnet coils, and consequently six alternate magnetic fields. The armature ring is constructed in the same way as in the preceding machine, and contains coils without iron. The commutator is composed of twenty-four plates. As regards the principle by which the currents are rendered continuous, it is absolutely the same as that employed in Hefner Alteneck's machine. It is alleged that Ball's machine was constructed before Hefner Alteneck's; but there can be no doubt that a description of the latter appeared before Ball's machine had ever been mentioned.

The Arago machine with six electro-magnets (Fig. 62) presents a special arrangement which does not exist in the former machinc. Each of the induced coils is divided into two magnetic fields, and a current is produced in the external circuit by those parts of the field which possess the highest intensity. The induction produced in the other parts of the field engenders a current which feeds the inductors. In consequence of this arrangement, the collector 


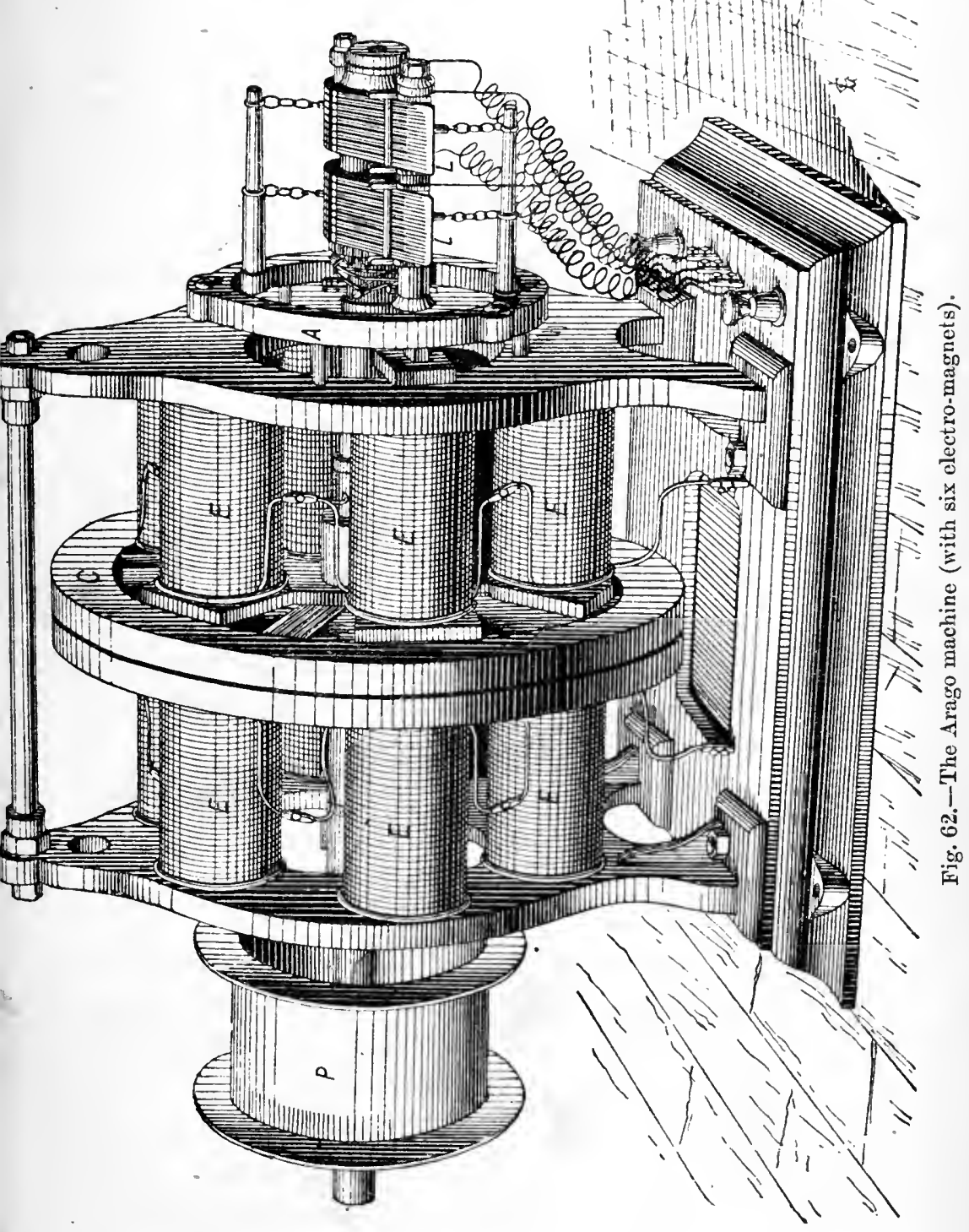


is divided into two distinct parts, each.corresponding to one of the circuits mentioned.

The brushes L L, too, have a special monnting. Their supports are fastened to a ring $A$ outside the frame of the machine, and the brushes themselves are, on the other hand, kept in position by springs starting from rods, which are likewise fastened to the ring.

It is stated that, with a speed of 1000 revolutions per minute and an expenditure of from five and a half to six horse-power, this machine can feed from ten to twelve arc lamps of average power.

Various Machines. - The number of new machines with continuous currents increases daily, in almost alarming proportions, and it is almost impossible to keep pace with them. We have restricted ourselves to the description of those apparatus which present any new or interesting details of construction.

Maxim, of New York, has constructed a generator with Gramme's ring and Siemens' inductor, which is chiefly characteristic for its mode of current regulation. We shall refer to this machine again in the chapter on the applications of electric lighting.

Gülcher uses for his lamps (with voltaic arc), which are all placed on shunts, a machine with four coarse-wire poles, with the view of producing quantity. The Gramme ring is flattened, and the two opposite inductors are connected by a U-shaped armature, which surrounds the ring to excite it on three sides.

We must here close the list of continuous-current machines. Their number is legion, and only part of them have been tested in practice; experience alone will enable us to select the really practical ones from amongst so many. 


\section{B.-Machines with Alternating Currents.}

If we consider a spiral of an induced coil during an entire revolution of the movable mass-inductor as well as armature-round its axis of rotation, we find that in all machines which we have considered till now, each spiral was traversed alternately by two or an even number of currents of opposite direction. These currents were collected, more or less successfully, in the external circuit, so as to obtain them always in the same direction, and to produce a continuous current. For certain applications it is absolutely useless to invert these currents. In certain machines, as for instance those of the Alliance, this inversion of currents could only be effected with great loss, which might easily be avoided if we content ourselves with collecting the currents without inverting them. Machines which realize these eonditions are alternatingcurrent machines, and are divided into two classes, like the continuous-current machines: into machines with permanent magnets and machines with electro-magnets.

a.-Magneto-Electric Machines with Alternating Currents.

It suffices to suppress the inverting commutator of the electro-magnetic machines of Pixii, Clarke, Page, Siemens, etc., and to replace it by a simple collector, in order to obtain apparatus with alternating currents, whose power will depend, as with continuous-current machines, on the intensity of the magnetic field, the velocity of rotation of the coils, their dimensions, number, etc.

Alliance Machines.-In 1849, Nollet, Professor of Physies at the École Militaire of Brussels, constructed a Clarke 
machine on a large scale, and this machine is now known under the above name.

After endless disappointments, of which the details are to be found in Count du Moneel's Exposé des Applications de l'Électricité, the Alliance machines, originally intended to give continuous currents, were, on the advice of Masson, Professor of Physics at the École Centrale, transformed into alternating-current machines. They gave, by this simple change, incomparably better results, and were adopted, as early as 1863, for the electric lighting of the lighthouses of La Hève.

The Alliance machine is well known now; we.shall simply indicate its principle. It is a Clarke machine of large dimensions, and its construction renders the multiplication of induction coils and induction magnets an easy matter.

For this purpose the coils are arranged on dises, and turn between the horse-shoe magnets, placed all round the circumference; each disc earries from eight to sixteen coils. The largest machine constructed up to the present has six dises of sixteen coils each, and fifty-six inducing magnets. For each revolution of the machine the wires of the coils are traversed by currents, which change their direction each time a coil passes before the poles of the inductors.

For a speed of 400 revolutions per minute, more than 100 ehanges of the direction of the current are obtained per second. The connections of the coils with the collectors can be arranged so as to satisfy either quantity or tension.

The Alliance machines with alternating currents are good machines, working very regularly, and of consider- 
able efficiency; they are not extensively used, because they occupy a large area in proportion to their power, because they are too heavy, and their price is too high for the work they transform into electricity. From this point of view they can be compared with low-pressure engines with condenser and low speed, of good efficiency, but to which we prefer engines of great speed, high pressure, and without condenser, because they are, with equal power, less heavy, less expensive, and less cumbersome.

De Méritens' Machine.-The machine invented in 1878 by De Méritens is also magneto-electric, but it differs from the Alliance machines by the shape of the coils, and by the way in which the induction currents are developed in the wires surrounding the armature ring.

We borrow the theory of this machine from Du Moncel's work on l'Éclairage Électrique-

"To understand the working of De Méritens' machines, let us suppose a Gramme's ring (Fig. 63) divided, for instance, into four sections, magnetically insulated from one another, and thus forming four arched electro-magnets, placed end to end. Let us suppose the iron core of each of these sections terminating at its two extremities in an iron piece A B, forming, as it.were, an enlargement of its poles; and let us suppose that all these pieces, united by means of a copper piece C D, constitute a solid ring, around which are placed permanent magnets, N S, N S, of alternately inverse polarity. Let us consider what will happen when this ring accomplishes a rotation round its axis. The enlarged pole $\mathrm{B}$, on its way from left to right, approaches $\mathrm{N}$; at this moment an induced magnetic current will be developed in the electro-magnetic helix $\mathrm{A} \mathrm{B}$, as in a Clarke's machine. This will be an instantaneous current of 
inverse sense to Ampere's particular currents of the inducing magnet; it will be very strong, on account of the proximity of $\mathrm{B}$ to pole $\mathrm{N}$. In the core A B a series of magnetic displacements will occur, which will give rise to a series of currents of inverse polarity, from $B$ to $A$; these currents will be direct with regard to the particular currents of $\mathrm{N}$, but they will not be instantaneous, and will go on increas-

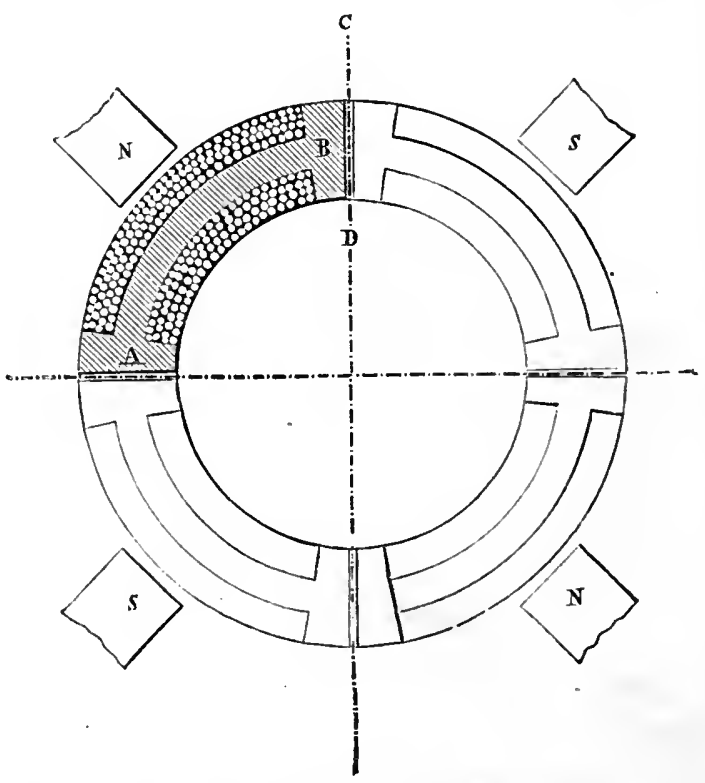

Fig. 63.-'Theoretical diagram of De Méritens' machine.

ing in strength from B to A. Dynamic induction currents, resulting from the passage of the spirals of the helix before pole $\mathrm{N}$, will be joined to these currents. When $\mathrm{A}$ leaves $\mathrm{N}$, a demagnctizing current will be produced, equal in strength and of the same direction as the magnetizing current resulting from the approach of the enlargement $\mathrm{B}$ to pole $\mathrm{N}$. The effect is in reality produced at a different extremity of 
the magnetic core, and the helix presents itself to the action of the inductor in an inverse sense. Hence the induced currents will be inverse to the inductor from the fact of the approach and withdrawal of the enlargements $A$ and $B$, and we have direct induced currents during the passage of the length of core A B S before the inductor, and direct induced currents resulting from the passage of

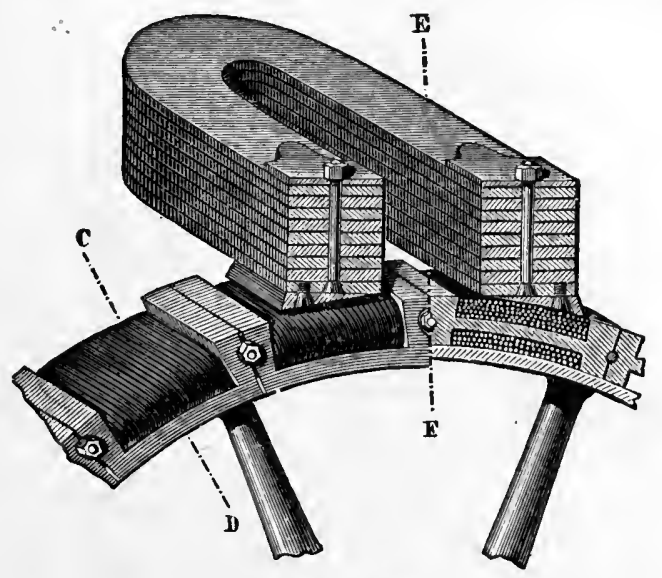

Fig. 64.-Details of De Mréritens' machine.

the spirals before $N$. All the causes of induction are thus united in this combination.

"The action which has been described for a single section of the ring can be effected at the same time for all the others, and to it will be added the currents resulting from the lateral reaction of the poles $A$ and $B$ on the neighbouring poles.

"In order to strengthen the effects of induction, M. de Méritens forms the core A B and the enlargements $A$ and $B$ with thin iron plates, cut out with a cutting machine, as shown in Fig. 64, and placed alongside of each other in bundles of fifty, of one millimetre thickness each.

VOL. I. 
"The wires of the helices are joined in such a manner that the indirect currents can be associated for tension, quantity, or series, according to the special requirements. In the theoretical diagram which we have given, we have only considered four sections, but in reality there is a

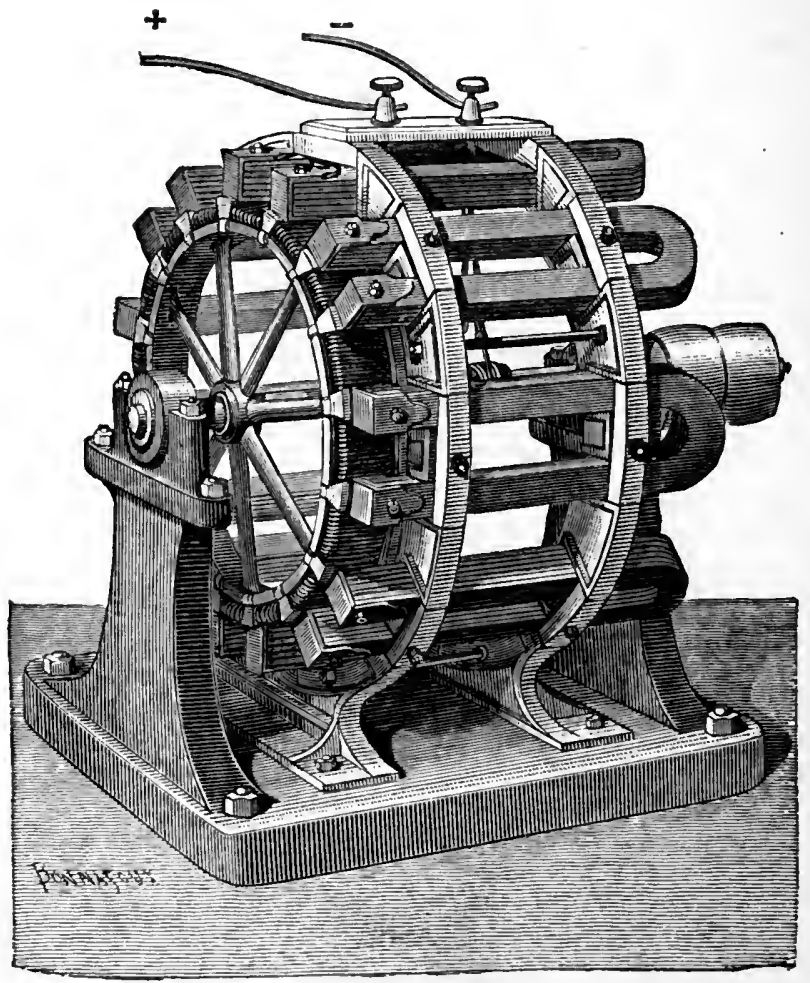

Fìr. 65.-De Méritens' machine.

much greater number, and in the model of which we have spoken, there are sixtcen, which can be easily distinguished (Fig. 65).

"They are mounted on a bronze wheel, which is fixed to the shaft of the engine. The inducing magnets are 
placed above this wheel; they are securely fastened to two bronze frames, and arranged horizontally. Fig. 65 will show how the different parts of this machine are mounted.

"It will easily be seen that the construction of the armature ring presents the most favourable conditions from a practical point of view. Each section being separate, it can be dismounted separately, and supplied with a coil of wire without any trouble. Whoever knows the difficulty which the coiling of Gramme's ring presents will be able to appreciate this advantage. The arrangement of the core, with the plates placed alongside of each other, and which can be cut by one single operation of the cutting machine, is another immense advantage, rendering unnecessary the great precision in the construction of these rings, which can only with the greatest difficulty be kept perfectly round."

For the lighting of lighthouses, De Méritens has constructed, on the same principle, a machine with five dises, very similar in appearance to the Alliance machine. The five movable armature rings, which can be joined for tension or quantity, or employed separately, according to the requirements, turn before the extremities of the magnets, instead of turning before their faces, as in the machine with one disc.

\section{b.-Dynamo-Electric Machines with Alternating Currents.}

Dynamo-electric machines differ from magneto-electric machines by the nature of the inductors, which with the former are electro-magnets, while permanent steel magnets are used for the latter.

In continuous-current machines the current of the 
machine itself is used for the magnetization of the inductors; this is not feasible with alternating currents, unless spccial arrangements are made which will be mentioned hereafter.

It follows that, to constitute a dynamo-electric machine with alternating currents, two machines are required: one of them is called the exciting, inducing, or magnetizing machine, is of comparatively small size, and is used for charging the electro-magnets of the second, which is called the distributing, division, or light machine; this latter furnishes alternating currents in one or several circuits.

- Lontin, Gramme, and Siemens all use two machines-an exciting machine with continuous currents, and a division machine; but each of these systems present certain distinctive peculiarities of construction.

Iontin's Alternating-Current Machine.-The first dynamoelectric machine with alternating currents and special induction machine was constructed by Lontin; he used the continuous-current machine described on p. 76 for this latter purpose. The current produced by it passes into the inductors of the division machine, which has the following construction :-

The current of the induction machine passes over to the pinion of the division machine by two brushes fixed to the terminals F F (Fig. 66). This pinion, which turns round an horizontal axis, is composed of twenty-four iron teeth A. Each of these teeth is surrounded by a spiral of copper wire; the current of the induction machine traverses this spiral, and magnetizes each tooth, by alternately changing the polarity of the poles in the external circumference.

A fixed crown, with twenty-four teeth fixed on the 
internal circumference, opposes the movable pinion, and thus forms a series of coils, which, by the extremities of the wires surrounding the core, are all connected with an apparatus called the manipulator, whose function we are going to describe.

By imparting to the magnetic pinion a rapid rotatory motion, the teeth of the pinion, in their passage before the coils $\mathbf{B}$, develop induction currents whose direction varies twenty-four times for each revolution. A machine with twenty-four coils, revolving at a normal speed of 360 revo-

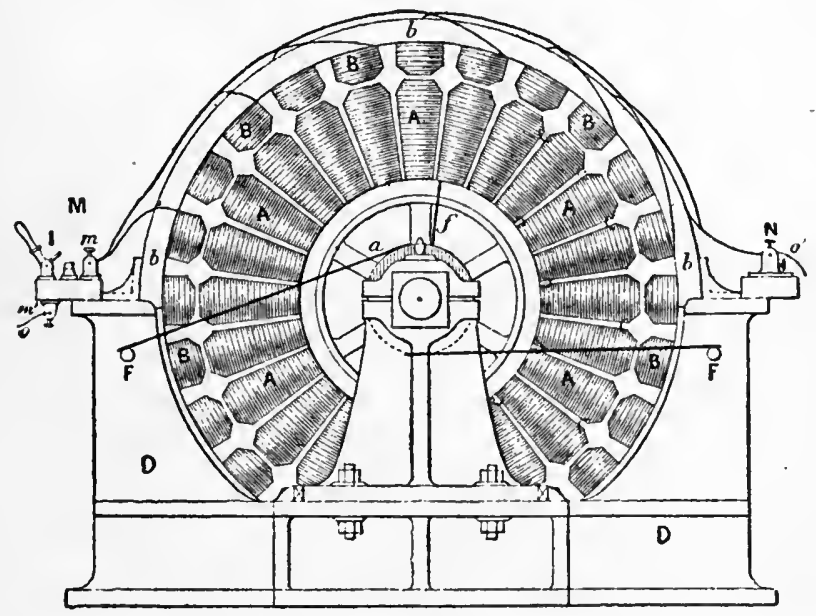

Fig. 66.-Lontin's division machine.

lutions per minute, produces 8640 changes of polarity in the core of the coils B; that is, 8640 currents of alternately inverse sense, or 144 inversions per second.

The extremities of the wires of the coils $B$ are fixed to the terminals $m$ of the manipulator M. By the help of the manipulator, the coils can be joined either for tension or for quantity, and in larger or smaller number. Twentyfour distinct circuits can be established, or the twenty-four 
coils can be arranged in series, on a single circuit, for the feeding of one single powerful focus.

In this machine the armature is fixed and the inductor is movable, as in Pixii's multiple machine, with this difference, that the movable magnet is replaced by an electromagnet which, for equal weight, has twenty times more power than a permanent magnet.

Gramme's Machine.-In this, as in Lontin's machine, which preceded it, the armature is fixed, and the inductors

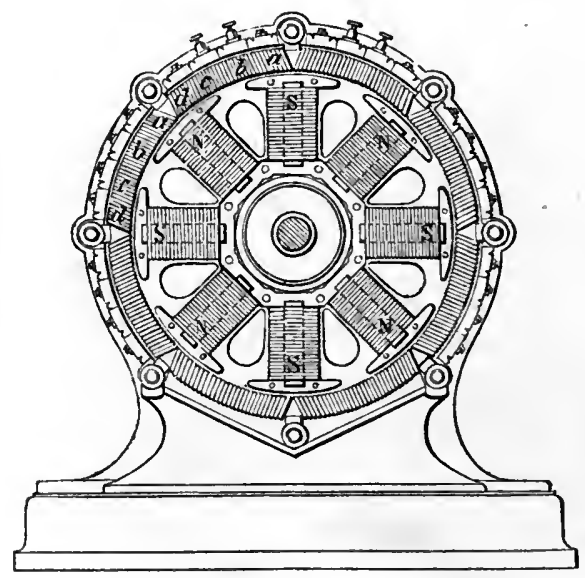

Fig. 67.-Diagram of Gramme's alternating-current marhine.

overcharged by a Gramme's machine (p. 70), are movable. The inductor (Fig. 67) consists of a magnetic pinion, formed of eight straight electro-magnets with alternating poles, which produce in each of the sections of the induced helix alternately inverse currents. The fixed armature is a long iron cylinder, arranged like the ring of the continuouscurrent machine of the same inventor:

It will be seen from the diagram (Fig. 67) that, the wires of the armature being fixed, they can be joined in different 
ways. Every eighth part of the circumference of the ring is divided into four sections; the thirty-two sections or elements consist of eight groups of four elements each; all the sections $a$ are joined to form one first group-for a reference to the diagram will show that all the sections $a$ are in the same position with regard to the eight poles of the movable inductor, and therefore associated; in the same

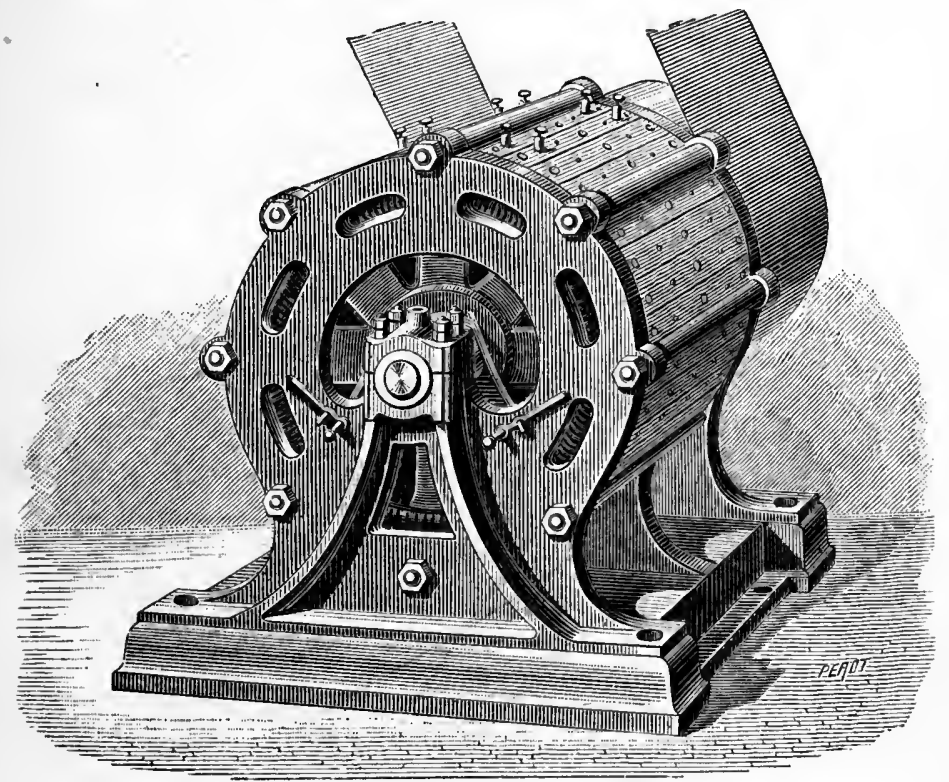

Fig. 68.-Gramme's alternating-current machine.

way, the sections $b$ are united in a second group, sections $c$ in a third, and sections $d$ in a fourth.

By placing sixty-four sections on the ring instead of thirty-two, many other combinations are possible.

As in Lontin's division machine, there is no commutator; two movable rubbers, in the shape of brooms or brushes, press against two distinct circles, and conduct the current from the exciting machine to the movable pinion. 
The machine is enclosed between two lateral cast-iron mountings, and encased by a number of mahogany planks, which effectively protect the exterior ring against all shocks.

The diagram shows the fly-wheel and strap for setting the machine in motion. Gramme constructs machines of this type of different sizes; from machines to feed four

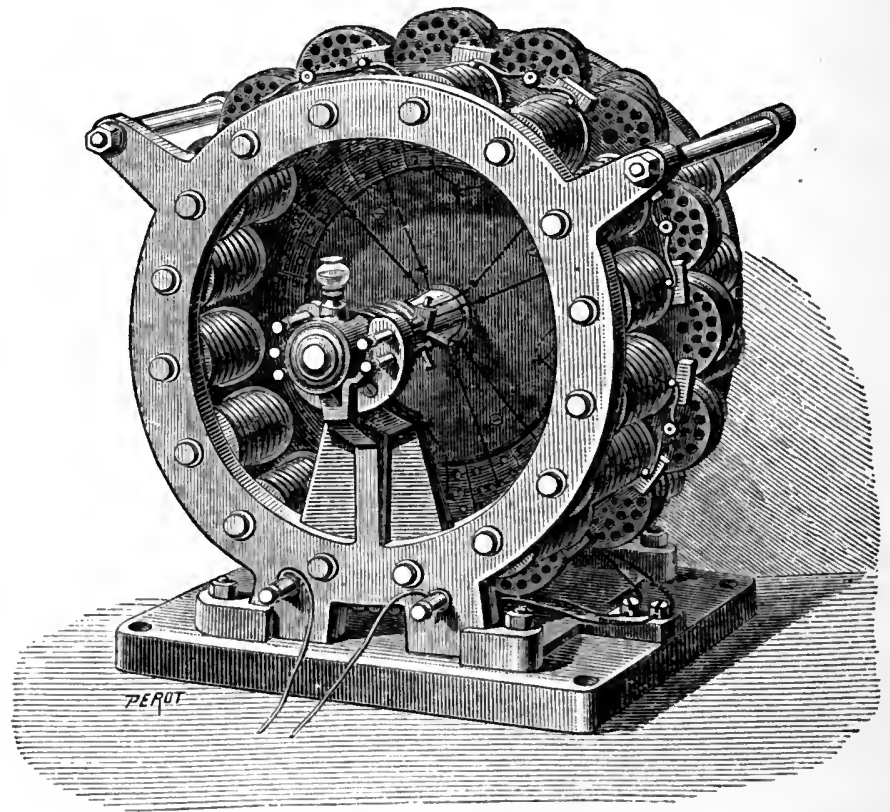

Fig. 69.-Siemens' division machine.

Jablochkoff candles in one circuit, to machines as used in the Hippodrome in Paris, which produce a current powerful enough to feed sixty candles, arranged in twelve circuits of five candles each.

Siemens' Machine.-Hefner von Alteneck's machine, constructed by Messis. Siemens, whose name it bears, differs from Lontin's and Gramme's machines by having 
fixed inductors and movable armatures. The exciting machine is Siemens' small-sized machine, represented in Fig. 38, p. 79. Its current passes to an inductor formed of thirty-two electro-magnets, which are fixed to two cast-iron rings, sixteen on each side of the movable armature.

The extremity of the core of each electro-magnet carries a little iron plate, which constitutes an enlargement of the pole, and thus facilitates induction; these poles are alternately of inverse sense. The armature consists of sixteen flat bobbins fixed to a plate, and revolving very rapidly in the circular space between the two series of inductors. The armature bobbins have no iron core as in other systems; the current is therefore developed in them merely by the passage of the spiral coils through the magnetic field of the inductors, and not by any magnetic overcharge or by the inversion of polarity of their cores.

The bobbins are previously grouped in the most convenient way for obtaining the desired results, and the currents are collected by means of brushes rubbing against the collectors, which are fixed to the extremities of the armature bobbins. A machine of this kind, with sixteen bobbins, is divided into two circuits; each of these circuits can feed ten of Siemens' differential lamps. Three conducting wires are sufficient for this purpose, for the return wire can be common to both circuits.

Gordon's Large Machine.-The dynamo-electric machines used for lighting have till now been of comparatively small dimensions; only Edison's machine, capable of feeding 1200 incandescent lamps, had attained larger dimensions. There can be no doubt that the future belongs to large machines, and with this conviction, Gordon has constructed his machine, the largest in existence, and which is repre- 
sented in Fig. 72. It is an alternating-current machine, with separate exciting machine, similar in principle to those of Siemens, Wilde, etc. The inductors are movable, and the induced coils are fixed. Two Bürgin machines are employed as exciting machines, and the motor acts direct upon the shaft without the use of any transmission.

The total weight of the machine is about eighteen tons, that of the movable armature is seven tons; the space occupied by the foundation of the machine is 4.06 metres by 2.13 metres, and the diameter of the armature is 2.67 metres. It is capable of feeding 5000 incandescent lamps.

Gordon's object being to construct a machine of great power rather than to invent a new one, the principle applied does not present any great feature of novelty. One interesting detail, however, deserves to be mentioned. In a previous apparatus Gordon had employed a number of armature coils equal to that of the inductors; he then noticed that an induction took place between the neighbouring coils, which led to a waste of electro-motive power. This was proved by feeding a certain number of lamps by means of the current of one of the induced coils and closing the circuit of the neighbouring coil : the intensity of the lamps fed by the former circuit was found to be lower by twenty or thirty per cent. This effect was owing to the fact that the poles of two neighbouring inductors being of opposite sense, two neighbouring induced coils were traversed at the same time by currents of opposite sense, which, by induction, acted upon one another. In the present machine Gordon has obviated this drawback by reducing by one-half the number of the inducing electro-magnets, so that there is only one to every two induced coils.

With this new arrangement, at the moment when the 
inductors exercise their maximum effect on the coils 1,3 , 5 , etc., the alternate coils $2,4,6$, etc., are sensibly at rest. The distance between the active coils, $1,3,5$, etc., is now sufficiently great to considerably diminish their effect upon one another; as regards the inactive coils, although they are influenced on each side by one of the active coils, the effects in this case are of contrary sense, and destroy one another.

The induced coils are arranged in two rings on each side

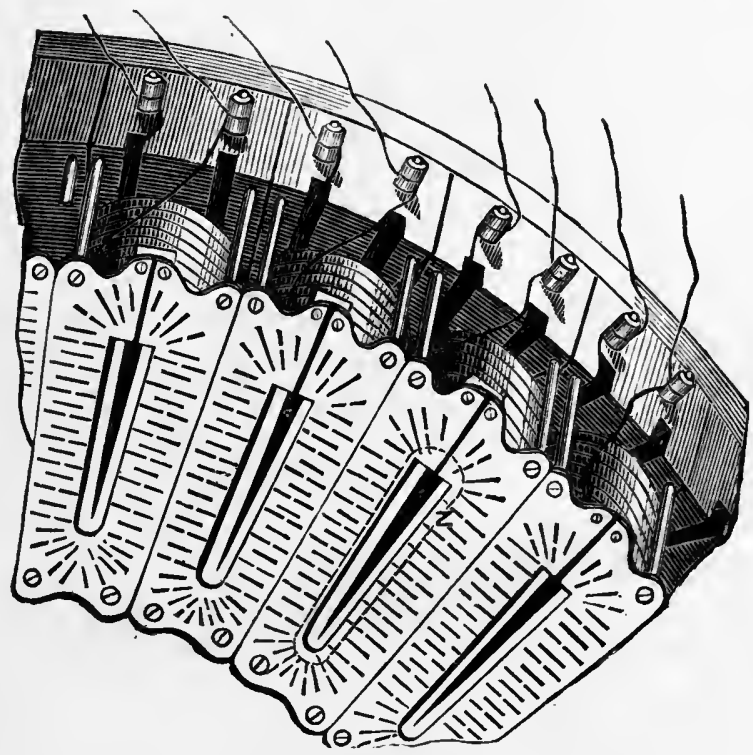

Fig. 70. - Gordon's machine (armature coils).

of the armature ; they are sixty-four in number on each ring, or 128 in all. They are oblong, as shown in Fig. 70, and have a U-shaped core. A sort of screw-bolt, the prolongation of this core, is fastened by a screw-nut to the iron ring which serves as support to the coils, and in order to avoid any deleterious induction, the latter are separated from the ring by thick blocks of wood, forming one of the faces of the 
coils. The other face is formed by a plate of German silver riveted on a shoulder of the core. This plate is perforated in many places to prevent any induction currents being produced in the metal itself. The coils are wound with

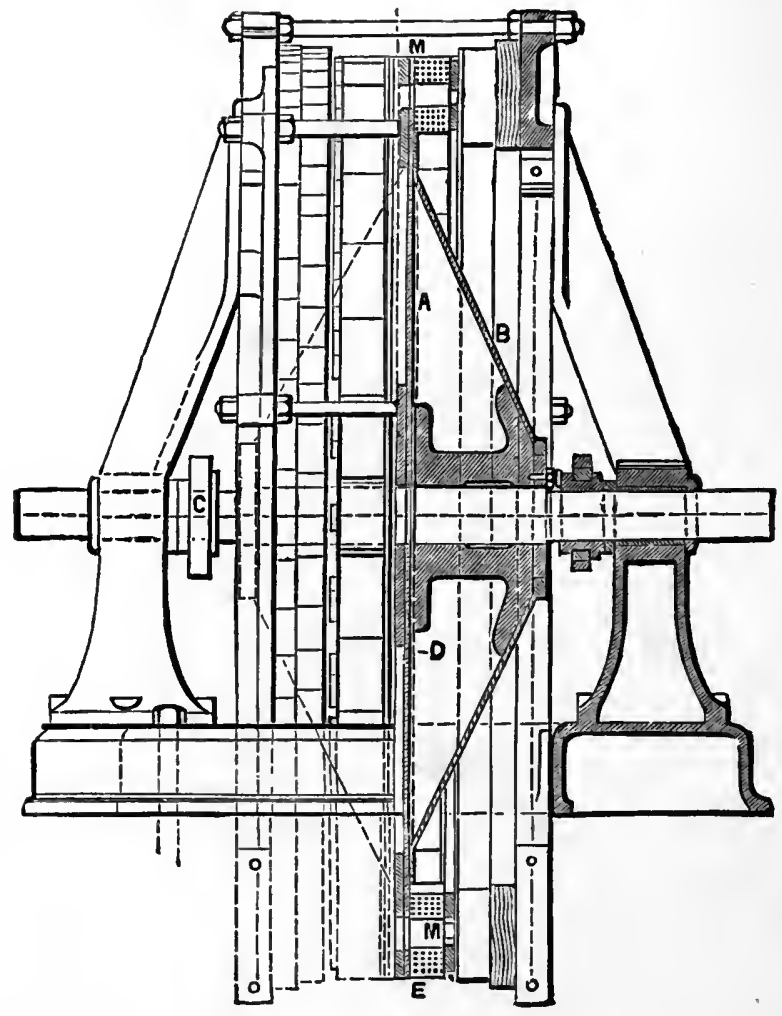

Fig. 71.-Gordon's machine (movable armature).

copper wire of four and a half millimetres thickness, in order to avoid the formation of Foucault's currents.

The movable armature consists of two discs A (Fig. 71). The base of the two cones B is fastened to the discs, whilst their summit is fixed to the shaft of the machine. The 
$\because=\quad \therefore$ 


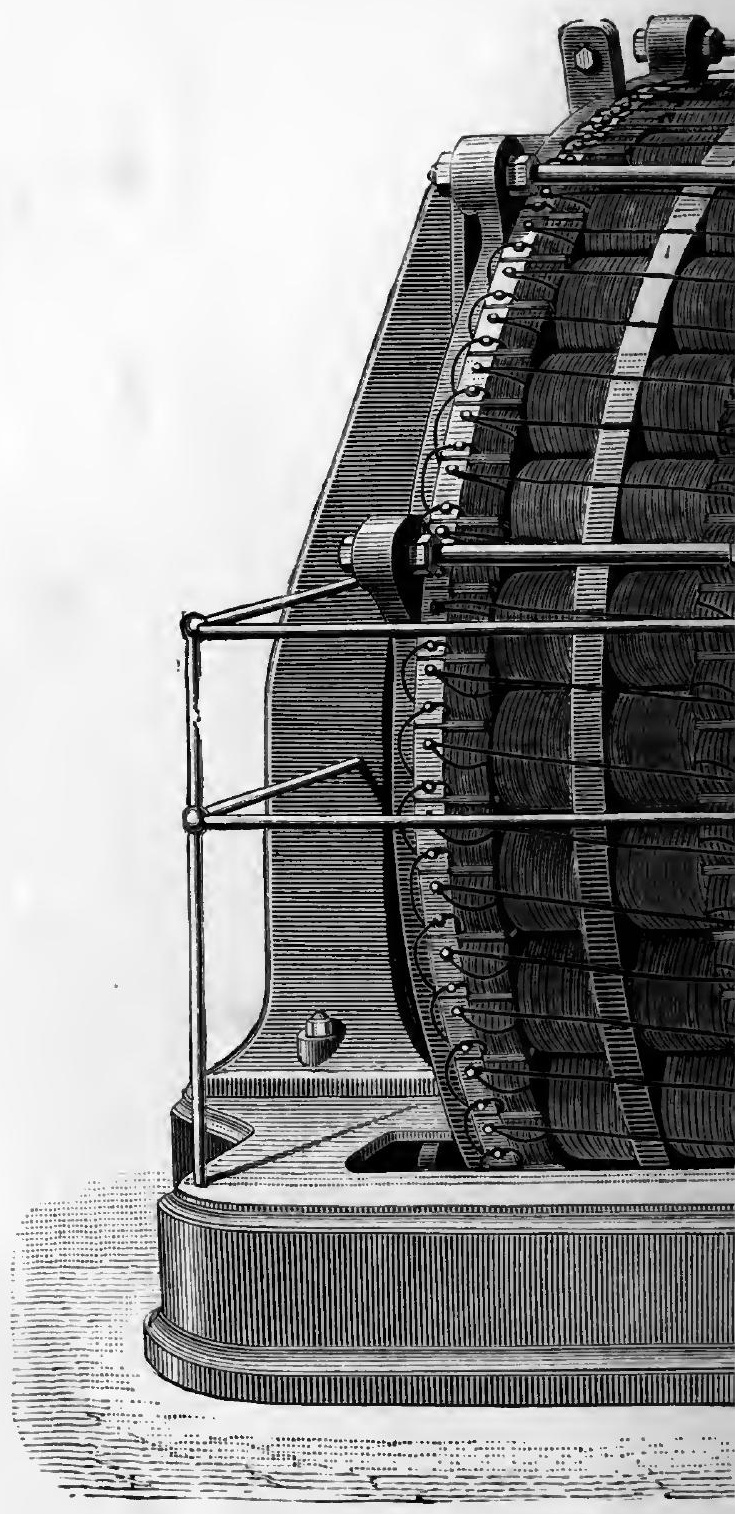

Fig. 72,-G (From 


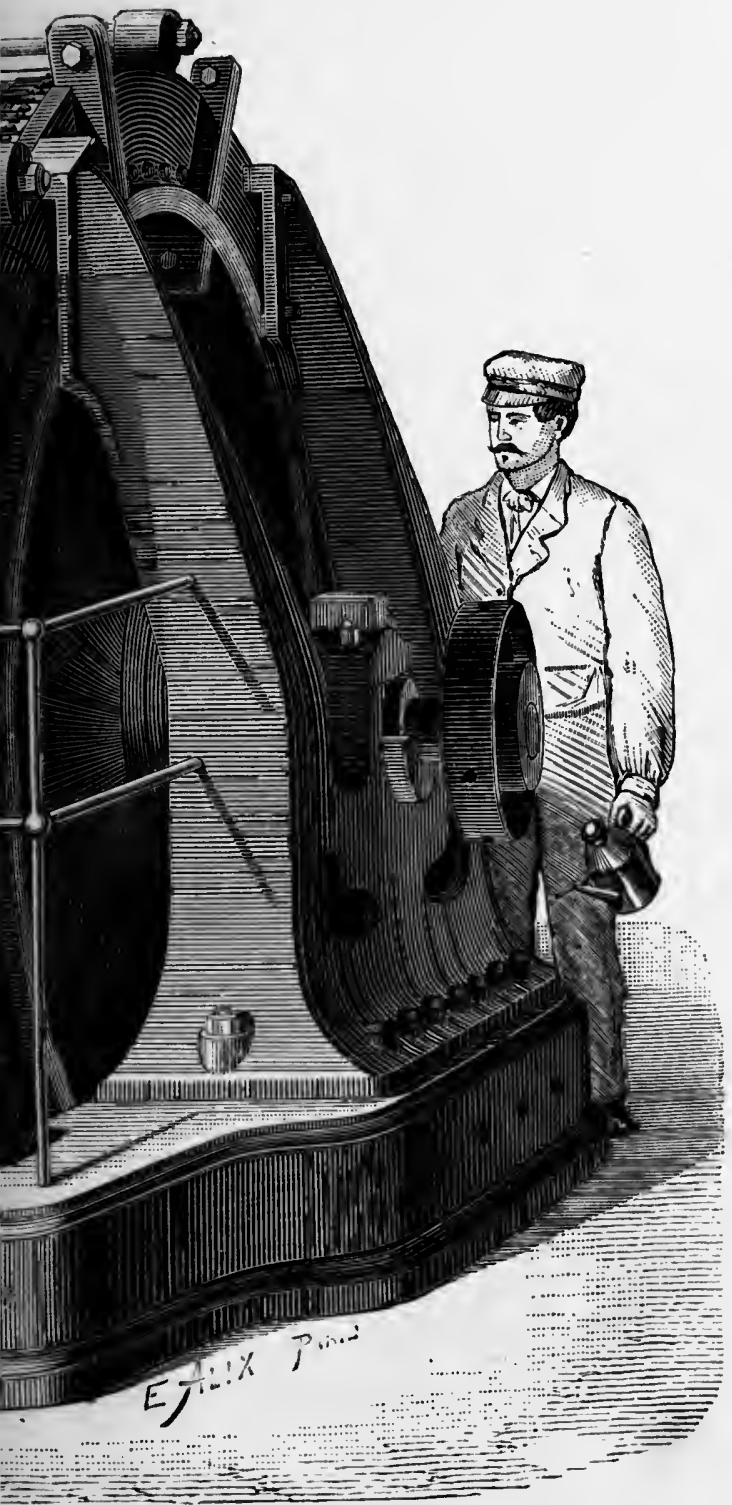

ge machine.

[Vol, I. p. 125. Éleclrique.) 

discs and the cones are made of segments of strong sheet iron, riveted together as in a boiler. The discs are kept apart in the centre by a wedge $\mathrm{D}$, and in the circumference by an iron ring. The base of the cones is smaller than the discs, so as to leave an annular space for the inductors: between the cones and the dises are strong shoulders fastened to the shaft, and of somewhat smaller diameter than those of the plate $\mathrm{D}$.

In the annular space surrounding the base of the cones are placed the cores of the electro-magnet inductors, to the number of thirty-two. Each of these inductors is formed of a core, which protrudes equally on either side of the two dises A. On each of the projections a brass bobbin is placed, and these bobbins are maintained in place by the polar pieces.

The shaft of the machine (Fig. 72) is supported by bearings of phosphorus bronze. There is a large opening in the foundation plate of the machine, and a pit in the ground beneath it, into which the machine works. The effect of this arrangement is to lower the centre of gravity and to give the machine greater stability.

The iron rings which carry the armature coils consist of three segments, of which one is smaller than the others. This facilitates repairs in case of an accident to one of the inductors. The small segment need only be removed and the armature turned till the electro-magnet in question comes into view, when it can easily be removed and repaired.

The induced coils can be coupled according to the requirements of the case; for instance, according to the number of Swan lamps to' be fed. With 1300 lamps the coils are grouped 4 for tension and 32 for quantity. The speed of the machine is then 140 revolutions per minute, which gives 
a linear speed of 18.3 metres per second for the cores of the inductors.

The current which passes into the inductors is equal to 19 ampères, and the electro-motive force obtained is 88 volts. The current in the armature wires is 27.5 ampères.

To feed 5000 lamps the 128 coils ought all to be connected for quantity; the speed of the machine would then be increased to 180 revolutions, a current of from 40 to 50 ampères would pass into the inductors, and lamps requiring 1 ampère and 60 volts would be placed in the circuit. A current of 40 ampères would be obtained in the armature wire, and the electro-motive force reduced to 60 volts.

The exciting machines which supply the current to the inductors are fed by a special steam-engine, and the current is regulated by acting upon the two motors. In a room adjoining the machine (Fig. 73) are placed some apparatus indicating the speed of the large machine, the intensity of the exciting current, the tension of the steam, etc. There are also two lamps, each belonging to one of the circuits of the machine, which indicate by a photometric method the variations of intensity of the current. The steam-pipes of the two motors pass into the same room, and one man can, by means of taps, regulate the speed at the same time that he observes the indicators.

It is stated that 94 per cent. of the work absorbed by the machine and the two motors are recovered in the lamps. In the experiment lately made at Greenwich the machine fed 1300 lamps of 20 candles cach, and spread over an area of about 13 acres.

Lachaussée-Lambotte's Machine.-This machine consists, like Siemens' alternating machine, of a dise of oval-shaped coils (Fig. 74) placed between two circular rows of clectro- 


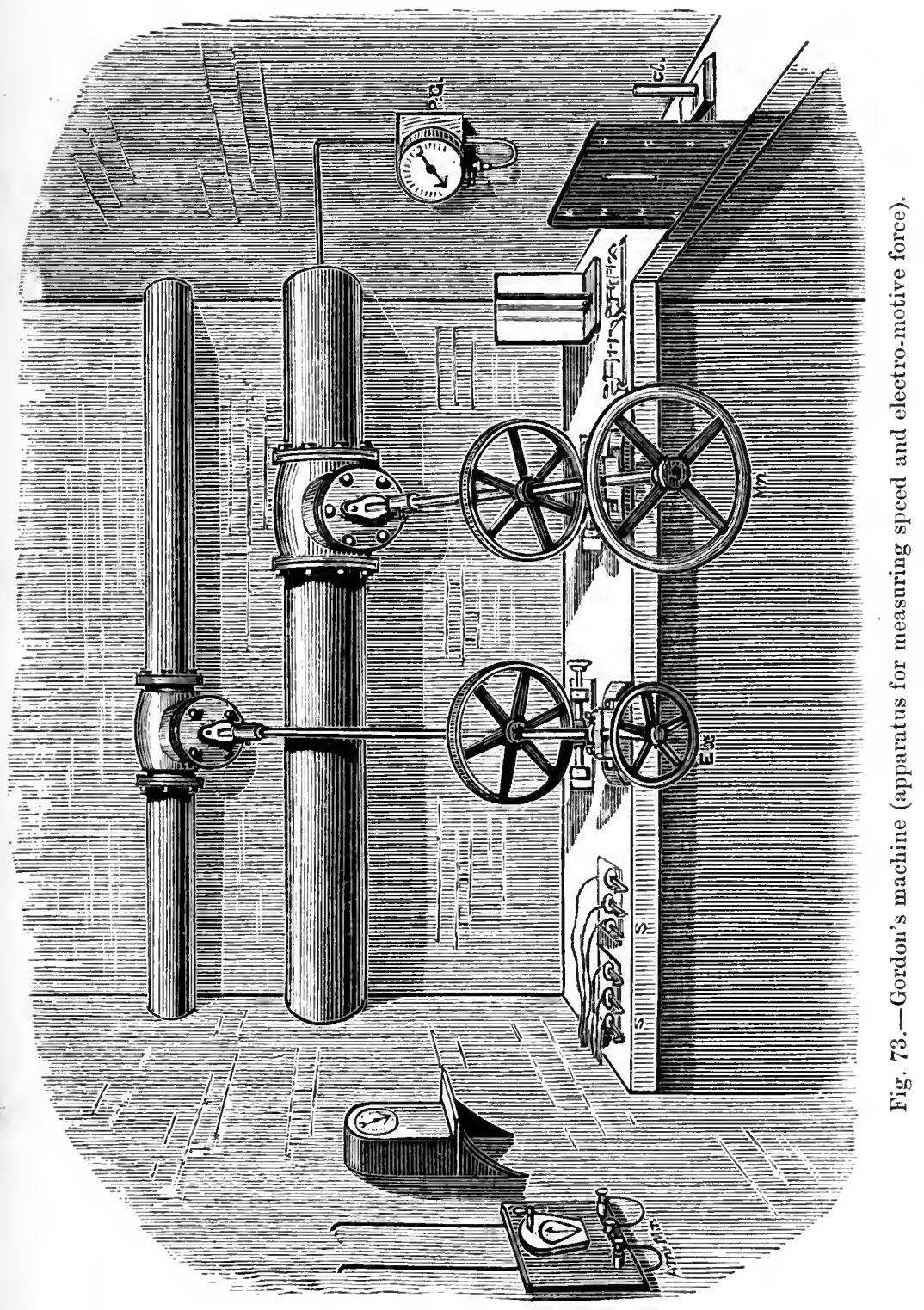


magnets. But in Lachaussée's machine it is the electromagnets which are movable, and not the oval coils; these latter have besides some soft-iron plates for their cores, and

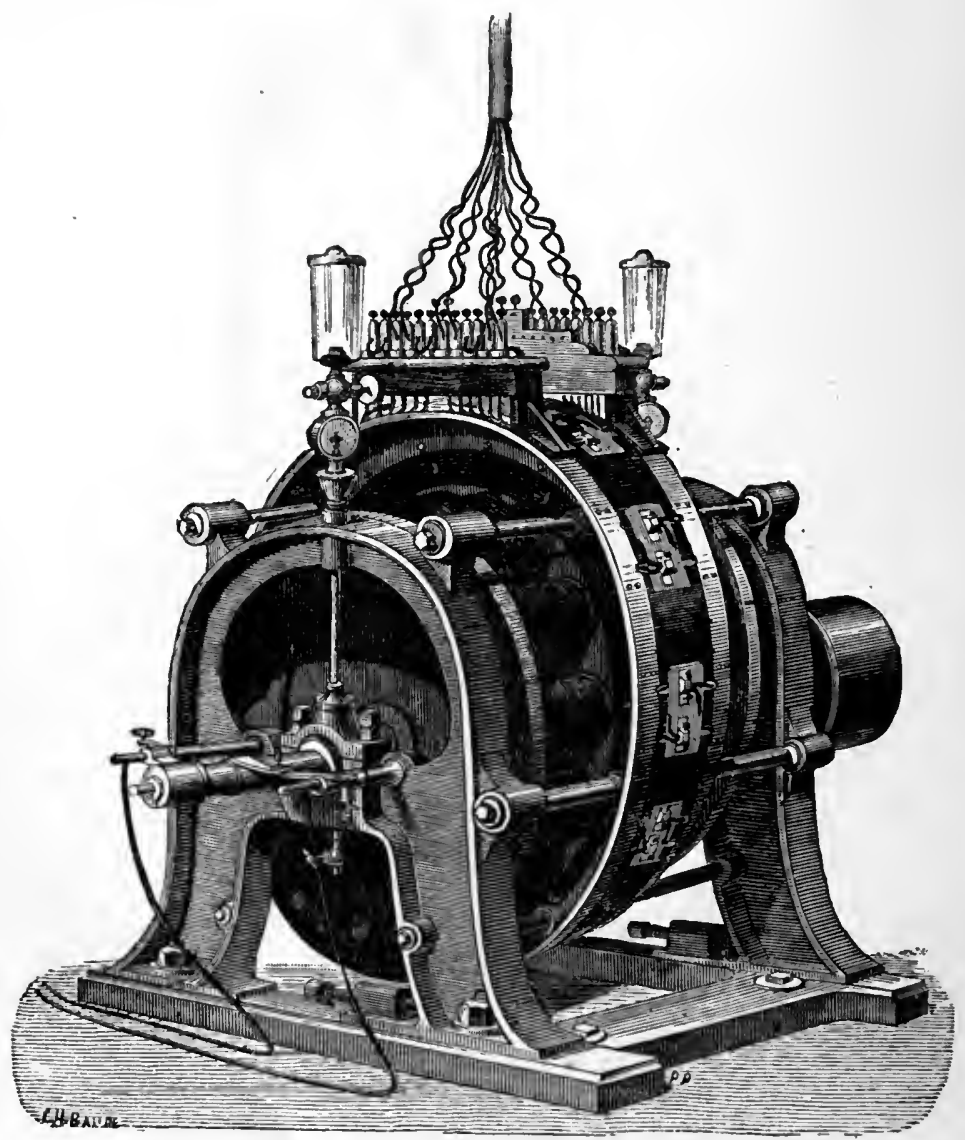

Fig. 74. - The Lachaussée-Lambotte machine.

constitute, therefore, by themselves veritable electro-magnets of special form.

The whole of these coils, with the necessary pieces for their support, form a drum, occupying the central part of 


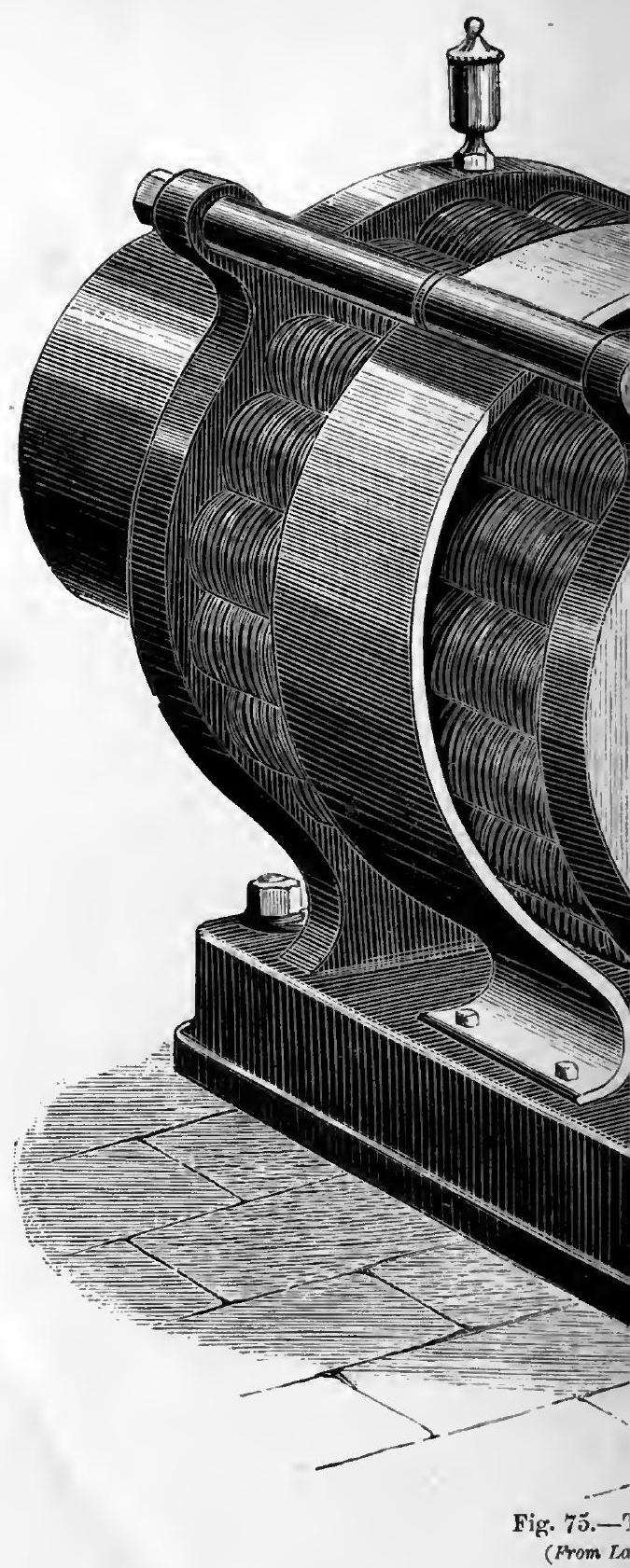




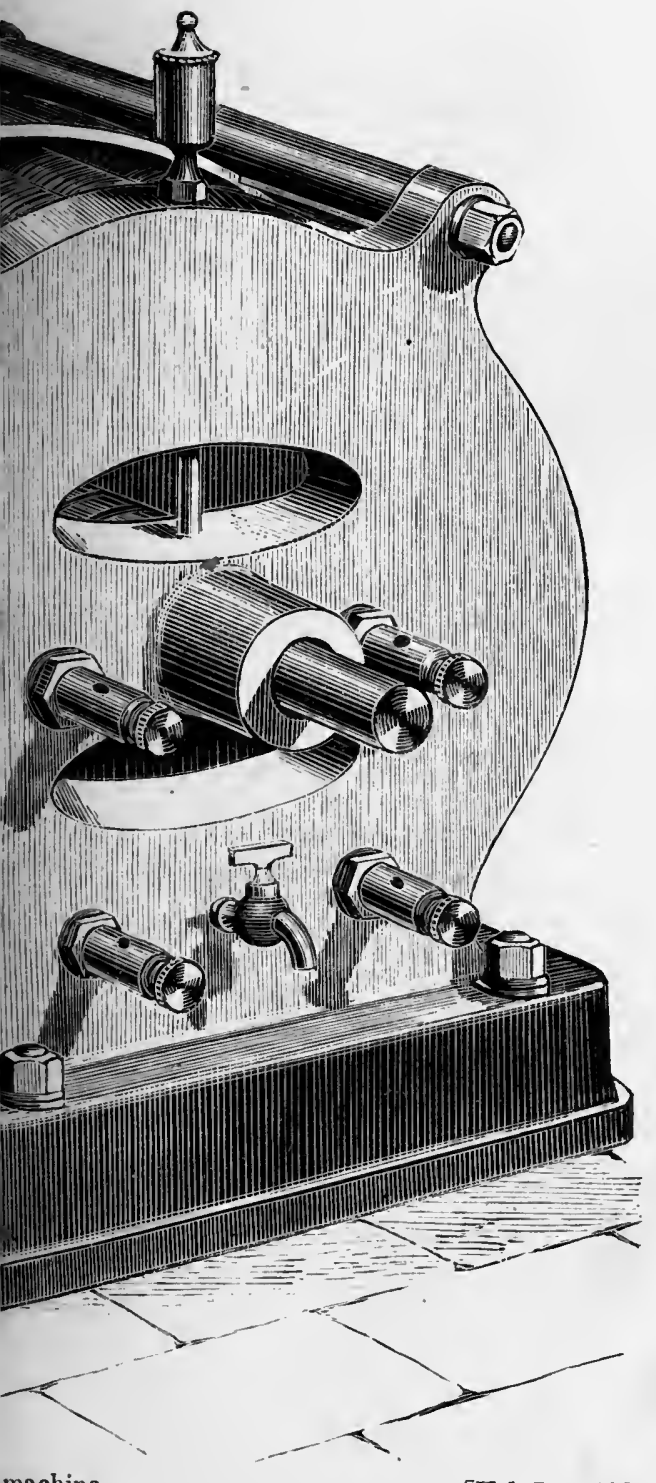

machine.

[Vol. I. p. 129. 

the machine. The oval coils are fastened sideways between two wooden discs screwed to a cylindrical centre-piece; towards the circumference they are let into movable blocks of wood (Fig. 74), which in their turn are fitted into the openings of the wooden ring that forms the exterior of the drum.

The wooden blocks can therefore easily be taken out, the coils removed from the drum, and changed or repaired as the case may be.

The extremities of the wire of each coil are fastened by screws to two copper pieces; to the same pieces are also attached the wires which are connected to the terminals fitted to a bar at the top of the machine; each of the coils is in the same way connected to a pair of corresponding terminals. Six strong ribs support the drum on each side and keep it at equal distance from the two supports forming the frame of the machine.

The electro-magnets, twelve on each side, are mounted on two copper plates fixed to the shaft and revolving with it. The current is conveyed to the inductors by two brushes, and these inductors are mounted for tension in such a manner that two consecutive electro-magnets are always of opposite polarity, and that the poles of two magnets, situated opposite one another on either side of the drum, are likewise of opposite name. A small Gramme machine feeds the inductors.

With an expenditure of twenty-four horse-power, one of these machines feeds twelve soleil lamps with an are of twenty millimetres.

Ferranti's Machine.-This machine is the joint invention of Sir William Thomson and Mr. Ferranti. In general aspect (Fig. 75) it resembles Siemens' and other alternatingVOI. I. 
current machines, and like these it has an armature turning between two circular ranges of electro-magnets of alternately inverse polarity. These electro-magnets, sixteen on each side, only differ from the inductors of most of the alternating machines by their ovoid shape, as will be seen in Fig. 76. The armature is the characteristic part of the machine. Instead of being formed of a series of coils, it is composed of a long copper strip (36 metres long by 12.5 millimetres in width and two millimetres in thickness) bent so as to form twelve equal horseshoe-shaped teeth $\mathrm{L} \mathrm{L} \mathrm{L,}$

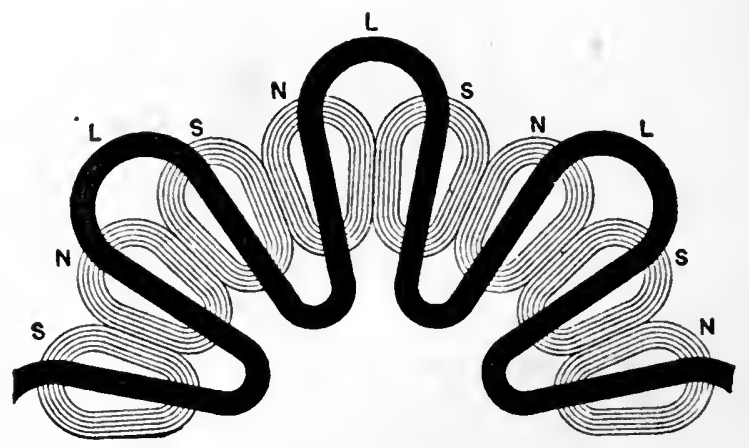

Fig. 76.-Armature of the Ferranti machine.

which are insulated from one another by indiarubber bands. The extremities of the copper strip are attached to two metallic collars, against which some brushes are made to rub. On considering two neighbouring radial parts of this armature, it will be seen that, when one of them approaches, say a north pole, its neighbour approaches a south pole; and the currents generated are therefore of opposite sense, that is to say, all the currents developed at a given moment are joined together. The principle of current generation in the radial parts is therefore the same as in the Siemens machine, as will be confirmed by a reference to Fig. 77, which shows 
the coiling of this latter; the difference between the two apparatus consists simply in the fact that in the Siemens machine the curved parts of the wire which unite the radial portions form distinct bobbins, while in the Ferranti machine the curved wires joining the radial parts are arranged so as to form a large circular zigzag. This coiling is similar to some arrangements indicated by.Sir William Thomson, in a patent of the 26th of December, 1881, and

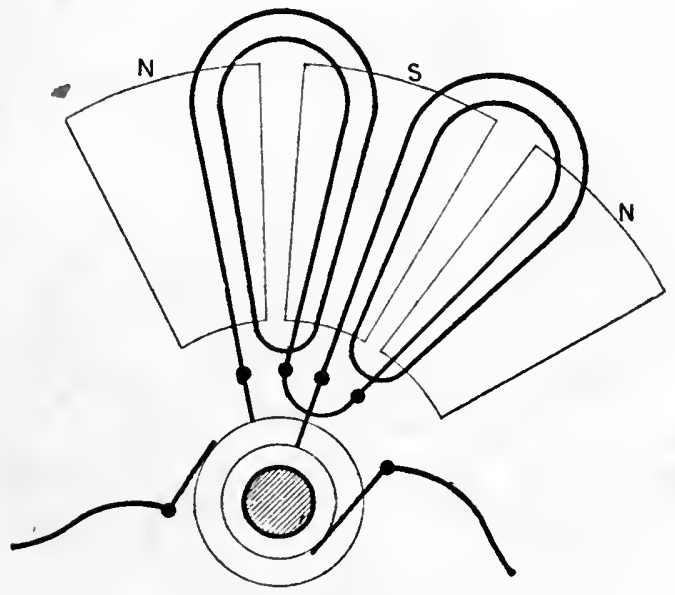

Fig. 77.-Armature of the Siemens machine.

this is the reason why the learned professor's name has been added to that of Mr. Ferranti. One of these arrangements will be easily understood by a reference to Fig. 78, where $\mathrm{W} \mathrm{W}$ represents the induced strip, and M M iron bolts by which it is fastened to an iron ring $K$. In the other arrangement (Fig. 79) the strip is applied to the circumference of a deeply grooved wooden wheel. The strip fits into the grooves and is fastened by bolts D D, which on either side pass beyond the wheel, and are kept in position by a ring $\mathrm{E} \mathrm{E}$ of steel wire. The Ferranti machine is sixty- 
two centimetres high, its foundation plate is fifty-five by sixty centimetres; its total weight is 587 kilogrammes.

The field-magnets are fifteen centimetres long; each core is surrounded by four coils of wire of 3.5 millimetres diameter; and the coils are connected in series, the resistance being $2.5 \mathrm{ohms}$; the vertical diameter of the cores is about
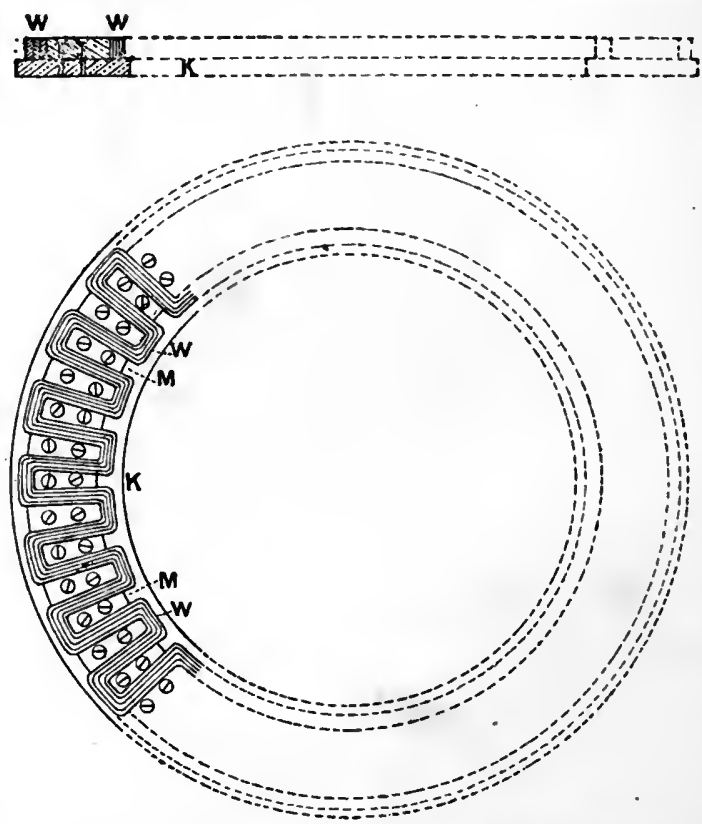

Fig. 78.-Sir William Thomson's coiling arrangement.

eleven centimetres; their largest horizontal diameter, eight centimetres. They are excited by a small Siemens machine giving a current of 21.5 ampères. The resistance of the armature is $0.0266 \mathrm{ohm}$.

At some recent experiments made in London, the Ferranti machine supplied the current for 300 Swan lamps of twenty candles each, three in series and each set of three in multiple arc. The expended force is stated to have 
been twenty-six horse-power, which would give twelve lamps of twenty Carcels each for one horse-power.

The advantages claimed for the Ferranti machine are its moderate price and the special arrangement of its armature

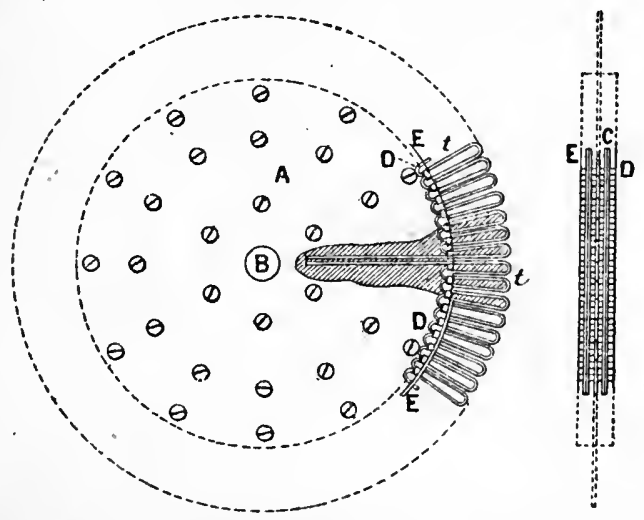

Fig. 79. - Sir William Thomson's coiling arrangement.

which renders the machine very light and of easy construction; also its great speed and the intensity of the magnetic field.

\section{c.-Self-Exciting Machines.}

All dynamo-electric machines which we have described require the use of two machines-an exciting or charging machine, and a machine properly speaking.

Machines which are worked without a distinct and separate charging machine are called self-exciting. The advantage of this arrangement consists in simplicity of transmission and construction, and also in cheapness.

Wilde's Machine.-The first alternating-current machine of this description was constructed by Wi'de, as early as 
1867 ; its use was discontinued for several years, but it is used now for the electric candles of the same inventor.

Wilde's self-exciting machine presents the same appearance as Siemens' alternating-current machine, without the enlargement of the polar extremities of the inductors and without the iron cores of the coils which form the armature in Wilde's model. To charge the inductors, Wilde employs the current produced by a part of the coils of the armature, after having passed it into a commutator of convenient construction.

The other coils are connected with a collector, similar to that of Siemens' machine. Wilde's machine is, therefore, self-exciting in so far as the inductors are fed without the use of a separate machine. It is, in fact, a dynamo-electric machine with direct and alternating currents at the same time.

Gramme's Machine.-In 1879, Gramme constructed a slightly different self-charging machine. It really consists of two distinct machines, mounted on the same frame, and set in motion by the same shaft, and consequently possessing the same speed. One of these is a Gramme dynamo-electric machine with direct currents, for the generation of electricity; the other an alternating-current machine, analogous to the one described on p. 118.

These machines cost fifty per cent. less than those with a separate charging machine. They have the same power, are less heavy, less cumbersome, and more easily set up. Although they are not an invention of great scientific value, they represent, nevertheless, a progress of a certain importance from a practical point of view.

Schuckert's Machine.-Schuckert, of Nuremberg, whose continuous-current machine we have already mentioned, 
has also invented a machine with alternating currents, in which the charging machine is altogether suppressed. For this purpose he reverses the alternating current on a certain part of the circuit, placing in this part the inductors, which are magnetized in the ordinary way by the current which has become direct.

De Méritens' Machine.-M. de Méritens has modified the magneto-electric machine which we have described on p. 111, and has transformed it into a dynamo-electric machine. For this purpose he surrounds the magnets with wire coils, and transforms them into electro-magnets, but, in order to avoid the use of a separate generating machine, he excites his electro-magnets by alternating currents without reversing them.

De Méritens obtains this result by surrounding each magnet with two coils, and, by a convenient distribution of the alternating currents, he transforms each magnet into a weak electro-magnet.

By means of two distributors, each carrying as many teeth as there are poles on the circumference of the inductors, the currents pass successively into each of the inductors, but in such a way that the current passing into a given coil at a given moment is invariably of a sense favourable to magnetic overcharge.

This is an ingenious contrivance, but it is doubtful whether a great enlargement of the magnetic field can be obtained under these conditions, for the point of magnetic saturation of steel is inferior to that of soft iron. On the other hand, the introduction of the coils of the magnets into the circuit produces an increase of resistance, which weakens the intensity of the current, and counterbalances the increase of power resulting from the magnetic overcharge of the inductors. 


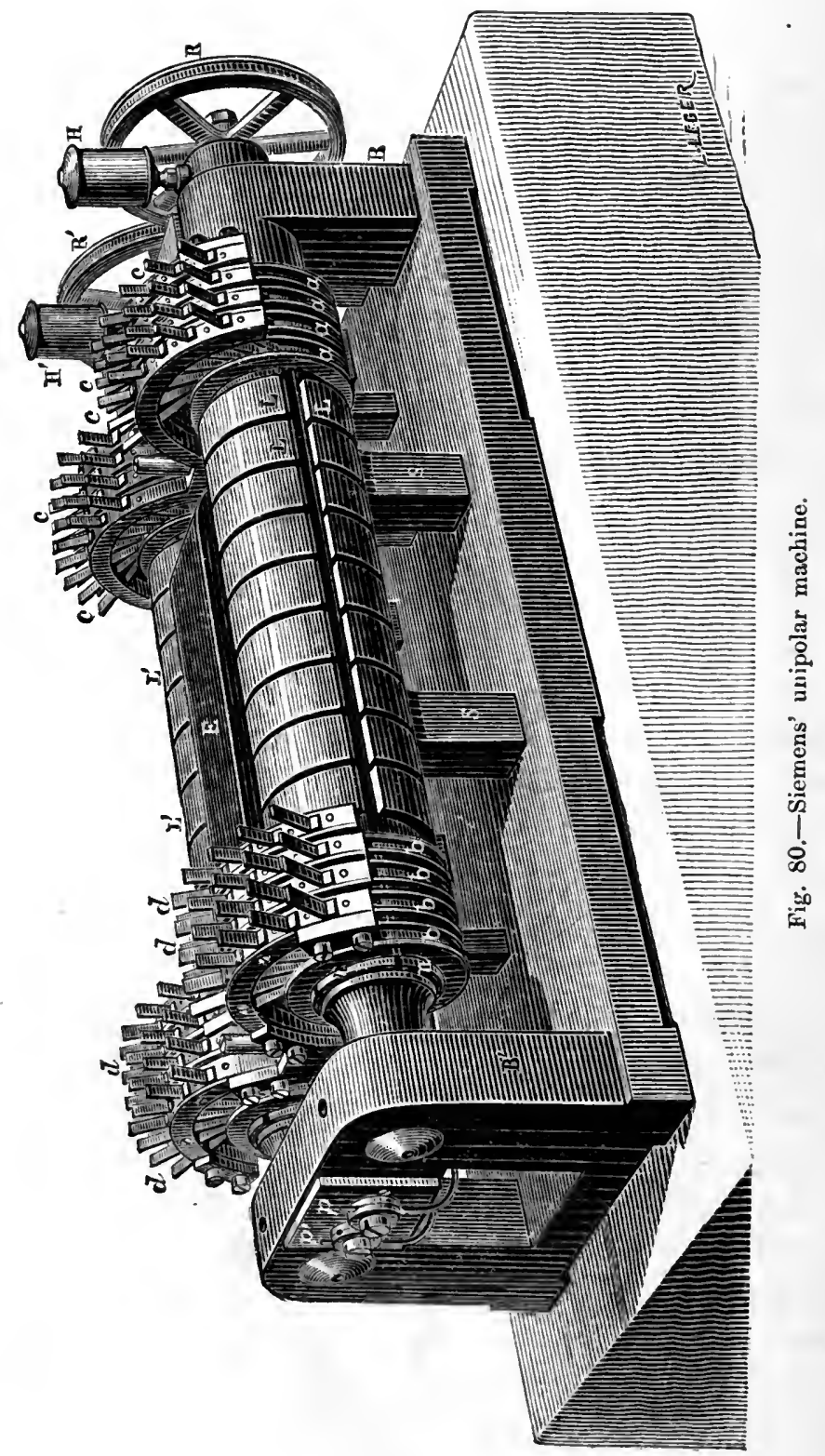


Bürgin's Machine.-Bürgin, of Bâle, has also invented an alternating-current machine; it consists of four rings, revolving in the magnetic field which is formed by the field magnet. In Bürgin's machine, each of the rings feeds the inductors during the fourth part of one revolution at the very time when the sense of the traversing current is favourable for magnetization, whilst the three other rings feed the external circuit. Thus Bürgin's self-charging machine acts the part of two separate machines-one for the inductors, the other for the external circuit.

Siemens' Unipolar Machine.-This remarkable machine, represented in Fig. 80, which really constitutes a class of its own, has a long electro-magnet $\mathrm{E}$ with a core formed of a number of iron plates, which protrude on each side of the coil, and taken in pairs represent an 8 , whilst taken as a whole they form on both sides of the coil two hollow cylinders L L, L' L'. Each of these hollow cylinders constitutes one of the poles of the electro-magnet. On the shaft in the interior are placed four copper plates $n n$, the extremities of which are connected to two ferrules $a b$, insulated from the other bands. We have, therefore, for each pole eight ferrules, and sixteen for the whole machine.

Above these ferrules metallic sectors are fixed to which collecting plates $c c, d d$, are fitted at regular intervals, in such a manner as to surround about one-fourth of the circumference of each ferrule. These collectors can be coupled so as to unite the movable plates either for tension or for quantity, and two wheels $R R^{\prime}$, set in motion by one and the same driving-belt, communicate to the two cylinders formed of the copper plates a motion in the same direction.

It is evident that by this arrangement the electro-motive force is considerably increased. 
Bréguet's Hand-worked Magnetic Exploder.-This is nothing but a magneto-electric machine of the simplest construction, and is based upon Faraday's fundamental experiment. By striking the handle of this apparatus, a sudden removal of the armature ensues, and a current is generated. A second current of inverse sense is produced by bringing the armature back into contact.

This instrument, which is chiefly used for the explosion of gunpowder, requires a current of great tension. The first-

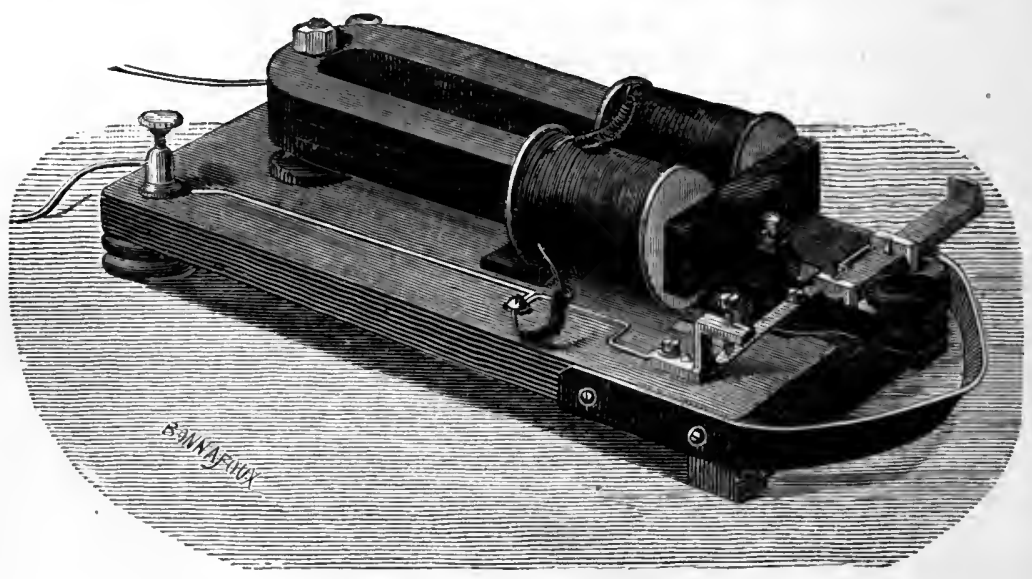

Fig. 81.--Bréguet's electro-magnetic exploder.

named current, the one produced by the sudden removal of the armature, is used in preference to the second, for the simple reason that the movement can be more rapidly executed than the contrary one. The following ingenious contrivance increases the tension of the current :-

The lever of the armature carries a little spring, as shown on the left front of the engraving, the extremities of which are in contact with a screw. When the armature is removed from the magnet, the spring no longer touches the screw. At the moment of departure, however, the 
spring is tightened, and therefore the contact between the screw and the spring only ceases after the armature has accomplished two-thirds of its movement. One of the ends of the conducting wire, which is wound round the arms of the magnet, is in communication with the lever of the armature, the other end communicates with the screw; consequently the current produced by the stroke remains shut up in the apparatus, at least during two-thirds of the time of its production. This arrangement, which at first sight seems to entail the loss of the greater part of the current, has the effect of increasing the tension, because the current produced by the apparatus is no longer a magnetoelectric induction current, but an extra current of this induction current, that is to say, an induction current produced at the moment of the severance of the local circuit from the magneto-electric current.

In fact, the simple addition of the spring and screw increases the tension of the current in the proportion of one to five. This is roughly shown by comparing the shocks received on placing two fingers on the terminals of the apparatus, and in a more accurate manner by the number of fuses which can be lit in either case.

This improvement, added to a careful construction of the machine, enables us to explode extra-fine sporting powder, placed between two metallic points at great distance from one another.

Exploders have been constructed which, by a single stroke, can light twenty Abel fuses; but this great power is only obtained by sacrificing the lightness of the apparatus (these powerful instruments weigh from twelve to fifteen kilogrammes). In most cases we content ourselves with smaller apparatus, weighing eight kilogrammes, which 
are capable of lighting from ten to twelve fuses in the laboratory, and from six to eight in the field.

Finally, in military engineering, experiments have been made with apparatus of very small dimensions and weight, capable of yielding only three or four simultaneous explosions, that is to say, a force sufficient to be absolutely certain of one explosion on the battle-field.

The exploder is the simplest magneto-electric apparatus that has ever been constructed, and we may add that it is impossible to conceive a simpler one, for it merely contains the three parts which are indispensable for repeating Faraday's experiment; they are-a magnet, an armature of soft iron wire, and copper wire surrounded with silk.

The permanent magnet of the new models of exploders consists of a laminated magnet, which, for equal weight, has a much greater power than the original magnet with three arms.

This apparatus, however, cannot be compared with Ruhmkorff's coil as regards its energy. The only advantage it possesses over that powerful instrument is that it is self-acting and always ready for action, whilst the induction coil necessitates the use of a charging battery.

Magneto-Electric Telephone Machine.-To complete the list of apparatus which transform mechanical work into electricity, we must mention the most sensitive and the most delicate of all, Graham Bell's magnetic telephone and its numerous imitations. All magnetic telephones are real electric generators, which, under the influence of the mechanical work resulting from the vibrations which constitute sound, engender undulating electric currents of an intensity proportionate to these vibrations. Thus the most powerful and the most feeble currents which we can produce have all the same origin : mechanical work. 


\section{CHAPTER IV.}

APPARATUS FOR TRANSFORMING ELECTRICITY.

AI.L the apparatus described in the preceding pages are veritable generators of electricity, for they engender electricity, either by means of chemical action as in liquid batteries, or heat as in thermo-electric batteries, or finally, work, as in electro-dynamic machines.

We designate by the term electric transformers apparatus in which electricity is no longer produced directly, but is transformed and changes its properties. No better parallel could be drawn than by comparing them with kinematic apparatus in mechanics. We have seen that a given source furnishes a certain volume or quantity of electricity at a certain pressure or tension. It may be of importance, in certain applications, to increase one of these properties at the expense of another, as it may be desirable in mechanics to transform speed into force or force into speed by means of fly-wheels or driving wheels.

Apparatus which allow of this transformation are called electric transformers.

It is clear that these apparatus can be divided into two large classes-such as transform the electricity they receive with a view to quantity, and such as transform it with a view to tension. 
To the first class belong Gaston Planté's secondary batteries, d'Arrsonval's voltaic condenser, and the batteries of Houston, Thomson, and Varley.

In the second class naturally figure induction coils, Planté's rheostatic machine, and the secondary batteries which, under certain conditions which will be mentioned hereafter, enable us to increase in very large proportion the tension of the original source.

All these apparatus, however, have a common character : they receive electricity and give out electricity, which they transform according to their individual properties.

All secondary couples are electric accumulators as well as transformers. They can be likened to apparatus which serve in mechanics for accumulation of work resulting from the action of forces, such as hydraulic accumulators, reservoirs of compressed air, springs which are appropriately named secondary motors, etc.

Secondary couples, when charged, represent a store of available electric work, which can be expended at will, and within a shorter or longer period; they enable us to obtain in a very short time effects of tension and of quantity much greater than those of the original electric source, or inversely, to produce less intense effects, but lasting for a much longer period. We refer our readers for further details to Gaston Planté's remarkable work, Recherches sur l'Electricité.

Planté's Secondary Batteries.-Planté has been led to the construction of his batteries by the study of the secondary currents which are produced within a battery. Batteries with two liquids and a constant current had been invented by Becquerel, in order to neutralize polarization. Planté, starting from another point of view, endeavoured 
to collect the secondary currents, and to put them to profit for the accumulation of the force of the voltaic battery. We give in his own words an account of the investigations which have led him to adopt the form of a secondary battery as it is used nowadays-

"We had found that the secondary electro-motive force of a voltameter, made of leaden sheets placed in dilute sulphuric acid, was more powerful and more lasting than that of all other metals, and that it even surpassed by one-half that of the most powerful voltaic element known, that of Grove or Bunsen. With such an electromotive force, the only question, in order to form a secondary element of great intensity, was to give it a very feeble resistance-to enlarge its surface as much as possible. This was the more easily accomplished, as the two sheets requisite for its construction were necessarily of the same nature and of an extremely flexible and malleable metal like lead. Thus we were led to construct, in 1860, a secondary element of great intensity, by using an arrangement similar to the one which Offershaus

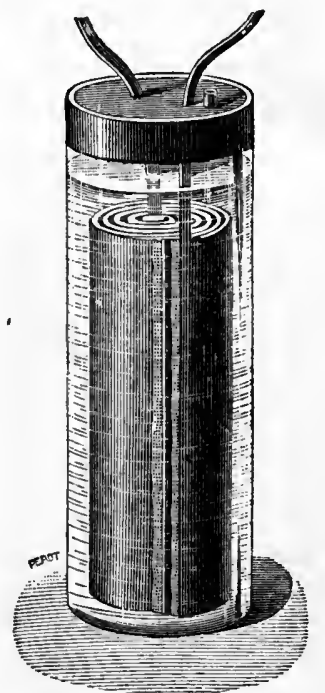

Fig. 82.--Flanté's element (1860). and Hare had used for the voltaic battery properly speaking, that is to say, by forming into a spiral two long and large sheets of lead, separated from one another by a piece of coarse cloth, and immersing them afterwards in a beaker full of water acidulated with one-tenth of sulphuric acid." 
Fig. 82 shows the arrangement of a secondary couple of this kind.

The cloth proved inconvenient, for it introduced an additional resistance, and became useless after a while. Planté then used batteries with parallel sheets, separated by insulating rods, and placed in indiarubber troughs. But these indiarubber troughs contracted after a time, and thereby brought the leaden sheets nearer to one another by curving them, and thus gave rise to contacts. The opacity of this substance, besides, prevented the experimenter from seeing what was going on in the interior of the secondary couples, which must be watched whilst they are charged. We give here Planté's own words-

"We have, therefore, come back to an arrangement similar to the one we described first, modifying, however, the mode of separation of the leaden sheets. We separated these sheets no longer by a piece of coarse cloth, but by narrow indiarubber bands, which possess the advantage of not being affected by the acidulated water, and of only covering a very small part of the surface of the electrodes. Two pairs of indiarubber bands, about one centimetre wide and half a centimetre thick, are necessary to prevent the sheets from touching each other. The opposite extremities of the leaden sheets terminate in two small plates, so as carefully to avoid any cause of contact, and to render uniform the distribution of the primary current over the surface of the electrodes, by holding apart from one another the two points through which the positive and negative electricity pass into the secondary couple. This arrangement, however, is not indispensable, if the leaden sheets are carefully and uniformly rolled round each other. The chemical action of the primary current is then equally distributed over the whole 
APPARATUS FOR TRANSFORMING ELECTRICITY. 145

surface of the secondary couple, even if the two poles of the battery are in close proximity to one another.

"The leaden sheets, separated by two or three indiarubber bands, are rolled round a wooden or metallic cylinder.

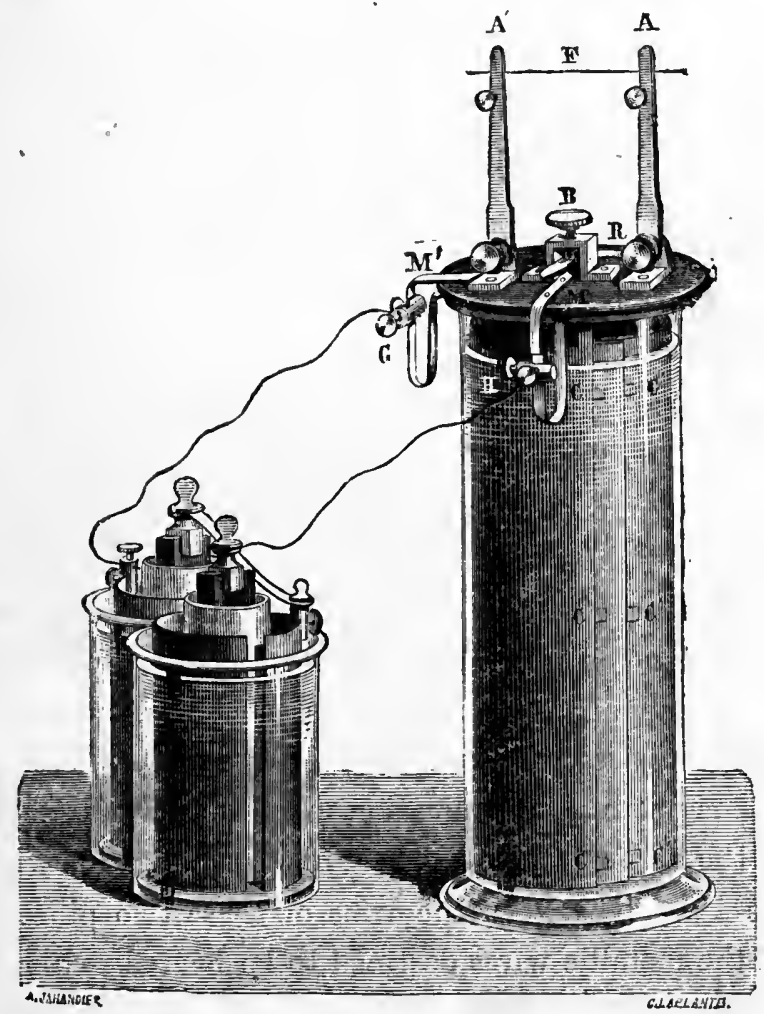

Fig. 83.-Plantê's secondary battery, charged by two Bunsen elements.

"On commencing the first spiral, it will be found convenient to place two small transversal indiarubber bands, of the same length as the cylinder, before the ends of the longitudinal bands, in order to keep apart the edges of the two sheets of lead, which might otherwise touch. When vor. I. 
the coiling is finished, the interior cylinder is carefully removed, and the spirals are kept in their places by means of small indiarubber cross-bars softened by heat. The couple is then placed in a cylindrical glass vessel, and fastened on the inside by small indiarubber slips. The vessel is filled with water acidulated with one-tenth of sulphuric acid.

"Fig. 83 represents an ordinary couple of rather large size, constructed in the way we indicated, and at the same time shows the system of communications for the charge or discharge of the couple and for exhibiting its effects.

"The earthenware vesssel which contains the leaden sheets immersed in acidulated water, is covered with a disc of hardened indiarubber, which carries the metallic pieces destined to close the secondary circuit, when the couple is charged. The extremities of the two leaden sheets communicate, by means of the binding screws $G$ and $H$, at the same time with a primary battery of two small Bunsen elements, and with the copper plates $\mathbf{M} \mathbf{M}^{\prime}$. The plate $\mathbf{M}$ is fastened to the upper surface of a second copper plate $R$, whose prolonged extremity forms a spring, and can be lowered and pressed down by the button $B$, whereby the plate $\mathrm{M}$ is put in communication with the pincers $\mathrm{A}$. Plate $\mathbf{M}^{\prime}$, on the other hand, is in constant communication with the pincers $\mathrm{A}^{\prime}$, and between the arms of these two pincers are placed the metallic wires destined to be heated red hot, and to be melted by the secondary current. The wires of any other apparatus into which this current is to pass can be fastened to the two pincers.

"This system of communications, fixed in the simplest possible manner to the cover of a beaker, does not break the primary current when the secondary circuit is closed. In order, therefore, to try the effect of the secondary couple 
alone, this latter is separated from the primary battery by detaching the wires $\mathrm{G}$ and $\mathrm{H}$; the button $\mathrm{B}$ is then pressed down. But even if these wires are left in communication with the secondary couple when discharging it, no inconvenience ensues; the action of the apparatus is not interrupted; its effects are even slightly increased by the action of the primary battery, which is added to that of the secondary couple; these two are in opposition when charged, and are associated for quantity at the time of discharge."

Chemical Action produced in the Secondary Leaden Couples. -The following is, according to Planté's account, the chemical action of a secondary couple: "When a secondary couple of large surface is new, and the current from two Bunsen couples is passed through it, oxygen gas appears almost immediately on the positive plate; part of this oxygen oxidizes the surface of the plate, which is very soon covered with a very fine layer of peroxide of lead.

"On the other hand, the hydrogen, after having reduced the slight coating of oxide with which the lead may be covered from having been exposed to the air, soon makes its appearance; and if after a few moments the secondary current is tested, it will be found very strong already from the vividness of the spark which is produced on closing and immediately breaking the secondary circuit with a copper conductor of small resistance. But the current thus produced is of very short duration.

"If the apparatus is charged a second time, after having closed the circuit so as to suppress the secondary current, the sheets are in a somewhat different state from what they were at the beginning. While the secondary current was closed, the oxygen, acting upon the sheet which was negative when the current was passing through it, has covered 
this sheet with a slight coating of peroxide; at the same time, the peroxide, formed on the other sheet during the passage of the principal current, was reduced by the hydrogen. We have therefore after a first experiment two sheets, whose surfaces have changed their molecular condition since the beginning of the experiment. They are covered with thin layers of oxide and of reduced metal, which will facilitate the ulterior action of the principal current on the secondary couple.

"If we first consider the sheet of lead which was negative while the principal current was passing for the first time, this shect is, as we have seen, covered with a layer of oxide after the passage of the secondary current. Consequently, if the principal current passes through it again, the hydrogen given off at first will reduce this coating of oxide instead of the slighter coating merely resulting from the exposure to air, as was the case before. Therefore a longer time will elapse before the appearance of hydrogen on the surface of this sheet; for this gas will only be given off when the oxide is completely reduced to the state of finely divided lead.

"During the second passage of the principal current through the positive sheet, the first particles of oxygen which rise to the surface meet with a layer of reduced peroxide, or finely divided metallic lead, on which the gas acts much more energetically than on the original leaden sheet; the gas is more easily absorbed, and a delay in the appearance of oxygen on this sheet will be noticed-a delay which corresponds to the time necessary for the reoxidation of the layer of reduced lead.

"When the secondary current is closed again, these phenomena are reproduced, and it is clear that, after these opera- 
tions have been repeated a great many times, the surfaces of lead of the secondary couple will be in a more favourable condition for oxidation or reduction; the layers of oxide alternately formed or reduced will become thicker, and the secondary effects which result from this will be more lasting and more intense.

"And this is really what takes place: the longer a secondary couple is exposed to the action of a primary current, and the longer it acts itself afterwards, the more lasting is the secondary current."

The formation of a secondary couple requires a number of precautions, which are minutely described in Planté's work. With a well-formed couple, twenty or thirty minutes may elapse during its charge with two Bunsen elements, provided the surface of the couple amounts to one square metre.

The whole work of the batteries is expended upon the oxidation of the lead on the one hand, and on the other on the reduction of the oxidized lead produced by the previous closing of the secondary current.

When once the gases are given off in a well-formed couple, it may be taken for granted that the battery no longer performs any work available for the production of the secondary current.

Power and Duration of the Discharge of the Secondary Couples.-The discharge of a well-formed secondary couple depends on the size of the sheets, the thickness of the deposits produced, and, finally, the exterior resistance of the circuit. The discharge is very constant as long as the battery contains electricity stored in the form of chemical work.

In the same way as in a very large and shallow vessel 
the liquid will flow for a long time and steadily from a small opening, and cease suddenly as soon as it stands below the opening, so a secondary couple of large surface, which heats a metallic wire to redness or produces a deflection of the galvanometer, will only show a diminution of intensity a few moments before the action ceases altogether.

The fact is striking, when the secondary current is discharged by making it traverse a platinum wire. The incandescence remains uniform for a long time, and ceases

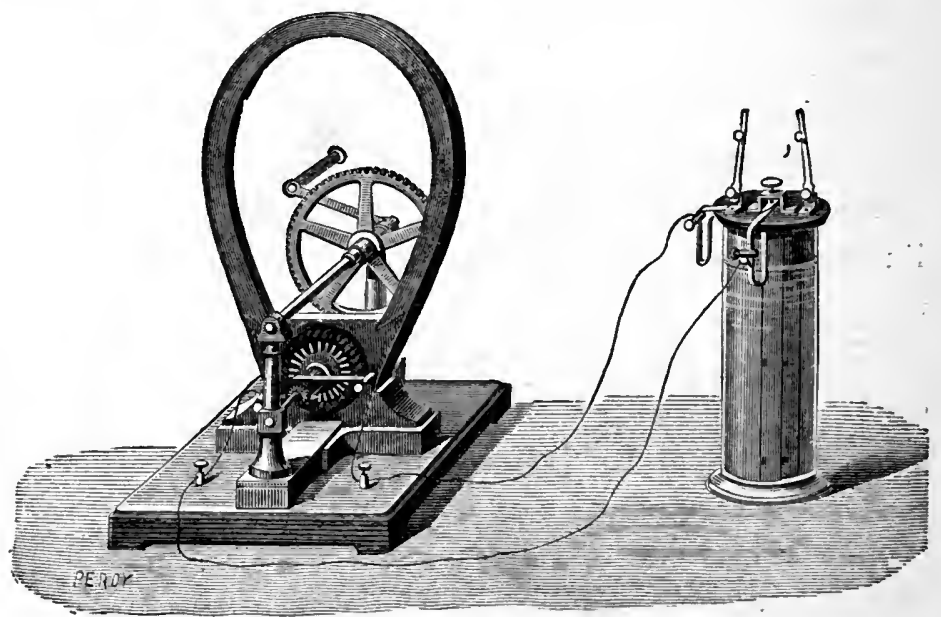

Fig. 84.-Planté's element charged by a Gramme's laboratory machine.

suddenly as soon as the store of chemical work accumulated in the couple is exhausted.

The electro-motive force of a well-formed secondary couple reaches about two volts and a half; this explains why at least three Daniell or two Bunsen elements are required for its complete charge.

The feeble interior resistance of the couples, which varies between $\frac{1}{20}$ and $\frac{1}{50}$ of an ohm, explains the quantity furnished by these instruments. Plante's elements can also be charged 
with Gramme's laboratory machines, and Fig. 84 represents this experiment; but in this case the speed must not fall below a certain point, or the battery would discharge itself into the machine to no purpose. To prevent this inconvenience, an automatic joining and disjoining arrangement has been constructed, which short-circuits the secondary battery as soon as the electro-motive force of the machine becomes too feeble to charge it, and places it back into the circuit as soon as the electro-motive force has become sufficiently strong to effect the charge. (See La Lumière Électrique, June 15, 1880.)

The secondary couples can preserve the accumulated charge for a long time. Thus a well-formed and wellcharged secondary couple can heat to redness for a few minutes a platinum wire half a millimetre thick, even two or three weeks after having been charged.

Planté has even obtained this effect with exceptionally well-formed couples more than a month after their charge.

The same observer has measured the proportion of the electric work restored by the discharge, to the electric work expended for the charge, by decomposing copper sulphate in a voltameter. The efficiency has been found to be from eighty-eight to eighty-nine per cent.; the secondary couple is, therefore, a rather perfect accumulator of the work of the voltaic battery.

Planté's Electric Tinder-box.-Fig. 85 shows a particular form which Planté has given to the secondary element, and which he has called "le briquet de Saturne" (Saturn's tinderbox). On the upper part of the box two small pincers are seen, between which a platinum wire is stretched. Whenever the two springs at the bottom are brought into contact by touching them with the finger, the battery current 
passes through the platinum wire, heats it to redness, and immediately lights a candle. A candle can be lit a hundred times with this apparatus before the latter requires to be

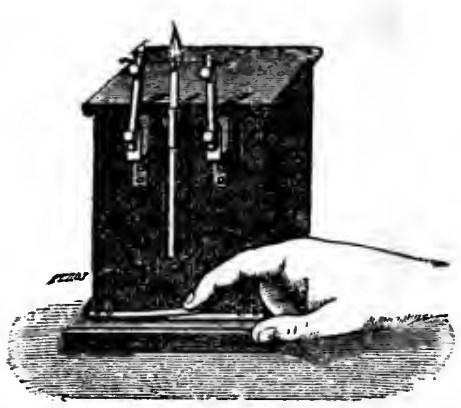

Fig. 85.-Electric tinder-box, with Planté's secondary lattery. charged anew with three Daniell elements. This is a new and very inexpensive way of obtaining a light, for the secondary couple itself expends nothing, and the charging battery only consumes a few grammes of copper sulphate for a very long period of work on the part of the tinder-box. This same apparatus can be used for tiring mines in civil and military engineering; experience shows that, with fuses of fine platinum wire (one-twentieth of a millimetre), a mine can be exploded at a distance of 900 metres, by means of a copper wire of that length and of three millimetres thickness:

Faure's Storage Battery.-This battery, which created a certain sensation when it was first brought out, is nothing but a slight modification of Planté's apparatus. Faure's aim was to increase the capacity of Plante's accumulator and to suppress the long and wearisome task of formation. For this purpose he coated the two leaden electrodes with a layer of red lead (which he fastened to the leaden sheets by rivets of the same metal), a sheet of parchment, and a piece of felt. In every other respect the construction of the apparatus is identical with that of Planté. After a relatively short period of formation-about a hundred hours-one of the layers of red lead is found to have changed completely into 
peroxide of lead, and the other, on the second electrode, has been reduced to lead. Faure also replaces the glass vessel by a leaden one, whose interior surface forms part of one of the electrodes.

The quantity of electricity stored depends, cceteris paribus, on the quantity of peroxide and of reduced lead deposited on the sheets. It would appear $\dot{a}$ priori that Faure's battery ought to have, for equal weight, an incomparably "larger storing capacity than Planté's; such, however, is not the case. Accurate experiments made by several French electricians have shown that of the work expended for the charge only sixty-six per cent. was returned.

Reynier's Modification of Faure's Battery. - Reynier found that in almost all the couples hitherto constructed contacts existed, giving rise to deteriorating local currents, and also that the felt used for the separation of the sheets became rotten after a short time; he therefore wrapped the leaden sheets, after having coated them with red lead, in a sort of woollen serge instead of felt, which gave infinitely better results. He also went back to the use of glass beakers instead of the leaden vessels, so as to be able to see what was going on inside the couple. Reynier thus supplies a battery of good efficiency and moderate price.

Kabath's Storage Battery.-Kabath forms his electrodes of leaden strips with perpendicular folds, and places from six to fourteen of them to a wooden trough at a short distance from each other. The odd plates are joined together and connected to a terminal, which forms one of the poles of the battery; the even ones are joined likewise, and connected to the other pole. The arrangement, therefore, is similar to that of the plates of a Faure battery of rectangular shape. Now, each electrode is composed of 
about a hundred thin vertical strips, alternately level and undulating, which are placed alongside of each other, and form a sheet from eight to nine centimetres wide, one centimetre thick, and forty centimetres long. The strips of lead are fastened together by leaden bands pierced with. holes.

Sellon and Volckmar's Storage Battery.-The leaden sheets employed in this battery are perforated, and the holes are filled up with spongy lead; the sheets are four millimetres thick, and the holes are about two millimetres apart. The active part of the plate is thus principally formed of the substance which fills the holes; the solid parts support the whole and participate, in a certain measure, in the electro-chemical action. De Chanzy employs masses of lead which, from their mode of preparation, present a slight weight and a very large surface. This result is obtained by melting the lead and projecting it in a particular manner on a cooled surface. The lead thus suddenly cooled forms very finely divided masses. De Chanzy places these masses into a vessel divided into two compartments by a porous medium.

Pezzer and Carpenter's Storage Battery.-This apparatus is constructed in the following manner:-A number of narrow strips of lead, ten to fifteen millimetres wide and 500 millimetres long, are cut out of leaden sheets of convenient thickness; they are pressed between the rollers of a foldingmachine, and come out crimped.

Each of them is folded in two, and a large number of these strips are placed on top of one another. The free ends are joined together by a soldering of lead, and a sort of fringe is thus formed, as indicated in Fig. 86.

These crimped strips are introduced in the place of 
carbon and zinc into a Bunsen element of rectangular shape, called Ruhmkorff's model. They fill the porous vessel and the intervals between it and the sides of the exterior vessel; the two vessels are filled with acidulated water. The ends

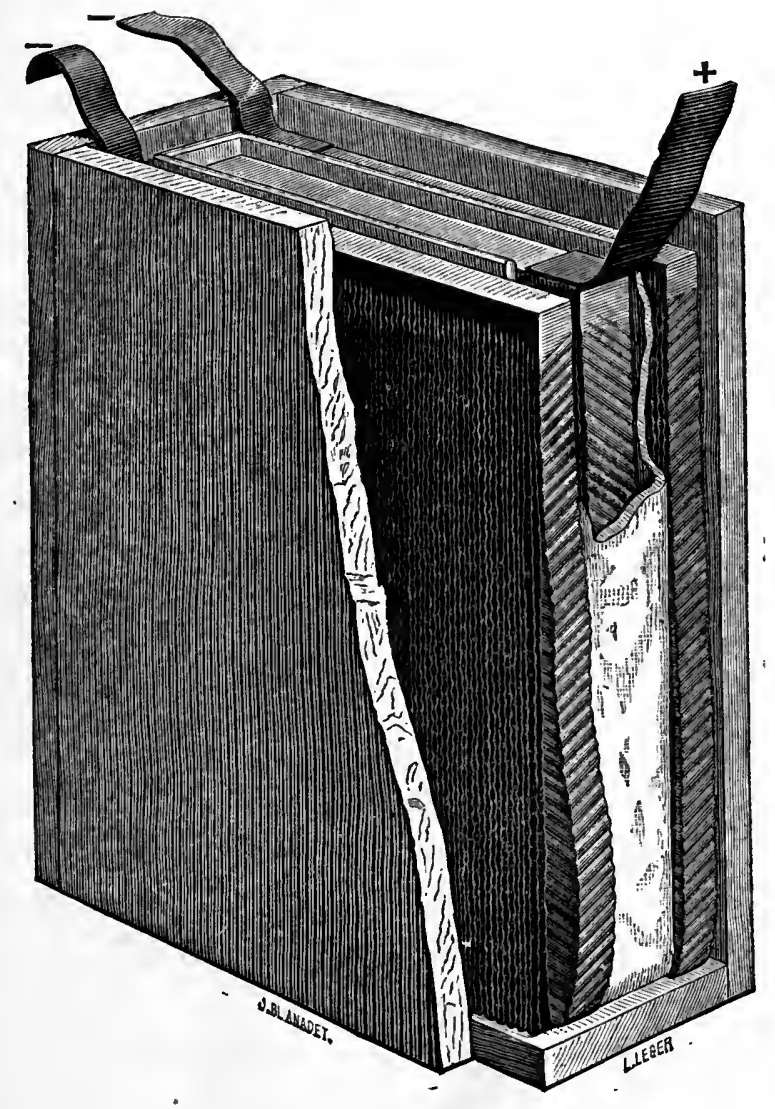

Fig. 86.-Pezzer and Carpenter's storage battery.

thickened by the solderings are at the top, the folds are below; plates soldered to the upper part serve as electrodes-positive in the interior and porous vessel, negative outside. By this arrangement, the negative electrode has 
a surface twice as large as that of the positive electrode. To obtain this result the positive strips are 0.5 millimetres thick and 15 millimetres wide, whilst the negative electrodes are 0.2. millimetres thick and 10 millimetres wide.

After the strips have been bent, their undulations make an angle of $45^{\circ}$ with the borders, and the inclination of the channels is alternate on the two halves brought into contact. This arrangement maintains and limits the distance of the neighbouring parts. The channels thus formed ensure the electrolytical action on all points, and their shape facilitates the generation of the gases at the moment when they are first given off.

Pezzer and Carpenter have also constructed a trough apparatus of two compartments, each of which is arranged in the same manner as the one shown in the figure. Excellent results have been obtained with this battery, but no figures are given to show the actual efficiency.

For all practical purposes, the source of electricity used for charging the secondary batteries would be some form of dynamo-electric machine. Now, for lighting by electricity directly with such a machine, the greatest eare has to be taken to prevent irregularity in the motion of the steam-engine which drives it, for every small jerk or other unevenness produces flickering in the light. The storage battery at onee provides a means of getting over this difficulty, for, if the dynamo-electric machine be employed to charge it, the battery may afterwards be connected with the lamps, and a steady current and unflickering light be produced, no matter how irregular the process of charging may have been. And this battery also provides a means by which waste energy can be economically utilized. It very often happens in factor.es that steam-engines are 
wasting steam, because less work than usual is being done by the machinery, and it is plain that if a dynamo-electric machine were arranged so that it could easily be put in gear, this waste need no longer take place, for the batteries could very well be charged at such times, and the stored energy would be available for lighting up or for driving motors.

Sutton's Electrical Storage Battery.-This storage battery, invented by Henry Sutton, of Ballaarat, Victoria, has a negative electrode of copper, and a positive electrode of lead amalgamated with mercury, immersed in a solution of cupric sulphate.

The chemical changes in this cell are exceedingly interesting and beautiful, the cell being composed of a sheet of lead cleaned with sulphuric acid and amalgamated thoroughly with mercury, and a sheet of thin copper a little shorter. The two sheets are perforated with a number of holes and then rolled in a spiral, separated by rubber bands cut every five inches; the holes in the plates and the cuts in the rubber bands being to allow free circulation of the solution (the short plate being uppermost before rolling). The combination is immersed in a solution of cupric sulphate, and the amalgamated lead plate made the positive electrode of a suitable source of electricity; the chemical action being that the oxygen of the decomposed solution combines with the lead, forming a perfectly even coating of the insoluble peroxide, the hydrogen replacing the copper of the solution, and the copper being deposited in the metallic state on the negative electrode. As the decomposition of the cupric sulphate proceeds, the solution gradually loses its azure blue colour, becoming more acid; and finally, when the whole of the copper is deposited, we have the solution colourless 
and transformed into hydric sulphate and water, the positive electrode peroxidized, and copper deposited on the negative electrode. During discharge, the peroxide is reduced and the copper element oxidized, the oxide combining with the acid and forming cupric sulphate, the solution returning to its original colour. This change of colour forms a beautiful means of telling when the cell is charged; it is a veritable charging gauge. The power of this cell is very great and very constant; it can be made to last for hours, the time being dependent on the quantity of cupric sulphate decomposed.

Sutton obtained, by the decomposition and recomposition of one pint of cupric sulphate, over two hours' effective work in heating to a red heat one inch of No. 28 iron wire, the cell measuring internally four inches deep and four inches diameter.

A practical form of cell for storing purposes may be made by fixing a series of amalgamated lead plates in a box in grooves, as in Cruikshank's trough battery, filling the intervals between the plates with solution of cupric sulphate, and passing a current through of sufficient tension to overcome the contrary electro-motive force of the series, the positive sides of the plates being peroxidized, and copper deposited on the negative sides. Each box contains twenty-five of these plates, and a number of these boxes can be coupled for quantity while charging and for tension during discharge.

This battery is a powerful and constant source of stored energy; the cell does not polarize itself during discharge, as is the case in both Planté's and Faure's cells,-in these cells the peroxide formed by the discharge produces a contrary electro-motive force. 
APPARATUS FOR TRANSFORMING ELECTRICITY. 159

Houston and Thomson's Storage Batteries.-In the apparatus invented by these two Americans, the secondary phenomenon on which the principle of accumulation is founded is nothing but the chemical decomposition of a salt-zinc sulphate in this particular case-by the electric current.

Fig. 87 represents the principle of their storage battery, and Fig. 88 a series of elements arranged for tension, to produce more intense effects.

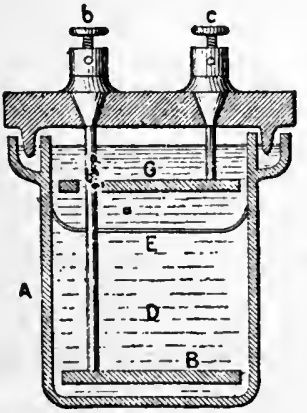

Fig. 87.-Simple element.

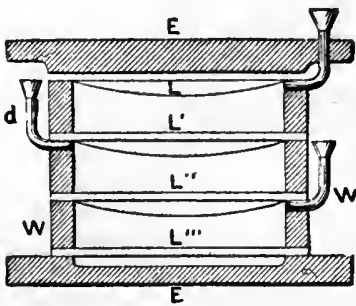

Fig. 88.-Series of elements.

Houston and Thomson's electric storage batteries.

In Fig. $87, \mathrm{~B}$ is a copper plate joined to the terminal $b$, and $\mathrm{C}$ a carbon plate connected with the terminal $c$. The vessel $A$, which contains the two plates, is filled with a solution of zinc sulphate; $\mathrm{E}$ is a porous diaphragm which separates the two plates.

If the current of a dynamo-electric machine traverses the apparatus in the sense $b c$, the zinc sulphate will be decomposed, and metallic zinc deposited on the carbon plate $\mathrm{C}$, whilst in the lower part of vessel A a concentrated solution of copper sulphate will be formed. The length of time during which the deposition or the charge lasts is only limited by the quantity of zinc sulphate contained in the 
vessel, and by the thickness of the copper plate $B$. This apparatus constitutes, therefore, a gravity element, similar to that of Callaud, and capable of furnishing an electric current, as long as the carbon plate $\mathrm{C}$ is covered with zinc.

Fig. 88 represents a series of couples mounted for tension. $\mathrm{L}, \mathrm{L}^{\prime}, \mathrm{L}^{\prime \prime}, \mathrm{L}^{\prime \prime \prime}$ are copper plates, separated by insulating rings ; the intervals between these plates are filled with a solution of zinc sulphate; the lateral tubes $d$ allow the expansion of the liquid.

The storage of electricity no longer depends on the surface of the plates, but on the quantity of zinc sulphate, which of course is a great advantage, but since the electromotive force of each element is equal to that of a Daniell element, and since the interior resistance must be very great, a considerable number of elements will be required to produce an appreciable effect, and even if there be a gain from the fact that the battery can continue its activity for a much longer time without requiring a fresh charge, yet it appears that, for equal power, this apparatus must be much more cumbersome and much heavier than Gaston Planté's secondary battery.

D'Arsonval's Voltaic Condenser.-At the very same time that Houston and Thomson were constructing their storage battery in America (October, 1879), M. d'Arsonval, Demonstrator at the Collège de France, was studying the same question, with the view of increasing the charge stored in Planté's secondary battery. In a memorandum presented to the Académie des Sciences on the 26th of January, 1880, he describes his secondary couple, which consists of a zinc plate and a carbon plate placed in a porous vessel full of granulated lead or small shot, the whole impregnated with a concentrated solution of zinc sulphate. 
If the couple is charged by a current passing from the carbon to the zinc, the zinc salt is decomposed, the oxygen forms peroxide of lead on the small shot, and the sulphuric acid remains in a free state. The deposition of the oxidizable metal zinc is therefore no longer limited, and the oxygen,can accumulate in larger quantity in the form of peroxide of lead. Manganese can be substituted for lead, silver for copper, and the results will be equally satisfactory ; but none of these can be compared to the peroxide of lead adopted by Planté.

Such is D'Arsonval's apparatus; a couple containing a kilogramme of small shot can keep in motion for four hours one of Marcel Deprez's small motors.

Mr. Varley has also invented secondary batteries constructed on a principle similar to that of Houston and Thomson.

Plante's, Faure's, and Sutton's secondary batteries, however, are without contradiction, the best of all, and the two former have already received important applications in practice. The question of storage of chemical work in secondary batteries, and its recovery in the form of electricity, presents the greatest interest and has the greatest future. We shall say a few words about it when we come to the electric motors; researches on this subject will be most remunerative in every respect, for a light and powerful electric accumulator would find a number of applications, the enumeration of which alone would fill a volume.

A method of accumulation brought before the meeting of the British Association at Sheffield, in 1879, by Mr. Ayrton, must also be mentioned here. He proposed to store the work of waterfalls, by decomposing water under pressure, and utilizing the hydrogen thus produced. We find VOL. $\mathrm{r}$. 
here, in another form, and with the additional principle of compression, the idea which led to the formation of the "Alliance" company, but as it is no longer a question of burning coal in order to produce gases, but rather to utilize natural forces, the question presents itself from a more philosophical point of view.

Induction Coils.-There is no necessity for giving here the history of these apparatus, which have rendered the name of Ruhmkorff celebrated, and have earned for this learned and unpretending inventor a prize of 50,000 francs awarded by the Académie des Sciences.

An induction coil is nothing else but an electric transforner, receiving electricity from a battery, and transforming it into electricity of high tension, similar in its effects to the so-called statical electricity. These currents are characterized by their high tension on the one hand, and by their feeble intensity, or quantity, on the other. That explains why these currents, although capable of overcoming great resistance, can only act very feebly when traversing electro-magnets in which the magnetic action is a function of the intensity of the current, unless these electro-magnets are surrounded by a very fine and very long wire.

The various applications of the Ruhmkorff coil are well known, as for the firing of mines and torpedoes, its application in medicine, the lighting of Geissler's tubes; its use for physical experiments, etc.

This interesting apparatus can, therefore, no longer be considered as a new application of electricity, but we must nevertheless mention here an induction coil remarkable for the effects which it produces, and which is probably the most powerful one constructed up to the present.

Spottiswoode's Induction Coil.-Mr. William Spottis- 
APPARATUS FOR TRANSFORMING ELECTRICITY. 163 woode, late President of the Royal Society, has had constructed by Mr. Apps an induction coil of remarkable power,

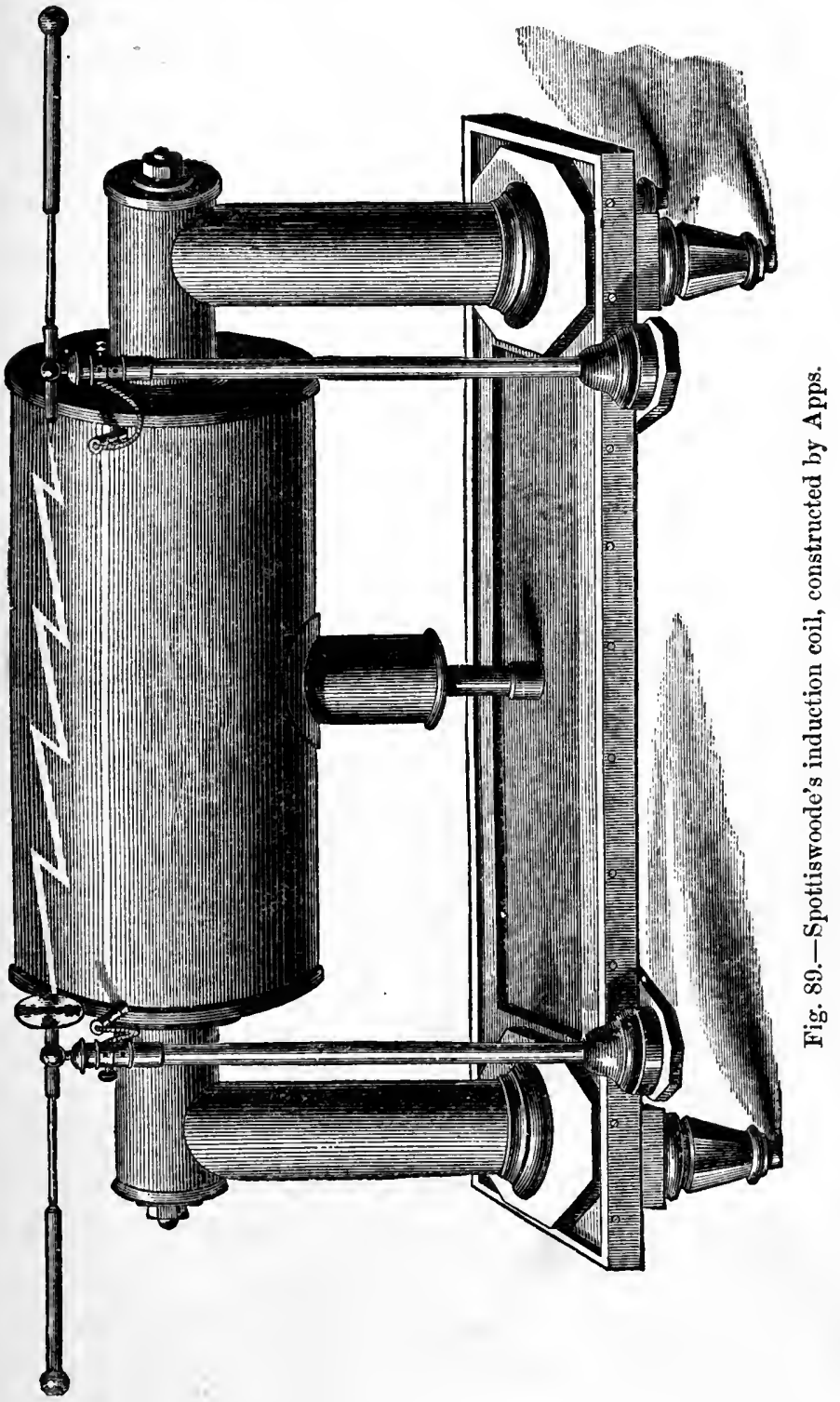


which has given sparks 110 centimetres long. The noise produced by each spark is like the report of a pistol.

The general view of the instrument is represented in Fig. 89. The coil is supported by two massive wooden pillars, covered with indiarubber and coated with paraffin at their two extremities. Besides these two supports, there is a third in the middle, whose height can be regulated by means of a screw, in order to resist the bending of the principal piece.

The coil can act with two induction circuits used alternately. Two persons can remove the one and substitute the other for it in a few minutes.

The first, which is use̊d for long sparks, and in fact for most of the experiments, has a core composed of a bundle of iron wires of $\frac{8}{10}$ of a millimetre each, and forming together a cylinder of 1.1 metre in length and nine centimetres in diameter. It weighs nore than 30 kilogrammes. The copper wire constituting the inductor has a length of 600 metres, a diameter of $2 \cdot 23$ millimetres, a conductivity of $\frac{93}{100}$, a total resistance of $2.3 \mathrm{ohms}$, and weighs about 25 kilogrammes. It is wound round the bundle of iron wires in 1344 spirals, which are arranged in six layers, and finally presents the form of a cylinder of 1.06 metre, and of an exterior diameter of 12 centimetres.

The other system of induction which is intended for batteries of larger surface, and to give shorter and broader sparks for experiments with the spectroscope, has a core of iron wires of $\frac{8}{10}$ of a millimetre, like the preceding ones, joined together, and forming a cylinder of 1.11 metre long and 97 millimetres wide. The weight of this core is 41 kilogrammes. The copper wire is the same as in the first circuit of induction. The conductor, which is 459 
metres long, is composed of two parallel wires. It is divided into three sections of two layers each; their resistances are $0 \cdot 181,0.211$, and $0.231 \mathrm{ohm}$ respectively. The length of the cylinder composed in this way is 1.01 metre, and its diameter 14 centimetres. The weight of the wire is 38 kilogrammes. Thanks to a new arrangement, these three sections can be joined for one single series, which gives a conductor of 4.87 millimetres diameter, or they can be placed on a shunt, which gives the equivalent of one single conductor of 14.63 millimetres diameter.

This conducting wire has given sparks 86 centimetres long (in the air, with a Grove battery of ten elements, whose platinum electrodes were 76 millimetres by 159$)$.

The secondary circuit is composed of 450 kilometres of wire forming a cylinder of 95 centimetres in length, 50 centimetres exterior, and 24 centimetres interior diameter. The conductivity of this wire is 94 per cent., and its total resistance $110,200 \mathrm{ohms}$. It is divided into four sections; the wire of the two middle sections has a diameter of about 0.24 millimetre, whilst in the two exterior sections its diameter is 0.28 and 0.29 millimetre respectively. The reason why the diameter of the induced wire is larger towards the extremities than towards the centre is the accumulated charge which these parts of the conductor have to support. Each of these sections is itself composed of flat dises, which have a thickness of about 200 layers of the wire of which they are formed. Finally, the total number of spirals in the induced circuit amounts to 341,850 .

It will be easily understood why such a great length of wire is required on considering that the exterior spirals are nearly a metre and a half long. It is admitted that the spark 
grows rather with the number of spirals than with the length of wire, provided that a good insulation is ensured in all the parts. To make sure of the result, each disc was separately and carefully tested; then they were tested in series, and all the results carefully registered. As a conclusive test, the coil was charged with seventy Grove elements, without interfering with the insulation.

The condenser belonging to this coil is much smaller than might be expected. After numerous experiments, Apps fixed upon a dimension which is used for coils giving a spark of 25 centimetres. These condensers are formed of 126 sheets of tinfoil, $4 \check{5}$ centimetres long by 21 centimetres wide, which are separated by a double thickness of varnished paper, and measure altogether 0.28 metre.

The following are some of the results obtained with this powerful apparatus (the first system of induction being used) :-

With five large Grove couples, the spark reached a length of 71 centimetres;

With ten couples, a length of 81 centimetres;

With thirty couples, a length of $106 \frac{1}{2}$ centimetres was obtainerl.

In some cases even 109 or 110 centimetres were obtained.

It is well known that an induction coil gives two induction currents-one at the moment of the closing of the inducing circuit, the other at the moment of breaking this circuit. The former possesses no tension; it is the second which produces the spark. Experiments made with the handsome apparatus mentioned have proved that the tension of the former can increase to such an extent that sparks of thirty-one millimetres are obtained by it. 
This fact, without precedent, deserves the greatest attention. The length of the spark of the current obtained by closing the circuit naturally depends on the intensity of the current of induction, and increases with it.

Flint-glass of seventy-five millimetres thickness has been pierced by this apparatus, without even using batteries which give the maximum effect.

Continuous High-tension Currents.-Induction coils furnish a current of very high tension, but intermittent and alternating, or, to speak more accurately, they furnish two perfectly distinct kinds of currents: the closing current, having little tension and much relative quantity; followed by an induced breaking or opening current, having an enormous tension, but much less quantity.

In certain experiments it may be desirable to have a continuous high-tension current.

Warren de la Rue has solved the problem by using 11,000 elements of his silver chloride battery, which has been described on p. 29. But the resistance of these elements is rather large, and, on the other hand, 11,000 elements occupy a certain space and represent a considerable sum of money.

Gaston Planté, who has made a number of the most interesting researches on high-tension currents, has used secondary batteries of 200 to 800 elements, and has obtained at the outset an effect equivalent to that of 300 to 1200 Bunsen elements. The tension of each couple, which always exceeds two volts, reached $2 \cdot 8$ volts at the beginning of the discharge; it is clear, therefore, that 800 secondary couples can furnish, within a very small compass, a tension superior to that of 2000 Daniell elements, and also of very superior quantity. 


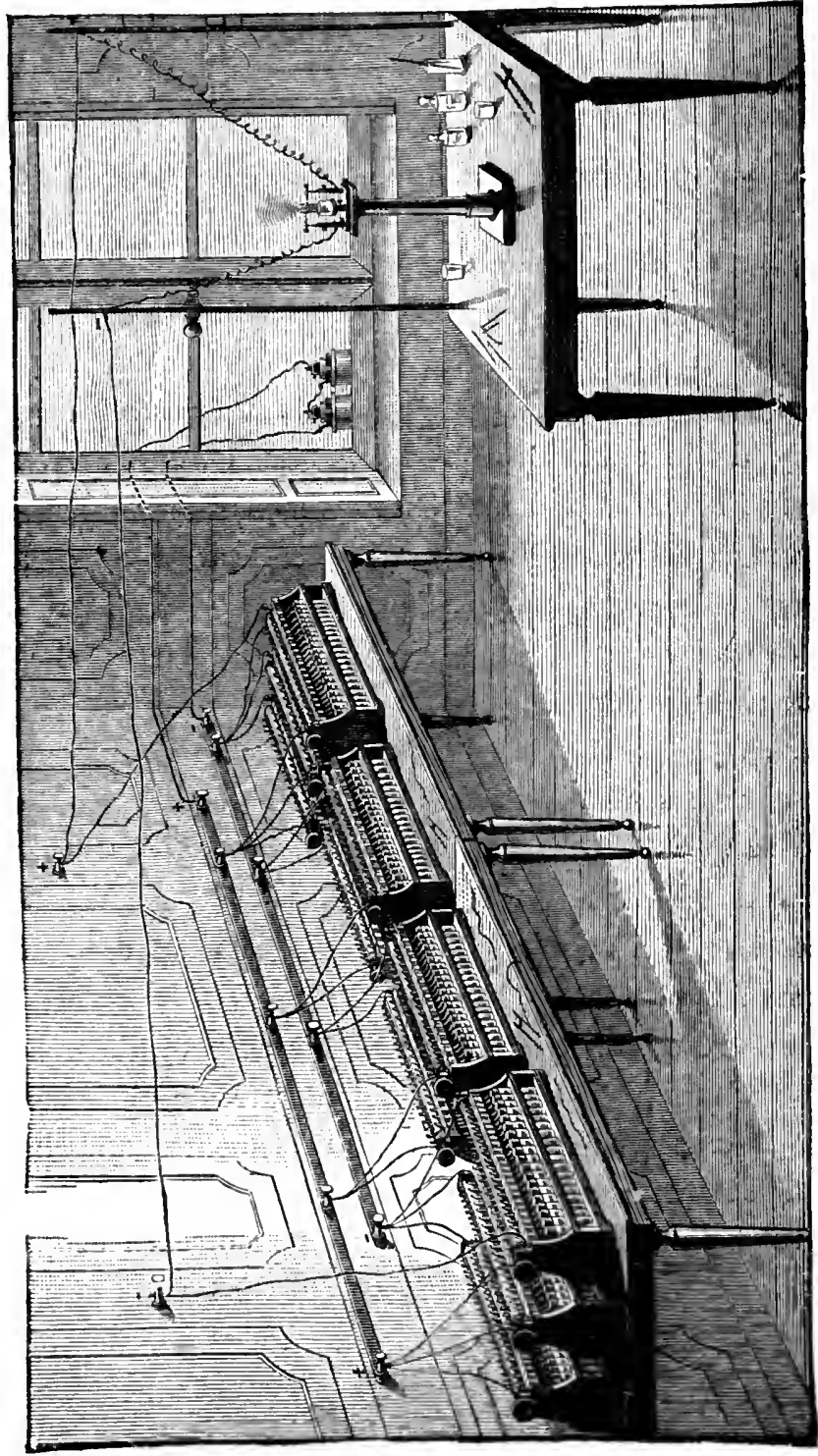

定 
Fig. 90 represents an arrangement of 400 elements, divided into ten batteries of forty couples. Two Bunsen couples are sufficient to charge each group for quantity; then, by means of a special commutator, they are grouped for tension, to effect the discharge. In a few seconds the work of several hours of the two Bunsen elements is expended, and this will explain the intensity of the effect.

These experiments, where the current possesses both tension and quantity, are very dangerous, and the apparatus must be handled with the greatest care. Planté received one day the discharge of 600 couples, and he describes the sensation as if a burning iron had been passing through his whole body. Fortunately, this accident had no fatal consequences; but, adds Planté, "it might have been otherwise if the 800 secondary couples had been in action at that moment."

Planté's Rheostatic Machine.-It has been mentioned that Planté had obtained tensions of more than 2000 volts with 800 secondary elements. These results even have been surpassed, and the rheostatic machine enables us more completely to transform the force of the voltaic battery, and to obtain a tension equivalent to that of apparatus of statical electricity. We again give his own account of the invention and construction of this interesting electric transformer-

"Having observed how easy it was to rapidly charge, with a battery of 800 couples, a condenser with a sufficiently thin insulating plate of glass, mica, indiarubber, paraffin, etc., I joined a certain number of condensers made of mica covered with tinfoil, and arranged them like the couples of the secondary battery itself, so as easily to charge them in quantity and discharge them in tension. 
"All the pieces of the apparatus must of course be carefully insulated. The commutator consists of a long cylinder

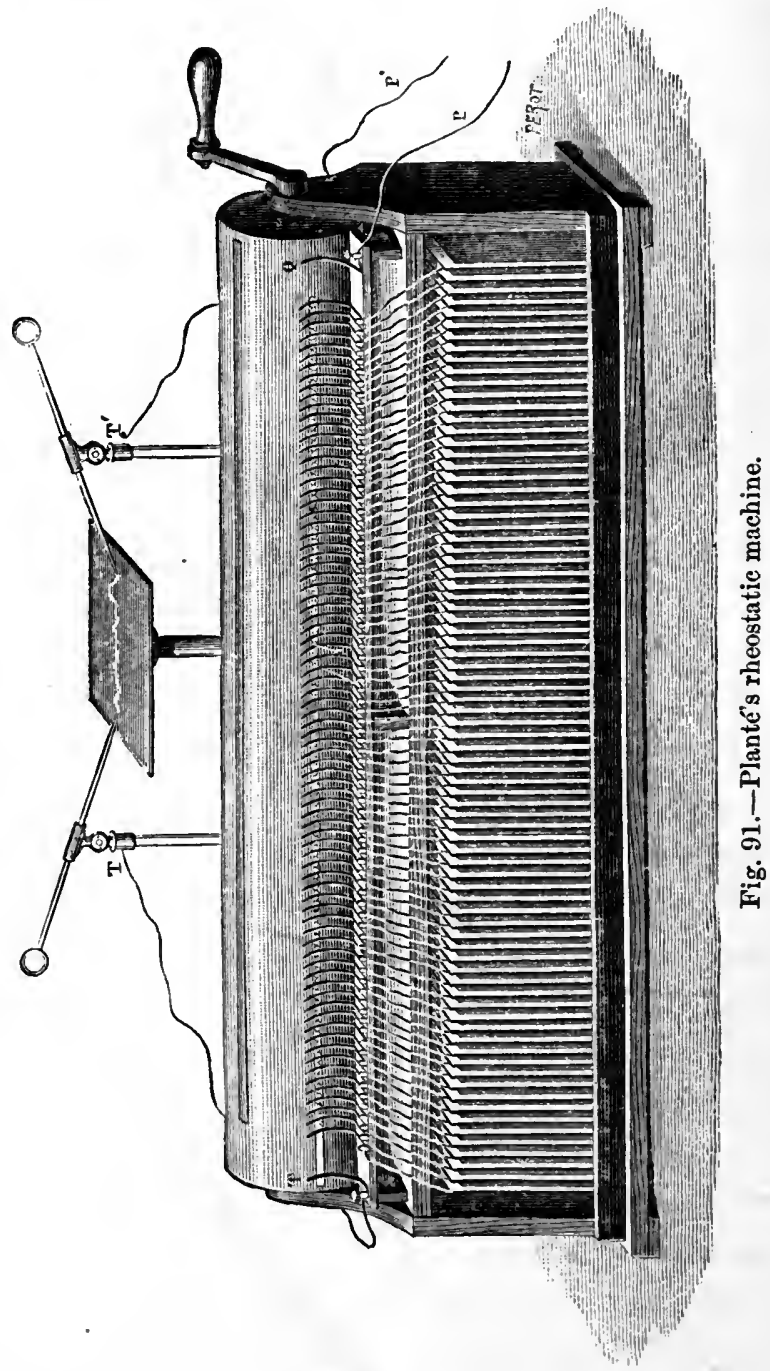

Q T (Fig. 91) of hardened indiarubber, and is provided with longitudinal metallic bands, which unite the con- 
densers for surface; copper wires, bent at right angles at their extremities, at the same time traverse the commutator to associate the condensers for tension. Thin plates or metallic wires in the shape of springs are in contact with the two armatures of each condenser, and fixed to an ebonite plate on each side of the cylinder to which a rotatory motion can be imparted.

"If the two terminals of the apparatus $\mathrm{T}$ and $\mathrm{T}$ " are connected with a battery of 800 couples, by means of the conductors $\mathrm{P}$ and $\mathrm{P}^{\prime}$, even several days after having been charged with two Bunsen elements, and the commutator set in motion, there appear, between the branches of the charging machine which are coterminous to the armatures of the last condensers, a number of sparks; similar to those of an electric machine which is provided with condensers.

"By using an apparatus consisting of thirty condensers only, each of which has a surface of three decimetres square, I have obtained sparks of four centimetres in length.

"This apparatus gives favourable results with less than 800 secondary couples; 200 couples gave a spark of eight millimetres, and, by diminishing the thickness of the insulating plates, and increasing the number of condensers, results might be obtained with an electric source of even smaller tension.

"It ought to be understood that the discharges of statical electricity in the apparatus are not alternately positive and negative, but always take place in the same sense, and that the loss of power resulting from the transformation must be less than in an induction coil, for the voltaic circuit does not close upon itself for a single instant and there is consequently no conversion of a part of the 
current into caloric effect. The apparatus can be kept in motion, and can produce a considerable number of discharges, without sensibly weakening the secondary battery.

"This is explained by the fact that each discharge only uses up a very small quantity of electricity, and that the circuit of the battery is not closed by a conductor. The electricity of the source simply spreads over the polar surfaces of the condensers while they are discharged. But this emission, which is constantly repeated, must finally carry off a certain quantity of electricity, and when the instrument is charged with a secondary battery, it seems quite possible at length to exhaust, in the form of statical effects, the limited quantity of electricity furnished by the current of the battery.

"The transformation of dynamical electricity is thus effected by another method than induction properly speaking, simply by a protracted statical effect, and this apparatus is properly designated by the name of 'rheostatic machine." "

After having enumerated the different methods by which electric currents are produced and transformed, we now pass to the description of some of the interesting applications of this mysterious force. 


\section{PAR'T II.}

\section{ELECTRIC LIGHTING.}

ELECTRIC lighting, both as regards its past history as also its future, ranks first among the applications of electricity, although several of these applications have been developed to a much higher degree.

The voltaic arc was first produced by Sir Humphry Davy, in 1813, with a battery of 2000 zinc and copper elements; but it was not till 1842 that Deleuil's and Archereau's experiments foreshadowed the possibility of its use as a source of lighting.

At that time the only batteries known were hydroelectric batteries; electro-dynamic machines were still in their infancy, and the means for regulating the light more than primitive.

In 1857 , the Alliance machines, perfected by Van Malderen, showed that electric light could be advantageously produced in certain special cases, and the application of these machines for lighting the lighthouses of La Hève, as early as 1863, brought the electric light for the first time within the reach of practical questions.

The invention of the Gramme machine in 1870 gave a new impetus to the electric light, which up to that time had 
only found a limited number of applications; and finally Jablochkoff's discovery, in 1876 , brought prominently to the front a new system of lighting, which is destined, not so much to do away with gas altogether, as to replace it in a great number of applications where the electric light has properties which recommend it in preference to all other systems.

Modes of Production of Electric Light.-All electric lighting depends on two essentially different factors-

1. The electric generator.

2. The electric lamp.

After having considered the different methods used for the production of light, it will be easier to appreciate the value of the different sources of electricity with regard to the apparatus they are intended to supply. The principal electric generators in use at the present time have been described in the first part of this work, and we shall refer our readers to the respective paragraphs whenever occasion requires.

The fundamental principle of the production of light in any system whatever, whether with oil, petroleum, gas, or electricity, is the following:-To bring a solid, liquid, or gaseous body to a high temperature, the quantity of light produced being the greater the higher the temperature of the body. In all lighting the rise of temperature is produced by the combustion of the oil, petroleum, or gas, which respectively supply the source of light. The high temperature of the luminous part of electric light is produced by the current passing through a medium which offers resistance to electricity; the electric current is thereby transformed into heat, within a very narrow space, by heating a relatively small number of molecules. When the clectric 
current heats a gaseous conductor, the light thus produced is called the voltaic arc. This voltaic arc, however, only becomes very luminous when the gaseous conductor contains. solid particles detached from the electrodes; these particles are raised to a very high temperature, and impart to the conductor its characteristic dazzling light. Conductors of gas carbon, or very dense carbon, are used for this purpose. The heat given off by the combustion of this carbon, in addition to that of the current, favours the production and preservation of a high temperature, and contributes in no small measure to the brilliancy of the light.

If a solid conductor of feeble conductivity is heated by the current and rendered luminous, the light in that case is produced by incandescence. Thus we have two distinct methods for the production of electric light, which we are going to examine successively.

\section{The Voltaic Arc.}

Whatever may be the tension of the electric source, it never is sufficient to cause the light to pass between the two electrodes, unless they are previously brought into contact. At that moment, the gas surrounding the electrodes is heated by the current, it becomes a better conductor, and the contact between the electrodes can now be broken, the passage of the current through the gaseous conductor being maintained all the while.

With high-tension currents, like those of Holtz's machine, and induction coils, the discharge can take place between two electrodes at a certain distance, and electric light is produced, but unfit for lighting purposes.

In an interesting historical essay on the light produced 
by electricity, published in the French journal La Nature, in April, 1878, M. Antoine Bréguet ascribes to Otto von Guericke (1602-1688), Mayor of Magdeburg, the first light produced by electricity.

In a paper read at the Royal Society, on the 6th of Febriary, 1746, Dr. Watson pointed out that electric sparks seemed to be of different colours according to the nature of the substances from whence they sprang-that the light seemed much redder when coming from substances with a rough surface, like rusted iron, oxidized copper, etc., than from substances with a smooth surface.

It was Watson who first made use of the electric light for lighting purposes; with four of his apparatus he obtained such large jets of flame, and so close to one another, that in a dark room the faces of thirteen persons present at the experiment could be distinctly seen.

All these experiments were made with statical electricity obtained by friction machines. The discovery of Volta's pile brought with it the realization of a powerful electric light - of the voltaic arc, properly speaking.

Sir Humphry Davy obtained by means of a battery of 2000 elements, of an active surface of more than eighty square metres, the poles of which were connected with two carbon ends, a dazzling and continuous light, without any perceptible noise.

Why cannot friction machines, which give a spark, give an intense light like batteries or electric generators ?

This question is easily answered by referring to what has been said about quantity and tension of electric currents.

The current of a battery generally presents great quantity and feeble tension, whilst electricity produced by 
a friction machine with a glass or ebonite disc, has on the contrary an enormous tension and small quantity.

The great brilliancy of the voltaic arc is due to particles of the material of the poles being raised to white heat in their passage from one pole to the other; it is clear, therefore, that a slender spark, which carries with it but very few particles from the electrodes, must be much more exposed to the cooling influence of the ambient air.

In 1850, Professor Masson, of the École Centrale, made numerous experiments on the voltaic arc, and showed that electricity produces no current in an absolute vacuum, which bears out the assumption of a ponderable matter, the nature and density of which greatly affect the tension of the electric fluid necessary for the production of a current. He proves that the electric spark is produced by a current, and propagated through the ponderable matter, which is heated by the spark in the same manner and according to the same laws as a voltaic current heats and renders luminous a metallic wire.

Matteucci, about the same time, investigated the conductivity of the arc passing between points of various substances, and found that it depended less on the conductivity of the metals forming the points than on their fusibility and volatility.

He also remarked the difference of temperature of the two poles; this difference increases with the non-conductivity of the materials the poles are made of, and also with the readiness with which these materials are dissociated or burned.

The great difference of temperature between two carbon points is chiefly due to the unequal heating of the two poles, the positive pole being nearly twice as hot as the negative vor. I. 
one; this unequal heating is accompanied by a difference in the disintegration of the substance, and this circumstance can, in its turn, modify the electrical resistance and the heat produced. The length of the arc varies with the tension of the current,-it can reach four to five centimetres with appropriate currents; its shape also depends on its length. When the are is short, it resembles a condensed sheaf of luminous threads, generally assuming a cylindrical shape.

A more rarefied atmosphere of violet colour surrounds

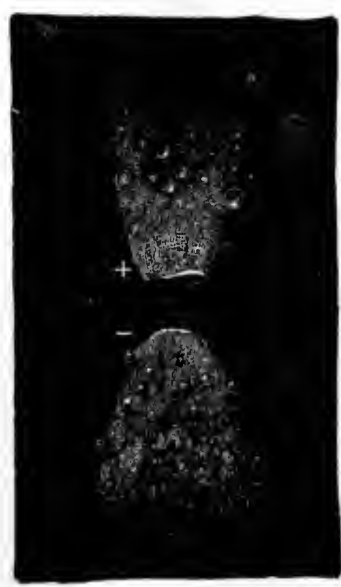

Fig. 92.-The voltaic arc. this first sheath like a case, and the base of this annular cylinder is enlarged on the positive carbon, whilst its opposite part is extinguished on reaching the negative pole. If the voltaic arc is lengtbened, the sheath becomes less condensed and more slender, and the violet sheath surrounding it assumes more the appearance of a flame. The central part is composed of incandescent particles, which detach themselves from the melted extremities of the points, seem to attract one another, and to form a continuous chain between the points. The exterior part of the arc consists of even more finely divided matter.

In the air, the voltaic arc rapidly but unevenly consumes the carbons, in the proportion of about one to two. The result of this uneven combustion is that the luminous point is displaced, and that the electrodes become deformed; the negative pole presents a sharp point, while the positive pole assumes a hollow, crater-like shape (Fig. 92), and sur- 
rounds the luminous point with a more or less conspicuous border.

These phenomena are produced with continuous currents. With alternating currents, the waste of the carbons is even and regular; both extremities assume a pointed shape, and the luminous point is thereby more completely disengaged.

It would, however, be a mistake to consider the craterlike shape of the luminous point as an inconvenience, for the hollowed electrode acts in this case as a reflector, and when the lamp is placed sufficiently high, the floor receives a larger quantity of light than the ceiling, which is a great advantage.

For the lighting of lighthouses, the decidedly concave shape of the positive electrode can supply, with a convenient arrangement of the negative carbon, a light emitted with much greater intensity in a given direction; continuous currents are, therefore, in this country preferred to alternating currents for the lighting of lighthouses.

The light thus produced is called condensed light; it is obtained by placing the lower negative carbon in such a position that its axis is in the prolongation of that side of the upper carbon which faces the horizon that is to be lighted. By this arrangement, if the light produced by the carbons is 100, the following intensities are obtained in different directions :-

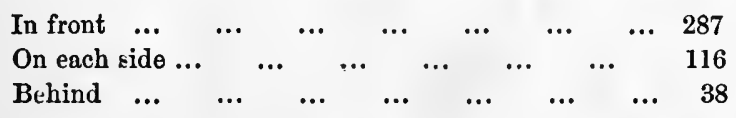

According to the different applications, it will therefore be advantageous to produce condensed light or ordinary light.

To produce electric light with the voltaic arc, three distinct conditions must be fulfilled-

1. To produce the electric current. The apparatus 
required for the fulfilment of this condition have been described in Part I.

2. To send the current into the two carbons. It will be seen, on examining the light-giving carbons, that it is not always easy to find carbon of a good quality, and that on this score research has not spoken its last word yet.

3. To maintain a convenient distance between the two carbons. This is the function of the regulators or arc lamps which are given in the following pages.

Carbon Points.-Sir Humphry Davy, who first observed the voltaic arc, used wood charcoal extinguished in water or mercury, but the combustion was extremely rapid, and this sort of carbon would never have supported the electric light for any length of time. A great progress was made when Foucault conceived the idea of using the carbon left in gas retorts, by cutting it into square sticks. This carbon lacks homogeneity, and contains silica which fuses, vaporizes, and shivers the carbon, to the detriment of the steadiness of the flame.

In 1842, Bunsen tried to obtain hollow cylinders of moulded carbon for his nitric acid batteries; for this purpose he cast a mixture of dry pulverized tar and glue into moulds, and baked them. He obtained thus a porous carbon cylinder of little compactness. To remedy this latter defect, he dipped the cylinder into treacle, and baked it a second time ; the sugar stopped up the pores, and left a very pure and compact carbon behind. The operation was repeated as long as there were any holes left, and till a faultless cylinder was obtained.

In 1846, Staite and Edwards took out a patent for a similar method of fabrication, specifying the use of these carbons for the production of the voltaic arc. 
In 1849, Le Molt patented a composition, formed of two parts of gas carbon, two parts of wood charcoal, and one part of liquid tar, the whole well powdered, mixed, compressed, moulded, soaked in treacle, baked for twenty-four hours, and purified by immersion in acids.

In 1852, Watson and Slater prepared carbon from the chareoal of twigs, which was purified with lime, baked, dipped in a solution of alum, baked again, soaked in treacle, and baked a third time.

In 1857, Lacassagne and Thiers dipped pieces of gas carbon into a solution of caustic potash or soda, which had previously been fused to transform the silica into soluble silicate. By washing the carbon sticks afterwards with hot water, and exposing them to a current of chlorine at red heat, compounds which had not been acted on by the potash were transformed into volatile chlorides of silicon, potassium, iron, etc. The light obtained with these carbons is a little steadier, but they produce a great deal of ashes and flake.

By using very pure materials, and forming his carbons with tar that had been distilled a second time, Jacquelain obtained plates which, when cut into sticks, gave a white, steady light, whose intensity, for equal currents, surpassed that of the ordinary carbons by twenty-five per cent.

In 1877, Archereau made excellent carbon points from compressed carbon mixed with magnesia.

Carré manufactures now, on a large scale, carbon points containing metallic salts which, according to their nature, give to the flame a greater steadiness or a more beautiful tint. The process of manufacture has improved considerably, and has become more rapid by the use of the drawplate recommended by Archereau as early as 1855. 
A mixture of very pure charcoal powder, coke, and lampblack is thickened with syrup of sugar; it is triturated and passed through the draw-plate under a pressure of about 100 atmospheres; the sticks thus obtained are baked, dipped while red hot into the syrup, baked again, dipped into syrup, and so on, a certain number of times. The carbon prepared in this way is a good conductor; it burns rather slowly, without cracking and withont giving too much flake.

The charcoal used by Gauduin, in 1877, in the manufacture of his carbons was obtained by the destructive distillation of tar, resin, bitumen, etc., which left behind sufficiently pure carbon after their decomposition. The moulding is effected at a very high pressure by a hydraulic press. Experiments made by Fontaine showed that the light produced by gas carbon was equal to 103 gasburners; the light produced by Archereau's carbons equal to 120 burners, and that Carre's carbons gave a light equal to 180 burners. Gauduin's carbons gave from 200 to 210 Carcel burners with the same electric source, and also lasted much longer than Carrés points.

Napoli, of Paris, produces carbons of high quality, and on a large scale. The materials he employs are gas-tar and the residue of coal slowly distilled at a dull-red heat. The coke is first reduced to an impalpable powder, and afterwards carefully screened. The screened materials are then mixed with tar, the proportions being seventy-five per cent. of coke and twenty-five per cent. of tar. When the mixing is completed, the compound, which is very compact and solid, is forced into its ultimate shape by hydraulic pressure. In baking the carbons, they should not be brought beyond the dull-red heat at which the tar has been distilled from 
the coal. They are placed in a retort, raised to a cherry red, and maintained at that degree for several hours, and then slowly cooled. After the first baking and cooling, the carbons are subjected to a second and similar operation, only this time they are raised to a white heat, which drives out the final traces of ' gas, and leaves the carbons of great hardness and with a fine fracture like that of crucible steel.

In this condition, and without any further treatment, the pencils can be used for lighting purposes; they burn with perfect steadiness and give a pure white light. It is claimed, moreover, that the relative consumption, as compared with the Carré carbons of similar size and in the same time, is as 1 to $3 \cdot 3$.

Cylindrical carbons are now almost exclusively used; their dimensions vary with the system of lighting.

For Reynier's incandescent lamps, sticks of two to three millimetres arc used. For Werdermann's lamps, Jamin's and Jablochkoff's candles, carbons of four to five millimetres are used.

For arc lamps fed by Gramme's machines or machines of similar power, carbons of seven to ten millimetres are used; finally, for powerful arc lamps for lighthouses and other naval purposes, carbons whose diameter sometimes reaches twenty millimetres are used.

Classification of Apparatus for Electric Lighting with the Voltaic Arc.-The number of apparatus for the production of the voltaic arc, and which enable us to regulate more or less accurately the distance of the carbon points, and the steadiness of the arc, is so considerable that it is impossible to establish an exact and complete classification. They can, however, be divided into two large classes, according to the relative position of the carbons- 
1. Arc lamps.

2. Electric candles.

In the electric lamp, the arc is produced between two carbon points, placed end to end, by a self-acting apparatus, which also keeps the points at a convenient distance.

Electric candles are apparatus in which the carbons are placed parallel to each other. They differ from the lamps by the complete absence of any mechanism, or by a mechanism the simplicity of which constitutes in practice no slight advantage. 


\section{CHAPTER I.}

ARC LAMPS.

Hand-worked Arc Lamps.-The light produced by the voltaic arc received no practical application till 1844; up to that time it was only considered an interesting lecture experiment and nothing else, for neither powerful batteries nor proper carbons were then obtainable.

In 1844, Léon Foucault used the Bunsen battery, which had just been invented, and replaced the sticks of common charcoal, which had been in use since Davy's time, by pieces of gas carbon.

He constructed a very simple lamp, worked by the hand, which enabled him to take photographs; whilst at the same time Deleuil made public experiments, by the same process, on the Place de la Concorde, at Paris. It is evident that the method of regulation by hand is as barbarous as it is primitive ; it is quite obsolete now.

Arc Lamps with Circular Electrodes. - It is worthy of note that the first self-acting apparatus had circular carbons, which are no longer used now. We shall only say a few words about them.

Thomas Wright, of London, constructed in 1845 a selfacting lamp, in which the arc passed between discs, bevelled on their circumference, and which was set in motion by a special mechanism. 
In 1844, Le Molt, a French physicist, patented a similar apparatus, which acted for twenty or thirty hours without necessitating any adjustment of the lamp.

In 18.57, Harrison constructed a self-acting arc lamp, in which there was a single circular electrode placed in the lower part, and connected with the negative pole of the electric source; the positive electrode consisted of a carbon pencil fixed to a spiral metallic tube, which advanced by means of clockwork in proportion as the carbon was used up. ${ }^{1}$

In 1877, Reynier invented an apparatus in which the carbon discs are carried by two systems of articulate levers, isolated from one another, and set in motion by two independent clockworks. The distance of the points is regulated by the action of a solenoid, and the discs move slowly under the action of a clockwork movement, to present successively all the points of their circumference to the source of light. This regulator is, however, too complicated; the carbon discs, moreover, throw too much shade, and have therefore been replaced by carbons of the shape of square or round sticks.

Classification of Arc Lamps.-All arc lamps utilize the variations produced in the current to act upon a mechanism which regulates the distance of the carbons. In certain arc lamps only one light is fed by a given electric source. In others two, four, and up to forty, are supplied by the same current. We accordingly divide all arc lamps into singlelight (monophotal) and polyphotal, or division, lamps.

1 See L'Éclairage à l'Électricite of H. Fontaine, 2nd edit. 


\section{Single-Light, or Monophotal, Arc Lamps.}

All single-light arc lamps present this common characteristic feature, that the electro-magnetic system which produces the adjustment of the distance of the carbons is placed in the same circuit as the arc itself. The regulating apparatus is therefore traversed by the whole of the current, and the variations in the intensity of the current cause the approach or the withdrawal of the carbons.

The first arc lamps were all constructed upon this principle; the variation of the current acted sometimes on a solenoid, as in Archereau's lamp, sometimes on an electromagnet commanding a clockwork movement, as in Foucault's. One of the oldest, which dates back as far as 1848, was constructed by Archereau, whose primitive apparatus has served as a basis for numerous other combinations.

Archereau's Lamp.-In Archereau's apparatus, the upper (positive) carbon is fixed, the lower (negative) carbon rests on a cylinder made half of iron and half of copper. This cylinder is placed in a solenoid, which is traversed by the current, and is kept in equilibrium by a counterpoise. The action of the solenoid produces and maintains the distance of the two carbons by exercising a magnetic attraction on the iron rod which terminates the negative carbon, provided the proportions between the power of the solenoid, the weight of the rod, and the intensity of the current are carefully established. Archereau's lamp is no longer used now, because the luminous point did not remain constant, and, on the other hand, because it was 
rather difficult to regulate the action of the solenoid without causing a rupture of the arc.

Jaspar's Lamp.-The apparatus consists of two movable carbon-holders $t$ and $t^{\prime}$ (Fig. 93). The negative carbon-holder

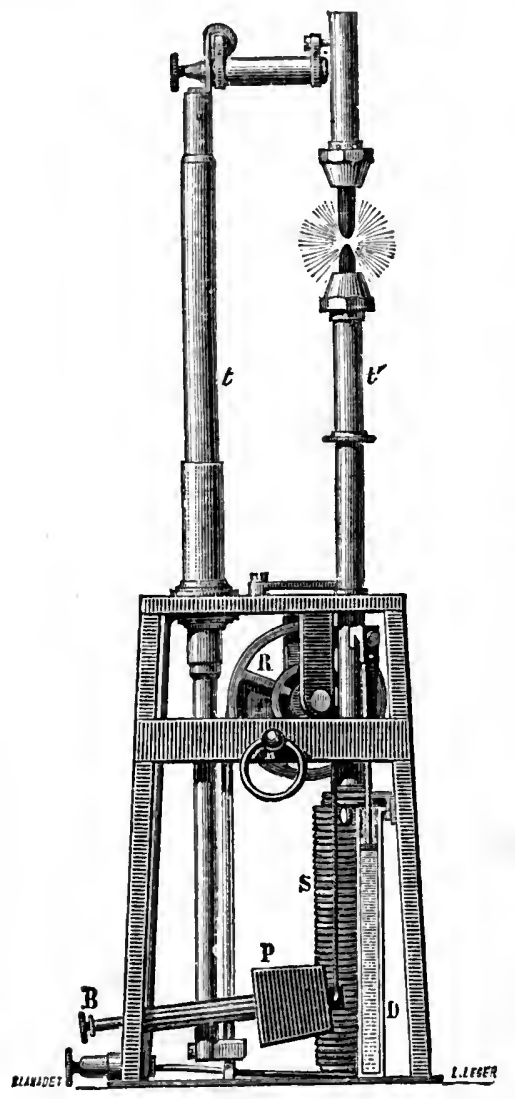

Fig. 93.-Jaspar's lamp.

$t^{\prime}$ is formed of a copper tube ending in a hollow cylinder of soft iron. In the middle the tube $t^{\prime}$ is pierced with a longitudinal slit, wide enough to allow a grooved pulley to pass; around this pulley is wound a cord which descends in the axis of the tube $t^{\prime}$ and carries a weight $P$ that keeps it stretched. This weight abuts upon a transverse piece in such a way that, when the cord is wound up, the weight in rising lifts the carbon-holder. The grooved pulley is fixed on a small drum, on which is also placed a second grooved pulley having a diameter double that of the former. On the latter is wound a cord attached to the lower extremity of the positive carbon-holder $t$, and it also carries a counterpoise. It will be seen that, by the direction in which the cords are wound, the weight of the positive carbon-holder tends to raise the 
negative carbon-holder, and that this will move through only half the distance of the former. This difference corresponds to the unequal wear of the carbons. The positive carbon-holder is formed of a copper tube $t$, furnished with a clip to which the carbon is attached. The carbon is held securely in place by a locking screw.

The negative carbon-holder carries a pendent rod, terminating in a small piston which can move within the tube $\mathrm{D}$ filled with mercury. The diameter of the piston is less than that of the tube, so that when the piston moves down by the motion of the carbon-holder, the mercury passes from one side to the other of the piston by the narrow angular space. This acts as a very efficient brake, and prevents any sudden action which would show itself in irregularity of the light. On the tube $\mathrm{S}$ is wound an insulated copper wire forming a solenoid, and intended to produce the separation of the carbons, and to counterbalance the effect resulting from the weight of the positive carbon-holder.

When at rest the carbons are in contact. At the moment when the current passes, the solenoid attracts the iron core of the lower holder and maintains the carbons at the proper distance. When the arc is lengthened, the current is weakened, and the carbons, acted on by the weight of the holder $t$, are drawn slightly nearer to one another. The counterpoise $\mathrm{P}$ acts in an opposite sense to the holder $t$, since, by the winding of the cords, it tends to produce a withdrawal. The adjustment is therefore easily obtained by the displacement of the weight $P$. The more intense the current, the nearer the weight $P$ must be to the point where the cord is attached.

The action of the solenoid on the carbon-holder $t^{\prime}$ is not the same at the end as at the beginning. This difference of 
action is compensated for by the small counterpoise $r$, which is regulated by its distance from the centre. At the beginning

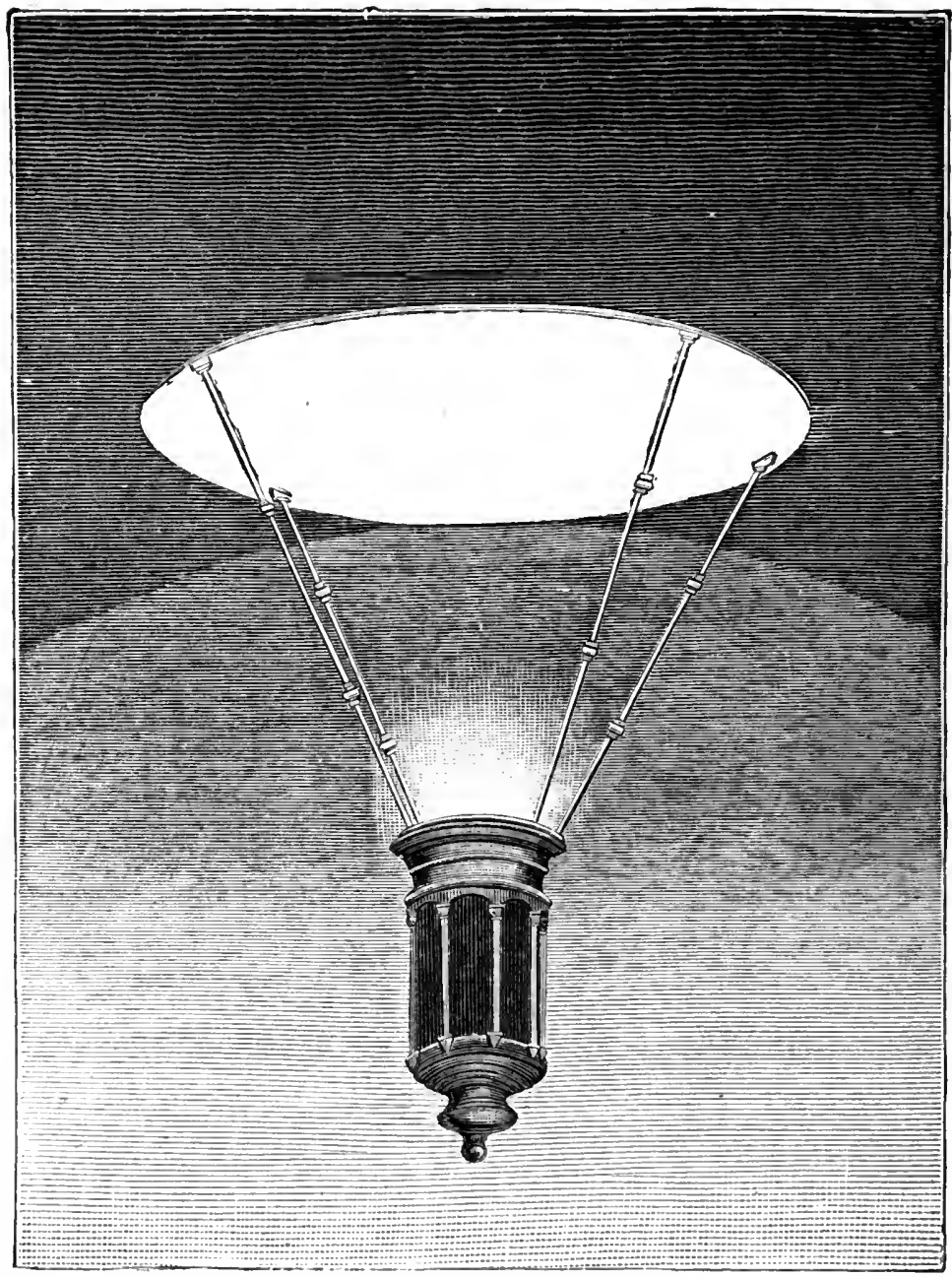

I'ig. 94.-Jaspar's suspension lamp.

the counterpoise $r$ has no action; at the end its weight is added to that of the weight $\mathrm{P}$ to counterbalance the action 
of the solenoid. Fig. 94 shows the Jaspar suspension lamp.

The Jürgensen Lamp.-In this lamp the movements of the carbons are regulated by the differential action of two solenoids, tending, the one to the withdrawal, the other to the approach of the carbons. The apparatus is represented in front elevation by Fig. 95 , and the diagram, Fig. 96, explains the principle of its working.

The two carbon-holders are arranged, as in Serrin's lamp, above the case containing the regulating mechanism, the prolongation of their rods penetrating into the case. On two columns $m n$, forming part of the frame, is fixed a transverse piece $e$, which serves as support to an axle $x$, attached in the same way on the other side of the lamp. To this axle are fastened two wheels $\mathrm{H}$ and $h$, whose movements control those of the carbon-holders; that is to say, if the wheels turn in an inverse direction to the hands of a watch, the upper

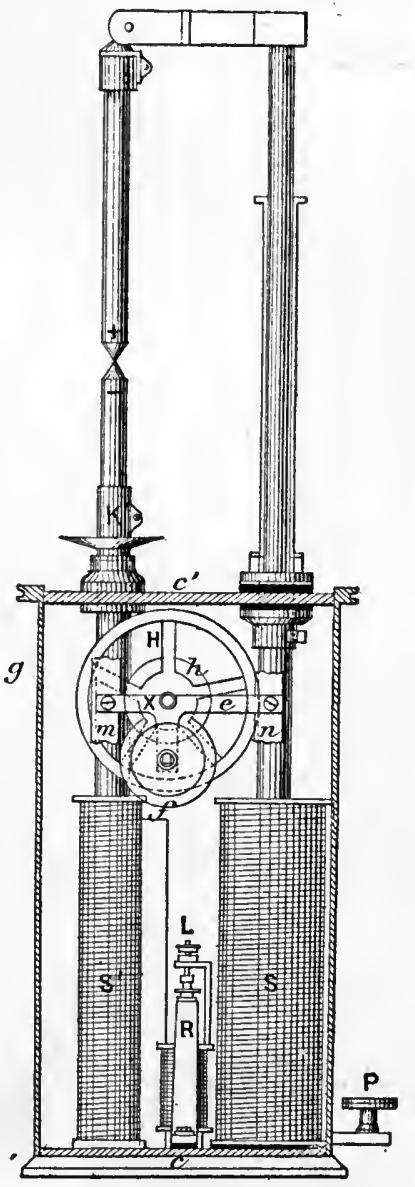

Fig. 95.-Jürgensen lamp (elevation). carbon is raised and the negative $\mathrm{K}^{\prime}$ is lowered, and a withdrawal will therefore ensue. If the wheels; on the 
contrary, turn in the direction of the watch-hands, an approach of the carbons takes place, and, in consequence of the different diameters of the two wheels, the quantities by which the two carbons advance are proportionate to their respective waste. In one word, the luminous point is fixed.

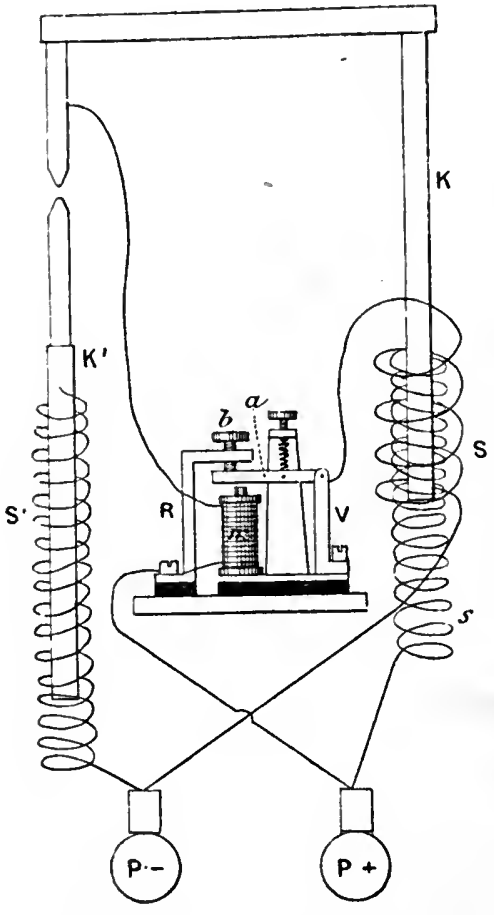

Fig. 96.-Jürgensen's lamp (diagram).

The two regulating solenoids are placed at $S^{\prime}$ and $S$; they have softiron cores, terminating in the carbon-holders. In addition to the solenoids, a small special arrangement is provided, represented in detail in the centre of Fig. 96, for lighting the lamp when the carbons are not in contact. This arrangement will be understood by referring to the diagram, which will also enable us to follow the working of the lamp.

Supposing the two carbons to be apart from one another, the current enters the lamp through the terminal $\mathrm{P}+$, and arriving at the piece $\mathrm{R}$ cannot energize the electro-magnet $m$; the armature $a$ of this latter will therefore remain apart, and the current, passing through the piece $R$, the screw $b$, the armature $a$, and the piece $\mathrm{V}$, will traverse the solenoid 
$\mathrm{S}$ and return to the terminal $\mathrm{P}-$. The solenoid $\mathrm{S}$, acting on the soft iron of the carbon-holder $\mathrm{K}$, will lower it, and $\mathrm{K}^{\prime}$ being raised at the same time, contact of the two carbons will be produced. At this mornent part of the current will pass through the electro-magnet $m$, the armature of this latter will be attracted, and the contact at $b$ broken, so that the current will no longer be able to pass from $R$ to $\mathrm{S}$, but its main path will be from $\mathrm{P}+$, through $m$, through the two carbons and the solenoid $\mathrm{S}^{\prime}$ to $\mathrm{P}-$. The solenoid $\mathbf{S}^{\prime}$ will then begin to act, and will bring about the withdrawal of the carbons and the production of the arc.

When once the arc is established, and as long as it is maintained, the lamp may be considered a differential lamp. On the upper part of the solenoid S is, in fact, branched another solenoid $s$ of great resistance. The two solenoids combined form, when the contact at $b$ is broken, a solenoid of great resistance placed in a shunt between the two terminals of the lamp. It will now be clear how the regulation is effected. When the arc acquires too great a resistance, more of the current passes into $\mathrm{S} s, \mathrm{~K}$ is lowered, and the carbons approach one another. In the opposite case the action of $\mathrm{S}^{\prime}$ becomes preponderating, and a withdrawal is the result.

The natural tendency of the carbons to effect an approach is further regulated by a little drum containing a spring. This spring has the same function as the adjusting weight of Jaspar's lamp, to which the Jürgensen lamp bears a great resemblance.

For intensity of current of 14 to 14.7 ampères, which is generally sufficient for the lamp, the difference of potential at the terminals was found to be 41 to $42 \cdot 5$ volts, and the work expended in the are 60.6 to 61.44 kilogram- 


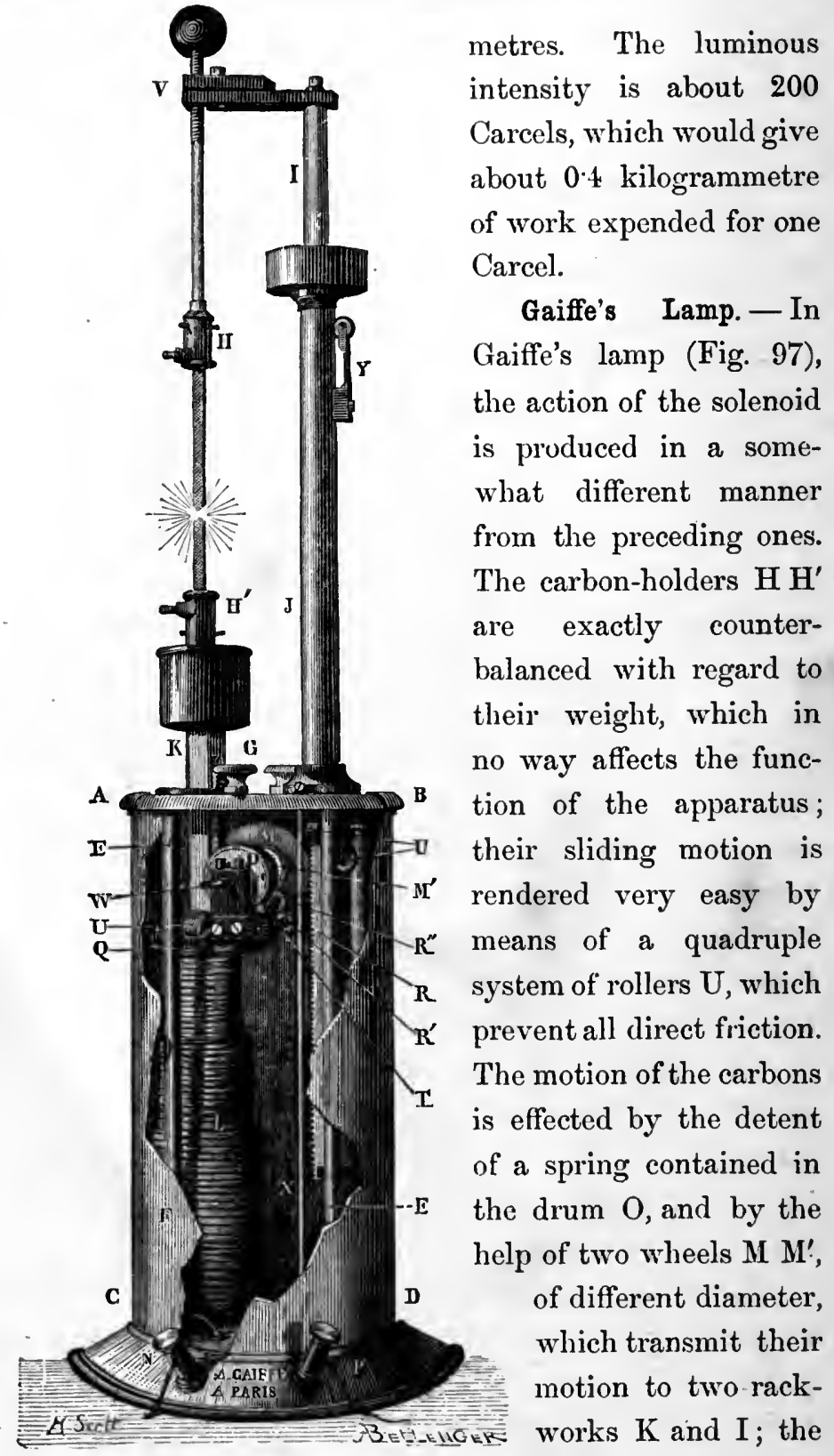

Fig. 97.- Gaiffe's lamp. 
two carbon-holders $\mathrm{H} \mathrm{H}^{\prime}$ are in direct connection with $\mathrm{K}$ and I. In its movement, the rod of soft iron penetrates more or less into a coil L, which is surrounded by an insulated spiral wire. The attraction of this spiral coil on the iron rod causes the necessary distance of the carbonholders for the production of the luminous point.

The spiral coil and the spring of drum $\mathrm{O}$ are arranged in such a manner that their antagonistic forces remain in the same relation during all the time that the carbon-holders are moving; consequently, if the voltaic arc has a length of three millimetres, for instance, at the moment of the lighting of the regulator, it will have the same length at the time of extinction caused by the waste of the carbons. The spring can be stretched more or less, and the apparatus can therefore be used for very different intensities of currents; its action is sufficiently regular with a Bunsen battery of from fifteen to twenty elements. By a special contrivance, the luminous point can be displaced at will, without being obliged to extinguish the apparatus or otherwise to interfere with the carbon-holders. This contrivance consists of a system of pinions $R, R^{\prime}, R^{\prime \prime}$, which under ordinary circumstances are kept apart from the wheels $\mathrm{M} \mathrm{M}^{\prime}$, but can be made to work into these wheels by a slight pressure, so as to allow, by the help of a key, of a simultaneous raising or lowering of the carbon-holders without altering their distance. The luminous point can thus be easily regulated -an indispensable requirement for optical experiments and for projections.

The apparatus is worked in the following manner:-The current enters by the terminal $\mathrm{P}$, follows the path $\mathrm{X}, \mathrm{J}, \mathrm{I}$, $\mathrm{V}, \mathrm{H}, \mathrm{H}^{\prime}, \mathrm{K}$ passes into the coil $\mathrm{L}$, and leaves by the terminal N. When no current circulates, the two carbons press 
against one another by the action of the spring of drum $\mathrm{O}$; but as soon as the electric circuit is closed, the coil attracts rod $\mathrm{K}$, and the combined movement of rod $\mathrm{K}$ and the second rod I brings about a withdrawal of the carbons and the formation of the voltaic arc. The attractive force of the coil must always be slightly superior to the action of the antagonistic spring; this condition is fulfilled by loosening or tightening the spring.

Reynier's Lamp with Spring and Solenoid.-Numerous experiments made by Saint-Loup and Reynier show that the attraction exercised by a solenoid on a bar placed along its axis can be depicted by a curve which, if the distance of the soft iron from a fixed point be represented by the abscissæ, and the magnetic attraction by the ordinates, starts from a point above the axis of $x$, and attains its maximum height when the lower end of the bar is at twothirds the height of the core, and from this point falls back to the axis of $x$. The descending portion of this curve deviates very little from the straight line joining its extremities.

The diagram of a spiral spring which unwinds on lengthening has the same form; in order to get a spring whose diagram coincides with that of the solenoid, it suflices that, when the spring is subject to a weight equal to the maximum attraction of the solenoid in question, it should be shortened by an amount which is equal to the length of the path traversed by the soft iron between the position corresponding with that maximum and the position where the magnetic attraction ceases.

A spring of this description, placed beneath the soft iron in the solenoid, and whose lower end is at a point determined by the coincidence of the diagrams, forms 
with the bar a system which enjoys the following adyantages :-

1. When the current which magnetizes the solenoid is of normal intensity, the spring counteracts the magnetic attraction which draws down the soft iron, whatever may be its position above the region of maximum attraction.

2. When the current is less intense, the action of the spring overcomes the magnetic attraction, and pushes the iron upwards, whatever its position.

3. When the current has greater intensity, the attraction overcomes the action of the spring, and draws down the iron, whatever its position.

A solenoid, a soft iron rod, and a spring combined under the conditions that have been described, form the principal parts of the lamp. The lower carbon-holder is mounted on the soft iron, and follows its movements ; the upper carbon-holder is immovable and attached to the body of the solenoid by a doublebent support. The lamp (Fig. 98) is represented before it is lit, the iron

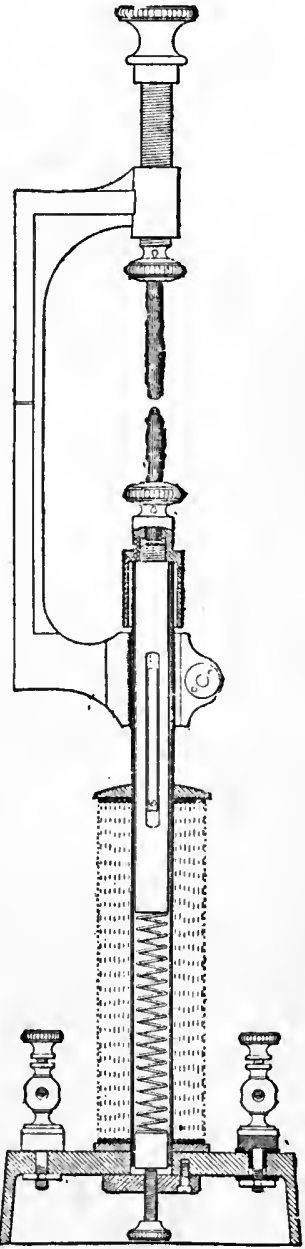

Fig. 93.-Reynier's arc lamp. being slightly above the position of maximum attraction.

As soon as the current passes into the apparatus, the iron is pushed downwards in the solenoid, and descends 
until the arc reaches its normal length. In proportion as the carbons are used up, the iron ascends in gradual stages, which are equal to the sums of the lengths of the wasted carbons, so as to maintain the length of the voltaic are constant.

The spring being constructed for a given intensity of the normal electric current, the diagram of the spring no longer coincides with the curve of the solenoid if a current of different intensity passes into the lamp-it will be above or below that curve. This diagram can be brought back to an approximate coincidence by altering its origin, which can be done by loosening or tightening the spring by means of a screw in the socket of the lamp. This adjustment can only be carried out within very narrow limits. The lamp gives a bad result with currents of very different intensity from that for which the spring has been originally constructed.

The lamp represented in Fig. 98 is a laboratory model, specially intended for projections and currents of average intensity. A rougher model still, with larger carbons, is used as a safety-lamp with the self-acting lighting apparatus of the same inventor (vide p. 213). It is set in action as soon as the carbons of the principal regulator are used up, and acts for twenty minutes, which allows time for supplying the first lamp with fresh carbons without interrupting the light. The lower carbon progressing alone, the luminous point rises continually; to counteract this the upper carbon is from time to time pushed slightly forward by turning the screw which supports the carbon-holder, and the light is thereby brought back to a convenient height. During all this time, the lower carbon-holder continues to move, and thus the length of the arc remains constant. Less complete than Duboseq's and Serrin's 
lamps, but also less costly, this little instrument is sufficient for most of the optical experiments.

Marcus, Dubos, etc., have also constructed lamps with solenoid action, but their description would lead us too far.

Arc Lamps with Motor Springs. - The first of these lamps was invented by Foucault, and afterwards improved by Duboscq, who uses it for his projection apparatus and for lighting the stage. In the original apparatus, the adjustment of the carbons had to be effected with the hand. In the improved apparatus, there are two distinct clockworks -the one for bringing the carbons together, and the other for their withdrawal. An electro-magnet placed in the circuit unclamps one or the other of these mechanisms, according as the current is more or less intense. The luminous point can be raised or lowered during the action-an indispensable requirement for projections-by turning with the hand one of the toothed wheels of a drum, which acts upon the carbons. This apparatus can be used in all positions, but it is rather delicate, and must be regulated for each special application.

Girouard's Electric Lamp with Independent Regulator.We give a description of this apparatus in the inventor's own words, after a memorandum which Du Moncel read at the Académie des Sciences, on the 24th of January, 1876.

"This new electric lamp has a regulator, which can be placed at a convenient distance from the apparatus in which the electric light is produced, and which can be rendered sufficiently sensitive for all requirements. The lamp, therefore, consists of two distinct apparatus, which are represented in Figs. 99 and 100. They are connected by two different circuits, giving passage to two distinct currents: the one, very strong, produces the voltaic 
are after passing through the electro-magnet of the relay; the other, rather feeble, only effects the unhinging of the clockwork which regulates the movement of the carbons.

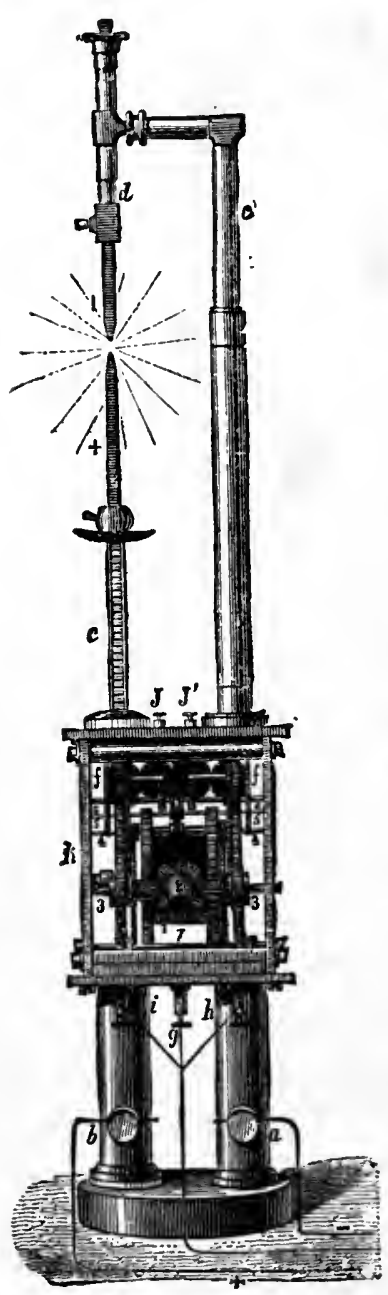

"The relay consists of an electro-magnet $b$ with a coarse wire, the armature $n$ being fixed to a lever, which is pulled in opposite directions by two springs $o$ and $o^{\prime}$; it can occupy a definite position, and consequently place a contact spring of the lever between two contact screws $p$ and $q$, which are in connection with the eleatromagnetic systems regulating the movements of the carbons. The tension of the springs $o$ and $o^{\prime}$ are calculated in such a manner that, for an intensity of current sufficient to yield a good light, the armature in question has no contact with the serews $p$ and $q$. Hence, if the current becomes too strong or too weak, the lever presses on one or the other of these screws and produces an unclamping, which causes an approach or a withdrawal of the carbons. It is elear that, if the carbons are in contact, the light-producing current must be Fig.99.-Girouard's electric lamp. of superior intensity to the normal current of the armature of the regulator; a contact will be established with the serew $p$, and a withdrawal of the 
carbons will ensue. On the contrary, if the distance of the carbons becomes too great, the contact will be effected by the screw $q$; a closing of the current will take place, and consequently an approach of the carbons. A convenient adjustment of the two springs $o$ and $o^{\prime}$ and the screws $p$ and $q$ will render the apparatus as sensitive as could be desired, and enable us to control it from a distance, without the necessity of touching the light-producing part.

"An interrupter permits the closing or the opening of the current which produces the light. The lamp consists, like

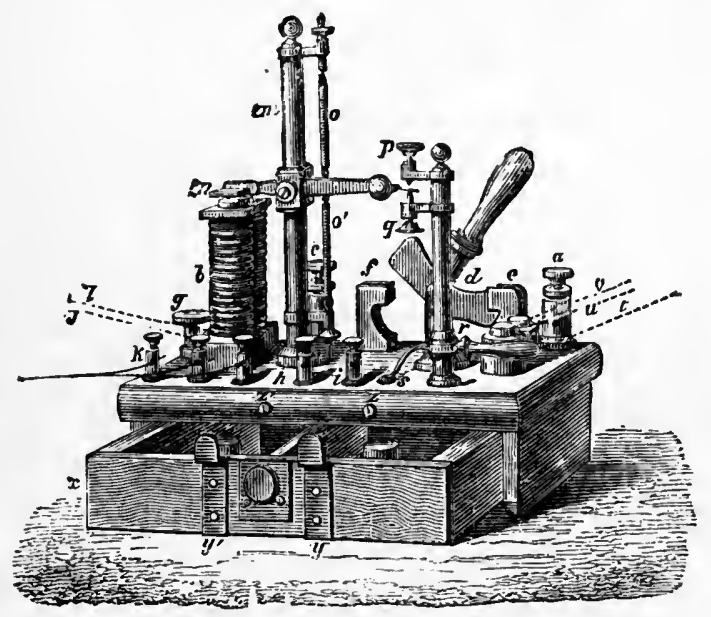

Fig. 100.-Relay of Girouard's lamp.

all ordinary electric lamps, of two long pencils of graphite carried by a rackwork and set in motion by two distinct clockworks, although directed by the same drum. The last movable wheel of each of these mechanisms is stopped by a detent of a particular electric system, which is connected to the screw $p$ and the screw $q$ of the regulator, and the wheelwork of the two mechanisms is constructed in such a manner that, at the moment of approach or withdrawal, 
the relative movement of the carbons maintains the luminous point in a fixed position.

"The arrangement of this apparatus, which can be worked in all positions, renders it convenient for a great number of applications-for instance, military operations, for navigation, for lighting the stage, for submarine research, and even for projections in physical experiments. For this latter purpose, there is a small mechanism fixed to the two rackworks, which allows of a vertical displacement of the luminous point without extinguishing the light, and consequently the light can be properly focussed.

"In the model represented (Fig. 100), the battery for charging the electro-magnets of the lamp is enclosed in the socket of the regulator. It is a small mercuric sulphate battery."

Régnard's Lamp.-In this regulator, the carbons are screw-shaped along their whole length, which makes them more expensive. They move forward or backward by the action of two female screws, which are set in motion by a spring and an electro-magnetic loosening apparatus, similar in principle to that employed by FoucaultDuboseq.

Very long carbons can thus be placed in the lamp without increasing the resistance of the circuit, since the contacts take place very close to the arc; but the manufacturing of the carbons presents serious difficulties, and raises their price considerably, and this renders the apparatus of little practical value.

Serrin's Lamp. - This regulator has found a great number of practical applications, for it fulfils almost completely, and in a comparatively simple way, all the requirements of an apparatus of this kind. 
Serrin's apparatus leaves the carbons in contact when no current passes; when the current is established they are removed to the required distance, and then approach one another gradually without coming again into contact. If the arc is broken from some cause or other-for instance, a strong wind or the breaking of a carbon-the apparatus brings the carbons again into contact, and then removes them to the necessary distance, so that the voltaic arc is reproduced with all its former brilliancy.

To realize all these conditions, the positive carbonholder (Fig. 101) consists of a rackwork, acting on a series of multiplying wheels for the increase of speed, the last of which corresponds with a wheel carrying a certain number of long teeth in the form of a star. When no current passes, and the carbons are separated, a motion of the wheels takes place until the carbons touch. At that moment the electro-magnet becomes active, attracts its armature, and causes the descent of a movable oblong check to which it is fixed; this catches the toothed wheel, stops the descent of the upper (positive) carbon, and lowers the negative carbon, which is fixed to this movable piece. If the arc is lengthened, the intensity of the current is weakened, the movable piece ascends under the action of two supporting springs, and sets free the large toothed wheel. The upper carbon can now descend a certain distance, until the are is sufficiently shortened and the intensity brought back to its normal strength, when a fresh stoppage ensues, and thereby a new state of equilibrium. If, on the contrary, the carbons are too close to one another, the intensity of the current increases, the movable piece is more strongly attracted by the electro-magnet, and continues to descend, producing a lengthening of the voltaic arc. On the 
left of the diagram is a screw which tightens or loosens the

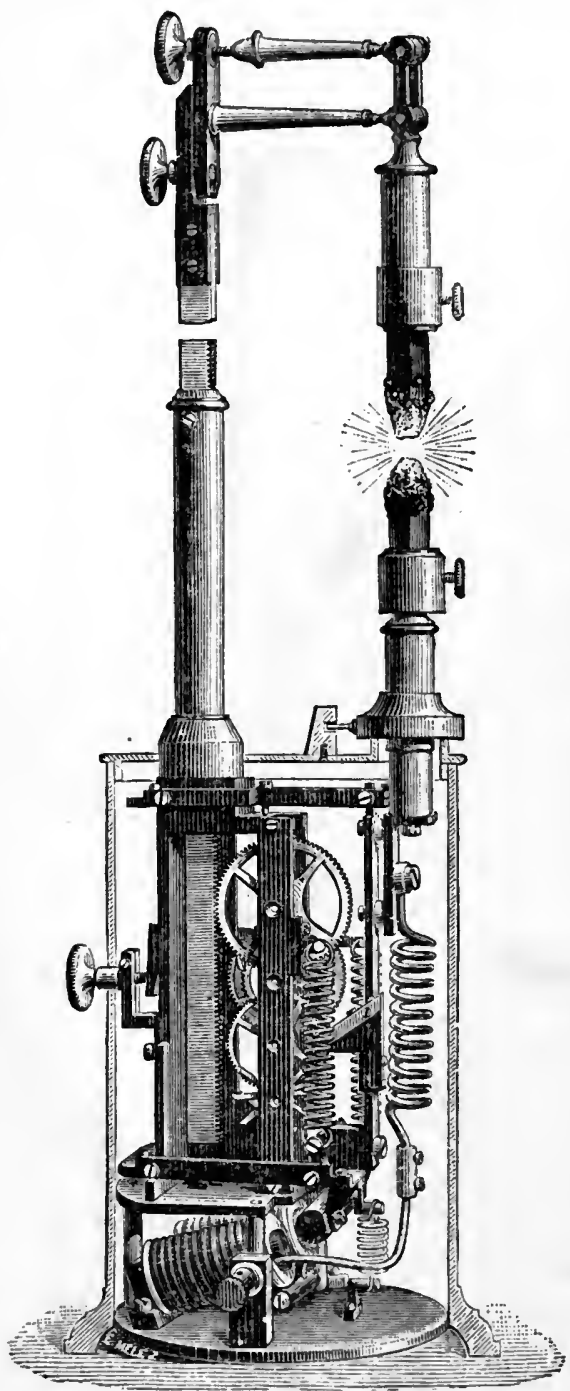

Fig. 101.-Serrin's lamp, constructed by Suisse. antagonistic spring of the electro-magnet.

In this apparatus, constructed by Suisse, the luminous point is displaced, and descends in proportion to the waste of the carbons. In an improved model, the luminous point remains constant, because the positive carbon descends, and the negative carbon ascends at the same time at a relative rate previously calculated, and which is nearly as two to one.

Serrin's lamps are most frequently used with Gramme's machine (p. 70). The extreme sensitiveness of Serrin's regulator constitutes, under these conditions, an appreciableinconvenience. When the carbons contain impurities, variations are produced in the power of the electro-magnet, which causes an oscillation of the lower 
carbon-holder. These oscillations increase or diminish the intensity of the voltaic arc, and consequently its resistance.

But as the current produced by dynamo-electric machines varies considerably with the resistance of the external circuit-represented here by the voltaic arcvariations of intensity ensue from this fact alone, which increase the effect of the first oscillations, and render the light very unstable. Here Serrin's regulator is at fault from the very reason of its excellent qualities.

With regard to this, it must even be remarked that, if the conductors have too great a resistance, the lamps, while giving less light on account of the weakening of the current by the resistance of the circuit, act nevertheless with more regularity.

This arises from the fact that the variations of resistance of the arc, although constant as regards absolute amount, have less influence on the total resistance of the circuit. With a good generator and very pure carbons, these defects can be avoided. From this point of view, Wheatstone's mounting (p. 61) as applied to dynamoelectric machines constitutes a great improvement, which we soon hope to see introduced for Gramme's electric generators, for in machines of that particular description, the variations of the electric elements consequent upon the resistance of the external circuit, are favourable to the regularity of action instead of being opposed to it.

The Crompton Lamp (01d Model).-The action of the lamp (Fig. 102) is as follows:-The bottom, or negative, carbon-holder is held up by a spring $S$, but upon the current entering at terminal $\mathrm{D}$ and passing round the electro-magnet $M$, it draws down the armature $\mathrm{L}$ which is attached to the negative carbon rod $A$, and thus pulls down 


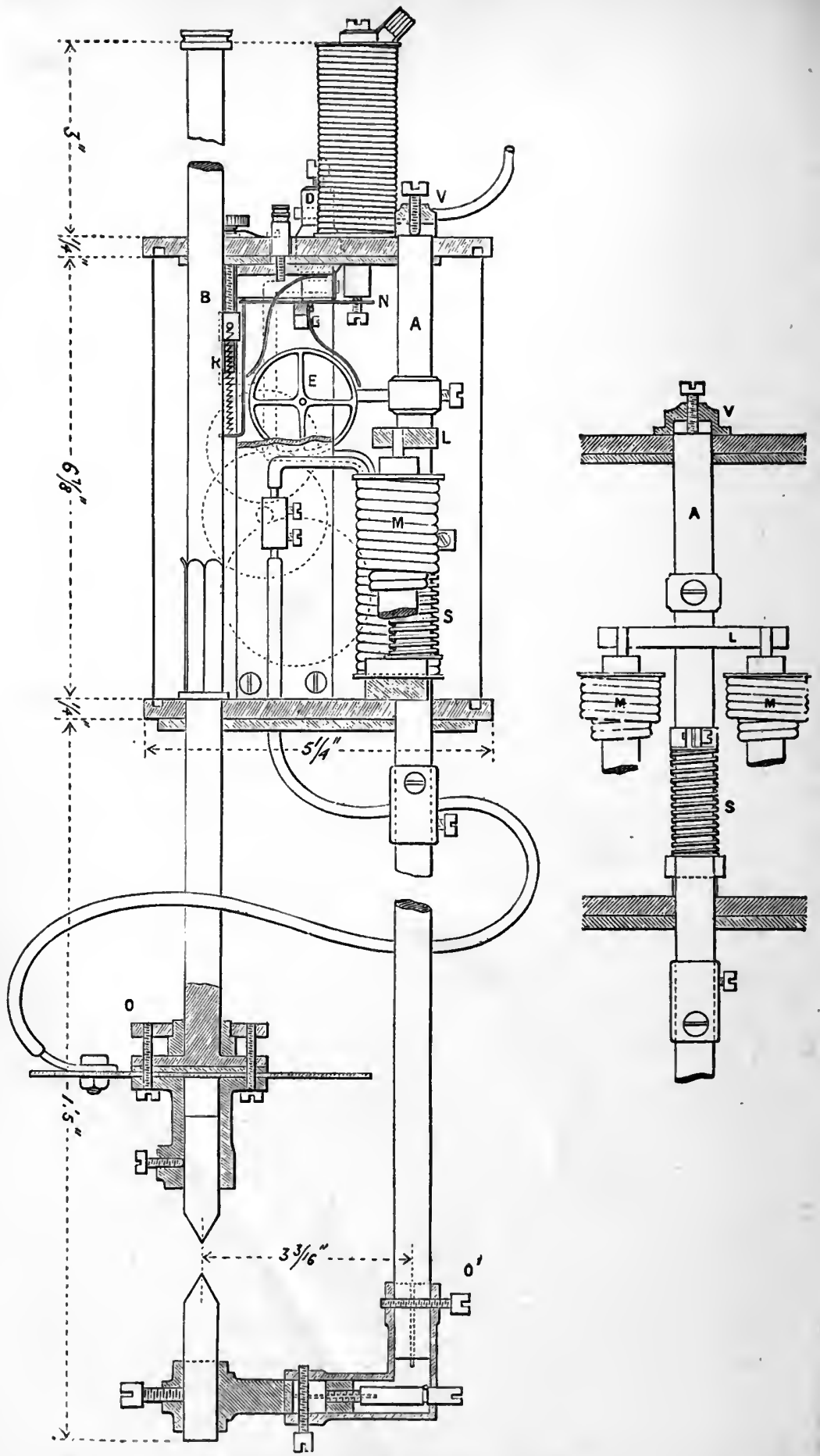

Fig. 102.-The Crompton lamp (old model). 
the negative carbon and establishes the arc, at the samc time releasing the brake on the right-hand side of wheel $\mathbf{E}$.

The feeding mechanism is actuated by a shunt magnet, which is shown at the top, and thus the lamp maintains a constant difference of potential between its electrodes. $\mathrm{E}$ is a brake-wheel controlling the movement of the clockwork; the brake is lifted up by the armature $\mathrm{N}$ of the shunt magnet.

The Crompton Arc Lamp.-The latest form of this lamp is represented in Fig. 103. The framework consists of two hollow rods A A, united at the bottom by a casting B, which contains two small grooved pulleys $\mathrm{P} P$, and also serves to hold the large tube $\mathrm{S}$, and at the top by another casting $\mathrm{C}$, which also contains two pulleys and forms the top of the lantern, having also two lugs for slinging it by. To one of the side rods $\mathrm{A}$ is fixed the solenoid $\mathrm{D}$, which is made in two dimensions: the upper one is wound with a high-resistance coil and forms a shunt between the terminals of the lamp; the lower division is in the main circuit. Inside the solenoid moves an iron core, which is attached at its upper end to a small clockwork arrangement $\mathrm{E}$ which is hinged at $a$; a dash-pot $\mathrm{G}$ below the solenoid serves to steady the up-and-down motion of the core. $\mathrm{H}$ is the positive carbon-holder, having a plate on which weights can be placed for regulating the lamp; it is attached to but insulated from one end of a flexible metallic cord, which passes, as shown, over the pulley $\mathrm{P}$, round the wheel $b$ in the clockwork $\mathrm{E}$, over the second pulley $\mathrm{P}$, and down the tube $\mathrm{A}$, eventually passing under the wheel $\mathrm{L}$ in the negative carbon-holder, the other end of the cord being nipped by a milled screw $n$. The positive carbonholder $\mathrm{H}$ is guided by two small rods $r r$, held at the top by a casting $M$, and insulated from it by small earthenware 


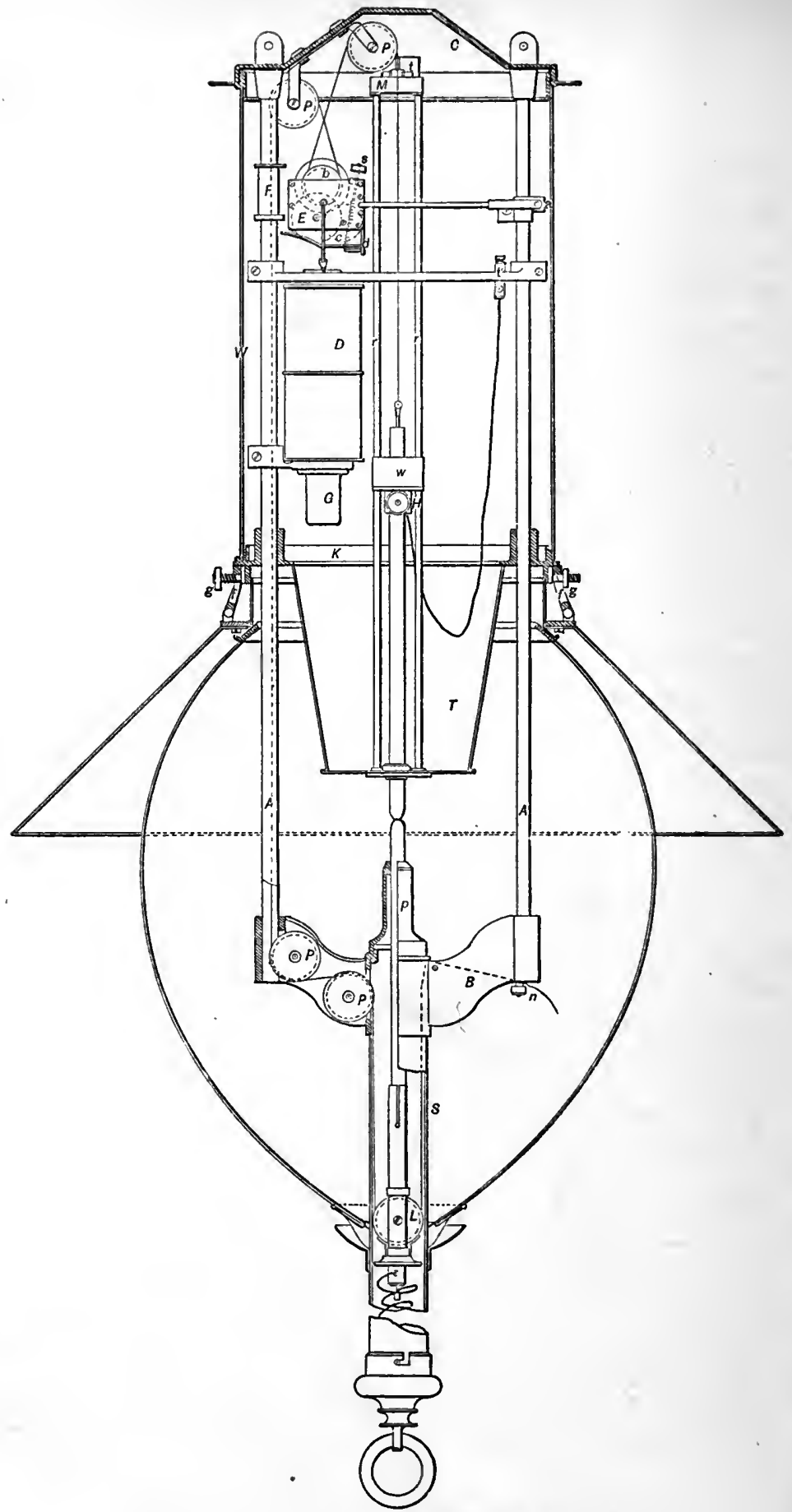

Fig. 103.-The Crompton are lamp (latest form). 
ferrules; at their other end they fit into the earthenware ferrules which are held at the bottom of a tin case $T$, which prevents all fumes from rising into the working parts of the lamp. The positive carbon passes through the bottom of $\mathrm{T}$, and is insulated from it by a large earthenware ferrule, The negative carbon rises from its holder through a nozzle $p$, and meets the positive carbon half-way between the nozzle and the bottom of the tin case $\mathrm{T}$. $\mathrm{F}$ is an adjustable stop clamped on the rod $\mathrm{A}$, and serves to release the brake $d$ from the brake-wheel $c$ when the iron core raises the clockwork high enough. The milled nut $s$ serves to put just sufficient pressure on the wheel $c$ to prevent its turning when away from the stop $\mathrm{F}$.

The action of the lamp is as follows:--The current enters at the terminal and passes round the solenoid $D$ to the terminal $t^{\prime}$, which is insulated from the frame of the lamp. From there, by means of a flexible cord, it reaches the positive carbon-holder $\mathrm{H}$; it then traverses the negative carbon, whose holder is in connection with the frame of the lamp, and so to the next lamp. When the current passes round the solenoid, its core is drawn down, and the carbons are separated. As the arc lengthens, the shunt coil draws the iron core up until the brake $d$ is brought against $\mathrm{F}$ and the wheel $c$ is released, the weight $w$ and with it the positive carbon then falls, at the same time raising the negative carbon exactly half the distance of its own fall. As soon as the lamp feeds, the current in the shunt coil decreases, and the main current pulls the brake $d$ just clear of the stop $F$, the arc again lengthens, the case rises, the brake again strikes $\mathrm{F}$, and so on.

The adjustment of the lamp is effected by two methods : either the weight $w$ can be increased or decreased according 
as the lamp burns with too long or too short an arc, or the position of the stop $\mathrm{F}$ can be altered by placing it higher or lower according as the arc is too short or too long.

The lantern is formed of a tin cylinder $\mathrm{W}$ which rests on a ring-shaped casting $\mathrm{K}$, to which is fixed the tin case $\mathrm{T}$; the casting is fixed to the rods $\mathrm{A} A$, and has a raised edge on the upper surface, ontside which fits the tin cylinder; a similar edge underneath has the tin reflector fitting it inside. To the reflector is fixed the glass globe, the bottom of which slides on the tube S. A hinged door in the cylindrical part of the lantern affords access to the clockwork, whilst access to the carbons can be had by sliding down the globe and reflector together; this must be done before changing the carbons; after the lamp is trimmed the globe and reflector are again raised, and the hangers $f f$ are thrown over the milled nuts $g g$, which, when screwed up firmly, fix them in their places.

Five such lamps, with a moderate length of conducting wires, can be maintained in one circuit, by Crompton's $\mathrm{C}$ machine running at a speed of say 1650 revolutions per minute.

The internal resistance of the machine is about 4 ohms. The current required to work the lamps is from 18 to 20 ampères. The electro-motive force of the machine is 280 volts, leaving 200 volts for external circuit, which makes it 40 volts per lamp. Intensity of light between 2000 and 2500 candles for horizontal, and between 2500 and 4000 candles for downward rays.

Siemens and Hefner Alteneck's Lamp.-This lamp (Fig. 104) with motor weight has been constructed with the view of working it with Siemens' and Hefner Alteneck's machines, which have been describerl on p. 78 . 
The approach of the carbons is effected by the weight of the positive carbonholder, which serves as motor. The withdrawal, on the other hand, is produced by a small electro-magnet.

The principal parts of this lamp are: The two carbon-holders $\mathrm{A}$ and $\mathrm{B}$, a clockwork movement driven by the weight of carbon-holder A, and whose last wheel is a ratchetwheel regulaterl by a fly I. The current of the machine enters by the terminal $\mathrm{C}$, traverses an electro-magnet $\mathbf{E}$ (one of its cores only is represented in the diagram), then passes through the body of the lamp to the positive carbonholder A, traverses the arc, and returns to the machine by the carbon-holder B, which has an insulated mounting, and by theterminal $\mathrm{Z}$, which is connected with the negative pole.

At the moment when the current passes into the lamp the carbons are

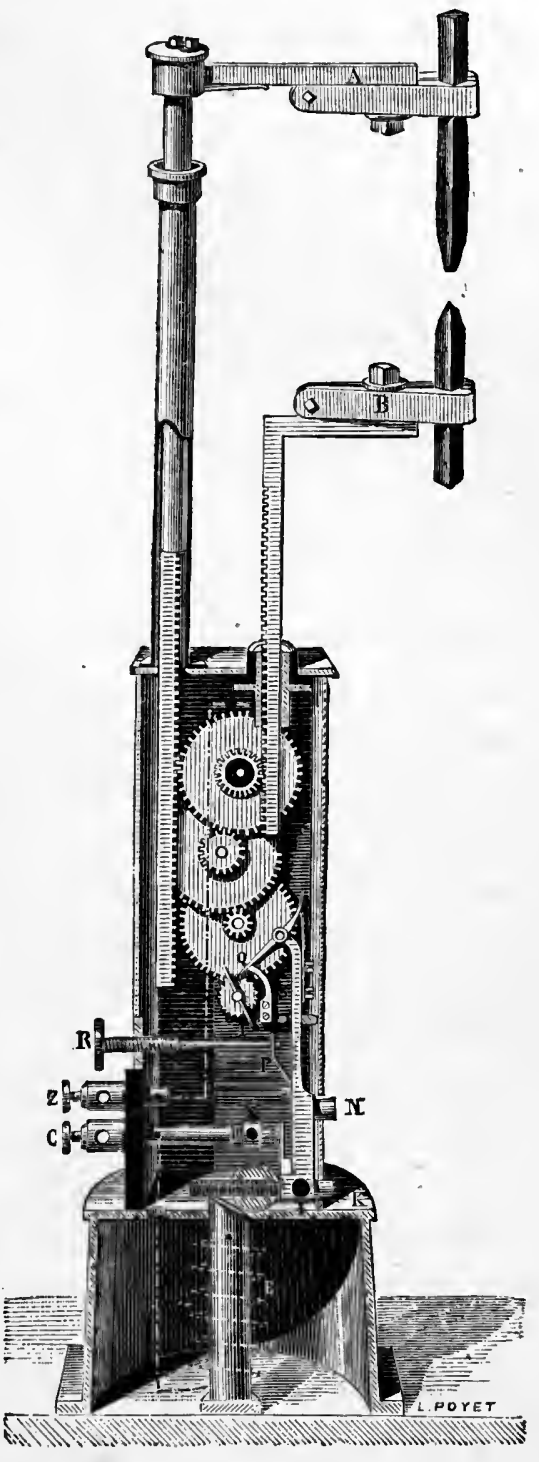

Fig. 104.-Hefner Alteneck's lamp with continnous current. 
in contact, and the current is very intense; the electromagnet attracts its cylindrical armature, which is fixed to a large vertical lever, pivoted about $\mathrm{Y}$. This lever rocks from right to left around $Y$, and a ratchet. $Q$, fixed to its extremity, turns the wheel I by one tooth in an inverse sense to the movement of the carbon, but at this moment a contact is produced at $\mathrm{X}$; the current now traverses directly the shunt of feeble resistance produced by this contact, and the electro-magnet becomes inert. Under the action of the spring $P$, regulated by the screw $R$, the lever takes up its original position; but then the contact in $\mathrm{X}$ is broken, and the magnet is energized afresh : it attracts its armature, and the wheel I turns again by one tooth in a sense favourable for the withdrawal of the carbons. A rapid succession of movements is thus produced, as in the vibrations of an electric bell, and the carbons are very rapidly removed to a normal distance for which the apparatus is regulated. When this distance is reached, the ratchet $Q$ causes a stoppage and maintains the carbons at the required distance. The lever is now close to the contact $\mathrm{X}$, and if the apparatus is well regulated, a sort of vibration is felt on touching $N$ with the finger, which is the safest indication of a good regulation. If the arc is lengthened, the armature tends to withdraw from $\mathrm{E}$, and gets free of the wheel I, which permits an approach of the carbons. Three methods of adjustment are practicable with this apparatus-

1. The tightening of spring $P$.

2. The distance of the armature from the electro-magnet $\mathrm{E}$, which can be regulated by means of a screw $\mathrm{K}$.

3 . The contact $X$, regulated once for all by the conductor according to the course of the lever and the length of the teeth of the ratchet-wheel. 
In practice, by acting on the spring $P$ by means of the screw $\mathrm{R}$, the apparatus gives good results; yet fault will be found with its delicate working and the noise produced by the movements of the lever.

For all that, the apparatus is frequently used in this country and in Germany, like Serrin's lamp is in France.

Carré's Lamp.-In Carré's lamp, which is more especially used with alternating currents, the weight of the upper carbon acts as motor, as in Serrin's apparatus; but the current acts upon a solenoid, containing a soft-iron rod, which is connected with the movement for the withdrawal or advance of the carbons.

Hiram Maxim's Lamp.-The characteristic feature of this apparatus is that the carbons approach one another very quickly when not traversed by a current, as for instance at the moment of lighting, and very slowly when the arc is formed. (See Fontaine's L'Éclairage à l'Électricité.) Any sudden approach of the electrodes is thus avoided, and a very steady light obtained.

Burgin's Lamp.-In this lamp (Fig. 105) the approach of the carbons is effected by the weight of the upper carbonholder, which, by the aid of two cords wound on the pulleys $\mathrm{P}, \mathrm{P}^{\prime}, \mathrm{P}^{\prime \prime}, p$, and $\mathrm{P}^{\prime \prime \prime}$, lifts the lower carbon-holder through half the distance by which it descends itself. The stoppage of the carbons is produced by a brake $\mathrm{K}$ acting on a large wheel $R$. This latter is mounted on the same shaft as the pulleys $\mathrm{P}$ and $\mathrm{P}^{\prime \prime}$, but this shaft is carried by an iron piece A A, fastened to the two parallel frames $B B^{\prime}$, and capable, therefore, of a motion parallel to itself when attracted by an electro-magnet M M. The wire of this electro-magnet is, of course; traversed by the current which circulates through the carbons. 
In a state of rest the carbons are in contact, but as soon as the current passes, the armature $\mathbf{A ~ A}$ is attracted and is

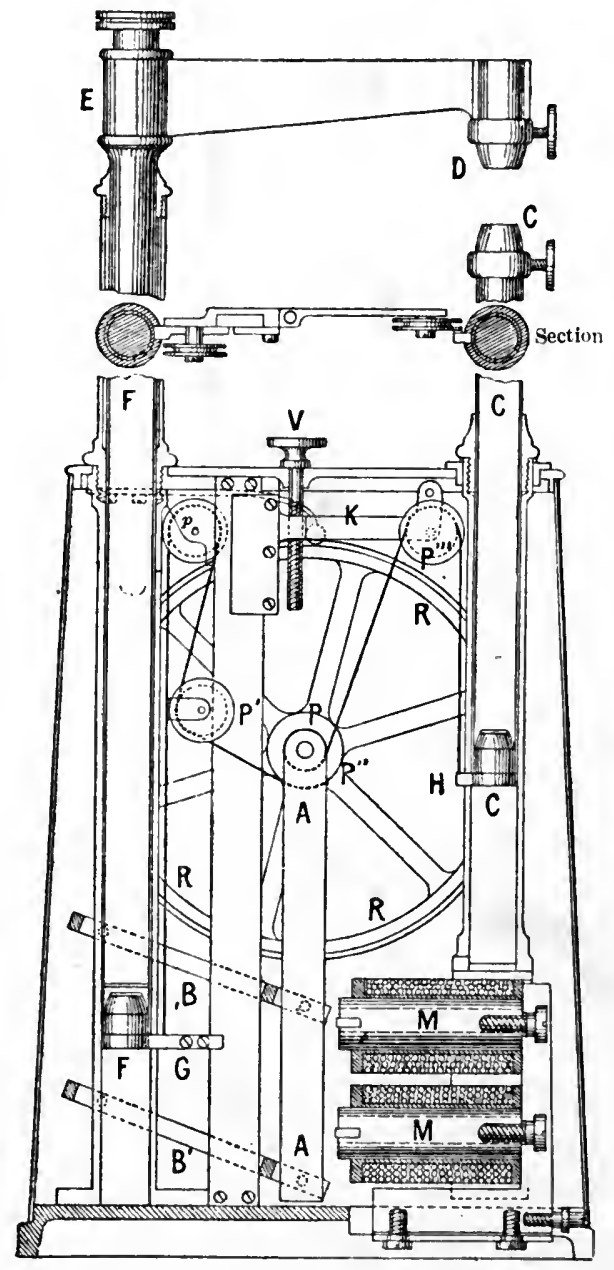

Fig. 105.-Bürgin's lamp (first model).

(From La Lumière Électrique.) suddenly raised; the cord $\mathrm{P}^{\prime \prime} \mathrm{P}^{\prime \prime \prime}$ is slackened, the lower carbonholderdescends by its own weight, and the arc is established. When the distance between the carbons becomes too great, the electro-magnet M M is weakened, and slightly relaxes its armature; the wheel $R$ no longer presses against the break $\mathrm{K}$, and the carbons again draw. nearer to each other, until the normal intensity. is restored, upon which the advance is checked anew. The regulation of the lamp is effected by slightly displacing the electro-magnet $M M$, so as to bring it more or less near its armature; there is also a screw $\mathrm{V}$, 
which allows the raising or lowering of the luminous point, which in this system is fixed.

In a simplified model, applicable to cases where the fixity of the luminous point is not required, the lower carbon only is movable; the pulleys $\mathrm{P}$ and $\mathrm{P}^{\prime}$ are suppressed, and $\mathrm{P}$ and $\mathrm{P}^{\prime \prime}$ are replaced by a little drum. The cord is attached to this latter, passes over the pulley $\mathrm{P}^{\prime \prime \prime}$, and is connected at $\mathrm{H}$ to the lower carbon-holder. It tends, therefore, constantly, as long as the brake does not press on wheel $R$, to bring the two carbons in contact. This type, represented in Fig. 106 , is chiefly intended for industrial purposes, while the former is fitted for ex: periments in which the luminous point is required to be fixed.

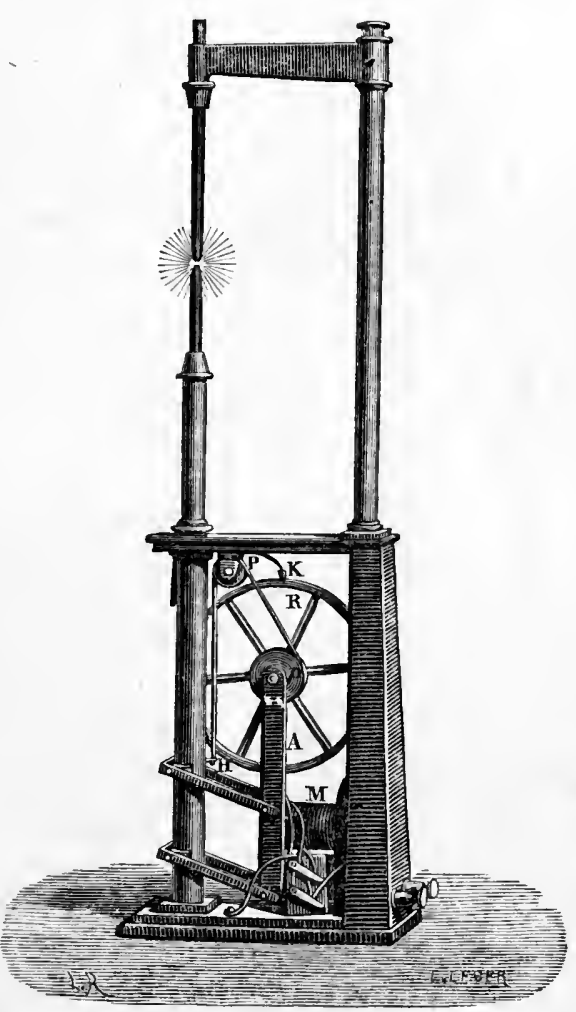

Fig. 106.-Bürgin's lamp (later model).

(From La Lumière Électrique.)

The carbons being in contact when the current passes, the first sudden elevation of the armature slackens the cord sufficiently so bring about a withdrawal, and after that the action continues as in the first model.

Figs. 107 and 108 show suspension lamps. It will be 
seen that the whole of the mechanism is in the upper part of the lamp, and occupies very little space. The positive carbon $B$ is fixed and supported in the lower part of the
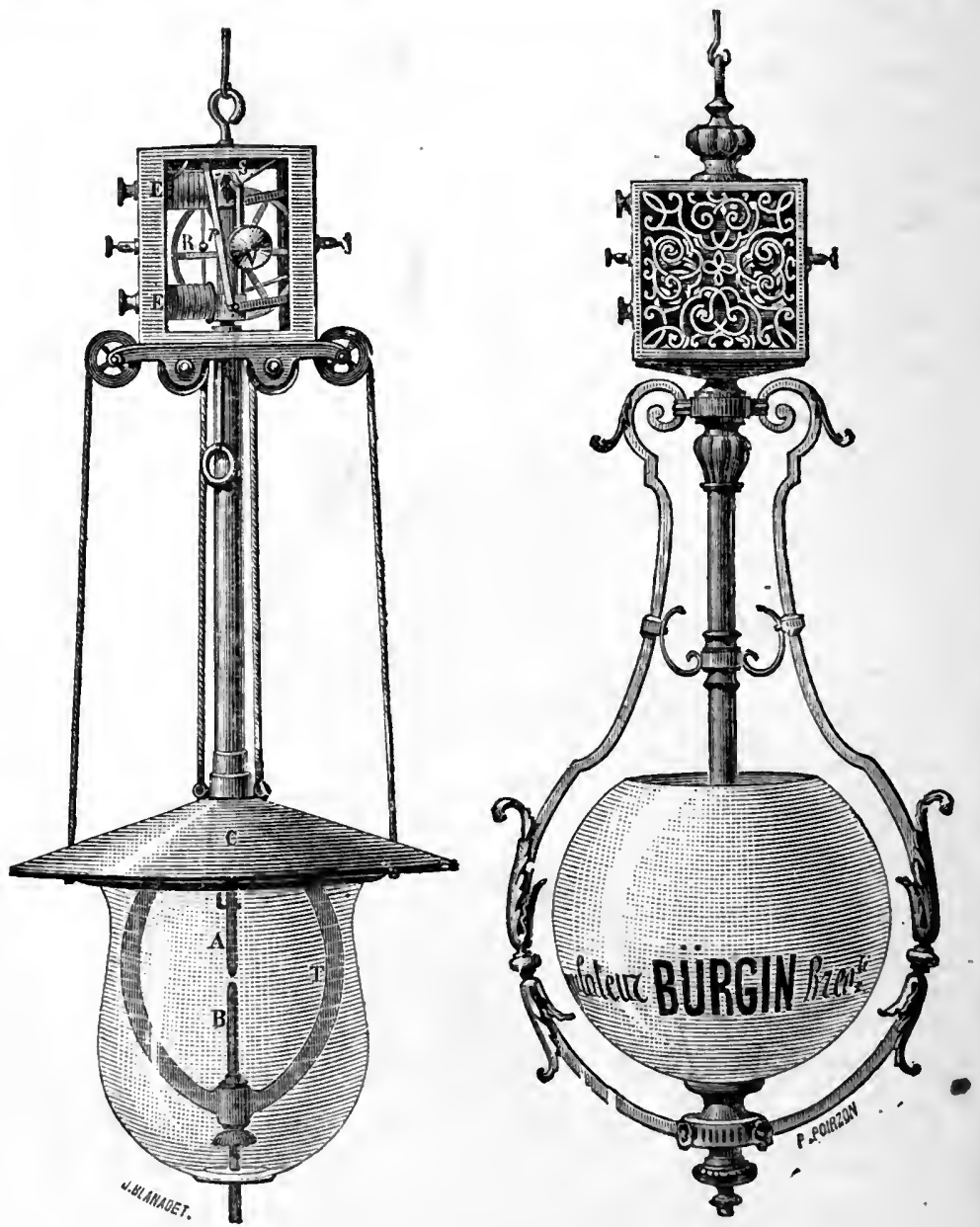

Figs. 107, 108.-Bürgin's suspension lamps.

(From La Lumiere Electrique.)

lamp by an oval frame $\mathbf{T}$. The negative carbon $\mathbf{A}$ tends to descend by its own weight, and a cord attached to its car- 
bon-holder at the same time tends to turn the wheel $R$. This latter is carried, as in the other models, by the armature of the electro-magnet $\mathrm{E} \mathrm{E.} \mathrm{When} \mathrm{the} \mathrm{lamp} \mathrm{is} \mathrm{started,}$ the first attractive movement of the armature raises the upper carbon and produces withdrawal, while the wheel $R$ at the same time presses against the brake; the adjustment is now effected as has been shown in the lamp represented in Fig. 105.

Dornfeld's Lamp.-The inventor-electric engineer at Messrs. Krupp's, in Essen-has constructed an apparatus similar to that of Bürgin, but in which the descent of the

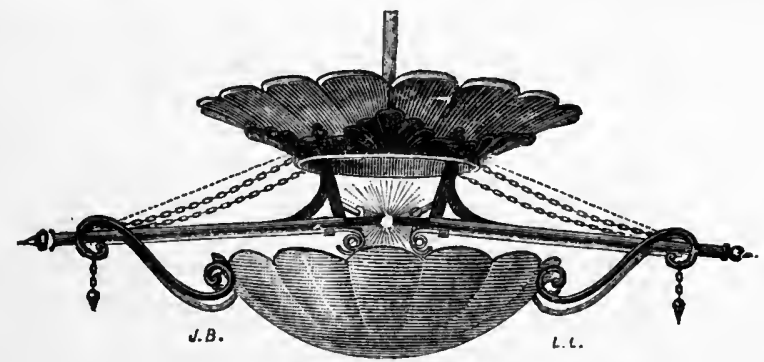

Fig. 109.-Solignac's lamp (perspective).

(From La Lumière_Électrique.)

positive carbon is retarded by a fly turning in a bath of mercury; the brake acts by means of a solenoid, which serves at the same time for the stoppage, the release, and the withdrawal of the carbons.

Solignac's Lamp.-In this system, which is shown in perspective in Fig. 109 and in detail in Figs. 110 and 111, the carbons F F, about nineteen inches long, are placed nearly horizontal, and with their ends in contact. Their movement is regulated by a system of chains $\mathrm{E} E$, which pass over the pulleys D D attached to the free end of the carbons, and over the pulleys $\mathrm{K} \mathrm{K}$ fastened to the frames C B, C B, and thence to a weight always tending to keep the ends of the 
carbons in contact. Beneath each carbon is a glass rod $G$, the ends being curved and butting against a nickel stop $\mathrm{L}$, the position of which can be adjusted. by the screw MI (Fig. 111). The current is brought to the carbons by the rollers I I, carried at the end of the bar $J$ attached to the frame. The distance of the carbons is regulated by means of the screw $H$.

The action of this lamp is as follows:-The arc being

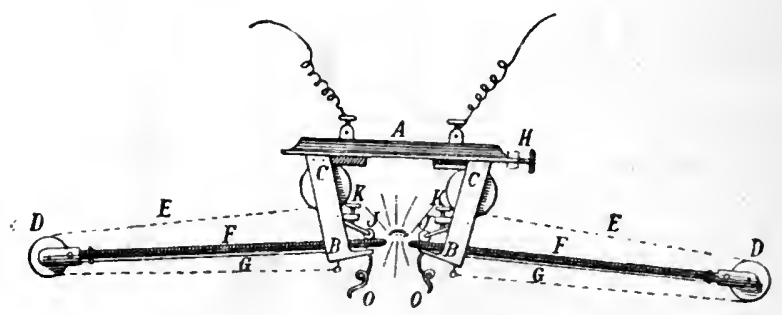

Fig. 110.-Diagram of Solignac's lamp.

(From La Lumière Éltetrique.)

established, the incandescent cone gradually approaches the ends of the glass rods, and softens them in such a way that they yield under the pressure of the weight attached to the system of cords E. Curling under the nickel stop $\mathrm{L}$ as shown, they allow the carbons to approach, and so the arc

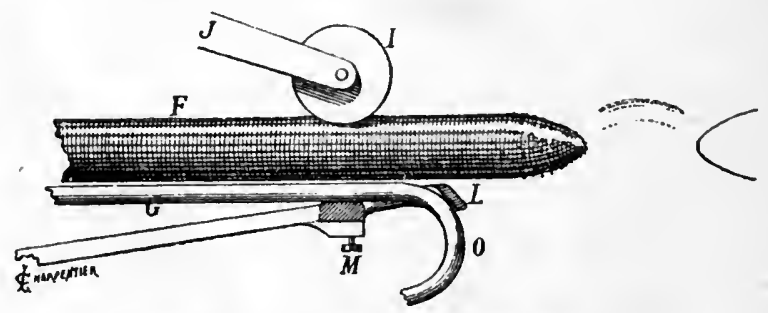

Fig. 111.-Details of Solignac's lamp.

(From La Lumière Électrique.)

is constantly maintained. That this is the simplest system of arc lamp that has yet been invented is beyond doubt; whether it will stand the test of practice remains to be seen. 
The trials so far have been very satisfactory, but the arrangement is so new that it has not yet emerged from the experimental stage.

Solignac has provided his lamp both with a relighting and a regulating apparatus, the latter being placed at a distance.

The re-lighter consists of a balanced rod carrying at one of its extremities a carbon, which, by the action of an electro-magnet, abuts in a parallel direction against the two carbons of the lamp, and thus closes the circuit; on the rod being raised the circuit is opened, and the lamp is started when the action of the electro-magnet has ceased.

The regulator consists of an axle $A$, carrying two pallets $\mathrm{B} \mathrm{C}$, which plunge into two beakers $\mathrm{B}^{\prime} \mathrm{C}^{\prime}$ filled with mercury; the axle is fastened to a winch, which is controlled by a shunt solenoid of great resistance.

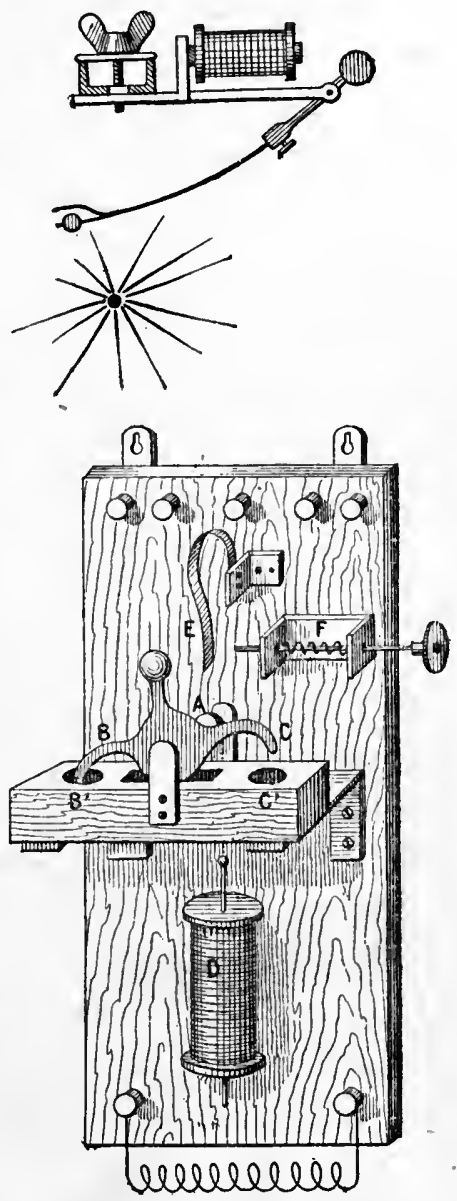

Fig. 112.-Solignac's lamp (automatic relighter). (From La Lumière Électrique.)

The beaker $\mathrm{B}^{\prime}$ conveys the current to the lamp, and the beaker $C^{\prime}$ to the corresponding resistance. The spring $E$, on being repulsed by the piston rod $\mathrm{F}$, replaces the pallet $\mathrm{B}$ 
into the beaker $\mathrm{B}^{\prime}$, and the current is ready to circulate through the lamp. This movement brings about the lighting of the lamp. When $\mathrm{F}$ touches $\mathrm{E}$, the current passes through the bobbin of the relighter. It passes, therefore, at the same time through the lamp, the solenoid, and the relighter, and this latter, being of much feebler rcsistance, absorbs nearly the whole of the current. When the $\operatorname{rod} F$ is drawn back again, it releases the spring $\mathrm{E}$, the current no longer passes through the relighter, this latter lifts the carbon by the action of a counter-weight, and the lamp is lighted. If, during the short period when $F$ is no longer in contact with $\mathrm{E}$, the circuit of the lamp is interrupted, the current passes into the solenoid, which plunges $\mathrm{C}$ back into $\mathrm{C}^{\prime}$ and reintroduces the corresponding resistance into the circuit of the lamp.

This arrangement requires no special attention or care for its working, it does not deprive the lamp of any of its advantages, for it does not introduce any deleterious resistance during the action of the lamp; it renders, moreover, the lamps independent of one another, and if the arc is accidentally extinguished or acquires too great a resistance, the solenoid no longer receives any current, and then acts to interpolate the resistance. But this only takes place in extreme cases, for, owing to its enormous resistance, the solenoid absorbs no current during normal action.

Polyphotal, or Division, Arc Layips.

When several monophotal arc lamps are placed in the same circuit, they soon get out of order.

It is easily explained why such must be the case with 
all these lamps, for the movement of the carbons is effected by an electro-magnet, which obeys the variations of intensity of the currents which are produced by the lengthening or shortening of the voltaic arc. If two apparatus are placed in the same circuit, the lengthening of one of the two arcs is sufficient to set the two electro-magnets in action, and at the same time to shorten both the voltaic arcs; upon this the second lamp immediately ceases to act, when it is merely a question of regulating the distance between the carbons of the first. In other cases one arc might be very long and the other very short without interfering with the equilibrium, since the intensity of the current will be the same as if the two arcs had the mean length necessary for their efficient working. The connection of the two regulators, therefore, entails in each of them changes which finally result in a complete disarrangement of the whole apparatus.

The independence of each lamp must, therefore, be ensured by acting on the resistance of the arc, shortening it if it is too long, and lengthening it if it is too short, independently of all the other lamps placed in the same circuit. The polyphotal lamps fulfil this condition. They are all founded on the law of shunted currents. Before even the division of light was thought of, this law was applied to arc lamps, at a time when batteries were the only electrical source for producing the voltaic arc.

Lacassagne and Thiers' Lamp.-In Lacassagne and Thiers' lamp with an overflow of mercury, patented in $185 \overline{5}$, a shunt bobbin is offered, whose function it was to shorten the voltaic arc when the carbons were too far apart, by an overflow of mercury which, by raising the lower carbon-holder, shortened the arc. In this apparatus 
we even find, besides the principle of shunts, the principle of differential action as in Tchikoleff's and Siemens' apparatus; but in reality it has never acted as a division apparatus.

Tchikoleff's Lamp.-To remedy the drawbacks which have been mentioned with regard to electric regulators mounted for tension in the same circuit, Tchikoleff proposed, as early as 1869 , to apply to the regulators the differential action of shunted currents.

Tchikoleff's first apparatus was a Foucault's lamp, in which the opposing spring was replaced by an electromagnet, which received only a very feeble shunted current on account of the great electrical resistance of the fine wire of which it was formed.

The armatures of the two electro-magnets were placed at the two extremities of a rocker, carrying a lever for the release of the mechanisms used for the approach or withdrawal of the carbons.

The apparatus was in a state of equilibrium when the resistance of the arc was normal.

If the arc was lengthened, its resistance increased, the action of the fine wire coil preponderated, and, by causing a rocking motion of the armature, set free the mechanism for the approach of the carbons. The inverse effect, on the other hand, set free the mechanism for the withdrawal. Another model consists of 'a Gramme's ring placed between two diametrically opposed electro-magnets; one of these, with coarse wire, is traversed by the direct current, and the other, with very fine wire, by a shunted current; the ring can turn in either sense, according to the predominating influence of either of the electro-magnets. This double movement of rotation can be utilized to produce the withdrawal or the approach of the carbons, with this advantage, 
that the movements are the more rapid the further the apparatus is from its position of equilibrium.

Tchikoleff's apparatus are arranged for continuous currents. They possess the advantage of acting without any clockwork, springs, or electric contacts; no previous adjustment is required either before or during the time of action, and the light remains constant in spite of any possible variations of the intensity of the current.

Lontin's Iamp. - This apparatus does not utilize the differential action of the current, but its action on an electro-magnet with fine wire, mounted in a shunt on the voltaic arc. Unlike Serrin's lamp, the pallet of the electro-magnet is arranged in such a manner that the lamp is always clamped, and the release is only produced when the arc attains too great a length; for then the current, passing into. the shunt, becomes more intense, attracts its armature, and releases the mechanism for the approach of the carbons. In other models, the electro-magnet is replaced by a solenoid, which does not affect the principle but prevents the vibrations of the armature when alternating currents are used.

With Lontin's apparatus, as many as twelve regulators have been placed in tension on one single circuit. With one single Lontin's division machine, as many as thirty-one Lontin's regulators have been fed and arranged in different circuits by means of the manipulator, described on p. 117 .

Mondos' Lamp.-In this lamp the lower carbon is fixed, whilst the upper carbon, which is carried by a movable rod, tends to descend by its own weight. This downward movement is regulated by the other parts of the apparatus. Fig. 113 is a diagram of the mechanism of the lamp, which is shown in perspective in Fig. 114. The upper 
carbon is fixed to the rod $t$, which moves without friction in a tube T. This latter traverses a ring $R$ and the platform $A$, and carries a bar $\mathrm{F}$, which on one side is fastened at $\mathrm{O}^{\prime \prime}$ to a lever $\mathrm{L}$, carrying a weight $\mathrm{M}$, and on the other side carries an iron cylinder $\mathrm{P}$, which can penetrate into an electro-magnet $\mathrm{E}$.

The descent of the carbon is regulated by the lever I', fastened at $\mathrm{O}^{\prime}$ by means of an articulated piece to the tube $\mathrm{T}$. The tubc $\mathrm{F}$ moves freely in the annular part of the lever

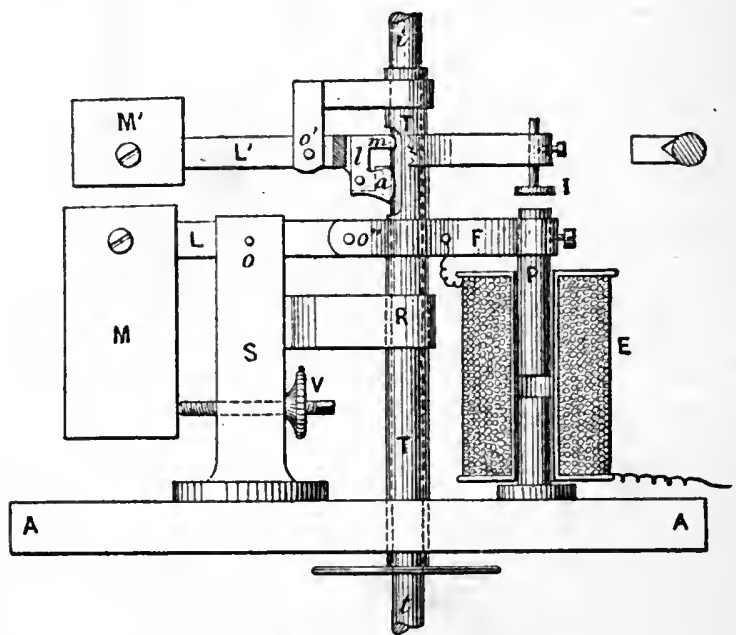

Fig. 113.-Mondos' lamp (mechanism).

(From La Lumière Electrique.)

$\mathrm{L}^{\prime}$, which carries a counter-weight $\mathbf{M}^{\prime}$, and terminates on the other side in an iron armature $I$, situated at a short distance above the cylinder $P$. The annular part of the lever $L^{\prime}$, moreover, carries a movable brake $m l a$, which is arranged in such a way that, if the lever $L^{\prime}$ is lowered on the righthand side, it presses on the rod $t$ through a notch in the tube $\mathrm{T}$, whilst, if the lever rises, it allows the free descent of rod $t$. This brake, therefore, connects the rod $t$, which carries the carbon, with the remainder of the apparatus: 
These two mechanisms for the lighting and the regulation are both controlled by the same electro-magnet $\mathrm{E}$, of whose core the movable cylinder $\mathrm{P}$ is a continuation. This electro-magnet is wound with very fine wire, and placed in a shunt between the two terminals of the lamp. We thus find here again an arrangement due to Lontin, which allows of several lamps being placed in the same circuit.

It will now be easy to understand the working of the apparatus. Supposing the carbons to be apart from each other, the current, arriving through the terminals $\mathrm{C} \mathrm{C}^{\prime}$, which are in communication with the two carbons, can only pass through the shunt of the electro-magnet. The cylinder $\mathrm{P}$ is therefore strongly attracted, and the bar F, in its descent, carries the tube $\mathbf{T}$ with it, and raises the weight $M$.

On the other hand, the cylinder $\mathrm{P}$ becomes a veritable electro-magnetic core, and attracts in its turn the armature I and the lever $\mathrm{M}^{\prime} \mathrm{L}^{\prime}$, which oscillates round $\mathrm{O}^{\prime}$, the brake $m l a$ releases the rod $t$, which descends till the carbons are in contact.

The current can now pass direct through the two carbons, and the attractive force of the electro-magnet $\mathbf{E}$ is weakened; the weight $\mathrm{M}$ now raises the tube $\mathrm{T}$, which carries the rod $t$ with it, because the armature $\mathrm{I}$ is partly released and the weight $\mathbf{M}^{\prime}$ presses the brake against the rod. This backward movement brings about a slight withdrawal of the carbons, and the arc is formed.

When, in consequence of the waste of the carbons, the arc is lengthened, the shunted current becomes stronger, and the tube $\mathrm{T}$, by a fresh descent, produces a first approach of the carbons. The brake now releases the rod $t$, and the carbons draw still nearer to each other; but if, after this second movement, the carbons are still too far apart, then 


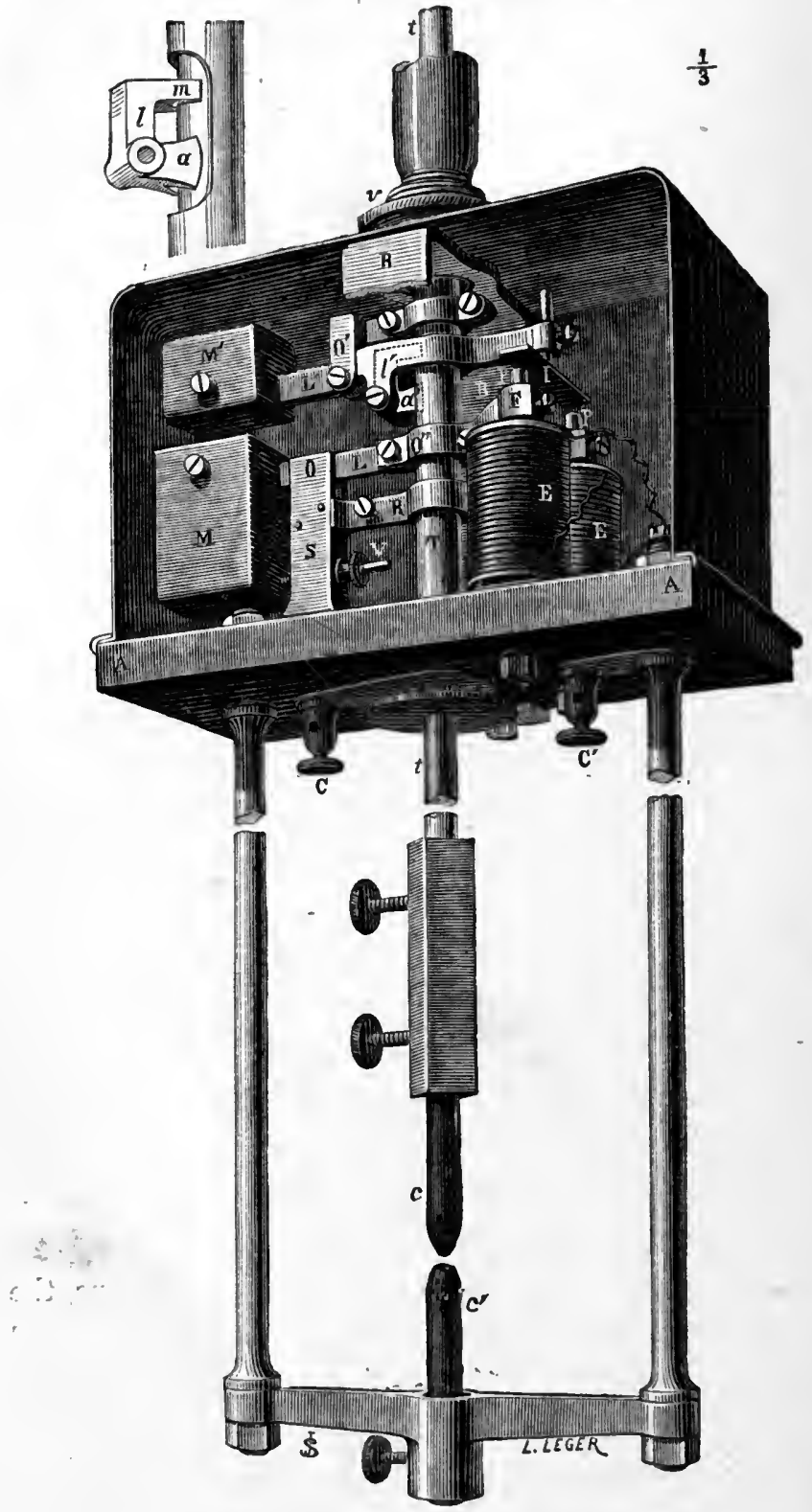

Fig. 114.-Mondos' lamp (perspective). (From Ia lumière Électrique.) 
the tube $\mathrm{T}$ is immediately raised and establishes the normal distance of the carbons.

We have supposed that at the moment of lighting the carbons are apart, and this is, in fact, the normal condition of the lamp. But if, from some cause or other, the carbons are originally in contact, the lighting can only be effected after the carbons are slightly wasted by incandescence. This is certainly a drawback, not a very serious one, since it can only occur in consequence of an accidental extinction produced by a gradual weakening of the current; but it is a defect which ought to be remedied, as it might retard the lighting by several minutes. According to the inventor, the illuminating power of each lamp is forty Carcels, and the force expended by the exciting and the alternating machines is twelve horse-power, or three-quarter horse-power for each lamp. The light itself is brilliant and steady.

Mersanne's Lamp.-This lamp has been constructed with the view of obtaining a light for at least sixteen hours, by using very long carbons; only part of this length, however, is actually in the circuit, so as not to increase the resistance.

A clockwork is used for the movement of the carbons.

When the arc has a tendency to lengthen itself, a larger part of the current passes into an electro-magnet placed in a shunt; the armature sets free the clockwork, which causes an approach of the carbons until the resistance of the arc has gone back to its normal value.

The necessary retrogressive movement for the formation of the arc is obtained by a second electro-magnet established in the same circuit of the shunt, which only acts when the whole current passes into this shunt, either by the breaking of the arc or because the are is not formed. At the moment when the current is sent into the apparatus, 
the whole current passes into the elcctro-magnets arranged on the shunts; the first sets free the mechanism for the advance, the second attracts its armature and pushes forward one of the carbons.

When the two carbons are in contact, the electromagnets of the shunt become almost inactive, as the greater part of the current passes through the arc, and they release their armatures; the armature of the first electromagnet arrests the advance of the carbons, that of the second produces withdrawal by the action of an opposing spring. Mersanne's lamp is used, with Lontin's division machine, for lighting the goods station of the Paris, Lyons, and Mediterranean Railway. at Paris. A single machine feeds eighteen lamps, arranged in six circuits of three lamps each. In spite of the complication of the movements of the clockwork and multiplying wheels, this lamp gives very satisfactory results.

Siemens' Differential Lamp.-This apparatus, constructed by Hefner Alteneck, enables the inventor to place as many as ten lamps in tension on the circuit of a Siemens' alternating-current machine, at the same time rendering the action of each lamp independent of all the others.

This apparatus, too, is an application of the law of shunted currents, and of the differential action which they exercise in proportion to their relative intensities upon two circuits. Although the current is in reality alternating, we may consider it as continuous at a given moment, in order to understand the working of the apparatus. The current, arriving from the machine at L (Fig. 115), is divided into two parts: one part of the current traverses a coil $\mathrm{T}$ formed of very fine wire and of great resistance, and leaves by $b L^{\prime}$ to enter the following lamp; a second part of the 
current tiaverses a coil $R$, with a coarse and short wire,-on leaving $R$, it trarerses the voltaic arc formed between the carbons $g h$, and passes from $b \mathrm{~L}^{\prime}$ over into the following lamp.

The current is thus divided into two very unequal fractions: the weakest traverses the coil $\mathrm{T}$, which has a fixed and very large resistance; the greatest part of the current traverses the voltaic arc, which constitutes a variable resistance with the withdrawal of the carbons. Let us place in the interior of each coil a soft-iron rod $s s^{\prime}$, connected by a lever $c d a$ with the carbon $g a$; and let us see what will happen.

Each of the coils attracts its corresponding solenoid with a force proportionate to the intensity and to the number of turns. It is clear, therefore, that for a con-

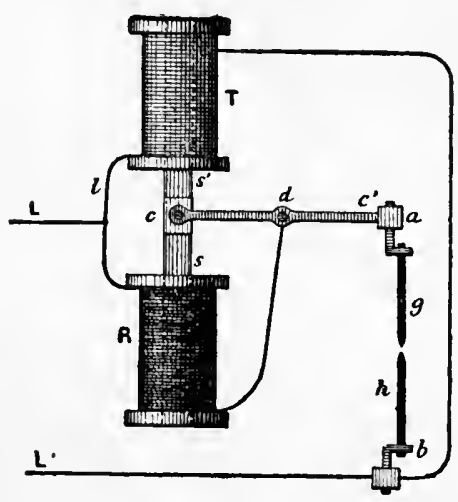

Fig. 115.-Diagram of Siemens' differential lainp.

venient resistance of the voltaic arc, the relative resistances of the two circuits will tend to equalize the actions of the coils on the rods $s s^{\prime}$, and the lever $c d a$ will take up a certain position of equilibrium. If the resistance of the arc increases, this equilibrium will be destroyed, the action of the coil $\mathrm{T}$ will become predominant, the lever c $d$ a will oscillate round $d$, and will cause an approach of the carbons. The opposite effect will be produced if the resistance diminishes, for then the coil $R$ will act more energetically on the $\operatorname{rod} s$, and a withdrawal of the carbons will ensue. The regulator no longer acts by the variations 
of the intensity of the current in the circuit, as in Serrin's lamp, but by the variations of resistance of the voltaic arc.

Such is the principle of the apparatus represented in its working condition in Fig. 116. If the action of the coil $\mathrm{T}$ preponderates, a small ratchet-wheel is released, which allows the upper carbon to descend by its own weight. In order to render that descent slow and regular, there is a sort of small pendulum, which only allows the escapement of one single tooth by oscillation, and as the descent can be arrested at each fifth part of an oscillation of the pendulum, the movements are absolutely imperceptible, and do not interfere in the slightest degree with the steadiness of the light.

The solenoid is connected with a little air-pump, in order to render its movements more gentle, and to prevent it from vibrating under the influence of the alternate currents. When the withdrawal of the carbons is produced, the mechanism is raised, together with the upper carbon, until the distance is reached for which the apparatus has been regulated. This regulation is very easily effected, without an opposing spring, by raising more or less the coil of fine wire, in order to vary its action.

The lower carbon being fixed, the luminous point descends in proportion to the waste of the carbons, but as the mechanism is placed altogether above the luminous point, no shade is thrown by the socket of the apparatus, and this displacement entails no inconvenience under these conditions, especially when the lamp is placed rather high; the mechanism is hidden in a copper cylinder, which preserves it and is used for its suspension.

One of the lamps can be extinguished without interfering with any other; a key has only to be introduced into the communicator, which establishes a direct communi- 
cation; on taking out the key, the extinguished lamp is immediately relighted.

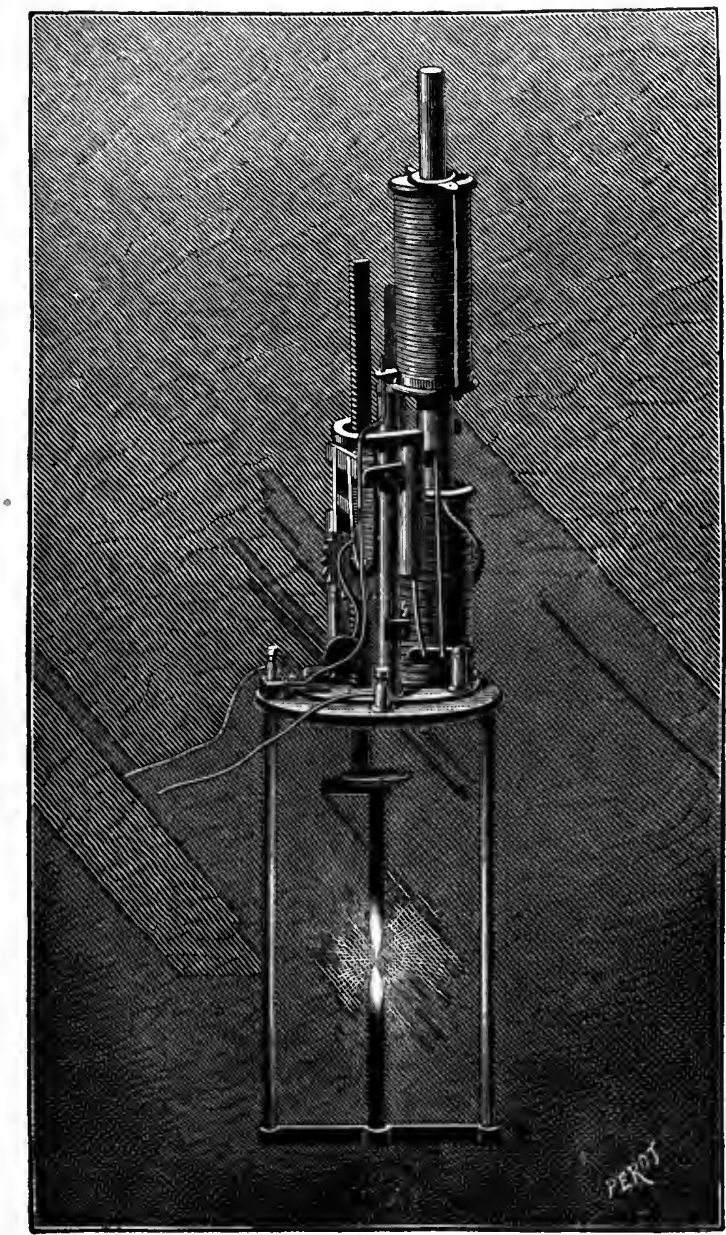

Fig. 116.-Siemens' differential lamp.

Finally, a simple and ingenious contrivance must be mentioned.

The rackwork of the movable carbon-holder carries a 
small cross-beam; when the rackwork is at the end of its course, this cross-beam presses against two small platinum contacts connected with the terminals; and establishes a direct communication. This prevents the extinction of all the lamps of one circuit when the carbons of one of these lamps are used up, and it has been forgotten to replace them in time. A dynamo with sixteen bobbins expends, with its charging machine, about ten horse-power, and feeds twenty lamps of a power of about twenty-five Carcel burners (400 candles) each.

Berjot's Lamp.--The lamp, shown in Fig. 117, consists of a casing (Fig. 118) enclosing the mechanism. Its clockwork is held in a frame, at the top and botton of which are the four flat springs $R \mathrm{R}$, suitably regulated and subject to the action, under varying conditions, of the two electromagnets $\mathrm{E} \mathrm{E}$. One of these electro-magnets tends to raise the frame, the other to move the mechanism. The first one $\mathrm{E}$ is covered with coarse wire and is placed in the main circuit with the carbon; the other $\mathrm{E}^{\prime}$ is formed of fine wire, and is placed in a shunted circuit. As shown, they are straight magnets, the cores of which are cut in the middle, one-half $\mathrm{N}$ constituting the armature; in this manner is combined the reciprocal action of a solenoid with the attractive action of the magnetic core. The frame containing the clockwork carries the weight of the upper carbon-holder, which is connected to a long rack $\mathrm{C}$, gearing with the first wheel of the clockwork, through the pinion $P$. A counter-weight $M$, acting in conjunction with the upper carbon-holder, tends to pull down the frame containing the clockwork; this is resisted by the electro-magnet $\mathrm{E}$, which retains it in a fixed position. The carbon-holder itself would always tend to fall if it were not held up by the clockwork 
of which the last wheel $\mathrm{D}$ is controlled by the brake $\mathrm{F}$ fixed to the case of the lamp. As this brake may be raised or lowered by the action of the electro-magnet $E^{\prime}$, the rack of the upper carbon-holder is free to move when the magnet $E^{\prime}$ is in action, the approach of the two carbons resulting from this action, which takes place when the arc is sufficiently weakened to give to the electro-magnet $\mathrm{E}^{\prime}$ an excess of power over the other. The approach of the carbons is, moreover, effected conjointly with the locking of the case containing the clockwork, but when the carbons are so near together as to restore the strength of the current of the arc, the magnet $\mathrm{E}$ raises the cage and the fine-wire magnet $\mathrm{E}^{\prime}$, releasing the brake $\mathrm{F}$, prevents the clockwork from moving until the arc again becomes weakened.

The apparatus is so sensitive that the core is in a constant state of vibration, and as the clockwork is never stopped abruptly on account of the delicate action of the brake, very

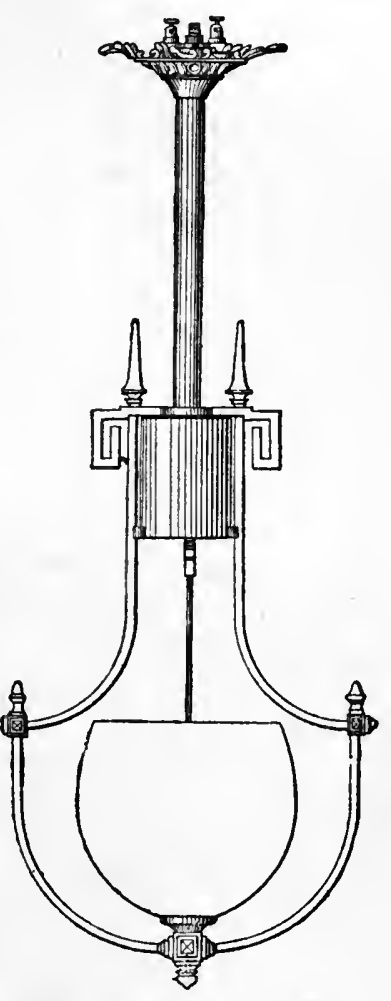

Fig. 117.-Berjot's lamp (suspended).

(From La Lumière Électrique.) slight differences in intensity can be corrected without any appreciable variations in the steadiness of the light. When the rack has arrived at the end of its travel, that is, when the upper carbon is consumed, a stud on the rack presses on a contact-piece, and shunts the current into the 
circuit of other lamps. In order to secure a prolonged service with this regulator, without renewing the carbons, a

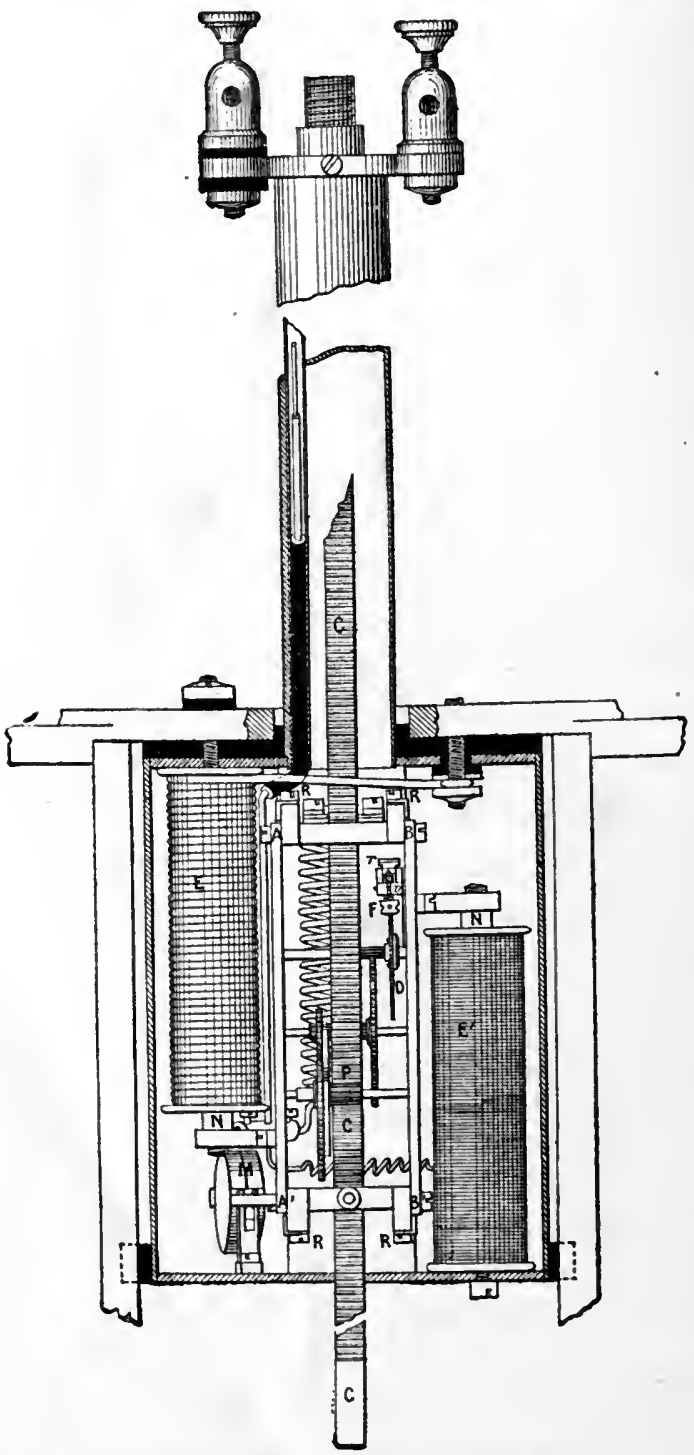

Fig. 118.-Berjot's larnp (mechanism). (From La Lumière Électrique.) 
modification is devised by Berjot, in which several racks are employed, one coming into action as the previous one is thrown out.

As will be seen, the mechanism of the lamp is simple, its action sensitive and certain, and its appearance is pleasing.

Standard Lamp for French Lighthouses.-The lamp which has been adopted for the French electric lighthouses, and which is shown in Fig. 119, is a combination of the Serrin and the Berjot lamps, which have both been deseribed in the preceding pages. It comprises the two electro-magnets of the Berjot lamp, the armatures of which form an internal core, one magnet having coarse wire and placed direct in the circuit, the other a fine-wire magnet mounted in a shunted circuit. The former acts on the articulated frame carrying the lower carbon; the other influences the disc brake that controls the clock train. In the drawing, $\mathrm{S}$ is the coarse electro-magnet acting through the lever $\mathrm{Q}$ on the articulated frame or cage, the two springs $R$ and $R^{\prime}$ tending always to lift this cage. The tension of these springs is regulated by the bent lever $\mathrm{L}$ hung to the frame of the lamp, and the position of which can be altered by the screw $V$, which can be turned by a key passing through a hole in the casing. The fine-wire coil is not shown in the drawing, but it occupies a position corresponding to that of the electro-magnet $\mathbf{S}$ on the other side of the clock train. The connection between the two carbon-holders and the first spindle of the train is effected by means of a steel ribbon $F$ attached to the lower ends of the two rods $g$ and $l$, the lower and upper carbon rods respectively. This ribbon is led over several pulleys and passes round the greater part of the circumference of a wheel on the first axis of the clock train, so that there should be no slip between the ribbon and the pulley. 
The rod $g$ slides in the tube $\mathrm{D}$ fixed to the articulated frame, and this tube has a vertical slot formed in it to allow the ribbon to be attached. This mode of connecting the two rods supporting the carbons, which replaces the chain of the Serrin regulator, allows the carbons to be set at their right height by a slight slipping of the ribbon. Another peculiarity in the lamp is the mode of connection adopted for the internal mechanism. The current from the large cable conductor is led to the upper carbou from the rails and the non-insulated part of the lamp. From the lower carbon it returns to the two insulated terminals $\mathbf{H}$ and $\mathbf{H}^{\prime}$, passing partly through the articulated cage, and partly through the electro-magnet $\mathrm{S}$. The connections between the pieces to which the current arrives are made with four spirals of thick copper wire, nickel plated: they are shown at M and $N$, placed above and below the coarse-wire magnet $S$. The tube D carrying the rod $g$ is not insulated from the articulated cage, but the latter is insulated from the rectangular bar by which it is carried. This arrangernent is convenient in construction, because it is more easy to insulate a rectangular bar than a round piece like the tube $\mathrm{D}$. An air-pump $T$ serves to deaden the motion of the cage, and prevents any violent oscillation. The ingenious details of the porous plate $v$ must be noticed. When large carbons like those necessary for lighthouses are employed, the turning-piece $a$, that serves to regulate the height of the arc, becomes heated as well as the rods $t^{\prime}$ and $t$ and the different parts exposed often causing them to bind. To avoid these inconveniences, a porous clay shield $v$ is introduced as shown. When the upper carbon-holder has reached the end of its travel, it acts laterally on a small inclined rod which draws back a spring, and cuts off the connection with the fine- 
wire electro-magnet, so that it cannot be injured by the passage of too strong a current.

Weston's Lamp.-This lamp is represented in perspective

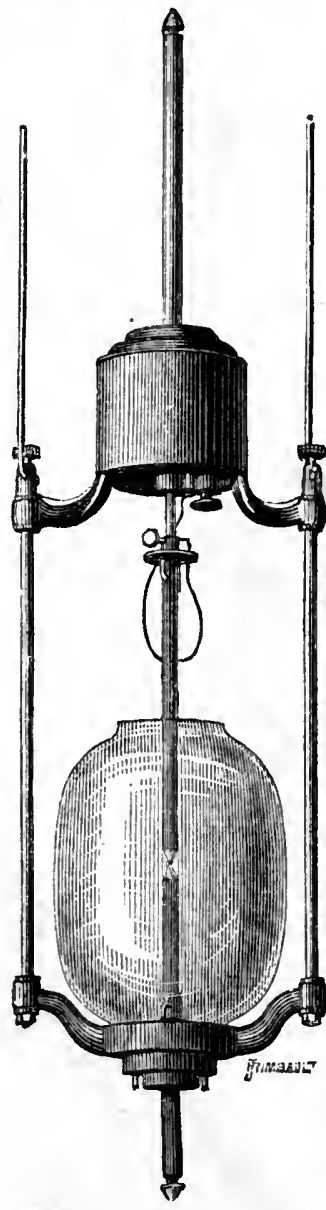

Fig. 120.-Weston's lamp (perspective).

(Brom La Lumiere Électrique.) in Fig. 120; Fig. 121 shows the details of the mechanism contained in the upper box. The tube at the top of the box contains the rod $R \mathrm{R}$ which carries the upper carbon. When no current circulates in the lamp, this rod descends by its own weight, and the two carbon points are in contact. When the lamp is started, the rod $R$ can be arrested by a curved lever $\mathrm{C} \mathrm{C}$, which raises an armature A A attracted by the electro-magnet M M ; this armature, being fastened to the two horizontal springs $\mathrm{O}$ and $\mathrm{N}$, can only move up or down. The electro-magnet is of special construction; one of its coils is shown in section in Fig. 121. It is wound alternately with coarse and fine wire, but the two wires are wound in inverse sense, so that the action of the electro-magnet depends on the differential action of the currents which traverse these wires. There is a spring at $\mathrm{S}$, whose tension can be regulated by a screw acting on an articulated lever.

The movement of the armature, therefore, depends on the difference between the tension of this spring and the 
attractive force of the electro-magnet. The lamp acts as follows:-The carbons being first in contact, the current arrives through the terminal on the left (Fig. 121), traverses the coarse wire of the electro-magnet, then the carbons, and ascends on the right of the lamp-frame to the terminal on the right. The electro-magnet, now being energized, lifts its armature, which carries with it the lever $\mathrm{C} \mathrm{C}$ and the rod $R$, and the arc is established. The fine wire of the electro-magnet is likewise connected to the terminals of the lamp, and constitutes, therefore, a shunt of the maincurrent. The intensity of the current circulating through this shunt will increase in proportion to the distance between the carbons, and consequently the resistance of the arc. As soon as this resistance increases, the current traversing the fine-wire coils is

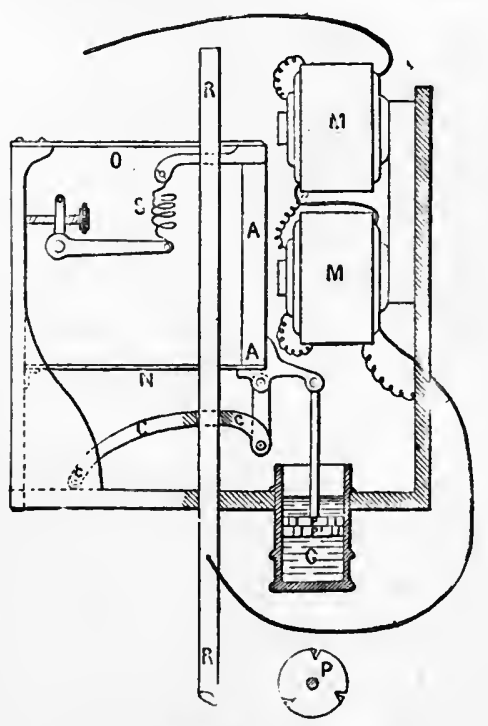

Fig. 121.-Weston's lamp (diagram). (From La Lumière Électrique). strengthened, the intensity of the magnet is lessened, the armature is lowered, the carbons draw nearer to each other, and the shunt current becomes less intense. If from some cause or other the arc is extinguished, the armature is entirely released, the two carbons touch anew, and the current is re-established.

To damp down the morements of the armature, it is connected to the rod of a piston $\mathrm{C}$, which moves in a cylinder filled with glycerine. This piston is formed of two discs 
$P$, each of which has three triangular notches on its circumference; one disc is fixed on the rod of the piston, the other capable of rotating around it. The notches can be made to coincide with or to pass by one another, and the movement of the piston is thus regulated with great nicety.

The lamp gives very satisfactory results, regulating itself with great accuracy according to the strength of the current, and producing a steady white light. It has the additional advantage of a very simple construction.

Gülcher's Arc Lamp.-This lamp is attracting much attention by the steadiness of the light it gives and the simplicity of its construction. The regulation of the carbons is effected by a single electro-magnet, rendering the lamp very cheap to manufacture and repair, and not liable to be easily put out of order.

Fig. 122 shows the lamp in perspective, and it will be seen that the two carbon-holders are connected by means of a cord which passes over a pulley $\mathrm{R}$; the upper one, whose $\operatorname{rod} \mathrm{T}^{\mathrm{T}} \mathrm{T}^{\prime}$ is of iron, is heavier and tends to descend at the same time as it draws up the other.

The rod of the upper carbon-holder moves before the pole of a straight, cylindrical electro-magnet $\mathrm{E}$, which is free to oscillate in a vertical sense round its axis; the other pole of the electro-magnet is pressed against a regulating screw $\mathrm{V}$ by a spring whose tension is adjusted by means of a lever.

At the outset the carbons are in contact; if afterwards the current passes through the carbons and the electro-magnet, one of the extremities of this latter adheres to the iron $\operatorname{rod} \mathrm{T}^{\mathrm{T}} \mathrm{T}^{\prime}$ of the upper carbon-holder, and keeps it in place, whilst the other is attracted by a small iron block $\mathrm{C}$ placed beneath. In consequence of this attraction, the electromagnet is lowered towards the block $\mathrm{C}$, and raised on the 


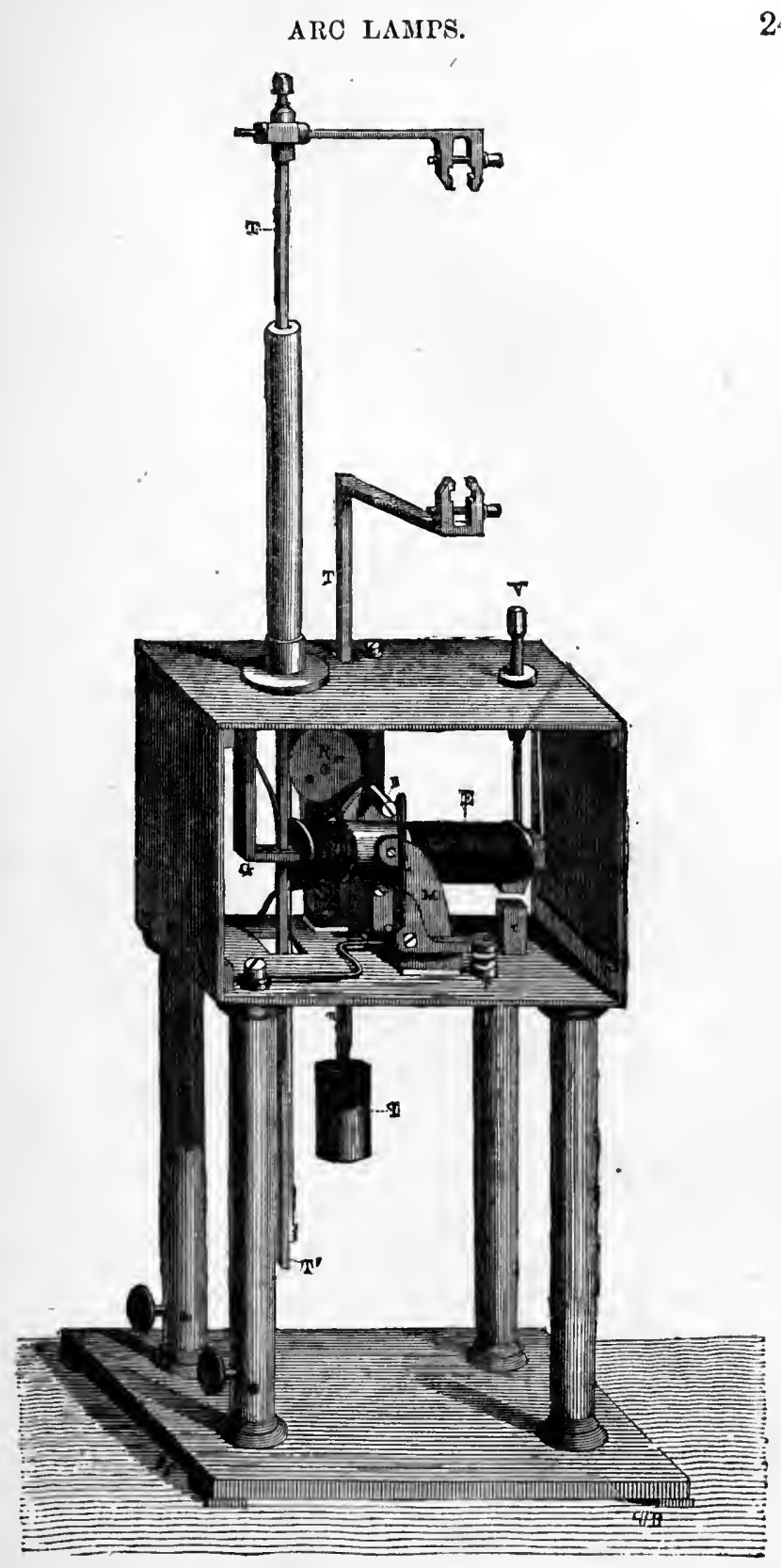

Fig. 122.-Gülcher's lamp (perspective). (From La Lumière Électi ique.) 
opposite side; the upper carbon-holder thereby ascends, and the voltaic are is produced. Now the electro-magnet begins to oscillate, and finally abuts against the adjustment

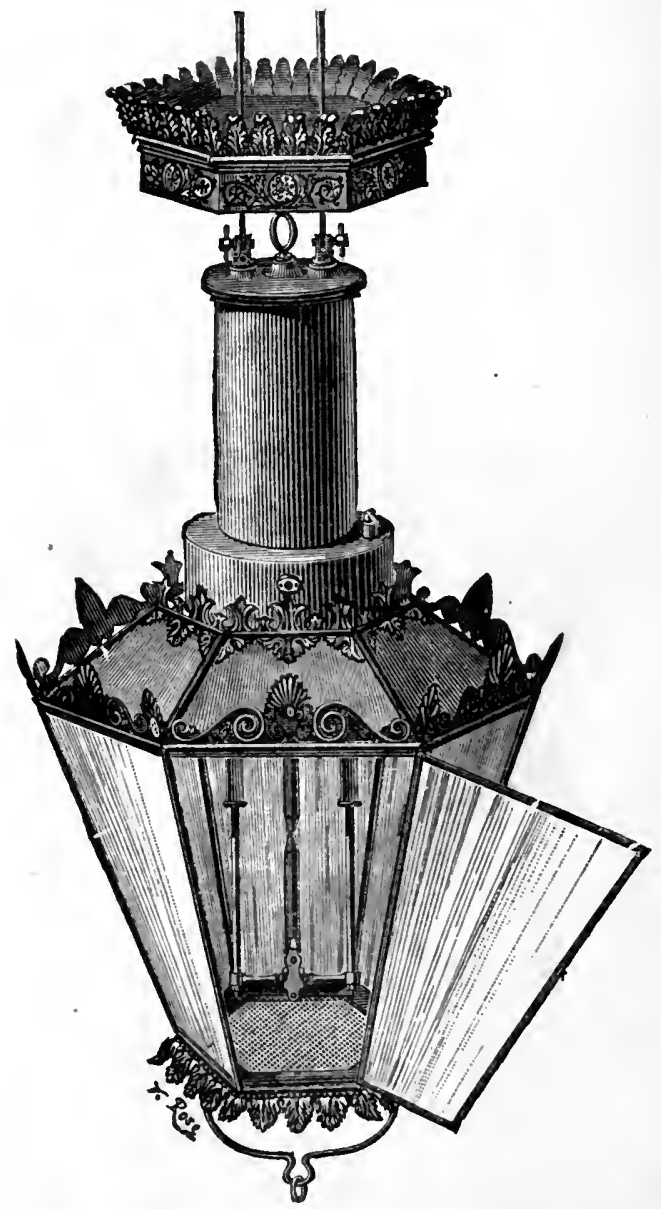

Fig. 123.-Gülcher's suspended lamp.

(From La Lumiere Électrique.)

screw $\mathrm{V}$; it remains in this position as long as the current preserves its normal intensity. However, in the same measure as the arc increases, the intensity is diminished, the 
current being weakened by the gradual waste of the carbons; this causes the electro-magnet to release the upper carbonholder, and the carbons approach each other. A little

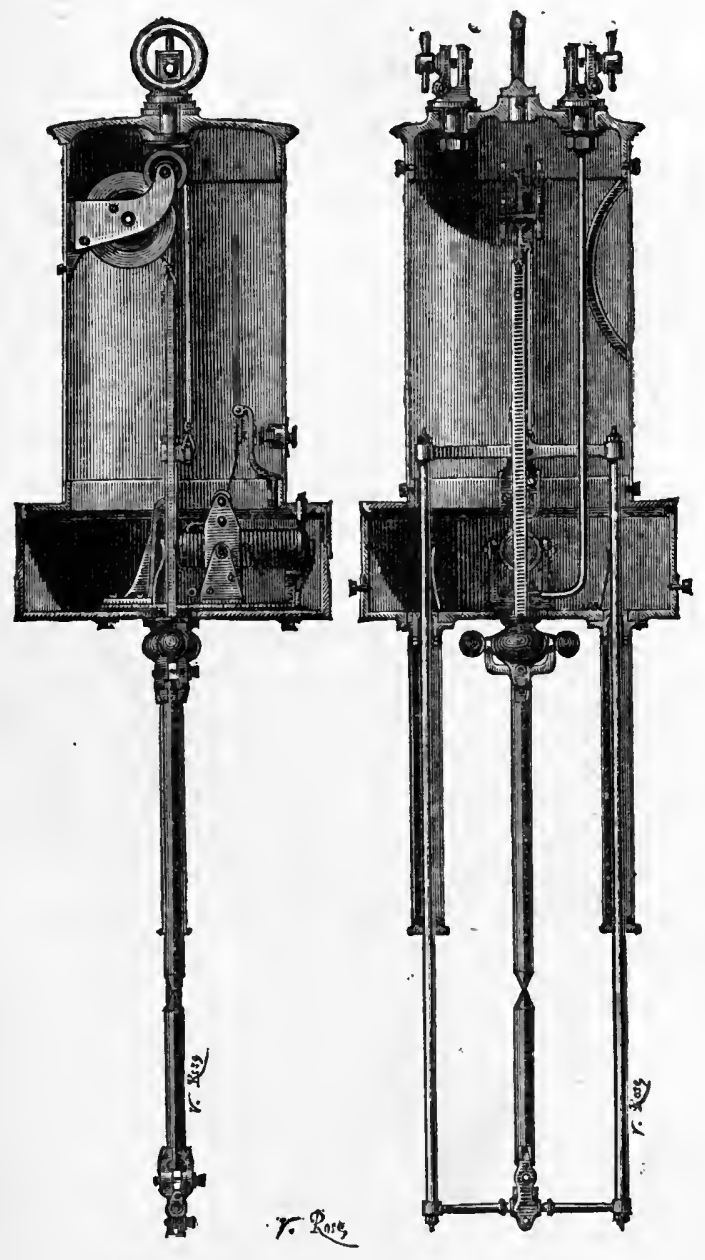

Figs. 124, 125.-Gülcher's suspended lamps.

(From La Lumière Électrique.)

iron block attached to spring $\mathrm{L}$ acts as brake, and damps down the action of the electro-magnet when the lamp is started or some adjustment becomes necessary. Under 
ordinary conditions, the electro-magnet remains immovable after it has taken up its original position, but it is always ready to resume its oscillations to counteract any irregularity which might occur. Figs. 123, 124, and 125 represent models of the suspension lamp.

Such is the arrangement of the Guilcher lamp. It now remains to be seen how the peculiarities of its arrangement render it suitable for being used in groups in the same circuit, and how one lamp acts as the regulator of the second, the first and second as the regulators of the third,

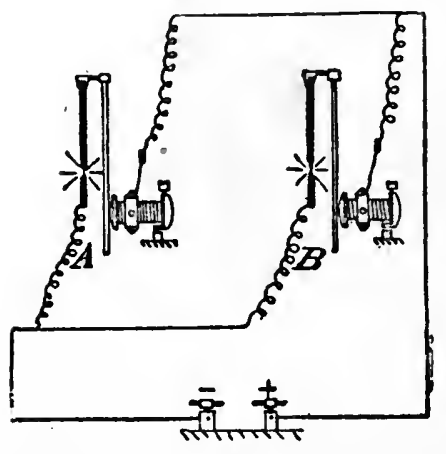

Fig. 126.-Connections of Gülcher's lamp.

(From La Lumière Électrique.) and so on. This regulating action depends on the positive motion of the electro-magnet, and upon the quantity of electricity flowing through the circuit. To take first the simple case of two lamps put in parallel circuit with the generator. If a lamp A (Fig. 126) be first lighted by connecting the branch wire from the trunnion of the electromagnet to the main conductor from the positive pole of the generator, the light will establish itself as has been already described; then, if the connection with lamp B be completed, the current is divided, and lamp B is lighted. At first the part of the current flowing through lamp $B$ is stronger than that passing through lamp $A$, because the carbon points of the former are in contact, and the resistance is less. The result of this inequality is that the electro-magnet of lamp $B$ is set in motion, the carbons are separated, and the resistance is increased; at the same time that this takes place, 
the current passing through lamp A is strengthened, and the carbons are brought somewhat closer together. From this it will be seen that if in either lamp the carbons become too far separated, the preponderance of current in the other will tend, through the action of the magnet, to raise its carbon-holder, increase the distance between the points, and so weaken the current in the first-named lamp; then the magnet will cease to maintain the carbon-holder, which will slide down till equilibrium is established or the same process is reversed, and so on, one lamp regulating the other, and

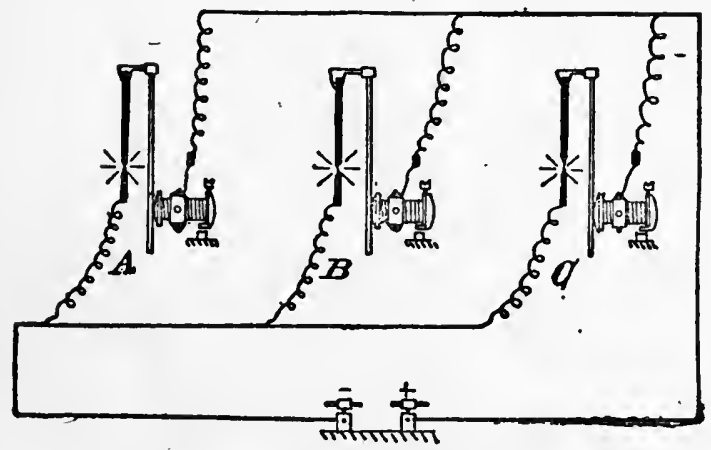

Fig. 127.-Connections of Gülcher's lamp.

(From La Lumière Électrique.)

consequently itself. With three lamps in parallel circuit, as shown in Fig. 127, the same processes take place; when all three are lighted, lamp $\mathrm{A}$ regulates lamps $\mathrm{B}$ and $\mathrm{C} ; \mathrm{B}$ regulates $\mathrm{A}$ and $\mathrm{C}$, and $\mathrm{C}$ regulates $\mathrm{A}$ and $\mathrm{B}$. Experiments made with this lamp have shown that 1.66 horse-power were required for each lamp of 135 Carcels.

The Brush Lamp.-The great simplicity of the Brush lamp consists in the fact that the feed is actuated by gravity alone, while it is controlled solely by the influence of a magnetic field upon a bar of iron; the intensity of the field varies with the strength of the electric current 
passing through the lamp circuit. The general external appearance of the three forms is shown in Figs. 128, 129, and 130 : the first representing the single-carbon, or eight-hour lamp; the second the double-carbon, or sixteen-hour lamp,

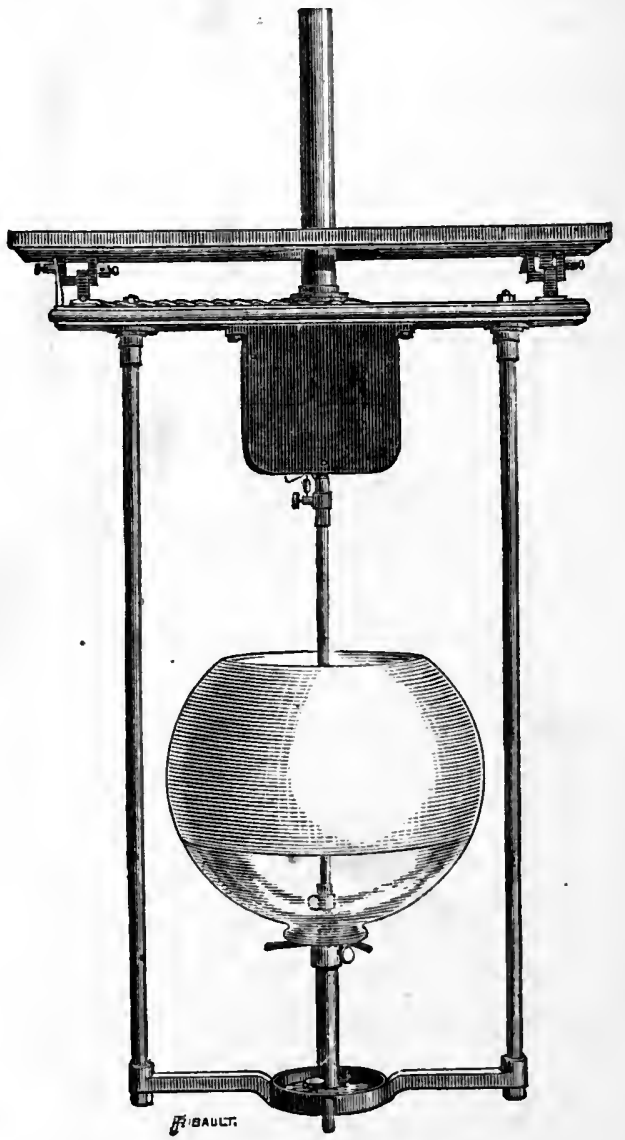

Fig. 128.--Single-carbon lamp.

with its glass globe removed; and the last is a sketch of a more ornate form of the double-carbon lamp, the controlling apparatus being enclosed in a nickel-plated case. In all these lamps, which are designed for general illumination, 
the lower carbons are fixed, and, as, they burn away, the upper carbons follow them, so that the arc gradually descends; but for certain special installations, such as light-

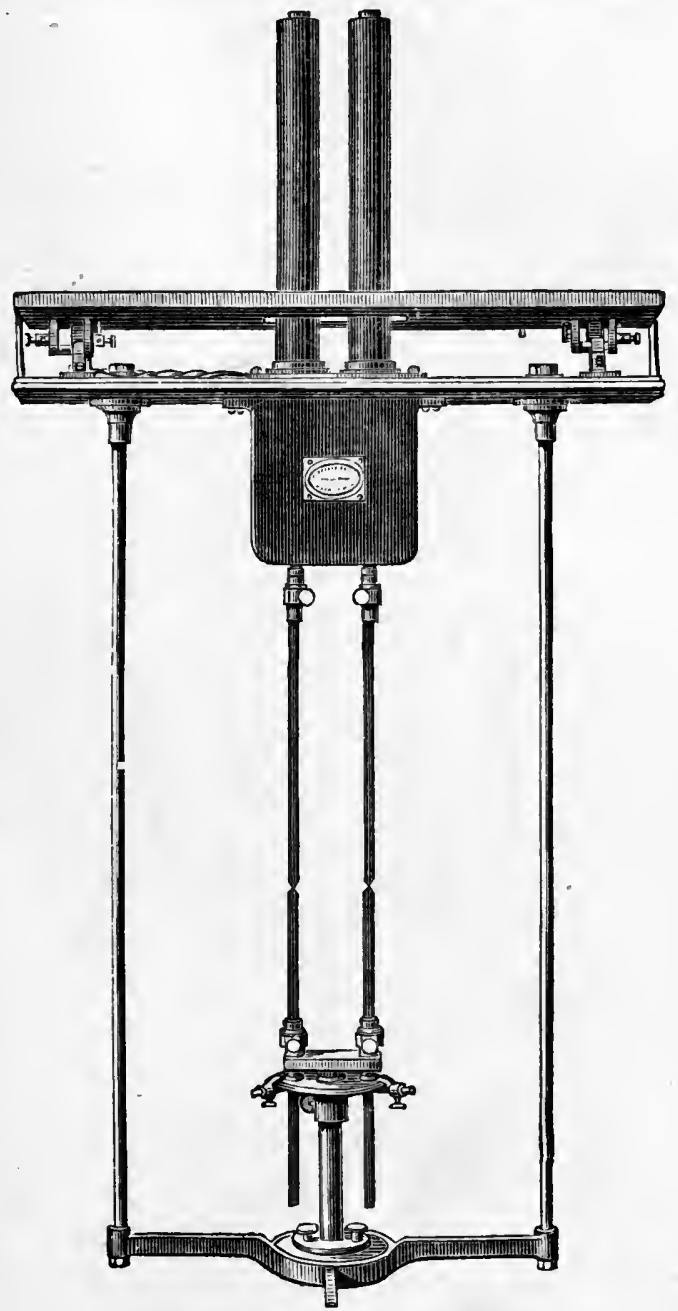

Fig. 129.-Double-carbon lamp.

houses or for purposes of projection, a focus-keeping arrangement has to be applied. 
Like most of the modern electric lamps, the upper carbon descends by its own weight until it touches the lower carbon, and the circuit is thereby completed; the effect of this in the lamp we are describing is to cause a soft-iron plunger to be drawn to a more or less extent within a hollow coil, or sucking magnet, and, through the

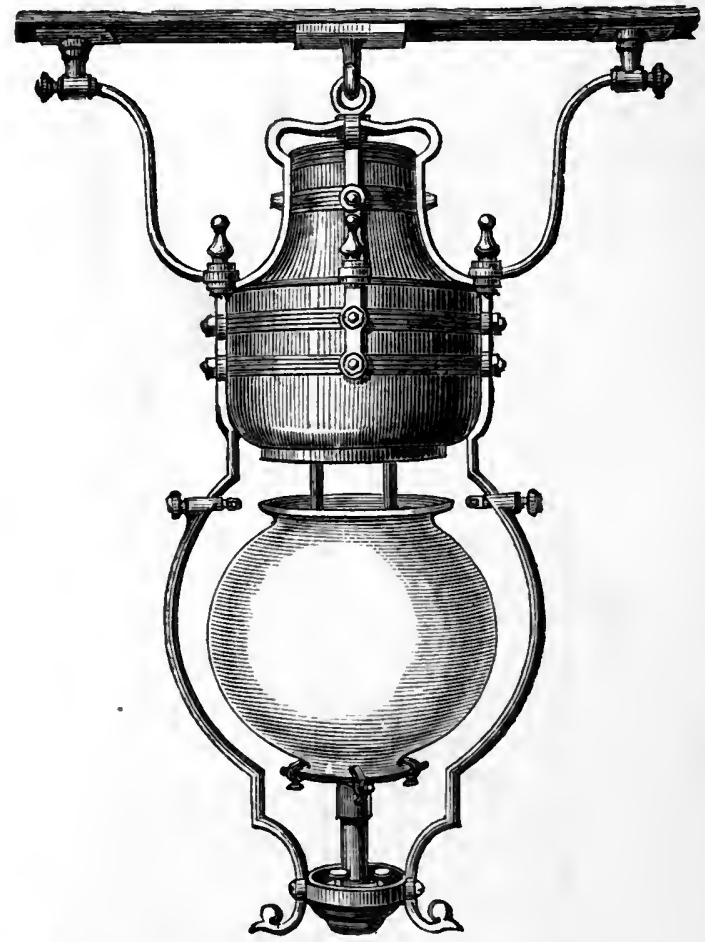

Fig. 130.-Double-carbon lamp, with plated case.

intervention of a lever and an annular clutch surrounding the rod of the upper carbon like a washer, the upper carbon is lifted away from the lower, and the arc is established. As the carbons burn away, the arc has a tendency to become longer and its resistance to increase, and this, by diminish- 
ing the strength of the current, diminishes the supporting power of the magnetic helix, allowing its plunger to descend, and, in so doing, to lower the carbon and shorten the are until the proper strength of the current is restored, when the rising of the plunger once-more holds the carbon in position. To ensure the individual independence of each lamp and a uniform light, each of the bobbins of the controlling magnet is wound with two distinct coils of wire, the first consisting of a number of turns of thick wire through which the current is transmitted to the arc, and the second of a much larger number of convolutions of fine wire which forms a secondary or shunt circuit between the terminals of the lamp, the connections being made in such a way that the electric current shall pass through it in a direction opposite to that in the primary or thick-wire circuit, forming a shunt circuit of high resistance through the lamp, which, being always closed, is independent of the arc. It will readily be understood that, as the current flows in opposite directions around the magnet, the influence of the fine-wire circuit will be to neutralize or weaken the attractive influence of the thicker helix; but the number of convolutions of the two coils, as well as their respective resistances, are so proportioned to one another, that the attractive influence of the primary helix (when the arc is of its normal length) shall overcome the influence of the secondary circuit. Owing to the greater resistance of the latter, not more than one per cent. of the main current is transmitted by the fine-wire helix, but its magnetic influence is rendered considerable by its greater number of convolutions.

From the above description it is clear that, since the electric current has two routes from one terminal of the 
lamp to the other-the one through the arc and the other independent of it-should the arc become too long, its resistance will increase, and a larger proportion of the current will be shunted through the fine-wire helix, while the strength in that of the thicker helix will diminish, the resultant magnetic influence on the plunger will be reduced, and the upper carbon will be brought closer to the lower; on the other hand, if the arc becomes shorter than its normal length, its resistance is reduced, more current flows through the primary helix, and less through the secondary, and the carbons are drawn further apart.

Fig. 131 is a diagrammatic sketch, showing the course of the primary circuit, and illustrating the general principle by which the are is controlled; it also shows the shortcircuiting contrivance by which any accident to one lamp or irregularity of working cuts it out of the general circuit, and does so without exercising any influence upon the other lamps in the series. In this diagram, $\mathrm{X}$ and $\mathrm{Y}$ represent the two terminals of the lamp, which in most cases consist of hooks which, by being dropped over pins attached to the ceiling-and which are connected with the line circuit - place the lamp in circuit with the machine. The current, entering at $\mathrm{X}$, is transmitted through the two hollow bobbins $\mathrm{H} \mathrm{H}^{\prime}$ in parallel circuit, the outcoming ends being joined together and connected to the upper carbon-holder $\mathrm{N}$, and if the carbons are in contact, the current flows through them and by the vertical rods of the lamp to the terminal hook Y. The effect of this is to convert the solenoids $\mathrm{H}$ and $\mathrm{H}^{\prime}$ into magnets, which, by drawing into themselves the two iron plungers $\mathrm{N}$ and $\mathrm{S}$, lift one edge of the washer clutch $\mathrm{W}$, which, by its oblique action, seizes the carbon rod (much in the same way as a tent-rope 
tightener grasps its cord) and lifts the upper carbon until its influence is balanced by that of the fine-wire helix, which, it must be remembered, surrounds the thicker coil.

The short-circuiting apparatus, shown to the left of the carbons $\mathrm{K} \mathrm{K}^{\prime}$ (Fig. 131), consists of an electro-magnet T, wound with a thick and fine wire coil similar to those of the regulating solenoids, but both wound in the same direction. When the thick-wire circuit of this magnet is complete, it forms a shunt of less resistance between the terminals, and therefore short-circuits the lamp, transmit-

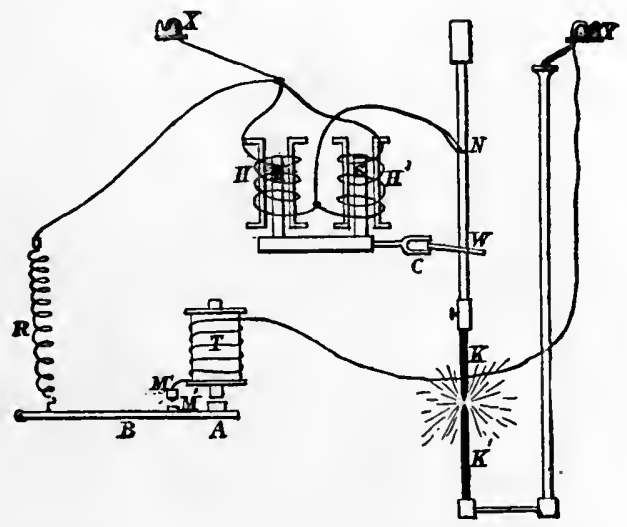

Fig. 131.-Diagram of the Brush lamp.

ting the current to the main circuit. Now, the fine wire wound upon this magnet is in circuit with the fine wire of the regulating solenoids; it follows, therefore, that if, through a failure of the arc, or through its becoming abnormally long, or through any considerable increase in its resistance, a larger proportion of current be diverted through the finewire circuit, the attractive force of the electro-magnet $T$ would be increased, and its armature A, which is attached to the pivoted lever $B$, would be attracted, the contact pieces 
$\mathrm{M}$ and $\mathrm{M}^{\prime}$ would thereby be brought together, and the terminals would be short-circuited through the thick coil $\mathbf{M}$ and the resistance spring $R$. By thus short-circuiting the terminals through a route altogether independent of either the fine or the thick wire solenoids, their magnetic action ceases, and either the upper carbon is dropped, or, if it is burned out or from any other cause the arc is not established, the contact-pieces $\mathrm{M}$ and $\mathrm{M}^{\prime}$ are held firmly together by the attraction of the magnet $\mathrm{T}$, and the current

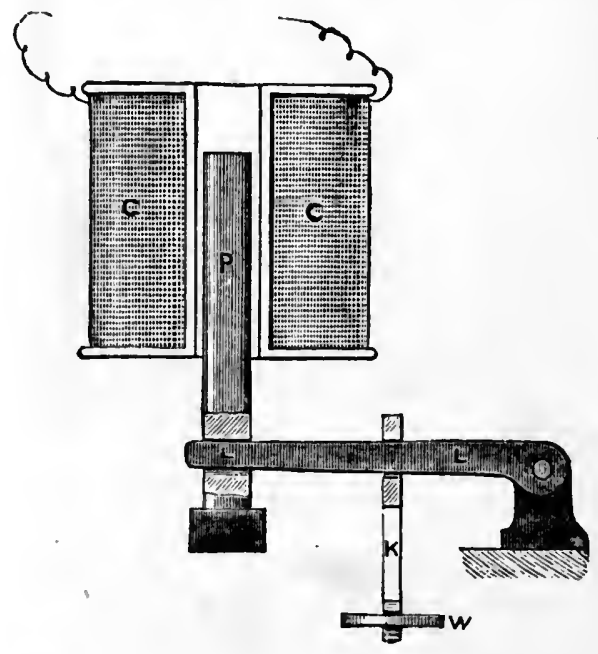

Fig. 132.-Two-wire solenoid of Brush's lamp.

flows past the faulty lamp to the others in the series, its extinction calling attention to it, while the other lamps become proportionately brighter. The carbon pencils are a foot long, and are electro-plated with a thin covering of copper; they last about eight hours, during which time about nine and a half inches of the positive and about four inches of the negative carbons are consumed. When, however, the lamps are required to burn for a longer period, 
double-carbon lamps are employed, fitted with two pairs of carbon-holders, as shown in Fig. 129, and each is controlled in the same way as in the single-carbon lamp, but the controlling apparatus is not duplicated. When the lamp is put into action, the arc is formed between one of the pairs of carbons, and when they have burnt out, the other pair automatically starts into action and continues to burn until it is in turn consumed; in this way the time of burning is doubled, and in order to extend the period of burning, it is only necessary to employ a lamp with a third pair of carbons, when it will be capable of illuminating for twenty-

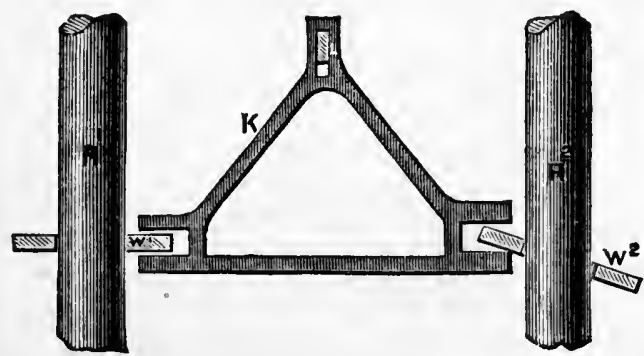

Fig. 133.-Arrangement for the change of carbons in the double lamp.

four hours, each additional pair of carbons extending the period of burning for another eight hours.

The change from the one pair of carbons to the new pair is effected by purely mechanical means, and by a contrivance as ingenious as it is simple. This little apparatus will be understood by reference to Figs. 132 and 133, which also more clearly illustrate the action of the washer clutch than the preceding figure. $\mathrm{CC}$ is the hollow solenoid, or "sucking magnet," which exercises a magnetic influence upon the soft-iron plunger $P$. The rising or falling of this plunger raises or lowers the little frame $K$ (shown also in Fig. 133), by the intervention of the lever $L . \quad R^{1}$ and $R^{2}$ are 
the carbon rods which pass vertically through the casing of the lamp, and $\mathrm{W}^{1}$ and $\mathrm{W}^{2}$ are the clutch washers which surround them. One side of each of these washers passes between a pair of jaws, forming part of the little frame $\mathbf{K}$, and when the latter is drawn upwards its action is first to tilt the washers and then to cause them to clutch the rod on diagonally opposite corners. By the very simple device of making one pair of jaws a little higher than the other, it takes its grip before the other begins to act, and consequently lifts its corresponding carbon higher than its neighbour; the consequence is that only one arc is established, viz. across the lesser distance, and in all consequent feeding and controlling the pair of carbons first started are alone affected, because, although both carbons are raised and lowered together, the lower end of the reserve carbon is always higher than the other by the difference in the height of the two pairs of jaws on the frame K. A time arrives, however, when, owing to the shortening of the consuming carbons, they can no longer meet when the frame is dropped, and the current by which they are again separated can only be transmitted by the reserve carbons coming into contact, the circuit is thereby completed, the new carbons are separated, and the arc between them continues to be controlled by the magnet and clutch in the same way as the first arc was.

The Brush system is capable of establishing a. greater number of large are lights in a single circuit (as many as forty) than any other arc light system. It possesses also another very special advantage, and that is that a series of lights may be maintained at a great distance from the machine, and with a remarkably small loss; and this is of especial advantage where several lights have to be em- 
ployed, because if there are as many long conductors as there are lamps, then the whole of the loss of current due to the resistance of the cables must be borne by each lamp, whereas if a number of lights can be included in a single circuit, the loss of current from the resistance of conducting cables is equally divided among all the lamps in the series. There are, of course, commercial advantages in burning a number of lights in a single circuit from one machine, for there must necessarily be an enormous increase in prime cost in the adoption of a number of dynamoelectric machines in the place of one, as well as in the multiplication of conducting cables, and the advantage in point of attendance and repairs is obviously in favour of the single-machine system.

The Lever Lamp.-In most lamps it is usual to have an electro-magnet, or solenoid, in circuit with the carbon points, to separate them, thus forming the arc; and also a shunt electro-magnet, or solenoid, to regulate the arc in each lamp, when several lamps are worked on a single circuit. This is true of the Brush, Weston, and other well-known systems. One of the advantages of the Lever lamp is that it dispenses altogether with one of these magnets, the one technically called the "main," and is regulated solely by the shunt magnet, or solenoid.

The latest form of this lamp is represented in Fig. 134. $A$ is the armature, $L$ the lever, $D$ the spring, $E$ is a nut for adjusting the tension of the spring $D$, and $I$ is a projection attached to the lever $\mathrm{L}$, which tilts the metal collar B by means of spring D. G is the set-screw for adjusting the length of the arc. The iron screw $\mathbf{J}$ with a large head, passing through the armature $A$, serves as an adjustable pole-piece, and can be secured by means of a lock-nut $J^{\prime} . \quad R$ is the dash- 
pot, and $S^{\prime} S^{\prime}$ are the binding screws from which the electromagnet F F is shunted. The current, entering at the positive binding screw $\mathrm{S}$, passes through the insulated flexible
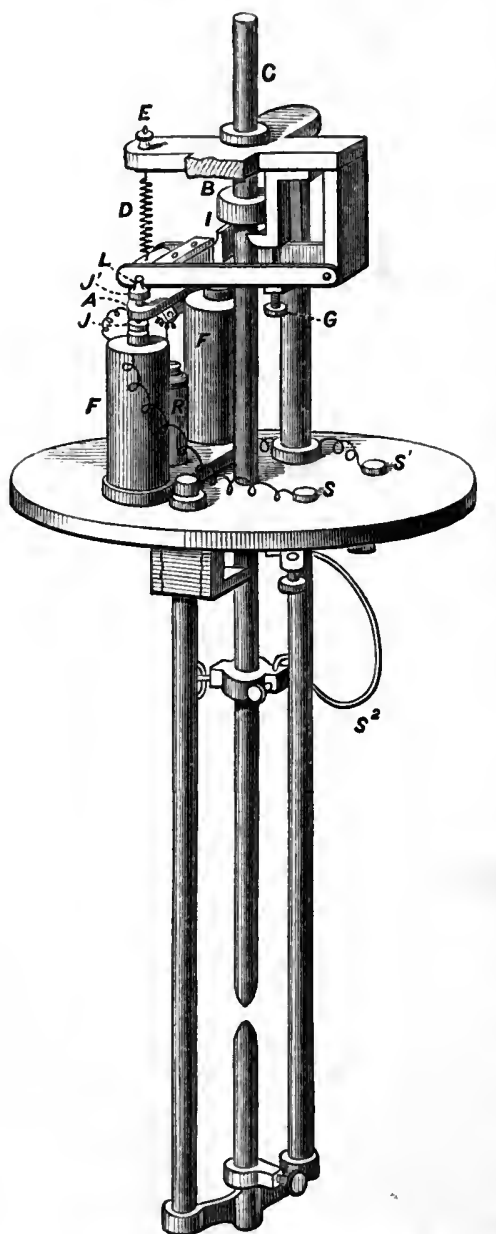

Fig. 134.-The Lever lamp.

wire or cable $\mathbb{S}^{2}$ to the uppercarbon-holder C; from thence, through the arc and lower carbon and through the frame of the lamp to the negative binding screw $\mathrm{S}^{\prime}$. The side rods are insulated from each other, and the ends of the coils of the electro-magnet $\mathrm{F} \mathrm{F}$ are connected to the binding screws $\mathrm{S} \mathrm{S}^{\prime}$, thus placing the electro-magnet $\mathrm{F}$ in derivation from the arc.

The action of the lamp is as follows:-The carbons in the lamp being separated, the only path for the electric current is through the coils of fine wire on the shunt electromagnet, and as the entire current will flow through these coils, the magnet will become very powerful and will attract the iron bar. When this takes place, the carbons come into contact with cach other; for the lever will allow the metal collar to loosen the carbon-holder, which slides through its bearings by 
gravity. The contact of the carbons, however, completes what is technically called the main circuit, i.e. it allows the current to pass through them, the proportion of the current in the two circuits-the main and the shunt-being inversely as their resistance. But the coils on the shunt electromagnet being wound with very fine wire, the resistance of the shunt circuit is much greater than the resistance of the main circuit through the carbons, so that nearly all the current goes through the carbons, and very little through the shunt circuit. This causes the electro-magnet to lose nearly all its magnetism, whereupon the spring again pulls up the lever, and with it the carbon rod, thus forming the arc. When the carbons burn away, the distance between them becomes greater, or in other words, the resistance in the main circuit is increased. This causes more current to pass through the shunt electro-magnet, which, becoming stronger, again attracts the armature and lowers the carbon rod. In practice it is found that there is scarcely any perceptible motion of the lever when the are is burning, for the shunt electro-magnet and the spring, acting on the lever in opposite directions to each other, make a perfect balance, or in other words, establish an equilibrium resulting in a steady and regular light: this is one of the advantages of the Lever lamp.

There is yet another important feature in connection with the working of the Lever lamp. The cheapest and simplest form of continuous-current machine can be used, and, in order that the machine may excite itself, although the resistance in the main circuit is too great for it to do so through that resistance when the carbons are separated, a simple electro-magnetic switch at the machine permits of its doing this in the following manner:-Connected to the terminals, or binding screws, of the dynamo are the ends voL. $\mathbf{I}$. 
of two coils of wire, which are wound over the limbs of a horseshoe electro-magnet to a metal stud fixed on a board, which, by means of a spring contact-piece moving through an armature capable of rotating a short distance opposite to the poles of the electro-magnet on a pivot, allows the dymano to excite itself through the coils on the electromagnet. Now, when the dynamo machine has excited itself and is ready to supply the current, the electro-magnet, becoming magnetized, attracts the armature, causing the contact-pieces to leave the metal stud, and thus to break the circuit formed by the coils. Immediately this takes place, the current goes through the shunt electro-magnet on the lamps, that is, when there are a number in series or single circuit, and causes them to form their arcs as already explained.

With a Biirgin dynamo which, when running at full speed, gives an electro-motive force of 190 volts, Mr. Lever works five lamps of 2000 candle-power each. There is no simpler arc lamp actually in use, and, it may be added, no more economical one, since it does away with the useless resistance of the main magnet, or solenoid. A greater number of Lever lamps can be worked in single circuit by the same current than in any of the ordinary type.

Gérard's Lamp.-The regulator is placed above the curbons, and is worked by alternating currents. It consists of a hollow electro-magnet (Fig. 135), allowing a free passage for the upper carbon. This electro-magnet, with a fine wire, is mounted in a shunt on the terminals of the lamp. An articulated brake, with a screw, keeps the upper carbon in position; the pressure of the brake against the carbon being regulated by a spring. The upper part of the brake carries an armature, and, when this armature is 
attracted, the brake frees the carbon and allows it to descend.

The lower carbon is supported by a movable vertical frame carrying an armature at its upper end, and this armature is placed at the lower extremity of the electromagnet. The movable frame is balanced by two spiral springs, which are seen on the right and left of the electro-magnet. When at rest the carbons are not in contact.

The apparatus is worked in the following way:-When the current passes into the lamp, the carbons are apart from one another; the current can therefore only pass through the fine wire. The electromagnet, consequently, becomes very powerful, and attracts both the upper and lower armatures.

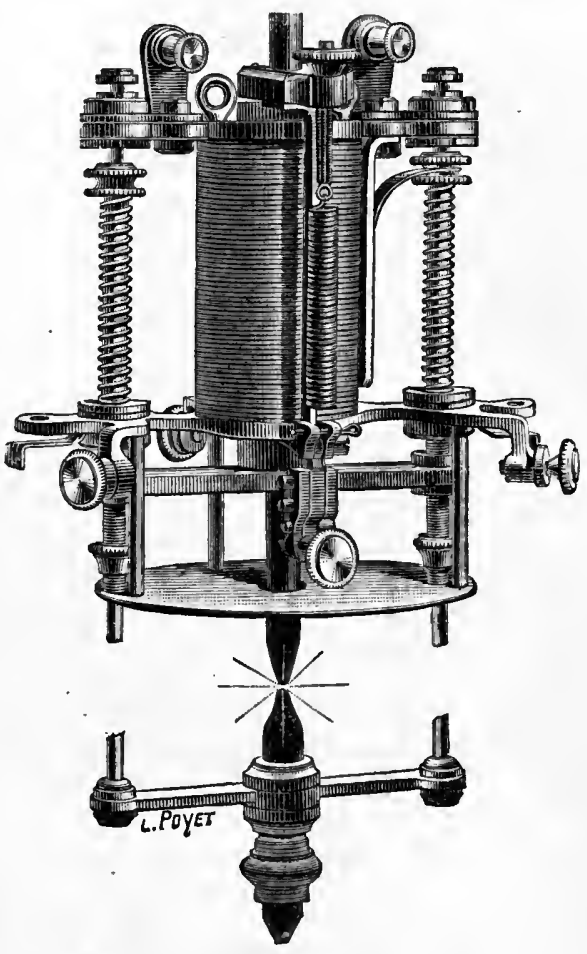

Fig. 135.-Anatole Gérard's lamp.

The upper armature, acting on the brake, frees the upper carbon and allows it to descend. The lower armature raises the frame and the lower carbon. As soon as the two carbons are in contact, the current passes into the main circuit, and that which passes into the shunt is sensibly weakened. 
The lower armature is released, the lower carbon descends, and the arc is formed. On the other hand, the armature of the brake is partly released, and the screw presses against the upper carbon and prevents it from descending.

If the spring is properly adjusted, balance is established; but in proportion as the carbons are wasted, the arc will be lengthened, and the intensity of the current in the electro-magnet will increase. The brake is now gradually loosened, the alternating currents will produce an oscillation of the armature, and the upper carbon slides down imperceptibly and continuously to maintain the requisite distance.

Gérard's Automatic Controller.-This apparatus instantly and automatically re-establishes the general circuit, and prevents the extinction of all the other lamps of the same circuit, when, from some reason or other, either accidentally or purposely, a lamp ceases to act. It consists of a small wooden block, to which an electro-magnet with fine wire is fixed; this electro-magnet is connected to the upper terminals, which themselves are connected to the lamp; it is therefore mounted in a shunt. The two lower terminals (Fig. 136) communicate with two beakers half full of mercury; the left-hand terminal is connected to the arrival wire, and the right-hand terminal to the return wire of the current.

Underneath the straight electro-magnet is an armature carrying a small hook, to which, by means of a second hook, a cross-bar is suspended; this latter supports two iron rods placed immediately above the beakers with mercury. When the current passes into the lamp, the electro-magnet, which is mounted in the shunt, does not receive sufficient current power to attract its armature. When the current-as, for instance, in the case of an accident-no longer traverses the 
lamp, the electro-magnet becomes powerful enough to attract its armature, frees the hooks, and causes the iron rods to fall into the beakers with mercury; a direct communication is established, and the lamp is short-circuited without interfering with the general circuit. $^{1}$

This apparatus, as simple as it is ingenious, works in the most perfect manner. The two beakers can be placed one above the other; the principle remains the same, but less room is taken up, and the apparatus can be placed on the narrow pillars of workshops and factories, for which Gérard's lamp and this automatic controller seem to be specially

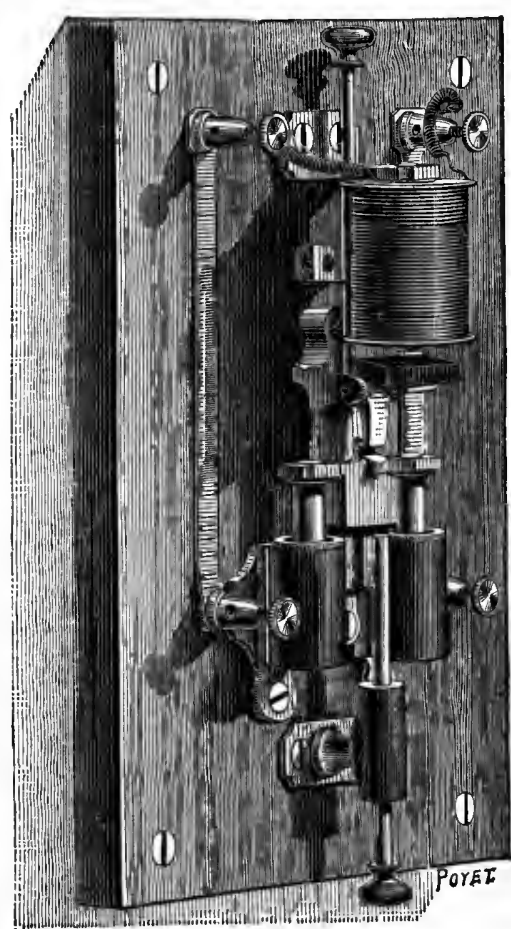

Fig. 136.-Anatole Gérard's automatio coutroller. adapted.

Abdank's Lamp.-This lamp has a separate regulator, which is placed at some distance from the lamp. The bottom, or negative, carbon of the lamp itself (Figs. 137, 138, 139)

I Strictly speaking, the lamp is not altogether withdrawn from the circuit, but in consequence of a direct communication being established between the two lower terminals by the beakers containing the mercury, the lamp only receives a current which is practically null. 
is fixed, but the top, or positive, carbon is movable in a vertical line. It is screwed at the point $\mathrm{C}$ to a brass $\operatorname{rod} \mathrm{T}$ (Fig. 138), which moves freely inside the tubular iron core of an electro-magnet $\mathrm{K}$. This rod is clutched and lifted by the soft-iron armature $A B$ when a current passes through
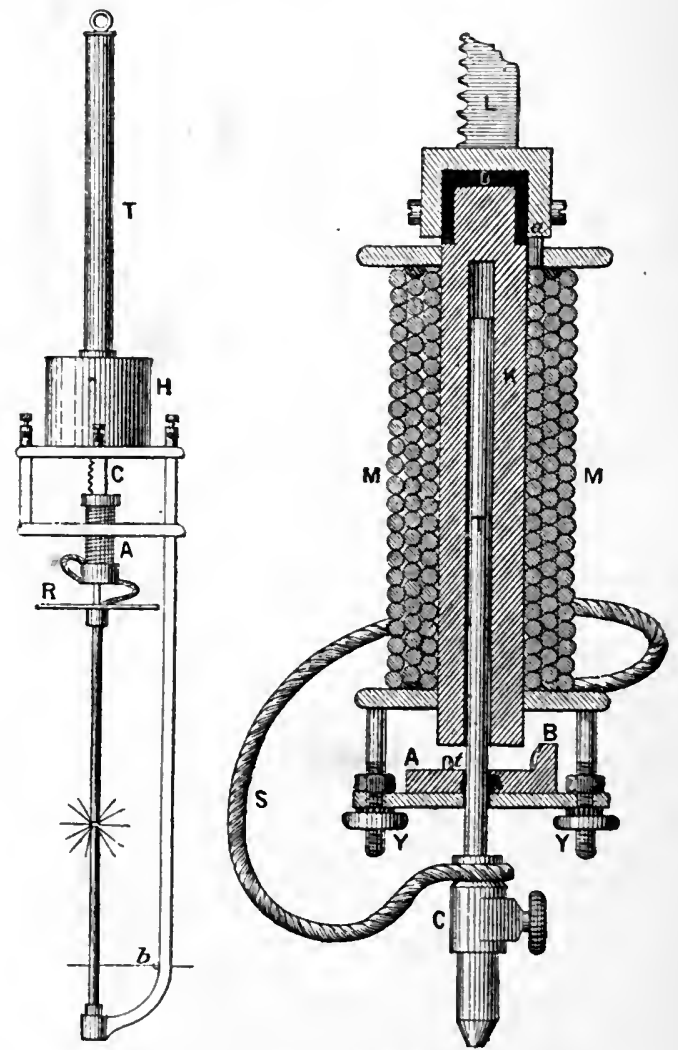

Figs. 137, 138.-Abdank's lamp.

(From La Lumiere Électrique.)

the coil M M. The mass of the iron in the armature is distributed so that the greater portion is at one end B, much nearer the pole than the other end. Hence this portion is attracted first, the armature assumes an inclined position, 
maintained by a brass button $t$, which prevents any adhesion between the armature and the core of the electro-magnet. The electric connection between the carbon and the coil of the electro-magnet is maintained by the flexible wire $\mathrm{S}$.

The electro-magnet A (Fig. 137) is fixed to a long and heavy rack $\mathrm{C}$, which falls by its own weight and by the weight of the electro-magnet and the carbon fixed to it. The length of the rack is equal to the length of the two carbons. The fall of the rack is controlled by a friction brake B (Fig. 139), which acts upon the last of a train of three wheels put in motion by the above weight. The

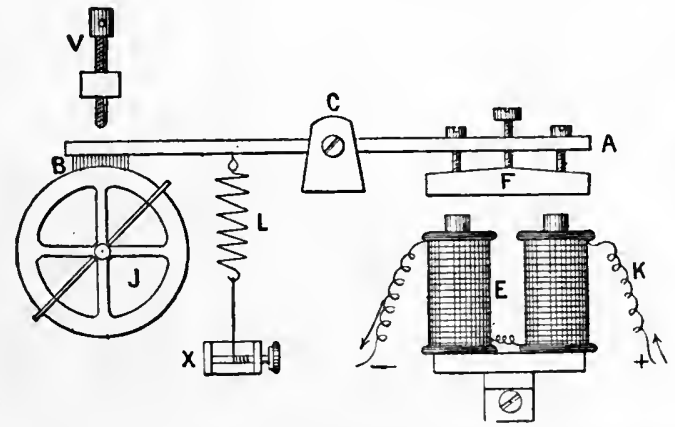

Fig. 139.-Abdank's lamp : clockwork movement enclosed in box H (Fig. 137).

(From La Lumière Électrique.)

brake $\mathrm{B}$ is fixed at one end of a lever B A, the other end carrying a soft-iron armature $F^{*}$, easily adjusted by three screws. This armature is attracted by the electro-magnet $\mathrm{E}$ (whose resistance is $1200 \mathrm{ohms}$ ) whenever a current circulates through it. The length of the play is regulated by the screw $\mathrm{V}$. The spring $\mathrm{L}$ applies tension to the brake.

The regulator (Fig. 140) consists of a balance and cutoff. The balance is made with two solenoids $S$ and $S^{\prime}$, whose relative resistances are adjustable; $\mathrm{S}$ conveys the main cur- 
rent, and is wound with thick wire having practically no

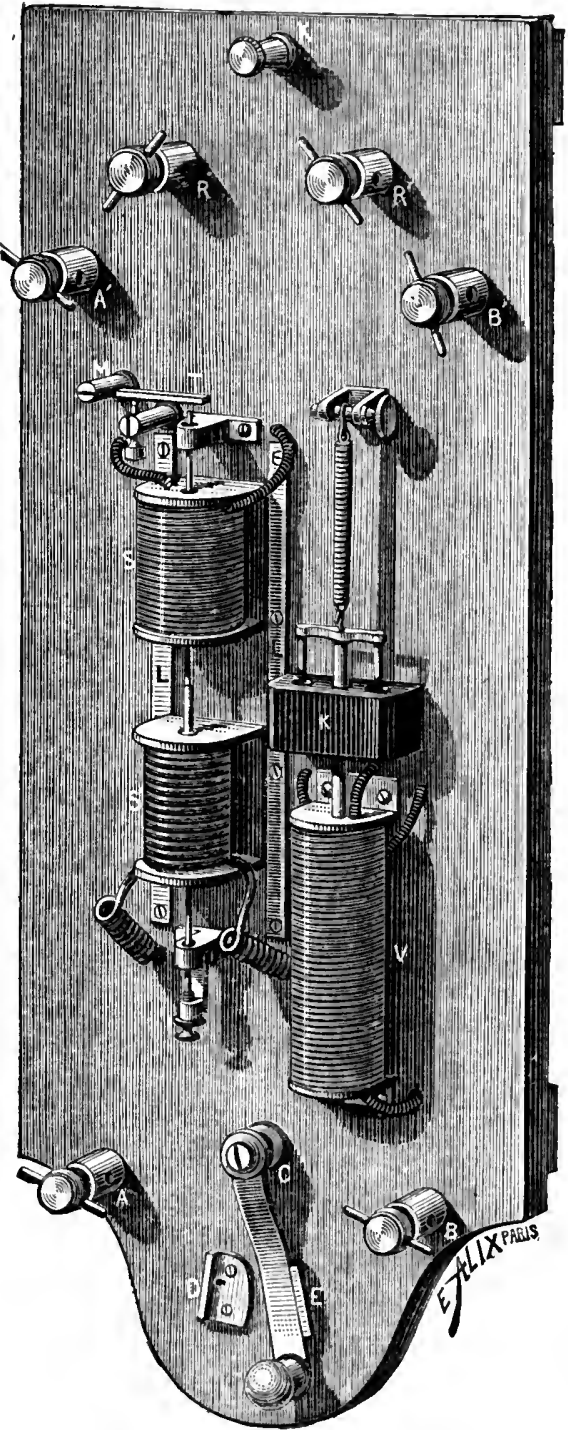

Fig. 140.-Abdank's lamp : regulator (perspective).

(From la Iumière Électrique.) 1esistance, and $\mathrm{S}^{\prime}$ is traversed by a shunt current, and is wound with fine wire having a resistance of 600 ohms. In the axis of these two coils a small and light iron tube (two millimetres in diameter and sixty millimetres in length) freely moves in a vertical line between two guides. When magnetized it has one pole in the middle and the opposite at each end. The upward motion is controlled by the spring $\mathrm{N}$ T (Fig. 141). The spring rests upon the screw $\mathrm{H}$, with which it makes contact by platinum 'electrodes. This contact is broken whenever the little iron rod strikes the spring $\mathrm{N}$ T.

The positive lead 
from the dynamo is attached to the terminal $\mathrm{B}$, then passes through the coil $\mathrm{S}$ to the terminal $\mathrm{B}^{\prime}$, whence it proceeds to the lamp. The negative lead is attached to terminal A, passing directly to the other terminal $\mathrm{A}^{\prime}$, and thence to the lamp.

The shunt, which passes through the fine coil $\mathrm{S}^{\prime}$, commences at the point $\mathrm{P}$ (Fig. 141). The other end is fixed to the screw $\mathrm{H}$, whence it has two paths-the one offering no resistance through the spring $\mathrm{T} N$, to the upper negative terminal $\mathrm{A}^{\prime}$; the other, through the terminal $J$ to the electro-magnet of the break $M$, and thence to the negative terminal of the lamp $L^{\prime}$.

The last part of the apparatus is the cut-off (Fig. 141), which is used when there are several lamps in series. It is brought into play by the switch $C D$, which can be placed at $\mathrm{E}$ or $\mathrm{D}$. When it is at E, the negative terminal $\mathrm{A}$ is in com-

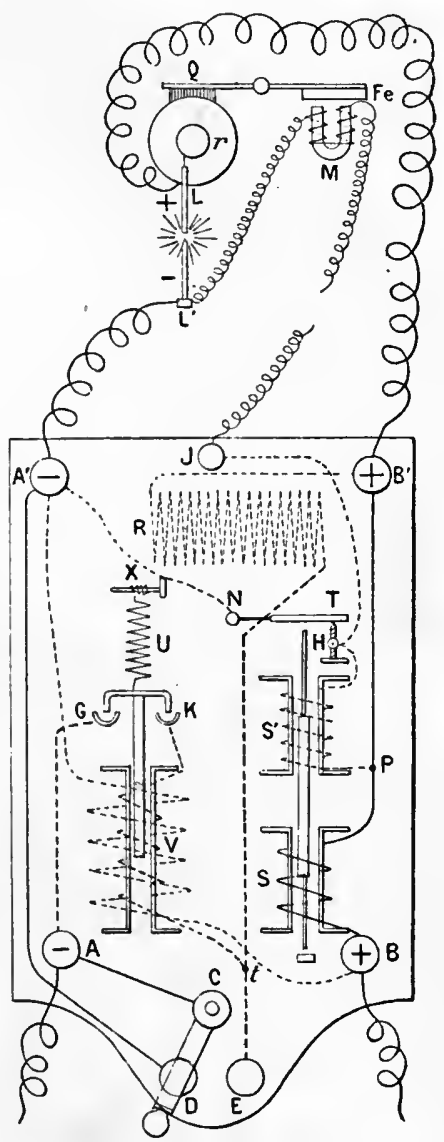

Fig. 141.-Abdank's lamp : regulator and cut-off (section). (From La Lumiere Électrique.)

munication with the positive terminal $B$, through the resistance $R$, which equals the resistance of the lamp which is therefore out of circuit. When it is at $\mathrm{D}$, the cut-off acts automatically, to do the same thing when required. This is 
done by a solenoid V, which has two coils, the one of thick wire offering no resistance, and the other. of 2000 ohms resistance. The fine wire connects the terminals A and B. The solenoid has a movable soft-iron core suspended by the spring $U$. It has a cross-piece of iron which can dip into two mercury cups $\mathrm{G}$ and $\mathrm{K}$, when the core is sucked into the solenoid. When this is the case, which happens when any accident occurs to the lamp, the terminal $\mathrm{A}$ is placed in connection with the terminal B through the thick

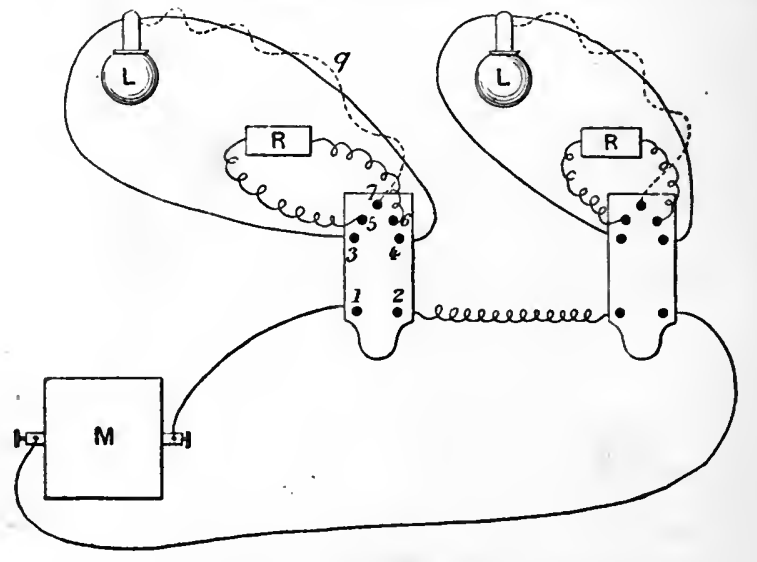

Fig. 142.-Adbank's lamp (arrangement in series). (From La Lumière Électrique.)

wire of $\mathrm{V}$ and the resistance $\mathrm{R}$, in the same way as it was done by the switch $C$ D. Fig. 142 shows the arrangement of the lamps in series.

Arc Lamps with Oblique Carbons.-They were first constructed in 1846, by William Edwards Staite. The two carbons were inserted in two directing sheaths, and descended in proportion to their waste by means of springs, and finally came to abut against a refractory non-conducting substance. The length of the voltaic arc is altered by displacing one of the carbons. 
Brockie's Lamp.- This lamp is also a division regulator, in. which the distance of the carbons is readjusted every minute or every half-minute, by automatically interrupting the circuit during a small fraction of a second. At the moment of interruption, the electro-magnet which maintained the carbons in position becomes inert, the upper carbon falls back into contact, and when a new current passes, the electro-magnet removes the carbons to their normal distance, and maintains them in that position until the next readjustment takes place. The eclipse is of so short a duration that it is almost imperceptible, and the waste of the carbons between two successive readjustments is so slight, that it does not sensibly affect the resistance of the circuit or the intensity of the passing current.

In the latest model, the readjusting electro-magnet is not placed in the circuit itself, but supplied by an auxiliary shunted circuit, and this circuit alone is affected by the interruptions of the current.

Rapieff's Lamp.-This lamp, invented in 1878, consists of four carbons, two for each pole.

The upper pole consists of two carbons of a diameter. of six millimetres, sliding between rollers, which direct their motion and establish a perfect electrical contact. These two carbons form a sort of $\mathrm{V}$, the length of which diminishes with the combustion, whilst its meeting point is fixed. A weight, attached to a string, as will be seen from Fig. 143, causes the carbons to descend; the meeting of the points causes the carbons to stop, and fixes the luminous point.

The lower pole forms a reversed V, lying in a plane perpendicular to that of the carbons of the upper pole. The same arrangement of rollers and strings with weights, raises the carbons, which, by their meeting, afford a second fixed 


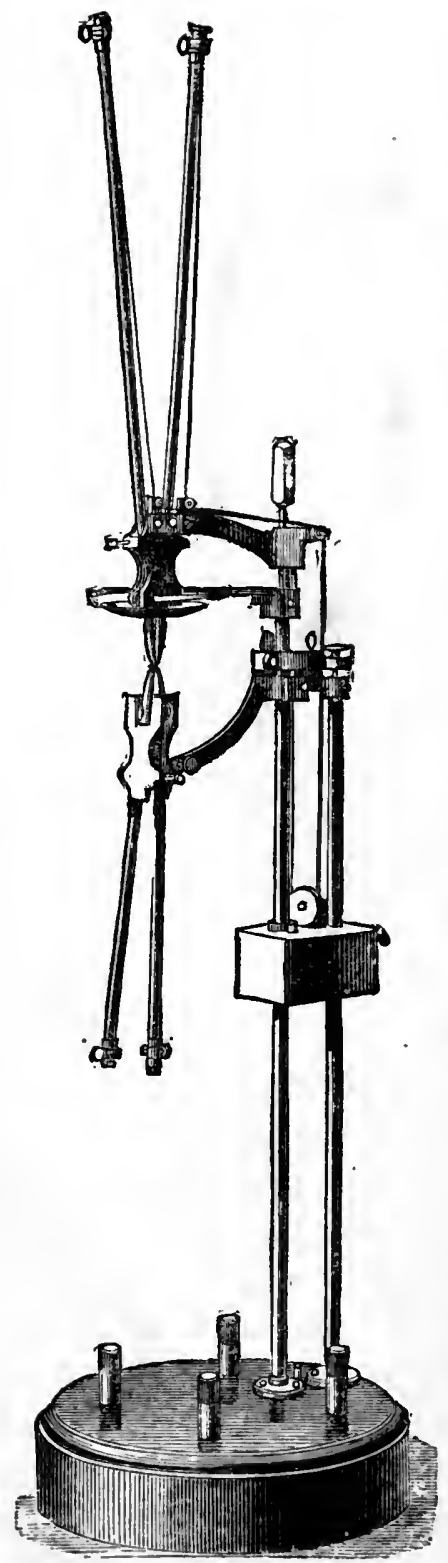

point as regards their support. This support, which is articulated at the point $\mathrm{O}$, is destined to maintain the carbons at a proper distance at the moment when the current passes. To obtain this result, a flat electro-magnet with two limbs is contained in the socket; one of these limbs is fixed, and the other articulated at one of its extremities. At the moment when the current passes, the opposite poles attract one another, and the displacement of the lower movable limb of the electro-magnet acts on a rod contained in one of the vertical supports of the apparatus, and causes a contraction of the support of the lower carbons; a withdrawal of the carbons which had originally been in contact, ensues, and consequently the formation of a voltaic arc, whose length is regulated by counteracting the effect of the electro-magnet by a spiral spring; a regulating screw keeps this spring Fig. 143.-Rapieff's electric lamp. properly adjusted. By this 
simple arrangement, an electric lamp is obtained in which the luminous point remains constant, and the carbons are of unlimited length; the resistance, moreover, which these carbons introduce into the circuit is very feeble, and independent of their length. In another arrangement, Rapieff replaces the two carbons of the upper pole by a single carbon of larger size. In this lamp with three carbons, the part of the electro-magnet is reversed: it only tends to bring the carbons into closer proximity for the purpose of lighting them. When the lamp is lit, this electro-

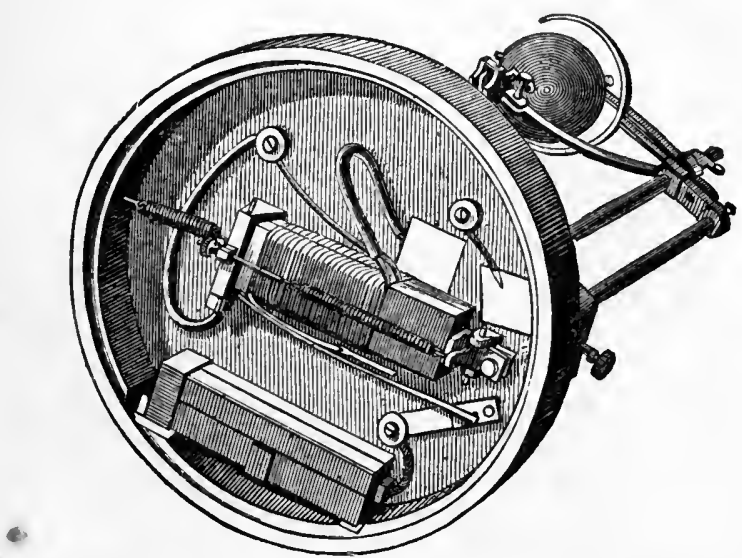

Fig. 144, - Rapieff's lamp (socket of the arparatus).

magnet ceases to act; the arc has then a constant length, which is regulated in advance, and only depends on the number of apparatus introduced into the circuit.

Each lamp is supplied with an ingenious contrivance to which Rapieff has given the name of safety apparatus, and which re-establishes the circuit directly between the borders of the lamp, if from some reason or other-for instance, by the accidental breaking of a carbon-the lamp is rendered useless. The Rapieff system is used in London 
for the lighting of the Times printing-office. A Gramme's machine with alternating currents (type of sixteen candles), of whose four circuits two only are utilized, supplies the current for two series of three lamps each. These lamps light up most brilliantly a surface of about 400 square metres, in spite of their opaque glass globes.

Rapieff's lamps are always used with alternating currents; they allow, to a certain degree, a division of light, since four of them can be placed in tension on a single circuit.

Killingworth Hedges' Lamp.-This lamp belongs to the Rapieff type, and is illustrated in Figs. 145 and 146.

$A$ and $B$ are two troughs, rectangular in cross section, and inclined to one another so as to form the letter $\mathrm{V}$. Within these troughs slide two carbon pencils of circular cross section, meeting, when no current is passing, at the lower point $\mathrm{E}$. The carbon-holder $\mathrm{B}$, to the right of the illustration, is rigidly attached to the framing of the lamp, but the trough $A$, which carries the negative carbon, is attached to the framing by a pivot shown in the figure, and on this pivot the carbon-holder can rock, its motion being controlled by the position of the armature of an electro-magnet above it, the coils of which are included in the circuit of the apparatus. By this means, the moment the current is established through the lamp the armature is attracted and the points of the two carbons are separated, thus forming the arc. The positive carbon $\mathrm{C}$ is held from sliding by the gentle pressure against it of the smaller carbon rod $C^{\prime}$, which also lies in a trough or tube fixed in such a position that the point of contact between the two rods is sufficiently near the arc for the smaller rod to be slowly consumed as the other is burned away; the latter in this way is permitted to slide gradually down the trough as long as the 
lamp is in action. The negative carbon-holder $\mathrm{A}$ is provided with a little adjustable platinum stop $\mathrm{E}$, which, by pressing against the side of the conical end of the negative carbon, holds the latter in its place, and prevents it sliding down the trough except under the influence of the slow combustion of the cone resulting from the wasting action of the arc. The position of the stop with respect to the conical end is determined by a small adjusting screw.

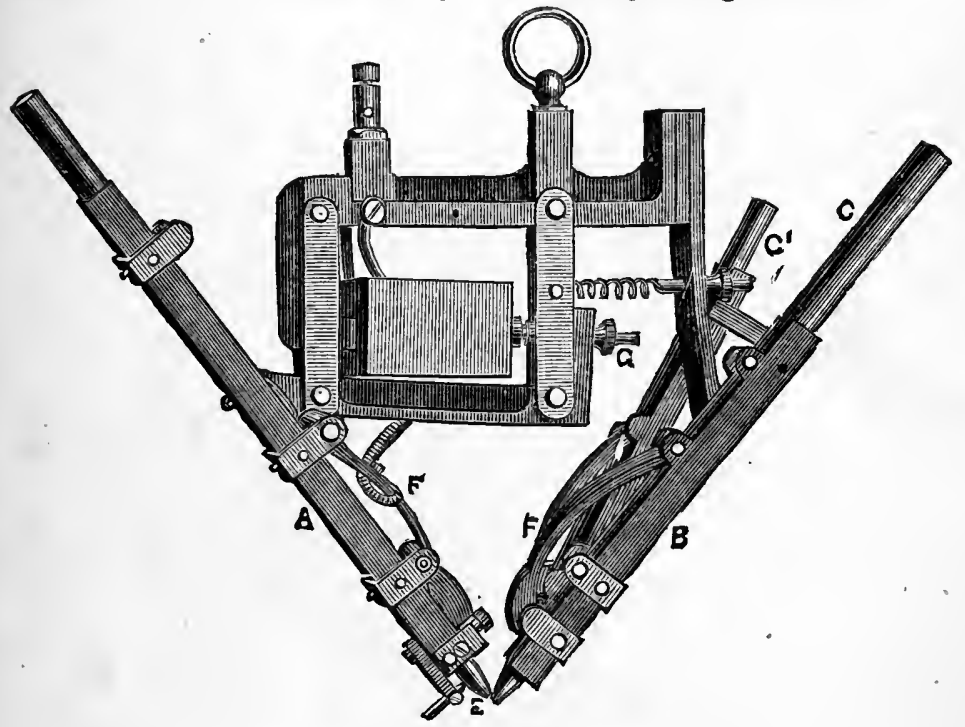

Fig. 145.-Killingworth Hedges' lamp.

(From La Lumière Électrique.)

In order to maintain good electrical contact between the fixed conducting portions of the lamp and the sliding carbons, Hedges fits to each carbon-holder a little contactpiece $\mathrm{F} \mathrm{F}$, hinged to its respective trough at its upper end, and carrying at its lower or free end a relatively heavy little block of brass grooved out to fit the cylindrical side of the carbon, against which it bears with an even pressure. By this arrangement the length of that portion of the 
carbon rods which is traversed by the current is always the same, notwithstanding the shortening of their total length by combustion; the resistance of the carbon electrodes is therefore maintained constant, and for the reason that the contact-pieces press against the rods very near their

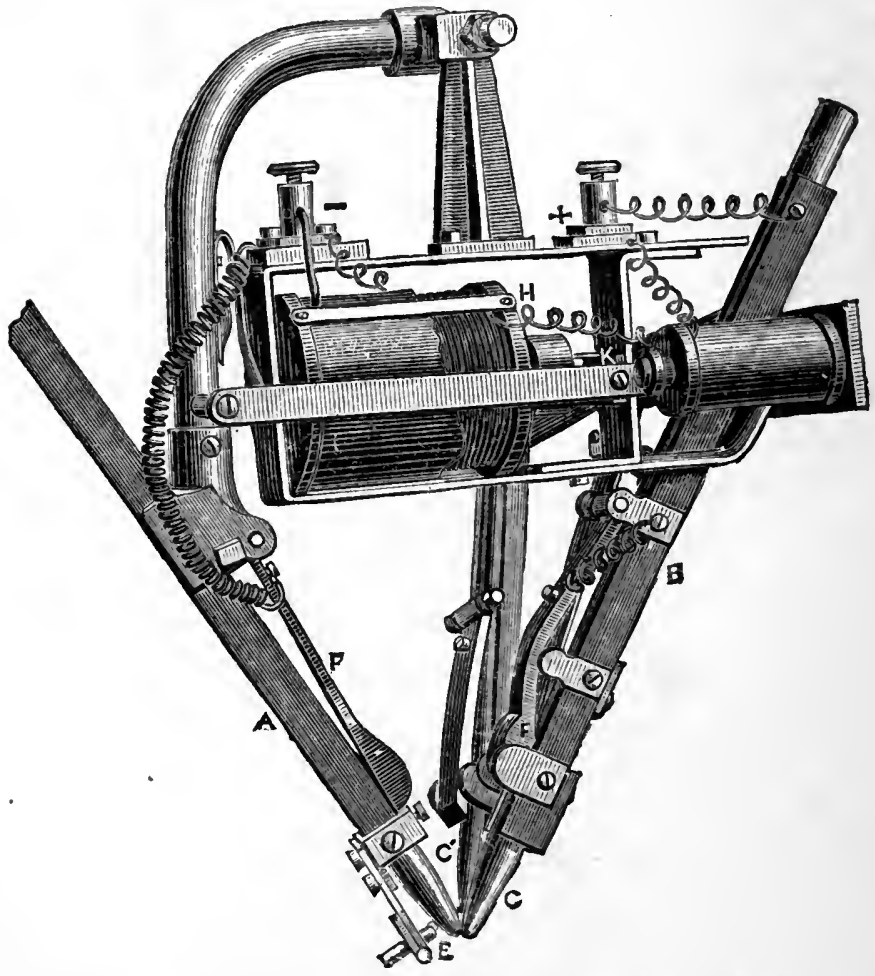

Fig. 146.-Killingworth Hedges' lamp (for working in series).

(From La Lumière Électrique.)

lower ends, that resistance is reduced to a minimum. The length of the arc can be determined by the adjustment of the screw G, by which the amount of movement of the armature is limited.

Fig. 146 represents a modified form of Hedges' lamp, designed for installations where it is desirable to burn a 
number of lamps in series. In this arrangement the carbons are separated by the attractive influence of a solenoid upon an iron plunger, to which is attached (by a non-magnetic connection) the armature of an electro-nagnet. The coils of the magnet, which are of fine wire, form a shunt circuit between the two terminals of the lamp, and so disposed with respect to the armature as to influence it in an opposite direction to the solenoid. When the circuit of the lamp is completed with the generator, the carbons are drawn apart by the action of the solenoid on the plunger, and the distance to which they are separated is determined by the difference of attractive forces exercised upon the armature by the solenoid and the magnet; but as the latter forms a short circuit to that of the arc, it follows that, should the resistance of the arc circuit increase, either through the arc becoming too long or through imperfections in the carbons or contacts, a greater per centage of current will flow through the magnet coils, and the arc will be shortened, thereby reducing its resistance and regulating it to the strength of the current.

Mr. Hedges' installation comprises a commutator, represented in elevation and section in Fig. 147, and intended to prevent sparking. The characteristic part of the apparatus is the circular shape of the contact-piece and also the fact that the space between the two fixed contacts is filled with compressed asbestos. The circular contact can be turned so as to present a fresh surface when any trace of oxidation manifests itself, and a spiral spring, represented at A ensures complete contact. The communication between the arc and the circular piece is effected by a spring $\mathrm{F}$.

The system is completed by a portable apparatus for the feeding of the lamps (Fig. 148). 
The dynamo-electric machines must revolve at a speed of from 900 to 1600 revolutions per minute, and
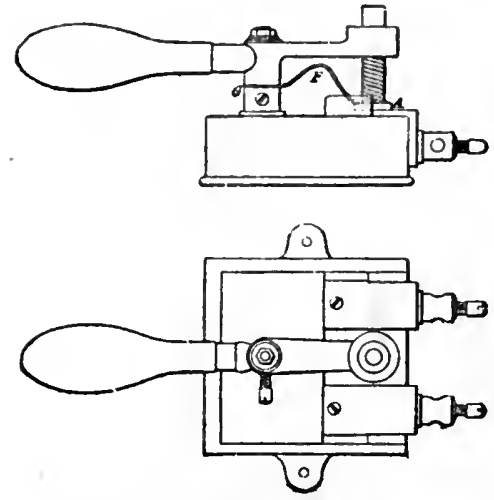

Fig. 147.-Killingworth Hedges' commutator.

(From La Lumière Électrique.) at this great speed it is almost impossible directly to connect the electric machines with an ordinary steam-engine. Multiplying wheels, on the other hand, are not very practical on account of the noise they produce, and ordinary transmissions in many cases take up too much room. Mr. Hedges' transmission is shown in Fig. 148. Tt consists of an ordinary friction pinion driven by a strong pulley or fly-wheel fastened to the shaft of the motor, and at the same time by a belt

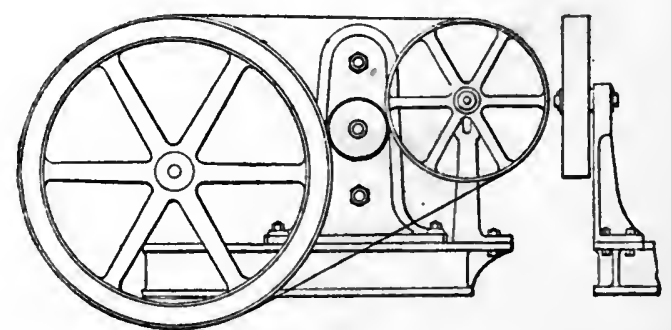

Fig. 148.-Killingworth Hedges' portable feeding apparatus. (From La Lumière Électrique.)

which passes over the fly-wheel and another pulley fastened to a special shaft. This latter pulley is pressed against the intermediary wheel by the belt. By lowering it the belt can be stretched.

The Pilsen Lamp.-This lamp, invented by Piette and 
Krizik, deserves attention especially because in it the two magnetic attractions arising from the same source regulate the light without any further mechanism, and without application of any other forces than those due to the current itself.

An extreme simplicity of construction has been obtained by this method, and the mechanical resistance and other deleterious obstacles have been reduced to a minimum. This result has been obtained in the following manner :-

The current, before entering the lamp, is divided into two parts, each of which traverses one of two superposed solenoids, in which an iron rod of peculiar shape slides up and down. The principal current traverses the first solenoid constructed of coarse wire, whilst the shunted current traverses a solenoid composed of a large number of very fine wire spirals. The relation between the resistance in the solenoid and the number of spirals has been calculated in such a manner that, as soon as the arc attains a fixed and determined resistance, the iron rod remains in equilibrium under the influence of the two solenoids. When the resistance increases, the equilibrium is broken and an adjustment takes place. The carbon could therefore be fixed directly to the rod, if the attraction of the solenoids for each position of the rod remained the same. But this is not the case. When an iron rod penetrates into a solenoid traversed by a current, the attraction is maximum when the end of the rod is at the same level as the medium line, of the solenoid. When the rod advances beyond that line, the attraction gradually decreases, and is equal to zero when the middle of the rod coincides with the middle of the solenoid.

This is the reason why a lamp constructed on the above 
principle would not act well, for the adjustment could only be effected for very short distances.

Piette and Krizik have overcome this obstacle by giving to the rod a peculiar elongated form represented in Fig. 149 A, B, C, D.

-Under these conditions, the attractive power of the solenoid remains the same almost for one-half of the rod,

A

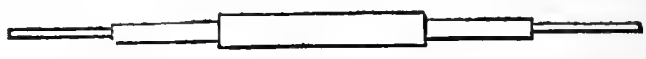

B

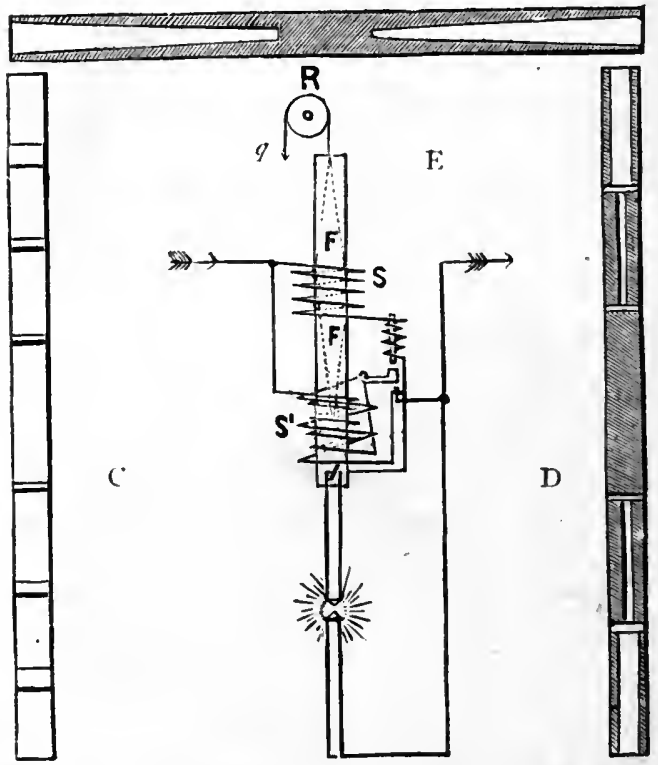

Fig. 149.-A, B, C, D, iron rods of Piette and Krizik's lamp; E, section of Piette and Krizik's differential lamp.

for different diameters of this rod correspond to differences in the attractive power of the solenoid.

A regular motion of the rod in the solenoid, for a considerable distance, can be obtained in this way, and the 
carbon can be fixed directly to the rod without the intervention of an intermediate mechanism.

Fig. 149 E shows a section of the mechanism of the lamp, and Fig. $1 \check{0} 0$ a view of the whole lamp as it was exhibited at the Paris Electrical Exhibition.

$\mathrm{F} \mathrm{F}$ is an iron rod enclosed in a cylindrical copper box sliding on two rollers. Rod F F is suspended to a pulley $R$ and balanced by a counterpoise $q$. The positive carbon, as will be seen from the figure, is fixed directly to the rod. The path of the current is as follows: - The current arrives in the solenoid $\mathrm{S}$ through the conducting wire, passes through the positive

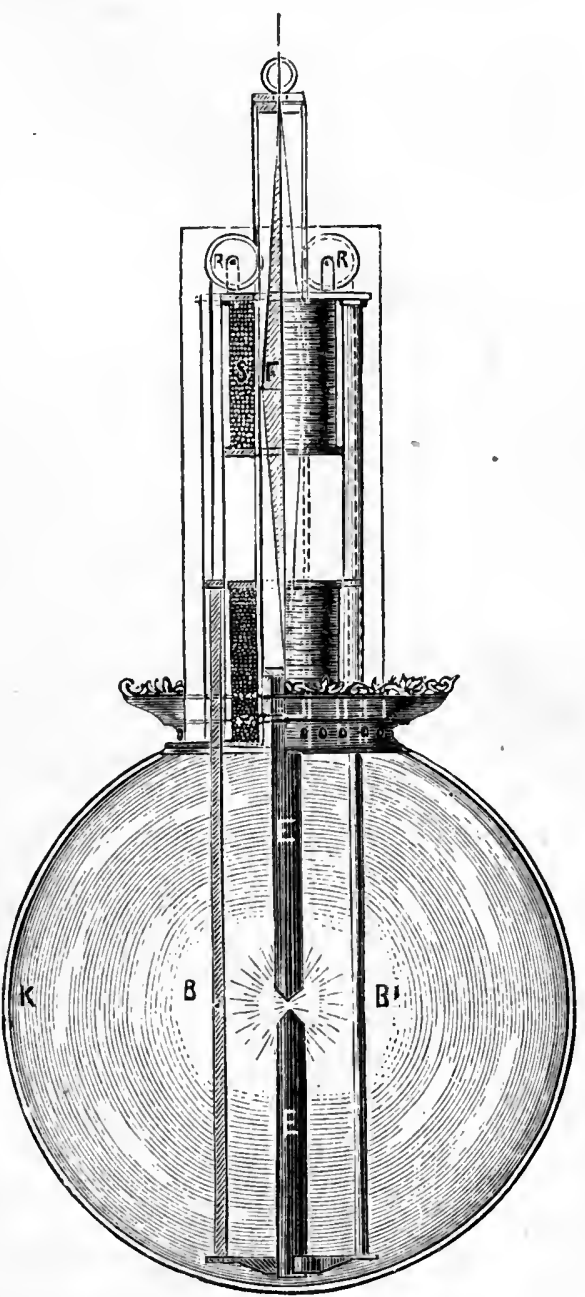

Fig. 150.-The Pilsen lamp. carbon, the voltaic arc, and the negative carbon. The shunted current traverses the lower solenoid $\mathrm{S}^{1}$, and returns to the circuit outside the lamp. 
For a given resistance of the arc, the rod is kept in equilibrium by the two solenoids. A waste of the carbons increases the resistance of the arc; the magnetic action of the solenoid in the shunted circuit increases, and the positive carbon descends with the rod.

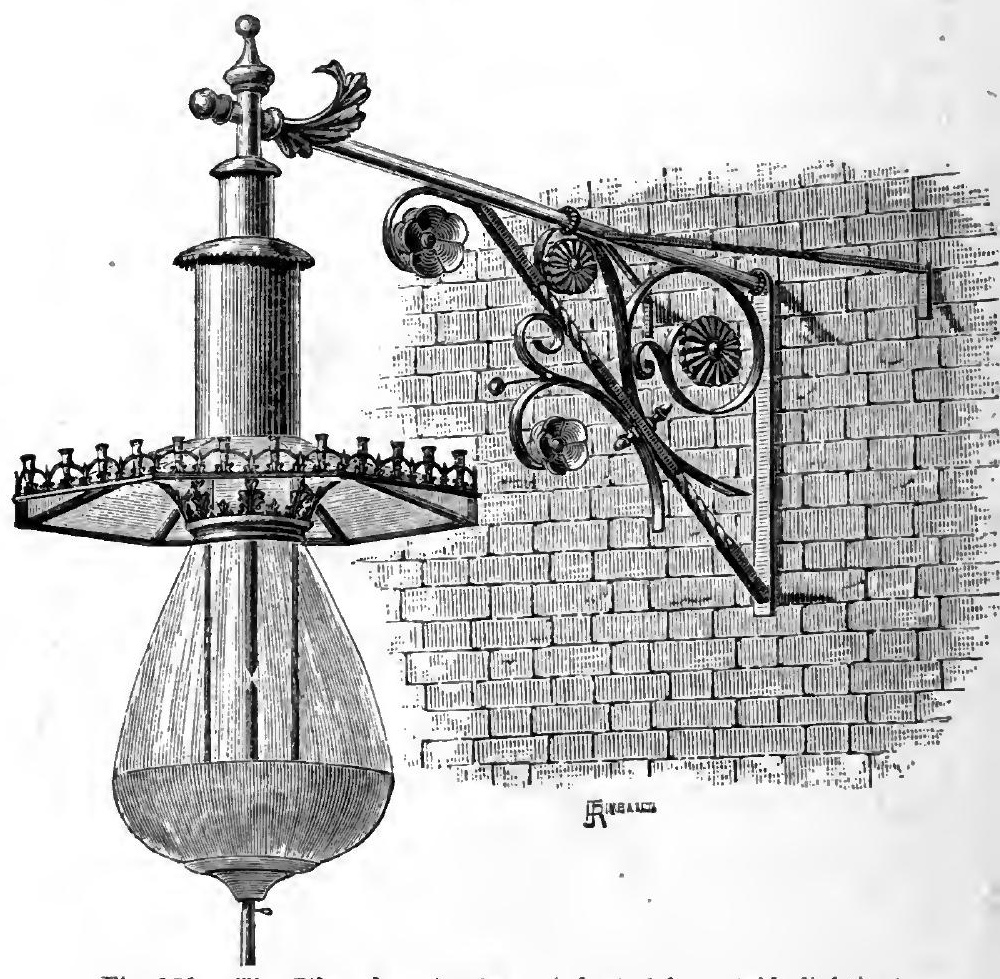

Fig. 151. - The Pilsen hanging lamp (adapted for outside lighting).

The solenoids are superposed in such a way that the distance between their centrcs is equal to half the length of the rod.

The action of the solenoid placed in the principal circuit keeps the carbons apart, that of the solenoid in the shunted circuit brings them together. When, from some cause or 
other, the current is interrupted, the carbons remain at a certain distance from one another, for they are balanced by the weight $q$. If after this a current passes, it only passes through the solenoid in the shunted circuit, and the carbons are brought into contact only to be instantly removed again to a fixed distance. But if many lamps have to be lit in the same circuit, and the carbons are not in contact, the current can only pass through the shunts, and the resistance becomes too great. In this case the lamps cannot be lit. To obviate this inconvenience, each lamp has its own automatic commutator, which, before the carbons actually touch,

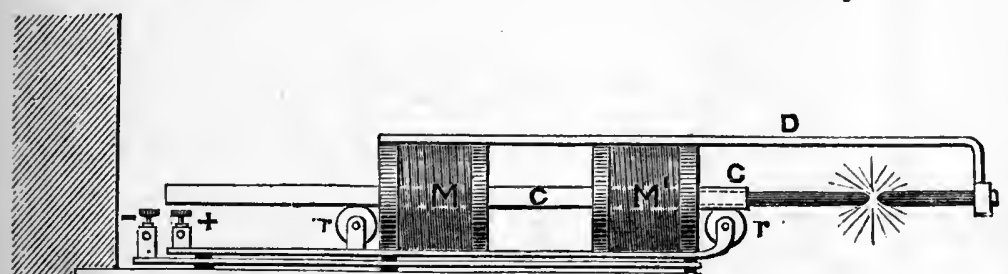

Fig. 152.-The Pilsen horizontal lamp.

sends the current into a spiral of coarse wire having a coil of fine wire at the lower end, and as soon as the carbons touch, this wire is excluded from the circuit.

The Pilsen lamp can be readily adapted to any fittings or application for lighting. Fig. 151 shows a lamp adapted for outside or street lighting.

Fig. 152 represents a horizontal lamp. The iron core $\mathrm{C}$ is fixed inside a brass tube, and is supported and free to 


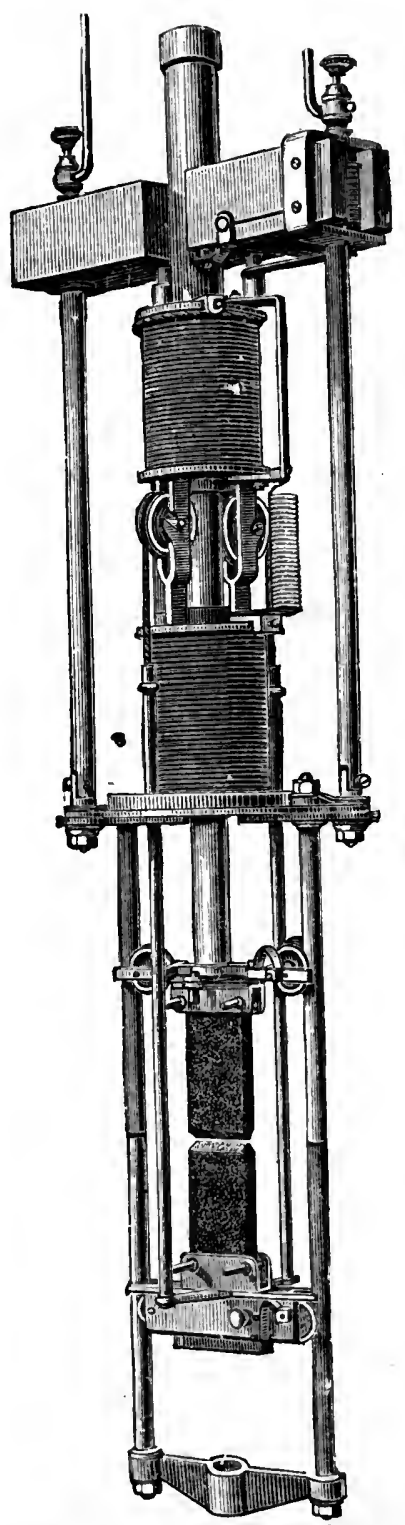

Fig. 153.-The Pilsen factory lamp (adapted to burn 100 hours consecutirely). move upon the contact rollers $r r$; one carbon is fixed to the end of the tube $\mathrm{C}$, and the other to the pivoted arm D; the coils $\mathbf{M}$ and $\mathbf{M}^{\prime}$ are practically arranged in the same way as in the previously described lamp.

The special advantage of this form of lamp is that it can be used in low-roofed factories close up to the ceiling.

Fig. 153 shows the Pilsen factory lamp. The electrical connections are the same as those in the lamp shown and described in Fig. 149. The small control electro-magnet in this lamp is fitted in the top wooden frame at the right side, and is then out of the way of harm. The mechanical construction of this lamp is much stronger than the hanging lamp; there are two sets of contact rollers-one set attached to the top and one set to the bottom carbon. The principal novelty in this lamp is the use of plate carbons. Fig. 154 shows the details of the carbon-holder. The spring clip $h$ is attached to the collar and arm $\mathrm{L}$, and the two are pivoted at $\mathrm{K}$, and are free to 
ARC LAMPS.

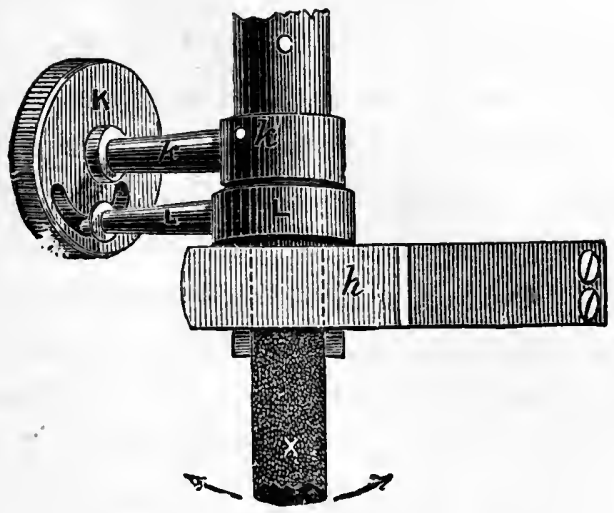

Fig. 151.-Details of carbon-holder.

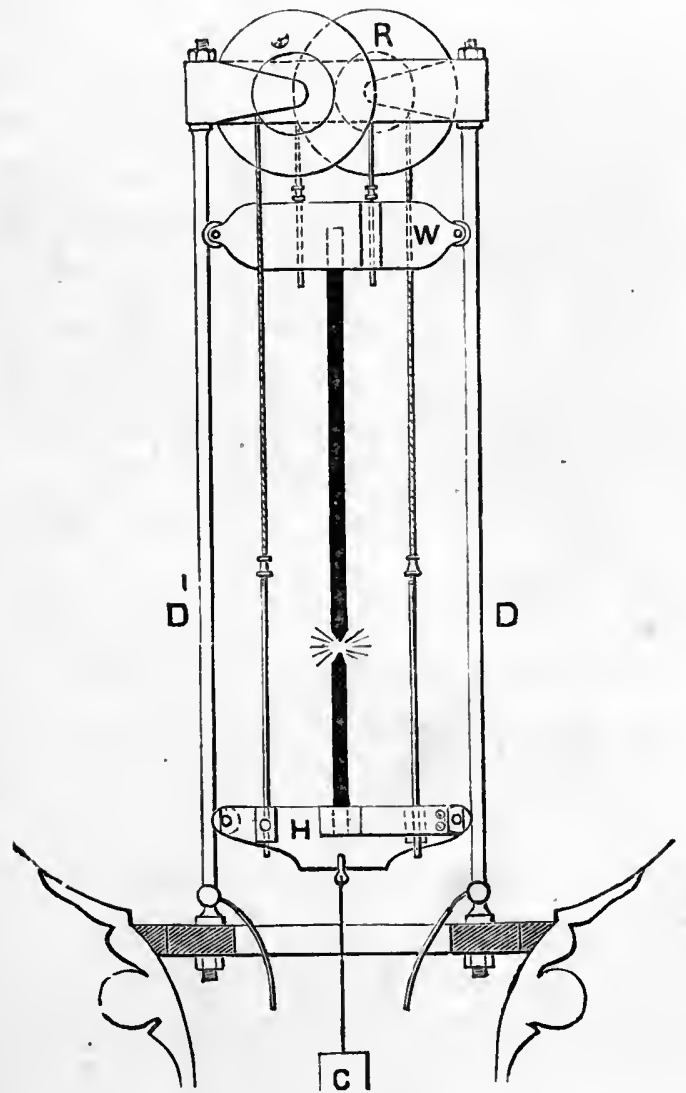

Fig. 155.-The Pilsen lamp adapted for street lamp-posts (interior view). 
move laterally. Above $\mathrm{L}$ is a fixed collar and $\operatorname{arm} k$, and on the end of that arm a milled head $K$ is free to turn. $K$ has an eccentric slot cut in its face, into which a pin projecting from the end of $\mathrm{L}$ is inserted. By turning the milled head $\mathrm{K}$, the arm $\mathrm{L}$ and carbon clip $h$ are moved backwards or forwards, as may be required. The core tube $\mathrm{C}$ can also be moved round. Fig. 155 shows the Pilsen lamp adapted for street lighting. In this lamp the counterbalance of the iron core is reversed, and the coils placed at

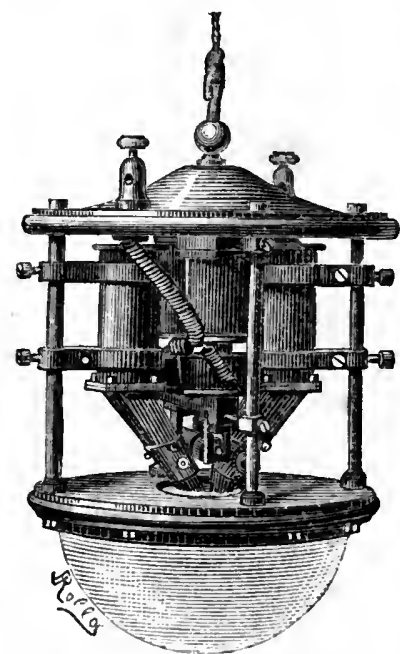

Fig. 156. - Somzée's lamp.

(From La Lumiere Électrique.)

the bottom and inside the tube of the top of the lamp; the side rods $\mathrm{D} \mathrm{D}^{\prime}$, weight $\mathrm{W}$, holder $H$, and rollers $R$ with their cords, are, except for being reversed, the same as the hanging lamp shown in Fig. 149.

This special arrangement of the lamp allows the coils to be placed in safety, and avoids shadows on the globes.

Somzée's Lamp.-In this apparatus, shown in Fig. 156, finely powdered charcoal is made to fall into the arc by means of the inclined tubes shown in the figure. The idea of thus increasing the quantity of solid particles carried to incandescence is doubtless very ingenious, but it remains to be proved that it will answer in practice.

Various Arc Lamps. - We are obliged to omit a great number of arc lamps, some of them very ingenious in principle, but which either practice has not sanctioned, or which have not had time yet to be practically tried. 
Wallace and Farmer have constructed an apparatus with flat electrodes, working a long time without renewal of carbons, but presenting the same drawbacks as the lamps with circular discs, which are now very rarely used.

Moléra and Cébrian have constructed are lamps worked by hydraulic pressure.

Others, like De Fonvielle and J. Van Malderen, utilize the reciprocal repulsion of the electrodes under the action of the passing current; but in this case the apparatus is too delicate, and is affected by the slightest motion, because, the repulsion being very feeble, the equilibrium is most unstable:

There are few inventions on which so much ingenuity has been expended as on arc lamps; but here, as elsewhere, many are called and few are chosen.. Our space has been devoted to the selected few, and also to some extent to the eligible ones. 


\section{CHAPTER II.}

\section{ELECTRIC CANDLES.}

AN apparatus with a voltaic are in which the carbons are placed parallel and not end to end, is called an electric candle.

In 1846, William Edwards Staite invented an apparatus in which the carbons were placed in an oblique position (an intermediate apparatus between the regulator and the candle). In 1874, Werdermann patented a sort of electric candle for piercing the rock in mining and tunnelling operations, but which was not intended to produce electric light.

But the genuine electric candle which, by a complete absence of any mechanism and its extreme simplicity, has gained such notorious success, is due to a Russian officer, Jablochkoff, and dates from 1876.

Jablochkoff Candle.-The following is an extract from the patent, which accurately describes this invention:-

"My invention consists in the entire suppression of all mechanism commonly used in electric lamps. Instead of effecting by mechanical contrivances the automatic approach of the carbons in proportion to their combustion, I place these carbons alongside one another, separating them by an insulating substance which is capable of being consumed at the same time as the said carbons-kaolin, for 
instance. The two carbons thus prepared can be placed in a.special candlestick, and as soon as an electric current from some source or other passes through them, an electric arc is produced between the two extremities. For the

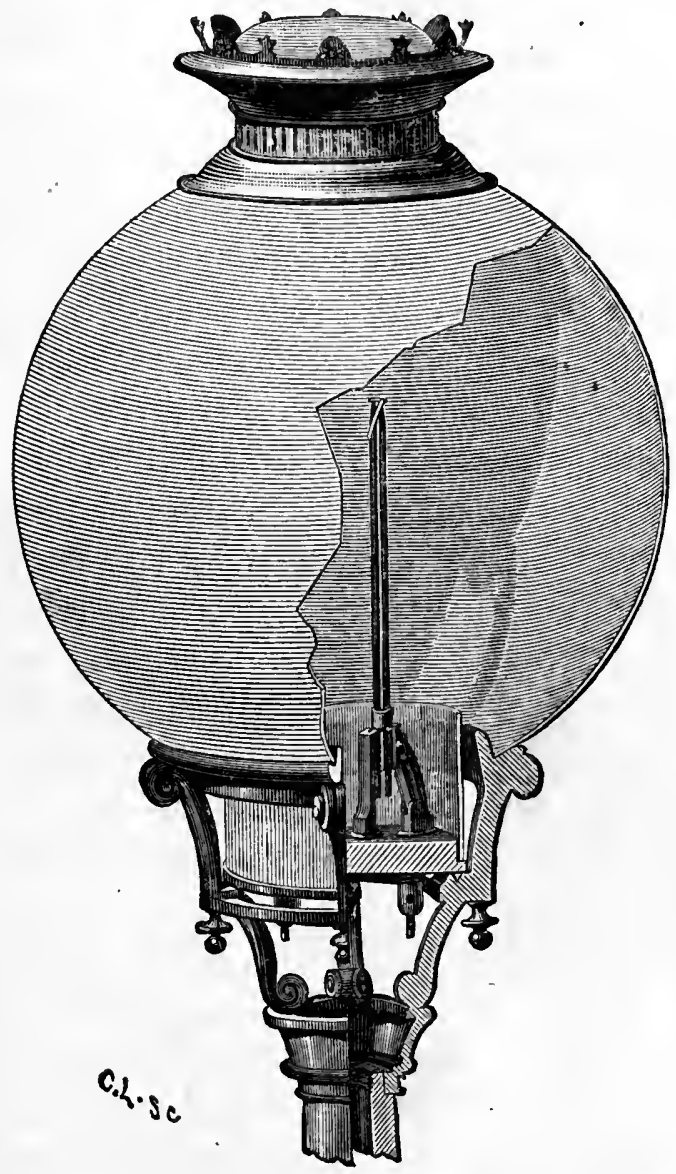

Fig. 157.-Jablochkoff candle (globes in the Avenue de l'Opéra, Paris). purposes of lighting, I join the two free extremities by a small band of carbon, which first becomes red hot, and serves as a prime of the voltaic arc." 
The candles, therefore, burn side by side, and are used up in the same time, like the wick of a composite candle.

The insulating matter maintains the arc at the extremity of the candle, and volatilizes in proportion to the combustion of the carbon.

It is a well-known fact that, in the voltaic arc, the positive carbon burns twice as quickly as the negative one. Jablochkoff has attempted to remedy this drawback, which would rapidly produce extinction on account of the unerqual length of the carbon points, by making the positive carbon twice the size of the negative one; but practice has not yet sanctioned this method. At present all Jablochkoff candles have their two carbons, and are exclusively fed by alternating-current machines. Fig. 157 represents the

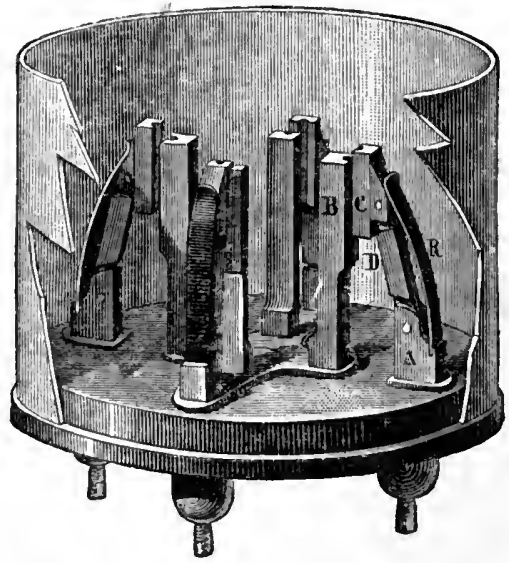

Fig. 158.-Lamp with four candles. Jablochkoff candle in its present form; the two parallel carbons have a diameter of four millimetres, and are twentytwo centimetres long, which ensures for the candles an average duration of one hour and a half. The lower ends of the carbons fit into two small brass tubes of five centimetres in length, separated by an insulating substance. In the Avenue de l'Opéra, four of these candles are placed in a globe, represented in Fig. 157, which softens the light, and makes it appear like a large luminous mass without any precise point of departure. 
At the front entrance of the French Opera, the globes are of frosted glass, which is less diffusive, but absorbs less light.

The four candles are placed at the four corners of a square, in pincers with springs B C (Fig. 158), whose insulated branches are connected with the electric conductors.

A commutator, placed within reach of the hand, and enclosed in a box to screen it from any meddling on the part of the public, turns the current from a nearly wasted candle into a new one. When the globe contains four candles, five conductors are sufficient; the first four are each connected with a candle, and the fifth forms the common return wire.

A rapid movement of the commutator turns the light from one candle to another, and this momentary interruption of the circuit has no appreciable effect upon the light of the other candles in the same circuit.

The two carbons being insulated, it is indispensable, for the purpose of lighting them, that there should be a previous communication for a few moments of the two points of the candle. At first, Jablochkoff lit his candle directly by placing between the two extremities a piece of charcoal, which he removed afterwards when the arc was formed. In order to be able to light from a distance, Jablochkoff has placed between the two carbons a piece of graphite, fastened by a small ribbon or paper, which establishes a complete conducting circuit.

When the current is sent into the candle, the little piece of graphite is heated, becomes red hot, and is consumed. The voltaic arc then appears, and is maintained as long as the candle lasts and the electric current is sufficiently powerful. The graphite can be replaced by a fine metallic wire or a piece of lead. 
At the Hippodrome, in Paris, Geoffroy effects the priming by means of a paste of special composition, with carbon as chief ingredient, which produces the same effect. By this means, half-burned candles can be used again by priming them afresh.

Gadot has used different substances for the insulating matter, to give it a certain conductivity, sufficient to allow the current to pass into all the candles at the same time. A larger quantity of the current passes into that candle which, from some difference in the mode of its manufacture, has the least resistance, and heats it; its conductivity is thereby increased to such an extent that light is produced. If the candle breaks, or burns to the end, it is extinguished, and the current lights that one of the remaining candles which possesses the least resistance, and so on. The drawback to this system is that it creates in the candle shunted currents causing an appreciable loss of electricity. The increase of conductivity of the insulating matter produced by heat is so large, that the circuit of a brightly burning candle can be interrupted for nearly two seconds without leading to its extinction or that of any other candle in the same circuit. After a certain time, the cooling destroys the conductivity, and the lighting becomes impossible from a distance; the candle must be primed anew.

The Jablochkoff candle has found a great number of applications, some of which are described in Chapter IV. Direct measurements of the work expended and the illuminating power obtained have repeatedly been made,

We quote some of the results of these experiments from a report presented in May, 1879, to the Metropolitan Board of Works, by Messrs. Bazalgette and Keates.

The first installation, which was made on the Victoria 
Embankment, consisted of twenty candles fed by a Gramme's machine with alternating currents, arranged in four circuits of five candles each.

The work expended by the machine was, deducting the work expended by transmission and by the machine working in open circuit-

$$
\begin{gathered}
\text { Total work } \\
\text { gross. }
\end{gathered}
$$

For 5 candles, or 1 circuit utilized, 13.57 horse-power, or 1.57 per candle.

$\begin{array}{llllllll}10 & " & 2 & " & 17 \cdot 91 & , & 1.27 & \\ 15 & " & 3 & , & 20.75 & " & 1.05 & ", \\ 20 & " & 4 & , & 23.53 & , & 092 & \end{array}$

The illuminating power has been found to be-

For one candle with open light...... 39.8 Carcel burners, or 640 sperm candles.

$\begin{array}{lllll}" & \text { frosted globes. } 27 \cdot 9 & & 450 & \\ \prime & \text { opaque globes } 16 \cdot 3 & " & 256 & \end{array}$

Opaque globes thus cause a loss of nearly sixty per cent. of the light emitted, and frosted globes nearly thirty per cent.

Of all the systems of electric lighting the Jablochkoff candle was the first to be extensively used for street lighting; more than 2500 of these candles were in use in Paris three years ago, and there is no doubt that, with successive improvements and lowering of price, it will even find a greater development in the future.

The only drawback to the Jablochkoff candle is the incessant variation of tint and brilliancy. This defect may be lessened by improvements in the construction of the candle and the manufacture of the carbons, but it is extremely doubtful whether it will ever disappear completely. An account of a Jablochkoff installation will be found in Chapter IV. (p. 439), in the article on the illumination of the port of Le Havre. 


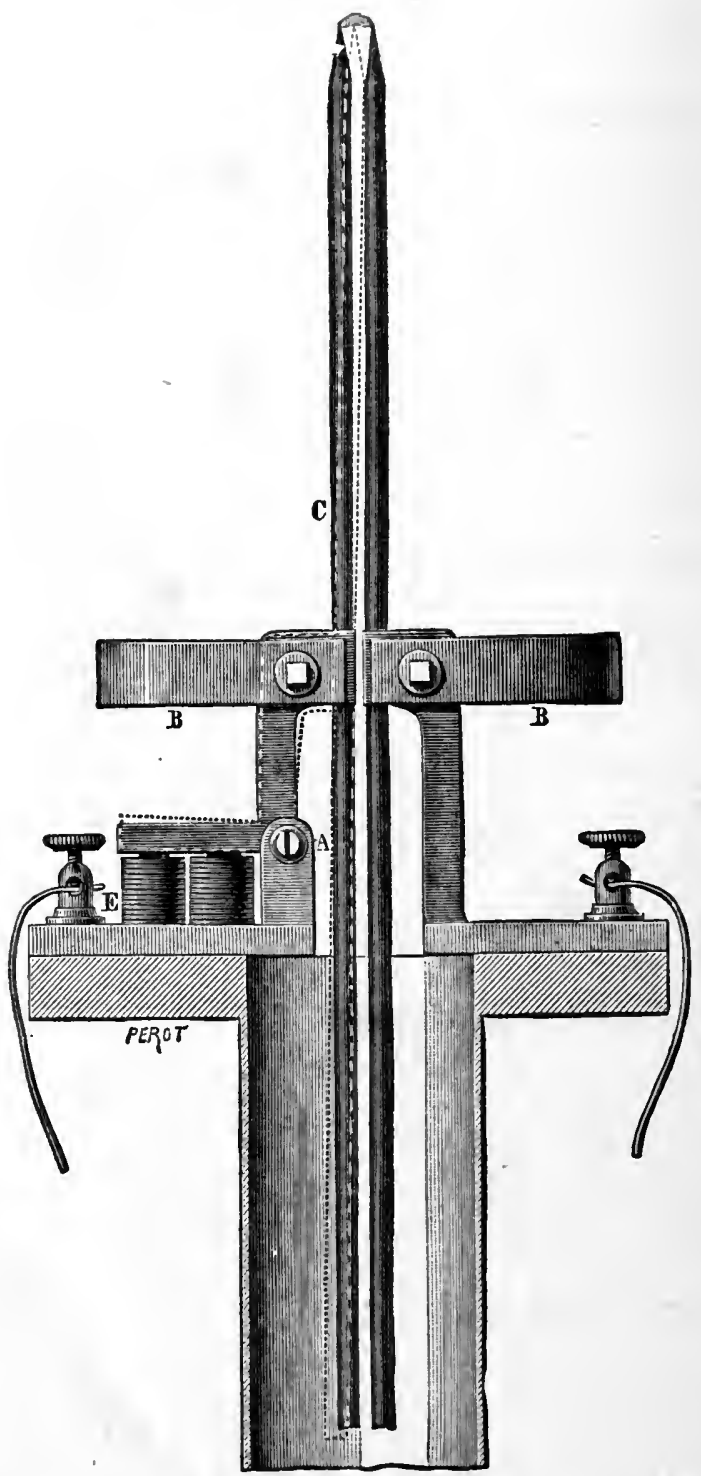

Fig. 159.-Wilde's candle (half natural size). C, movable carbon; A, ax:s of rotation of the support of the movable carbon; $B$, springs of the rollers; E, clectro-magnet. 
Wilde's Candle.-We have seen that in the Jablochkoff candle an insulating substance is used placed between the two carbons of which it is formed. In the candles we are going to describe, and which have hitherto found only a small number of practical applications, the insulating matter is entirely absent.

Wilde's candle (Fig. 159) consists of two parallel carbons of four millimetres diameter, placed at a distance of about 3.5 millimetres from one another. Each carbon is fixed to its support by means of a flat, $U$-shaped steel spring $B$ (Fig. 160), to whose extremity is fixed a roller D, which keeps the carbor in position by pressing it against the support M, allowing it at the same time to slide upwards. The carbon on the right is fixed; the support of the left

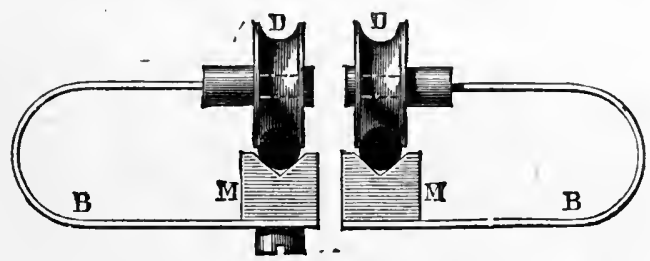

Fig. 160.-Pincer with rollers of Wilde's candle. M, fixed supports; D, copper rollers; $B$, springs.

carbon is articulated in A. If the current does not traverse the apparatus, the left carbon takes up the position marked by dotted lines; the circuit is then closed by the electromagnet $\mathrm{E}$, the two carbons, and the terminal fixed to the right hand support. But now the electro-magnet becomes active; it again attracts its armature which had gone to join the rocking carbon support $\mathrm{C}$, and the carbons are placed in a parallel position. This simple arrangement completely realizes the conditions of a self-acting apparatus. 
If, from some reason or other, the candle goes out, the electro-magnet becomes inactive; the carbon $\mathrm{C}$ again takes up its inclined position, and produces automatic relighting, if the circuit is complete in all the other parts of the systern. Wilde's candle burns, therefore, like a Jablochkoff candle without an insulating substance, at the rate of ten to twelve centimetres per hour, with an illuminating power varying from thirty to eighty Carcel burners (270 to 720 sperm candles), according to the power of the passing current.

Carbons of sixty-five centimetres length represent about five hours' lighting; the luminous point is, at the moment of lighting new carbons, at a distance of about twenty centimetres from the supports.

At the end of about an hour and a half, the arc has sunk down to $\mathrm{C}$ by the waste of the carbons; these are now pushed upwards by raising a small disc inside the tube with the help of a screw fixed on its side; the carbons slide upwards in their support, by the intervention of the rollers D (Fig. 160), and the candle is lengthened by a fresh amount. This operation is effected very rapidly, without extinguishing the candle, and resembles the raising of a candle in a common candlestick.

For long periods of lighting, Wilde places on a socket a series of candles in the form of a crown, the size of the candles being calculated to give from one and a half to two hours' light. By turning this socket either by the hand or by a self-acting apparatus, the candles are successively placed in the circuit. The luminous point is thus always placed in the centre of the diffusing globe, and the shade of the unconsumed candles is projected on the side where it interieres least with the light.

The candle can be turned upside down, and burns as well 
as in its natural position; the waste of the carbons is slightly increased in this second position. If the arc is extinguished, Wilde's system produces automatic relighting; the extinction does not last for any appreciable length of time.

Rapieff's Candle.-Rapieff's apparatus was invented about the same time as Wilde's, which it resembles in all its essential parts. We mention it here in its historical order, without entering upon the question of disputed priority of which we find many instances in the history of electricity.

Jamin's Candle.-In this candle, which was invented by Jamin, in 1879, and whose first model is shown in Fig. 161, the two parallel carbons are placed in the holder; they are surrounded by a wire in which parallel alternating currents circulate which feed the candle. To light the candle, a piece of charcoal, used for the priming, is placed between the extremities of the two carbons. If the priming is effected midway between the carbons, the voltaic arc produced at this point is rapidly carried to the extremity of the carbons, where it remains, whatever the position of the candle. The whole novelty of Jamin's invention lies simply in this removal of the arc, which is brought about by the directing action of the currents surrounding the apparatus.

The current circulates in the parallel carbons and the wires surrounding them in such a manner that, at a given moment, it ascends through the right hand carbon and descends through the carbon on the left; it ascends equally through the wires on the right and again descends through those on the left. The voltaic arc, which is nothing but an element of the current, is displaced in the sense indicated by Ampère's law.

It is at the same time attracted upwards by that part 
of the current which passes above, through the attractive force of parallel currents of the same sense; and pushed upwards by that part of the current which passes beneath, through the repulsive force of parallel currents of contrary sense; it is likewise pushed upwards by the tendency of the right hand current to turn the movable current in a parallel sense; and finally pushed in the same direction by the same tendency of the left hand current.

The four actions are concurring, exactly as in a galvanometer the four parts of the wire (two vertical and two horizontal ones) concur to cause a deflection of the needle.

The energy of this action can be varied ad libitum: By increasing the number of coils which surround the apparatus, the energy in question increases in the same ratio. On the other hand, the directing action of an element of the surrounding current on the movable element-the voltaic arc-being proportional to the simple distance, a surrounding circle acts in the same way, whatever may be its diameter. (If it becomes too large, the action of each element decreases as the radius increases, but the number of these elements increases as the radius, and an accurate compensation is the result.)

It may happen that the action is too strong, the current being carried upwards with such violence that its course can hardly be followed with the eyes, and that, on arriving at the end of the carbons, it is still pushed further, is lengthened, and breaks. The remedy in this case is simple : by diminishing the number of the surrounding coils, the forces are brought back to a stable equilibrium.

Jamin has constructed a second candle, where, by means of magnetization of a contrary sense produced by alternating currents, an oscillating movement is communicated to 
the carbons, simultaneous with the passage of the currents. When no current passes, the carbons are in contact, and are lit as soon as the circuit is closed.

This oscillating motion of the carbons leads, according to

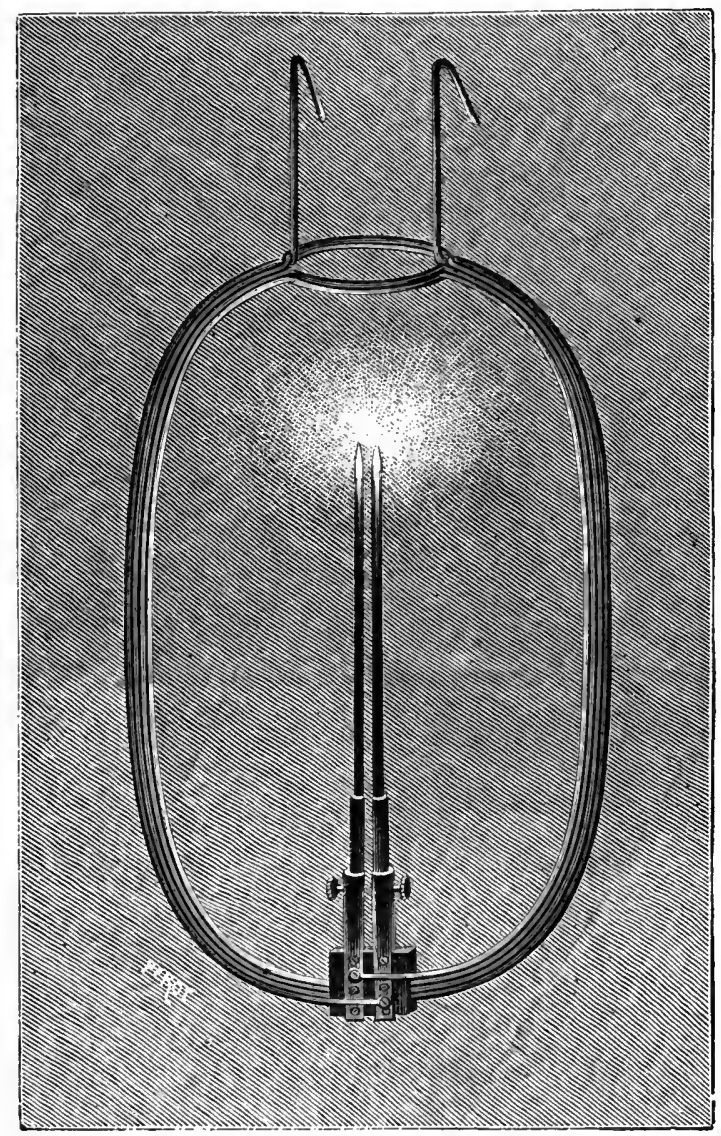

Fig. 161.-Jamin's candle (1879).

Jamin, to a more thorough utilization of the alternating current, which can only traverse the arc if its intensity is sufficient to overcome the latter's resistance.

By an approach of the carbons when the intensity 
diminishes, the current is made to pass during a longer time at each oscillation, and the utilization is from this cause increased to a certain extent. The motion of the carbons causes a more rapid circulation of air, and thus facilitates the combustion of the carbons. But supposing even this
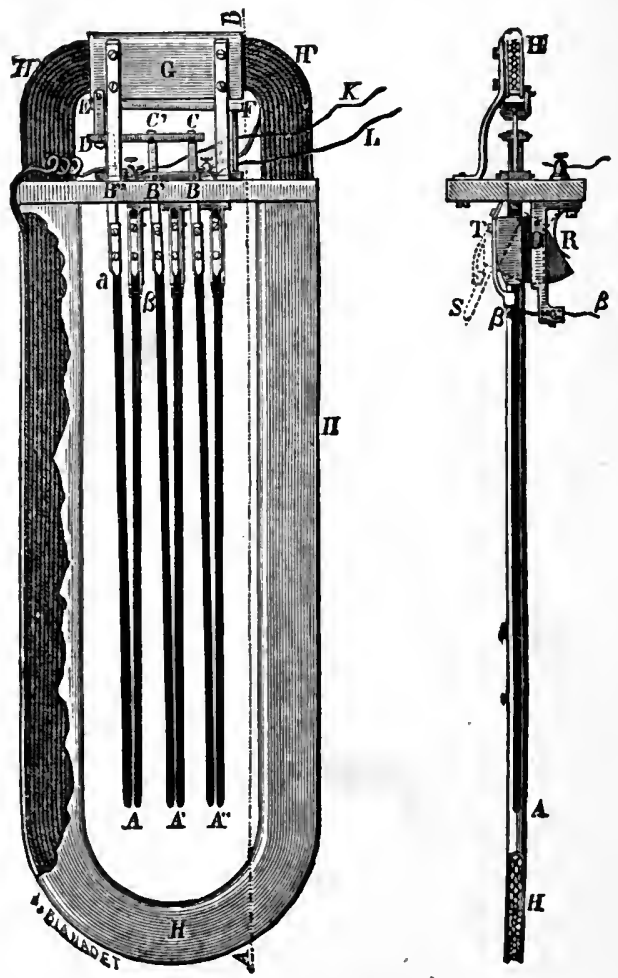

Fig. 162.-Jamin's candle (1880).

vibratory motion of the carbon possessing all the advantages attributed to it by Jamin, there is the drawback of a considerable noise, which renders the candle inconvenient in a room. All electric apparatus worked with alternating currents are subject to this inconvenience, and this constitutes an advantage in favour of continuous currents. 
A third model of Jamin's candle proved to be nothing else but an identical reproduction of the relighting of a Wilde's candle. The electro-magnet G (Fig. 162) causes the withdrawal of the carbons by attracting a small armature $\mathrm{E} \mathrm{F}$ at the moment of the passage of the current; by a combination of levers $\mathrm{E} \mathrm{D,D} \mathrm{C}$, this armature removes the left hand carbon of each candle $A, A^{\prime}, A^{\prime \prime}$. The arc is at first developed on the three candles, and after a short delay is fixed to that candle which has the least resistance. When one of the candles is consumed, the arc burns a small brass hook B placed at the upper end; the carbon support, through the action of a spring $R$, then assumes the position $\mathrm{O} \mathrm{S}$, the arc is broken, the carbons fall down, and a new candle is lit. The carbons must, therefore, be most carefully prepared, and the adjustment most accurate, if the three candles are to act each in its turn, and burn to the end without any accident.

Jamin's candles thus possess all the drawbacks of a regulator, without its corresponding advantages, for it is impossible so to regulate the proper resistance of each apparatus that the current it receives should be regularly expended.

At the moment when the arc is lengthened, it consumes more electrical energy at the expense of the other lamps; the reverse takes place when the arc is shortened. These changes in the resistance are also shown by differences in the intensity of the light. Wilde's candle has this drawback in common with Jamin's candle. In Jablochkoff's candle the kaolin keeps the carbons parallel, and partly compensates these defects; but the compensation is obtained at the expense of notable changes in the tint of the light.

In all electric candles, therefore, simplicity of construction 
can only be obtained at the expense of the quality of the light, its steadiness, or its tint.

To maintain the arc at the extremity of the carbons, Jamin always uses his guiding frame. This guiding frame $\mathrm{H} \mathrm{H}$, formed of forty coils (instead of six used in the first model), introduces a very appreciable resistance into the circuit, and thereby produces an unfavourable result, which to a great extent counteracts the advantage arising from the reversed position of the carbons. In short, Jamin's third apparatus is far from realizing all the advantages that were expected of it.

Solignac's Candle.-Solignac has constructed candles with four carbons. The two on the outside have four millimetres diameter; the two in the centre, two millimetres and a half. The four carbons are placed in the same plane, at a distance of half a millimetre, without any intermediate insulating material. The current passes into the candles by the two exterior carbons, which are fastened to two copper sockets. These candles work very well, but are rather delicate in their construction and their manipulation. They are not used in practice.

Debrun's Candle.-Debrun's candle, with a self-acting relighting arrangement, consists of two parallel Carre's carbon pencils of six millimetres diameter, and twenty to thirty centimetres long, two to three millimetres apart from one another, and maintained in their parallel position by a small glass plate placed at the foot between the two carbons. The whole is secured by insulating varnished paper and two bands of iron wire. For longer periods of lighting, two candles, instead of one, can be placed in a specially constructed lamp, and these two candles are lit successively and automatically. 
At the foot of the candle is a sinall electro-magnet, whose armature carries a small piece of carbon; when traversed by the current, the electro-magnet attracts the armature and presses the carbon against the foot of the candle. Contact is thus established, and the arc is formed at the foot of the candle. As soon as the current traverses the candle, a small electro-magnet, in the same circuit as the candle itself and set up apart as a relay, acts on an armature connected with a mercury commutator, and breaks the circuit of the electro-magnet which was placed in a shunt.

The current, therefore, traverses the candle alone, and is swiftly carried to the upper extremity by the current of hot air which is produced at the foot. As long as the candle burns, the electro-magnet of the relay keeps the lighting circuit open and the small piece of carbon away from the candle. If the candle is extinguished, the commutator of the relay is released, closes the circuit of the candle electro-magnet, and the same series of phenomena repeats itself.

When the first candle is consumed, the arc burns a small brass wire at the bottom, a spring of coarse brass wire is released, and conveys the current from candle No. 1 to candle No. 2, which is lit in the same way.

All electric candles possess two very serious drawbacks, which render them inferior to regulators: the first is the noise produced by the alternating currents which are obligatory for the uniform waste of the two carbons; the second results from the very position of these carbons and their fixed distance. The arc passes between the points, and a part of the light is thus eclipsed; from want of regulation in the distance of the carbons, this distance may, 
at a given moment, not be proportional to the intensity of the current, hence the want of stability of the light of all these candles.

In order not to use them up too quickly, they are fed by currents of feeble intensity-eight or nine ampères for candles of four millimetres. In proportion as the regulators are improved and simplified, the electric candles lose their importance, always excepting the Jablochkoff candle, the extreme simplicity of which will never be equalled.

The Soleil Lamp.--This apparatus, invented by Clerc and Bineau, is intermediate between a Jablochkoff candle and

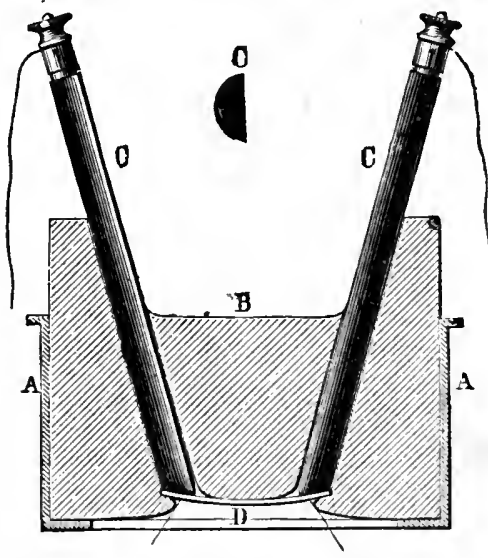

Fig. 163.-Diagram of the soleil lamp. an incandescent lamp. Although its form only vaguely reminds us of a candle, we mention it here so as not to have to assign to it a special class.

Figs. 163 and 164 show its construction. Two carbons C C, slightly deviating from the vertical position, are placed between a central piece $B$ of white marble or condensed nagnesia and an exterior piece of some stone or other; they descend by their own weight in proportion to the waste of their lower part, and are kept back by a small ledge or shoulder which is seen on the exterior stone. The whole of this arrangement is placed in a cast-iron box A A open at the bottom. This box is suspended by a large stirrup, to which the conducting wires are connected.

The conducting wires convey the current to the upper 
end of the two carbons. Before lighting, the carbons rest on a small and fine piece of carbon D, which is heated by the passing current, is rapidly consumed, and prepares the

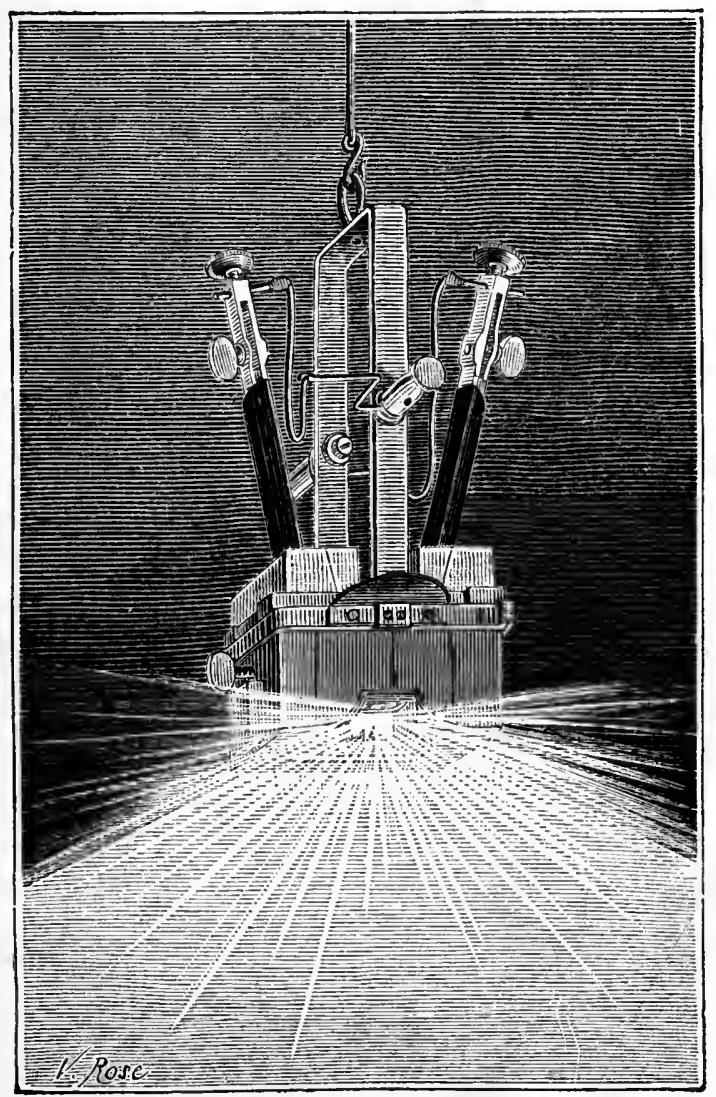

Fig. 164.-Soleil lamp.

(From La Lumière Électrique.)

production of the voltaic arc, like the prime of the Jablochkoff candle.

When once the arc is established, it is maintained in spite of the great distance between the carbon ends, because 
the surface of the marble, or the magnesia between them, has had time to become a conductor, through being heated during the preparatory period of lighting.

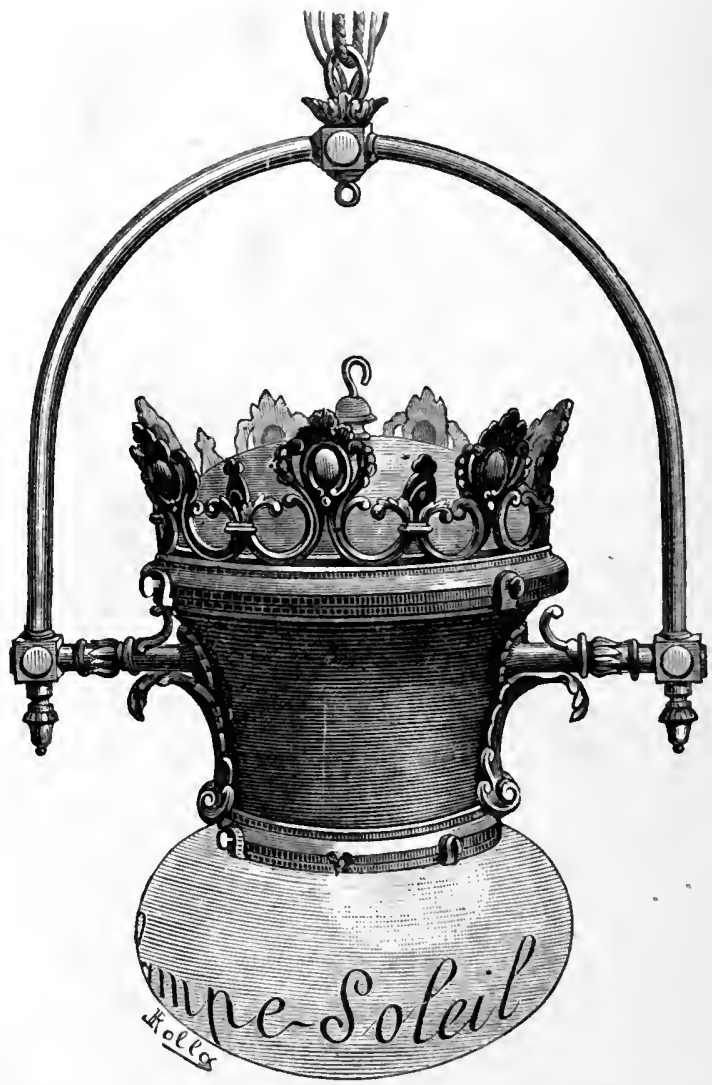

Fig. 165.-Soleil lamp (enclosed in hermetical lantern). (From La Lumiere Électrique.)

This arc of great length heats the marble, and produces an intense light of yellow tint, to which the lamp owes its name.

The soleil lamp (Fig. 164) throws all its light downwards, which is as a rule desirable, but the total suppression 
of light in any other direction is sometimes objectionable. Moreover, the different pieces surrounding the luminous part of the marble throw a shade and assign to the luminous rays a limited area.

When the light has to be thrown upwards, to light up the ceiling, for instance, the lamp is simply reversed and the carbons are pushed by the aid of springs. By an ingenious contrivance, however, the springs can be dispensed with. If the movable carbons are replaced by carbon blocks of comparatively large size, these blocks wear gradually, until the are becomes too large, and the lamp is extinguished. This arrangement was used for the experiments in lighting the lobby of the Paris Opera.

The lamp is generally worked with alternating machines, like Gramme's or especially Lachaussée-Lambotte's (p. 126), and, to stifle the noise of the alternating currents, it must be placed in a hermetically closed lantern (Fig. 165).

The luminous point is absolutely fixed, and the light is perfectly steady-two very important advantages of this lamp. A further advantage is the very considerable time during which the lamp can act without stoppage, without renewal of carbons, and without any personal attention. The length of the carbons can vary from five to thirty centimetres, and the lamps can act for fifteen or sixteen consecutive hours, if necessary.

The marble block between the two carbons is likewise wasted; the very high temperature to which its lower part is exposed causes a decomposition of the calcic carbonate, quicklime is formed, which is rendered incandescent by the arc. A marble block can last for twenty consecutive hours, or for two lighting periods of ten hours; it does not last so long if the lighting periods are of two or three hours 
respectively, and the lamp is thus subjected to a series of lighting and extinction.

The length of the arc can vary from ten to sixty millimetres, according to the resistance for which it is intended. For a short circuit, greater intensity and a thinner marble are required; for a long circuit, a higher tension, less intensity, and a thicker marble must be employed. The lamps commonly used in practice have a slab of marble or magnesia of ten to twenty millimetres thickness.

Latterly, condensed magnesia has been substituted for marble, because it lasts much longer; it is not decomposed by heat, and can last for a hundred hours, but gives a white light.

It is of great importance that the blocks of refractory matter should last for a long period, and the inventors have devoted special attention to this point. They have succeeded in constructing blocks which will last from thirty to thirty-five hours, and for this purpose have subjected the refractory material to a previous trial in an apparatus which reproduces as near as possible the conditions to which they are subjected in the lamp itself.

This apparatus, which is represented in perspective in Fig. 166, consists of a block of refractory stone $M$, on which is placed a second refractory block $P$, similar to that of the lamp itself, but much larger, and having a cavity in the lower part. The refractory material is placed in this cavity, and two carbons $\mathrm{C} \mathrm{C}$ allow the formation of a very powerful arc. The refractory material is thus subjected to the same temperature as that of the lamp, and its capacity for endurance can be tested in this way.

By using the Lachaussée-Lambotte machine, the current can be divided into several circuits, each of which contains 
several lamps in series; by means of a commutator the current can easily be switched off to an adjacent lamp. Although there is very little chance of an extinction, an automatic commutator constructed by Maguire provides even against this contingency.

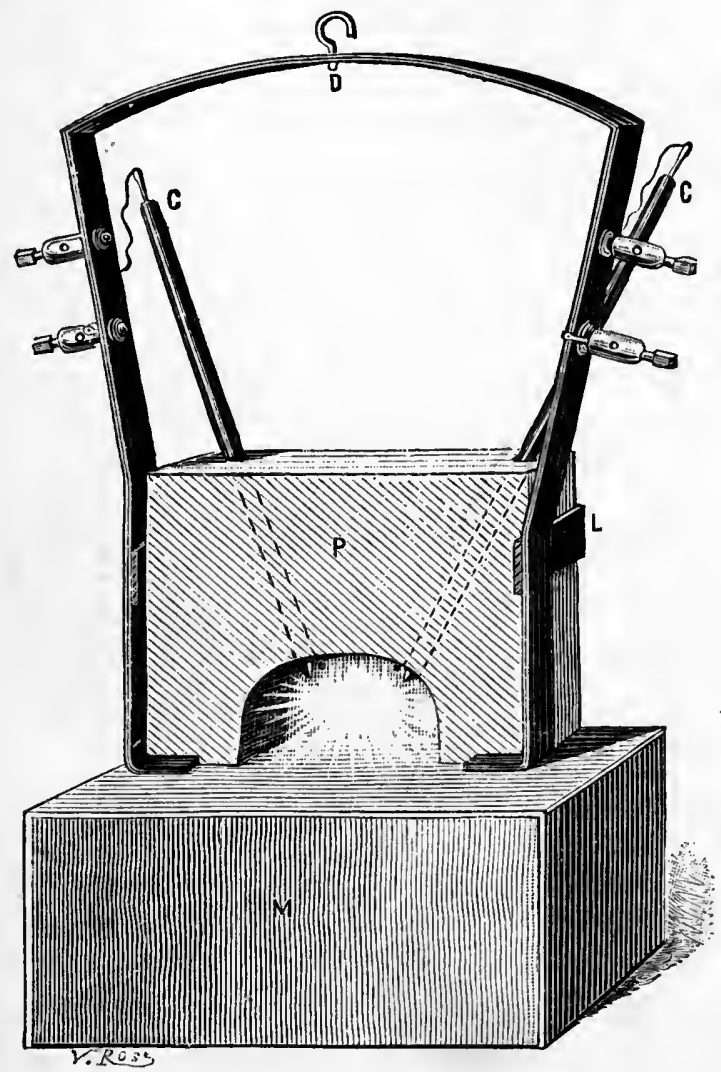

Fig. 166.-Soleil lamp (apparatus for testing blocks).

(From La Lumière Électrique.)

It will be seen that the soleil lamp fulfils all conditions required for good working, and we have only to consider now the economical part of the question. 
The material consumption, both of carbons and marble block, may be estimated at three centimes (not quite a halfpenny) per hour.

As regards the force expended, a number of trials were made with an alternating Gramme machine, fed by an exciting machine which was driven by an engine of 4.5 horse-power.

The luminous efficiency per horse-power increases with the size of the lamp : a lamp of eleven millimetres, fed by a current of ten ampères' intensity, gave seventy Carcels per horse-power, whilst a lamp of thirty-five millimetres and twenty-two ampères' intensity yielded as many as $\mathbf{1 3 8}$ Carcels per horse-power. It must, however, not be forgotten that the luminous zone of each lamp occupies only a portion of the circumference, and that the results recorded are measured in the direction of the maximum intensity. 


\section{CHAPTER III.}

\section{LIGHTING BY INCANDESCENCE.}

Is all apparatus which we have hitherto described, the light is produced by the voltaic arc, which implies a longer or shorter distance between the two conductors. This space is occupied by gas raised to a very high temperature, and by particles of carbon detached from the electrodes. In the systems about to be examined now, the light is produced by a solid body raised to a very high temperature by the passage of an electric current.

In electric lighting by incandescence, two large classes must be distinguished, founded on different principles.

1. Incandescence with combustion; systems, Reynier, Werdermann, etc. The light is produced by a carbon point which is traversed by the current and more or less quickly consumed.

2. Incandescence alone. In these apparatus, the electric current passes through a substance of comparatively small conducting power, and heats it till it becomes luminous, but without destroying the substance itself.

For these apparatus, either carbon filaments or metals difficult of fusion, such as platinum, an alloy of platinum and iridium, or iridium by itself, are used. 


\section{Incandescent Lamps with Conbustion.}

The first incandescent lamp dates back as far as 1845, and is due to De Moleyns; but the principle of an incandescent lamp with combustion was patented as late as 1876, by Varley; and even his lamp is only an intermediate arrangement between a real incandescent and an arc lamp. These apparatus are sometimes ealled lamps with imperfect contact.

Varley's Lamp.-The following is the description that Varley gives of his lamp in his patent of 1876 :- "A carbon

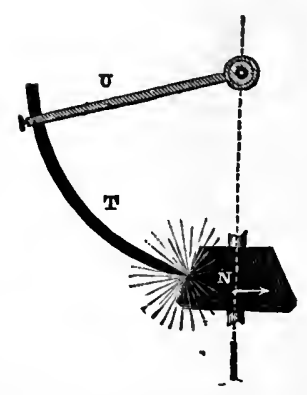

Fig. 167.-Varley's lamp. point $T$ (Fig. 167) presses slightly by its weight and its mounting $U$ against the periphery of a roller of gas carbon $\mathrm{N}$; hence results imperfect contact, and, consequently, incandescence and combustion of the carbon point at its extremity."

It seems, however, that the light was produced in this lamp by a series of sparks, which owed their formation to the composition of the roller $\mathrm{N}$, which consisted of alternately conducting and insulating parts, as well as to its rapid rotatory motion.

Reynier's Lamp.-F́mile Reynier constructed, in 1877, the first electric combustion lamp founded on the incandescence of a carbon point which was traversed by an electric current. Reynier was led to the construction of his lamp by making experiments on pure incandescence with some Russian lamps which will be mentioned hereafter. All these lamps had one and the same serious defect-the 
waste of the central part of the carbon, which brought about its rupture, and necessitated the substitution of a fresh carbon.

Reynier was led to believe that, if the extremity of the carbon point touched another massive carbon, the waste would be produced at the point of imperfect contact where the temperature was highest, on account of the electrical resistance due to this imperfect contact.

If the carbon was burned at its extremity, it only

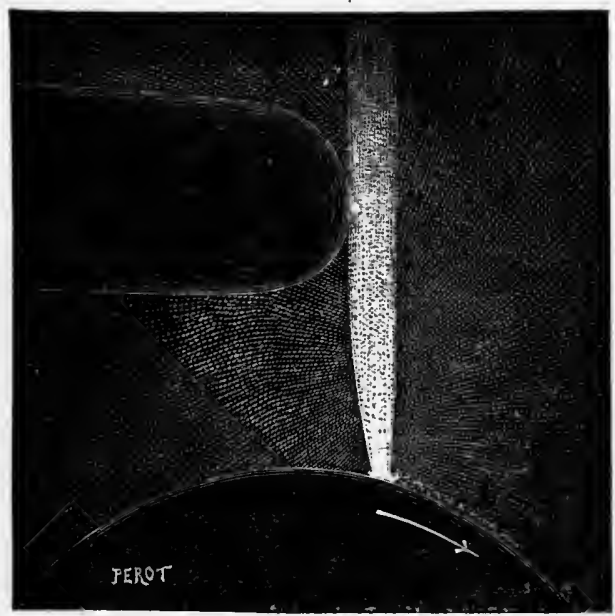

Fig. 168.-Enlarged image of the carbon of an incandescent lamp (Reynier's system).

required pushing upwards, like the candle in a carriage lamp. The combustion of the carbon even increased the light, the heat produced by it being instrumental in keeping up a very high temperature.

The characteristic part of Reynier's apparatus is, therefore, a carbon point pressing against a fixed contact (Fig. 168), and rendered incandescent between this point of contact and another, situated at some distance from 
the first. The first lamp was worked with four Bunsen elements (Ruhmkorff's model), a Gramme's laboratory machine, and even with a secondary battery of three Planté elements.

In his first experiments, Reynier found that the fixed, immovable carbon against which the incandescent carbon pressed, did not answer its purpose. He therefore gave it the shape of a disc, which slowly turned round on its horizontal axis. La Nature of the 24th of August, 1878, represents two different models of Reynier's lamp, which was afterwards simplified by the inventor in the following manner:-

The carbon pencil falls downwards by its own weight and that of the carbon-holder, directed by guiding rollers; it comes to press against a carbon disc, which is somewhat in advance of the vertical line passing through the centre of the disc, and the descent of the pencil arising from its waste thus turns the disc without any mechanism. The carbon is maintained at a short distance by a cylinder; and another carbon contact, which determines the incandescent length, presses against it from beneath. By a very simple contrivance, the pressure exercised by the carbon on the disc is transmitted, and acts as a check on the motor carbon-holder. The weight of this latter is, therefore, effectually kept back as long as the carbon retains its original length and presses against the disc; and, on the other hand, is set free as soon as the lower support gives way through the waste of the carbon pencil. It is worthy of note that the highest temperature is at the lower point of contact, and that the slow combustion in air lessens the size of the carbon towards its lower end, and thus facilitates its advance. Instead of being a draw- 
back, as was the case in the old lamps, this accessory circumstance has been turned to profit in Reynier's system.

The lamp can be introduced into the circuit of a Gramme's machine supplying an arc lamp, and can thus furnish an additional light, without sensibly interfering with the principal light furnished by the voltaic arc.

Reynier's lamps present a certain elasticity as regards their illuminating power, for, according to the intensity of the traversing current, they can give a light varying from four to forty Carcel burners (36 to 360 candles).

Reynier's Lamp, Model of 1879.-In this latest form of Reynier's incandescent lamp, the carbon C (Fig. 169), pushed by some mechanism in the direction of the arrow, presses against the contact in $\mathrm{B}$; the lateral contact $\mathrm{L}$, mounted at the extremity of a lever, presses against the carbon by the action of a spring $r$, and confines the incandescence of the carbon point to the limits $i$ and $j$.

The contact with the end-piece B is placed in a holder, fixed in the lower socket of the burner. To introduce the carbon into the lamp, this holder with the end-piece is taken out, the carbon is pushed into the open tube, and the holder put back into its place. The apparatus is now ready to act.

The incandescence, as we have mentioned, is limited to the space between $i$ and, $j$, and its length can vary from four to eight millimetres. The light obtained is from five to twenty-five Carcel burners (45 to 225 candles) for each apparatus, according to the intensity of the electric current and the length of incandescence determined upon. With a battery of eight Bunsen elements (Ruhmkorff's model), a light equivalent to about twelve Carcel burners (108 sperm candles) is produced. If the apparatus is worked by magneto-electric machines, it can produce, per horse-power, 
from three to five light-giving centres of eight to fourteen burners each. This gives an illuminating power of thirty to forty Carcel burners (about 270 to 360 sperm candles) per horse-power.

A given burner can easily be adapted for lamps of different forms. Reynier has constructed apparatus with a
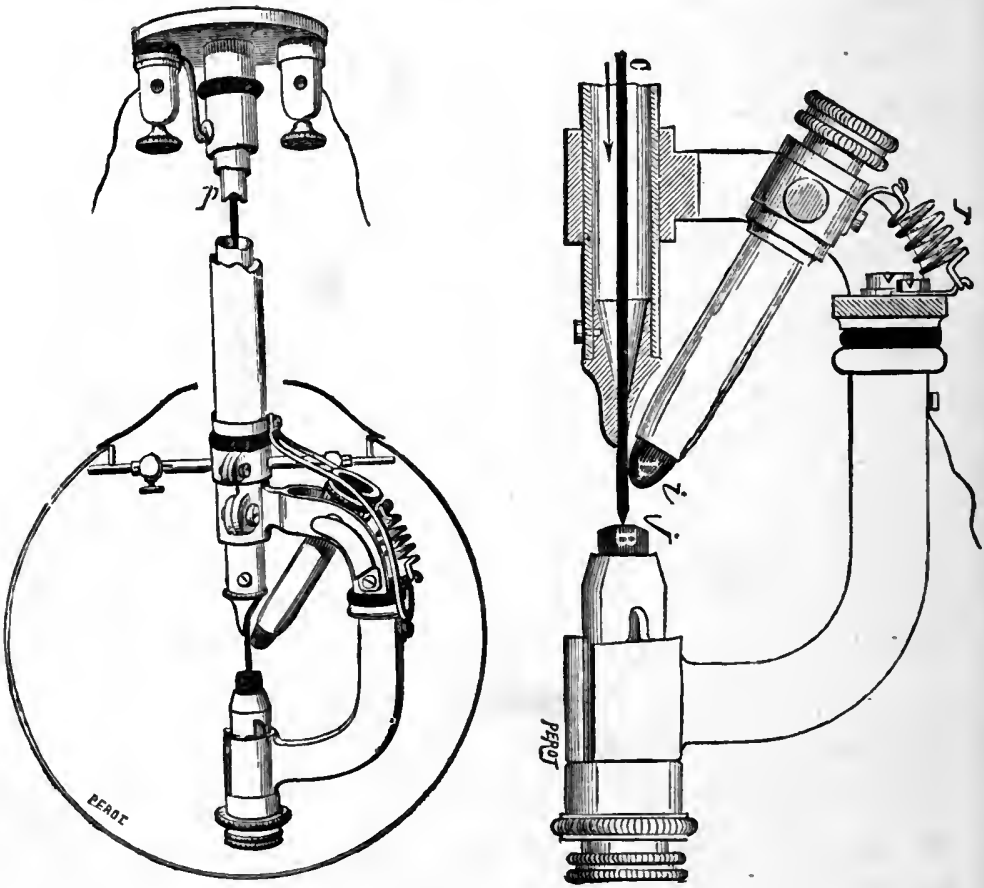

Fig. 169.-Reynier's lamp (1879).

downward action, by means of a counterpoise or of hydraulic pressure; a lamp with a drum, acting in any position; and, simplest of all, a suspended apparatus, which is represented in Fig. 169. Here the advance of the carbon is obtained by the descent of the cylinder $p$, which presses directly against the carbon. 
Reynier's Self-acting Lighter.-If from eight to twelve lamps are placed in tension on an ordinary Gramme's machine, as in the applications of Reynier's incandescent lamp, the accidental extinction of one lamp causes the extinetion of all the others; and in the sudden darkness it is impossible to find out which lamp is at fault. This of course, is a very serious drawback, and accidents of that kind will be the more frequent the larger the number of light-giving centres.

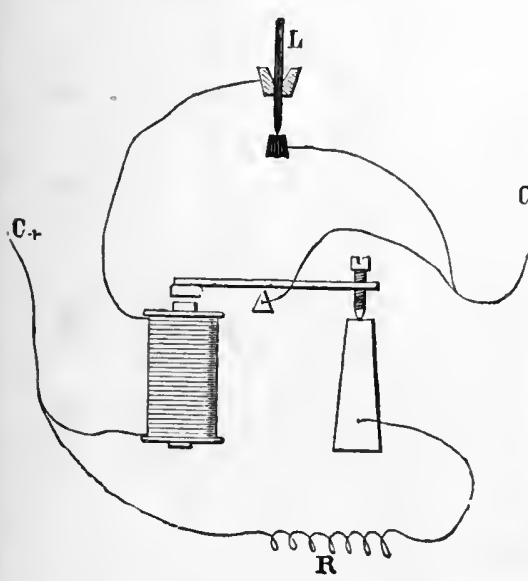

Fig. 170.-Diagram of Reynier's self-acting lighter.

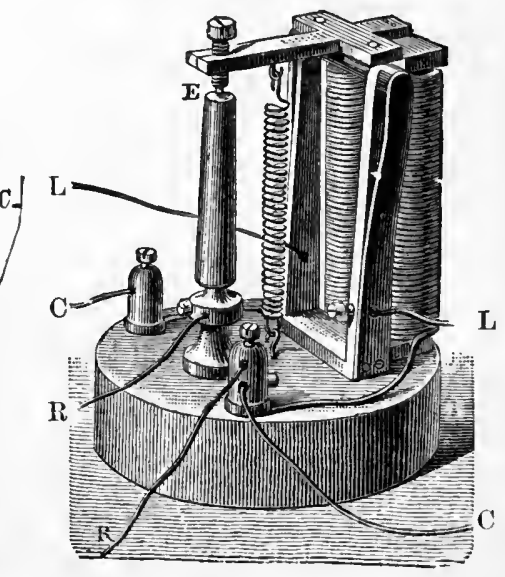

Fig. 171,-Practical form of Reynier's self-acting lighter.

To guard against this serious inconvenience of a partial extinction, Reynier employs a self-acting apparatus, called an allumeur (lighter), whose construction will easily be understood by a reference to Fig. 170 .

The principal conductor $\mathrm{C} \mathrm{C}$, instead of being in direct connection with the terminals of the lamp, is fixed to the lighter, where it is shunted into two circuits of equal resistance, namely, lamp $\mathrm{L}$, and a spiral $\mathrm{R}$ of German silver. The shunted current, passing through the lamp, magnetizes 
an electro-magnet, whose function it is to break, by means of a lever, the shunted current in the wire $R$; this latter current is closed again automatically by the same lever when the electro-magnet is demagnetized.

Fig. 171 represents the lighter in its practical form. $\mathrm{C} \mathrm{C}$ are the main wires; $\mathrm{L} \mathrm{L}$, the conductors connected with the lamp; $R \mathrm{R}$, the connections of the shunted circuit. The following contingencies may arise in this system :-

1. The lamps being all supplied with carbon, the circuit is closed : the current is for a moment divided between the wires of the shunt and the lamps; but immediately afterwards the electro-magnets are magnetized, open all the shunted currents, and the lamps alone remain in the circuit.

2. At the moment of lighting, one or several lamps are without carbon: the lighters of the lamps supplied with carbon act in the way that has been described; the currents of the lamps without carbons pass into the shunt wires, and the carbons can be replaced without interfering with the intensity of the current or the illuminating power of the lighted lamps.

3. A lamp which has been acting, stops from want of carbon or some other accident : the lighter connected with the lamp closes the shunted circuit, and all the other lamps are not affected.

4. During the action of the other lamps, a lamp is supplied with carbon: the current is for a moment divided between this lamp and the resistance of its lighter, whose electro-magnet is immediately magnetized, opens the shunted current of the resistance and short-circuits the lamp.

The lighter, as will be seen, fulfils all requirements. It renders the lamps practically independent of each other, and requires no rersonal attention. 
Werdermann's Lamp.-Certain arrangements of Reynier's lamp are reproduced in this lamp, but the most characteristic point consists in the reversed position of the apparatus.

In Werdermann's first model, patented a few months after Reynier's system, the negative carbon, placed in the upper part, consists of a carbon disc of large diameter; the positive carbon is an ordinary carbon pencil of four and a half millimetres diameter.

A simple spring ensures a permanent contact of the carbon with the positive electrode; the carbon is pushed by a weight in proportion to its waste.

Does Werdermann's lamp produce a voltaic are or no? This question, which is often asked, resolves itself simply into a matter of definition. If by the term "voltaic arc" is meant a material interval between the two electrodes, an interval filled with gaseous molecules and particles of carbon raised to a very high temperature, Werdermann's lamp does not produce a voltaic arc. The very fact of the carbon point being pushed upwards must necessarily bring about a contact between the disc and the point, although this contact can be rendered very slight by means of a check employed in the last model.

But in incandescent combustion lamps, the high temperature produced by the electric current strongly heats the atmosphere surrounding the luminous point, while particles of carbon, detached from the positive electrode, are consumed in it.

We find, therefore, in this part of the lamp a halo of very high temperature, of great conductive power, and full of particles of carbon-in short, all the necessary conditions for the formation of the voltaic are properly speaking.

In Werdermann's first experiments, the lamps, ten in 
number, were mounted in shunts on the same circuit. A Gramme's machine was sufficient to feed the ten lamps. In his new apparatus, Werdermann arranges them for tension, with a machine which gives less quantity but a greater electro-motive force.

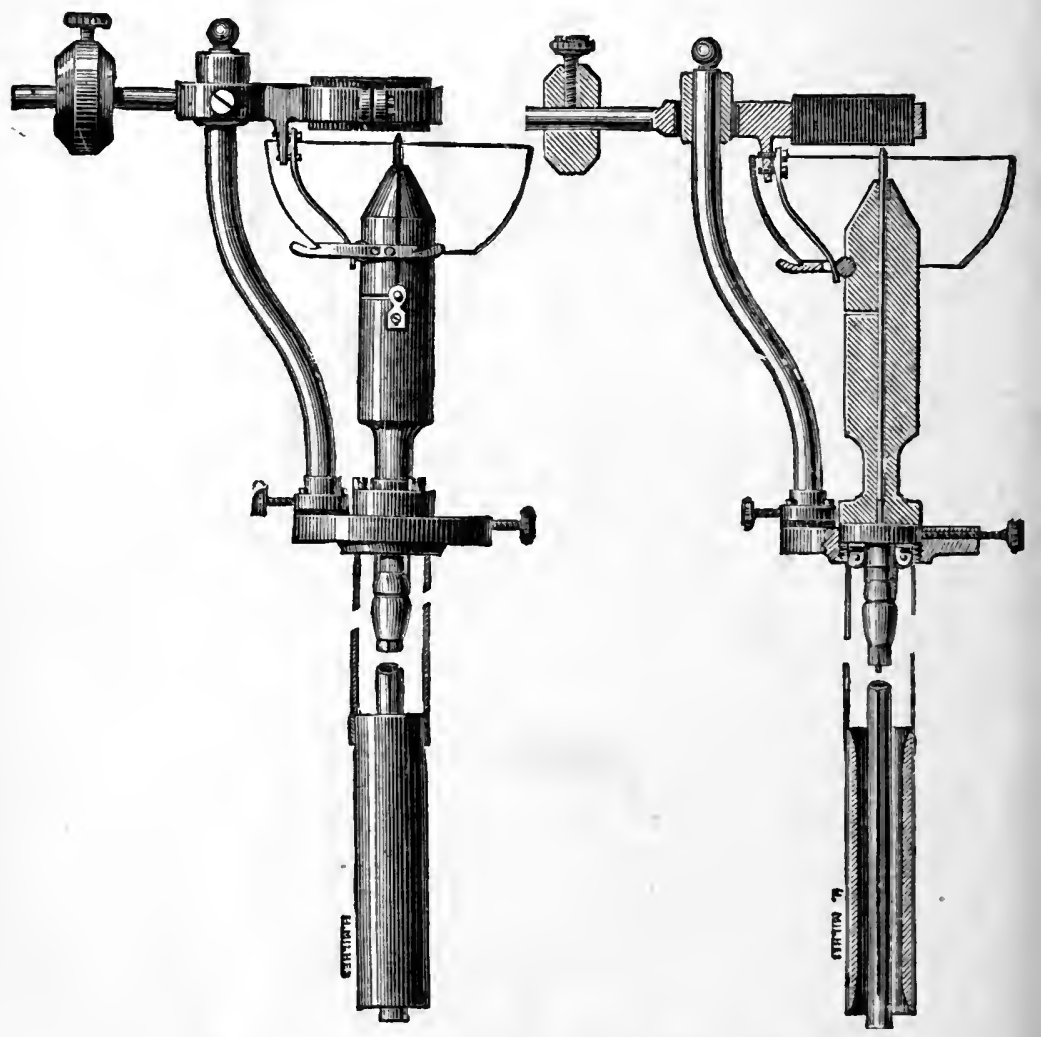

Fig. 172.-Werdermann's lamp (elevation and section).

In his new lamps (Fig. 172), the inventor has regulated the pressure of the lateral contact on the carbon point according to the pressure which this point exercises on the upper disc.

For this purpose the disc is placed on a beam kept in 
equilibrium by a counterpoise sliding on a horizontal rod; this rod is situated on the left of the point of suspension, and fixed in place by a screw. The beam carries a small arm, placed inside the globe, which exercises on the lateral contact a pressure in proportion to the pressure of the vertical carbon itself; this pressure opposes the ascending movement of the carbon point by wedging it between two jaws, of which one is fixed and the other movable.

This system formis, therefore, a check, whose equilibrium depends on the primitive adjustment of the counterpoise. In proportion as the carbon is wasted, the pressure on the disc diminishes, the lateral contact is slightly slackened, and permits the ascending motion of the carbon point.

Very slight contacts between the dise and the carbon point are obtained in this way; the pressure is never strong enough to produce the breaking of the point; the waste of the carbon is very regular; and the light shows a steadiness which is not to be found in the same degree in any other system.

The lamps act in tension on the same circuit. If one of them goes out from some reason or other, it must immediately be withdrawn from the circuit, and direct communication be established between the two terminals of the lamp, in order to prevent the extinction of all the other lamps in the same circuit. This result is obtained by the lever which presses against the lateral contact. When the carbon point no longer presses against the disc, this latter begins to rock, and the lever presses against a metallic stop, which establishes a direct communication and short-circuits the lamp.

Joël's Improved Werdermann's Lamp.-The light is pro- 
duced, as was the case in Werdermann's system, by the heating to incandescence of the end of a small rod or pencil of carbon forming one electrode, which protrudes through a pair of contact jaws, and abuts upon a fixed cylinder of copper forming the other electrode. The carbcn pencil consumes at the rate of two and a half to three inches per hour, for lights of 100 candle-power and upward, and is pushed forward according to the consumption. The length of carbon in circuit between the contact jaws and the fixed electrode is about three-quarters of an inch, and this, by the passage of the current, is rendered highly incandescent, chiefly, however, at that part near the copper eleetrode, where the pencil becomes pointed, and therefore more intensely heated. There is also, in addition to this, a glow or flame-like appearance from the sides of the consuming carbon to the copper electrode, the light thus apparently taking an intermediate position between the purely incandescent system and that of the arc. The heated point of carbon becomes curled at the tip in a peculiar manner, as though it were viscous, in shape somewhat like a mushroom where it wastes away, and is replaced by the gradual forward motion of the pencil.

The fixed electrode, which may be entirely of copper or with a graphite insertion, remains intact without any appreciable wear.

The chief improvements in this lamp consist in the simplification and certainty of action of the mechanism in connection with the contact jaws for clamping the carbon pencil, by which means the lateral pressure of the jaws and the feeding of the carbons are attained by the combined action of one actuating weight, as shown in the 

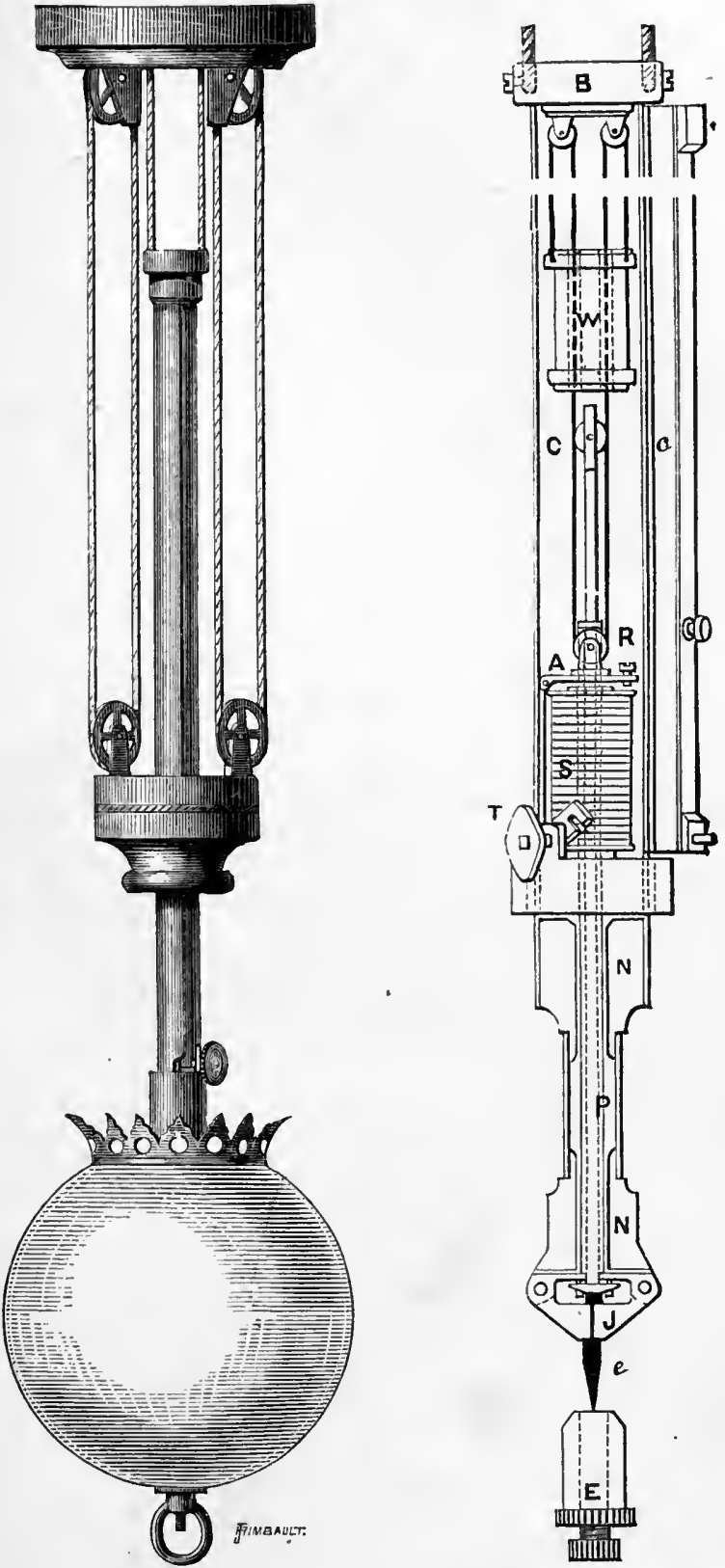

Fig. 173.- Joël's improved Werdermann's lamp (elevation and section). 
diagram (Fig. 173). It will be seen from this that the lateral pressure is always proportional to the downward pressure, and may be varied to suit any conditions. The details devised for rendering the lamps in the same circuit independent of each other, and its general adaptability for interior and domestic lighting, constitute an important advance on anything which has gone before.

On referring to the sectional view of the lamp, $\mathbf{E}$ is the fixed copper electrode on which abuts the point of the carbon $e$, which is rendered intensely incandescent by the passage of the current between the jaws and the copper cylinder. The jaws are seen at $\mathrm{J}$, clamping the carbon pencil. The actuating weight $W$, which gives both the feeding motion to the pencil as it consumes, and the lateral pressure to the jaws, is suspended by continuous cords to the top of the lamp B; the cords thus passing down through the weight and under one of the rollers at $R$, up again through another roller at $R$, and ending at the weight. The rollers $R$ are attached to a light tube $P$, which passes down through a nipple $\mathrm{N}$, and terminates in a flange under the horizontal arms of the jaws, and lifts them according to the leverage, thus producing the lateral pressure on the pencil. The top of this tube has also attached to it the armature $\mathrm{A}$ of an electro-magnet $\mathrm{S}$, wound with fine wire, and arranged in a shunted circuit in such a manner that, as long as the normal condition of the light is maintained, it is neutral; but if an arc should be accidentally formed between the carbon $e$ and the copper $\mathbf{E}$, the electro-magnet comes into action in opposition to the controlling weight, and frees the jaws from lateral pressure, thereby allowing the carbon pencil to descend freely and establish contact. 
The carbon-holder (Fig. 174) is also arranged so that when the pencil is very nearly consumed, the lamp is automatically eliminated from the circuit by the lever at $\mathrm{L}$ making contact with the arm carrying the copper electrode. The stem or body of the lamp forms an important part of the whole, being formed of metal tubing in two semicircular halves, each half forming part of the electrical circuit, the current traversing one side, of which the jaws form part, passing through the carkon pencil to the copper electrode, and returning by the other side of the lamp.

The two sides are kept closed mechanically (but insulated electrically from one Fig. 174.-Caranother) by the latch and knob T (Fig. 173), Joël's lamp. which also automatically short-circuits the lamp when opened for the purpose of putting in fresh carbon, thus rendering it perfectly safe for handling. There are ingenious arrangements attached to this system for switching a lamp on or off, with resistances equal to that of the lamp; but upon these it is unnecessary to dwell, these adjuncts being common to many systems.

Professor Adams stated, at the Society of Arts, that with this system an illuminating power of $71 \check{\text { candles per }}$ horse-power could be obtained.

Incandescent lamps are very capricious. Difficulties arise from the extreme thinness and delicacy of the glass employed, leakage from defective sealing or fractures, the liability of the incandescent material to shake loose in its supports, and the great care required in manipulation. The Joël lamp is free from these objections, and as in 
lamps that are purely incandescent the heat is produced by the current only (the carbon not undergoing combustion by reason of the absence of oxygen), it follows that the incandescent portion cannot attain to so high a temperature as when the carbon is consumed, and therefore the light must necessarily be of less power than that in the lamp described. In the workshops of the Electric Light Agency two of these lamps take the place of fifteen gas-jets, with highly satisfactory results. The carbons employed are five millimetres in diameter, and about one metre in length. The lamp burns for seven or fourteen hours, according to the dimensions of the carbon.

Various Lamps.-A number of lamps have been constructed since Reynier's and Werdermann's, founded on the incandescence of carbons with combustion. We only mention a few which differ from the two prototypes simply by some details of construction or arrangement.

Trouvé has constructed a lamp in which we find the ascending carbon, the rotating carbon abutment, and the carbon point wasted at its pointed extremity, as in Reynier's lamp. By employing carbon points made of very fine carbon, a light of several Carcel burners can be produced with six Bunsen elements.

In Ducretet's lamp, the carbon is pushed upwards by a column of mercury into which it is immersed.

This apparently simple arrangement, which is already indicated in Reynier's patent, has the drawback of giving off mercurial vapours, in spite of all the precautions that have been taken to prevent the heat of the incandescent carbon from reaching its surface.

Finally, Tommasi, in 1879, constructed a Reynier's lamp 
under the name of revolver lamp, in which a certain number of short carbons are arranged in a circle round an iron tube, three centimetres in diameter and turning on a pivot, A clockwork machinery successively places each of these carbons in the circuit, which gives to the light a certain duration.

\section{LAMPs With INCANDESCENCE ALONE.}

These lamps are constructed on the principle of raising the temperature of a moderate and infusible conductor by the passage of a powerful electric current. The intensity of the light emitted by a heated body increases in a very large proportion with the temperature, and we need, therefore, only employ a substance with a very high point of fusion, to be able to raise it to a very high temperature. It is further necessary that the heat produced by the current should be concentrated within a very small space, to avoid the cooling which is so prejudicial to the quantity of the light produced.

The substances most commonly used for the production of light by incandescence are-platinum, an alloy of platinum and iridium, iridium, and carbon.

The first lamp with incandescent platinum dates back as far as 1841, and was invented by Frederick de Moleyns, of Cheltenham.

The idea of employing iridium and its alloys is due to Pétrie, in 1849. In his patent, Pétrie gives the method of preparation of iridium intended for electric lamps.

The idea of using carbon for producing incandescence belongs to King, who patented it in 1845. According to 
Giffard, King was simply the agent of the real inventor, J. W. Starr, of Cincinnati.

Starr was the author of several philosophical works, and it was the great philanthropist, Peabody, who supplied him with the necessary funds for his experiments, which were made in this country.

Starr set up a candelabrum with twenty-six lights, symbolical of the twenty-six states of the Union. Faraday admired this first experiment very much, and Starr and King started for the United States soon afterwards. The day after their departure, Starr was found dead in his bed.

King pointed out in his patent the advantages of gas carbon, but in order to prevent its combustion, he placed it in a closed vessel from which the air had been previously exhausted. Two or more of these apparatus could be placed in the same circuit of a current supplied either by batteries or by magneto-electric machines.

In 1846, Greener and Staite took out a patent for a lamp similar to King's. To free the carbon from its impurities, it was treated with aqua regia.

After that, lighting by electricity completely fell into oblivion till 1873, when a Russian physicist, Lodyguine, took up the question. His researches earned him a prize from the St. Petersburg Academy of Sciences.

The advantages of carbon were then very clearly defined by Wilde, who was entrusted with the report on the award of the prize. "Carbon has at equal temperature a greater radiating power than platinum, its thermal capacity is much smaller, so that the same amount of heat raises a carbon point to a much higher temperature than a platinum wire. Besides, the electrical resistance of carbon is about 250 times 
greater than that of platinum; the carbon may, therefore, be made much larger while raising the temperature as much as the metal. Finally, carbon is infusible, and its temperature may be raised without any danger of fusion."

Kosloff, of St. Petersburg, who came to France to work Lodyguine's patent, slightly improved the lamp, but without obtaining any satisfactory results.

In 1875, Konn constructed a similar lamp; in 1876, Bouliguine constructed another, with a single carbon.

In 1879 appeared the American (Sawyer's) lamp, in which the incandescent carbon is placed in an atmosphere of nitrogen, in order to prevent its combustion.

In all these lamps, although the carbon does not burn in the true sense of the word, there is yet a sort of disintegration or evaporation which gradually destroys the incandescent carbon. This evaporation is, moreover, clearly proved by a deposit of sublimated carbon, in the form of a very fine powder, on the interior surface of the globes and the different interior parts of the apparatus.

Incandescent Lamps with Platinum Wire.-It has been mentioned on p. 323 that the first lamp of this kind was constructed by De Moleyns, in 1841, and a similar lamp by Pétrie, in 1849.

In 1857, De Changy invented a system, permitting the divisibility of the electric current. According to a letter addressed by M. Jobard, Director of the Musée Industriel Belge, to the Académie des Sciences in Paris, Changy obtained, with a battery of twelve Bunsen elements, twelve lights, of great steadiness and independent of each other; but the terms of the patent are so vague, that it is impossible to understand the exact working of Changy's apparatus. 
Edison's Lamp with Incandescent Platinum Wire.-This lamp consists of a spiral platinum wire ${ }^{1}$ traversed by an electric current. To prevent the fusion of the wire, a metallic rod, placed inside the spiral and heated by it, establishes by its expansion a direct communication; a short circuit is formed between the two terminals of the apparatus, and weakens for a short period the current traversing the luminous spiral. The spiral is cooled, and also the metallic rod, which contracts and thereby breaks the short circuit. The current traverses anew the spiral platinum, and the same series of phenomena are exhibited. But as these phenomena of heating and cooling are very rapid, Edison's apparatus was little else but an electrical vibration instrument.

In Lontin's lamp the adjustment was effected by the variation of intensity of the current, that is to say, by the cause and not by the effect.

Maxim's incandescent platinum lamp was similar to that of Edison; the adjustment was likewise effected by the expansion of the foil.

Edison's Incandescent Carbon Lamp.-After having unsuccessfully attempted to produce light for practical purposes, by the incandescence of platinum wires traversed by a current, Edison has had recourse to specially prepared carbon, and this special preparation constitutes the very essence of his invention.

Edison's lamp, patented in 1879 (Fig. 175). consists of

1 Most of the incandescent metallic lamps hare spiral wires. The object of this arrangement is to concentrate the heat of the current within a small space, in order to raise the temperature of the body to its maximum, and consequently to obtain from it a large quantity of light. A platinum wire, traversed by a current of given intensity, is carried to red heat when stretched out, whilst it reaches white heat when coiled into a spiral. 
carbon in the form of a horse-shoe of five to six centimetres in length, and three to four millimetres in width. This carbon is placed in a globe which has been so completely exhausted that the atmospheric pressure inside, according to Edison, does not exceed the $\frac{1}{100000}$ th part of one atmosphere. The extremities of this carbon are fixed to two platinum pincers $a a$, which terminate in two wires of the same metal, connected with the exterior terminals.

This carbon is prepared by cutting small sheets of brown paper in the form of a horse-shoe; a certain number of these sheets are placed in an iron mould, which is gradually heated in a muffle; at the end of the operation, the muffle is maintained at a very

Fig. 175.-Edison's lamp (patent of
high temperature during 1879). 1, Paper carbon in form of a horsea certain time.

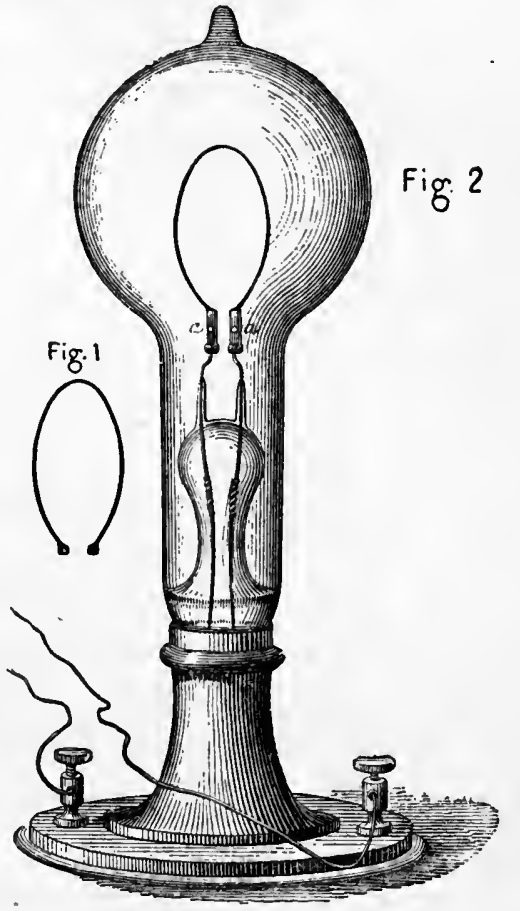
shoe; 2 , the entire apparatus.

The volatile parts of the paper disappear, and a small texture of rather fragile paper carbon remains in the mould, which, when placed in the vacuum globe and traversed by an electric current, can, according to Edison, remain incandescent for an indefinite period, without breaking or deteriorating. 
In 1880, Edison took out. a fresh patent for an improved lamp, and this is the one used at the present moment. The chief improvement is the substitution of carbonized bamboo filaments for carbonized paper. These filaments are prepared in the following way:-Canes of this material are first sawn into pieces of the required length, which are from the character of the plant naturally tubular. One of these pieces is then split longitudinally down the middle into two half-tubes, each of which is again split into three narrower

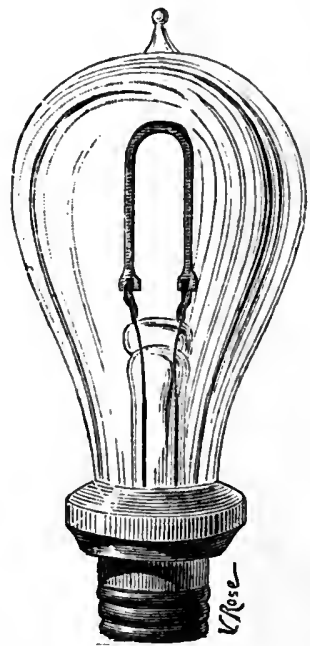

Fig. 176.-Edison lamp (patent of 1880). (From La Lumière Électrique.) strips; the hard silicious outer covering peculiar to the plants of the bamboo family is then removed, and the straight fibrous portion is shaved down till it is perfectly straight, flat, and of uniform thickness throughout, when it is cut to the required length. The next step in the process consists in pressing one of these flat strips between two metal blocks-which are accurately surfaced to one another-so as partly to project throughout the whole of its length; this projecting portion is then cut away with the exception of a little piece at each end, so as to have a fine thread of bamboo fibre having enlarged portions at either end, or, if the intended filaments are for the half-lamps, an enlarged portion is left in the middle as well as at the two ends, so that two filaments may be constructed out of the same bamboo strip. The threads so prepared are then laid in moulds consisting of nickel plates, in which grooves are cut of the required horseshoe form; a flat nickel plate is then fixed over them, so as 
to enclose them and prevent the admission of air during the process of carbonization, which is effected by raising the moulds with their contents to a high temperature by placing them in a muffle and heating them to incandescence. When the moulds are opened, the filaments are in a condition to be electro-plated to their platinum supports, and introduced into the lamp-bulbs; but yet another process is required to confer upon them their perfectly homogeneous and elastic character, as well as their refractory nature at high degrees
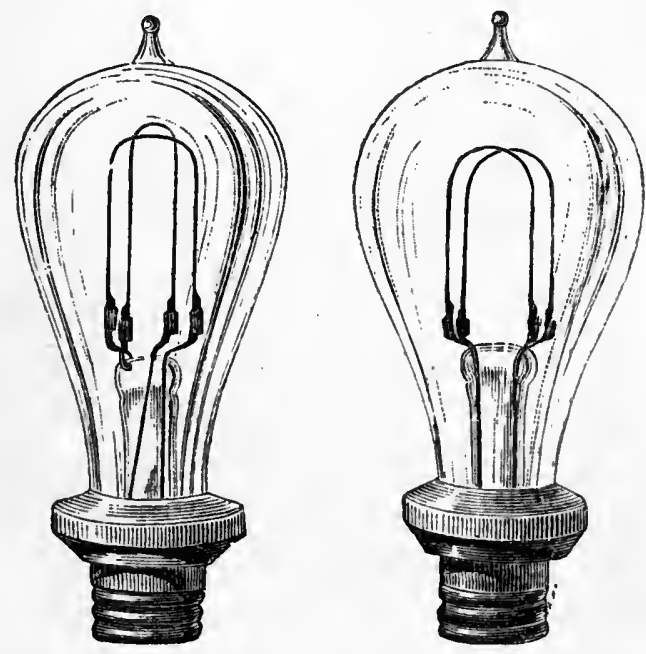

Figs. 177, 178.-Different forms of the Edison lamp.

(From La Lumiere Électrique.)

of incandescence, which are such essential characteristics in an incandescent lamp. This further process is applied when the lamp is attached to the pump, and while ex-・ haustion is going on; it consists in alternately heating and cooling the filament in the vacuum created by a Sprengel pump, by passing currents of electricity through it of increasing strength, until high degrees of incandescence are reached, and between each increase of current allowing 
it to cool down, exhaustion going on all the time. By this process, not only are all the occluded gases eliminated, but, as the fibre is subjected to a far more severe test than it can ever be subjected to in working, none but the "fittest" survive, and a healthy "generation" of lamps is ensured.

The different forms of the Edison lamp and the methods adopted for mounting them are illustrated by Figs. 175-180.
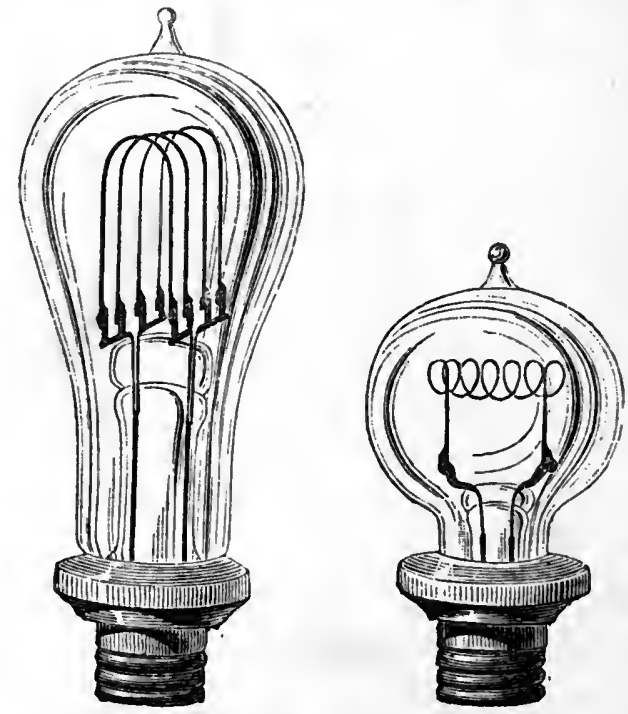

Figs. 179, 180.-Different forms of the Edison lamp.

(From La Lumière Électrique.)

In the lamp (Fig. 176) the two ends of the carbon filament are connected respectively, by means of platinum wires, to a screw and a cone, each stamped in thin brass, held at the proper distance apart, and insulated from one another by a. filling of plaster of Paris, which surrounds the neck of the envelope and forms a firm and rigid attachment. The socket into which this fitting screws, and which is in permanent connection with the conducting mains, consists of 
a cone of thin brass, below which is a hollow screw, both being embedded in a cylindrical mass of plaster of Paris, and forming a matrix or counterpart of the fitting of the lamp. The action of screwing a lamp into its socket is to draw the cone on the lamp firmly into its seat, and at the same time to press the threads of the screw against the corresponding threads of its helical socket, thus ensuring very perfect metallic contact between the terminals of the lamp and the conducting mains.

A considerable portion of the work of manufacturing the lamps is performed by machinery, by which greater uniformity is ensured; but the chain of operations by which the rough materials are converted into a finished incandescent lamp is as follows :-

The closed end of the bulb is heated and drawn out into a hollow cone. To this little cone is then fused a short fine tube, by which the envelope is afterwards to be attached to the exhausting apparatus. The next process is to prepare the glass stem, by which the carbon filament is held in its place within the globe, and within which the platinum conductors are fixed that are to join it to the connecting fitting. A piece of tube is taken, and its two ends are drawn or tapered off. It is then thickened up in two places, between which it is divided into two separate tubes; two platinum conducting wires are then passed through the tube, care being taken that they do not touch one another. The large end is then heated nearly to its point of fusion, and is squeezed flat, so as to cause the platinum wire to be closely embedded within its substance. The little cranked pieces are then soldered to the ends of the wires, and to these are attached, by an electro deposit of pure copper, the ends of the carbon loop described above. The glass stem, with the carbon 
filament attached, is then introduced into the neck of the bulb, to the mouth of which its thickened portion is attached by fusion, so as to form with it one piece of glass. The drawn-down end is trimmed off square. The exhausting tube is drawn down in two places, so as to form two capillary contractions. This tube having been attached by a mercury joint to a Sprengel pump, the bulb is exhausted, and when the requisite exhaustion is complete, the contraction nearest the pump is sealed up, leaving a short length of tube attached to the capillary neck, but exhausted to the same degree of internal residual pressure. A second sealing up of this neck renders the hermetic closing of the lamp complete, the object of the double sealing being to guard against any leaking into the envelope at the moment of detaching it from the pump; and, by fusing a little globule of glass upon the sealed-up point, all possibility of an opening being left into the outer air, however minute, is removed. Nothing now remains but to attach the terminal fitting to the stem of the bulb, and the lamp (shown in Fig. 176) is complete.

The different methods adopted by Edison for applying the lamps to commercial or to domestic use, the connections, and otner details of installation, will be found in the next chapter, p. 464.

Swan's Incandescent Lamp.-This lamp was constructed several years ago, but has only given satisfactory results since Swan has made certain improvements in the preparation of his carbon filaments, which finally led to a complete success. Swan's lamps are now extensively used in this country. As regards their efficiency and illuminating power, it appears that fifteen lamps can be fed by one horsepower, and give a whiter and more brilliant light than 
other lamps of similar construction with the same motive power. Swan does not employ a special generator of his own; he uses sometimes Faure's accumulators, Siemens' or Brush's machines, and also De Méritens' alternating-current machine (p. 111). This latter seems to give particularly good results; for with continuous currents of great intensity the carbons sometimes break off at the positive pole.

Fig. 181 represents the lamp with its mounting, which can easily be adapted for a supporting bracket or chandelier. It consists of a glass globe containing two platinum carbon-holders, with jaws and tightening rings, exactly like the brass crayon-holders which were formerly used for drawing. The carbon filament, much thicker at the two extremities, is bent in form of a flat helix, so as to form a ring in the centre of the glass globe, where an accumulation of heat is required.

The preparation of these filaments of carbon is somewhat different from that of the Edison carbons. They are made of strings of cotton about ten centimetres in length, the ends of which are thickened by winding some additional cotton round them.

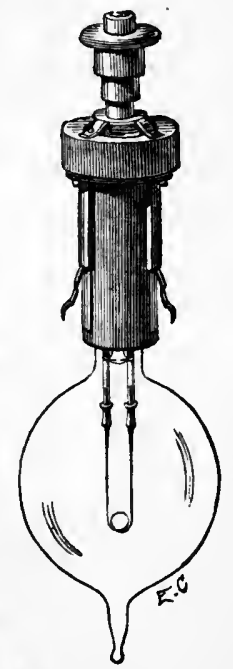

Fig. 181.-Swan's lamp. These strings are inmersed in dilute sulphuric acid (one part of acid to two of water), and, after a certain time, assume the compactness and hardness of parchment. They are then placed in an earthenware vessel full of carbon dust. This vessel is hermetically closed and raised to white heat for a certain time, after which the carbons are placed in the lamps, which have been exhausted by means of the Sprengel pump, and electrically heated to incandescence in order to 
drive out all the gases which might be contained in the pores of the carbons. After this operation, which may last

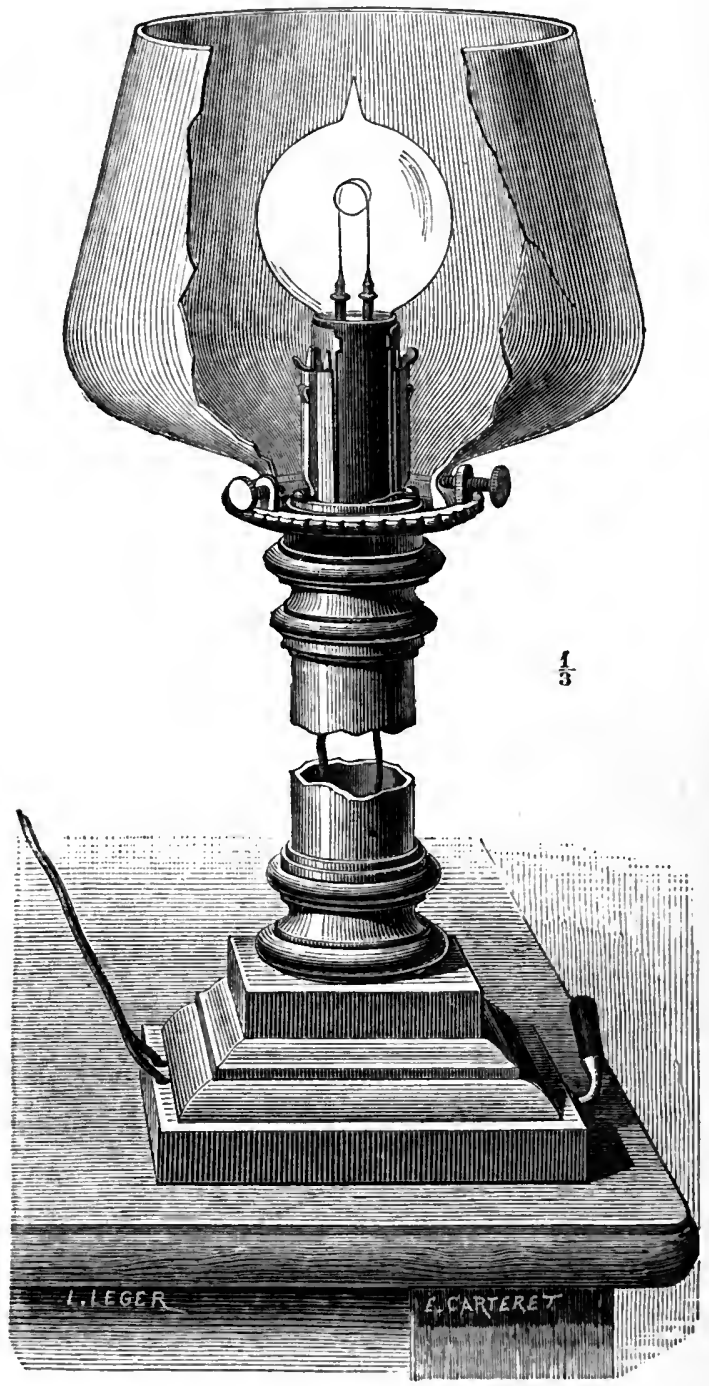

Fig. 182.-Swan's portable lamp. 
Fig. 182 shows how Swan has adapted his lamp to a portable stand, and Fig. 183 shows the reversed side of this stand, with the electrical connections. Fig. 184 shows Swan's lamp for lighting mines. The latest form of the Swan safety-lamp will be found in the next chapter on p. 400.

The most recent form of lamp devised is that illustrated by Figs. 185 and 186, which is due to Mr. Gimingham, of
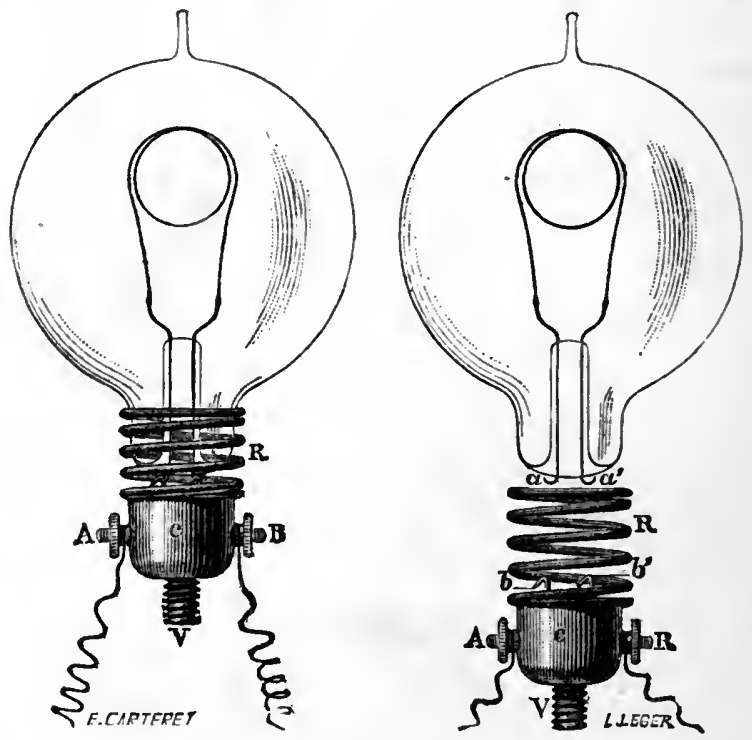

Figs. 185, 186.-Swan's lamp (latest form).

(Fiom La Lumière Électrique.)

the Swan Electric Light Company, and which, while being neater and simpler than those of the older types, can be produced more cheaply. The exterior of the lamp is entirely of glass, and from the short stem at the top project two sinall platinum loops $a a^{\prime}$, the terminals of the platinum wires carrying the carbon filament. 'The lamp connection is shown in Fig. 186, and is about as simple as can be 
desired. It is an ebonite stud $c$, with a screwed plug $\mathrm{V}$ for attachment to a gas-bracket or other stud; on each side of the stud are the binding screws $\mathrm{A}$ B, and in connection with them are the two small platinum hooks $b b^{\prime}$ projecting from the stud. Attached to this latter is also a light spiral spring R. Fig. 185 shows the lamp connected up, and from this figure it will be seen that the loops of the platinum terminals are passed over the platinum hooks on the stud, compressing the spiral spring round the neck of the lamp, and thus making a most efficient fastening, in which the electrical contact is perfect, and at the same time there is a total absence of rigidity. Not even the fittings of Edison's lamp can compare with this graceful and simple device. The exhaustion of the bulbs, which must be very perfect for the successful working of the lamp, is effected by means of a modification of the Sprengel pump, constructed by Mr. Gimingham.

Some further details of installation will be found in the next chapter, p. 424, and also an account of the latest form of Swan's mining lamp on p. 399.

Lane Fox's Lamp.-Fig. 187 gives a sectional view, and Fig. 188 a perspective view, of the lamp. The light results from the incandescence of a continuous conductor made of a filament of carbon. This conductor is of high electrical resistance, so that a comparatively small amount of electricity driven through it develops sufficient heat to produce incandescence. The filament is enclosed in a glass globe, from which all deleterious atmosphere has been as completely removed as possible.

In the diagram of the lamp, the curved line A represents the luminous conductor, which consists of a carbon filament, prepared by baking a strong cotton thread or string in a 
hermetically sealed vessel at white heat. Conductors may be made in this manner with a resistance of several thousand ohms for only a few inches length.

In order to reduce this resistance and to produce a harder and more durable carbon, Lane Fox adopted a plan, first suggested by Sawyer, of New York, of heating the

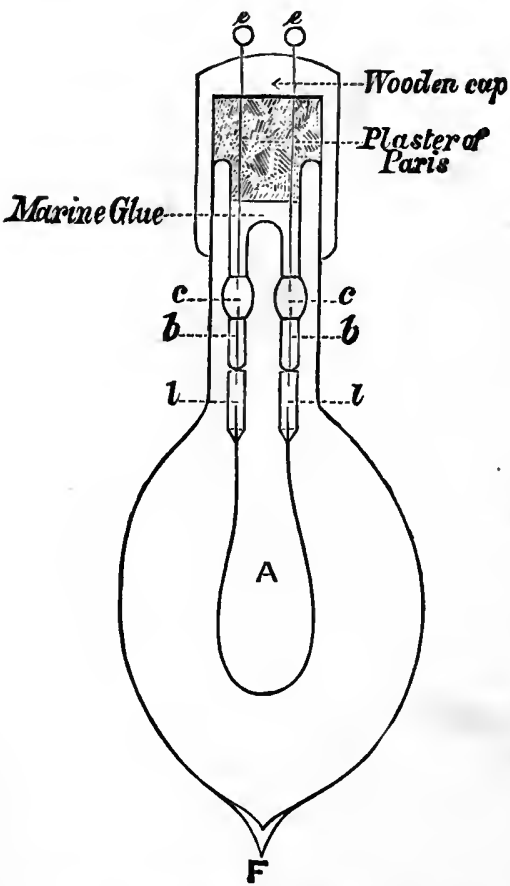

Fig. 187.-Lane Fox's lamp (sectional view).

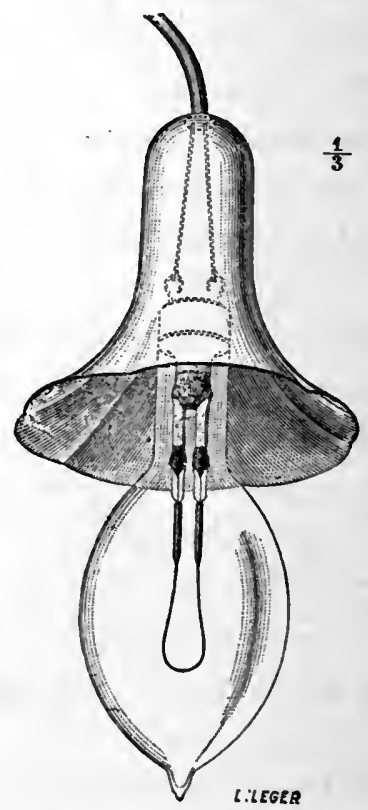

Fig. 188.--Lane Fox's lamp (elevation).

conductor to incandescence whilst immersed in a hydrocarbon liquid or gas; but this process is not by any means essential. Referring again to the diagram, $l l$ are carbon cylinders, into which the ends of the carbon filaments are secured. A small hole is drilled through these cylinders, and into these holes the ends of the carbon thread fit 
tightly. A very good cement for fixing these carbons may be made out of plumbago and Indian ink. The ends of the platinum wires are beat into eyes $e e$, into which the hooks of the holder are inserted.

Figs. 189 and 190 show the latest form of the Lane Fox lamp.

The filament employed in these lamps is made from grass fibres, preferably that known as French whisk, or bass broom, and used in making certain kinds of carpet brushes. The fibre is first cleaned by boiling in a strong solution of caustic soda or potash, and the outer skin scraped off. The soda or potash is then boiled out of it, and a number of fibres are stretched round a mould or shape of plumbago, and are then baked in a plumbago crucible at a white heat. After being baked in this manner, the fibres are further carbonized by depositing carbon upon them from a rich hydro-carbon gas, such as benzole. For this purpose they are suspended in large globes filled with benzole or coal-gas, and then heated to incandescence by the current. The white-hot filament decomposes the gas, and carbon
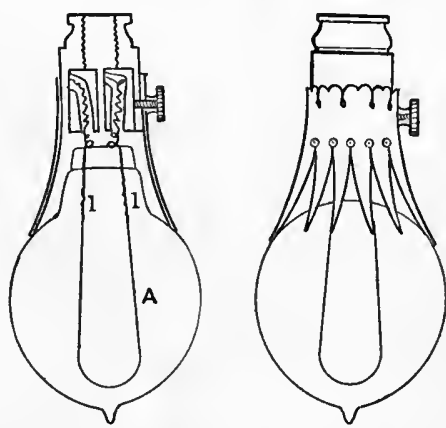

Figs. 189, 190.-Lane Fox's lamp (latest form). is deposited on its surface, especially at the thinner parts, where the temperature is highest. In this way the fibre is covered with a hard skin of carbon, which brings it to the required resistance and renders it more uniform throughout-a point of some importance as affecting the durability of the filaments. Instead of employing the electric current in this way, Mr. Lane Fox carbonizes his 
filaments by raising the benzole receptacle to a white heat in a furnace.

After being carbonized in this manner, the fibres are classed according to their thickness, and are ready for mounting in the lamps. Slight differences of thickness

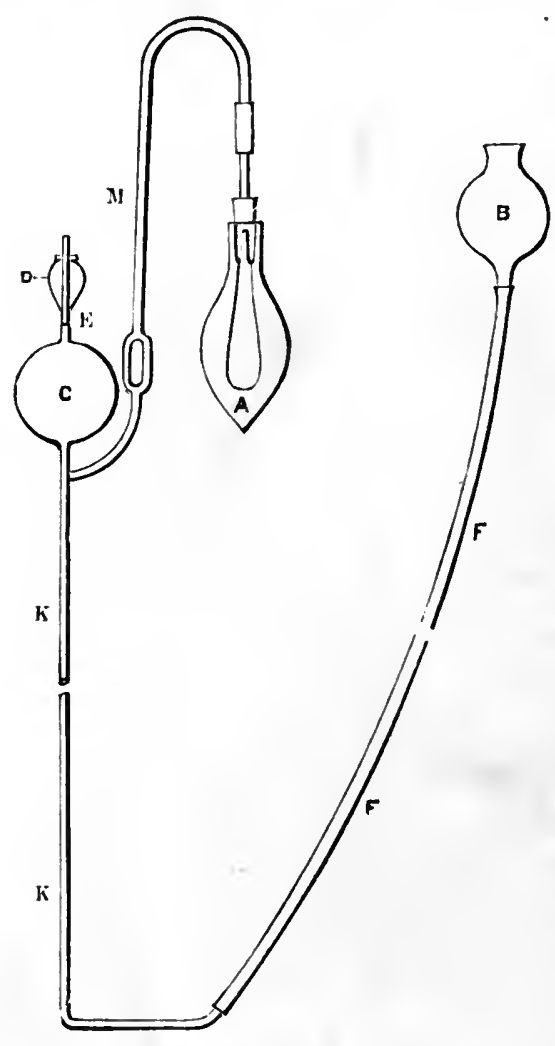

191._Lane Fox's air-pump. occasion great differences in resistance, and the thicker specimens are reserved for lamps of thirty to sixty candle-power, whilst the smaller ones are kept for lamps of ten to twenty candle-power.

The exhaustion of the bulbs is effected by a very simple and ingenious mercurial airpump, invented by $\mathrm{Mr}$. Lane Fox. It consists (Fig. 191) of a vertical glass tube $\mathrm{K}$, terminating in a bulb $\mathrm{C}$, at the upper end of which is a ground neck $\mathrm{D}$ with stopper E. At the lower end of the glass tube $\mathrm{K}$ is attached a strong flexible tube $\mathrm{F}$ of greater length, to the other end of which is fitted an open glass vessel $B$. To the glass tube $K$, just below the bulb $C$, is connected a vertical tube $M$, which is connected with the lamp $\mathbf{A}$ to be exhausted. 
The action of the pump is as follows:-The vessel B having been filled with mercury when in a lowered position, is raised to such a height that the vessel $\mathrm{C}$ is filled. The neck of $\mathrm{C}$ is then closed with the stopper $\mathrm{E}$, and the vessel $B$ lowered to allow the mercury to fall well below the junction of tube $\mathrm{M}$, which produces a partial vacuum. The vessel $\mathrm{B}$ is then raised, the stopper $\mathrm{E}$ being removed to allow the air to escape.

This operation is repeated till a complete vacuum is produced in the lamp, and is continued while the filament

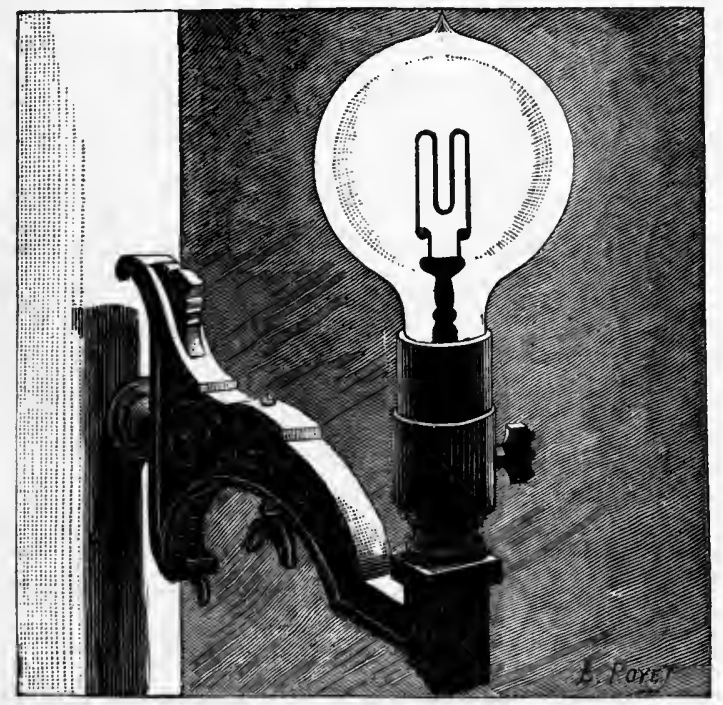

Fig. 192.-Maxim's lamp.

is maintained in a state of incandescence, when the tube connecting the lamp is fused off by the glass-blower.

Mr. Lane Fox's arrangements for distributing, regulating, and measuring the current will be found in the next chapter, p. 480.

Maxim's Incandescent Carbon Lamp.-The filament, which 
is of the shape of an M (Fig. 192), is made of carbonized cardboard. The globe is exhausted, and a certain quantity of hydro-carbon vapour (gazoline) introduced, then the globe is again exhausted whilst the current passes, and the operation repeated until all the air is expelled. It bas been proved by experience that by the passage of the current the rarefied vapour of gazoline has a renovating effect, by depositing its carbon on the most incandescent, that is to say the thinnest, parts of the filament. The filaments are connected to the platinum pincers by means of soft carbon, which gives an excellent contact. The conductors are sealed into a special enamel which has nearly the same coefficient of expansion as platinum; this prevents cracks and contributes to the tightness of the apparatus. The lamps are fixed to an ebonite mounting, which terminates in a pedestal of German silver; this latter can be screwed on a gas-bracket instead of an ordinary burner.

Maxim's arrangements for distributing and regulating the current are given in the next chapter, p. 489.

Radiwanowski's Incandescent Lamp. - This lamp, quite recently invented by Captain Radiwanowski, of the Russian Engineers, consists of a simple small tube of lime or magnesia, enclosing a certain quantity of carbon, or of a carbon filament covered with a layer of lime or magnesia. The electric current, passing through this tube or carbon, first raises the carbon to red heat, and afterwards the magnesia, which gives, according to the inventor, a beautiful light, similar to the Drummond light, but more intense. The quantity of electricity required for feeding this new electric incandescent candle is less than that required for the Edison and Maxim lamps. The lamp is, moreover, much 
simpler and requires no exhaustion. It remains to be seen how it will operate in practice.

Various Systems of Lighting.-In certain special cases, particular systems of electric lighting have been used, of which we must say a few words.

Gaiffe has combined a lighting system for mines, consisting of a Ruhmkorff coil and a Geissler's tube, surrounded by a second thicker tube to protect it against shocks. The miner carries on his back the battery and the coil packed in a small knapsack. The lamp is held in the hand by flexible wires of a certain length; it can also be suspended, if required.

Planté has proposed the use of his secondary battery for the same purpose. Jablochkoff used kaolin plates in 1878, which he heated by high-tension currents, developed in an induction coil by the alternating currents of a Gramme's machine. The light is very steady and very beautiful ; it varies, according to the power of the source, between two and fifteen Carcel burners. It is, in reality, an incandescent light, but the plate must previously be heated to make a conductor of it, by joining the two electrodes which are connected with the kaolin plate, with a piece of gas carbon. A spark is passed at a given point, the carbon becomes red hot, transmits its heat to the nearest particle of kaolin, and the current passes, first on a small area, which gradually enlarges as the carbon slides along the kaolin. Radiation makes the luminous band appear much larger than it really is. The waste of the kaolin plate is imperceptible, and does not exceed one millimetre per hour.

Jablochkoff has obtained even better results with this system by interpolating in the circuit of an electro-magnetic Alliance machine a condenser and a kaolin plate. In this 
case, he does not employ an induction coil ; the kaolin plate is fed by successive discharges of the condenser.

Some inventors have proposed the use of certain powders rendered incandescent by their passage through a voltaic arc. Way lit up threads of mercury traversed by the current; the mercurial vapours given off by this lamp poisoned the inventor.

Of all these more or less successful attempts, there remain at present only two general methods of electric lighting-

1. The voltaic arc, by arc lamps and candles.

2. Incandescence, by carbon, platinum, iridium, and other bodies of moderate conductivity. 


\section{CHAPTER IV.}

\section{THE APPI.ICATIONS OF ELECTRIC LIGHTING.}

THE applications of the electric light daily assume more importance, and it would lead us too far to enumerate all the experiments made in this direction since Deleuil and Archereau first attempted, in 1842, to use it for practical purposes.

We shall confine ourselves to the description of some of the most important, from the powerful light of lighthouses, equal to 2000 Carcel burners or more, to incandescent light whose illuminating power scarcely attains one-tenth of a candle, and which is used in surgery for lighting obscure cavities.

The E'lectric Sources considered from the Point of View of their Application for Lighting Purposes.

After having described the different modes of producing the electric current, and the apparatus which transform the electricity they receive into light, we have to examine the value of each of these electrical sources with regard to the luminous centre which it is destined to feed, before entering upon the description of the various applications which have been made. 
We shall follow, in this short review, the same order that we have adopted for the description of electric generators.

Liquid Batteries.-Although, theoretically, electric light can be produced by any battery, provided we have at our disposal a sufficient number of conveniently arranged elements, there are very few liquid batteries capable of producing light easily and inexpensively. The great strides electric lighting has lately made only date from the use of powerful machines-about ten years ago. ${ }^{1}$ The introduction of the Jablochkoff candle, in 1876, gave a new impetus to inventors, and especially to speculators and speculations. Before the employment of machines, the Bunsen battery in its different forms was used for lighting on a large scale, with Serrin's regulator; for optical projections and stage purposes, with Foucault's and Duboscq's regulators. It is still used at the Paris Opera-House for scenic effects, but there is very little doubt that it will, before long, be replaced by an electric machine. The use of batteries for electric lighting is now exclusively restricted, with a very few rare exceptions, to the lecture-room, the laboratory, and for optical projections.

Quite apart from the question of expense, which forbids the use of batteries, the manipulation of a large number of elements-forty at least of moderate strength are required to produce a somewhat stable voltaic arc-presents serious difficulties and drawbacks, and the vapours given off are very injurious into the bargain.

In spite of these numerous drawbacks, Tommasi attempted, in 1879, to render the Bunsen battery available

1 The application of the Alliance machiues for the electric lighting of the lighthouses of La Heve has existed since 1863. 
for domestic lighting. Tommasi's element, which presents no particularly novel features, in spite of the high-sounding name of "perpetual battery" given to it by the Abbé Moigno, does not in any way fulfil the aim of its inventor, or of the company working the patent. We have not heard of a single application of this system of lighting, which was established with a capital of two millions of francs.

The same may be said of Cloris Baudet's so-called impolarizable battery. Émile Reynier, more than any one else, was interested in finding a practical battery which, if applied to his lamps, would have opened for them an immense field of application. After having tried all the known batteries capable of giving any results, he has come to the conclusion that none of them could satisfy the conditions required for the application of electricity for domestic lighting. Tommasi's and Cloris Baudet's batteries have not modified this conclusion. The field, therefore, remains open to inventors, who may be more successful in the future.

Thermo-Electric Batteries.-The application of thermoelectric batteries for lighting is of a very recent date. The credit of having first used these appliances for the said purpose, in 1879 , is due to Clamond.

With the battery of 6000 elements, described on p. 48 , Clamond has worked two Serrin's regulators specially constructed for this battery, and has obtained a light varying from thirty to fifty Carcel burners for each regulator.

This is a very promising result, although only moderate from an economical point of view.

Secondary Batteries.-Although the secondary battery is not an electric generator in the proper sense of the word, 
it can render services in several special applications, of which we will only quote a few.

Secondary batteries can produce, after having been charged for a few minutes with two Bunsen elements, a voltaic arc of a few seconds' duration and of great intensity, on employing a sufficient number of secondary couples. This light can be used for signals, photography, and generally in all cases where only a short period of lighting is required.

To state the problem from a more comprehensive point of view, secondary batteries will enable us to utilize natural forces, such as waterfalls, winds, and tides, for electric lighting.

It is no mere Utopian fancy which holds out the promise of similar applications; for they rest on a philosophical principle-the turning to account of wasted, or rather non-utilized forces; and on a series of apparatus which all exist in reality-windmills, turbines, electrical machinery, and storage batteries.

The size of these batteries, and with the size their storing power, has been increased to such an extent that successful attempts. have recently been made to use them for the illumination of large buildings.

The Théatre des Variétés in Paris is lighted by 250 Swan lamps, and the electricity necessary for this installation is supplied by 270 Faure accumulators of sixty kilogrammes each. In these batteries Faure has replaced the felt by asbestos paper; it remains to be seen whether the apparatus has gained in durability by this change. They are discharged in series of thirty; eight of them are sufficient for the purposes of illumination, the ninth being held in reserve.

A gas machine of twelve horse-power, working twenty- 
two hours per day, charges the accumulators by means of three Siemens machines, type $\mathrm{D}^{2}$, a fourth being held in reserve.

The economical side of the question is naturally of the greatest importance, and it appears from exact calculations made by M. Fr. Geraldy, that the expense of the electric light at the Variétés is six centimes (a little more than a halfpenny) per Carcel-hour (light of nine candles per hour), while with gas the expense would be three centimes per Carcel-hour.

From another experiment made at the Grand Hotel, Charing Cross, it appears that, for the feeding of eighty Swan lamps during six hours, eighty Faure accumulators had to be charged during fourteen hours by two Edison machines, type Z. Now, each of these machines would feed directly sixty Edison lamps of nine candles each. Let us suppose the light of the Swan lamps to be likewise of nine candles each; we shall find that, by supplying the lamps from the machines direct, without the intermediary of storage batteries, 120 lamps can be fed during fourteen hours by the same mechanical work which supplies eighty lamps during eight hours only ; by the direct employment of machines we therefore obtain an illumination of one and a half times greater intensity and two and a third times longer duration. These figures speak for themselves.

Storage batteries, of course, can only give back, with a certain loss, the electro-motive force which they have received through a generator, and as a transformation of force cannot be effected without loss, the employment of accumulators for electric lighting cannot be advantageous as regards efficiency.

But-and this is a very important consideration-accu- 
mulators may render excellent service as reserve apparatus, in the case of partial extinctions or other accidents, and as additions to an established service. As long as storage batteries retain their present conditions and prices, they will hardly be available by themselves for large installations, except in cases where their charging can be effected by some natural force.

Magneto and Dynamo Electric Machines.-The different electric generators-magnetos and dynamos, continuous and alternating current machines-adapt themselves almost equally well for electric lighting. In practice, however, certain machines are specially constructed to suit certain lamps.

Monophotal lamps work equally well with all currents, provided the intensity and the electro-motive force of the current are sufficient to maintain the arc.

Polyphotal lamps, or division regulators, also employ both kinds of currents, but they are generally worked with machines of a special type, constructed, in most cases, by the inventor of the lamp. Instances of this kind are the Gramme, Siemens, Weston, and Brush machines.

For all candles alternating currents are used; the necessity of an equal waste of the two carbons renders their use absolutely obligatory. Now, alternating currents produce a very disagreeable noise, and this is in many cases a fatal objection to their employment.

Reynier's, Werdermann's, and Napoli's incandescent lamps are equally well adapted for either kind of current, but it appears that continuous currents give a higher efficiency.

The incandescent lamps, properly speaking, generally employ continuous currents, but adapt themselves almost equally well to alternating currents. 
The number of lamps fed by one single machine is also very variable. A single lamp fed by one machine constitutes a very expensive installation, and is now only resorted to in special cases; the division lamp allows two, four, six, ten, and even as many as forty, arc lamps to be placed in tension on the same circuit.

Electric candles are mounted in series, by dividing the current supplied by the generator into several distinct circuits.

Incandescent lamps also are mounted in series according to the qualities of current supplied by the machine. The tendency at present seems to be to divide the light by placing the lamps on shunts, and thus securing the absolute independence of each lamp.

The following tables, showing the efficiency of different continuous and alternating current machines, contain the results of experiments made by MM. Allard, Joubert, Le Blanc, Potier, and Tresca, at the Paris Electrical Exhibition.

These tables require a few explanatory remarks.

What is called electric horse-power and arc horse-power represents an electrical work of seventy-five kilogrammetres per second (amount of work required to raise a weight of seventy-five kilogrammes through one metre), generally calculated from the intensity, resistance, and electro-motive force; total mechanical efficiency is the ratio between the total electrical work and the effective motive power; mechanical efficiency of the arc means the ratio between the work actually measured in the are and the same effective motive power; finally, electrical efficiency of the arc means the ratio between the electrical work of the arc and the total electrical work.

As regards the values given for luminous efficiency 
corresponding to the expenditure of one mechanical horsepower, of one electric horse-power, and of one arc horsepower, it must be clearly understood that the photometric intensity designated by the term mean spherical intensity ${ }^{1}$ is used throughout these tables; it is, in fact, the only one which represents the luminous phenomenon in its entirety, and which, therefore, can be compared in a rational manner to the different elements producing this intensity. It must, however, be remarked that, by taking the requirements of public illumination as the basis of their estimates, the constructors have naturally been led to much higher estimates of the power of their apparatus.

After a long series of observations, it was decided slightly to slacken the speed of the machines ; the photometric result thereby became more favourable. The figures given in the tables correspond to these results.

As regards more particularly the employment of alternating currents, the result of only three experiments is given; these hardly allow of any comparisons being made, and in one of them no electrical measurements or calculations are given. With regard to the two others, the efficiencies are almost identical, although the photometric intensities per horse-power are in themselves very different; but this difference is explained by the fact that the light was respectively divided amongst lamps of very different intensities.

It will be seen that the number of Carcels per mechanical horse-power keeps on decreasing in the same measure as the intensity of the lamps diminishes.

1 The mean spherical intensity of a sonrce of light, for instance an aro lamp, is obtained by taking the middle point of the aro as the centre of a sphere, and dividing the sum of the illuminations of the areas constituting the surface of the sphere by the total number of areas. 


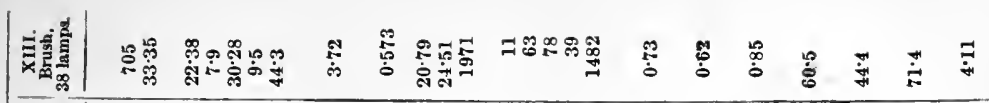

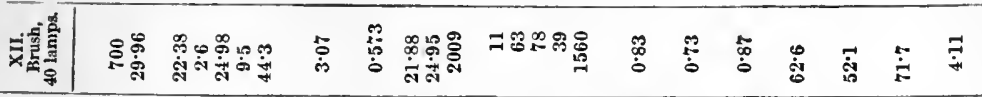

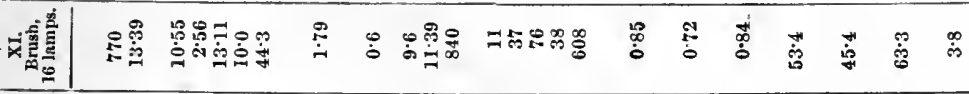

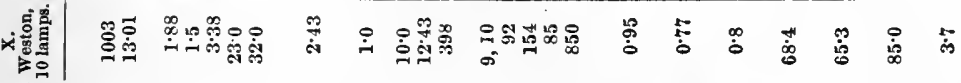

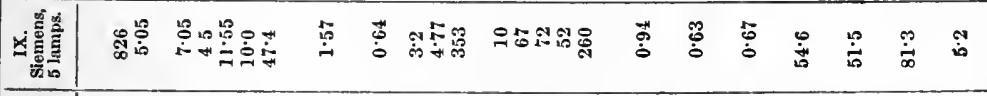

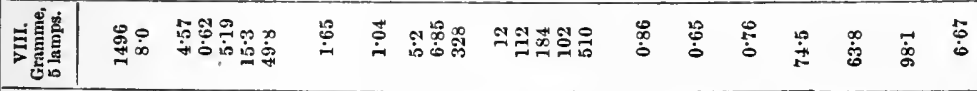

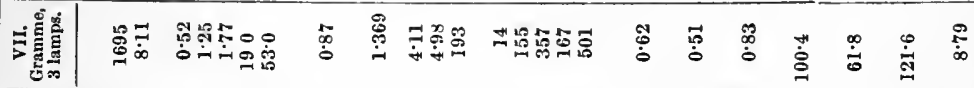

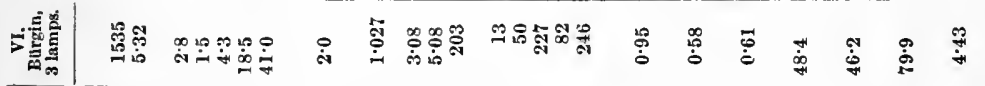

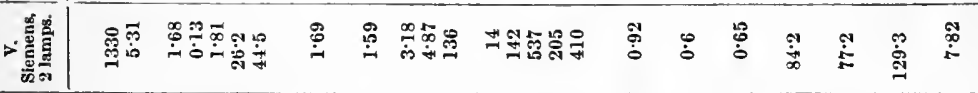

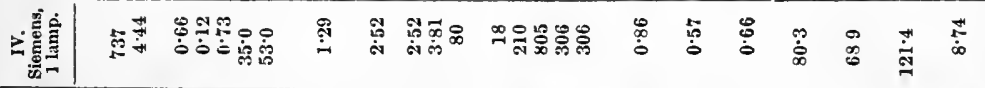

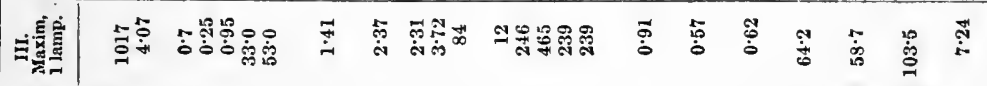

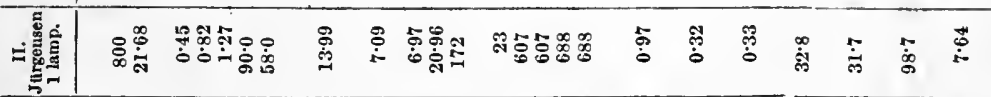

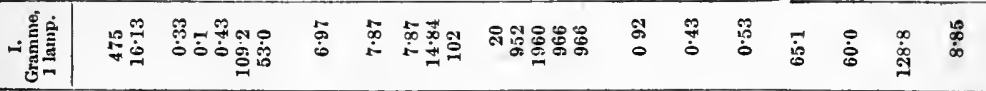

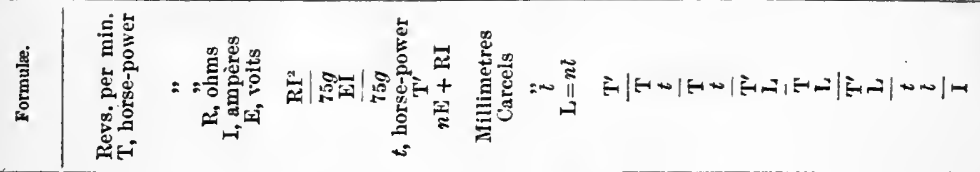

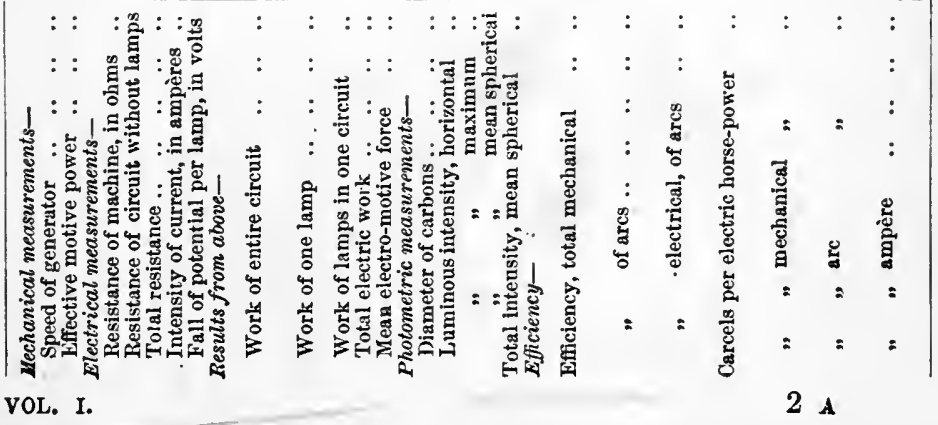


Table II.-Experinents on Alternating-Current Machixes FEEDING ARC LAMPs.

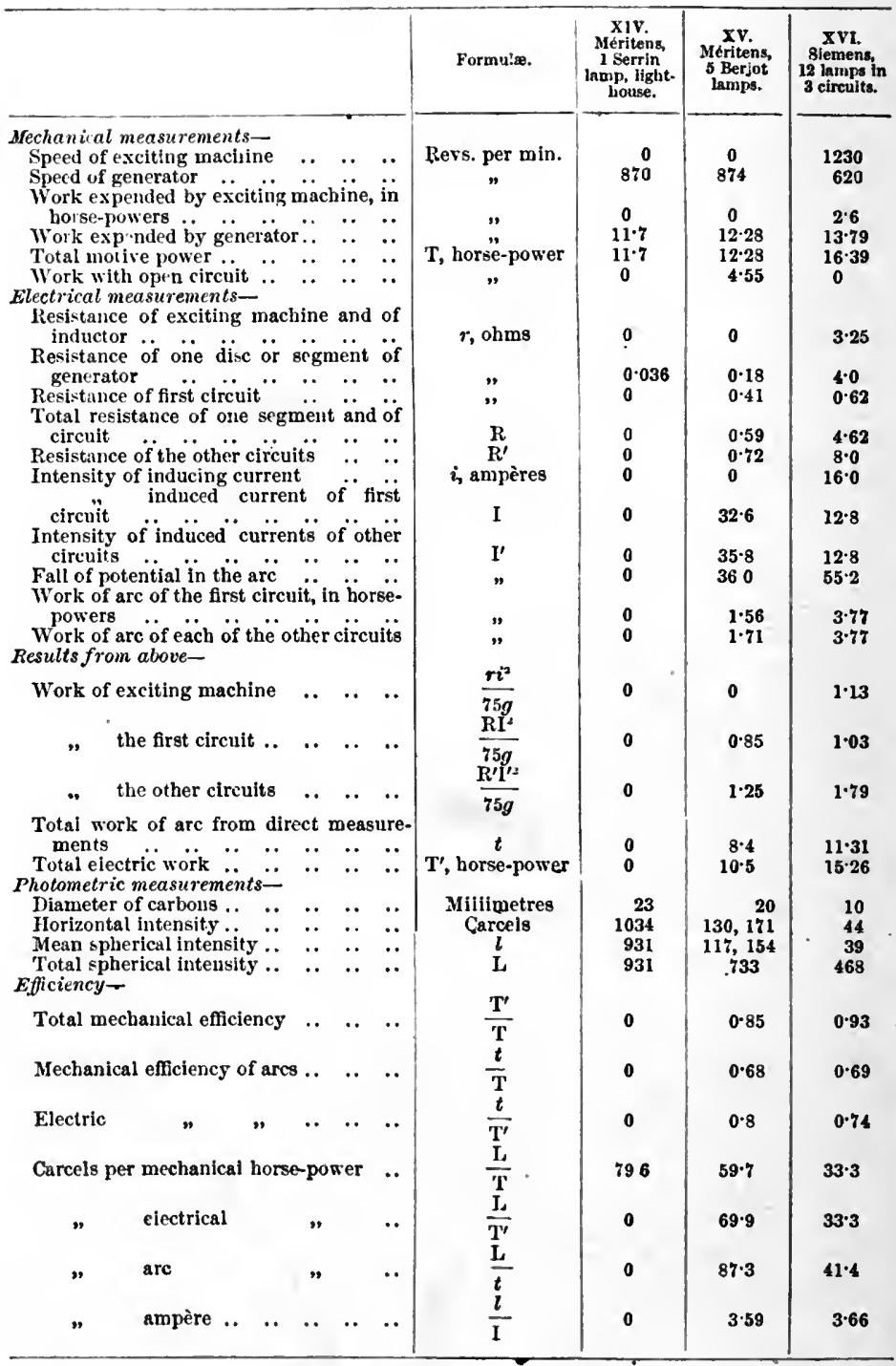


Table III.-Comparison of Mean Efficiencies of Continuous-Current Machines according to the Intensity of the LaMps.

\begin{tabular}{|c|c|c|c|c|c|c|c|c|c|c|c|}
\hline & & & & & & & \multirow{2}{*}{ Formulæ. } & \multicolumn{4}{|c|}{ Mean efficiency. } \\
\hline & & & & & & & & 1 lamp. & $\begin{array}{l}2 \text { to } 5 \\
\text { lamps. }\end{array}$ & $\begin{array}{l}10 \text { to } 40 \\
\text { lamps. }\end{array}$ & General. \\
\hline \multicolumn{4}{|c|}{ Efficiency, total mechanical .. } & $\cdots$ &.$\cdot$ & .. & $\frac{\mathbf{T}^{\prime}}{\mathrm{T}}$ & 0.89 & 0.86 & 0.84 & 0.87 \\
\hline$"$ & mechanical, & of ar & $\mathrm{rcs}$ & .. &.. & .. & $\frac{t}{\mathrm{~T}}$ & 0.47 & 0.59 & $0 \cdot 71$ & 0.59 \\
\hline$"$ & electrical, & $"$ & & $\cdot \cdot$ & $\cdot \cdot$ & $\cdot \cdot$ & $\frac{t}{\mathrm{~T}^{\prime}}$ & 0.53 & 0.7 & 0.84 & 0.69 \\
\hline \multicolumn{6}{|c|}{ Carcels per mechanical horse-power .. } & .. & $\frac{\mathrm{I}}{\mathrm{T}}$ & 55 & 60 & 50 & 54 \\
\hline$"$ & electrical & & " & &.. & .. & $\frac{\mathrm{L}}{\mathrm{T}^{\prime}}$ & 61 & 72 & 59 & 63 \\
\hline$"$ & arc & & $"$ & & $\cdot \cdot$ & .. & $\frac{\mathrm{L}}{t}$ & 113 & 102 & 71 & 93 \\
\hline$"$ & ampère & .. & 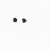 & .. & $\cdot \cdot$ & $\cdot \cdot$ & $\frac{\imath}{\mathrm{I}}$ & $8 \cdot 1$ & $6 \cdot 6$ & 3.8 & $6 \cdot 0$ \\
\hline
\end{tabular}

As regards the two series of experiments made with different arc lamps, it must be borne in mind that the object here is not to establish the superiority of one or the other of these lamps,-such a comparison could only be the result of a very large number of determinations, sufficiently varied as regards each of these lamps to establish with certainty the conditions which produce the maximum effects. The only aim was to state in a more authoritative manner the results usually obtained in practice. From this point of view, the columns relating to the different efficiencies commend themselves to our attention.

It will be seen at once that the total mechanical efficiency is exceedingly high, and this means that the currents generated are very perfectly collected in all actual machines; the small losses of work, of course, arise from the passive mechanical resistances of the different modes of installation. 
It is, therefore, beyond doubt that the work transmitted to the coil is, to all intents and purposes, represented in its totality by the available work of the electric currents themselves, excepting the work incumbent upon the iron armatures of the machines.

As regards this work, it is likewise represented integrally by the work of the ares and that of the resistances: the former only is utilized in the voltaic arc in form of heat and light; the latter is always lost in heat dispersed through the different parts of canalization.

The work of the arcs appears somewhat more favourable as regards mechanical work for machines of large resistance feeding a large number of lamps, and it is exactly with these machines that the electrical work is utilized to the greatest advantage; the electrical efficiency of the arc may thus be increased to double its value, or even more.

The number of Carcels produced by each electrical horse-power expended in the ares diminishes regularly with the falling off of luminous intensity in the lamps.

As regards the total mechanical efficiency, that depends entirely on certain local conditions, and is in no way characteristic for each of the different systems, as a reference to Table III. will show.

Before leaving the subject of the efficiency of machines, we have to mention some important researches made on this subject by M. Marcel Deprez. He has shown, by numerous and accurate experiments, that large machines are very superior to small machines, both as regards absolute production as well as relative power per unit of weight. We shall refer again to this question in the chapter on the distribution of electricity. 
It will be seen, from all that has been said, that there is no electrical source which could be recommended for universal application. Special requirements must determine the choice, as, for instance, the number of light-giving centres, their intensity, the greater or lesser need of steadiness, of simplicity, or economy.

\section{Il.LUMination of Lighthouses.}

ON the 26th of December, 1863, the lighthouses of La Hève were for the first time lighted by electricity with the

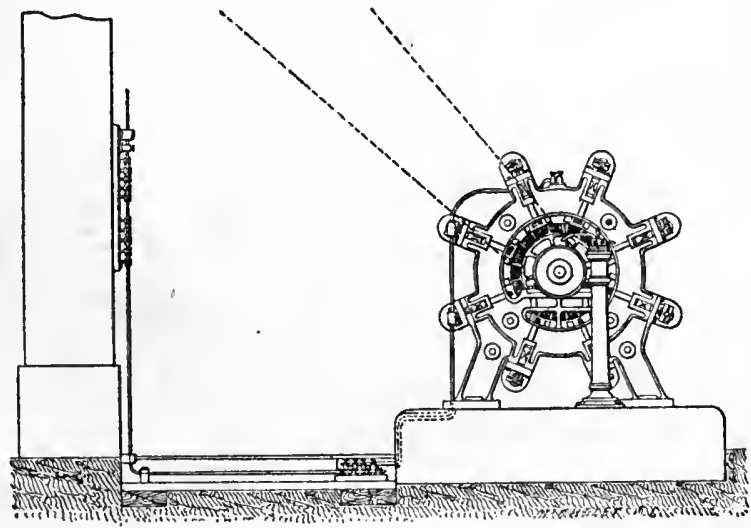

Fig. 193.-De Méritens' machine.

(From La Lumière Électrique.)

Alliance machines, and since that time all the more important French lighthouses have followed suit; and also in England and the Colonies the electric light has to some extent ousted, not only the old oil-lamp, but also the Drummond light.

The Lighthouse of Planier, near Marseilles. - Two De Méritens machines are used for the production of the light; they are not intended to act at the same time, but the second 
one is always ready, in case of any accident, to replace the other. Figs. 193 and 194 represent one of the machines. Each machine is divided into two circuits, represented by four terminals placed in the upper part of the frame-two at each extremity. The two terminals placed alongside of each other at each end of the machine are, at a given moment, of equal polarity. From each of them starts a copper conductor, which descends to the foot of the machine, passes

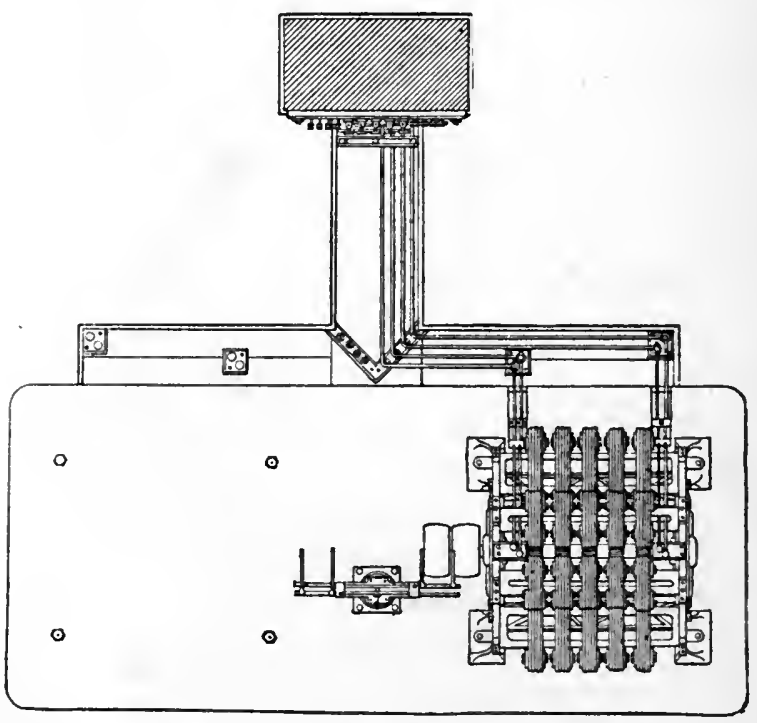

Fig. 194.-De Méritens' machine.

(From La Lumière Électrique.)

round the brickwork foundation, follows the ground as shown in Figs. 193 and 194, and reaches the commutator (Fig. 195) placed on the pillar of brickwork which forms one of the supports of the transmissions.

The commutator is intended, on the one hand, to transmit the current at will, either to machine 1 or to machine 2 , and on the other hand, to join, for tension or quantity, the two 
circuits of each machine. The four possible combinations of this commutator are represented in Figs. 196 and 197. On referring to these figures, it will be seen that the apparatus is composed of fixed contacts and of movable contacts. The former, slightly more tinted in the figures, are fourteen in number; four of them, placed on the left, are connected to the terminals $1,2,3,4$, to which are connected the conductors of the machine on the left, or machine 1. Four others, symmetrically situated on the right, are connected to the terminals corresponding with machine 2 .

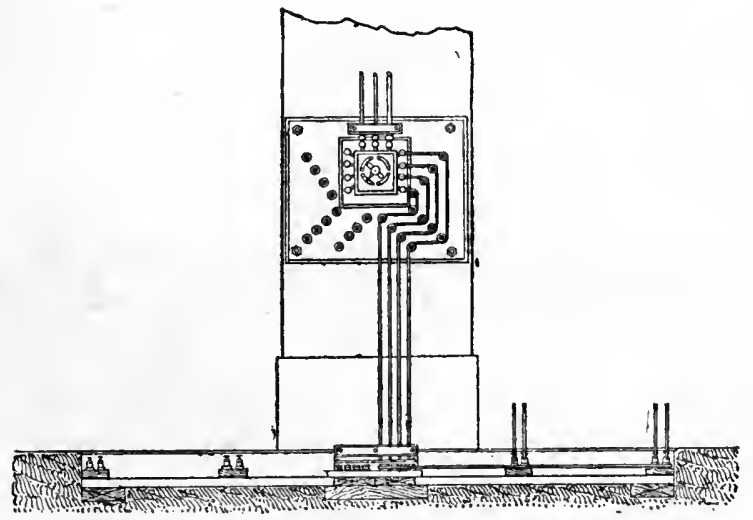

Fig. 195.-Connections of commutator with the machine.

(From La Lumière Électrique.)

The three upper contact-pieces are connected to the terminals communicating with the cables of the regulator. Here it must be remarked that the current arrives in the lamp through a stout cable, and, after traversing the arc, passes into two smaller cables, one of which leads to the electro-magnet of the regulator, the other being a plain wire. Of the three contact-pieces, the one on the left communicates with the terminal $\mathrm{E}$, to which is connected 


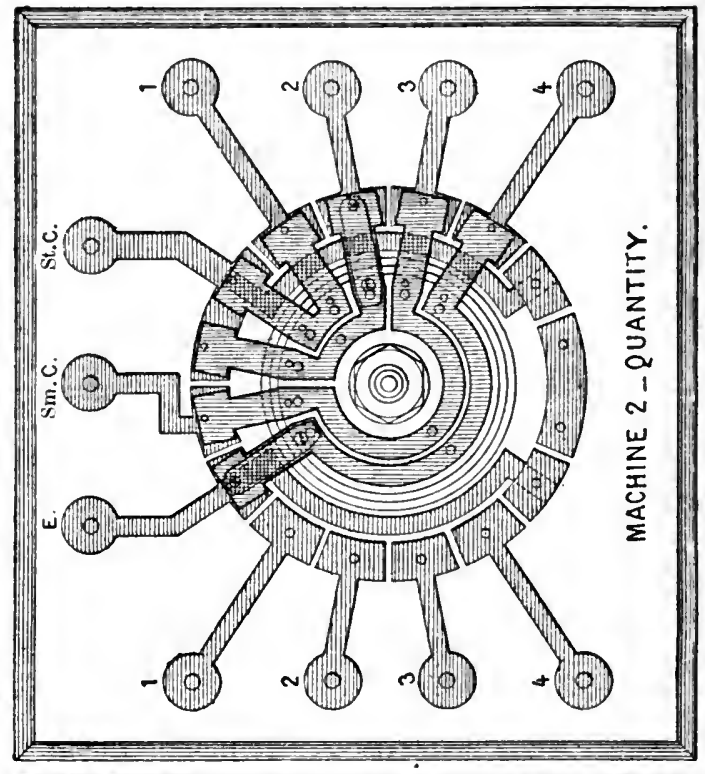

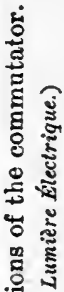

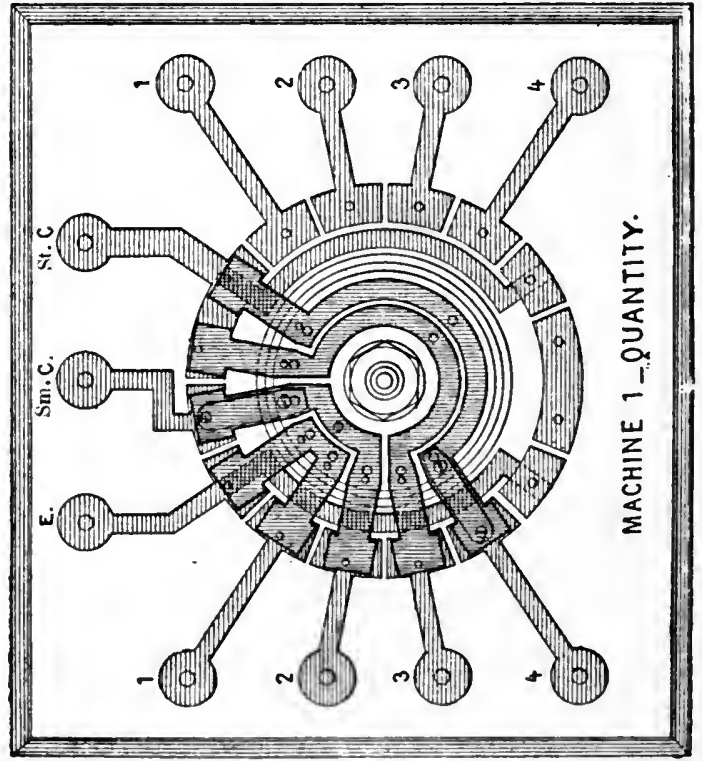

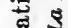

ณ

है 8

I

g 
THE APPLICATIONS OF ELECTRIC LIGHTING. 361
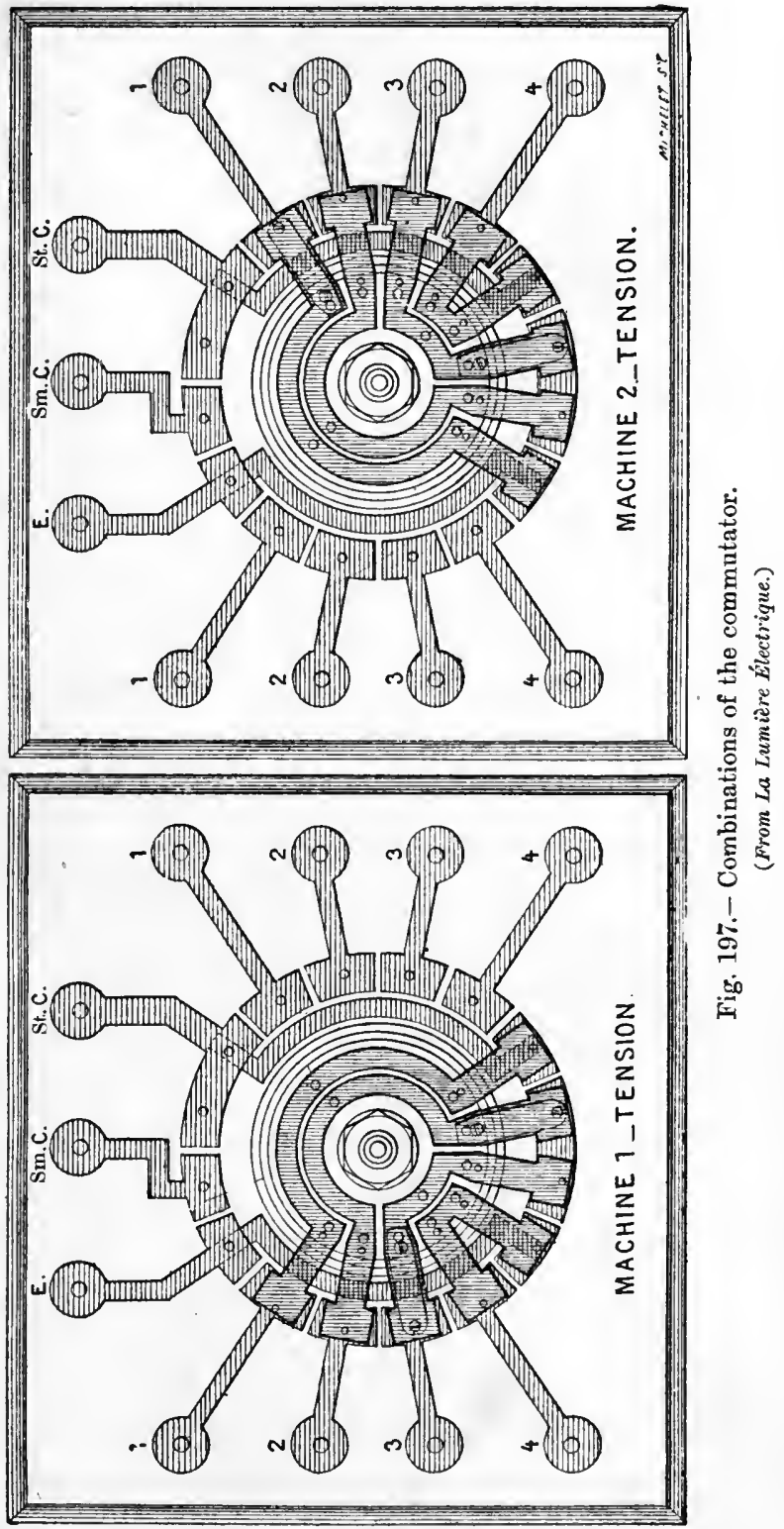
the cable of the eleetro-magnet; the following one is connected to the terminal Sm. C of the small plain wire; finally, the piece on the right, half as large again as the others, is in communieation with the terminal St. C of the stout cable. The system of fixed contacts is completed in the lower part by three pieces, of which the middle one is twice as large as the others. The two lateral ones are connected by supplementary conductors: that on the left with the piece of the terminal $\mathrm{E}$, that on the right with the piece of the terminal St. C. The movable contacts, eight in number, are represented in Figs. 196 and 197 by a stronger tint than the fixed contacts. They are all fastened to the same disc, movable round the centre of the machine. Two adjacent contacts are joined together so as to form a sort of $U$; the two following, situated on either side of the two first, form an enlarged $U$, as do also the four others, with this difference, that their junction is enlarged into the form of a segment of a circle. By means of a handle fixed to its centre, the movable disc can be made to take up different positions.

Let us suppose, for instance, that the movable contacts are in the position indicated in the figure by the words machine 1 quantity. The terminals 1 and 2 being at the same moment of the same polarity, the current enters at the same time through the two contacts corresponding to these terminals, and thence passes simultaneously into the small wire and into the electro-magnet wire. On reaching the carbon, it is united in one cable, and returns through the stout cable to the terminal St. C. On the fixed double-sized contact in connection with this terminal press two movable contacts, through which the current returns to the terminals 3 and 4. 
THE APPLICATIONS OF ELECTRIC LIGHTING. 363

When the same machine is mounted for tension, the current starting from the first circuit of the machine through the terminal 1, traverses the most open of the movable contacts, and arrives at one of the fixed interior pieces connected by a supplementary conductor to the terminal St. C; thence, following the stout cable, it passes through the carbons, the electro-magnet wire, and the terminal $\mathrm{E}$, or to the smallest

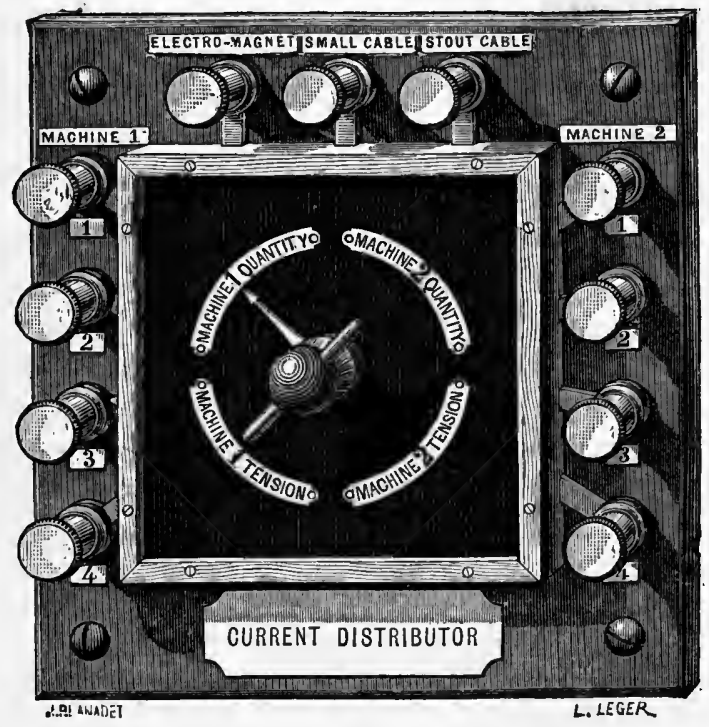

Fig. 198.-Commutator: perspective (French). (From La Lumière Electrique.)

U-shaped movable contact and to the terminal 4. It then traverses the second circuit of the machine, returns to the terminal 3, passes through the second movable contact, and arrives at the large fixed lower piece, whence it is conveyed through the third movable contact to the terminal 2 , that is, to the first circuit of the machine.

By examining the position of the movable contacts 
indicated for the two mountings of machine 2 , the path of the eurrent can be followed in a similar manner. Fig. 198 shows the perspective of this commutator, and it will be seen that the contacts are covered with an ebonite plate,

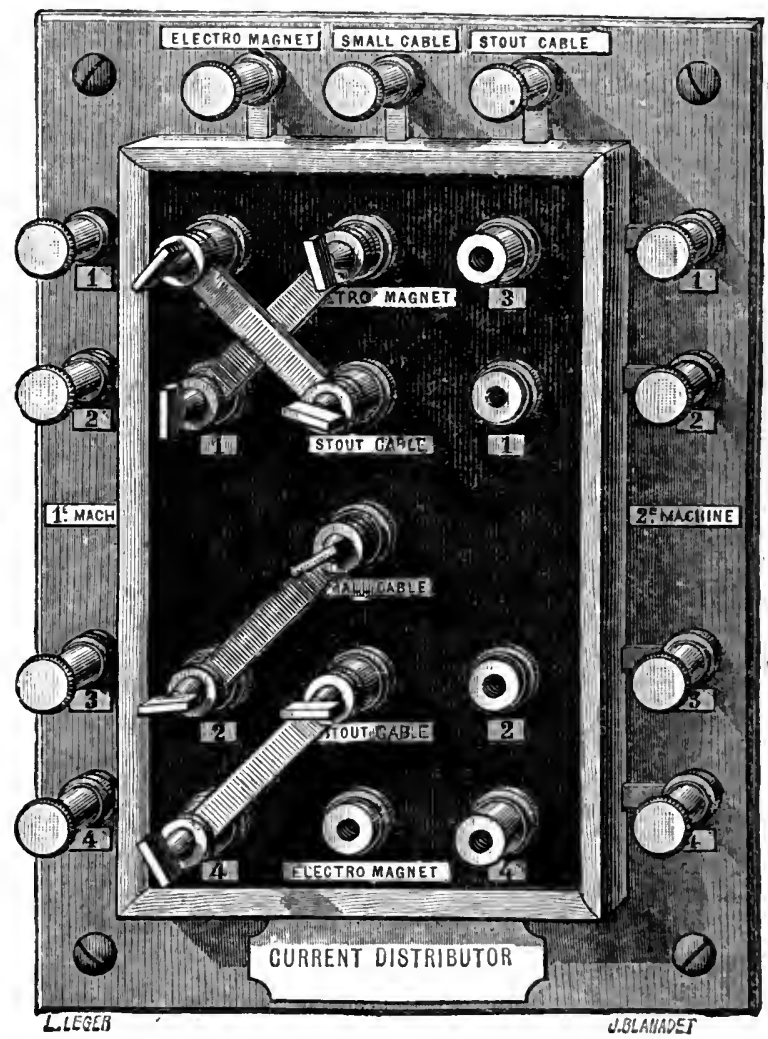

Fig. 199.-Commutator: perspective (English).

(From La Lumiere Électrique.)

which is traversed by the handle turning the movable disc. The ebonite plate carries besides four inscriptions corresponding to the different combinations of the commutator, and a movable index points to the actual combinations. 
THE APPLICATIONS OF ELECTRIC LIGHTING. 365

This system of commutator permits an instantaneous change in the grouping of the two circuits of the same

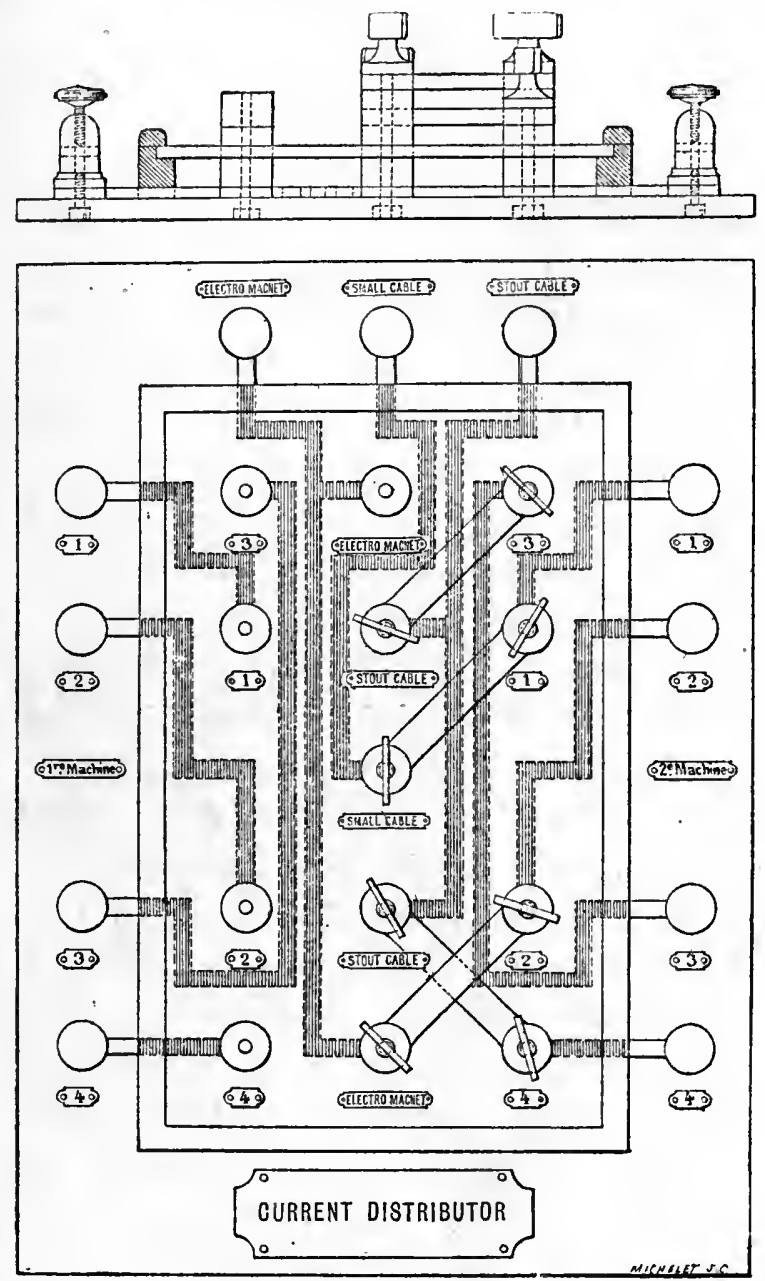

Fig. 200.-Commutator : diagram (English). (From La Lumière Électrique.)

machine, and also the immediate substitution of one machine for the other. 
The lighthouses in this country where the De Méritens machine has been adopted employ another arrangement, represented in Figs. 199 and 200. The terminals of the cables of the regulator and the conductors of the two machines have sensibly the same relative position as in the preceding case. These terminals are, by means of copper plates, connected to tightening screws, carrying the same numbers as the terminals. The tightening pieces in communication with the terminals of the machine can be connected by strong copper plates with the tightening screws corresponding to the cables of the regulator, and, in order to facilitate this connection, the tightening pieces are more or less raised, so that the plates can cross each other without touching. In this way absolutely perfect contacts are obtained, but a change of combination requires a longer time. A reference to Figs. 199 and 200 will clearly show this arrangement. The lighthouse lamp itself has been described on p. 235 .

The Dungeness Lighthouse.-The electric light was finally installed at this lighthouse on the 6th of June, 1862. The light is fixed and white. The machines used were Holmes', and the first trial with these machines was made at Blackwall, in 1857, under Faraday's supervision. The success of these experiments, and Faraday's favourable report, induced the Elder Brethren of the Trinity House to order two machines for trial at the South Foreland. The dimensions of these machines, which appear to us enormous on comparing them with the modern apparatus of equal power, were as follows:-Length 2.8 metres, width 1.7 metre, height 2.9 metres; they weighed five tons and a quarter, and carried sixty magnets of twenty-two kilogrammes each, arranged in three circles, and 160 bobbins on two wheels. The 
machine received these large dimensions because its speed was not to exceed ninety revolutions per minute; the permanent magnets were fixed, and the currents were inverted by a commutator. Each machine absorbed about two and three quarters horse-power. These machines were used for thirteen years at Dungeness; the results obtained, however, were not very favourable, and the electric light was, in 1876, rep'aced by oil-lamps.

The electric lighting apparatus at Dungeness consisted (Figs. 201 and 202) of two lamps placed above the original oil-lamps, which remained available in case of accident. Each of the former was provided with a dioptric ${ }^{1}$ lantern of 300 millimetres diameter, specially constructed by Chance Brothers, of Birmingham. Two reserve lamps were ready to act by a simple contact.

Holmes' lamps differed from Serrins' (vide p. 202) by the

1 Dioptric apparatus are those which group the light in parallel bundles of rays by means of refraction; such, for instance, are Fresnel's graduated lenses. Apparatus which act by reflection, as, for instance, parabolic reflectors, are called catoptric; they present, compared with the others, the

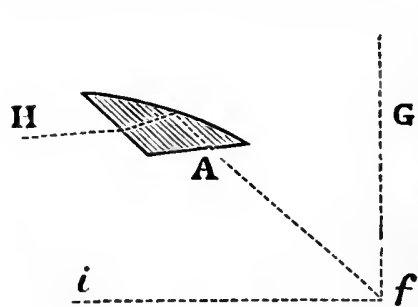

Fig. 202a.

(From La Lumière Électrique.) drawback of absorbing more light, of imparting too much divergence and less brilliancy to the reflected rays, and of G lending themselves with difficulty to a uniform distribution of light over the whole or a part of the horizon.

Catadioptric apparatus are those which act on the light at the same time by refraction and by total reflection; such is the case with Fresnel's prism or ring, represented in Fig. $202 a$, which renders the rays emitted from the focus $f$ parallel at $\mathrm{H}$. If the ring turns round $f \mathrm{G}$, it distributes the light over the horizon, and constitutes an element of fixed light; if it turns round $f i$, it groups the rays in bundles, like the lenses of eclipse lighthouses. Catadioptrio apparatus are employed as soou as the angle of the ray $f \mathrm{~A}$ with the horizontal line $f i$ attains about $40^{\circ}$. 
substitution of pulleys and cords for the pinions and the rackwork in the regulating apparatus. The carbons were six millimetres thick; the upper carbon was 305 millimetres and the lower carbon 150 millimetres long. The mean focal intensity of the light was 670 candles (75 Carcels); the mean intensity of emitted light was, under the most favourable conditions, 19,000 candles, or more than twelve and a half times higher than the old catoptric apparatus.

The steam-engines and generators were placed in the basement of the lighthouse, and the boilers in an adjacent shed, and required an increase of the staff. The expense of lighting was seven shillings per hour with the electric light, and only two shillings and threepence per hour when oil was used. It must, however, be remarked that the old apparatus only gave a focal intensity of 250 candles, whilst the new one gave an intensity of 670 candles, so that in reality the electric light was only 1.12 more expensive than the oil.

South Foreland Lighthouse.-The two lighthouses guarding the passage of the Goodwin Sands were erected in the year 1843. The focal plane of the larger one is 113 metres, that of the smaller is 89 metres above high tide. Four Holmes machines (Figs. 203 and 204) are used for the electric light, and driven by two engines of ten horse-power. The engine-shed is about half-way between the two lighthouses. The length of cable of the higher one is 210 metres, that of the lower one is $\mathbf{1 8 0}$ metres. The optical apparatus of the higher lighthouse (Figs. 205, 206, and 207) is fixed, and shows a white light, the refracting prisms impart to the rays different vertical divergences, graduated in such a manner as to extend their luminous range as far as possible on the sea, voL. I. 
starting from the two zones contiguous to the medium zone, whose divergence is $5^{\circ} 24^{\prime}$, and whose rays cover a distance of 1100 metres from the lighthouse. The back light-which would be lost in the angle of $134^{\circ}$ indicated in Fig. 206-is utilized for the illumination of the horizon by refracting it on two systems of five prisms, by means of two holophotal

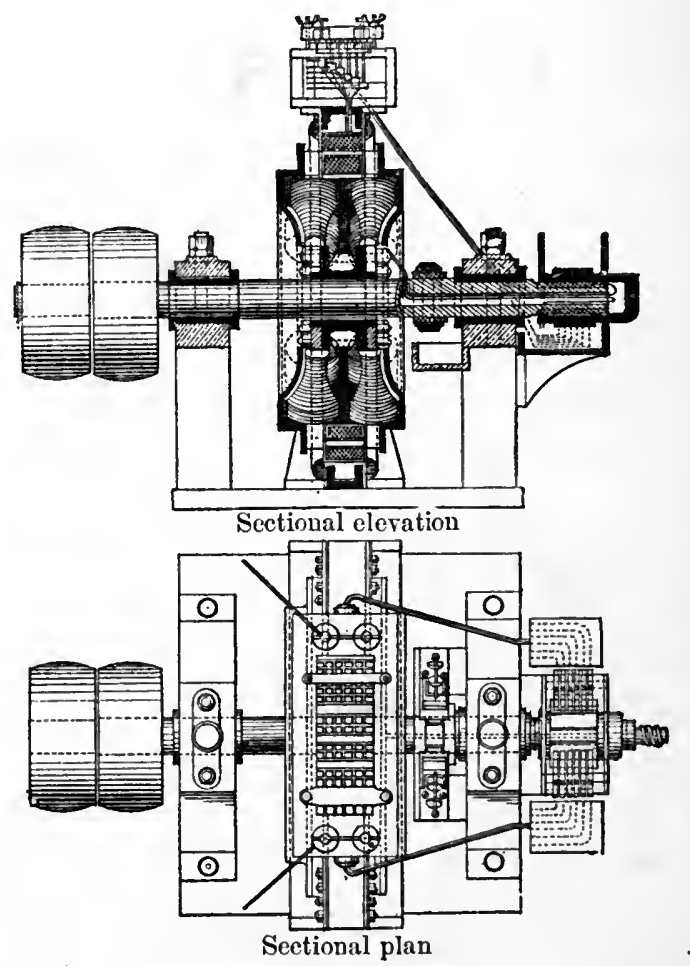

Fig. 203.-Holmes' machine.

(From La Lumière Électrique.)

half-lenses. The focal distance of the two half-lenses is $\mathbf{1 8 7} \cdot 5$ millimetres; the prisms are 533 millimetres high. The intermediary prisms are catadioptric, and of the ordinary Fresnel type; the first prism is a refractor, and the last has a trapezoidal section, which enables it to 
THE APPLICATIONS OF ELECTRIC LIGHTING. 371

reflect the light at an angle of more than $90^{\circ}$. These prisnis reflect and refract the light on half of the illuminated horizon. The angles of emergency of each of these prisms are independent of each other, and calculated in such a manner as to give to the luminous bundle a uniform intensity. This auxiliary apparatus is by Messrs. Stephenson and Swan.

The optical apparatus of the smaller lighthouse (Fig.

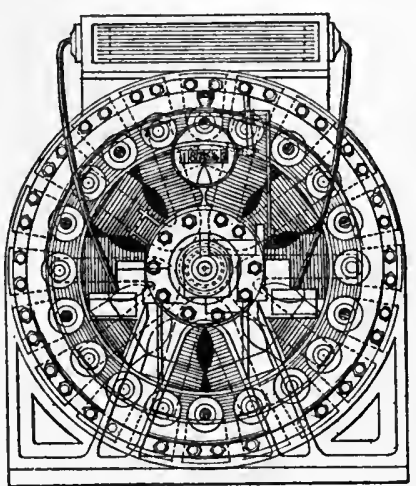

ELEVATION

Fig. 204.-Holmes' machine.

(From La Lumière Électrique.) 205 ) is similarly arranged, only the medium zone has been divided into four zones, whose divergence beneath the horizon reaches $17^{\circ}$ $23^{\prime}$, so as to cover a distance of 300 metres from the lighthouse.

The electric light of these lighthouses has worked without interruption since its installation on the 1st of January, 1872. Its expense is one-fourth that of the oil-lamps.

Lizard Point Lighthouse.-The electric light

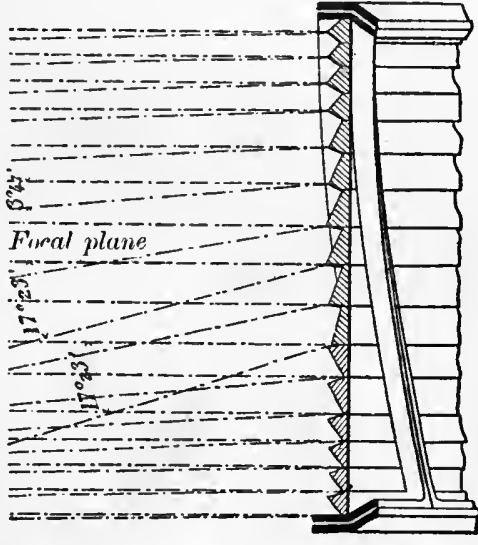

Fig. 205.- South Foreland lighthouse (section of the central cage).

(From La Lumiere Électrigue.)

was installed in the two lighthouses of Lizard Point on the 22nd of March, 1878. In consequence of numerous trials 
made under the direction of Professor Tyndal, the Trinity Board adopted, for the lighting of the Lizard Point lighthouses, Siemens' machines with longitudinal bobbins. These machines, six in number, make 850 revolutions per minute, and can each produce a light of 3620 candles. They are driven by three of Brown's hot-air engines of ten horse-power.

Macquarie Lighthouse.-The well-known firm of Chance

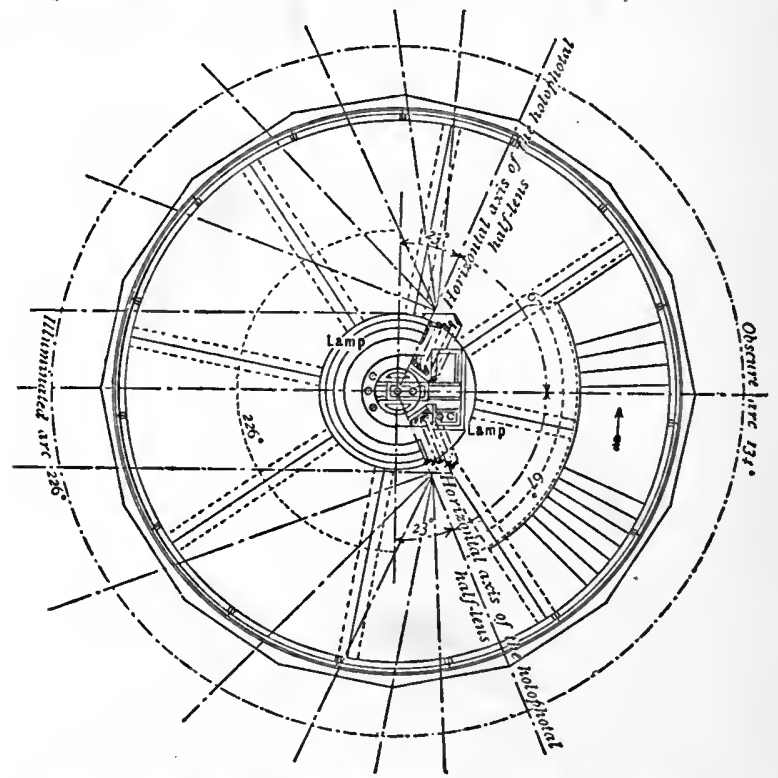

Fig. 206.-Plan of the apparatus of South Foreland. (From La Lumière Électrique.)

Brothers, of Birmingham, has lately established at Macquarie, Port Jackson, in the Bay of Sydney, the optical apparatus represented in Fig. 208. This apparatus, specially constructed for the application of electric light, has a ray of 0.92 metre in the focal plane. It consists of sixteen panels, each carrying eleven prisms in the upper part and eighteen in the lower. These panels at the same time concentrate 


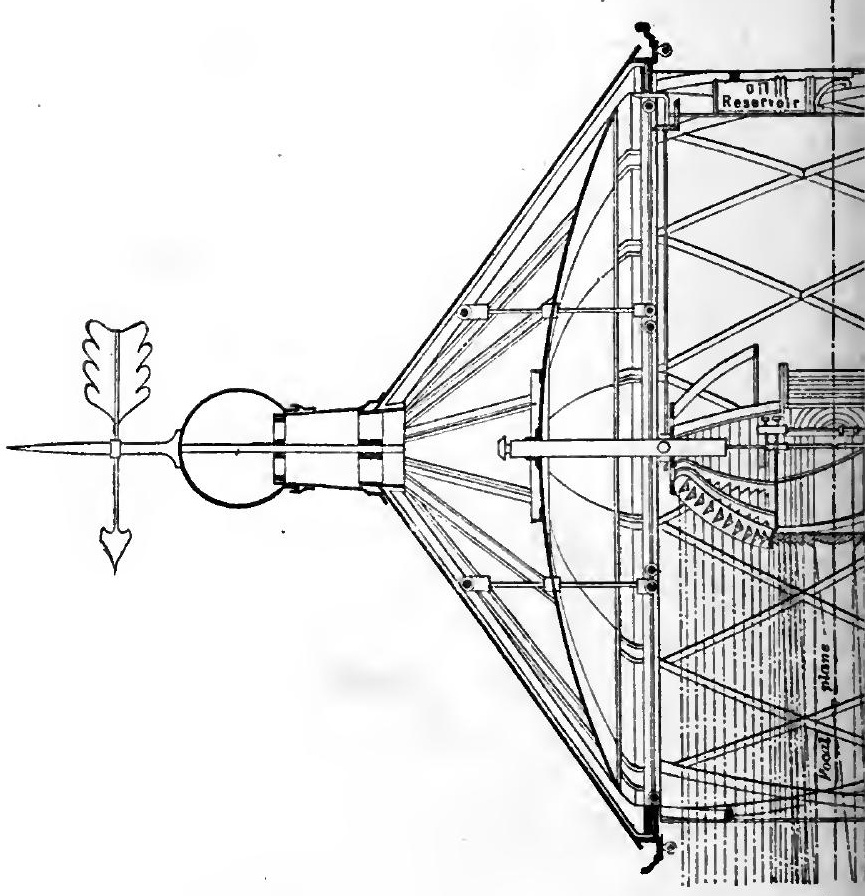




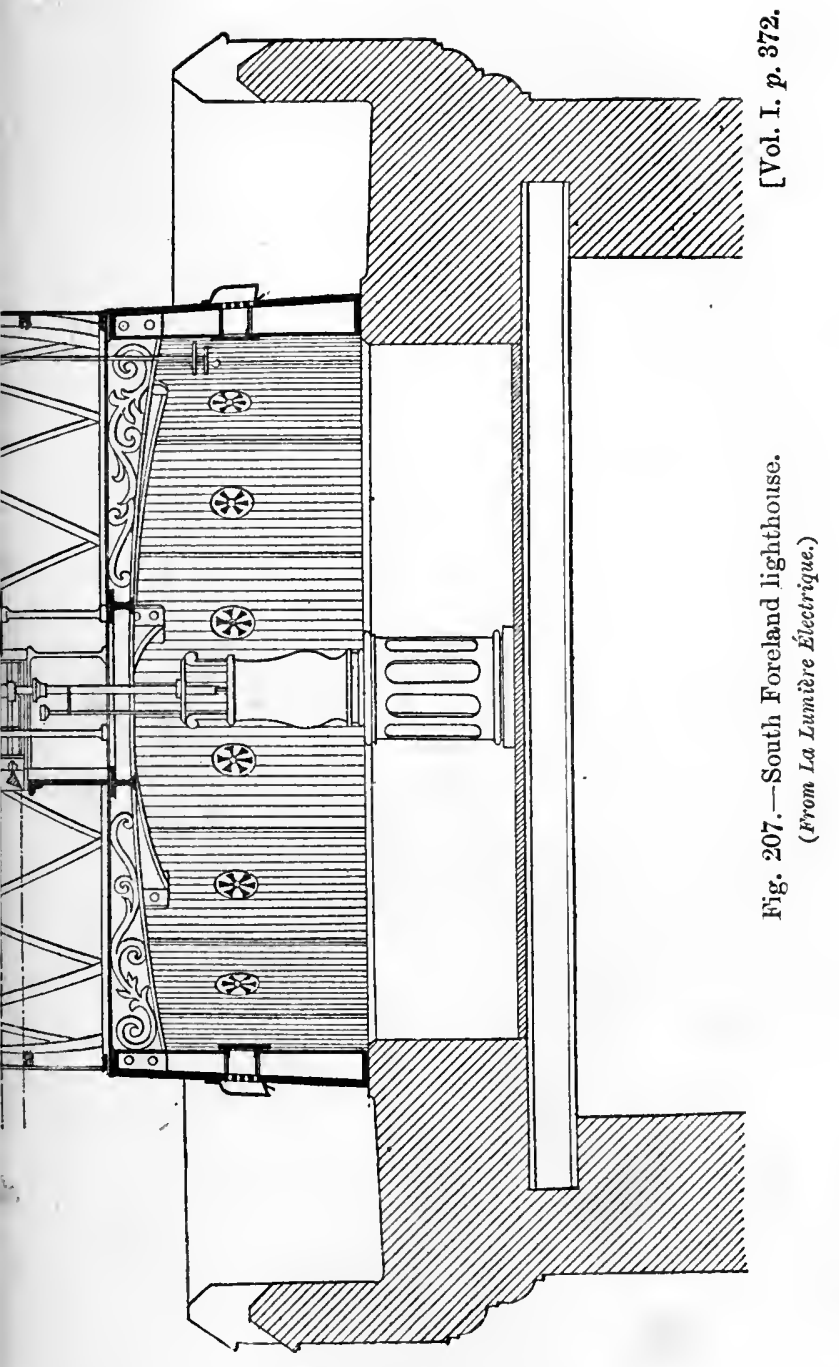




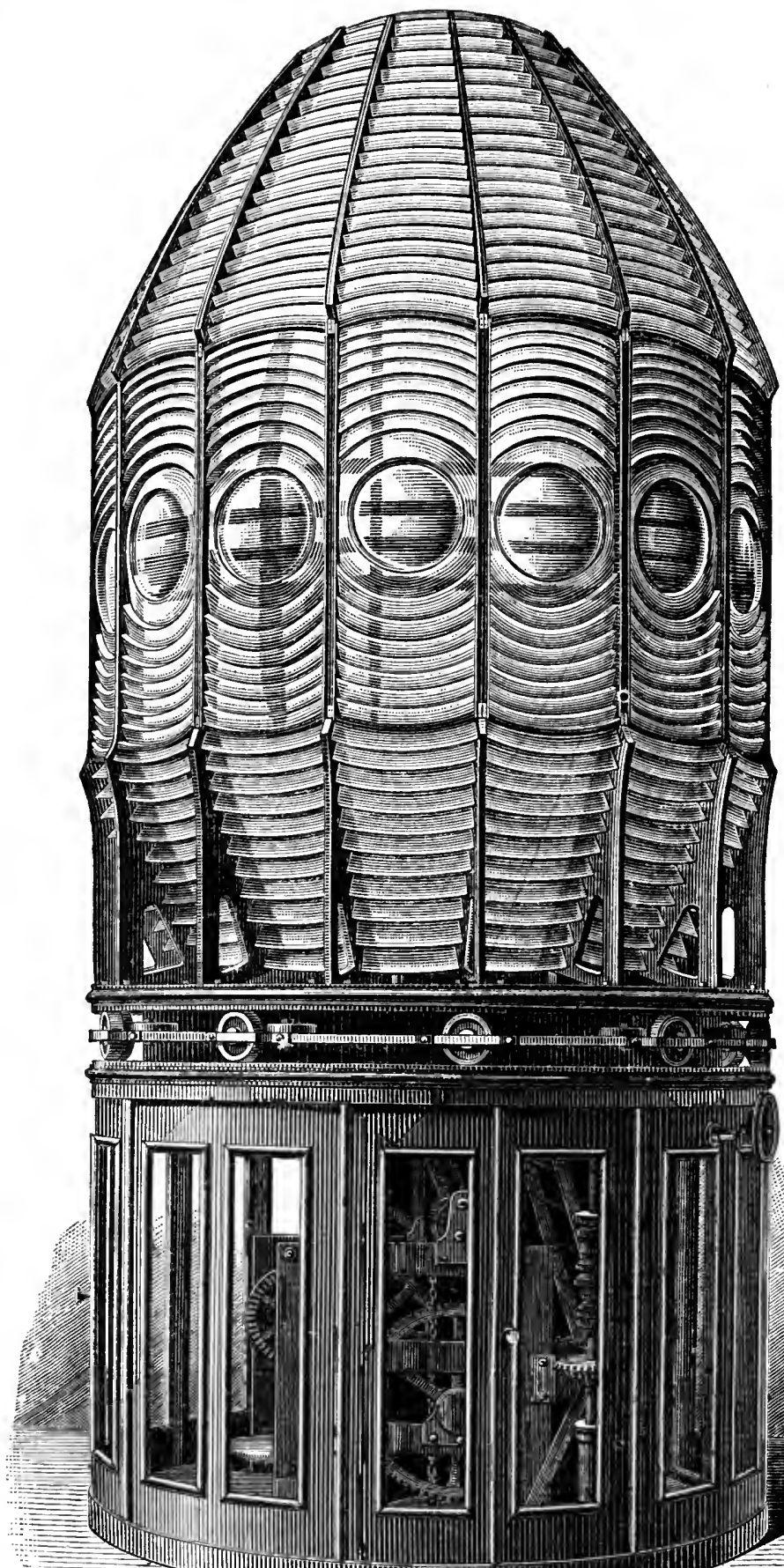

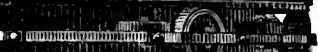
(ivin 
the light and impart to it the requisite divergency, and give every minute a flash of eight seconds, without any intermediary apparatus. The height of the optical apparatus is 27 metres; the intensity of light in the focus is 12,000 candles. The lighthouse only illuminates one-half of the horizon, on which the rays of the obscure arc are concentrated by means of a dioptric mirror. The bronze cage of the apparatus is arranged so as to offer the least possible obstruction to the light.

The lamps are of the modified Serrin type (vide p. 235). The lamp in action can, in three or four seconds, be replaced by a reserve lamp. The carbons are twenty-five millimetres in diameter.

The electricity is supplied by two De Méritens alternating magneto-electric machines. These machines weigh 2500 kilogrammes each, give a current of 110 ampères, and expend work of about seven horse-power; they are driven by two of Otto and Langen's gas-engines (Crossley's type) of eight horse-power.

In case of accident, the electric light can in ten seconds be replaced by a gas flame; this change is sufficiently rapid, considering that there is an interval of one minute between each flash.

The whole apparatus is mounted on a cylindrical socket, which allows access to the lantern without interfering with the rotation.

In order to enable the person in charge easily to maintain the electric arc in the focus of the apparatus, two curved prisms have been fixed to the frame of the dioptric mirror, so as to form on the floor two very distinct images of the carbon points of the lamp; the person in charge has only to shift the lamp until he has caused these images to 
coincide with two marks traced on the floor. He can perform this very simple and accurate manipulation without looking at. the electric arc, which is always trying to the eyes.

From accurate experiments made by Wigham, it appears that for installations where only a moderate intensity of light is required, gas can compete with electric light, because the latter requires a larger staff, but as soon as the required intensity reaches (as it does at Lizard Point) 8000 to 16,000 candles, the price of electric light is only three-fifths or fourfifths of that of gas or mineral oil, and this economy increases with the intensity of the light. The most economical installation is that at Lizard Point, where the electric light costs seven shillings per candle per year, against eleven shillings at La Hève.

The Lighthouse on the Isle of Razza.-The apparatus of the Isle of Razza (Bay of Rio Janeiro), which has lately been constructed by Messrs. Sautter and Lemonnier, of Paris, is remarkable for its exceptional dimensions-it measures 1.4 metre diameter in the focal plane-and by its novel and.ingenious mechanism.

The electrical apparatus at Razza replaces an old catoptric apparatus. The lighthouse is built on a rock seventy metres in height. The tower of the lighthouse itself (Fig. 209) is twenty-six metres high, so that the focal plane of the optical apparatus is at a height of ninety-six metres above the sea-level.

The machine-room is represented in Figs. 210 and 211. The electricity is supplied by two Gramme machines (type C T, continuous current), capable of feeding a light of about 18,000 candles, at a speed of 700 revolutions per minute, and expending seven horse-power per machine. 


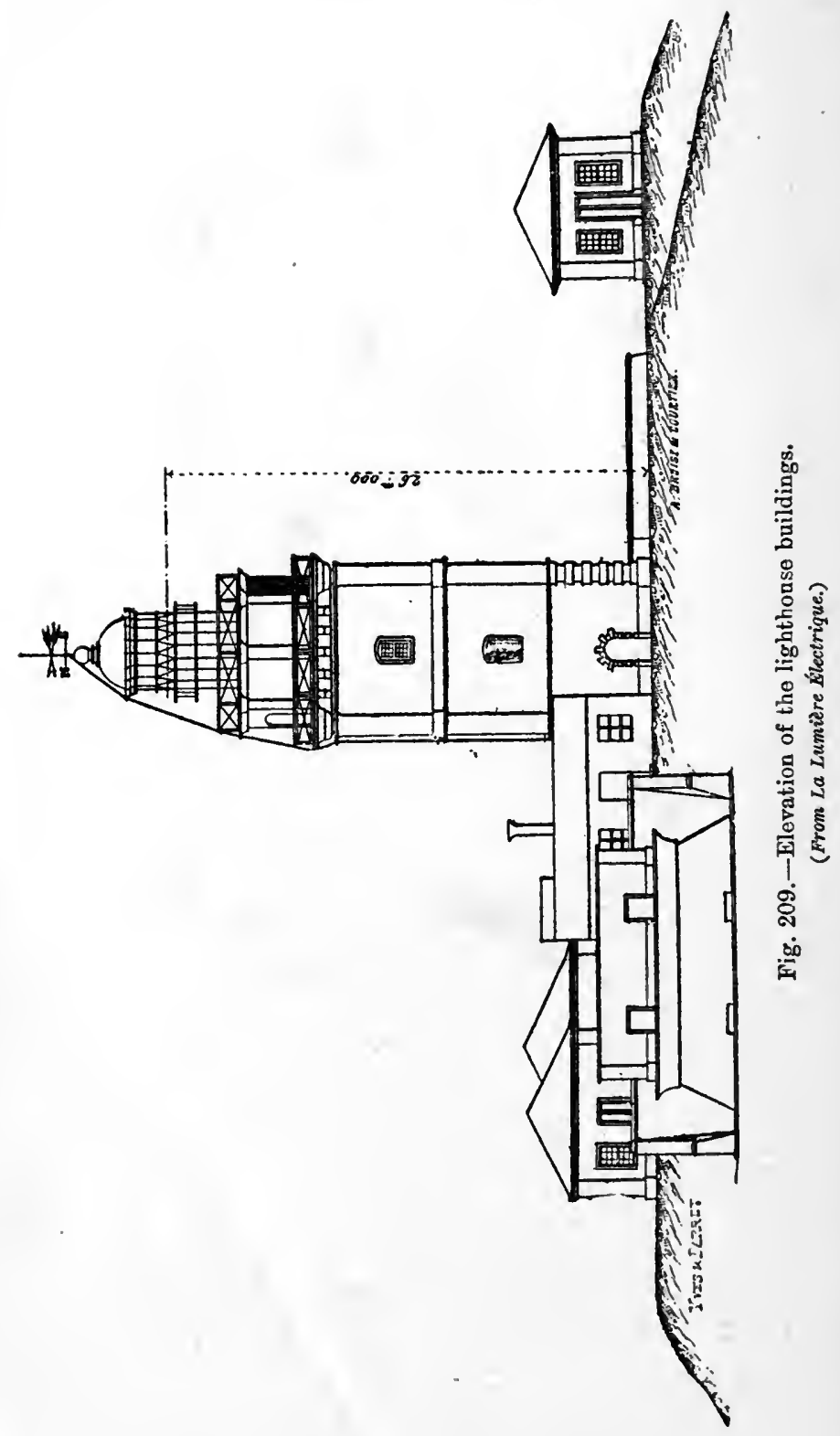


THE APPLICATIONS OF ELECTRIC LIGHTING. 377 One of these machines suffices for the normal illumina-

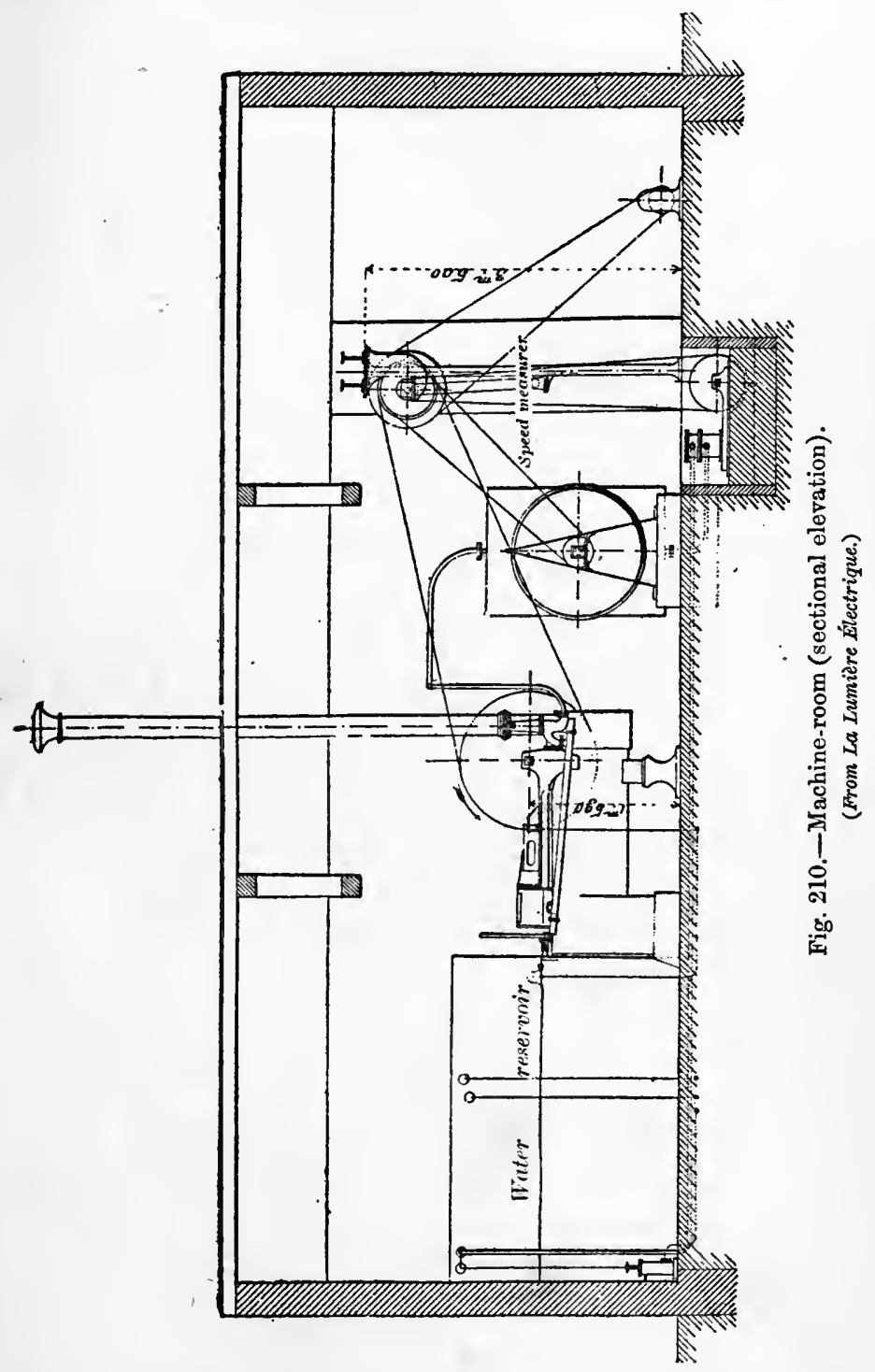

tion of the light-house, the other is held in reserve. The 
whole of the installation is double, to provide against accidents.

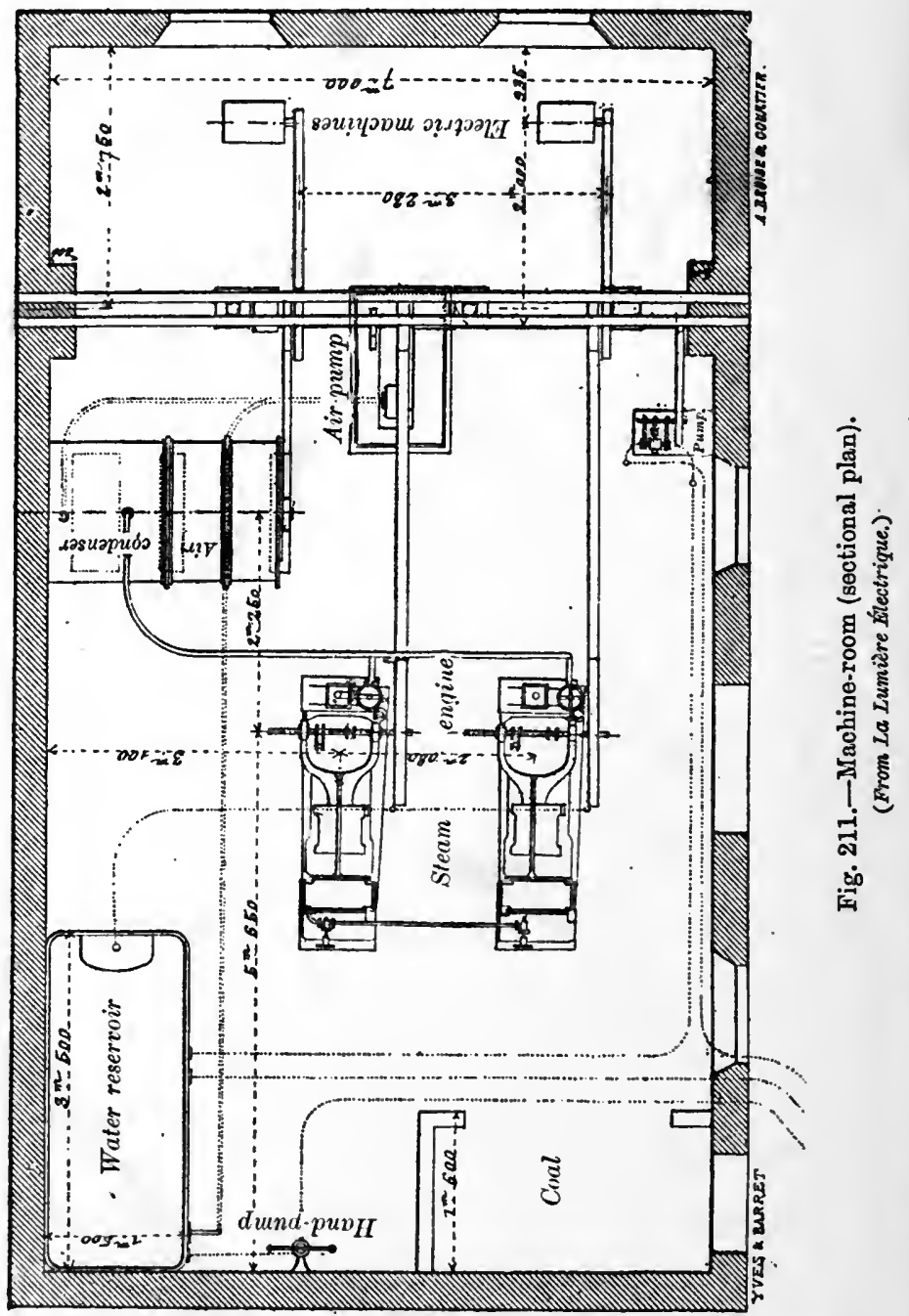

The !Gramme machines are driven by two semi-fixed compound steam-engines of ten horse-power each. An air- 
condenser connected with the steam-engine supplies the lighthouse with fresh water.

The arrangement of the lantern enclosing the optical apparatus is represented in Fig. 212. The space occupied by the optical apparatus is marked in dotted lines. The whole arrangement and the principal details of the optical apparatus are represented in Figs. 213, 214, and 215.

The lantern is 3.5 metres in diameter; its mountings are vertical above and below the apparatus, and arranged crosswise in the central part. The employment of vertical mountings and convex mirrors, which are difficult to replace, is thus avoided. The optical apparatus is arranged in such a manner that all the necessary manipulations for ensuring the steadiness and precision of the illumination can be carried out without entering the lantern.

The revolving action of the apparatus is controlled by the pinion $p$ (Fig. 213) of the axle $a$ gearing into a fixed clock-train; to stop the revolving action, it is sufficient to release, by means of the handle $\mathrm{A}$, the pinion $p$ of the clock-train. The studs $G$ are used for centring the apparatus by the tightening screws of its platform. A change of lamps, in case of accident, is effected by the fly $\mathrm{B}$, which drives, by means of the pinions $p^{\prime} p^{\prime \prime}$, the interior clocktrain $c^{\prime}$ fixed to the platform of the lamps; this platform, by pivoting round its axis $d$, replaces a disabled lamp by a fresh one.

To provide against any accident, an oil-lamp, always ready to act, is installed on a table $\mathbf{T}$ (Fig. 214), which, by pivoting round its axis, brings the rails, which carry the oil-lamp, into the prolongation of those of the electric lamps, and this allows of their being replaced in a few minutes. The 


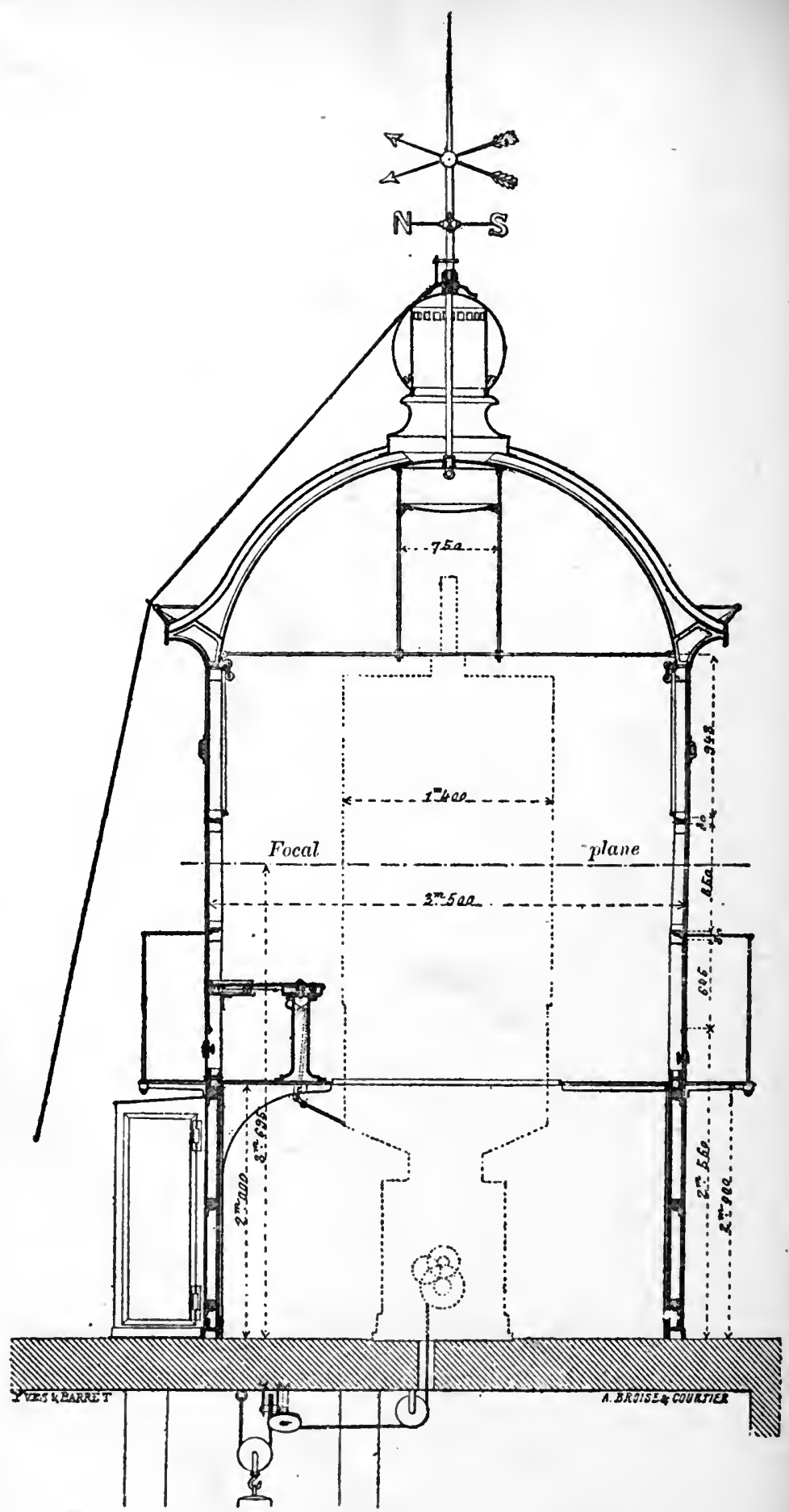

Fig. 212.-Arrangement of the lantern. (From La Lumiere Electrique.) 


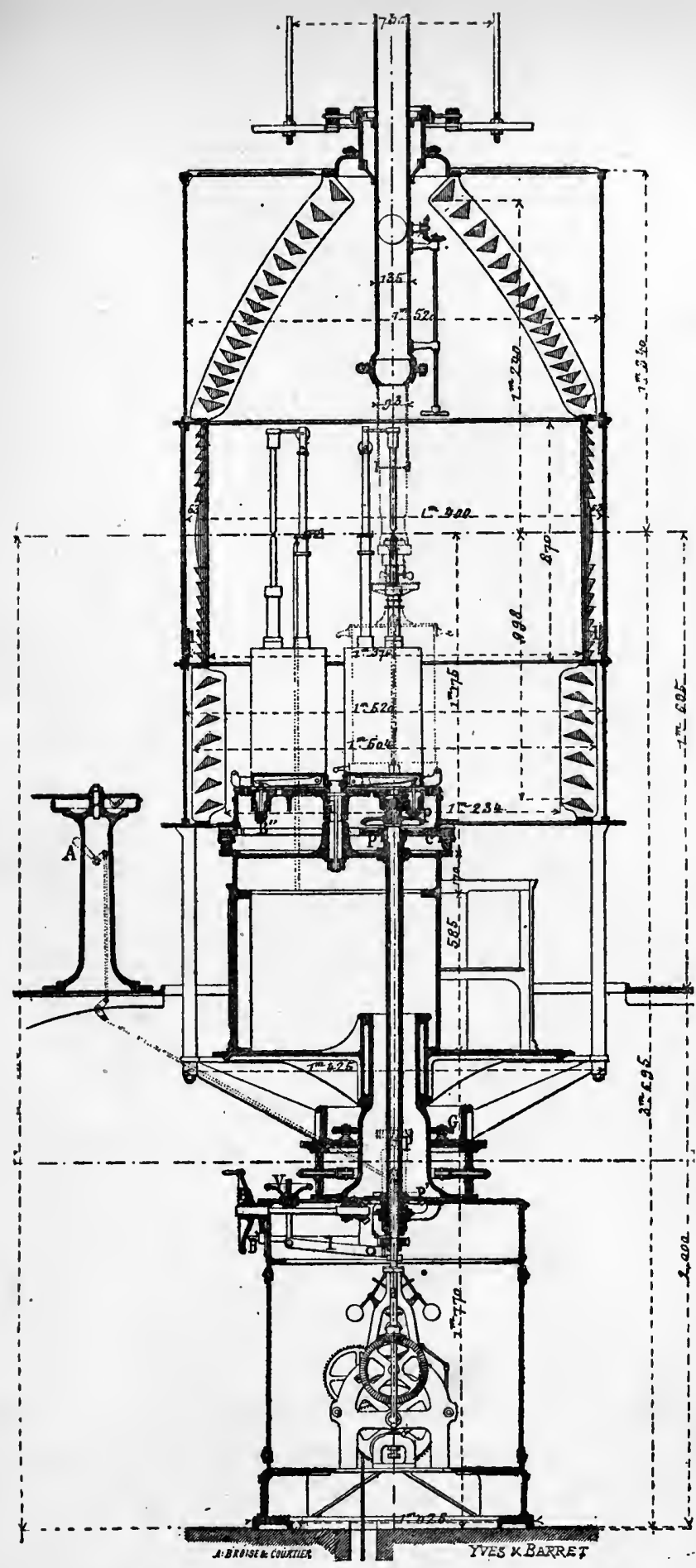

Fig. 213.- - Vertical section of the optical apparatus. (From La Lumiere Electrique.) 
focussing of the lamp is most ingenious. The lamp is first of all raised to the level of the focus, by means of the mechanism V I (Fig. 213), which raises or lowers the platform $\mathrm{P}$; then it is placed in the focus itself by turning the shaft $\mathbf{B}^{\prime}$; this shaft controls, through a clock-train and an endless screw, the tube of the platform $\mathrm{P}$, to which is bolted an eccentric, which displaces the lamp in an horizontal direction.

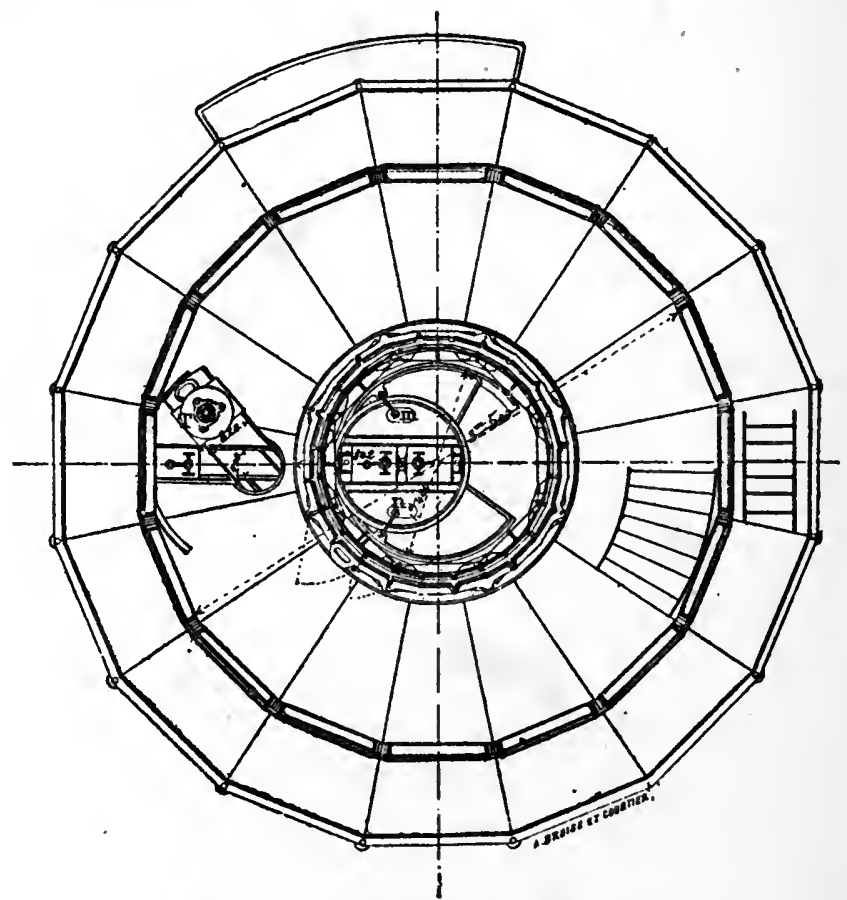

Fig. 214.- Section through the focal plane.

(From La Lumiere Électrique.)

The electric arc is found to be in focus when its images refracted by the prisms at $90^{\circ} \mathrm{m}$ and $n$ (Fig. 214) coincide with the tracings on two ivory plates fixed to the socket close to the lighting handles $\mathrm{V}$ and $\mathrm{B}^{\prime}$ (Fig. 215).

The lighting is thus effected from the outside of the 
THE APPLICATIONS OF ELECTRIC LIGHTING. 383

apparatus, with the greatest simplicity and precision, and without looking at the dazzling light. The dotted lines of Fig. 213 indicate the substitution of an oil-lamp for one of the electric lamps.

The electric lamps, three in number (two of them being reserve lamps), are reversed Gramme arc lamps, so as to obtain a fixed luminous point. In the lamp adopted at the Razza

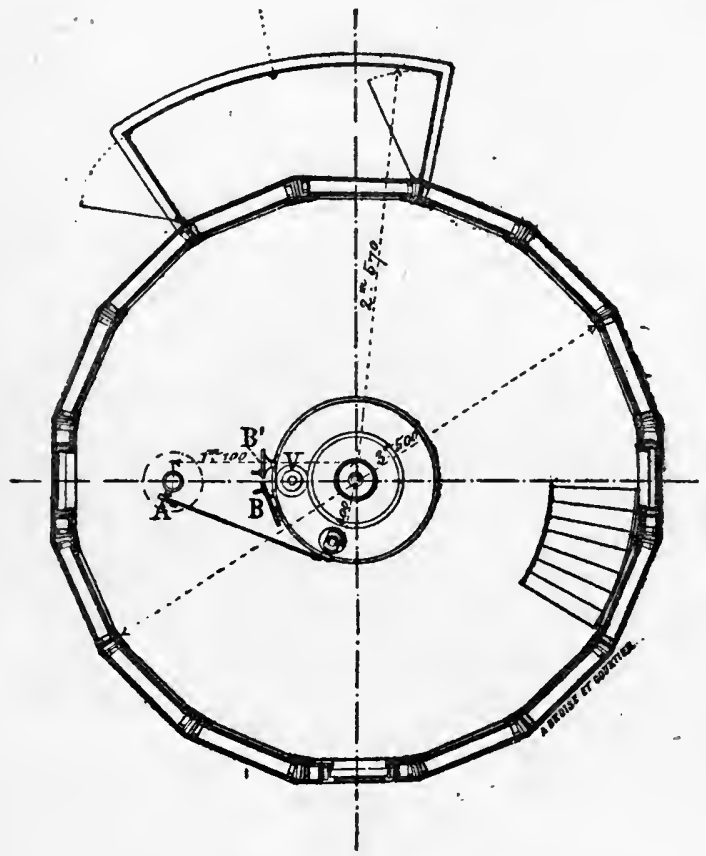

Fig. 215.-Section throngh the sliding rollers.

(From La Lumiere Électrique.)

lighthouse, and which is represented in Fig. 216, the upper carbon remains fixed. In this figure will be seen the last of the train of clockwork $R$ and its brake $S$ controlled by the shunt magnet $O$. This brake is only released when the lamp is brought into focus. The current arrives in the 
lamp through the supporting rails, and only traverses the lamp when the elastic screw-plug B" (Figs. 213 and 216) is pressed against the platform $P$, and thus closes the circuit through the carbons, which approach each other on the lever $\mathrm{B}^{\prime \prime \prime}$ being pushed back.

The light consists of two white flashes and one red flash, succeeding one another after an interval of fifteen seconds. For this purpose, the central cage is divided into twenty-four annular panels, the cupola and the lower part into eight panels only. The white flashes are produced by sixteen of the panels of the central cage, the red flashes by the remaining eight, as well as by those of the cupola and of the lower part. This arrangement ensures for the red flashes the same intensity as for the white ones, although the red glass reduces by nearly one-third the intensity of the luminous rays which traverse it.

The large diameter of the optical apparatus, joined to the small dimension of the electric arc, presents the inconvenience of giving to the emitted rays a very feeble divergency, and consequently considerably to reduce the duration of the flashes; to remedy this defect a slightly curved cylindrical lens has been placed before each of the lenses of the cage, which ensures for the rays a horizontal divergence of $3^{\circ} 45^{\prime}$. These lenses are red for the red flashes, and white for the white flashes.

The cupola and the lower part of the apparatus are entirely surrounded by red glass. The cylindrical lenses of the cage and the red glass are mounted on hinges, so as to facilitate the cleaning. The mechanism of the optical apparatus is calculated so as to throw all the rays on the horizon, with the exception of those emitted by the lower part of the cage, which are inflected on the sea by the lenses $l^{\prime}$ (Fig. 213),

VOL. I. 
in order to light up the neighbourhood of the lighthouse. The apparatus makes one revolution in six minutes.

\section{Illumination OF Vessels.}

On board a steamship, electricity can be economically produced, because it is always easy to take a few horsepowers from the engines to drive the electric generator; the question of expense, therefore, does not stand in the way of the application of electric lighting to steamers. We find, in fact, that the large steamship companies are introducing electric light for the illumination of their saloons and cabins.

The Swan lamp has been extensively introduced by some of the largest companies. The Inman Company's steamer, City of Richmond, was the first ship which was lighted with the Swan lamp. They have been regularly in use since June, 1881, and have given universal satisfaction.

There are sixty lamps installed; forty-eight are hung in plated pendants with obscured globes. Each pendant is fitted with two lamps. The lamps at the companion staircase and in the passages are hung from plated brackets of ornamental design. The lamps are attached to the pendants and brackets by the spring holders (vide p. 336), which are specially adapted for use on board ship ; for whilst they keep the lamp in perfect contact, they allow a certain amount of movement, which prevents the lamp being affected by the vibration of the ship. The wires conveying the current are attached to each side by the binding screws, which are in contact with the hooks which hold the lamp.

The electricity is generated by a Siemens alternating machine, driven by an ordinary horizontal steam-engine, 
placed in a small room adjoining the main engine-room, and supplied with steam either from the main or donkey boilers. From this machine two insulated main leading cables are laid to the saloon, etc., with branch wires to each of the lamps. At each point where these branches are attached a switch is fixed for turning out the lamps when desired; it is so arranged that it cannot be turned except with a peculiar kind of key, which is in possession of the steward, and the form of the key makes it necessary for the switch either to be fully off or fully on before the key can be removed, thus preventing sparking. These switches are also each fitted with a safety fusing connection to prevent risk of fire.

Since the lighting of the City of Richmond, the electric light has been introduced into large numbers of steamers.

But not only for mercantile shipping, but also for the navy, the question of electric light is of the greatest importance. The new engines of naval warfare have brought about a complete change in naval tactics; the powerful ironclads have called into existence an enemy, the torpedo. The torpedo is fired by electricity, but, in order to produce the desired effect, it must first of all be brought close to the enemy's vessel. This is done by means of a torpedo-boat, a small steam-launch of the highest possible speed, which has to place the torpedo under the hulk of the vessel without being seen, and to make off immediately afterwards. It is, therefore, indispensable for large ironclads to have an electric beacon on board, which allows them to explore the horizon and to discover the approach of a torpedo-boat at night.

Another use of the electric beacon on board a man-ofwar is to light up the coast where a landing is to be effected, and also forts which are to be bombarded. The landing of 
the French on the coast of Tunis, of the English in the late Egyptian war, the bombardment of Sfax, Gabès, etc., by the French, and of Alexandria by our navy, are wellknown instances, still fresh in our memories, of the immense importance of electric light in naval warfare.

Experiments made on board the French ironclad Richelieu with two different types of lamps, the one regulated by the hand, the other automatic, have resulted in the adoption of hand-regulated lamps by the French navy. In these lamps the distance of the carbon points is regulated by means of a screw and a little fly-wheel in proportion to the waste of the carbons. The lamps are provided with metallic contacts, which establish electrical communications with the conductors of the projector, and with two terminals for experiments made independently of the projector. The lower carbon is insulated from the frame, and receives the conductor of the negative pole. The upper carbon is in communication with the frame, and receives the conductor of the positive pole. The automatic lamps, constructed after Serrin's model, have a much more sensitive and complicated mechanism; they are intended to dispense with personal attention to the beacon by maintaining a constant distance between the two carbons. The construction and working of these lamps has been explained on p. 202. An improved model of Serrin's lamp, capable of being worked in any position and of resisting the rolling or pitching of the vessel, was shown at the late Paris Electrical Exhibition, but the advantages of this lamp are absolutely illusory, since the direction of the luminous rays has to be changed incessantly, and since the person manipulating the projector can easily attend to the lamp as well.

The advantages of the hand-regulated lamp are the following :- 
TIIE APPLICATIONS OF ELEC'TRIC LIGHTING. $3 \& 9$

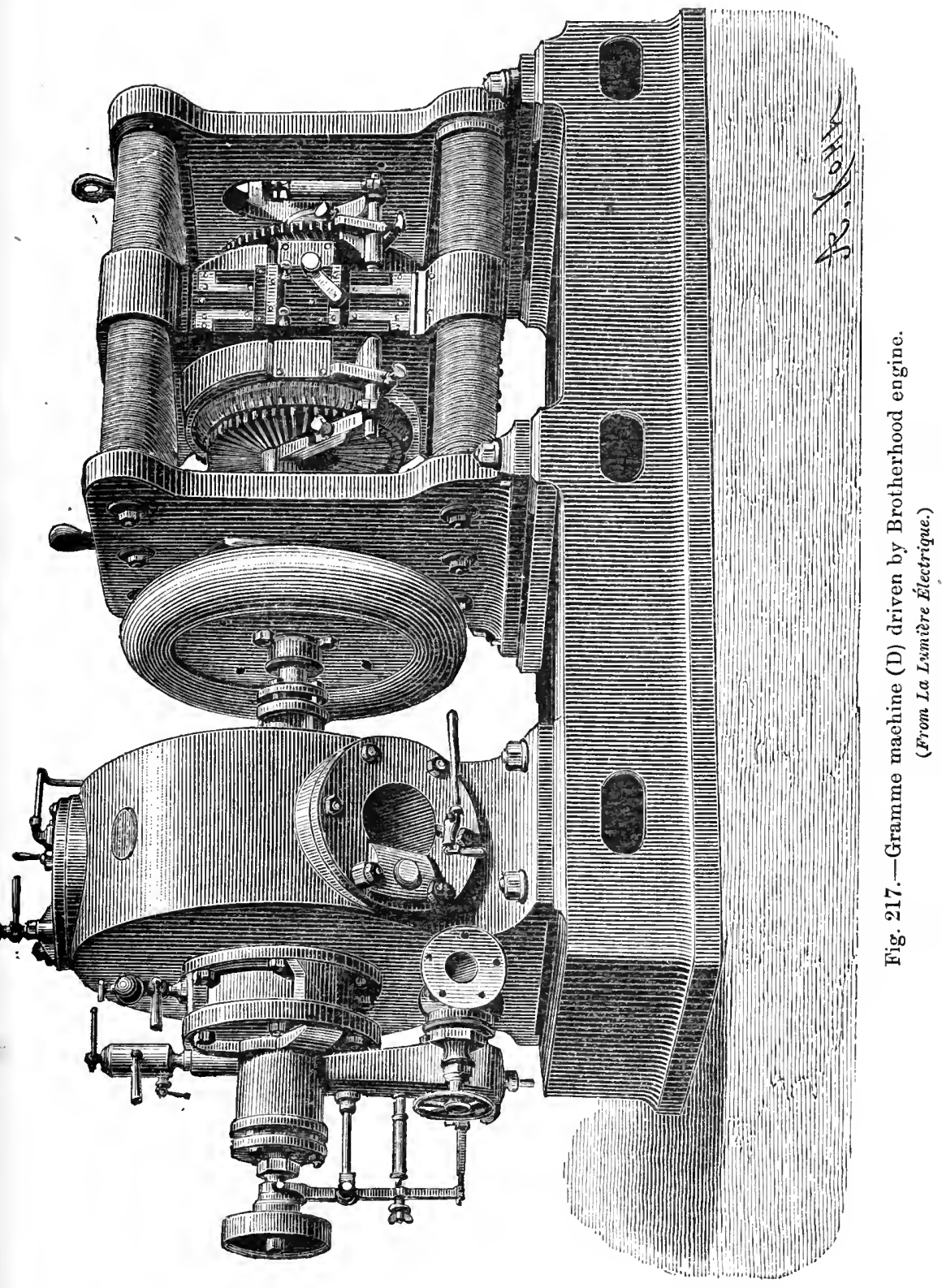


Facility and rapidity of priming and lighting.

Possibility of instantly obtaining the required length of arc.

The carbons can be withdrawn as much as the speed and power of the motor will allow, and can thus furnish the highest possible luminous intensity.

Independence of the apparatus of the movements of the projector and the vessel.

Possibility of pressing the carbons against one another, so as to remove their excrescences.

The mechanism is simple and solid and comparatively inexpensive ( $£ 12$, instead of $£ 32$ for the automatic lamp); the repairs can easily be executed on board.

The carbon-holders need not be vertical; they can be held in an inclined position, and this reduces to a considerable extent the necessary displacement of the upper carbon.

The ironclad ought, therefore, to be provided with one hand-regulated lamp for each projector, one reserve lamp of the same kind, and, if occasion requires, one automatic lamp for special cases where the lighted beacon has to be maintained in a perilous position which would endanger the life of the person in charge. The electric current is generated by a Gramme machine (type D) driven by a Brotherhood three-cylinder engine, to which it is coupled as shown in Fig. 217. From experiments made at Chatham, the Gramme machine has proved the most advantageous, as well as regards the total quantity of light produced $(27,500$ candles for fifteen horse-power expended), as also for good utilization of work. As a light apparatus, Mangin's projector is in universal use.

Mangin's Projector.-This projector has been adopted in 
THE APPLICATIONS OF ELECTRIC LIGHTING. 391

this country as well as in France, and is represented in Fig. 218. The hand-worked lamp described above is placed inside the apparatus; the reflecting spherical surface of the glass mirror is on the inside, and the ray must therefore

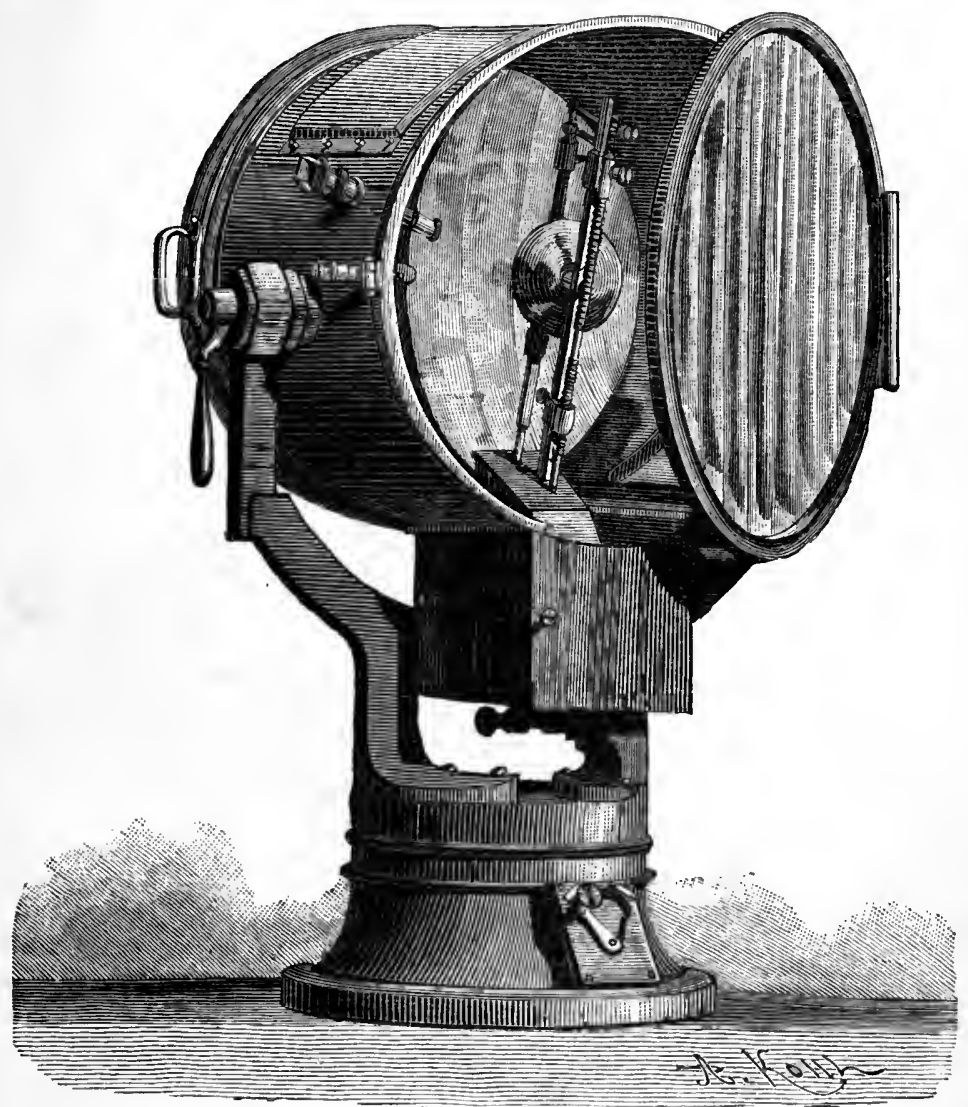

Fig. 218.-Mangin's projector.

(From La Lumière Électrique.)

twice traverse the thickness of the mirror before receiving its final direction. The two surfaces are not parallel either, the interior surface being a sphere, which has not the same radius as the other. The different thicknesses which the 


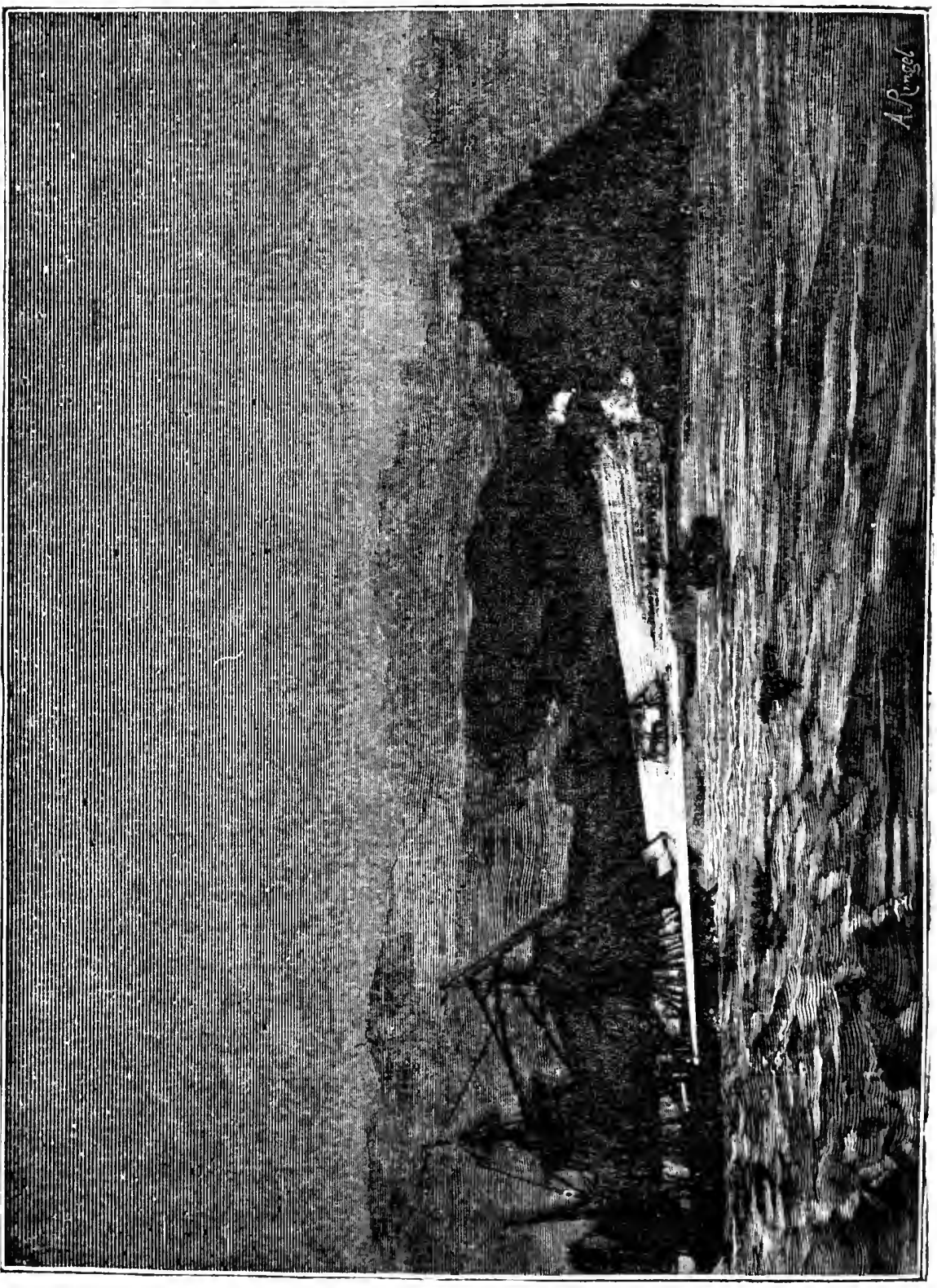


respective rays have to traverse, according to the angle they form with the axis, modify their direction so as to bring them back to strict parallelism, provided the radii of the two spherical surfaces are of proper proportion. As regards the inclined position of the carbons, it has been found that, on employing a continuous current, the ray of maximum luminous intensity does not lie in the plane perpendicular to the line of the carbons, but about $30^{\circ}$ below it. Fig. 219 shows the electric light projected by this apparatus on the Isle of Tabarca, in the Tunisian war.

But not only for men-of-war, but also for our transatlantic steamers the use of an electric light-beacon and projector might be of the greatest value. It is well known that, towards the autumn equinox, immense masses of ice are detached from the northern coast of Greenland and carried south by a powerful current. The track of the outgoing transatlantic steamers takes them very near to Cape Farewell, the southern extremity of Greenland, and their meeting with the floating icebergs is by no means a rare occurrence. The approach of these formidable enemies at night constitutes a great danger for the vessel, and this danger could easily be averted by the employment of an electric light-beacon.

\section{Night Signals.}

The signals actually in use are of two different kindssignals intended to ensure the safety of the vessel, and signals intended to form a system of optical telegraphy, enabling the vessels to communicate during the night, either with one another or with the shore, as easily as they communicate in the day-time by means of the flag-signals. The 
former have been described in the preceding article, and we have here only to occupy ourselves with the latter kind of signals. Successful experiments have lately been made on board the French ironclad Colbert, with electric light signals.

In the ordinary system ten oil or petroleum lamps are employed, which are hoisted in two groups of five along the mainmast-the first group to the masthead, the second below the mizzen-yard; and the different signals constituting the international code are obtained by varying the number of lighted lamps or their respective positions.

In order, therefore," to give a signal, a certain number of lamps must be lighted and hoisted, which requires a certain time; then, since a signal must not be changed until it has been repeated by the other vessel, a certain time must be allowed for the answer. For the second signal the lamps must be lowered again, the new signal prepared, the lamps hoisted, and again time allowed for the answer, and so on. It will easily be understood that this mode of correspondence is very slow, and it is therefore only employed for communication with vessels at a great distance, and when one and the same signal has to be transmitted to several vessels, as, for instance, in squadron manœuvres. and in this case the transmission is even slower, each vessel having to repeat the signal.

The electric installation on board the Colbert has been carried out by De Méritens. The source of electricity is a small De Méritens magneto-electric machine placed on the hind quarter-deck and driven by eight men. The current of the machine is conveyed to a commutator placed in the pavilion of the foot-bridge, and thence can assume three different directions. 


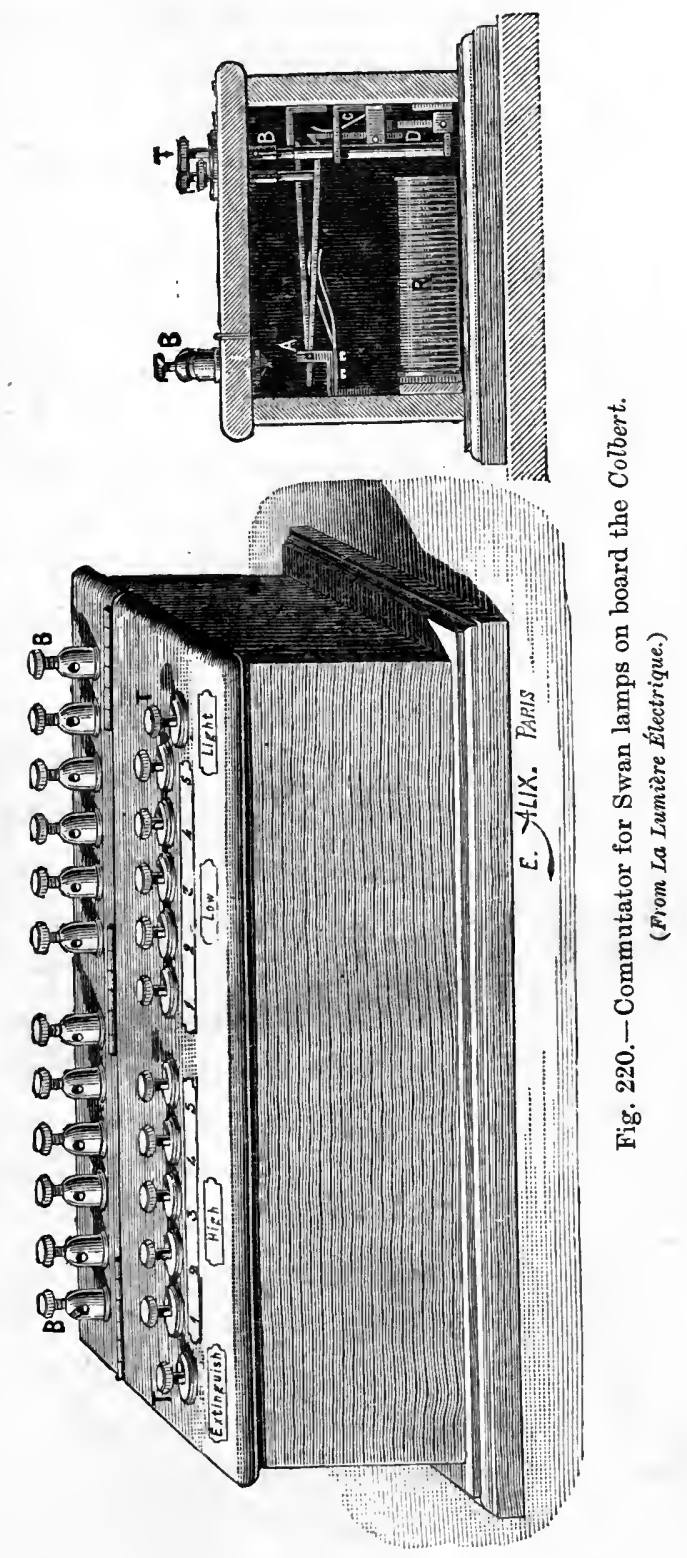


One of these directions leads to a second commutator, represented in Fig. 220. In this apparatus the current, which enters through one of the extreme terminals $B$, can leave through ten shunts, starting from the intermediary terminals and leading each to a Swan incandescent lamp. These lamps, placed in ordinary ship-lanterns, are hoisted to a fixed position, and occupy the habitual place for signals, that is to say, five at the main-masthead, where they are maintained by the cord used for hoisting then, and five below the mizzen-yard. The current is conveyed from the commutator to the lamps by a cable of eleven wires; the last of these wires serves for closing the circuit of each of the lamps with the machine; the total length of this cable is about seventy metres.

The apparatus being thus arranged, the production of all the signals is effected by simply pressing the studs of the commutator (Fig. 220). Of the twelve studs, the ten in the middle establish the shunted current for each of the lamps, and the two studs on the outside serve for their lighting and extinction.

To produce a signal, therefore, the studs corresponding to the lamps to be used, and also the stud bearing the inscription "light," must be pressed down; the signal disappears on simply pressing the stud with the inscription "extinguish," when all the shunts are immediately broken. The signals can thus be produced without any loss of time except the interval required for the repetition of the signal by the other vessel.

When the current does not pass through the lamps, the stud "extinguish," instead of breaking the circuit, causes it to pass into some resistance bobbins equivalent to the lamps, so that the men driving the machine are not affected by the 
closing or opening of the circuit. The details of the commutator will be seen from the section of the apparatus given in Fig. 220. It has been stated that the current passed from the ruachine to a commutator, and thence branched off in three different directions; and we have now seen that one of these branch currents leads to the second commutator and supplies the Swan lamps.

Another branch conveys the current to a lantern suspended from the gaff, which is intended to light up all the points of the horizon. The lantern contains a Berjot lamp (p. 232), indicates the position of the vessel, and secures it against collisions.

Finally, the third branch conveys the current to a Coulomb lantern placed on the foot-bridge, and intended for a sort of optical telegraphy. This lantern, represented in Fig. 221, is only visible from one quarter of the horizon, and is, moreover, provided with a screen A, Fig. 221.-Coulomb lantern, for incan. which, by means of a rod

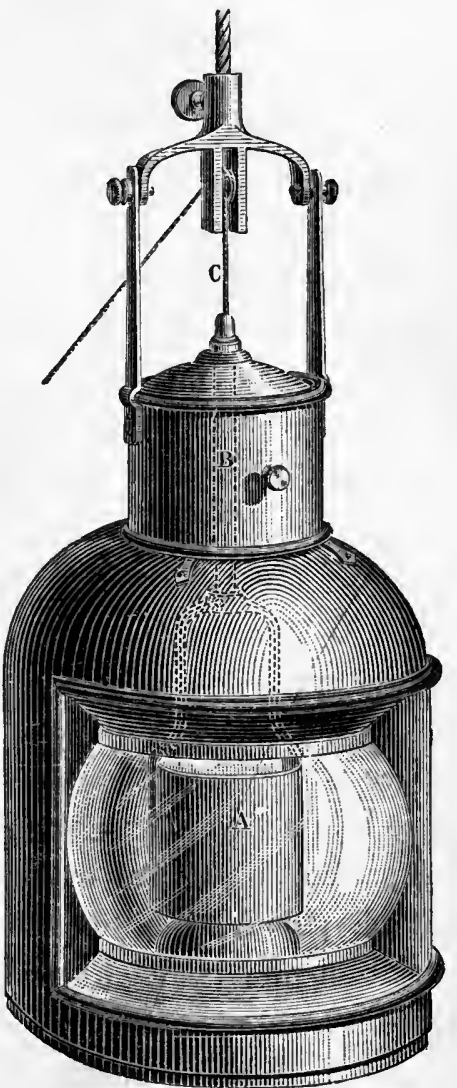

(From La Lumiere Électrique.) and a cord, can be raised or lowered, so as to produce veritable optical Morse signs. The lantern can be fitted either with a large incandescent lamp or with a Jablochkoff or Wilde candle. 


\section{Electric Light in Collieries. ${ }^{1}$}

The first practical application in the United Kingdom of the electric light to the illuminating of coal-mines was made on the 9th of August, 1881. The Garnock Colliery, near Hamilton, Lanarkshire, belonging to Mr. J. Watson, has been fitted with Swan's incandescent lamps specially arranged with outer lanterns of stout glass, air-tight, and provided with steel guards (vide p. 335). The workings in which the lamps are fixed are 118 fathoms, or 708 feet, below the surface. Twenty-one brilliant little lights placed at the pit-bottom, in the roads, and at the actual face of the seam where active operations are in progress, supply an illumination of a very different character from the dismal glimmer of an occasional Davy. The electricity was generated by a dynamo-electric machine at the surface, worked by a special twelve horse-power engine, and conveyed by two cables, first along telegraph poles to the pitmouth, then down the shaft to the workings, in one section to a distance of half a mile. The overhead wires are naked copper wires of three-eighths of an inch diameter, while those below ground are carefully insulated, and in the shaft are protected with an outer tube of galvanized iron. At suitable points of the circuit, safety air-tight switches, the invention of Messrs. Graham, of Glasgow, are inserted to afford control over individual lamps.

An experiment was made with a lamp to test whether, in the event of its being broken by accident, a surrounding atmosphere of explosive gas would or would not be kindled by the strip of red-hot carbon before it had time to cool. Into a box containing about three cubic feet of explosive gas, a single lamp, removed from its outer protecting 
case of stout glass was placed, and the current was turned on. The fragile bulb enclosing the incandescent carbon thread was then purposely broken, when the gas enclosed in the box immediately exploded. No such occurrence could possibly happen if the protecting case of stout glass is properly constructed. The risk of accident must be considered immensely less than that of the ordinary Davy lamp, especially when it is remembered that, with the brilliant light of the electric lamps, they need no longer be carried in the hand or set down upon the floor near the actual spot where the coal is being got, but can be fixed overhead at a safe distance against the wall of the mine. The ease with which the lights can be turned out during the firing of a blast is another point in their favour.

Another successful experiment of lighting coal-mines with electric light was made in 1881, in the presence of the Royal Commissioner for Reporting on Accidents in the Coal-Mines of the United Kingdom. The mine chosen for this experiment was that of Pleasley, near Mansfield. The pit is about 1000 feet deep; the light was only applied to three of the galleries, and here two Swan lamps (shown in Figs. 222 and 223) were used, enclosed in lanterns of an ingenious construction, due to Messrs. R. E. Crompton and Co.

The lamps, which are calculated to give the light of two or three candles, are so attached to the conducting wires that good contact is made with them, and the renewal of the lamp can be effected with great ease. As to the lantern, it is compact and light. It consists of few parts, and these are of a simple and inexpensive character. It has a ring (Fig. 223) at each end for suspension in either an upward or a downward position, and there is a second central contact to connect the lantern 
with the cable containing the conducting wires in communication with the apparatus generating the electric current.

In connection with the lamp there is a portable secondary
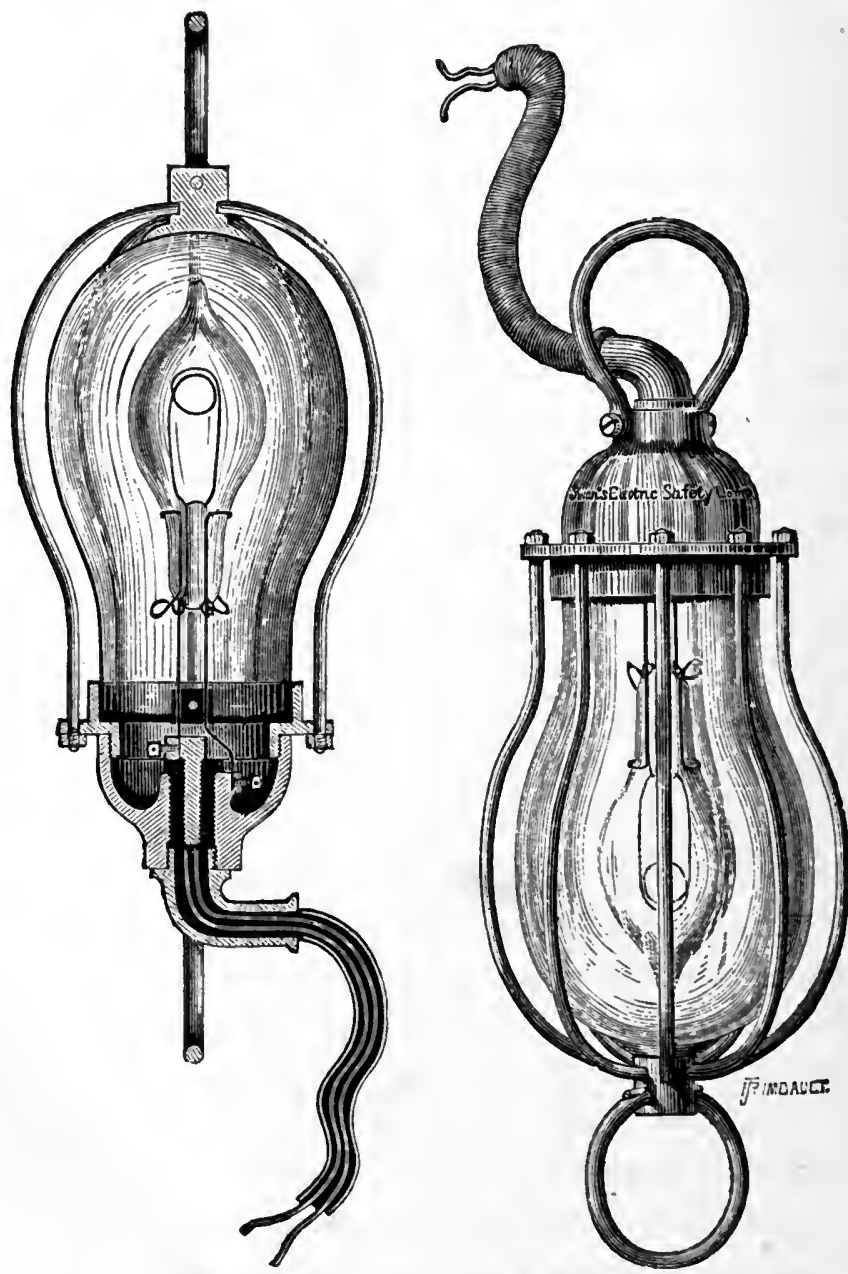

Figs. 222, 223.-Swan's mining safety-lamps.

battery contained in a small wooden box, which renders the safety-lamp independent of the main wires conveying the current from a distant dynamo-electric machine. 
The dynamo-electric machine would, however, still be required, even with the portable cells; for what is contemplated is that the portable secondary cells contained in the box should be taken to the dynamo to be charged by its action, and that, after being so charged, these portable stores of energy should be sent into the pit-workings on trucks, there to be connected with the lamps.

A set of cells, weighing about twenty pounds, would keep a lamp lighted for about eight hours, and the actual cost of supplying the current and keeping the lamp lighted would be very small-probably less than the cost of oil for producing an equal amount of light by means of the ordinary pit-lamps. The weight of the lamp is a pound and a half, and the weight of the box nine pounds and a half.

These lanterns may be transported without fear of accident. The globes are of very fragile glass; at the same time any explosion consequent upon the breaking of a lamp is prevented, since the air inside the lantern would be sufficient for the instantaneous combustion of the carbon filament before the flame could be communicated to the outside air. Ninety-four lamps were lighted; they were fed by the current of a portable machine placed near the entrance of the pit.

A number of other collieries have introduced electric light; but the progress in this direction is very slow, which is the more to be regretted as it cannot be doubted that the universal adoption of electric light in fiery mines would reduce the death-roll of persons killed by explosions of firedamp in Great Britain to one-tenth of its terrible proportions, which in 1880 reached 499 persons. It is to be hoped that before long the adoption of electric lighting for coal mines will be made compulsory by Act of Parliament. 
The colliery owners themselves will discover, when driven to this course by compulsion, that in the long run they effect an economy by discarding the clumsy and unsafe "safety"-lamp, which will soon be numbered, with the "flint-mill," among the relics of the past.

\section{Application to Military Operations.}

A number of these applications, as, for instance, the discovery of the approach of a torpedo-boat at night, the illumination of a coast-line where a landing has to be effected, of forts to be bombarded, of night signals on board a man-of-war, have been given in two of the preceding articles. The light can further be used for reconnoitring fortifications, for watching the erection of defensive works, firing batteries to prevent a surprise on the opening of a trench, for lighting up a breach, etc.

For the purposes of reconnoitring, for instance, a Gramme machine, with the driving Brotherhood motor and Mangin's projector, is placed on a strong carriage, and by a detachment of cavaliy taken to the designated spot. Steam is got up before starting, and on arrival the projector is conveyed to a short distance, if possible to elevated ground, and placed on a four-wheeled support or a strong pivoting saddle. The conducting wires are at the same time fixed to connect the projector to the machine, and the light is either directed to all parts of the horizon or fixed on a definite object as, for instance, the fortifications of a town to be bombarded. These apparatus have a considerable power of projection, and render the buildings of a town visible at a distance of 
9500 metres (about six miles). This apparatus can, however, on account of its weight, only be used on comparatively level ground; for mountainous regions, Messrs. Sautter and Lemonnier, of Paris, have constructed a carriage with a small Gramme machine and projector which can easily be drawn by one horse, and can, moreover, be entirely taken to pieces; the whole can be carried on a mule's back, and the light can therefore be produced at any spot to which mountain artillery can be taken.

\section{Illumination of RaILway Trains.}

It would appear that, on account of the unavoidable vibration and the frequent shocks to which a railway train is exposed, incandescent lamps only could be used for illumination, and, in fact, the ordinary arc lamps are wholly unfit for this purpose. An arc lamp has, however, been constructed by Sedlaczek and Wikullil which has been successfully used for locomotive head-lights. This system was tested at Paris during the Electrical Exhibition, and more recently on the Chemin de fer du Nord, between Paris and Dammartin, and also on the Kronprinz Rudolf Bahn, in Austria.

The lamp (Fig. 224) consists of two vertical tubes A and $\mathrm{B}$ of different diameters and connected at the bottom; two pistons, carrying the carbons, move in these tubes, which are filled with glycerine. In the lower part of the tube $\mathbf{B}$ is a small hollow piston $\mathrm{P}$ connected to a lever, $\mathrm{L}$ and maintained in position by means of a regulating spring $R$; at its other extremity the lever $\mathrm{L}$ terminates in an armature, which is opposed to an electro-magnet $\mathrm{E}$ of feeble resistance, 
through which the current feeding the lamp passes constantly. It will be seen that the two tubes $A$ and $B$ communicate by means of the small opening in piston $P$, and the pistons moving in A and B consequently take up a

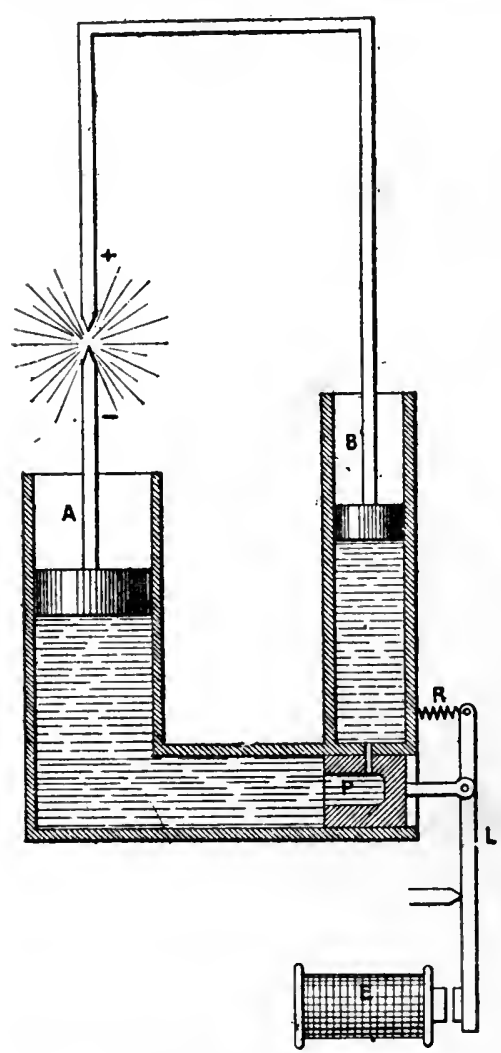

Fig. 224.-Sedlaczek and Wiknllil lamp (diagram).

(From La Lumière Électrique.)

position of equilibrium, which is regulated in such a way that, when at rest, the two carbons are in contact.

As soon as the current passes, the arc offering no resistance, the electromagnet $\mathrm{E}$ is strongly energized and attracts the lever $\mathrm{L}$. The small piston $\mathrm{P}$ moves to the right, and closes the communication between the tubes $\mathrm{A}$ and $B$; at the same time it causes a slight lowering of the level of the liquid in tube $\mathrm{A}$, and consequently a withdrawal of the lower carbon.

The electric are is now formed, and from this moment the piston $\mathrm{P}$ acts as a regulator, establishing or closing the communication between the tubes $\mathbf{A}$ and $\mathbf{B}$, that is to say, bringing about an approach or a withdrawal of the carbons in proportion as the arc increases or diminishes.

Fig. 225 gives a perspective view of the lamp, showing 
the essential parts as indicated in the diagram, with the only exception of the electro-magnet $\mathbf{E}$, which occupies a lateral position.

As used for a locomotive head-light, the lamp is mounted in a wooden case attached to the front of the smoke-box, so that the bottom of the case is on a level with the highest point of the boiler. In front of the bottom of the boiler are

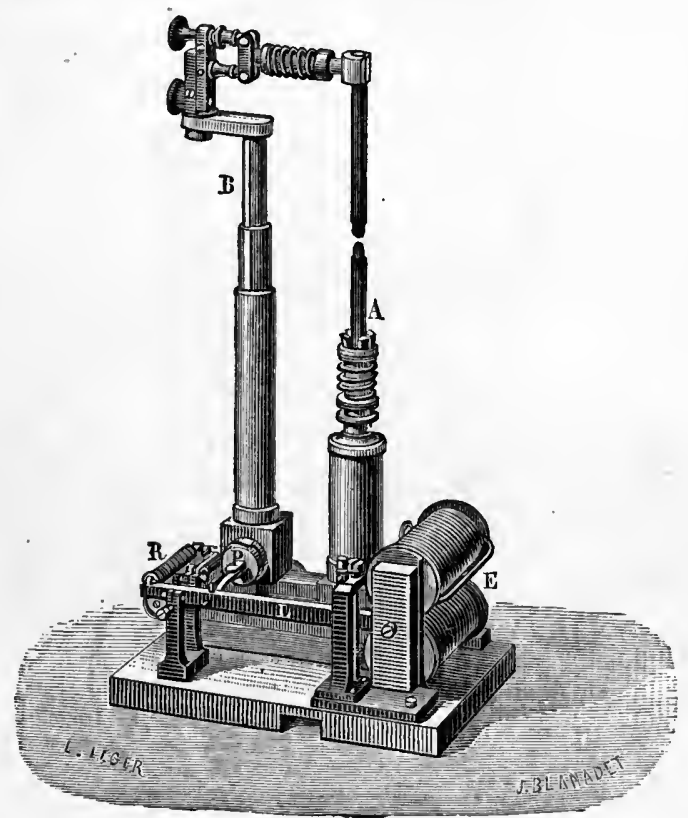

Fig. 225. -Sedlaczek and Wikullil lamp (perspective). (From La Lumière Électrique.)

placed a Schuckert dynamo (for experiments made in Austria) or a Gramme dynamo (for experiments made in France) and a three-cylinder Brotherhood engine (Figs. 226, 227, and 228). Such a lamp was found sufficiently powerful to illuminate a space of about one-third of a mile, and its efficiency was particularly evident in tunnels and for distinguishing signals. By a very simple arrangement the 
lamp can be rotated round its axis by the engine-driver, so as to throw a light in various directions.

Fig. 229 shows a train issuing from a tunnel, at the mouth of which a landslip has taken place, and Fig. 230 shows the illumination of the line by the locomotive lamp.

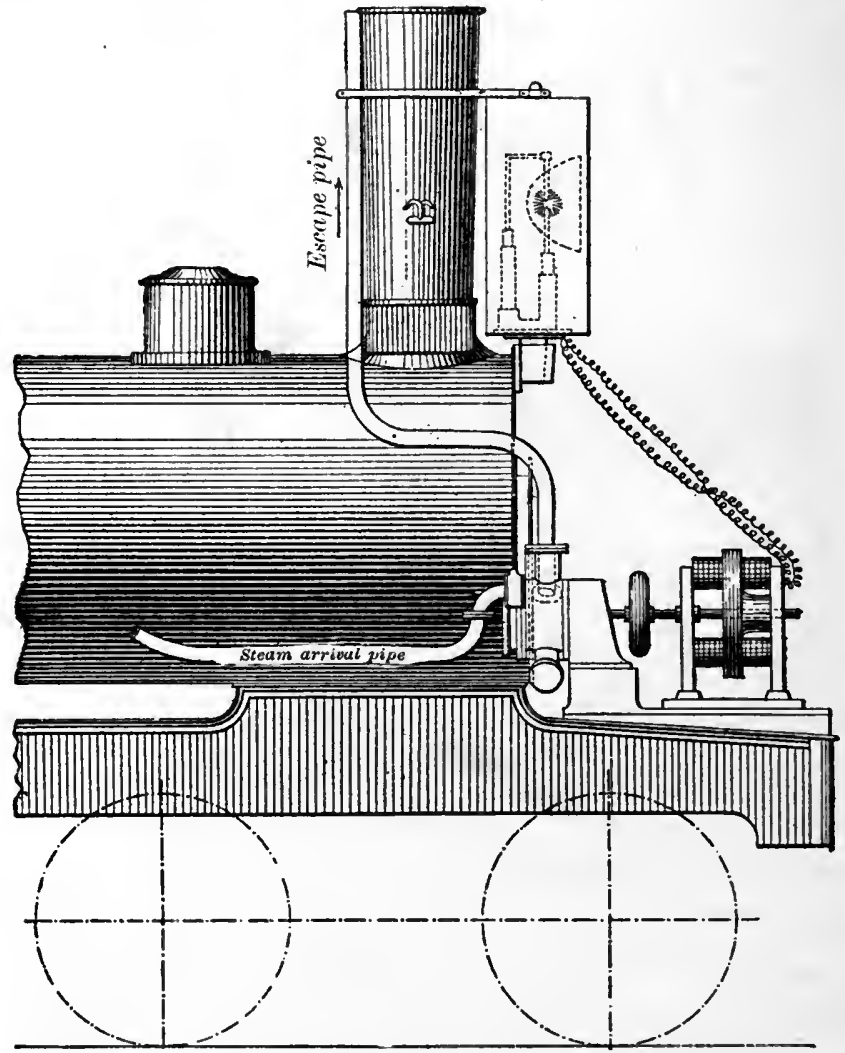

Fig. 226.-Locomotive with electric lamp (sectional elevation). (From La Lumière Electrique.)

This is one of the two tasks electrie light is called upon to fulfil with regard to railway travelling; the second task is the illumination of the carriages, and for this purpose we cannot do better than give an account of the installation 
adopted for the Pulman limited express between London and Brighton, which has lately called forth such a lively controversy in the daily papers.

The train contains four Pulman cars, and can accommodate 113 passengers. The front luggage-van contains two batteries of thirty-two Faure accumulators. Each of these batteries can be placed in communication with two cables extending from one end of the train to the other, and on which the Edison lamps are mounted in shunts. These two

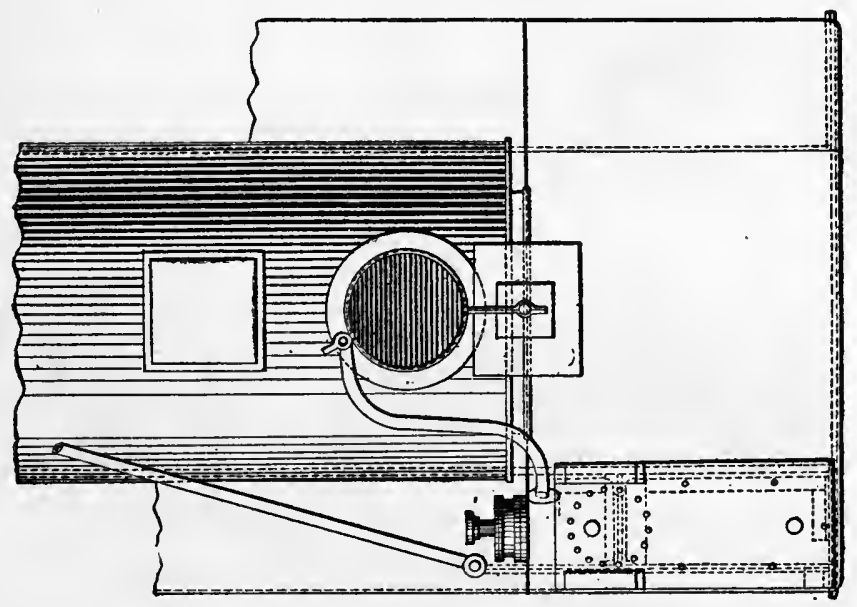

Fig. 227.-Locomotive with electric lamp (sectional plan).

(From La Lumière Electrique.)

groups of accumulators are enclosed in boxes of 1.8 metre in length, 60 centimetres in width, and 1.4 metre in height, the whole weighing about 600 kilogrammes.

As a rule, one of the batteries is sufficient to maintain the incandescence of the twenty-five lamps of the train, but if after some time one of the batteries becomes insufficient, some of the elements of the second or reserve battery are used. These batteries are charged at Victoria before the departure of the train. The electricity is produced by two 


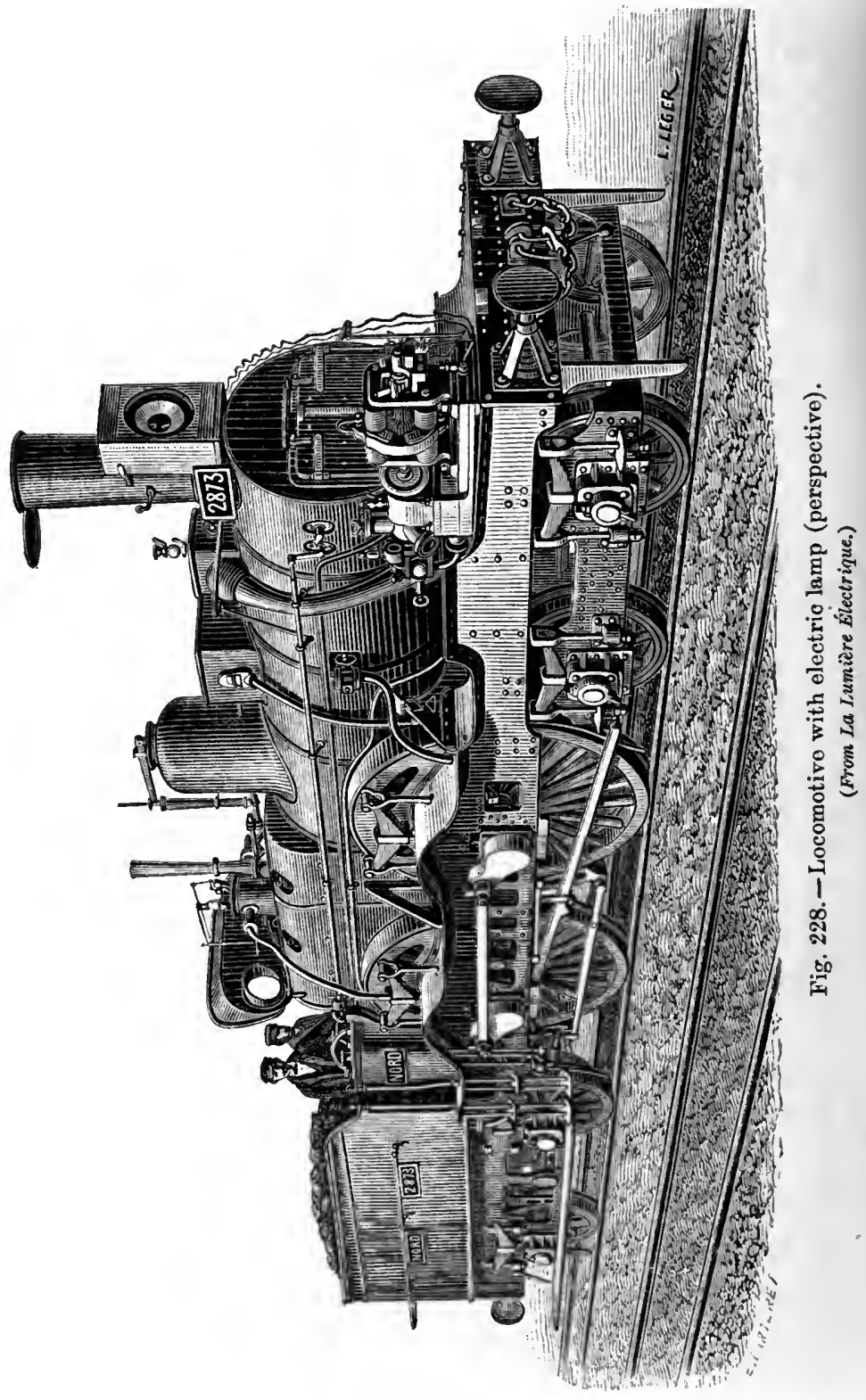


THE APPLICATIONS OF ELECTRIC IIGHTING $46 !$

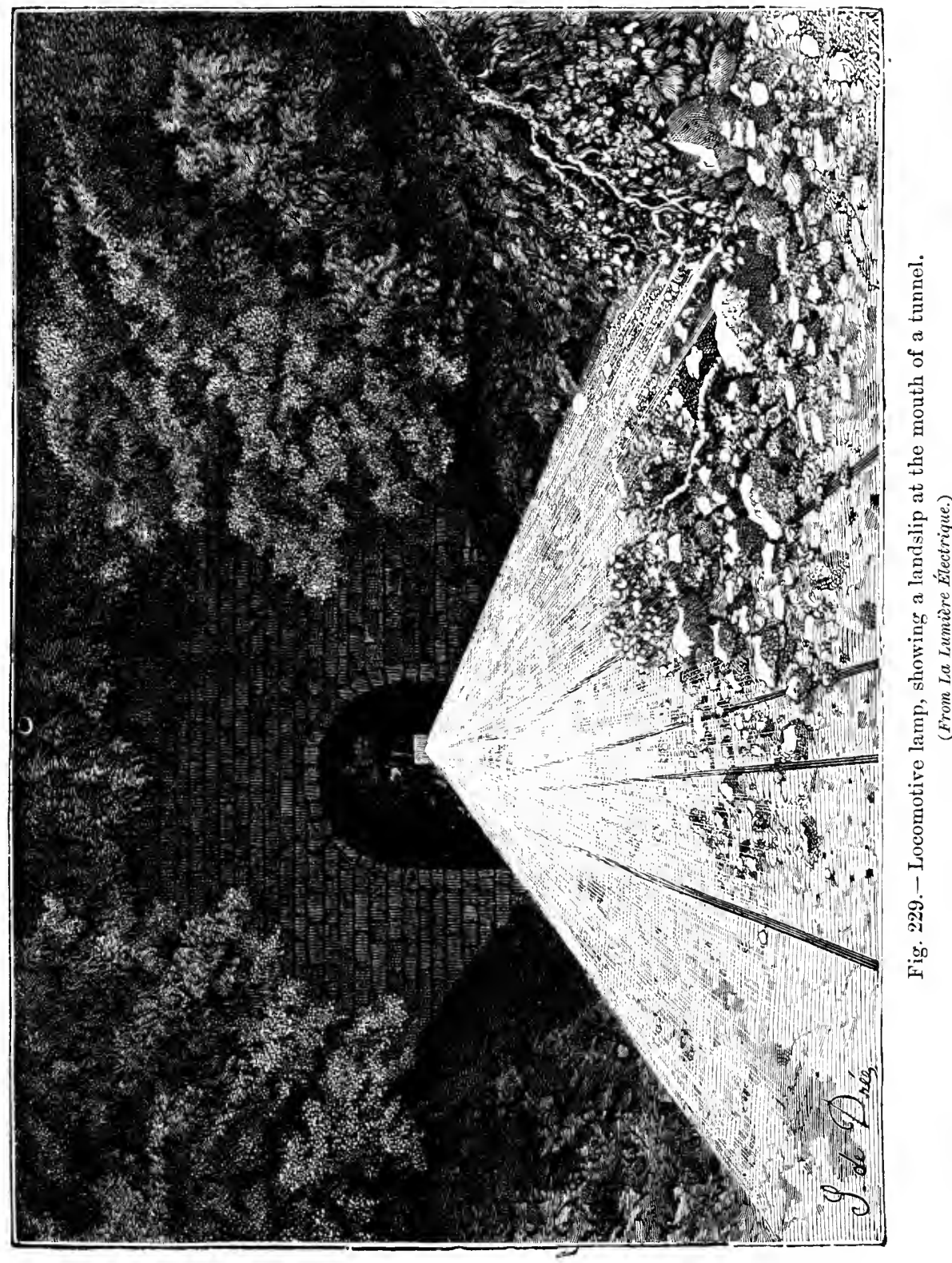


Gramme machines driven by a gas-engine of six horsepower. The charging of the batteries requires about ten hours. When it is finished, the machine charges other accumulators placed in the same room, and which serve for lighting one of the platforms of the station with Maxim lamps.

In the train, during the day-time, the lamps are lighted when passing through a tunnel, at night the lamps remain constantly lighted. The light is steady, and not fatiguing to the eyes.

The Edison lamps are fixed to the ceiling by means of the old oil-lamp supports, and were originally provided with the old lamp-shades. The engineer of the Electric Power and Light Company which has made the installation thinks that the batteries can maintain the incandescence for five hours. This is apparently the minimum obtained with the apparatus employed.

In this particular instance, the solution of the problem of electric lighting is comparatively easy, for the carriages are coupled for a considerable time, and need, so to say, never be uncoupled. The cables are continuous from one end of the train to the other, without any special coupling between the carriages; but this would be otherwise if the carriages had to be frequently separated. Even in this case, however, the necessity of keeping the train for ten hours near the charging machine presents a serious inconvenience. To remedy this defect, a Siemens machine is placed in one of the luggage-vans of the train (Fig. 231), and is driven by one of the wheels of the van. A small hand-worked commutator permits the change of the direction of the current simultaneously with any change in the direction of the train. Under these conditions, the current of the Siemens 

.

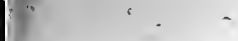




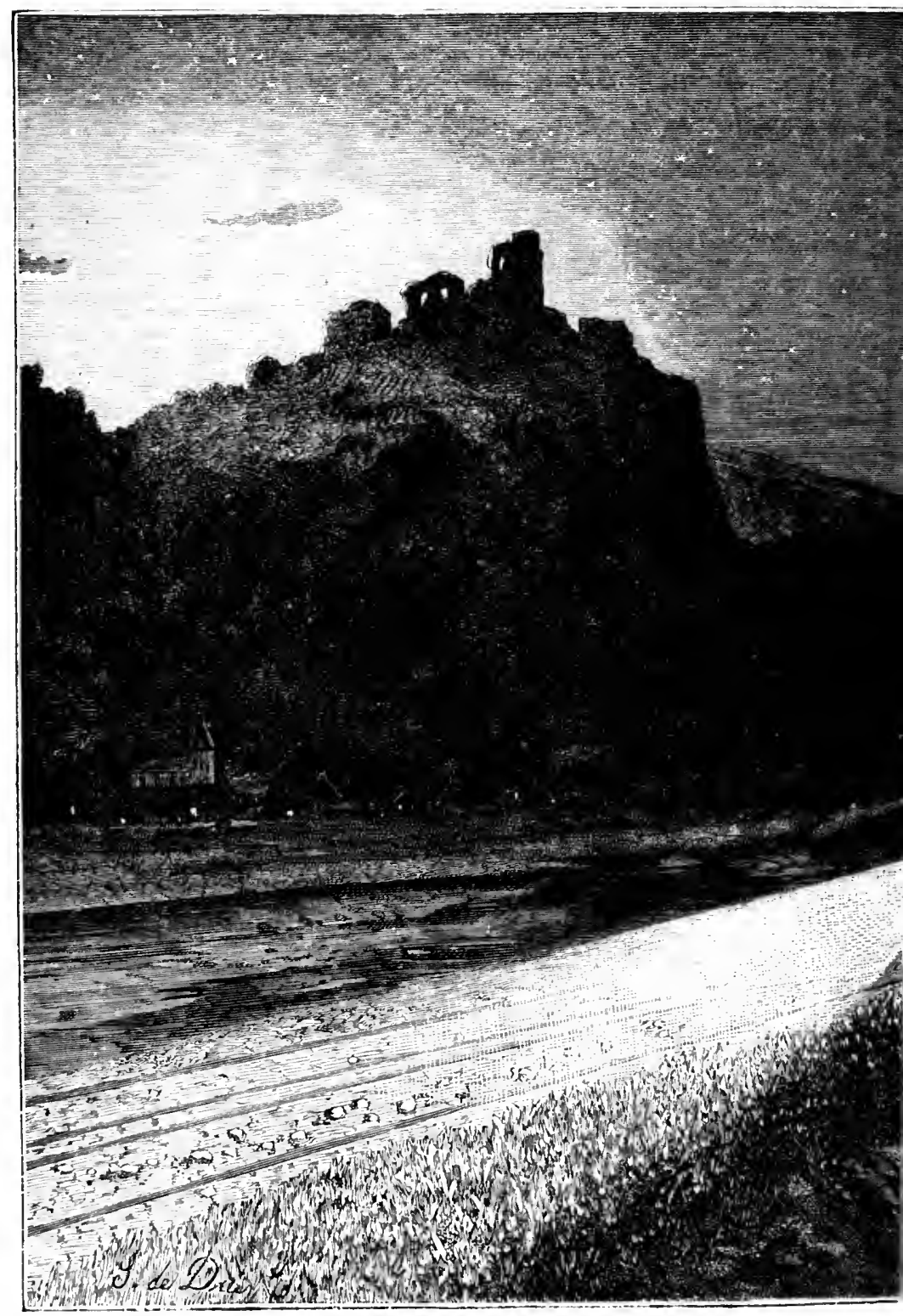

Fig. 230.-Illumiuation of (From $L a$ 


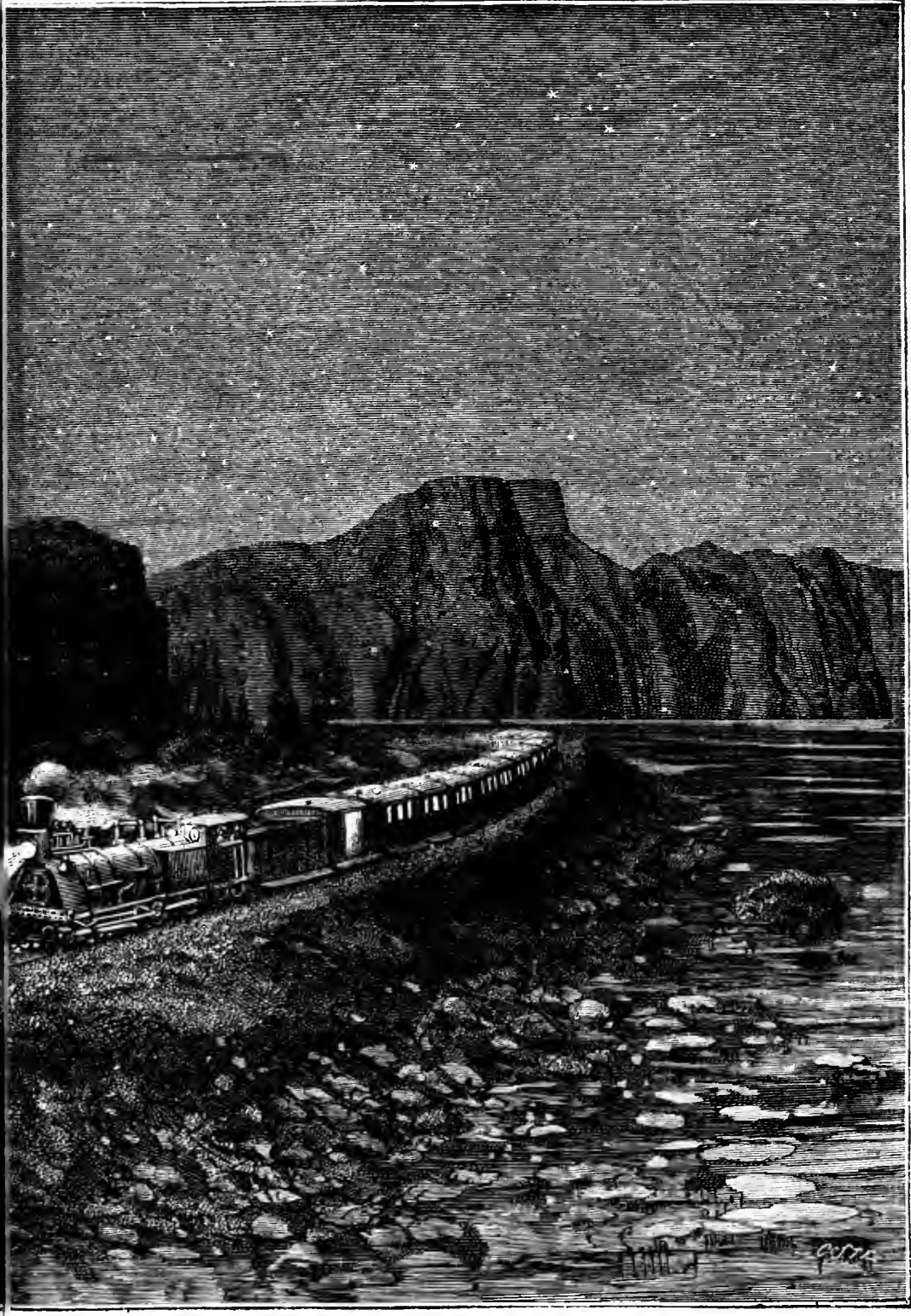

ne is the locomotive lamp.

[Vol. I. 1. 4.10. : Électrique.) 

THE APPLICATIONS OF ELECTRIC LIGHTING. 411

machine will pass constantly through the accumulators as well as through the lamps. When the speed of the train is slackened to such a degree that the current produced by the generator is feebler than that of the accumulators, an automatic commutator interrupts the communication between

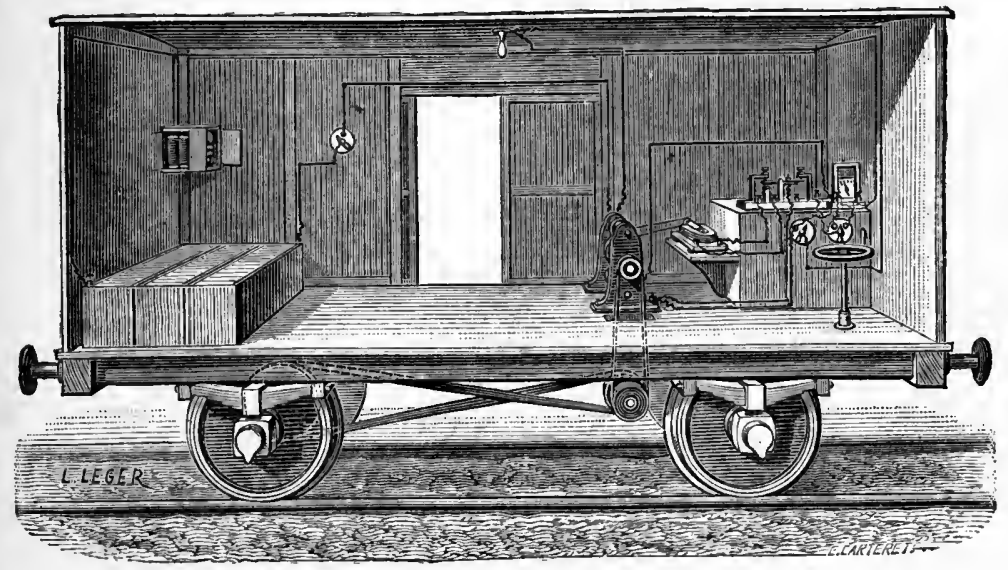

Fig. 231.-Luggage-van containing a Siemens machine.

(From La Lumiere Electrique.)

the machine and the accumulators, and the latter only feed the lamps.

Lighting of Workshops, Factóries, Offices, and Dwelling Houses.

For the illumination of large areas the are light is the most convenient, and has lately found a large number of applications in this direction, more especially so where the factory has already a powerful steam or hydraulic motor, from which a few horse-powers can be taken for the purposes of lighting; the light, of course, ceases at the same time as the work itself. The first installations were made with monophotal arc lamps, and with Gramme's or Siemen's 
machines; a separate machine and transmission were then required for cach lamp. During the last few years powerful machines and division are lamps have come into more general use-a machine sufficiently powerful to supply all the centres, either, as is most generally the case, in one circuit or in a small number of circuits, is used in preference; a considerable saving is thereby realized in the installation of transmissions, of conductors, and in the working expenses. The light-centres thus obtained are less powerful than the original monophotal regulators, but for equal expense they are preferable, because they allow of a more perfect division and diffusion of light.

This diffusion is almost indispensable in most of the applications, as will be seen from the account of a number of installations which we are going to place before our readers.

Illumination of the Brussels Telegraph Office.-The lighting is effected by three Jaspar arc lamps (vide p. 188), which, it will be remembered, are holophotal, that is to say, allow of no division of current, and require each a separate generator. Three Gramme machines, driven by a gas-engine, supply the electricity. The lamps are provided with suitable reflectors. Two of them are placed in the large room, and the third lights a smaller room. There remained another small room to be provided for, which is used for sorting the telegrams, and this was done by borrowing a part of the light of the latter lamp. Fig. 232 shows a plan of the two last-named rooms. The electric lamp numbered 1 and placed in the small room is provided with a system of lenses. This lamp carries a horizontal circular reflector and a conical reflector, nickel-plated inside, which fulfil the double function of hiding the lamp from direct view and of throwing its rays towards the ceiling. The lenses are 
arranged at the foot of the apparatus with movable supports, by means of which they can receive the necessary direction to throw the rays on a desired point. The plan shows that two bundles of rays were intended for the small sorting-room-one was reflected by a mirror at $\mathrm{D}$ and thrown on a white blind $b$, the other went on direct to $\mathrm{C}$.

Each of the generators expends on an average work of two and a half horse-power, and the lamps give a light of 1670 candles.

As regards the expense, the comparison with gas is

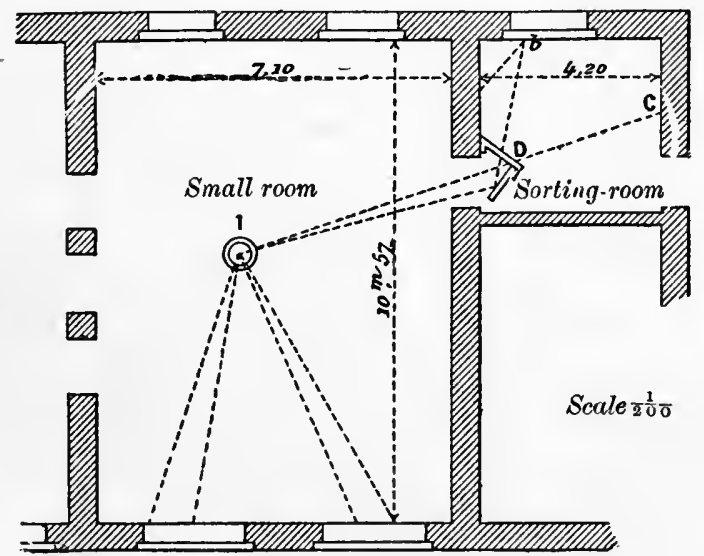

Fig. 232.-Plan of the Brussels telegraph office.

(From La Lumiere Électrique.)

interesting. It appears that the former lighting by seventy gas-burners came to one franc eighty-six centimes (exactly eighteen-pence) per hour, while the electric light costs three francs eighty-two centimes (a little over three shillings) per hour. On taking into account, however, the superior intensity of electric light, these figures would have to be considerably modified. One thing is certain, that electricity has accomplished two desiderata which could otherwise not have been fulfilled. The illumination of the rooms 
was insufficient, and at the same time the heat was intolerable: the light has been increased and the temperature has been lowered. With gas these results were impossible. The success of this illumination has led to the installation of

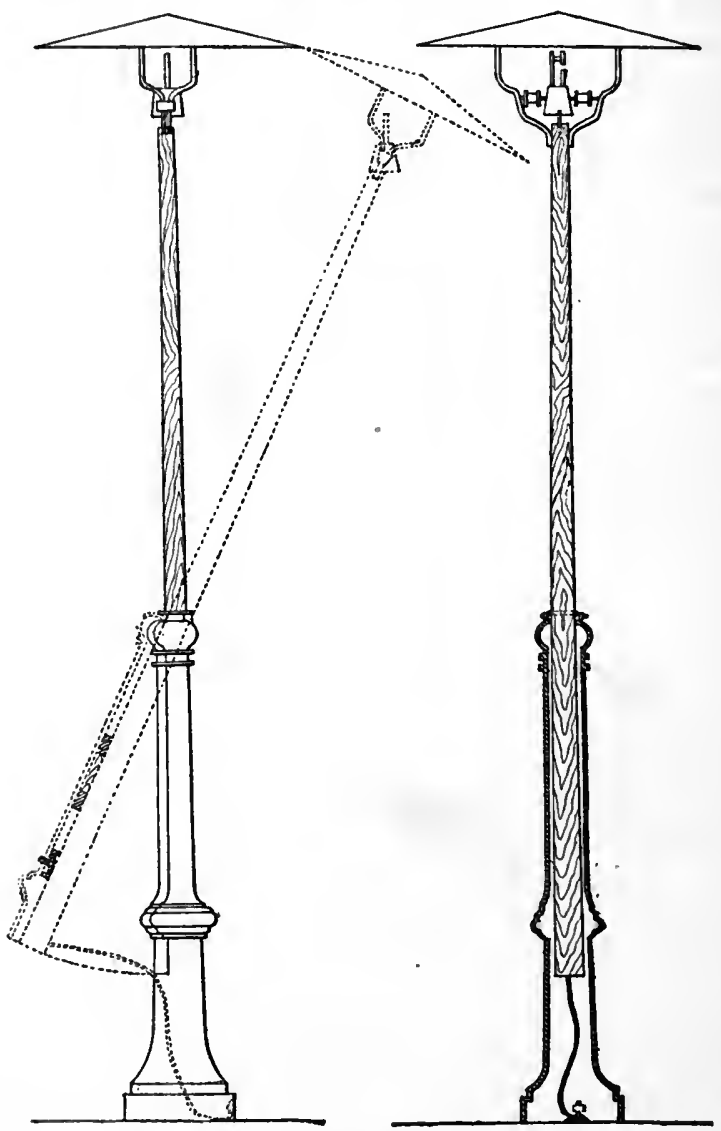

Fig. 233.-Jaspar's lamp (Gare du Nord, Brussels). (From La Lumiere Érectrique.)

three arc lamps on the Place des Nations, before the Gare du Nord, where the telegraph office is. These lamps are placed on oscillating posts similar to those used by Jaspar at the 
Paris Exhibition in 1881 (Fig. 233). The two branches are balanced in such a manner that the manipulation requires very little force. The lamp is carried by a small axle, so as to remain vertical during the movement. These three arc lamps replace twenty ordinary gas-lamps and two Sugg burners. The result is acknowledged to be very good; the price for gas was 1.02 franc per hour; for the electric light it is 2.55 francs. No comparison is given as to intensity:

The Magasins du Louvre.-Corliss steam-engines are used for driving five alternating Gramme dynamos with independent exciting machines, and supplying twenty-four Jablochkoff candles each ; one Méritens machine for twentyfive candles; a second machine of the same inventor for five candles and four arc lamps,-which make altogether 150 Jablochkoff candles and four arc lamps; to these must be added sixty Edison lamps for the illumination of the counting-houses, fed by a special electric machine.

The expenses of first installation were considerable, but this is mainly owing to the many tentative experiments which had to be made for this, the first installation on a large scale effected in Paris or elsewhere. The electricity generated by the machines at the Louvre serves besides for the transport of motive force to a workshop of the Rue de Valois.

The Magasins du Printemps.-These shops, which have lately been entirely rebuilt, have in the basement three steam-engines, together of seventy horse-power, driving three alternating Gramme dynamos of twenty lamps each, and two continuous-current Gramme machines for feeding fifty-six Maxim incandescent lamps. Seventy-five electric candles have been placed in the ground floor, nine in the 
entresol, sixteen in the first, four in the second, and fifty Maxim lamps in the upper stories.

The principal hall is to be lighted by additional electric candles and 400 Maxim lamps.

The Bon-Marché.-Here the Edison system has been chosen. The installation consists at the present moment of 400 Edison lamps, type A, equally divided amongst the basements and shops. These lamps are fed by two Edison machines, type $\mathrm{K}$, making 900 revolutions per minute, and intended to supply 250 lamps each at ordinary speed.

One of these machines works during the day for lighting the basements, and both work in the evening to supply the light for the shops; they are driven by a compound Veyher and Richmond engine, of sixty horse-power nominal. In one part of the shops, where each gas-burner has been replaced by an Edison lamp, the illumination is satisfactory enough as regards quantity, but leaves much to be desired with regard to quality. The lamps flicker, indicating all the irregularities in the movements of the driving machine.

The Comptoir d'Escompte.-It will appear strange that at the present time, when electric machines are so numerous and have been brought to such perfection, recourse should have been had to a liquid battery for an installation on so large a scale as that of the Comptoir d'Escompte. The special requirements of the case, however, were of such a nature that the problem admitted but of a few solutions.

The lighting by machines would have required a motor of about a hundred horse-power, and as the interior arrangements of the buildings may necessitate at any hour of the day the lighting of a larger or smaller number of lamps, according to the clearness of the sky, it would have been 
necessary to keep the motor in constant action. This difficulty, however, might have been overcome if the installation of a steam-engine had not been pronounced unsafe. Storage batteries would have suggested themselves, but their employment was rejected, on what grounds we cannot tell. There only remained now the use of the liquid battery, and it was Grenet's bichromate (vide p. 33) battery which was selected for the purpose. This battery presents a feeble interior resistance and an electro motive force superior to the Bunsen; unfortunately, its energy decreases very rapidly, and it can only be used for effects of short duration. This weakening of the current arises, not from polarization, but from a rapid alteration of the liquid, and especially from a deposit of chromic oxide formed on the positive electrode. Grenet has remedied these two defects by a slow but continuous renewal of the liquid, and by allowing air to enter at the bottom of the battery, which, by the agitation it produces, detaches the chromic oxide.

At the Comptoir d'Escompte the batteries are formed of forty-eight elements grouped for tension. Sixty of these batteries feed one arc lamp, or eight to ten Swan lamps each. They are placed in the attics of the building.

For the distribution of the current, all the batteries have one common wire, which is carried through all the rooms to be lighted; and the second pole of each battery is connected to a commutator, from whence start the wires which complete the circuit of each burner or a group of burners. This arrangement makes it possible to feed each of the circuits from any one of the batteries, and allows of a large number of combinations. To light one of the lamps it suffices to lower the zines of a battery, to open the taps for the circulation of the liquid and of the air, and finally to voL. I. 
establish conmunication by means of one of the studs of the commutator marked with the number of the lamp to be lighted. For extinction, the reverse process has to be adopted. By means of an index placed above the commutator, each bureau can demand the lighting or extinction of its lamps. All manipulations are thus effected at the proper time, without confusion and without useless expense. The lighting apparatus consists of fifty Siemens and Gravier arc lamps and a hundred Swan lamps, distributed over the different parts of the building. In the bureaux the arc lamps are hidden from view, and the lighting is effected by, reflection on the ceiling, under the most favourable conditions of steadiness and softness. The light of the large entrance-hall is especially beautiful ; a large plate-glass ceiling covers the hall, and above this ceiling are sixteen Siemens arc lamps, provided with large shades. The light from these lamps, softened and diffused by the glass ceiling, produces in the hall the effect of daylight. This effect is enhanced by four Siemens pendulums placed in bull's-eye windows in the corners of the hall, and by the light from the lamps in the adjacent bureaux penetrating into the hall through large bay-windows.

The result obtained by this installation is all that could be wished; but from an economical point of view it can hardly be satisfactory, for the battery consumes zinc and potassic bichromate-both rather expensive articles.

The Royal Library at Brussels.-The difficulties in lighting public libraries are casily understood. There is no difficulty in providing sufficient light for the tables in the reading-room, but this leaves the book-shelves comparatively in the dark-the title or number of a particular work cannot be discerned without the use of a hand-lamp, and 
this constitutes not only an inconvenience, but a serious danger; which has excluded all artificial light from most of the public libraries. And here we may well say that the electric light has come to the rescue. The readingroom of the British Museum was lighted with electric light in 1881, and, more recently, one of the large rooms of the Liverpool Library has been fitted up with soleil lamps.

At the Brussels Library the electric light has been applied to the reading-room where the periodicals are kept, and to the ante-room leading to it. In this latter, nine incandescent lamps (Müller-Nothomb's system) have been placed on brackets in groups of three. Three other lamps of the same system light up a passage leading to the machine. Finally, a soleil lamp with a globe completes the installation for the ante-room. The reading-room, with its semi-cylindrical ceiling, has been fitted up with three inverted soleil lamps (p. 300). These lamps, provided with opaque globes half hidden in flower-baskets, throw a diffused light on the ceiling, whose cylindrical surface disperses the light received, and thus illuminates even the remotest corners of the room. The titles of the books can be easily read on all the shelves, and the light hidden from direct view is soft, and possesses that pleasant tint characteristic of the soleil lamp.

The current is supplied by an alternating Gramme dynamo, which is driven by a locomotive engine. The machine feeds the four. soleil lamps, as well as the twelve Müller-Nothomb lamps, which are joined for tension in the same circuit.

The Müller-Nothomb lamp, which is very little known in this country, differs from the other forms of incandescent lamps, not so much by the nature of the carbon filament, 
which is made of carbonized parchment, as by the way in which this filament is attached to the two platinum wires protruding into the lamp. The filaments are flattened out perpendicularly towards their ends, and attached to the platinum wires by a special cement. The lamp, instead of being completely exhausted, is filled with nitrogen gas.

One of the characteristic features of this lamp is the resistance it opposes to sudden changes of current; it only requires a current of two ampères, and is stated to give an intensity of from twenty-five to thirty candles.

From all the installations described above, it clearly follows that a mixed system of lighting has to be adopted for the individual requirements and special arrangements of different buildings. The number of these installations increases daily, but the electric light will only receive the general application it deserves when, by the establishment of central workshops, or by the reduction in price of storage batteries, electricity can be supplied cheaply, in any quantity and at any time.

\section{The Illumination of Theatres.}

The Electric Light at the Paris Opera.-Electric light was used at this theatre for the first time in 1846, for lighting up the skating scene in Meyerbeer's Prophet, and created great astonishment and admiration.

For nearly twenty years Duboscq has been in charge of the electric lighting department at the old Opera-House in Paris, and it is he who has organized, only on a much larger scale, the same department at the new Opera.

The electric light is produced in that magnificent theatre, built by Charles Garnier, by 360 Bunsen elements 
set up in a large room on the ground floor of the building. This is one of the few instances where the battery is still used as an electric generator on a large scale.

Three different systems are used for lighting the lobby of the Opera and two smaller adjacent rooms : Maxim lamps are used for the two rooms, soleil lamps and Edison lamps for the lobby itself. Two candelabra on the mantel-piece carry the Maxim lamps, and the light is, if anything, too brilliant. The lobby itself was, in the first instance, lighted by Edison lamps alone, but it was soon found that this left Baudry's handsome fresco-paintings on the ceiling entirely in the dark, and two inverted soleil lamps have since been added, and the effect is so far satisfactory. There can be no doubt, however, that it would be still better if the chandeliers containing the Edison lamps were replaced by lamps attached to the walls, so as not to impede a full view of the ceiling.

The circular entrance-hall, situated beneath the auditorium, which is called the subscribers' lobby, is brilliantly lighted by a chandelier carrying sixteen Werdermann lamps. These lamps are incontestably amongst the most effective for the illumination of theatres and similiar buildings of large area. The drawbacks of the lamp are the slight variations which it exhibits when the pointed extremity of the incandescent carbon breaks off instead of being regularly consumed; besides, as the lamp presents a very feeble resistance, and consequently requires currents of a great intensity, stout cables have to be employed, and it is difficult to establish circuits of large extent.

On the staircase, Brush lamps and gas combined give a very favourable result. The Brush lamps, thirty-eight in number, were distributed over the entrance-hall and staircase, and received their current from the Palais de 
l'Industrie, through a cable six kilometres in length, on which they were all placed in series.

In the auditorium, a brilliant crown of seventy-two Jablochkoff candles, placed in the frieze, strikes the view. A number of Swan lamps are placed on the gaselier; of these the lower ones only are lighted, and produce, together with the gaslight and the Jablochkoff candles, a very brilliant effect.

The Electric Light at the Hippodrome.-The electric lighting of the Hippodrome consists of 120 Jablochkoff candles and twenty-one Serrin arc lamps, which in full work expend a force of 160 horse-power. The application of two different systems, candles and arc lamps, necessitates the employment of two systems of machinery, both invented by Gramme.

The 120 candles are fed by three alternating-current machines of twenty candles each, and by a machine of sixty candles. Each of the arc lamps is fed by a continuous-current Gramme machine. Each continuous-current machine can only feed one regulator at a time; but, by means of a commutator, different parts of the circus can be lit up according to the requirements of the performance. For carriage races and equestrian exercises, the are lamps of the circumference are lit; for ordinary performances, the arc lamps light up the centre of the ring; for performances in the air, another system is used.

All these changes are effected with remarkable rapidity and ease. An arrangement must be mentioned by which some saving has been effected in the original outlay. The machines working the regulators have no return wire to complete the circuit; the return is effected by the framework of the Hippodrome, which, wholly of iron, serves as a conductor and completes the circuit. 
The transmissions are so arranged that the speed of each machine is rendered independent of the others, and consequently proportionate to the distance which separates the lamp from the electric generator. For this purpose, the diameter of the driving wheel need only be specially adapted to each machine.

The division machines used are alternating Gramme dynamos. The bobbins are arranged in four circuits, feeding five candles each. These machines make 660 revolutions per minute; their inductors are fed by a Gramme machine (workshop type); the charging machines make 600 revolutions per minute.

The machine supplying sixty candles feeds twelve circuits of five candles each, and only performs 540 revolutions; its charging machine has a speed of 1100 revolutions; whilst the machines supplying twenty candles each expend sixty horse-power. The chief engineer, M. Geoffroy, estimates that the machine supplying sixty candles only expends fifty horse-power. This proves again the marked advantage of powerful machines over small ones. The work expended varies between 160 and 170 horse-power. The motive power is supplied by two compound steam-engines of 120 horse-power each. Two flywheels of 2.6 metres diameter transmit the motion to two shafts by means of indiarubber straps of forty centimetres width; the shaft carries a drum, and over the drum passes the horsehair strap which transmits the motion to the electric machines. Three boilers of superior make, of seventy-five horse-power each, complete the system. The extinctions, so frequent at the outset, become more and more rare every day, thanks to M. Geoffroy's exertions.

A few words must be said on the question of expense, 
which, of course, is a very important factor. The electric lighting of the Hippodrome, whose brilliancy and beauty are undeniable, costs 250 to 260 franes per night. The lighting with gas amounted to 1100 to 1200 francs per night a few years ago, and did not produce a very brilliant effect.

The considerable saving effected in this instance is due to the large scale on which the new system is carried out; for smaller operations, electric light would be more expensive than gas.

The Electric Light at the Savoy Theatre-CThis theatre is lighted by 1158 Swan lamps of Gimingham's improved model (vide p. 336). Of these 114 are placed in the auditorium, arranged in groups of three, and supported by elegant brackets along the different galleries. Each lamp is enclosed in a globe of ground glass, and gives a very soft and pleasant light. Fig. 234 represents one of these brackets, designed and executed by Messrs. Faraday and Son, of Berners Street, London.

Two hundred and twenty lamps are employed for lighting the numerous corridors, passages, and boxes of the theatre, while 824 are placed on the stage. These latter are distributed in the following manner:-

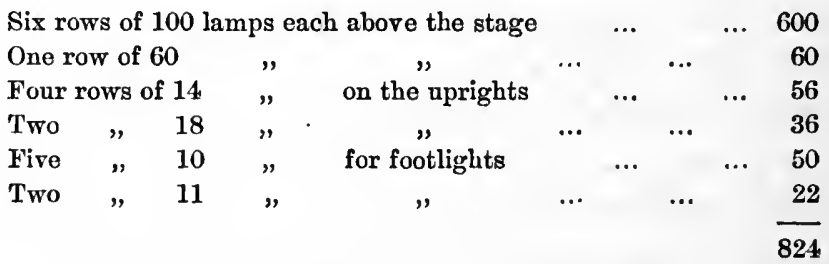

Besides the lamps mentioned, eight test-lamps are placed in the machine-room; they serve for lighting the room, and, as they are in the same circuit as some of the lamps of the auditorium, they indicate, by the change of their lighting 
power, when the lamps on the stage are extinguished or lighted. Fig. 235 represents a chandelier formerly used at the Savoy Theatre.

The lamps are mounted in shunts in six groups, five of which consist of 200 lamps each; the sixth of 170 lamps. The current of each group is produced by an alternating Siemens dynamo, type $W_{1}$, whose field-magnets are excited

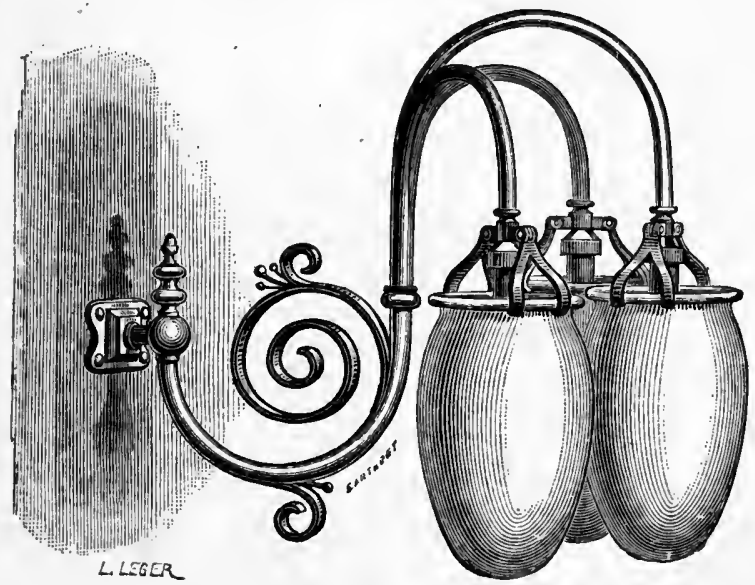

Fig. 234.-Swan lamps at the Savoy Theatre.

(From La Lumiere Électrique.)

by a continuous-current Siemens machine, model $D_{1}$. The machines and driving engines are placed in a shed erected on an open space near the Victoria Embankment, and the current is conveyed to the theatre by means of insulated underground cables. The six alternating machines $W_{1}$ make seventy revolutions, and the six exciting machines $D_{7} 1150$ revolutions per minute. They are driven by three steam-engines-a Garrett movable engine of twenty horse-power, a Marshall movable engine of twelve horse-power, and a semi-portable Robey engine of twenty 


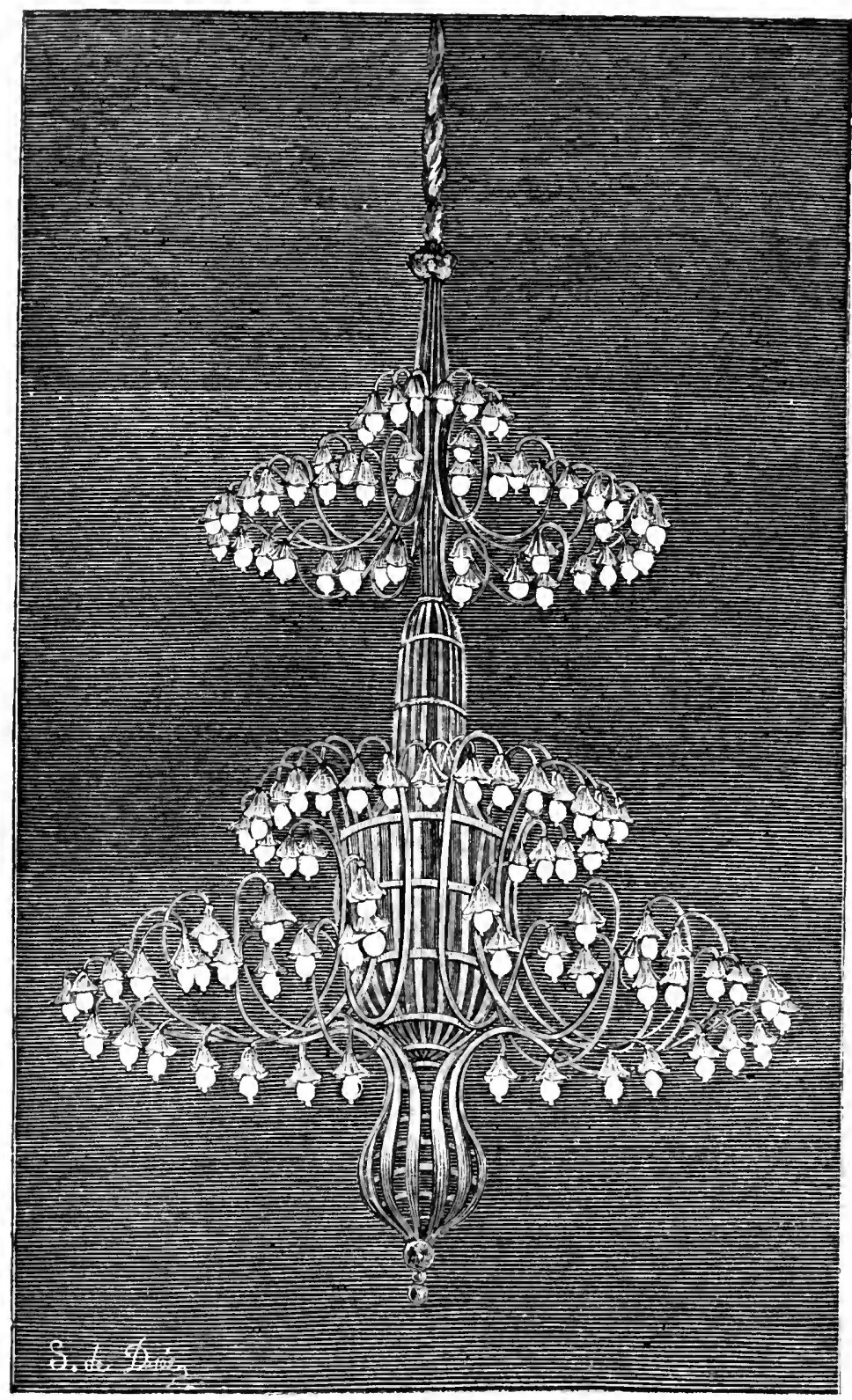

Figr. 2.35. - Chandelier for Swan lamps.

(Frm Ia lomisre fluctrique.) 
horse-power; but the horse-power actually expended, measured by Hefner Alteneck's dynamometer, is 120 to 130 . It must, however, be mentioned that, in addition to the twelve machines for feeding the 1166 incandescent lamps, there is also a Siemens dynamo $D_{2}$ which feeds the powerful arc lamp suspended outside the theatre above the main entrance, and that the force for driving this machine is included in the above figures.

The most interesting feature, however, from a scientific point of view, of this installation is the method by which the lamps in all parts of the establishment are worked; for any one of the series of lamps can be turned on at any moment to full power, or gradually lowered to a feeble red glimmer, as easily as if they were gas-burners, by simply turning a handle. There are six of these regulating handles-corresponding to the number of machines and circuits-placed side by side against the wall of a closet on the left of the stage. Each of these handles acts on a sixway commutator, by means of which a greater or lesser resistance can be introduced into the corresponding circuit (gradually increasing or diminishing it by six successive charges); the intensity of the current traversing the lamps is hereby diminished or increased by as many degrees. The special interest attached to this part of the installation consists in the fact that the lowering of the lamps is accompanied by a corresponding economy in the motive force of the machine, as the variable resistance which is introduced by the regulating handles is not introduced in the external circuit, either of the lamp or of the alternating machine, but in the exciting circuit; therefore, when a series of lamps is lowered, an increased resistance is produced in the circuit of the machine which excites the inductors of 
the alternating dynamo corresponding to this particular series of lamps, the intensity of the magnetic field of this latter machine is thus reduced, and consequently the intensity of the currents of this machine is diminished; but, through the weakening of the magnetic field, the mechanical resistance is reduced in proportion, and the machine expends less force. This special arrangement has been applied to four out of the six circuits, but it works so well that it will doubtless soon be applied to the two others, and this will considerably diminish the working expenses. The resistances introduced into the circuits are of two kinds: the four commutators to which we have alluded cause the current to pass into long spirals of iron wire, supported by a frame and having a free circulation of air around them in order to reduce the heat produced by the current, and the commutators of the two other circuits act in a similar manner on resistances composed of zigzag-shaped iron bands arranged in the same way.

The Electric Light at the Théatre des Variétés, Paris.The details of the installations at this theatre are almost identical with those at the Savoy. Swan lamps are used and the same kind of commutator; the gas, however, is not entirely replaced by electricity, and the current is supplied by 270 Faure accumulators. These are discharged in series of thirty, coupled for tension; four series being joined for quantity. There are, therefore, altogether nine series; eight are sufficient for an evening performance, the last being kept in reserve.

The driving machine is a gas-engine of twelve horsepower, which consumes about ten and a half cubic metres of gas per hour, and works twenty-two hours a day to charge the storage batteries. Four Siemens dynamos $D^{2}$, of 
which one forms a reserve, supply the electricity. By an ingenious arrangement, the excitation of the inductors of these machines is obtained by means of a special circuit branching off to the storage batteries. By this means the inversions of current, are avoided which occur sometimes when the machine in direct connection with the storage batteries slackens speed. On each of the exciting circuits a resistance box with commutator is placed, which allows the regulation of the magnetic field. It has already been stated (p. 349) that, from an economical point of view, the employment of storage batteries is by no means a success, the electric light at the Variétés being twice as expensive as the old gaslight.

Application of Electric Light to Horticulture by William Siemens.

On the 1st of March, 1880, Siemens communicated to the Royal Society a paper "On the Influence of Electric Light upon Vegetation, etc.," in which he arrived at the conclusion that electric light was capable of producing upon plants effects comparable to those of solar radiation; that chlorophyll was produced by it, and that bloom and fruit, rich in aroma and colour, could be developed by its aid. The experiments also went to prove that plants do not, as a rule, require a period of rest during the twenty-four hours of the day, but make increased and vigorous progress if subjected (in winter-time) to solar light during the day and to electric light during the night.

During the whole of the winter of 1880-81, Siemens continued his experiments on an enlarged scale. The arrange- 
ment used for the experiments consisted of a high-pressure steam-engine of six horse-power, which gave motion to two dynamo machines (Siemens D) connected separately to two electric lamps, each capable of emitting a light of about 5000 candle-power. One of these lamps was placed inside a glass house of 2318 cubic feet capacity, and the other was suspended at a height of twelve to fourteen feet over some sunk greenhouses. The waste steam of the engine was condensed in a heater, whence the greenhouses take their circulating supply of water, thus saving the fuel that would otherwise be required to heat the stoves.

The experiments were commenced on October 23, 1880, and were continued till May 7, 1881. The general plan of operation consisted in lighting the electric lights, at first at six o'clock, and during the short days at five o'clock, every evening except Sunday, continuing their action until dawn.

The outside light was protected by a clear glass lantern, whilst the light inside the house was left naked in the earlier experiments, one of Siemens' objects being to ascertain the relative effect of the light under these conditions. The inside light was placed at one side over the entrance into the house, in front of a metallic reflector, to save the rays that would otherwise be lost to the plants within the house.

The house was planted in the first place with peas, French beans, wheat, barley, and oats, as well as with cauliflowers, strawberries, raspberries, peaches, tomatoes, vines, and a variety of flowering plants, including roses, rhododendrons, etc. All these plants being of comparatively hardy character, the temperature in this house was maintained as nearly as possible at $60^{\circ} \mathrm{Fahr}$.

The early effects observed were anything but satisfactory, and it.was only after a lantern of clear glass had 
been put round the light, for the double purpose of discharging the chemical products of the are and of interposing an effectual screen between the are and the plants under its influence, that satisfactory results were obtained. Thus peas which had been sown in October produced a harvest of ripe fruit on February 16, under the influence, with the exception of Sunday nights, of continuous light. Raspberry stalks, put into the house on December 16, produced ripe fruit on March 1; and strawberry plants, put in about the same time, produced ripe fruit of excellent flavour and colour on February 14. Vines which broke on December 26, produced ripe grapes, of stronger flavour than usual, on March 10. Wheat, barley, and oats shot up with extraordinary rapidity under the influence of continuous light, but did not arrive at maturity; their growth, having been too rapid for their strength, caused them to fall to the ground, after having attained the height of about twelve inches.

It has likewise been proved that plants grown and brought to maturity under the influence of continuous light, are capable of reproduction.

The result of these experiments is that, although periodic darkness evidently favours growth in the sense of elongating the stalks of plants, the continuous stimulus of light appears favourable for healthy development at a greatly accelerated pace through all the stages of the animal life of the plant, from the early leaf to the ripened fruit. The latter is superior in size, in aroma, and in colour to that produced by alternating light, and the resulting seeds are not, at any rate, devoid of regerminating power. Further experiments are necessary before it would be safe to generalize, nor does this question of diurnal rest in any way bear upon that of annual or winter rest, which probably most plants that are not so-called annuals do require. 
Siemens has found useful employment during the daytime for the power-producing machinery by applying the electric energy to farming operations (including the pumping of water, the sawing of timber, and chaff and root cutting), at various distances not exceeding half a mile from the source of power; thus reducing indirectly the cost of the light during the night-time. Siemens has calculated that the total cost per light of 5000 candles amounts only to sevenpence per hour, provided the power of the dynamomachine is also utilized during the day-time.

The Electric Light applied to Building Purposes AND Agriculture.

When, in large towns like London, Paris, New York, and others, business premises are destroyed by fire, or extensive alterations have to be made on these premises, it is often of the greatest importance that the reconstruction or the alterations should be carried out with as little delay as possible, and in this case it will be found necessary to carry on the work during the night as well as by day; and here again the electric light has rendered signal services.

A number of buildings in Paris, as, for instance, the premises of the Crédit Lyonnais, the Transports Parisiens, the foundations of the Eden Théatre in the Rue Boudreau, owe their speedy termination to the use of electric light.

It will be in the recollection of our readers that the enormous shops situated at the corner of the Boulevard Haussmann and Rue du Havre, called les Magasins du Printemps, were entirely burnt down at the beginning of 1818 ; within six months of their destruction these shops 
had been entirely rebuilt-a truly marvellous feat, which could only be accomplished by the aid of electric light.

The Jablochkoff candle was used for illuminating the works at night; from the beginning a movable engine of six horse-power was employed for driving an alternating Gramme dynamo and its exciting machine, which fed eight lamps set up in different parts of the scaffolding. Four of the candle-holders had opaque globes; in the four others the candles were exposed, but a reflector was placed above these candles to throw the light on the particular spot where the workmen were employed. For the work of the masons the electric light was only used till six o'clock p.m., but for the erection of the iron framework it was kept up till nearly midnight.

The Jablochkoff candle has also been used for building the Hotel Metropole in Northumberland Avenue, London.

In districts where large crops of wheat or barley have been cut and are sufficiently dry to be carried, it becomes of the utmost importance, in the case of a threatened change of weather, that the carting should be performed within the shortest possible period, and there can be no doubt that before long the electric light will be called upon to lend its powerful help to the operations of carrying crops of corn and hay. If the individual farmer cannot afford to set up an installation for himself, associations will be formed, as is already the case for ploughing, threshing, etc., by steam, for the purpose named. Movable engines, which are extensively used for agricultural work during the day-time, will drive a generator during the night, posts will be established at convenient distances, connected by conducting wires, and carrying at the top arc lamps with powerful reflectors, which will throw their light on the

vol. I. 
surrounding area, and will enable the farmer to save the threatened crops.

\section{The Electric Light applied to Submarine Explora- TIONS AND Constructions.}

The electric lamp is admirably suited for submarine work, as, for instance, for constructing, at some distance beneath the level of the sea, the foundations of a lighthouse, a signal-tower, or some such building. After having determined, by means of previous explorations, the spot where the foundation is to be laid, barges with the necessary. material are taken to this spot, a steamer conveys to it the men to be employed in the work, and also supplies the necessary motive power for driving the electric machines that produce the light; the workmen, of course, are supplied with diving dresses, and telephones are used for communications. Under these conditions, and provided the sea be calm enough, the work can be carried on quite regularly and the most solid foundations constructed.

According to the work to be done,each workman can either carry his own lamp-and in this case incandescent lamps, specially constructed for the purpose, must be employed-or a single powerful centre can be used for lighting up the whole of the submarine building area. In this latter case Bazin's apparatus gives the most favourable results.

This consists of an electric lamp, which is nothing. but Duboseq's regulator, and was first used in 1864 to examine the wreck of the Confederate steamer, the Alabama, which was sunk in sight of Cherbourg. The lamp is enclosed in a thick cylinder of more than one metre diameter, which 
carries an enormous glass plate at the bottom. The lower part of the cylinder is filled with a solution of alum to counteract the pressure of the sea-water, which increases very rapidly with the depth. In the upper part is a powerful electric lamp, whose luminous rays pass through the solution of alum and the glass plate, to light up below it a circular space of more than thirty metres diameter. It is clear that this apparatus can be used with the best effect for the salvage of cargo of a foundered vessel.

\section{Illumination of Public Thoroughfares.}

Most of the well-known arc lamps, such as the Brush, Siemens, Crompton, Gülcher, Weston, etc., have been used for this purpose, and, in addition to these, the Jablochkoff candle and Edison's, Swan's, and Lane Fox's incandescent lamps have also found numerous and successful applications.

The Electric Light in the City.-The Street Committee, in conjunction with the Bridge House Estate Committee, entered, in 1880, into a contract with three electric light companies for the illumination of the principal streets of the City, as well as of London, Southwark, and Blackfriars Bridges. The three companies were the Brush, Siemens, and Electric Light and Power (Weston-Lontin) Companies. The lamps replaced in each of the three districts into which the City had been divided for the purpose, 156, 157, and 139 gas-burners of fourteen candles each, or 6328 candles altogether, and the intensity of the new installation was 103,400 candles or 16.3 times larger than that of gas.

The Brush lamps gave on an average fifteen times more light than the gas-burners, the Weston lamps fourteen times 
more, and the Siemens lamps twenty times more light. Through accidents, imperfect installation, and want of attention, the Brush lamps lost twice as many hours as the Siemens, but, on the other hand, the Siemens lamps lost thirty times as many hours as the Brush through the defective action of the carbons.

These experiments seem to have proved that most of the derangements to which the Brush or Siemens systems are liable arise from causes which can easily be avoided, with the exception of those accidents which are caused by the mechanism of the lamps themselves; most of the delays in the working of the Siemens lamps being attributable to a want of attention on the part of the persons in charge of the lamps, while those of the Brush lamps must be attributed to defects in their mechanism. Of the 3225 lamps which light the City, 2509 defective ones had to be removed in 1881 , which is, of course, a very considerable proportion. The Street Committee has decided, in consequence of these trials, that the Brush Company shall continue to light the first district of the City at a reduced price of $£ 800$ (against $£ 1410$, the amount of the first tender), and has rejected the offer of Messrs. Siemens of lighting the third district at a cost of $£ 3000$ per annum. Some figures on the comparative price of gas and electric light will be found in the chapter on the expense of electric lighting.

We only know of one town in this country where gas has been entirely replaced by electric light, and that is Chesterfield, in Derbyshire. For another instance of the same kind we have to go to the far West-to San José, in California.

The Electric Light at Chesterfield.-Twenty-two Brush lamps and one hundred Lane Fox incandescant lamps are 
used for lighting the streets of this town. The whole length of the streets is between fourteen and fifteen kilometres (about nine miles), and the number of lamps mentioned is amply sufficient for the requirements of the town. The posts from which the arc lamps are suspended are of variable height, according to the locality to be lighted; their length varies from four to eight metres. In streets where the houses are high (rather an exception at Chesterfield), and for the lighting of squares, the arc lamp is placed right at the top of the post. Crown-glass globes protect the eyes of the passer-by, and a large reflector placed above the lamp projects the rays on the ground and on the front of the buildings on either side. The cables for feeding the different lamps are all air-wires and attached to wooden posts, like telegraph wires. The arc lamps, as has already been stated, are suspended from variable heights, whilst the incandescent lamps are all placed at the same height, about four metres from the ground.

The Brush lamps are distributed over the area of the town in such a way that the incandescent lamps can be extinguished at midnight without plunging the town into darkness.

The current is produced in a central workshop by two dynamos, which could feed 40 arc lamps and 320 incandescent lamps, if necessary.

The Electric Light at San José.-This installation is of somewhat novel character, and is well worth mentioning.

San José is a town in the state of California, with a rapidly increasing population, and was formerly lighted with gas. Through the liberality and enterprise of Mr. Owen, the editor and proprietor of a daily paper, the town is now illumined in the most satisfactory manner by electricity. 
The first electric tower which was erected is a foursided pyramid; the framework is wholly of iron, not very elegant, but fulfilling its purpose, namely, that of supporting a number of large electric lamps, which by their radiation light up a very large area. The quadrangular figure at the base is about twenty-five metres in the side, whilst the pyramid at its pointed end is only 1.25 metre. The four supports which start from the corners of the streets are formed of gas-pipes of ten centimetres diameter up to a height of thirty metres, whilst the next fifteen metres are only seven centimetres, and the last portion only five centimetres in diameter. The total height is sixty metres, and all the oblique and circular branches are formed of narrower tubes. At the summit the lamps are surmounted by a large circular plate, which serves for their protection, and at the same time as a reflector for the diffusion of the light.

The lamps supplied by the California Electric Light Company have a lighting power of 400 candles each. Six of them are at the summit of each tower; they are fed by a Brush machine requiring a motive force of nine horsepower. The effect produced resembles the light of the moon on a very clear night; and the light, being at such a great height, can never fatigue the eyes of the spectators.

The light thus diffused in all directions is more intense at a distance of 800 metres from the foot of the tower than the light which would be produced by ordinary gas-burners regularly placed at twenty-five metres distance from one another; in fact the whole length of the two streets, at the intersection of which the electric lamps are placed, can be seen from end to end, a distance of three kilometres (nearly two miles). 
The results obtained with the first electric tower being so highly satisfactory, five new towers have been erected since, which now illumine the town in a marvellous fashion, and even throw their luminous rays into the suburbs, which formerly could not boast of even a single gas-burner.

Illumination of the Port of Le Havre.-This is a tidal harbour, and vessels can only enter it at high tide. When both the tides happen during the day-time, the vessel which misses the first can enter with the second, and has therefore only eleven hours longer to wait in the roadstead; but at those periods when one of the high tides occurs at night, the vessel which misses the day tide has sometimes to remain in the roadstead for twenty-three hours. Now, this is all well and good in calm weather, but when a gale is blowing the vessels have to weigh anchors, and it has happened more than once that steamers have had to disembark their passengers at Cherbourg.

A decision was therefore arrived at in 1880, in order to enable vessels to enter by night as well as by day, to light the harbour with electric light, and a contract was made with the Jablochkoff Company for ten years.

Whenever there is a night tide, the piers, the outer port, and the main docks are illumined one hour before and two hours after the moment of high tide, and the largest vessels can now enter by night as well as by day.

The installation comprises thirty-four lamps, of which thirty-two are indicated in the plan of Fig. 236; two more have been added since, and the existing machines can supply as many as forty. Independent of this installation, a number of lamps are used for lighting the quays used by the transatlantic steamers. 


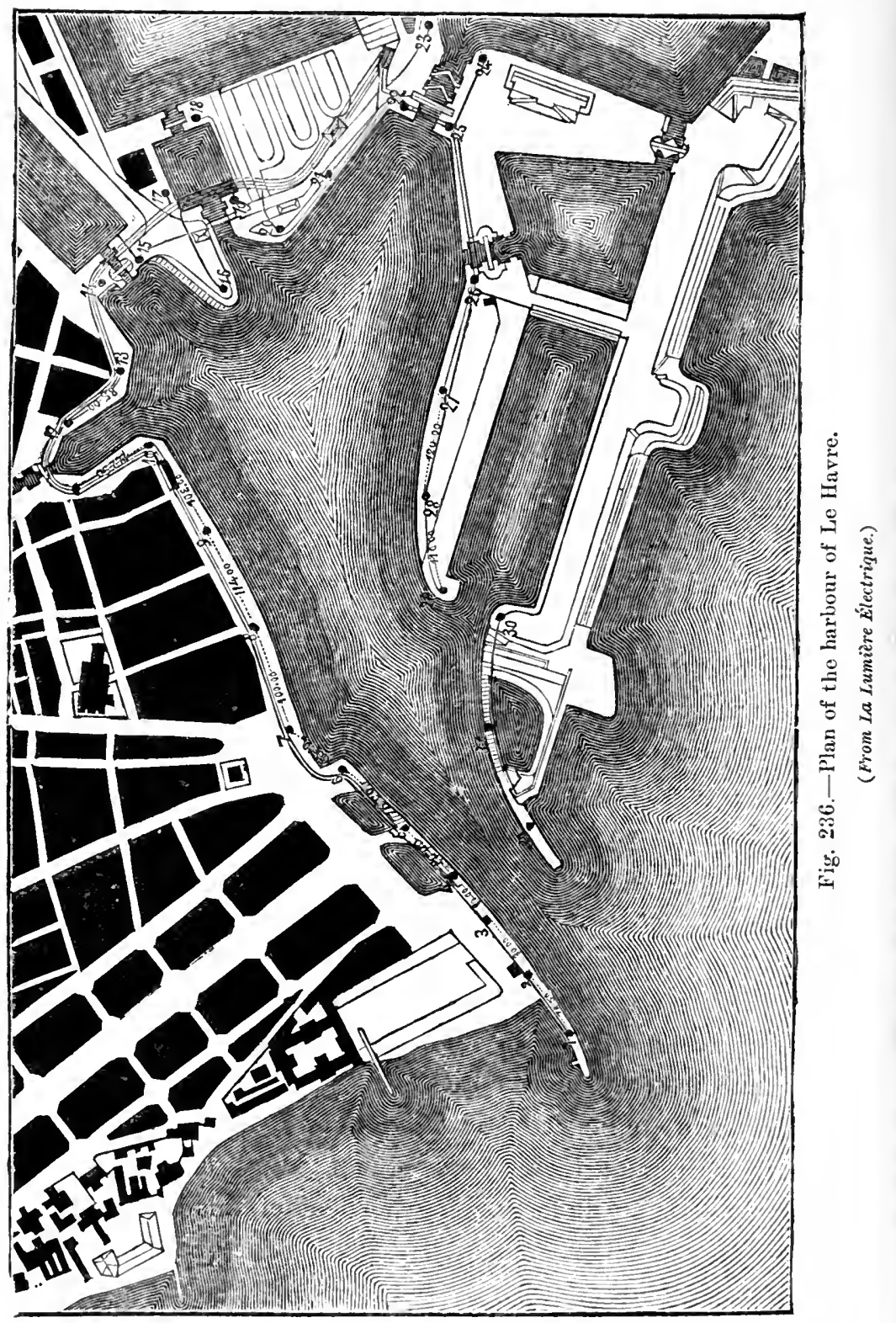


The thirty-two lamps which we have mentioned are divided into six circuits. The first circuit, of four lamps ( 1 to 4 ), lights the north pier, and is 3900 metres in circumference. The second circuit, of five lamps ( 5 to 9 ), supplies the large quay, and measures 2900 metres; a sixth lamp in a red lantern has been added to this circuit, intended for a signal. The third circuit, of six lamps (10 to 15), comprises the docks of Notre Dame and the docks of De la Barre; it is 1900 metres. The fourth circuit, of six lamps (16 to 21), lights up the lower part; it comprises a red light, and measures 1250 metres. The fifth circuit, of six lamps (22 to 27), lights the Transatlantic and Florida Docks; it measures 1400 metres. The sixth circuit, of five lamps (28 to 32), lights the south pier; it.measures 2900 . metres, and comprises a red light.

The machines are installed not far from the Transatlantic Jocks; two steam-engines of thirty-five horse-power each drive four self-exciting Gramme machines of type 2. One of these is held in reserve in case of accidents; the three working machines supply two circuits each. The arrangement of all the circuits is identical, and we need, therefore, only consider one of them. It is represented in Fig. 237, which does not so much preserve the proportions of the different parts, as indicate their respective positions.

$M$ is the self-exciting Gramme dynamo supplying the two circuit $C$ and $C^{\prime}$, the former of which we will now consider.

The current of this machine is to some extent regulated by means of the resistances $R$ connected to the terminals $\mathrm{B}$ and $\mathrm{B}^{\prime}$. This apparatus is placed between the exciting machine and the inductors of the generator, so that, by varying its resistance, the intensity of the magnetic field, 


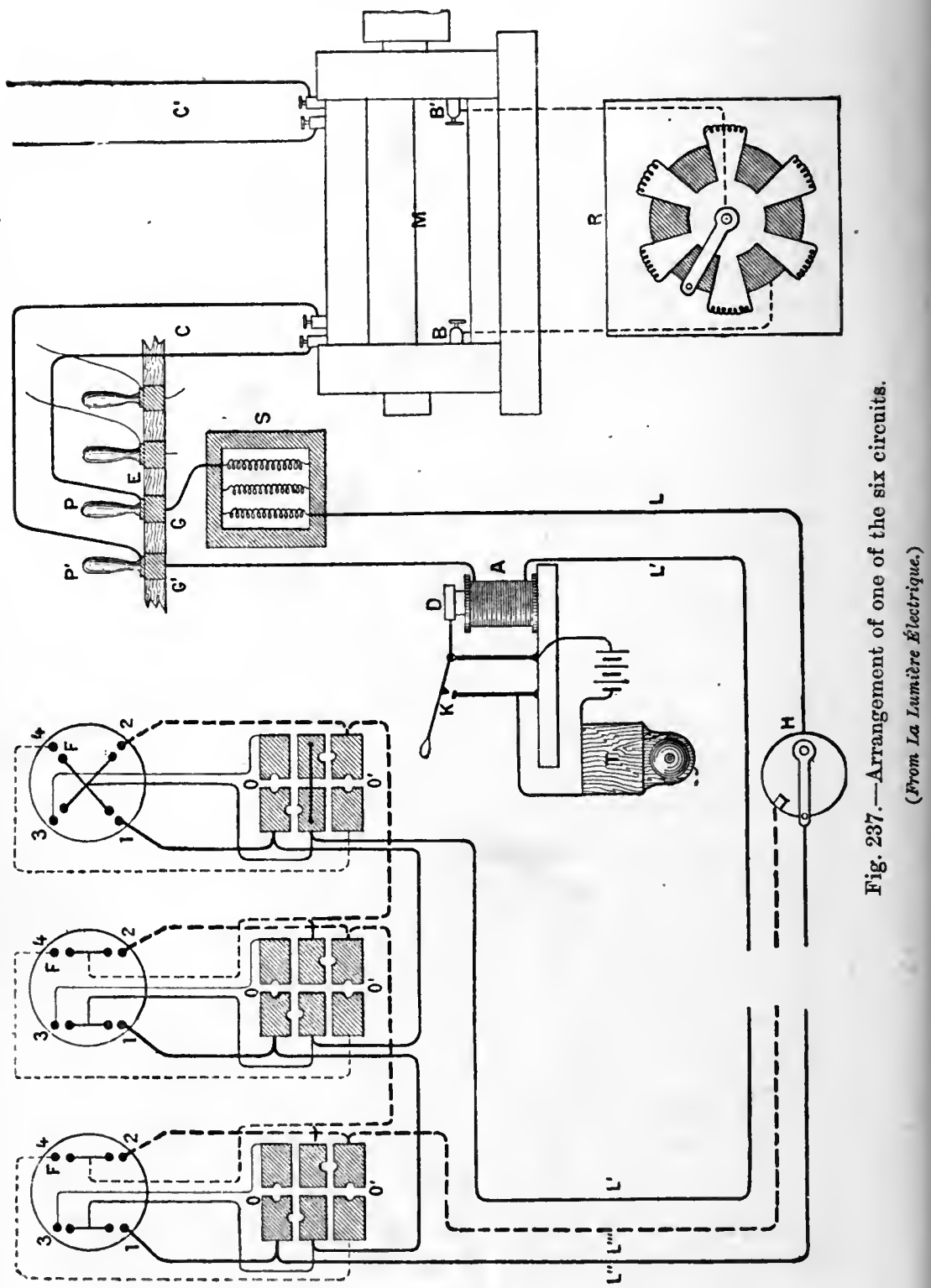


and with it the current produced, can be regulated. The conductors of the circuit $\mathrm{C}$ first go to two handles $\mathrm{P}$ and $\mathrm{P}^{\prime}$, terminating in metallic pieces, which fit into two other metallic pieces $G$ and $G^{\prime}$, fastened on the beam E. The following is the object of this arrangement :- If an accident happened to the machine, the handles $\mathrm{P} \mathrm{P}^{\prime}$ would be taken off and replaced by two similar ones, which terminate the conductors of the reserve machine, so that the current would only be interrupted for a very short time.

On the passage of the conductor $\mathrm{L}$, starting from $\mathrm{G}$, is a resistance frame $\mathrm{S}$, intended to render the circuit $\mathrm{C}$ equal in intensity to the circuit $\mathrm{C}^{\prime}$ of the machine. The inequality of intensity of the two circuits is due to two causes-first of all to the inequality of their length, and secondly to the fact, confirmed by experience, that the circuit $\mathrm{C}$, which is nearest to the exciting machine, has a greater electro-motive force than the other. By means of the frame $\mathrm{S}$ the two intensities can be rendered equal.

The conductor $\mathrm{L}$ then goes to the two-way commutator $H$. The two conductors $L^{\prime \prime}$ and $L^{\prime \prime \prime}$, which pass through $\mathrm{H}$, start from the machine-room, and transmit the current to the lamps $F, F, F ; L^{\prime}$ is the return wire.

Before returning to $G^{\prime}$, this return wire traverses an electro-magnet $A$, which during the passage of the current keeps an armature D constantly attracted. If extinction occurs, the current no longer passes, the armature is released, and closes the contact $\mathrm{K}$ and rings a bell $\mathrm{T}$. As will be seen later on, an immediate relighting can be effected on receiving this warning.

It has been stated above that nearly all the circuits comprise six lamps; for the sake of simplicity we have only represented three in our diagram. All these lamps contain 
four Jablochkoff candles, with carbons of six millimetres; but the first five contain each two candle-holders of two candles each, and have therefore two return wires, whilst the last contains a candle-holder of four candles, of the ordinary form, with a single return wire. Finally, at the foot of each lamp-post is a commutator $\mathrm{O}^{\prime} \mathrm{O}^{\prime}$ with six plates, through which the wires pass before going to the lamp. This arrangement, though apparently complicated, ensures two results: first, under normal conditions, by a simple manipulation in the machine-room, the current can be made to pass from the candles 1 to the candles 2 , by means of the commutator $\mathrm{H}$; secondly, in case of extinction, after having replaced the candles 1 by the candles 2 , the candles 3 can, by a simple manipulation of the commutator $\mathrm{O} \mathrm{O}^{\prime}$, be prepared for a fresh passage of current.

To understand how this is done, let us first suppose the commutator $\mathrm{H}$ to be placed in such a way that the current follows the conductor $L^{\prime \prime}$; it arrives first at the upper piece on the left of the commutator $\mathrm{O} \mathrm{O}^{\prime}$ of the first lamp, and goes from thence to candle 1; passing afterwards through the return wire of candle-holder $1-3$, it goes to the central piece on the same side, and starts again from thence to arrive at the commutator of the second lamp. In this, as in the following lamps, the current follows the same path, but with this difference, that there is only one return wire for the last lamp; finally, the current returns to the machine through the wire $L^{\prime}$, traversing on its way the electromagnet $\mathrm{A}$.

It will be seen that in this passage the current follows the full lines of the diagram. If now, the candles 1 being burned, the commutator $H$ comes to be changed and the current arrives through $\mathrm{L}^{\prime \prime}$, it will follow a path absolutely 
symmetrical with the first, indicated by the dotted lines, and will feed the candles 2. The return will be effected, as before, through the wire $L^{\prime}$. Now, let us suppose that, the current arriving through $\mathrm{L}^{\prime \prime}$ (full lines), and burning the candles 1 , a sudden extinction takes place, the alarm-bell $\mathrm{T}$ will ring in the machine-room. The first duty of the person in charge will be to place the commutator upon $\mathrm{L}^{\prime \prime}$, and this will light all the candles 2 , so that the extinction will only last a very short time. After this, in every lamp the hole which separates the two upper pieces from the commutator is stopped up with.a screw-plug. Now, the piece on the right, as will be seen from the drawing, communicates with candles 3 ; therefore, if at the moment when the candles 2 are burned the commutator is again put on the conductor $L^{\prime \prime}$, the current will pass through all the candles 3 .

If afterwards the two lower pieces of the commutators are equally joined at $\mathrm{O}$, the piece on the left being connected to the candles 4 , a new change of the commutator will light all the candles 4 . The four candles of one lamp can thus be lighted during the same evening; but at Havre this is unnecessary, - the period of lighting only lasts three hours, and two candles are amply sufficient. Under normal conditions, the current from the machine-room passes from one to the other, and the arrangement which we have described is only of value in case of accident.

In each of the commutators, the upper piece on the left and the lower piece on the right can each be joined by a screw-bolt to the corresponding centre piece. These two communications are intended to remove the lamp from the circuit, if the current passes either through $\mathrm{L}^{\prime \prime}$ or through $\mathrm{L}^{\prime \prime \prime}$.

The arrangement of the six circuits is the same as the one just described. All the wires go first of all to the beam 
$\mathrm{E}$, which carries all the handles; the electro-magnets $\mathrm{A}$ and the commutators $\mathrm{H}$, all provided with the same numbers, are arranged side by side in the machine-room, and the manipulation is thereby rendered very easy. All the conductors start together, and diverge afterwards towards their destination. As, at different points, these conductors had to be immersed under water or placed under sluices, they had to be specially protected against shocks. The ordinary

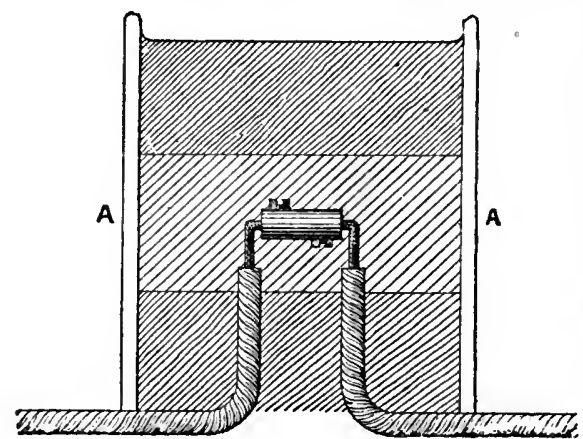

Fig. 238.-Junction-box. (From La Lumière Électrique.) conductor is, therefore, first of all covered with hemp, then with a coil of galvanized iron wire, then again with hemp, and finally with Clarke's composition.

At those places where the cables had to be joined underground, the splicing has been avoided by a special arrangement represented in Fig. 238. A A is an earthenware cylinder, about fifty centimetres high. It is placed in the ground, and the two cable-ends which are to be joined enter at the bottom, and are bent upward, as is shown in the figure. Plaster of Paris is then cast into the cylinder to about one-third of its height; above the plaster the cable is laid bare, and the two conductors are joined by a binding screw; the whole is then covered with a coating of paraffin, and the cylinder filled up with rosin or glue. Each of these cylinders contains several junctions; the number of the latter varies from three to twelve. A cover of sheet iron, pierced by a hole in the centre, closes the cylinder, and 
is level with the ground. To examine the junctions, the cover is removed and the upper coating is broken till the paraffin is reached. A successful innovation has been made in the lamps themselves. The globe, which used to be of one piece, is replaced by a spherical lantern divided into four parts by iron ribs. One of these spherical sectors acts as a door, and permits an easy renewal of the candles. The lanterns, as a rule, are of crown-glass, with the exception of those which are red or green and serve as signals.

The installation is a perfect success, and vessels enter now during the night tide nearly aseasily as during the day tide.

\section{The Electric Light applied to Photography.}

To obtain good photographic results with electric light, the lighted field must be large and very uniformly illumined. The light produced need not be fixed for a long period, since it has often to be extinguished every six or seven minutes, but during this time a surface of several square metres must be illumined in the most brilliant manner possible.

Dujardin has obtained this result by employing two arc lamps, with progressive and continuous movements, interpolated in the same circuit; the lamps are fed by two Siemens dynamos (medium size) united for tension and performing 850 revolutions per minute. With carbons of fourteen millimetres diameter arranged in such a way that the lower carbon is slightly in advance of the axis of the upper one, whereby the crater of the upper (positive) carbon presents itself under an angle of $45^{\circ}$, without any other reflector than the crater, a projection of light has been obtained equal in intensity to pale sunlight. The value of 
this illumination has been estimated by Dujardin at 1800 Carcels or 900 Carcels (8100 candles) for each lamp.

Dujardin uses the Serrin lamp, removing its electromagnetic check. The lighting is effected in the usual way, but the clock-train, when once released, causes a continuous advance of the carbons, which has been calculated according to their duration, and which prevents all the slight irregularities existing in all electric lamps, however perfect they may be. Under these conditions, the flame of the are envelops the upper carbon in a perfectly regular manner, and assumes a tulip-like shape, which is the most convenient for a steady brilliant light. With the ordinary lamps this flame is flickering and irregular. Dujardin is also of opinion that, by placing the two lamps in the same circuit, he contributes to this regularity in consequence of the compensations which result from their reciprocal action. He has also found that the resistance of the are is much larger than is generally believed, on account of the electro-motive force of polarization which is developed on the carbons, and this is confirmed by Edlund and quite lately by Jamin. $\mathrm{He}$ therefore placed his two dynamos in tension on the same circuit, and found that in this way their action was more regular. Dujardin uses the horizontal Siemens dynamo in preference to any other, and drives it by a steam-engine of nine horse-power.

The installation of the light apparatus is most simple. At the end of a long corridor painted in dark colour is a reflecting sheet to which are fastened the engravings, photographs, or drawings to be reproduced, and facing this surface, about two metres apart, are installed on the sides of the corridor the two lamps which are, on the side opposed to the sheet, covered by a sort of circular 
screen painted white. This screen serves at the same time for hiding the luminous points from the photographic apparatus and for throwing the light on the reflecting sheet. Behind the lamps and in the middle of the corridor is the dark chamber and its accessories for photographic reproductions. Dujardin is so well satisfied with the results obtained by this installation, that he never makes use. of daylight for his photographs.

\section{Electric Light applied to the Microscope.}

The lamps constructed for this purpose are Swan lamps, and were adapted for the application named by Mr. Stearn, Mr. Swan's colleague; they are shown in full size in Fig. 239. The length of the incandescent filament is $\frac{1}{10}$ of an inch, its diameter $\frac{1}{166}$ of an inch. Two Bunsen cells or four Leclanchés are sufficient to render them fully incandescent; but for general purposes it will be best to use an additional cell, regulating the (natural size).

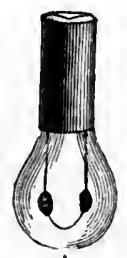

A

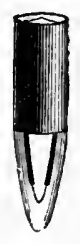

$\mathrm{B}$

Fig. '239. - Swan lamps for microscope intensity of light by means of the adjustable resistance D attached to the base of the microscope (Fig. 240). It is possible to obtain a light of $2 \frac{1}{2}$ candles from the tiny surface just mentioned, with an electro-motive force of $3 \frac{1}{2}$ volts and a current of $1 \frac{1}{4}$ ampère. It would, however, at a safe temperature, give a light equal to one candle.

For the illumination of opaque objects the lamp $\mathbf{A}$ is attached, by a jointed arm $\mathrm{E}$, to an insulated collar $a$, which screws on above the objective. The source of light can thus be rotated around the object while under examination, 
so that delicate surface-markings can be readily brought out.

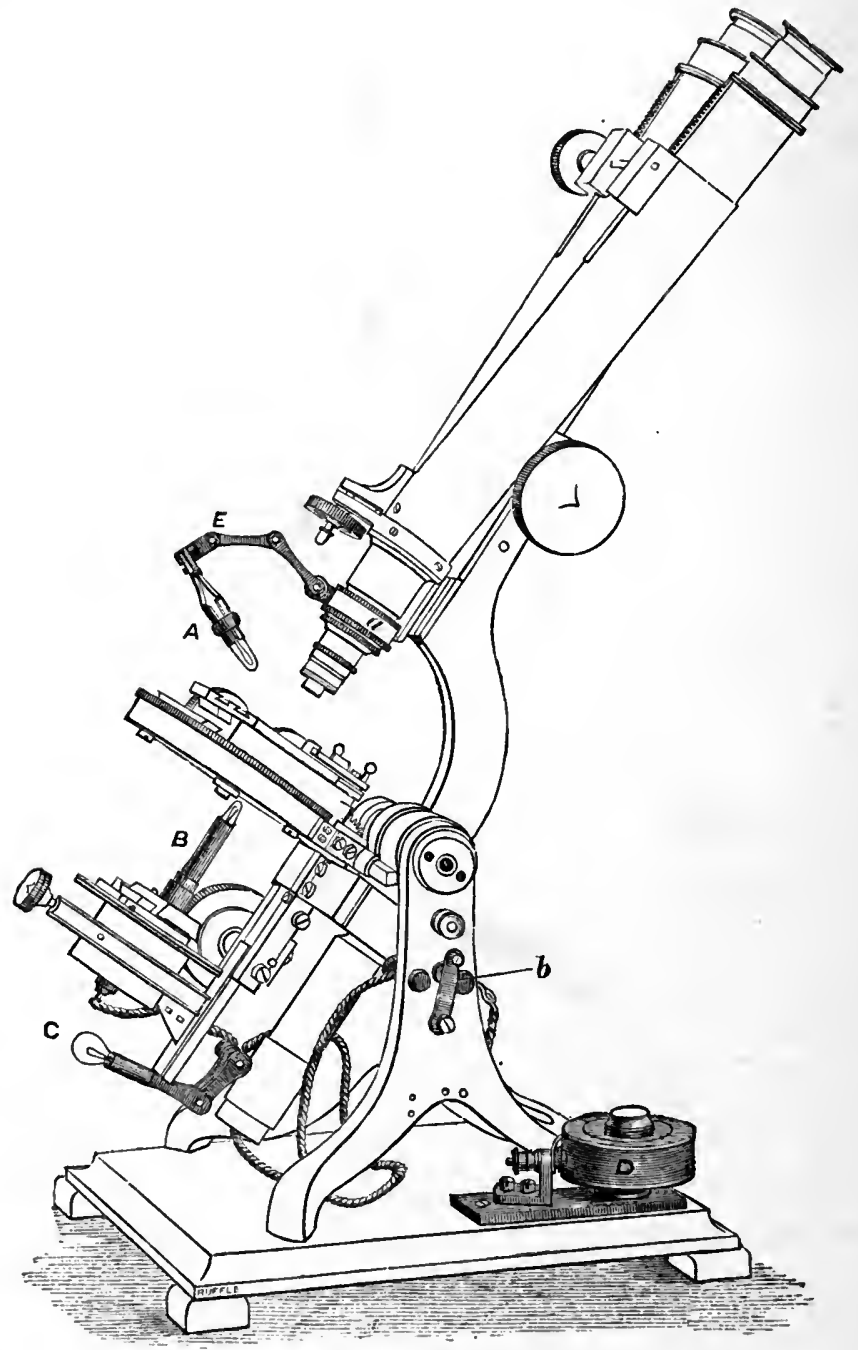

Fig. 240.-Microscope fitted with three Swan lamps.

By simply turning a switch $b$, the current can be made to pass to another lamp B, mounted on a platform 
fitting into the sub-stage, and capable of rotation and lateral adjustment, so that direct or oblique illumination at any angle may be obtained.

As the source of light is almost a point, and the lamp can be brought very nearly into contact with the slide, a higher degree of obliquity of the illuminating rays can be obtained than by almost any other method; and hence black-ground

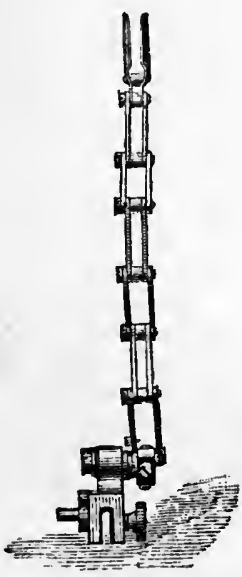

Fig. 241.-Fitting for attachment to the microscope stage.

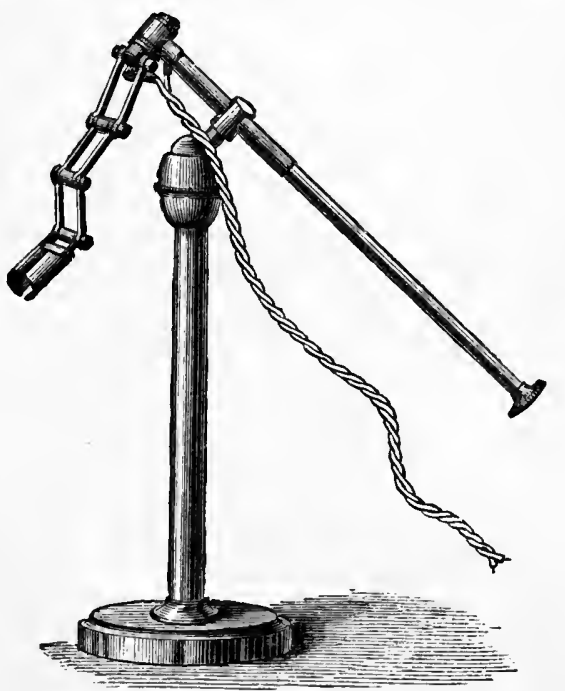

Fig. 242.-Adjustable stand for carry. ing lamp when used separate from the microscope.

illumination is shown with greater beauty and many of the diatoms display diffraction colours with unusual splendour.

For use with the polariscope, a third lamp C, of slightly larger size, is placed in the position of the usual mirror. This requires an additional cell, so as to develop a light of about four candles.

As the sockets of the lamps are all made to a standard size, it is easy, if a larger light than is given by the smaller lamp be required; to transpose the larger one to either of 
the above positions, and use the full strength of the battery. If it is found desirable with the lower powers to give parallelism or convergence to the rays, a very small lens can be mounted in front of the lamp, as in Fig. 241.

If a more simple mounting be desired, the form shown in Fig. 242 may be adopted; and the lamp can thus be placed in any position above or below the stage.

If it is required to maintain the lamps for several hours at full incandescence, then the most satisfactory battery to use would undoubtedly be Bunsen or Grove. If, however, the switch is turned off whenever an observation is completed, a modification of the Leclanché answers admirably. It is best to use five of the modified Leclanché cells, controlling strength of the current by means of the resistance, and the diminishing it as the potential of the battery falls.

Application of the Electric Light for Galvanocaustic and for lighting of Dark Cavities.

The property of the galvanic current of heating inferior metallic conductors to redness was utilized in surgery by John Marshall in 1851, by Leroy d'Etiolles in 1853, Mideldorpf in 1854, Broca in 1856, etc.

The lighting was attempted at a more recent date; in 1867, Dr. Bruck, a dentist at Breslau, brought out an apparatus for lighting up the buccal cavities, to which he gave the name of stomatoscope. A little later, Dr. Millot, in France, made numerous experiments for lighting up the stomachs of animals at the École Pratique, in Paris.

The attempts were not successful, on account of the inconstancy of the electric source (Grove's and Bunsen's 
couples), which necessitated the use of very thick platinum wires, in order not to expose them to a permanent volatilization. Luminous effects were obtained, it is true, but at the same time calorific effects of such intensity as to render this method of lighting impossible. A circulation of water was established to counteract the heat produced, but the apparatus were too cumbersome and difficult to handle, and have not been adopted in practice.

Gaston Planté indicated, as earily as 1868, the application of the secondary battery for this purpose, and carried it into effect in 1872.

In 1873, Dr. Onimus performed, with Plantés apparatus, cauterization of the lachrymal gland on seven or eight individuals, without re-charging the apparatus. Trouvé has added to Plante's apparatus a rheostat with a platinum wire for the graduation of the intensity of the current, according to the diameter and the length of the platinum wire used for lighting or for cauterizing. He has also added a galvanometer with two circuits, to control the charge of the secondary couple and the condition of the cliarging battery.

This apparatus is called a polyscope, and is represented in its box in Fig. 243.

By means of the rheostat, platinum wires of from $\frac{1}{15}$ to $1 \frac{1}{2}$ millimetre in diameter can be heated to the point of fusion for several hours without ever exceeding that point. Trouvé uses, instead of platinum spirals, small wire flattened in the middle, so as to form a small incandescent disc. The luminous power is thereby sensibly increased, as has been proved by Captain Manceron, in his experiments of lighting up the interior of guns at Saint Thomas d'Aquin.

For lighting of cavities, Trouvé employs a series of spherical reflectors, concave or parabolic, with or without 
mirrors. A handle and conducting wires connect these reflectors with the reservoir at $C$ and $D$.

Fig. 24.4 represents a reflector with a handle for lighting up the throat and other obscure cavities.

At the fiftieth anniversary of the École Centrale, in 1879, Trouvé illuminated for the first time, with a special polyscope, the interior of a pike. Nothing could be more interesting than to see this transparent

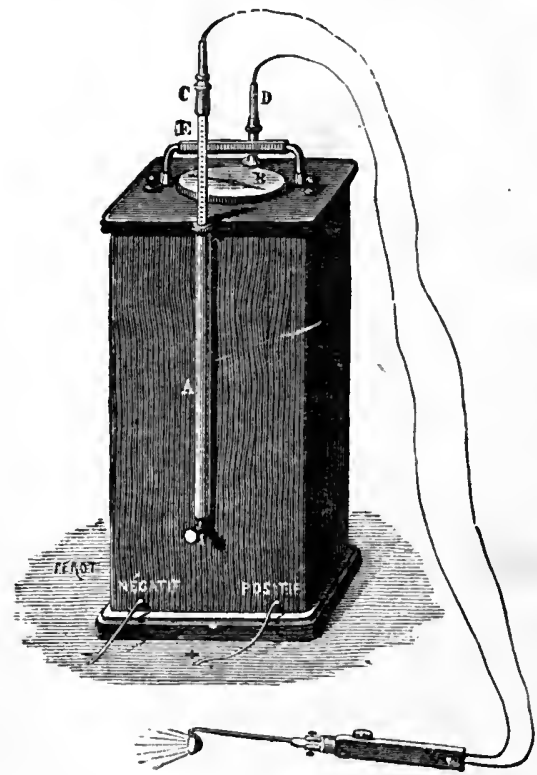

Fig. 243.-Trouvés polyscope.

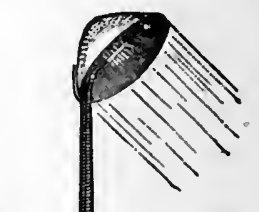
(1)

$$
\text { (1) }
$$

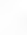


Payne's Apparatus for Illumination of Internal Cavities by Electric Light.-Remembering that Sir Henry Thompson explored the bladder by means of the electric light, and that Leitz, of Vienna, devised instruments for illuminating the throat, bladder, uterus, etc., Mr. Payne first made an instrument by means of which the throat could be examined. He subsequently succeeded in constructing an apparatus which enabled him, on performing the operation of abdominal section with incision of the liver, to light up the interior of the cyst by means of the electric light. The instrument consists of a brass tube electro-plated, $9 \frac{1}{2}$ inches in length and $\frac{11}{16}$ of an inch externally. One end of this tube is funnel-shaped, and the other is closed by a piece of glass. A Swan electric lamp is used, which is hermetically sealed in a glass shade; and water conveyed to and fro through very small brass tubes is made to circulate round the lamp. For prolonged observation, either a Grove or Bunsen battery is preferred, but for ordinary purposes four cells of a modified Leclanché battery are employed. It is advisable to have as great a pressure as possible for the water supply, so as to ensure perfect circulation; and for this a tin can containing water is suspended from a hook fixed near the ceiling of the room, connecting it with the brass tubes by means of lengths of indiarubber tubing.

\section{Advantages and Drawbacks of Electric Light.}

Fontaine, in the preface of his work on electric lighting, justly remarks, "It is incontestable that when a light of great intensity is required at a certain point, as, for instance, 
in a fort for watching the enemy, in ports for preventing the destructive effects of torpedo-boats, in lighthouses for guiding the navigators, electric light is not only the most economical of all, but often the only practicable one. It is equally certain that for a large dockyard as that at Havre, or for a vast enclosure like that of the Hippodrome, where it is impossible to suspend lighting apparatus and to place candelabra in the ring, the electric light is the only possible one, the only one which can replace the absent sun.

"We may, therefore affirm, that electric lighting has its own domain, where it need fear no opposition from any other system."

Another advantage of the electric light is the feeble quantity of heat produced by the voltaic arc. Laurbé has calculated 1 that for equal light the voltaic arc produces 150 to 200 times less heat than a gas-burner. This is a very valuable property, which is very much appreciated in a great number of applications where the heat produced by the gas is really unbearable. An additional advantage is that it deprives the surrounding air of a very small quantity of oxygen-the oxygen required for the combustion of the carbons-and, therefore, does not vitiate the atmosphere. It has been calculated that, at the Magasins du Louvre (vide p. 415), 369 times less air was vitiated by electric light than by gas, and it will be easy to understand what enormous benefit, as regards health, is conferred both on the employes and on the public by the additional 2613 cubic metres of pure air available in an hour. For tunneling it will prove invaluable, as has already been proved in the excavations for the Channel tunnel. For this application the question of expense of the ' See La Lumière Électrique of the 15th of November, 1879. 
motive force will be of secondary importance, because natural forces are generally utilized in these cases, and more compressed air is allowed to escape for the ventilation of the galleries than the electro-dynamic machines necessary for the feeding of these divided centres would consume.

Another advantage of the electric light-an advantage which is, however, contested by a great many impartial persons-is its tint, which permits the distinction of the most delicate shades of colours. Contrast alone imparts to it that pallid tint which is considered objectionable, and we have only to look at the same time at a gas flame and a Jablochkoff candle to see how little foundation there is for this objection. It is altogether the fault of the gas, which has a decidedly yellow tint; but our eyes are so accustomed to this coloured light, that electric light, which, like sunlight, contains all the colours of the spectrum, seems to us inferior, because it is on the contrary too rich, and objected to from the very excellency of its qualities.

Further advantages are that electric light has no smell, renders explosions impossible, diminishes the chances of conflagrations, enables us to obtain a light at long distances from the place of its production-an advantage which gas possesses at least to the same extent-and finally, if no direct but reflected light is required, it yields a splendid diffused light which can, from an economical point of view, successfully compete with gaslight.

And now let us consider the drawbacks. The first drawback, which is of a transitory nature, and is gradually disappearing in proportion as the apparatus are improved, arises from a want of constancy of the light, either with regard to its intensity or its colour. 
With the apparatus described in this work, great constancy can be obtained, provided they are well constructed and well regulated. Most of the arc and incandescent lamps afford all the necessary constancy. As regards candles, the changes of colour and of intensity are owing to the fact that these apparatus, as has been mentioned in Chapter II., have sacrificed the quality of the light to the simplicity of construction. The inconvenience arising from extinctions of short duration owing to the rupture of the arc, or of longer duration owing to accidental causes, disappears more and more, in proportion as the staff entrusted with the manipulations becomes more experienced.

Certain electric lamps make a very tiresome noise, which excludes them from localities where silence is desirable. This particular noise may be very slight in candles and arc lamps which employ alternating currents, but it cannot be altogether avoided: it arises from the very nature of these currents, and can only be completely obliterated by the use of continuous currents: these currents find altogether more frequent applications for electric appliances than the former.

We have reserved for the last the most serious objections to the use of electric light.

The hydro-electric battery is not a practical or an economical apparatus for this application; the thermoelectric battery is still in the state of an experiment, so that electric generators alone are available for producing the current; this necessitates, in most cases, the installation of a steam or gas engine, and involves all the drawbacks inherent to these apparatus, such as employment of a stoker, engine-man, keeping in repair, etc.

This is the most serious drawback, and in fact the only 
serious one. It disappears, however, in certain factories where the motive force can be borrowed from the general transmission. In this case, electric lighting only presents advantages. The solution of this question has to some extent been found in the erection of central electric workshops, distributing the electric current under similar conditions as the gas supply. We need only mention the Edison installation at the Holborn Viâduct, which is entirely supplied by two Edison machines set up in the basement of Messrs. Edison's office. Steam-engines, gas-motors, hydraulic-motors, are now specially and exclusively employed for electric lighting; their number increases every day, which is the best proof we can adduce for this system of lighting.

Expense of Installation and Working.-Nothing is more variable than the cost price of electric light; it may be ten times less expensive than gas or ten times more, and depends entirely on special conditions and individual requirements.

In a factory possessing a steam or hydraulic motor, a couple of horse-power for driving a Gramme machine, which feeds either an arc lamp or a series of incandescent lamps, can be spared, without involving any additional outlay.

The expense in this case is almost entirely confined to the first cost of the machine and the lamps, and to the combustion of the carbons. Under these conditions, electric light, if well arranged, is in every way superior to gas. For the same reasons, it will be economical to use it in steamships, where there is always an abundance of steam, lighting with candles or oil being very expensive. If, on the contrary, a movable engine is set up purposely for driving a single Gramme machine and feeding a single lamp, the expense is quite out of proportion with the result, and the use of gas is clearly indicated. 
By multiplying the number of centres to be supplied by one single motor engine, the disproportion gradually disappears, and if a powerful electric workshop is fitted up, as is the case at the Louvre and the Hippodrome, electric light, from an economical point of view, is preferable to gas. If a battery is used-the Bunsen and Grenet batteries are up to the present the only two that have been used for lightingthe original outlay becomes enormous; it amounts, according to Becquerel, to two shillings and sixpence per hour for sixty elements, without counting labour. The battery is only used now in a few exceptional cases where, from some reason or other, the employment of 'a steam-engine is objected to.

Gas-motors, which are extensively used now, have rendered electric lighting economical, on account of the facility with which they can be set going and also stopped, and because they only consume when they are at work.

If the gas-burner is a simple and convenient lighting apparatus, it cannot be denied, on the other hand, that its luminous efficiency is detestable. By direct combustion of four cubic metres of gas, forty Carcel burners ( 360 candles) at the most can be obtained. By expending the same quantity of gas in an Otto's motor, a force of four horse-power would be produced, which, transformed into electricity by a Gramme machine and into light by a Serrin lamp, would produce a luminous power of more than 300 Carcel burners (2700 candles), with 150 times less heat. This result is not at all surprising when we consider that common coal gas contains about twelve per cent. of light-giving hydro-carbons, whilst the remaining eighty-eight per cent. are for the most part gases which give no light, but only heat, by their combustion. 
These figures show that, if gas-burners divide the light, this division is not effected without a certain loss nor without a considerable waste, which is as a rule not sufficiently taken into account.

According to Picou, whose estimates seem very accurate, a Serrin lamp fed by a Gramme machine can light 250 to 500 square metres of a workshop where delicate operations are carried on, or 500 to 1000 square metres of an ordinary mechanical workshop, or 2000 square metres of a dockyard.

The capital invested for this purpose would be $£ 92$, and the expense per hour, assuming 500 hours of lighting per year, would be--

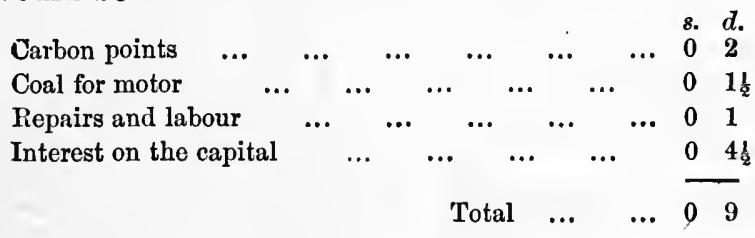

If the light is used every night-that is to say, 4000 hours per year-the expense only amounts to fivepence. With an hydraulic-motor, it is only sevenpence halfpenny for 500 hours, and fourpence for 4000 hours.

These figures, of course, only apply to one special case ; they differ for the various systems of lighting.

\section{The Division of Ligh't.}

Although electric lighting has made rapid strides within the last few years, improvements of a much more important nature must be in store for it, and these improvements are closely connected with the question of the division of light. 
It is evident that, if we succeed in creating small, economical centres, and, on the other hand, if electric workshops are established which deliver the current at the place where it is wanted, and in any quantity that may be required, as we can do with gas, all the drawbacks of electric lighting will at once disappear, and nothing but its advantages will remain.

No one would be considered over-sanguine now who predicted the realization of this industrial revolution. The manifold solutions which have been proposed show that it is possible; the important results already obtained point to a satisfactory and complete solution in the nearest future. The improvements to be made are of two very different kinds. On the one hand, the division of the current must be kept in view, and, on the other, a perfect utilization of this current with properly organized centres. The present tendency is, as we can state to our great satisfaction, no longer to separate these two modes of research, and most of the inventors aim at constructing machines whose special qualities fulfil the requirements of the lamp.

Thus, for instance, the Jablochkoff candle, which was formerly worked with Alliance machines, is now worked almost exclusively by Gramme machines with alternating currents; and this affords a most remarkable instance of division, since one single machine can, according to its dimensions, supply from four to sixty candles, arranged in this latter case in twelve series of five candles each.

The alternating currents of Lontin's machine feed as many as twelve Mersanne lamps; Siemens' machine supplies twenty centres, arranged in two circuits only. Wilde's and Jamin's candles, Rapieff's and Gérard's lamps, give similar results from the point of view of division. 
Continuous-current machines, also, allow of a division of light, either with the voltaic arc or by incandescence.

The Brush machine feeds forty lamps mounted for tension in the same circuit. This is the most remarkable result obtained as yet with arc lamps placed in one single circuit. All differential arc lamps, however, allow of this division, provided the current supplied by the machine is strong enough to overcome the resistance offered by the arc.

Even more remarkable results are obtained with incandescent light. . A Gramme machine (workshop type) can easily feed twelve Reynier lamps in one single circuit, and twelve Werdermann lamps in two circuits of six lamps each. Edison's large machine feeds 1000 lamps, and Gordon's machine is constructed to supply the current for 5000 incandescent lamps. In an experiment made at Woolwich it actually fed 1300 lamps of twenty candles each, distributed over an area of thirteen English acres.

The methods hitherto employed for the division of centres are defective in this one point-they do not realize, as a rule, an essential condition of a perfect system for division of light, and they are wanting in individual independence of the working centres from one another. The extinction of one Jablochkoff candle, for instance, causes the extinction of all the others placed in the same circuit; the extinction or the lighting of one centre influences all the others, and either increases or diminishes their illuminating power.

Nor does the voltaic arc allow of a proper division of light; in fact, for the supply of a voltaic arc a certain minimum tension is required, and a volume of electricity depending on the size of the carbons, and if either of these two conditions is not fulfilled, the arc is completely extinguished. 
It seems, therefore, a well-established fact that the division of light into small centres is reserved, in spite of its higher price, for the incandescence of carbon or any other substance of analogous properties.

An account of the different incandescent systems will show better than anything else how far the division of light has advanced, and how near we are to a complete solution of the question.

The Edison System.-The Edison lamp which has been

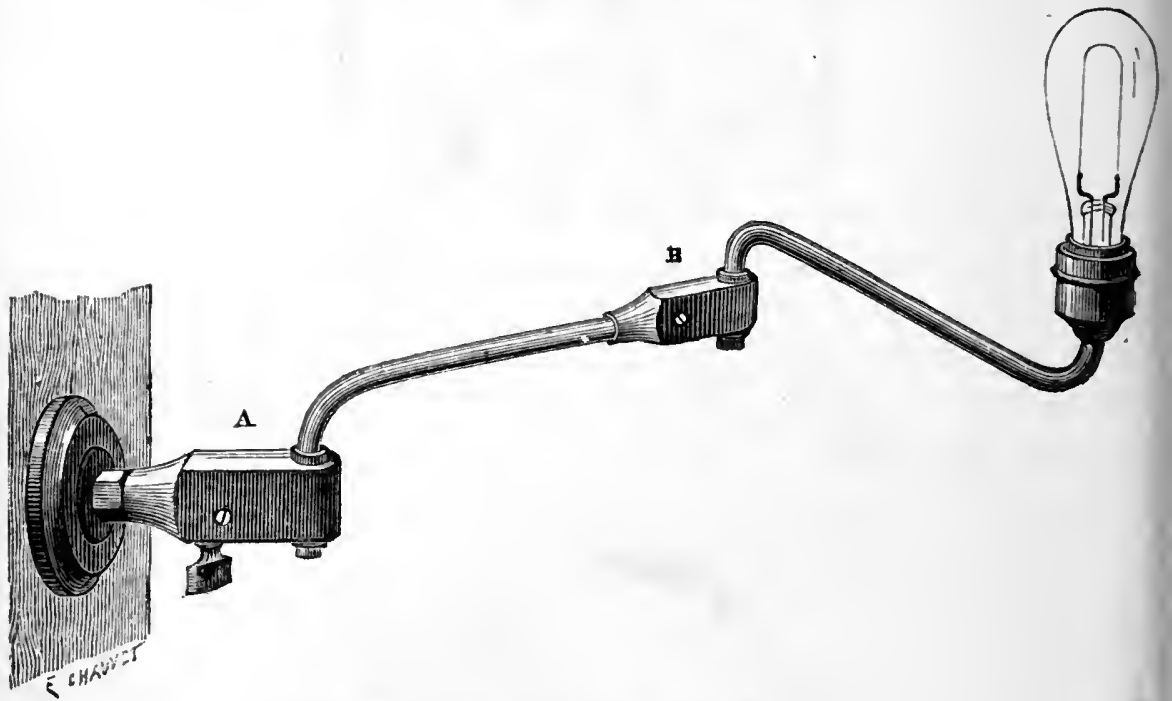

Fig. 245. -Edison's double-jointed bracket lamp.

(From La Lumière Électrique.)

described on p. 326, has been adapted for domestic use by various ingenious contrivances.

Fig. 245 is a double-jointed bracket lamp, which can be turned about and turmed on and off like a double-jointed gas-bracket. The interesting feature of these brackets is the construction of the joints on which the arms of the brackets are pivoted, and which are so arranged that the 
current is transmitted to the lamp in all positions of the movable arm. Fig. 246 is an enlarged view of the joint in Fig. 245. It will be seen that the two conducting wires connected with the main circuit are attached respectively to two metallic springs, which press against two

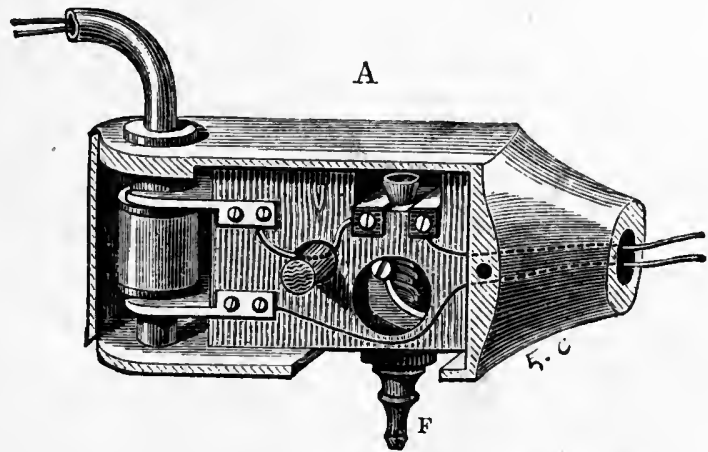

Fig. 246.-Larger elbow-joint of bracket lamp.

(From La Lumiere Électrique.)

brass collars, insulated from one another, and which are in connection respectively with the insulated wires that pass through the tubular arm of the bracket and lead to the lamp. It will also be seen that by this arrangement the wires of joint $\mathbf{A}$ are always in metallic connection with the wires of joint $B$, through the springs and collars which are always in contact in whatever position the arm may be. $\mathrm{F}$ is a

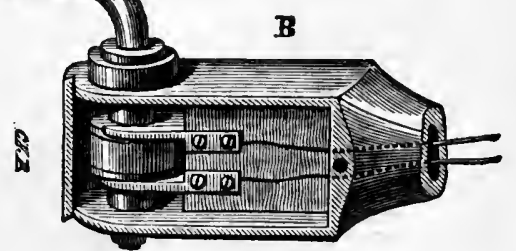

Fig. 247.-Smaller elbow-joint of bracket lamp.

(From La Lumière Électrique.) small quick-acting switch, by which the current can be shut off or turned on by an action precisely similar to the turning of a gas-tap.

Fig. 247 is an enlarged view of the smaller elbow-joint of VOL. 1. 
Fig. 245, and explains itself. It is, in fact, similar in construction to that shown in Fig. 246, but without the switch.

Fig. 248 shows the application of an Edison light to a portable lamp, which is connected with the conducting main by a pair of wires enclosed in a flexible covering, and which can be put in or out of circuit by the turning of a little switch in the upper part of the lamp.

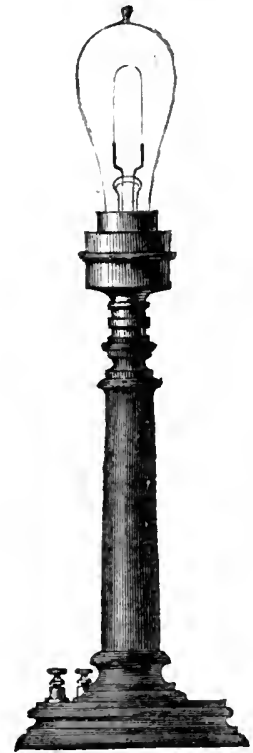

Fig. 248.-Portable lamp.

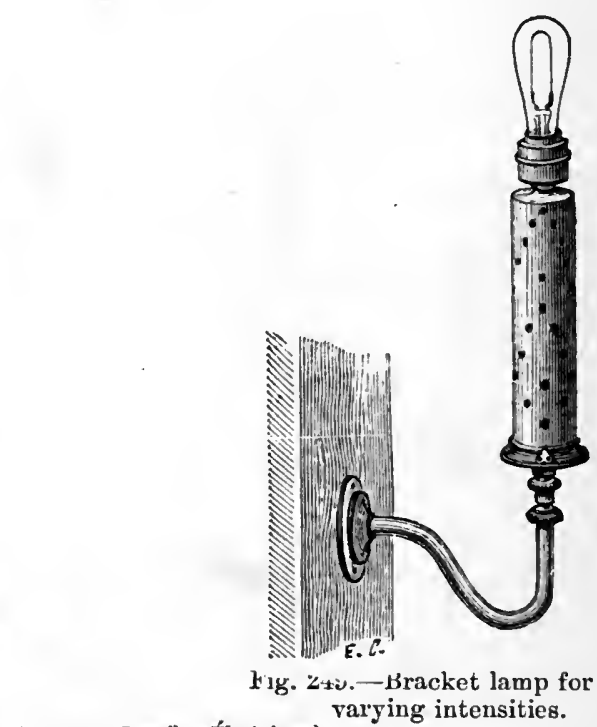

(From La Lumière Électrique.)

Fig. 249 represents a bracket lamp, which is capable of being turned gradually up or down, so as to produce varying degrees of illuminating power. Around the vertical supporting stem of the lamp are fixed a number of vertical rods of carbon, shown in Fig. 250, forming a sort of rheostat, or resistance box, the resistance in the circuit of the lamp depending upon the number of rods which are included in it, and this number can be varied at will by turning the 
circular base of the resistance box through a portion of a circle. The rods are protected from injury by being covered with a cylindrical metal tube, which is perforated with holes to admit a free circulation of air, and thus to dissipate the heat generated by the resistance offered to the passage of the current.

Fig. 251 is an illustration of a form of Edison lamp, or

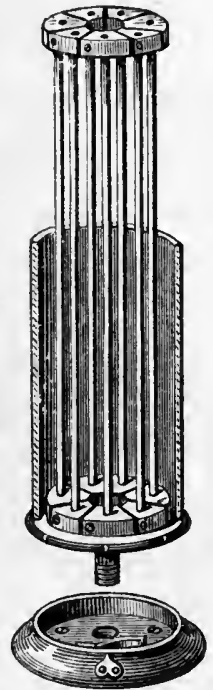

Fig. 250,--Interior arrangement of Fig. 249.

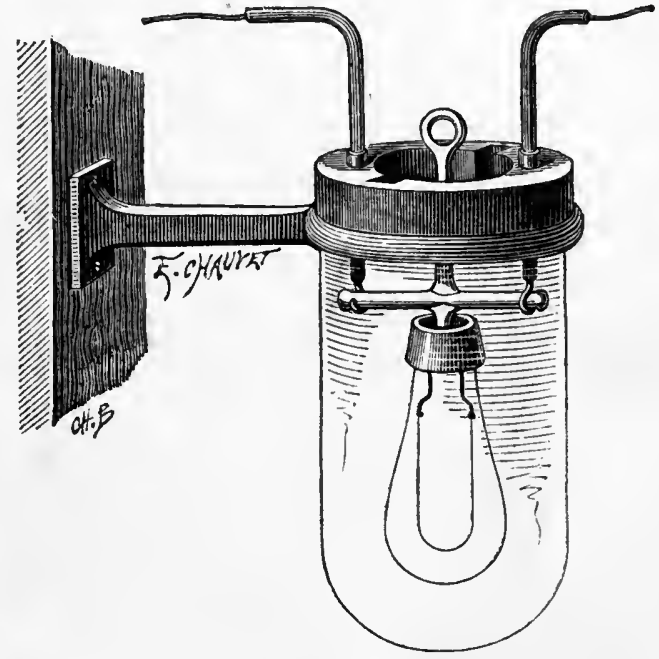

Fig. 251.-Edison's electric safety-lamp.

(From La Lumière Électrique.)

rather lantern, designed for illuminating mines or other places where there is danger of fire or explosion from the ignition of inflammable or explosive vapours. This very simple apparatus consists of an incandescent lamp supported in the middle of a glass vessel of water, with which it is entirely surrounded, the light emitted by the lamp passing through the water and through the glass sides of the water-vessel.

We now pass on to consider the conductors adopted 
under the Edison system for the canalization of the current, and by which electricity is distributed over a district to be lighted from a central station. We shall then refer to some other details connected with the distribution; and, lastly, to the apparatus devised by Edison for regulating the strength of the current to the work it is required to do, and the instruments by which the extent to which the current is utilized by subscribers or lost through defective insulation is indicated and recorded.

The conductors which carry the great outgoing current beneath the streets and in the sub-ways are of peculiar

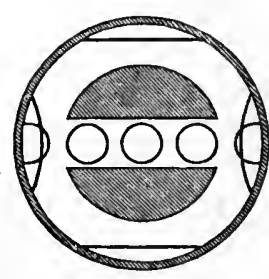
construction; while the conductors for shunts and single insulated wires for placing lamps in circuit are of the ordinary kind. The former consist of two solid segmental copper bars (Fig. 252), which are protected from injury and

Fig. 252. Main con- moisture by being passed through the (From La Lumierétectrique.) middle of a wrought-iron pipe, where they are held in position by diaphragms of stout millboard, placed at intervals along the length of the tube. The whole of the intervening spaces are filled with a bituminous insulating compound, which performs the threefold duty of keeping the conductors in the middle of the tube throughout their length, of serving as an insulating material protecting the conductors from damp, and of so completely fitting the tube that the latter, with its conductors in place, can be bent to any desired angle without displacement of its contents. As copper rods of such a length must necessarily expand and contract under variations of temperature, it would naturally follow that, unless some provision were made to allow for variations of length without distorting the mains, 
they would in a short time destroy themselves, or at least would soon be injured in both conduetivity and insulation. To meet this difficulty, a box (Fig. 253) is placed at the

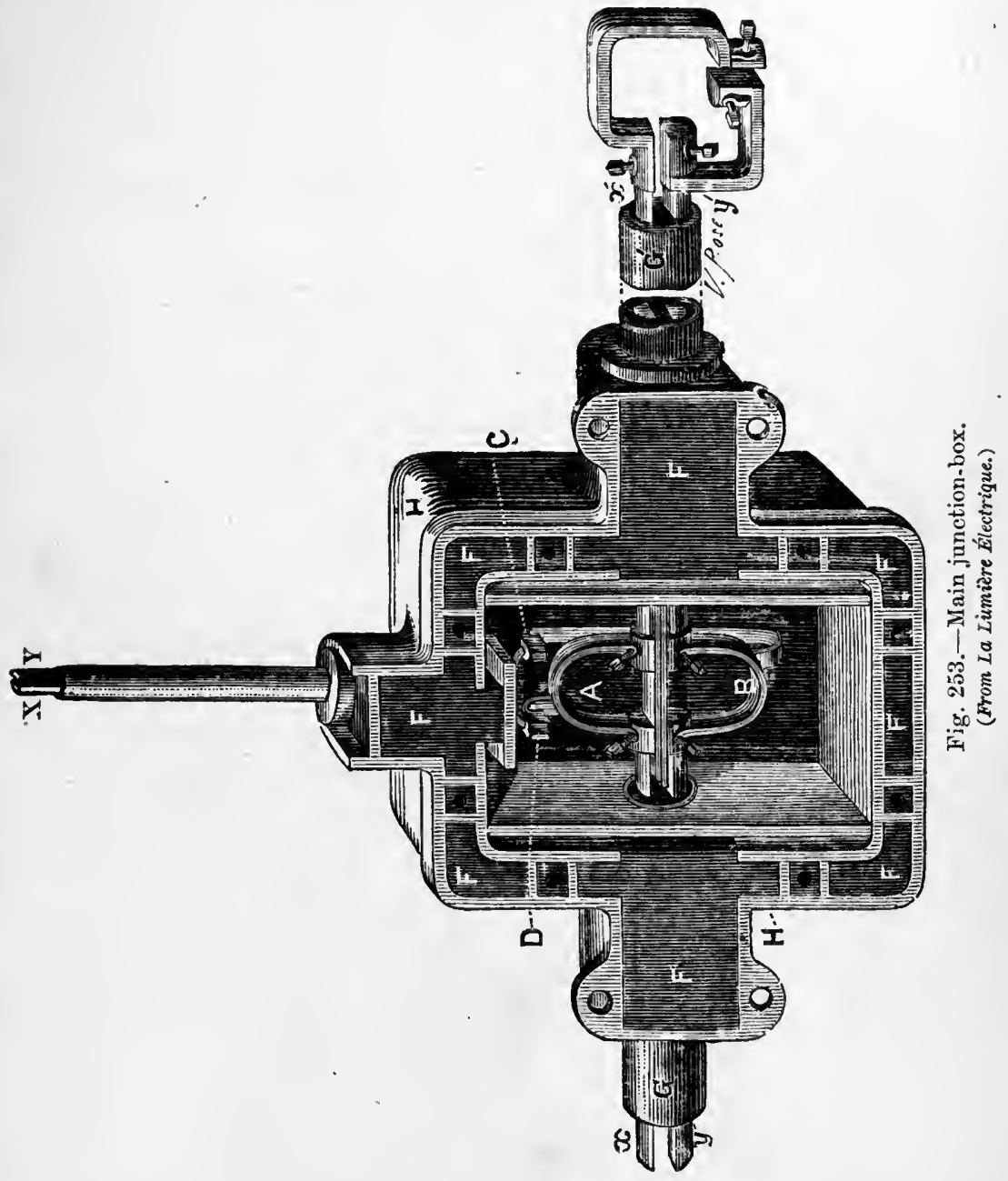

main junctions, made of cast iron $\mathrm{H} \mathrm{H}$, with double walls, through the side of which pass the ends of the two pairs 
of conductors $x y$ and $x^{\prime} y^{\prime}$ to be joined. The connection between them is made by two horse-shoe or U-shaped strips of copper A, B. These strips are of equal sectional area to that of the conductors, and are, on account of their form, capable of closing or opening under the elongation or shortening of the straight conductors. The figure also shows the method of connecting a branch sub-main X Y with the main circuit, the conductor $\mathrm{X}$ being connected to the loop $\mathrm{A}$ by the copper strip $\mathrm{D}$, while $\mathrm{Y}$ is a metallic connection with B by the bent strip C. Every branch circuit throughout the system is, moreover, protected against any sudden increase in the strength of the current by being provided with a safety-guard, consisting of a short length of lead wire, through which the whole of the current passing into a branch circuit is transmitted; and as long as this current is of normal strength, it acts simply as one portion of a conductor : but its cross section is so determined that it is incapable of transmitting a current of a strength above that at which the branch circuit can be worked in safety. The moment this strength is exceeded, the lead wire fuses and the branch to which it is fitted is cut out of the circuit. This safety-guard is applied, not only at every junction of sub-mains with the main, but at every branch leading from the sub-mains, and at every derived circuit or group of lamps, branching again within a house. The arrangement is clearly shown in Fig. 254, which represents a junction-box for connecting the cables for a house service with a sub-main. In this case the conductors of the two sub-mains are coupled together by the curved copper rods, $\mathrm{A}, \mathrm{B}, \mathrm{C}$, and $\mathrm{D}$; and the house cables $\mathrm{X}$ and $\mathrm{Y}$ enter the box by the tubes shown on the top of the figure, being connected respectively with the blocks $\mathrm{G}$ and $\mathrm{H}$, the former 
THE APPLICATIONS OF ELECTRIC LIGHTING.

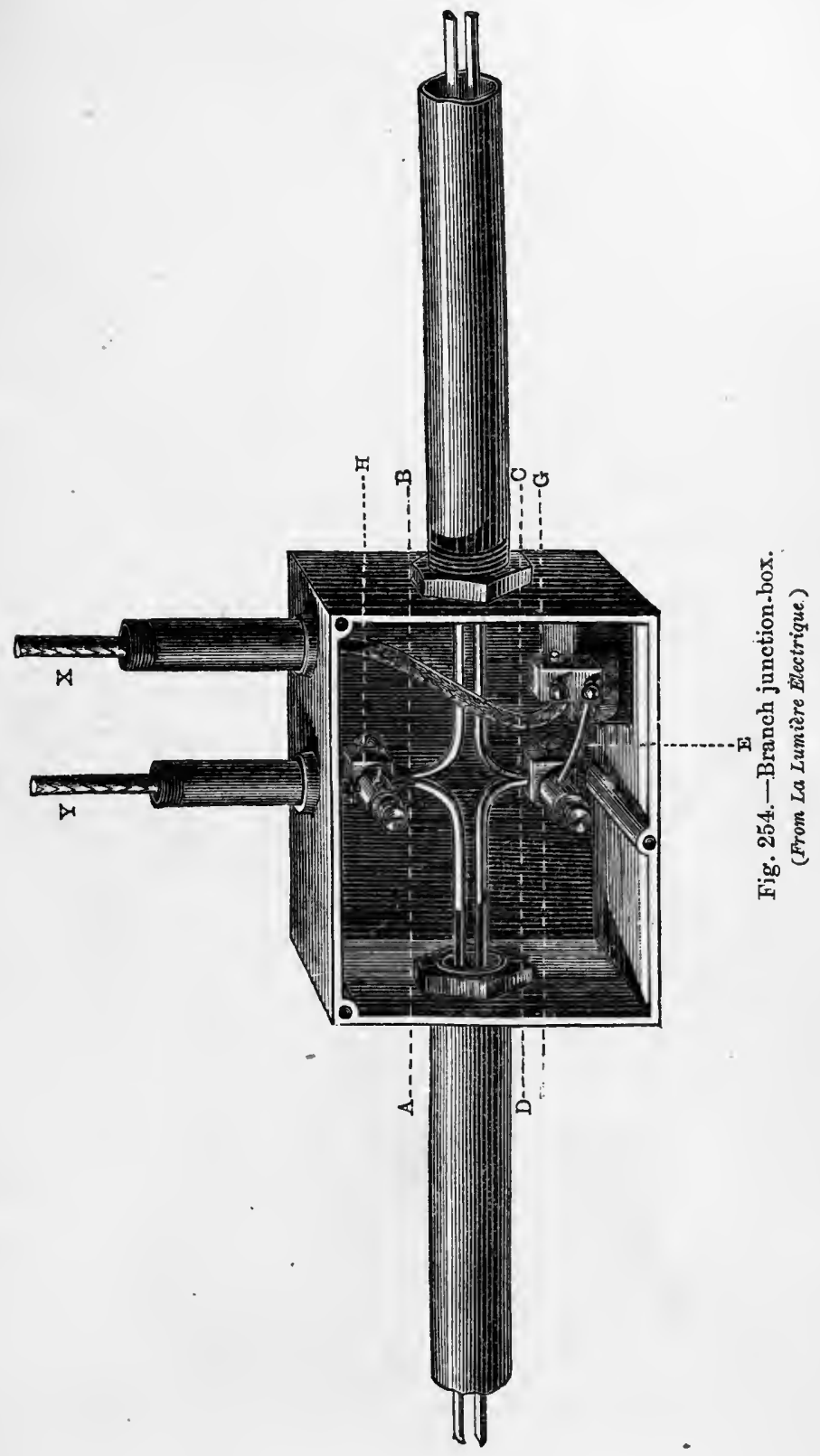


of which is coupled to $\mathrm{C}$ and $\mathrm{D}$ through the block $\mathrm{F}$ by the short length of lead wire E, which, by fusing, if by any chance the strength of the current rises beyond a certain point, cuts out the branch without affecting the main current passing through the box from right to left.

There are also special branch-blocks and safety-guards for the smaller branches, such as those within a house leading to one room or to a separate group of lights, and by which the lamp itself is protected by a short lead wire which fuses the moment that the current approaches a strength dangerous to the safety of the lamps.

To show how careful Edison is in avoiding any source of danger or of useless wear and tear of his apparatus, he has constructed a special switch for interrupting strong currents. It is clear that, however rapidly a contact may be broken, an electric arc must be momentarily produced, and this means consumption of the metal of which the contact surfaces are constructed. Edison divides, by the arrangement of his switches, the spark due to interruption over two, three, or more interrupters at the same time, thus reducing the arc, increasing the area of the surfaces in contact, and ensuring extremely rapid making and breaking of the circuit.

The strength of the current produced by the generator is regulated to the work it is required to do by the apparatus shown in Fig. 255. This current-regulator consists of a rectangular table supporting beneath it a number of coils $\mathrm{R}$ of German silver wire wound upon skeleton bobbins, between which and between the convolution of each helix, there is a free circulation of air, so as to keep the whole system cool. All these coils are coupled together in series, the first being connected to one attachment screw, the 
THE APPIICA'TIONS OF ELECTRIC LIGHTING. 473

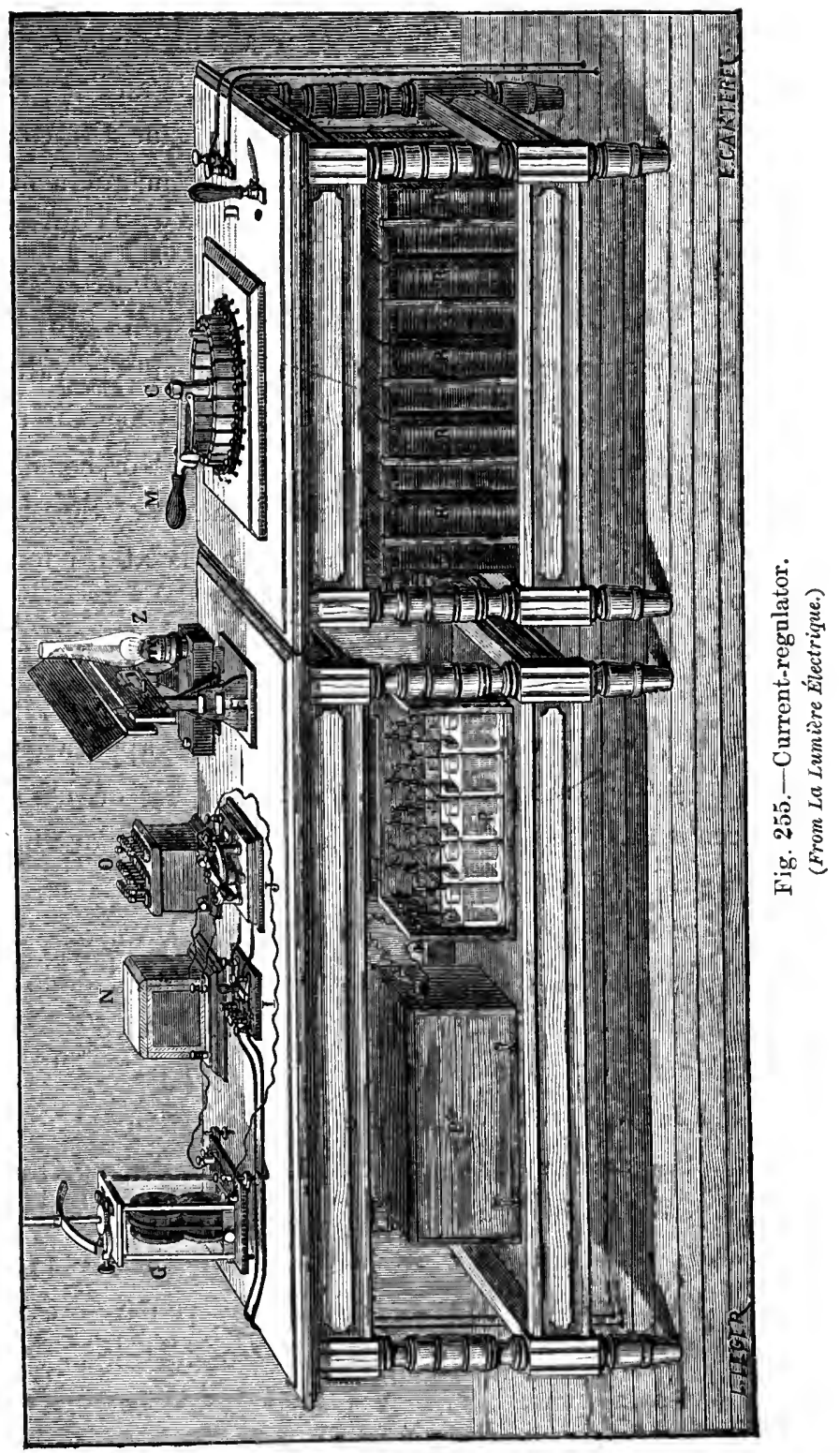


other attachment screw being connected to the regulating lever M, which, by being moved over a number of contactpieces arranged in a circle on the upper table and connected severally with the resistance-pieces underneath, can be made to throw into the circuit of the field-magnets of the generator or generators any number of the resistance coils, from one to the full number in the regulating apparatus. In permanent installations, the regulating-room is provided with a photometer $Z$, by which the photometric value of one lamp in any one circuit can continually be observed; and there is also an electro-motive force indicator, consisting of a reflecting galvanometer $G$, the resistances of the circuit being so balanced that, when the current is at its normal electro-motive force, the indicating spot of light rests at the centre of its scale, but moves to the left or right according as the electro-motive force diminishes or increases.

Finally, we have now to refer to the apparatus for measuring the amount of current made use of by each "consumer," as well as the total amount passing out of the central station of a district.

The principle of action of the instruments employed depends upon the fact that the weight of metal deposited per unit of time in an electro-plating bath by electrolysis is proportioned to the quantity of electricity passing through it in the same period, or in other words, to the strength of the current; and as the amount of this electro-deposition is equal at all points of a circuit, it follows that, by placing an electrolytic cell in any part of a circuit, the amount of electricity transmitted by the cell in a given time can be determined by observing the quantity of metal deposited within the same period.

Edison's Current-Meter.-The apparatus employed in 
the Edison system in connection with the service of a house is illustrated in Fig. 256. $\mathrm{V}$ and $\mathrm{V}^{\prime}$ are the two glass cells, hermetically closed by the stoppers $\mathrm{C}, \mathrm{C}^{\prime}$, and filled with a solution of zinc sulphate, in which is immersed a pair of amalgamated zinc plates. The two cells are precisely alike, but, from the fact that the one cell is short-circuited by a shunt having one-fourth the resistance of the shunt by

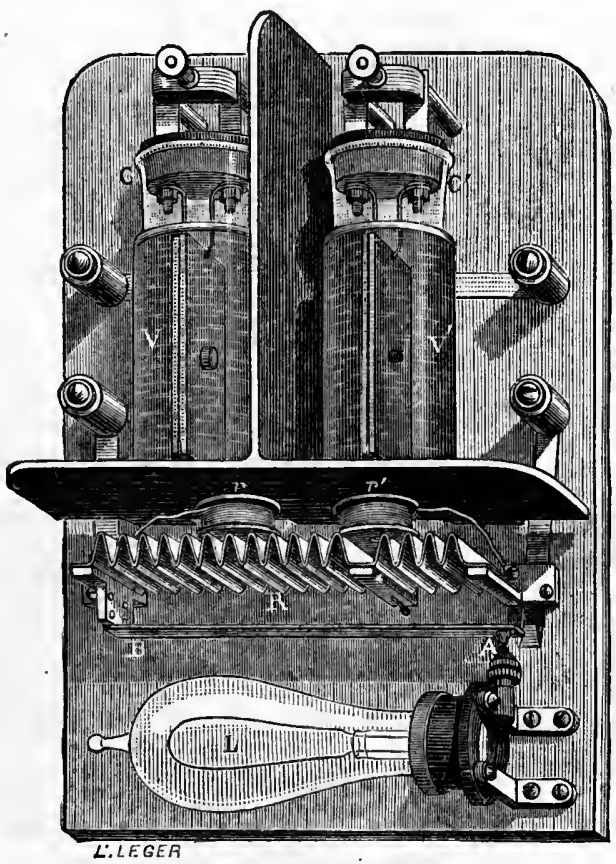

Fig. 256.-Edison's current-meter.

(From La Lumière Électrique.)

which the other is short-circuited, the quantity of electricity passing through the former in a unit of time is four times that which is transmitted by the other, and therefore, within a given period, the quantity of metallic zinc.deposited on the receiving plate of the one cell is four times that which is deposited on that of the other. 
The short-circuiting shunts are shown at $R$, and consist of a corrugated band of German silver, of which the twelve corrugations towards the left of the figure constitute the shunt of the cell $\mathrm{V}$, while the remaining four corrugations belong to the right-hand cell $\mathrm{V}^{\prime}$. An ordinary Edison incandescent lamp $\mathrm{L}$ is thrown into the circuit whenever contact is made at the point $A$, and this is effected by the bending downwards of the compound expansion bar A B, which is so constructed and adjusted as automatically to throw the lamp into circuit whenever the temperature within the instrument falls below a certain point. The radiant heat from the lamp then raises the temperature of the air within the apparatus until, by the upward curling of the compound bar, the lamp is again cut out of the circuit. $\quad r$ and $r^{\prime}$ are two compensating resistance coils of insulated copper wire, which are included in the branch circuit of the electrolytic cells, their object being to keep the resistance of the apparatus uniform under variations of temperature; for, as the resistance of the zinc solution within the cells is diminished by a rise of temperature, the quantity of electricity transmitted by it would be greater as the temperature was increased, unless this increase were compensated or neutralized. To effect this, a coil of copper wire is inserted in the circuit of each cell, and as the electrical resistance of copper increases with a rise of temperature, while that of the zinc solution diminishes, it is only necessary to make the length of the copper wire such that its resistance rises under an increase of temperature as much as that of its corresponding cell diminishes, in order to compensate the apparatus for variations of temperature.

The object of having two cells instead of one is to enable the supplying company to keep a check upon its 
inspectors, and also to guard against fraud on the part of dishonest consumers; for while the left-hand division of the meter is accessible to ordinary inspectors, and in some cases to the consumers themselves, the right-hand portion is under the sole control of the company, through their superior inspectors, and the readings of the two cells should always be in the same proportion to one another. Again, the object of causing the one cell to deposit slower than the other is that, while the ordinary inspectors go their rounds once a month, the upper inspectors visit the meters once a quarter; the one cell is, therefore, depositing for four weeks between two inspections, while the other is in action for thirteen weeks before its plates are weighed again; and by causing the three-months' cell to deposit slower than the one-month cell, the amount deposited in each between any two inspections is more nearly equalized. The cells are removed every month and fresh ones are substituted in their place, and from the amount of zinc deposited the consumer is charged so much per weber of current passed through the meter per hour.

Edison has also constructed a more elaborate automatic electric counter, which is rather suited to the laboratory of the central station than to house or factory installations, where it might soon be put out of order.

The second apparatus, represented in Fig. 257, consists likewise of two voltameters, but these voltameters have a

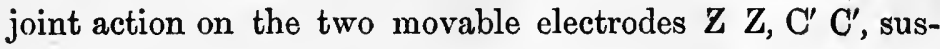
pended by rods $t$ to the two extremities of a balance-beam. The current which flows through them is of such direction that these electrodes act alternately the part of soluble electrode and reduction electrode ; from this results an alternating movement of the balance-beam for a given weight of 


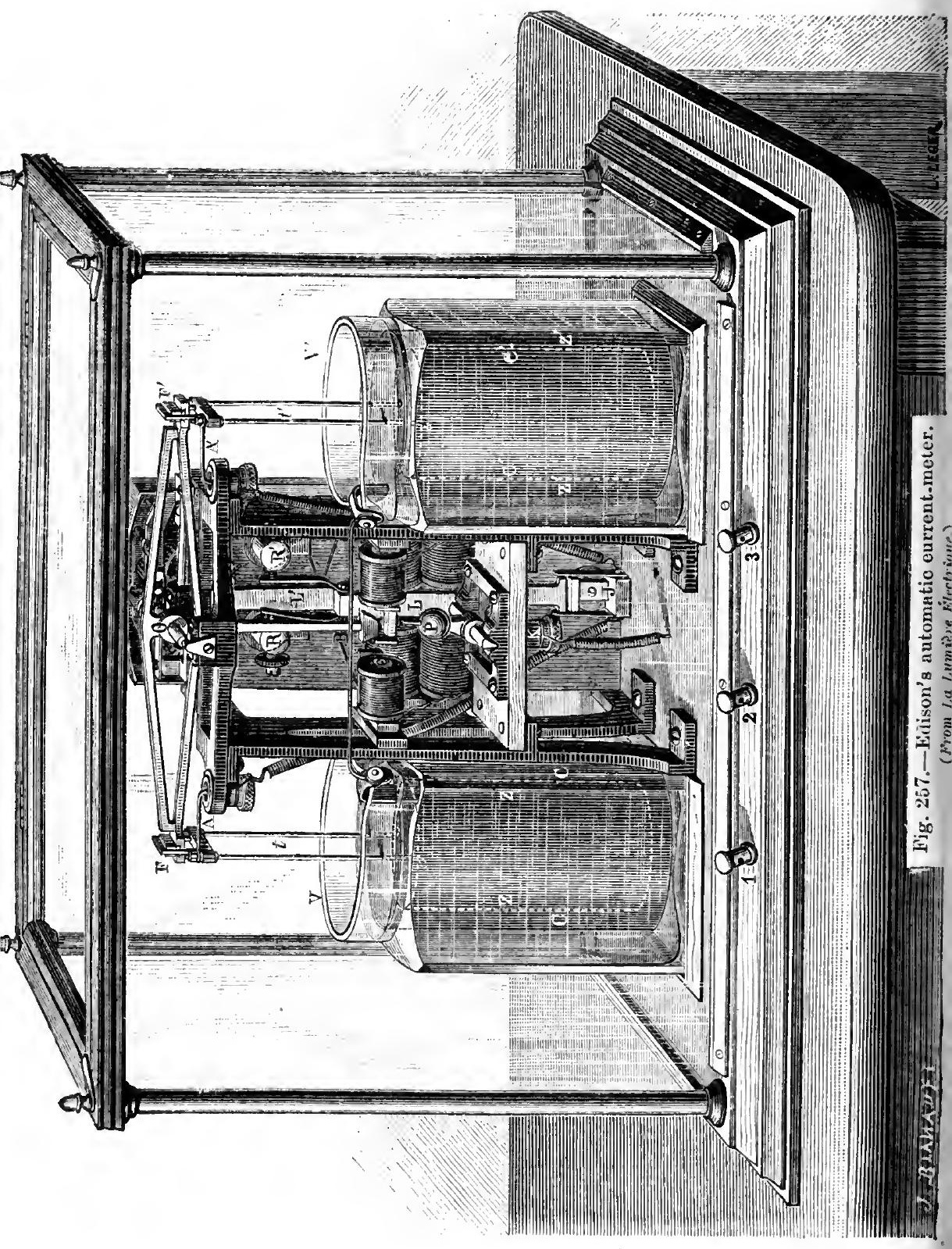


deposited metal, and this movement acts on a counter which registers the number of times this quantity of metal has been reduced within a given time, and indicates consequently the quantity of electricity supplied. This result is obtained in the following manner:-

The movable electrodes $\mathrm{Z} Z, \mathrm{C}^{\prime} \mathrm{C}^{\prime}$, are surrounded by two others $C C, Z^{\prime} Z^{\prime}$, which are maintained fixed in the vessels $\mathrm{V} \mathrm{V}$, containing a concentrated solution of cupric sulphate. The movable electrodes suspended to the extremities $F \mathrm{~F}^{\prime}$ of the balance-beam are, by means of an iron hook, placed in communication with capsules $\mathrm{A} \mathrm{A}^{\prime}$ filled with mercury and conveniently insulated from the main frame of the apparatus. The fixed electrodes $C \mathrm{C}, \mathrm{Z}^{\prime} \mathrm{Z}^{\prime}$, on the contrary, are in direct connection with two points of the working circuit representing the positive and negative poles of the electric generator. The current which passes through the apparatus is, as in the preceding apparatus, a shunted current of known intensity. The counter-weight $B$, which terminates the needle $\mathrm{O}$ B of the balance-beam, oscillates between two small pallets of soft iron, which terminate an oscillating lever $P$, fastened to a transverse piece which oscillates on knifeedges and plunges into a beaker $\mathrm{K}$ filled with mereury. By a counter-weight $P$ this lever is brought into unstable equilibrium, so that the least displacement to the right or left of needle $\mathrm{O} B$ is sufficient to cause an attraction of the pallets carried by the needle by one of the electro-magnets placed opposite the pallets. As soon as the pallet touches one of the electro-magnets, a current traverses this electromagnet, which firmly ensures contact, and at the same time a second electro-magnet placed behind acts on a large vertical lever $L L^{\prime}$ controlling a current-inverter. This inverter is formed by the four springs which are to be seen 
at the two extremities of the lever, and the two contact screws $R R^{\prime}$. By means of this inverter, which is connected to the capsules $\mathrm{A} \mathrm{A}^{\prime}$, to the fixed electrodes of the voltameters, and to the main frame of the apparatus, the direction of the current through the two voltameters is inverted, and as this current only takes effect at the moment when the balance-beam causes the lever $\mathbf{P}$ to oscillate, it is clear that these inversions are effected as often as the weight of deposited metal which serves as a unit is reached by the movable electrodes.

The large lever L L' has yet another important function, namely, that of working a mechanical counter placed in the upper part of the transverse piece against which it abuts. This counter is at $G$, but is not visible in the figure, and the lever acts on it by means of an excentric, which terminates its upper extremity. The discs of the counter are placed at the back of the apparatus, and are distinctly seen through the glass case which covers the whole.

The Lane Fox System. ${ }^{1}-$ None of the systems which are actually in use for electric lighting has been brought to greater perfection than Mr. Lane Fox's.

"A central station is chosen with a due regard to the area over which the electric current has to be supplied, and dynamos are set up there. To put them in motion, one or more steam-engines are required or a convenient fall of water. The central station will then comprise a source of mechanical motion for setting the dynamos in action, and the dynamos themselves. The external circuit, the vehicle of the electric current, has now to be distributed, and secondary batteries have to be set up at various points of

1 From a lecture delivered by Mr. Lane Fox before the United Service Institution, May 17, 1882. 
the circuit. The external circuit in its ramifications will very much resemble the mains, sub-mains, and pipes of a system of distribution for gas, only that the mains, sub-mains and branches and wires of the external circuit will be solid, much smaller, and, to prevent the waste of the current on its way to the lamps, insulated. A wire, precisely as in the case of gas, will proceed from a main, sub-main, or branch, to each point in a street or house at which it is proposed to set up a lamp. At the entrance into a house the size of the wire will depend upon the size of the house and the number of lights intended to be used, just as at the central station the size of the mains will depend upon the extent of the area over which it is proposed to distribute the current, and the number of points at which it is proposed to utilize it in the production of light. At the points in a house to which the lamps are attached, the wires will be about the size of an ordinary bell-wire, and as easily manageable, and, we may add, as perfectly harmless.

"There is no danger either of shock or of fire to be apprehended in a properly arranged system. The tension of the current to be used would not exceed, say, a hundred volts, and at this tension, or indeed-as has lately been proved before a Committee of the House of Commons-at a tension of twice that amount, a shock would be absolutely out of the question, and fires in such a system could happen only from 'short-circuiting,' which is perfectly avoidable, and need not ever happen."

In order to avoid the necessity of adjusting the plant at the central station and the mains and sub-mains to a maximum, which would entail a great deal of useless expense, and to avoid also the entire dependence of a district upon the central station for supply, Mr. Lane Fox proposes to set voL. I. 
up at various points in the district, and in connection, on the one hand, with the mains and sub-mains, and on the other hand, with the earth, secondary batteries as reservoirs of electrical energy. These batteries may be distributed at pleasure, and according to the exigencies of the case. Their action, assuming the plant at the central station and the mains and sub-mains to be adjusted to an average supply only, would be as follows:-The dynamos would work night and day. The current in the day-time, when not many lamps are in use, would enter the secondary battery, where its energy would be stored up. Later in the day and in the evening, when more and more lamps are brought into use, the current will cease to seek an entrance into the batteries, and will make its way through the new openings afforded to it at the various lamps. Finally, at night, when a great demand is made upon the current for all the lamps which may then be lit, a demand which the engines as adjusted could not alone meet, the pressure at the batteries will be removed, and the electrical energy which has been stored up in them during the day will issue out, and will supplement the current which is being supplied to the lamps from the central station. This supplemental current is now independent of the central station, and will flow on, though the latter should be entirely disconnected from the station.

The system of distribution is shown on the sketch plan Fig. 258. $G$ is the generating station, where the engine and dynamos are fixed, the latter being connected in parallel circuit. The position of the various appliances are shown diagrammatically on the plan. $\mathbf{M}$ are the mains or conductors, $\mathrm{A}$ accumulators (secondary batteries), $\mathrm{L}$ the lamps, $\mathrm{R}$ the returns, and $\mathrm{X}$ the meters. The lamps are all in mul- 
THE APPLICA TIONS OF ELECTRIC LIGHTING. $4 \& 3$

tiple arc, and a constant electro-motive force of about 100 volts is kept up in the circuit. This constant electro-motive Main
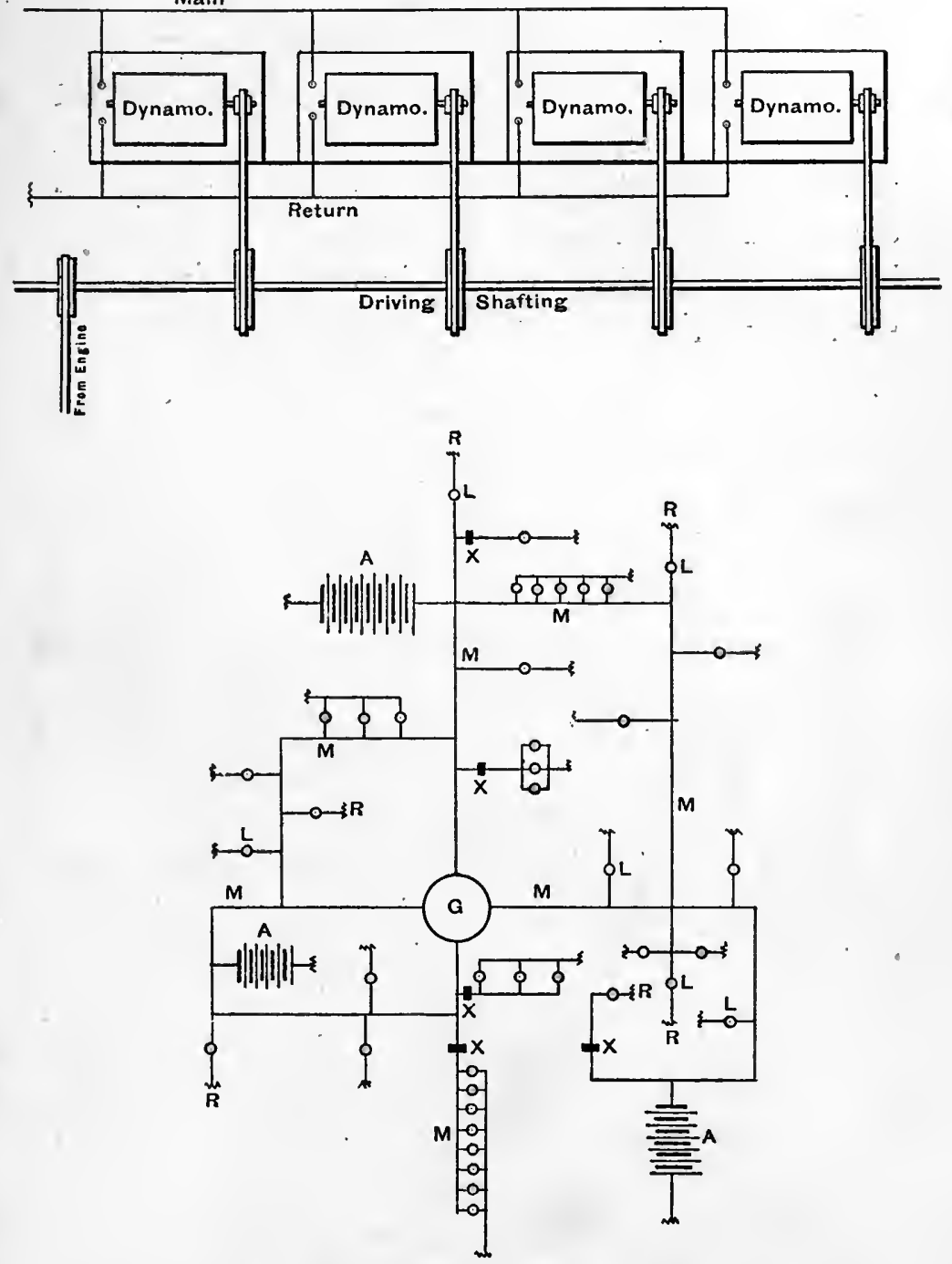

Fig. 258.- Lane Fox installation (diagram)

force is an important feature in the Lane Fox system, and is 
obtained by the employment of secondary batteries and by an automatic governor for controlling the generators, which is included in a circuit between main and earth.

The Regulator is shown diagramatically in Fig. 259. E is an electro-magnet through which the current passes, and when at its normal potential allows the lever $\mathbf{L}$ to remain midway between the contact points + and - ; when the current falls below its normal potential, the electro-magnet becomes weaker and allows the lever $\mathrm{L}$ to rise till it touches the point -, giving notice of the same through the wire

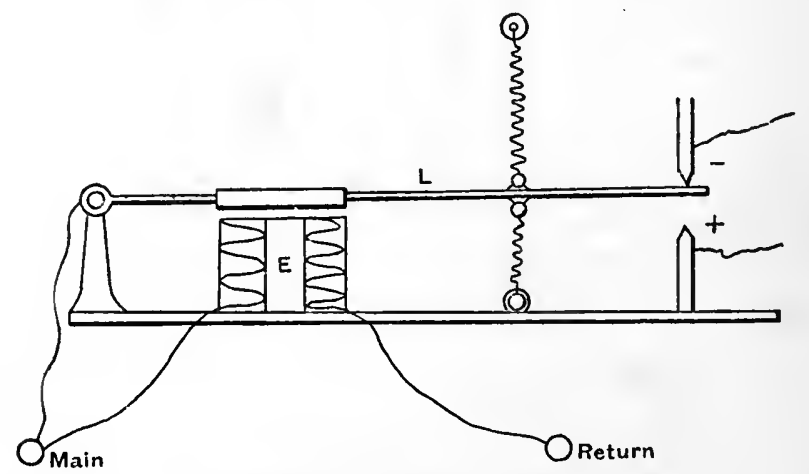

Fig. 259.-Lane Fox Current-regulator (diagram).

connected with this point, which can be made to communicate either with the person in charge or to work some automatic apparatus with the engine, dynamo, or secondary battery, so that the current may be increased. On the other hand, if the current passing through the electromagnet should be above the normal potential, the lever is attracted and contact made with point + , when the reverse of the foregoing operation takes place.

The whole of the regulating apparatus is represented in Fig. 260. It consists of an oscillating lever $G$, similar to the lever of an electric bell, which acts on a ratchet- 
wheel $\mathrm{N}$. The axle of this wheel carries a toothed pinion placed between two toothed wheels $R R^{\prime}$ having a common axle. This axle carries at its extremity a rubber $\mathbf{F}$, which moves over a circular range of contacts $c c$, corresponding to regularly increasing resistances, so that, according as the wheels $R R^{\prime}$ turn this rubber in one direction or another, a greater or less resistance can be introduced into the circuit or withdrawn from it. The axle of the two wheels moves longitudinally, and is directed by an electromagnetic system consisting of two electro-magnets $E^{\prime} E^{\prime}$ with a common armature A. This system is energized by a sort of relay with double contact $E^{\prime \prime}$ I, which sends through one or the other of the two electro-magnets $\mathrm{E}^{\prime} \mathrm{E}^{\prime}$ a local current, and thus causes the toothed pinion to work into one or the other of the two wheels $R R^{\prime}$. The relay being placed in a shunt of the current supplying the lamps, it can act on the resistance of the circuit by means of the rubber $\mathrm{F}$, which always re-establishes the original intensity. When this intensity increases beyond the useful point, the wheel moving the rubber in the direction of the increasing resistances is set in motion. In the opposite case it is the other wheel; so that the apparatus always oscillates between rather narrow limits.

The Secondary Batteries are of the Planté type, and are formed of two or more compound lead plates, each plate consisting of layers or laminæ of lead-foil, separated by asbestos or some other porous material. The laminæe of lead are soldered with lead solder at each end to a strong strip of lead forming the terminals and also serving as handles for removing the plates.

Lane Fox Electric Meter.-Mr. Lane Fox has completed 
his system by the construction of a very simple currentmeter, which is shown in Figs. 261 and 262.

$A$ is a solenoid, the coil of which forms part of the circuit or a shunted portion of the circuit of the conductor, the current passing through which is to be measured. The movable core $\mathrm{B}$ of this solenoid is attached to one end of a lever C, which is pivoted at D, and whose other end controls a conical valve or plug $\mathrm{E}$, which is made to work vertically in guides, and is lifted by the small spring $\mathrm{S}$, which is made just strong enough for this purpose; the valve $\mathbf{E}$ controls the flow of water through an outlet or
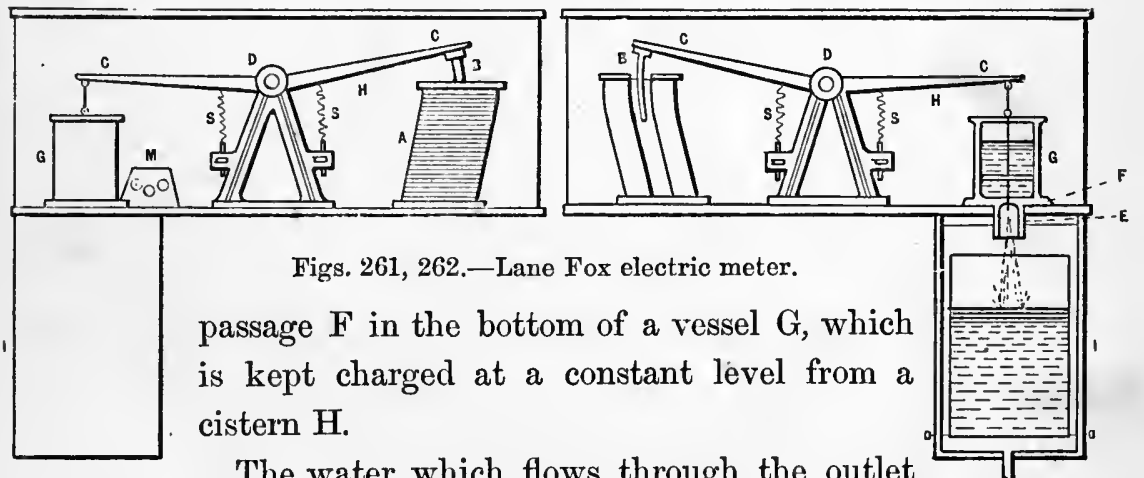

Figs. 261, 262.-Lane Fox electric meter.

passage $\mathbf{F}$ in the bottom of a vessel $G$, which is kept charged at a constant level from a cistern $\mathrm{H}$.

The water which flows through the outlet

$\mathrm{F}$ is received in a tank $\mathrm{I}$, containing two small oscillating tanks, which are filled and emptied alternately. The movement of these tanks works the index $M$.

The action of the apparatus is as follows:-When no current passes through the coil of the solenoid, the core $\mathrm{B}$ is in its highest position, and therefore the passage $\mathbf{F}$ is completely closed by the valve $\mathbf{E}$; but when, on the other hand, a current is passing, the core B is more or less drawn down according to the strength of the current, and therefore the valve $\mathrm{E}$ is more or less raised by the action of the lever $C$ on the spring $S$, and the flow 
of water from the vessel $G$ into the tank $I$ is more or less great.

By making each tooth of the index $\mathrm{M}$ indicate a given number of units of quantity, this index, combined with the quantity of water in the tank I, will enable the amount of electricity which passes through the solenoid up to any given time to be at once ascertained. In places where water would be liable to freeze, some other suitable fluid, such as dilute glycerine, should be used.

Finally, we have to mention an arrangement for regulating the strength of a local current-for instance, in a house, factory, etc. This arrangement is-

The Carbon Resistance Box.-This apparatus (Fig. 263)

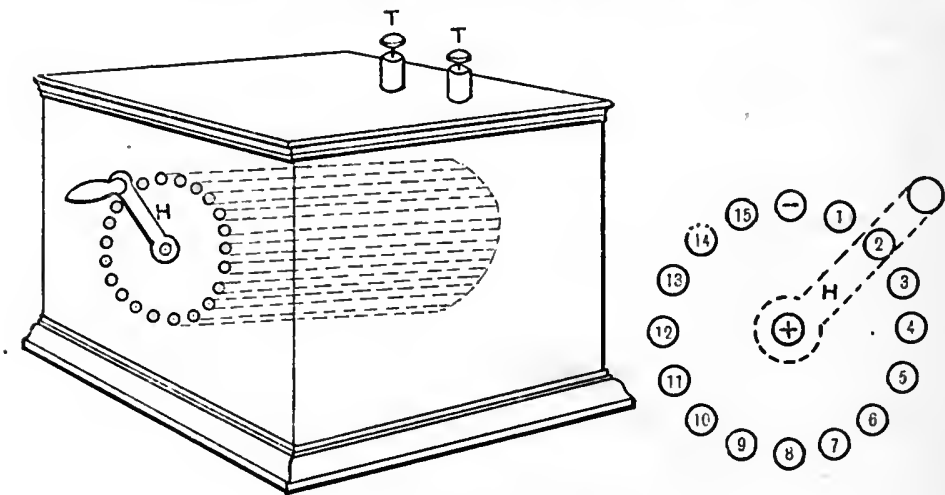

Fig. 263.-Carbon resistance box.

is composed of a number of carbon rods arranged in a circle round a central rod of copper, one of the outside circles being of the same metal. The terminals $\mathrm{T} T$ are connected with the back ends of the copper rods; the ends of the carbon rods, which are electro-plated and brass-tipped, project through the face of the box. The front end of each rod is connected with the back end of the next. The mode of action is as follows :- 
When the handle $H$, which is attached to the central rod, is made to connect the two copper rods + and -, no appreciable resistance is interposed. When, however, it is moved to carbon rod 1 , this resistance is added, as the current, passing from + through the handle $H$, must traverse rod 1 before reaching the terminal.

By moving the handle round, each additional carbon is brought into play, thus increasing the resistance, so that, when it is moved to rod 15 , there is fifteen times the resistance of rod 1.

In the diagram, fifteen rods are shown, but this number may be increased or diminished according to the requirements of the case, and they may be made to vary in resistance by altering the size of the rods.

The handle is shown to be worked by hand, but it can be arranged to be worked automatically by the current.

Maxim's System.-Hiram Maxim's system is more com-

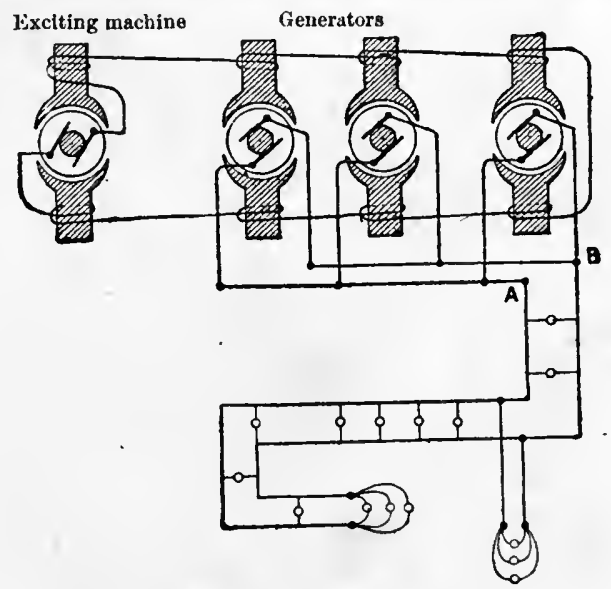

Fig. 264.-Mounting of Maxim's machine.

plete than Edison's, in so far as it includes an automatic 
regulation effected by the machine itself according to the variation in the number of the circuits supplied.

The diagram Fig. 264 shows the mounting, and Fig. 265 the whole of the so-called regulator. Maxim excites a certain number of dynamo-electric machines-three, for instance-by a fourth separate machine. The three generating machines are placed on shunts $\mathrm{A} B$, and the lamps themselves are on shunts of the principal conductors. To understand the action of Maxim's regulator, let us suppress it for a moment, and displace the collecting brushes of the exciting machine by the hand, whilst both generating and exciting machines are in motion.

On referring to the working of Gramme's machine, it will be seen that the current reaches its maximum when the brushes touch the collector in a vertical direction. When the brushes are placed in a horizontal direction, that is to say, when they touch the neutral point, there is no current at all. By moving, therefore, the brushes to an angle of about $90^{\circ}$, the current produced by the exciting machine will pass through all stages of intensity, from 0 to maximum. The magnetic fields of the generators will vary in intensity in the same proportion as the current produced by the exciting machine. The electro-motive force of the generators for a given constant speed being dependent on the intensity of the magnetic field in which the armature moves, any displacement of the brushes of the exciting machine must immediately act on the generators, and increase or diminish their intensity.

Maxim's regulator is an apparatus which automatically changes the bearing of the brushes, and renders the intensity of the magnetic ficld of the generators at each moment proportionate to the number of centres supplied. 
THE APPLICATIONS OF ELECTRIC LIGHTING. 491

It will now be easy to understand how the regulator (Fig. 265) acts. The upper part of the exciting machine

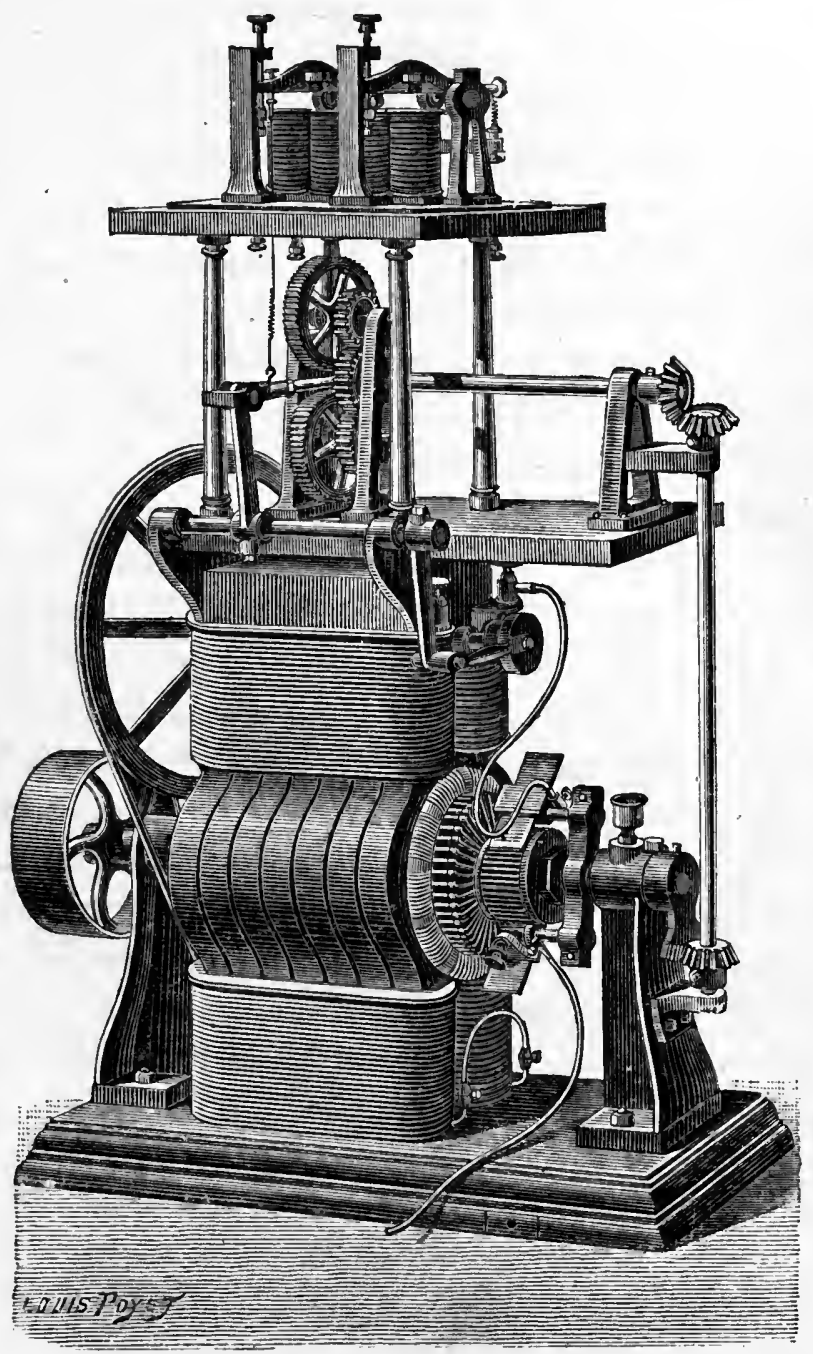

Fig. 265.-Exciting machine and regulator of Maxim's system.

carries two electro-magnets. We will only consider one of 
thein for the present, the second one having an entirely distinct function. This electro-magnet is coiled with fine wire, the extremities of which are connected to the two terminals of the generator, the latter being in direct connection with the lamp wires. The armature of the electromagnet is attached to a horizontal lever, whose path is limited by two abutment screws represented on the right; an antagonistic spring, regulated by a screw, balances the attractive force of the electro-magnet. The extremity of the lever of the armature is attached by a slightly elastic metal wire to a horizontal lever which carries two small teeth, and receives a rocking motion by means of a small connecting-rod and some transmission cords which connect the rod to the shaft of the exciting machine and impart to it a comparatively slow rotatory motion. This horizontal lever moves between two wheels toothed like ratchetwheels.

In the normal position of the armature and the lever attached to it, this lever moves freely in the space between the toothed wheels without acting upon either of them. Let us now suppose that two or three lamps are extinguished. The current thereby becomes too intense for the remaining lamps. The electro-magnet attracts its armature, the toothed lever is lowered, and the lower tooth works into the lower toothed wheel; the rocking motion imparts to this latter a rotation in a definite direction, so that for each turn of the small connecting-rod a slight rotatory motion is produced, which is transmitted to the brushes by a series of multiplying wheels, represented in Fig. 265. Under these conditions the brushes change their bearing; they approach the neutral point; the exciting current, and consequently the current produced by the 
generator, is weakened. These changes take place until the current recovers the requisite intensity for supplying the remaining lamps. The electro-magnet then releases the armature, and the toothed lever takes up again its normal position at an equal distance between the two toothed wheels. If any additional lamps are lit, the current is weakened, the armature acted on by the antagonistic spring is still further removed, the toothed lever this time acts on the upper toothed wheel, the multiplying wheels change the bearing of the brushes in an inverse sense and bring them near the maximum points, the intensity of the inductors and consequently the electro-motive force of the generator is increased.

The general adjustment of the whole system is thus in reality effected by the antagonistic spring. It suffices to slightly increase its tension to regulate the intensity of all the centres supplied by the machine. By diminishing the tension, the intensity of the lamps can be lowered to any degree, even to complete extinction.

Maxim's regulator is very sensitive, but unfortunately its action is also very slow. If too large a number of lamps were suddenly extinguished, the intensity would be too great in the remaining lamps, and some of them would undoubtedly break, being overheated by the sudden increase of intensity of the passing current.

In Maxim's system, the remedy, or rather a palliative, lies beside the evil. This palliative consists in a veritable safety-valve, which the inventor has added to his regulator, in the form of the second electro-magnet, which is to be seen in the upper part of Fig. 265. This second electromagnet is similar to the first, and is placed in the same circuit as the first, which we had, in order to facilitate the 
explanation, supposed to be directly connected to the terminals of the generating machine. Its armature is also mounted on a similar lever, and is likewise regulated by a spring with a tension slightly superior to that of the regulator. If the current which simultaneously traverses the two electro-magnets is intense enough to attract the armature of the second electro-magnet, it will depress the lever. The lever will then directly join together the two brushes of the same exciting machine; it will form a shunt, and short-circuit the coil. Under these conditions the inductors of both the exciting and generating machines will no longer receive any current, the generator itself will supply no current, the intensity of the lamps will be gradually diminished, a partial extinction of a few minutes will occur, but the lamps will be saved. Maxim must have thoroughly realized the fact that the regulator could not act effectually in all possible cases, to have endowed it with such a radical means of protection. It can, however, not be denied that, practically, the safety-valve is very rarely called upon to act, and that it is especially useful in cases of the sucl den rupture of a main conductor or of some similar accident.

The Efficiency of Incandescent Lamps.-From a report made by the Commission which had been appointed for this purpose at the Paris Electrical Exhibition, the following conclusions are to be drawn:-

1. The maximum efficiency of incandescent lamps, according to experimental evidence, cannot exceed 300 candles per current horse-power (current produced by a mechanical effect equal to one horse-power).

2. The economy of all the lamps of this kind is greater for high incandescence than for moderate incandescence.

3. The economy in the luminous production is greater: 
with lamps of great resistance than with apparatus of low resistance, thus coinciding with the economy in the distribution.

4. The relative efficiency of the four lamps examinedthe Edison, Lane Fox,Swan, and Maxim, expressed in Carcels and produced by one horse-power current, is the following:-

A. For sixteen-candle lamps-

Edison, 26.5; Swan, 24.0; Lane Fox, 23.5; Maxim, 20.4.

B. For thirty-two-candle lamps-

Edison, 41.5; Swan, 35.5; Lane Fox, 37.4; Maxim, 32.4 .

To double the light given by these lamps, the energy of the current had to be increased-for the Lane Fox and Maxim lamps, by twenty-six per cent.; for the Edison lamp, by twenty-eight per cent.; and for the Swan lamp, by thirty-seven per cent.

In a general way, and for a mean spherical intensity of 1.2 Carcel, which is quite practical, an effective light of twelve to thirteen Carcels per are horse-power, or about ten mechanical horse-power, can be obtained by means of incandescent lamps. Electric candles give forty Carcels per arc horse-power; arc lamps give about 100 Carcels. We can therefore say, in a general way, that the economical values of the three systems are as $1: 3: 7$, observing at the same time that, for each of the systems, the lamps of the highest intensity are always the most favourable in this respect.

Dr. Van der Ven has also made a number of experiments on the comparative efficiency of incandescent lamps, and has obtained the following results :-

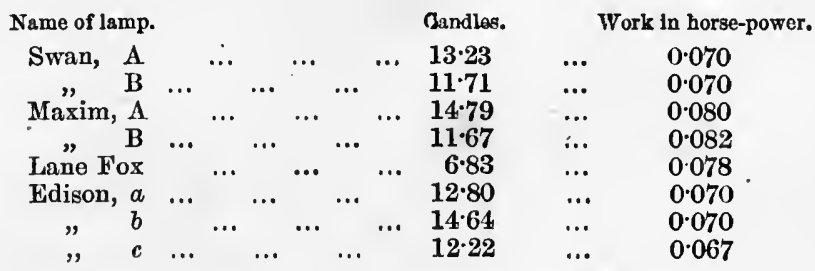


The results already obtained show that the division of light and of current power no longer presents any serious difficulties, and it is to be hoped that within a very short time electric lighting will have become one of the necessities of domestic life. The past here promises well for the future. 


\section{N D E X.}

$\mathbf{A}$

Advantages and drawbacks of electric light, 455

Alternating-current machines, 109

- dynamo-electric, 115

\begin{tabular}{l}
- Ferranti's, 129 \\
\hline Gordon's, 121 \\
\hline Gramme's, 118 \\
- Lachaussée-Lambotte's, 126 \\
- Lontin's, 116 \\
- Siemens', 120 \\
magneto-electric, 109 \\
- Alliance, 109 \\
De Méritens', 111
\end{tabular}

Apparatus for transforming electricity, 141 142 accumulators, storage batteries,

chemical action of, 147
Faure's, 152
Houston and Thomson's, 159
Kabath's, 153
Pezzer and Carpenter's, 154
Planté's, 142
power and duration of dis-
$-\quad$ of 149
- Regnier's, 153
Scllon and Volckmar's, 154
- Sutton's, 157
continuous high-tension currents,
160

D'Arsonval's voltaic condenser,

- Planté's electric tinder-box, 151

Planté's rheostatic machine, 169

Spottiswoode's induction coil, 162

Applications of electric light, 345

- agriculture and building purposes, 432

VOL. I.
Applications of elctric light, collieries, illumination of 398

—_ Swan's mining lamp, 400

electric sources for, 345

— batteries, 346

- liquid, 346

- secondary, 347

—— thermo-electric, 347

- generators, 350

- efficiency of, 351

galvano-caustic, and lighting of dark cavities, 452 Payne's apparatus, 453

- horticulture, 429

- lighthouses, illumination of, 357

— Dungeness, 366

- Isle of Razza, 375

— Lizard Point, 371

- Macquarie, 372

- Planier, 357

—_ South Forcland, 369

- microscope, 449

- military operations, 402

— night signals, 393

— pliotography, 447

public thuroughfares, illumination of, 435

- Chesterfield, 436

- City, the, 435

— Le Havre, 439

—_ San José, 437

- railway trains, illumination of, 403

- - submarine explorations, 434

- theatres, illumination of, 420

—- Hippodrome, 422

- Paris Opera, 420

- Savoy 'Theatre, 424

- Théatre des Variétés, Paris, 428 
Applications of eleetric light, ressels, illumination of, 386

—— Mangin's projector, 390

- workshops, factorics, dwellinghouses, illumination of, 411

\begin{tabular}{c} 
Bon Marché, Paris, 416 \\
\hline+ Brussels Royal Library, 418 \\
\hline $416-$ Brussels telegraph uftice, \\
\hline- Magasin dú Louvre, 415 \\
\hline Magasin clu Priutemps, 415
\end{tabular}

Are lamps, 185

circular electrodes, with, 185

- monophotal, 187

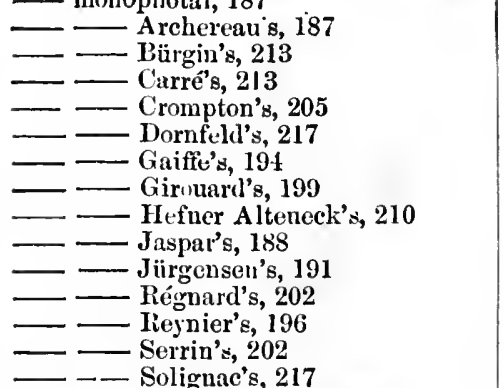

- oblique carbons, with, 266

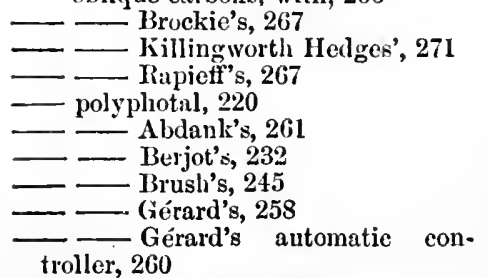

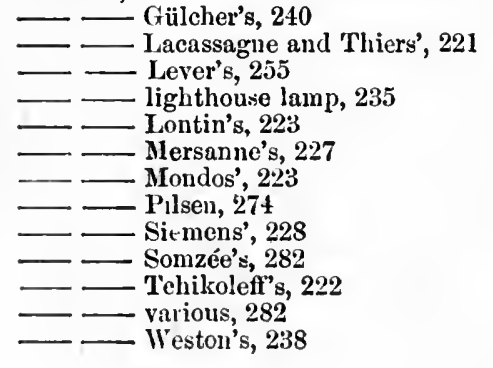

\section{B}

Batteries, electric, 5

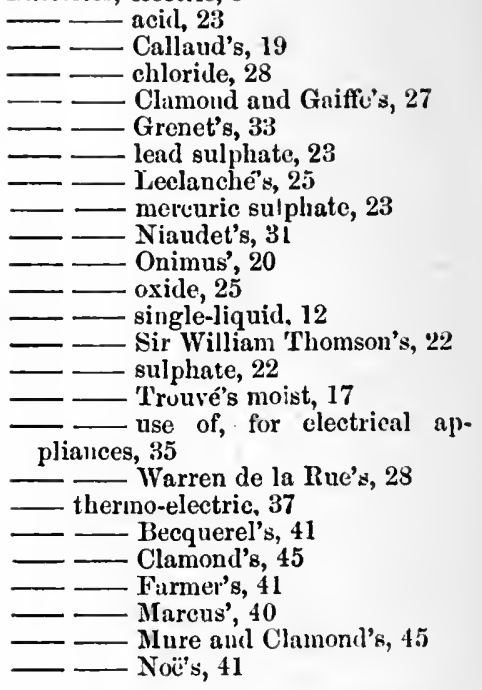

\section{C}

Carlon points, 180

Candles, eleetric, 284

- Debrun's, 248

_ Jablochkoff's, 281

- Jamin's, 293

Rapieff's, 243

Soleil lamp, 300

Solignae's, 298

Wilde's, 291

Continuous-current machines, 54

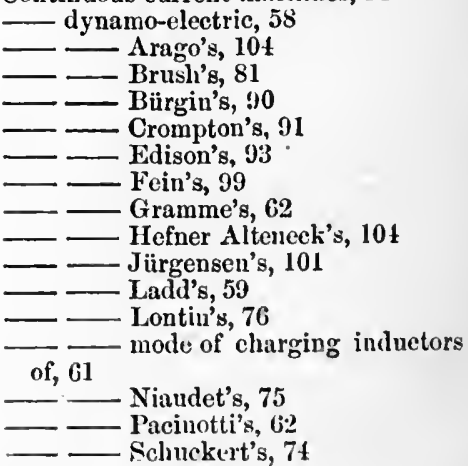


Continuous-current machines, dynamoelectric, Siemens', 78

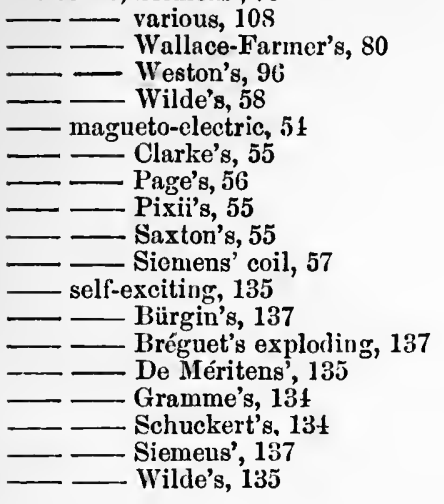

D

Division of electric light, 461

\section{$\mathbf{E}$}

Edison's system, $46 t$

Electrical units, 8

\section{G}

Generators, clectric, 52

\section{I}

Incandescent lamps, 307 Edison's carbon, 326

Elison's platiuum, 325
Incandescent lamps, efficiency of, $49 t$

—_ Lane Fox's, 337

- Maxim's, $3+1$

— M üller-Nothomb's, 419

- Swan's, 332

— Radiwanowski's, 342

various, 343

with combustion, 307

—— Joël's, 317

—— Reynier's, 308

313 Reynier's self-acting lighter,

—— various, 322

- - — Varley's, 308

—_... Werdermaun's, 31 ई

$\mathbf{J}$

Jablochkoff's installation, 439

$\mathbf{L}$

Lane Fox's system, 480

Light, electric, 173 expense of installation, 459 production of, $17 t$

M

Maxim's system, 489

$\mathrm{S}$

Swan's iustallation, 424

V

Voltaic arc, 175 


\section{INDEX OF INVENTORS' NAMES.}

* * Abrreviatioss: Cont.-cur. mach. = continuons-current machine; alt.-cur. mach. = alternating-current machine; selt-exc. mach. = self-exciting machine; incand. = incandescent; mach. = machine.

$\mathbf{A}$

Abdank, arc lamp, 261

Arago) (Ball), mach., 104

Archereau, arc lamp, 187

\section{B}

Bccquerel, lead sulphate battery, 23; thermo-electric battery, 41

Berjot, arc lainp, 232

Brégnet, exploder, 137

Brockie, arc lamp, 245

Brush, mach., 81 ; arc lamp, 213

Bunsen, battery, 24; carbon points, 180

Bürgin, arc lamp, 245 ; cont.-cur. mach., 90; self-exc. mach., 137

\section{C}

Callaud, battery, 19

Carré, arc lamp, 213; carbon points, 181

Clamond, thermo-electric batterv, $\mathbf{4 5}$

Clamond and Gaiffe, battery, 27

Clarke, mach., 55

Clerc and Bineau (Soleil), lamp, 300

Coulomb, lantern, 397

Crompton, arc lamp, 205 ; mach., 91

\section{D}

D'Arsonval, voltaic condenser, 160

Debrun, candle, 298

De Méritens, cont.-cur. mach., 111 ; self-exc. mach., 135

Dornfeld, arc lamp, 217
Dujardin, electric light applied to photograplyy, 447

E

Edison, carbon lamp, 326 ; installation, 461 ; mach., 93 ; platinum lamp, 326

\section{F}

Farmer, thermo-electric battery, 41

Paure, accumulator, 152

Fein, mach., 99

Ferranti, mach., 129

Foucault, arc lamp, 185

\section{G}

Gaiffe, arc lamp, 194

Gauduin, carbon points, 182

Gérard, arc lamp, 258; automatic controller, 261

Girouard, arc lamp, 199

Gramme, alt.-cur. mach., 118; cont.-cur. mach., 62 ; self-exc. mach., 134

Grenet, battery, 33

Grove, battery, 23

Gülcher, arc lamp, 240

\section{H}

Harrison, are lamp, 186

Hefner Alteneck, cont.-cur. mach., $10 t$

Holmes, mach., 368

Honston and Thomson, accumulator, $15^{9}$ 


\section{J}

Jablochkofi, candle, 284 ; installation, 439

Jamin, candle, 293

Jaspar, are lamp, 188

Joël, Iamp, 317

Jürgensen, mach., 101 ; arc lamp, 191

K

Kiillingworth Hedges, arc lamp, 270

Kabath, accumulator, 153

$\mathbf{L}$

Lacassagne and Thiers, arc lamp, 221 ; carbon points, 181

Lachaussée-Lambotte, mach., 126

Ladd, mach., 59

Lane Fox, installation, 480 ; lamp, 337

Léclanché, battery, 25

Le Molt, carbon points, 181

Lever, arc lamp, 255

Lontin, alt.-cur. mach., 116 ; arc lamp, 223 ; cont.-cur. mach., 76

\section{M}

Mangin, projector, 390

Marcus, thermo-electric battery, 40

Maxim, are lamp, 213 ; incand. lamp, 341 ; installation, 489

Mersanne, arc lamp, 227

Mondos, arc lamp, 223

Müller-Nothomb, incand. lamp, 419

Mure and Clamond, thermo-electric battery, 45

\section{$\mathrm{N}$}

Napoli, carbon points, 182

Niaudet, battery, 31 ; mach., 75

Noë, thermo-electric battery, 41

\section{O}

Onimus, battery, 20

\section{$\mathbf{P}$}

Pacinotti, maclı., 62

Page, mach., 56
Payne, electric light applied to surgical operations, 455

Pezzer and Carpenter, accumulator, 154

Piette and Krizik (Pilsen), lamp, 274

Pixii, mach., 55

Planté, accumulator, 142 ; electric light applied to galvano-caustic, 453; electric tinder-box, 15l; rheostatic mach., 169

\section{$\mathbf{R}$}

Radiwanowski, incand. lamp, 342

Rapieff, arc lamp, 267; candle, 293

Régnard, arc lamp, 202

Regnier, accumnlator, 153

Reynier, arc lamp, 196; incand. lamp, 308; self-acting lighter, 313

\section{S}

Saxton, mach., 55

Schuckert, cont.-cur. mach., 74 ; selfexc. mach., 134

Sedlaczek and Wikullil, are lamp, 403

Seebeck, experiment, 37

Sellon and Volckmar, accumulator, 154

Serrin, arc lamp, 202

Serrin and Berjot, lighthouse lamp, 235

Siemens, alt.-cur. mach., 120 ; coil, 57 ; cont.-cur. mach., 78; differontial lamp, 228; electric light applied to horticulture, 429; unipolar mach., 137

Siemens and Hefner Alteneck, arc lainp, 210

Solignac, arc lamp, 217; candle, 298

Somzée, arc lamp, 282

Spottiswoude, induction coil, 162

Staite and Edwards, carbon points, 180

Sutton, accumulator, 157

Swan, incand. lamp, 332, installation at the Savoy Theatre, 424; lamp applied -to the microscope, 449 ; mining lamp, 400

\section{$\mathrm{T}$}

'Tchikoleff, arc lamp, 222

Thomson, Sir William, battery, 22

'Tommasi, lamp, 322

'Trouvé, moist battery, 17; mercuric bisulphate battery, 23 
V

Varley, incand. lamp, 308

W

Wallace-Farmer, mach., 80
Warren de la Rue, battery, 28

Witson and Slater, carbon points, 181

Werdermann, lamp, 315

Weston, are lamp, 238 ; mach., 96

Wilde, candle, 291 ; cont.-cur. mach., 58; self-exc. mach., 133

Wright, arc lamp, 185

END OF VOI. I. 



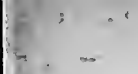

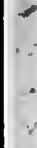

$\because$

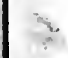




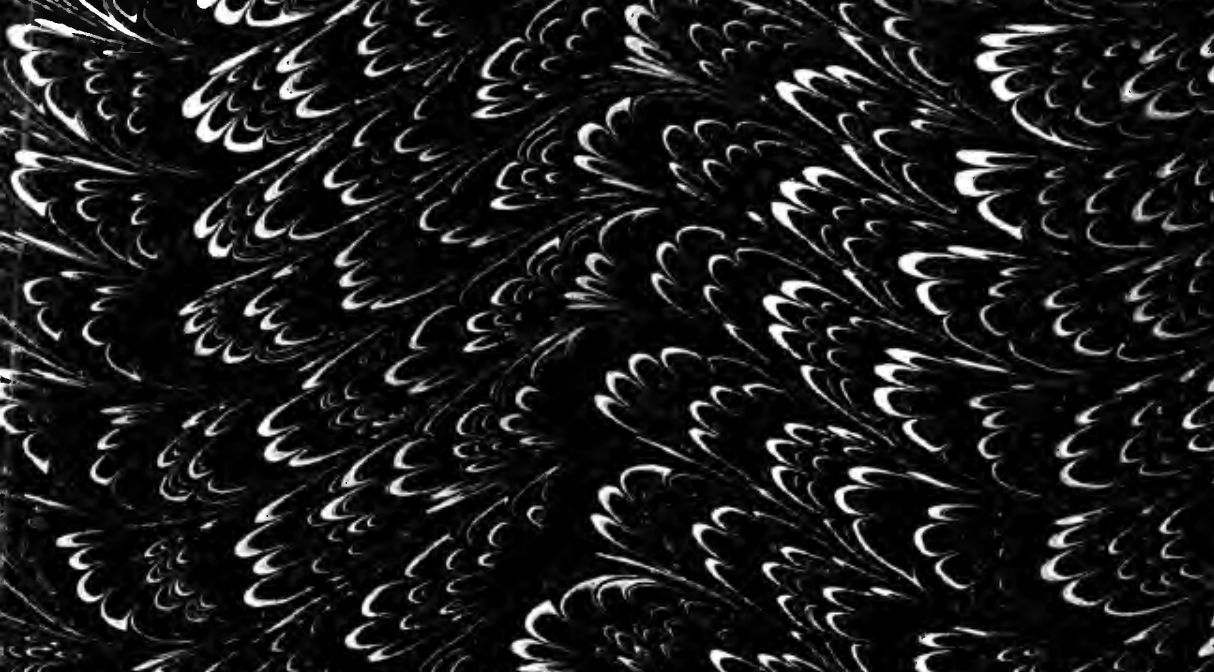

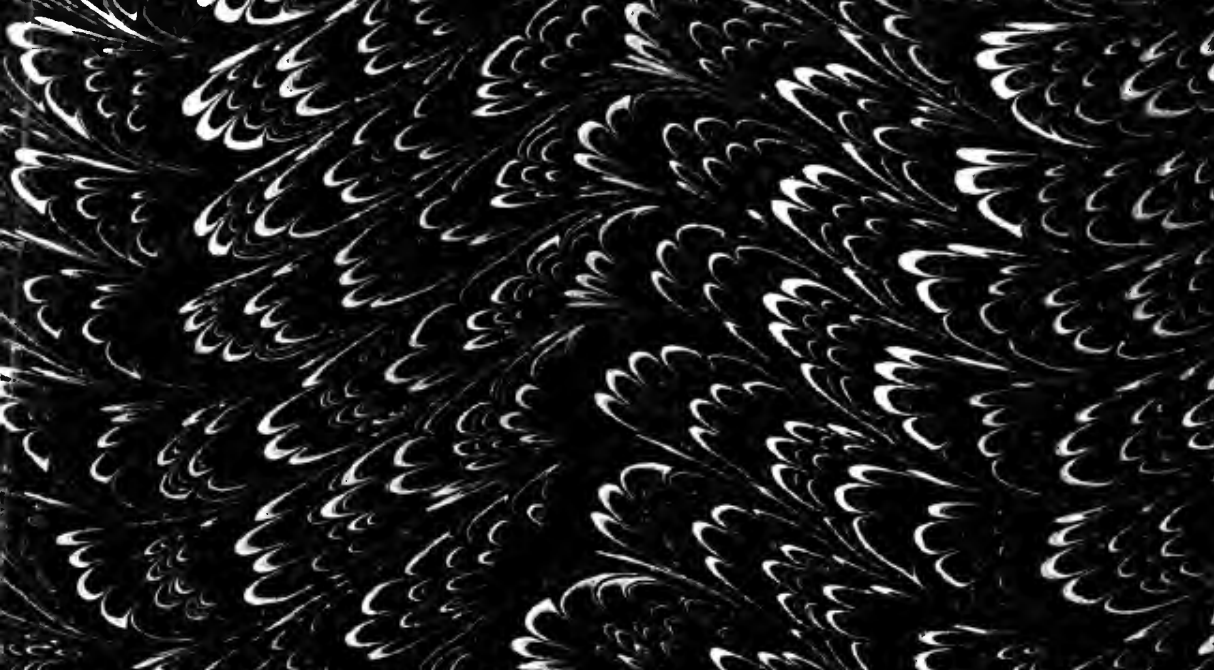

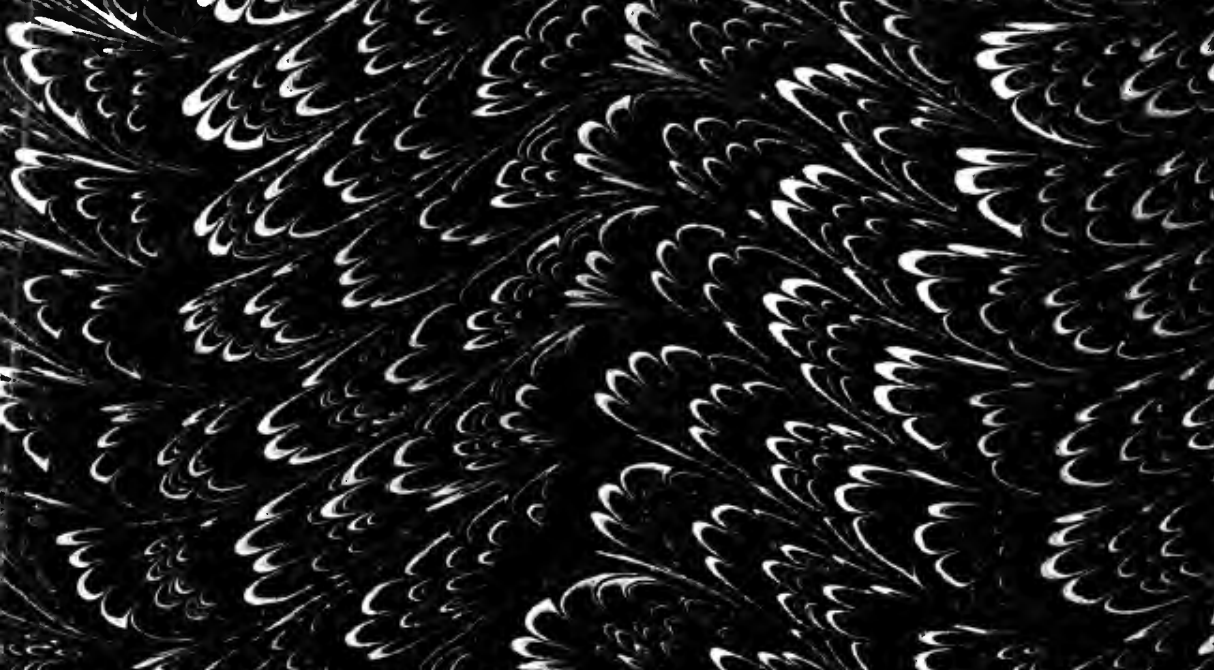

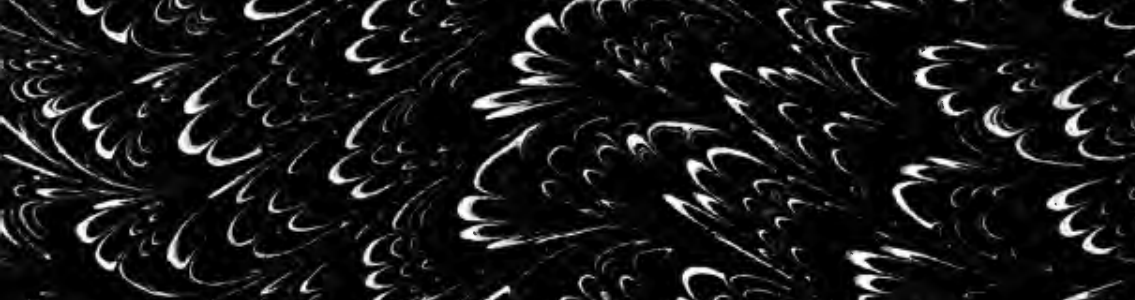
\& $\{$ की है

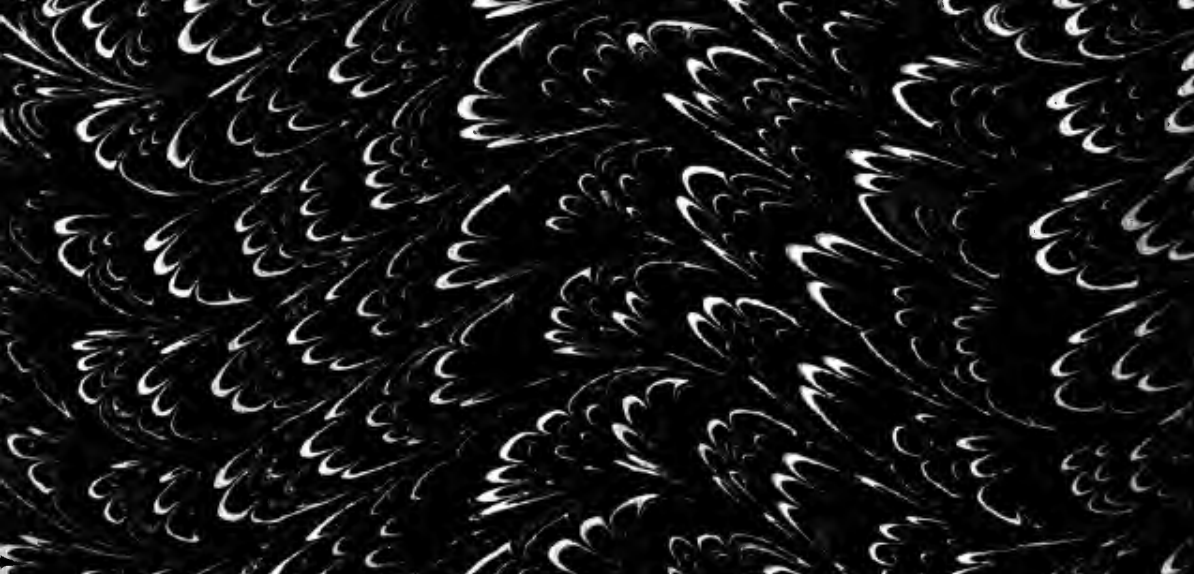

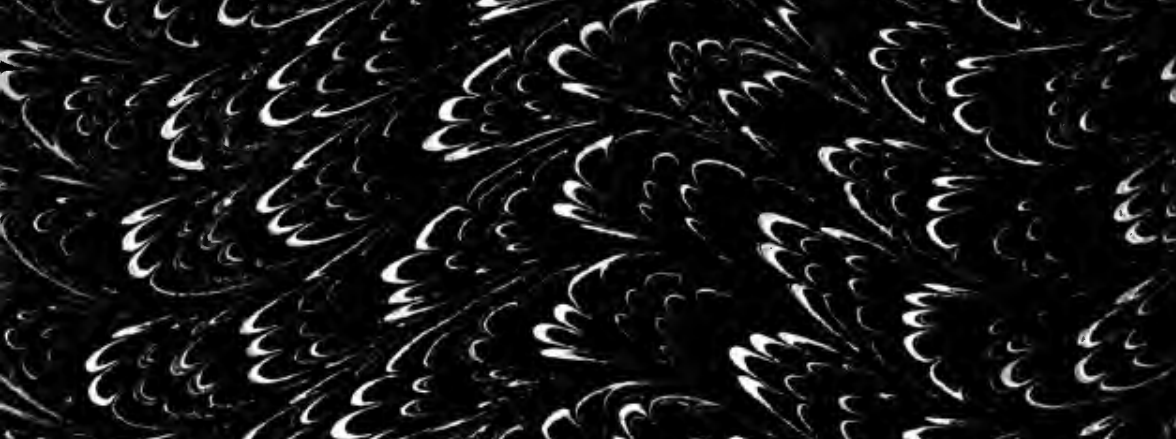
ccick

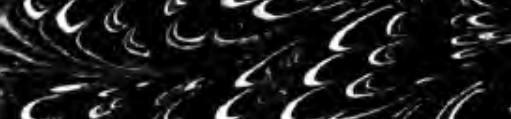
cacece बर्ती है हैं $\{\varepsilon$

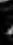

at 70 cas 


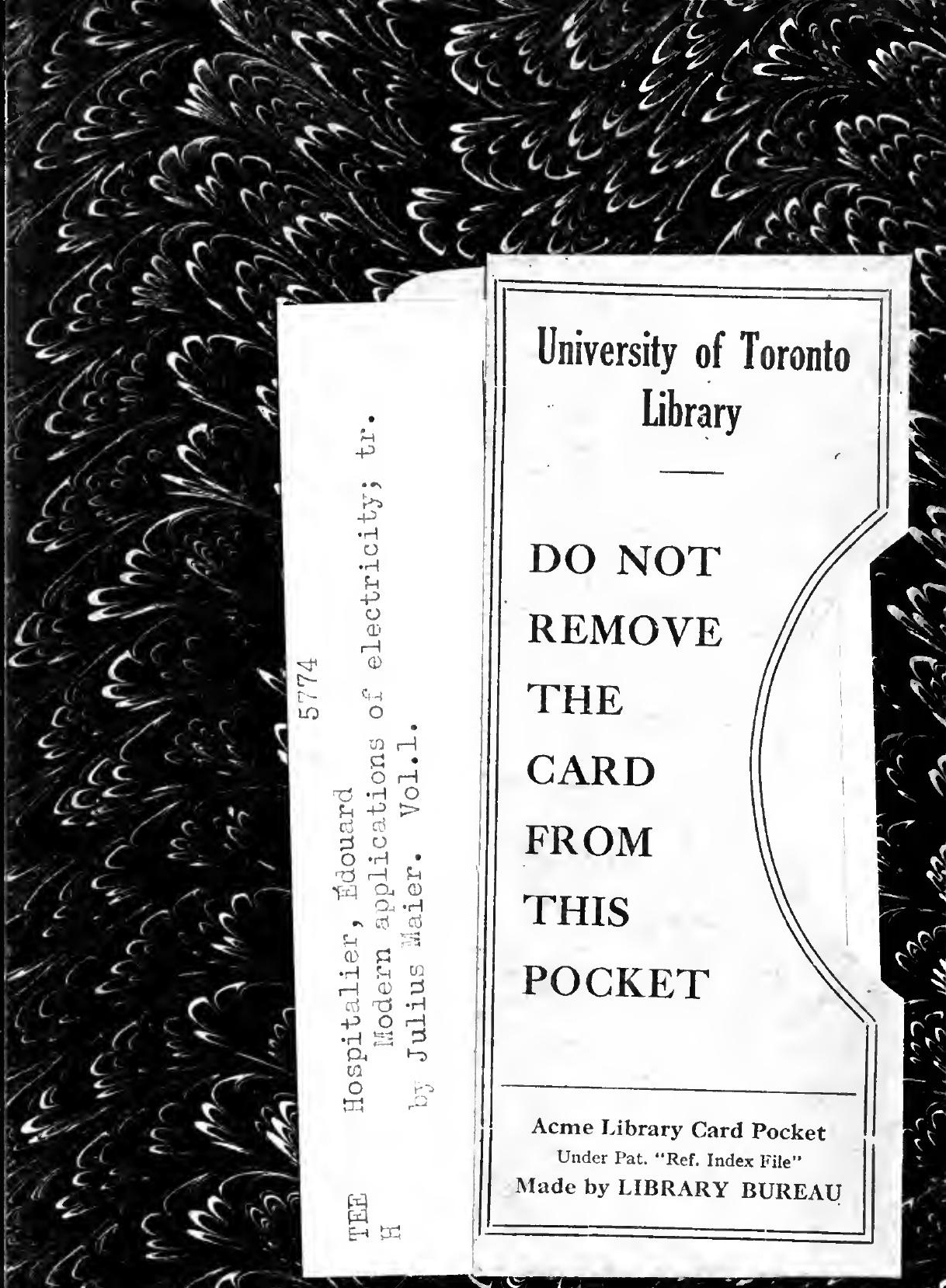


2.

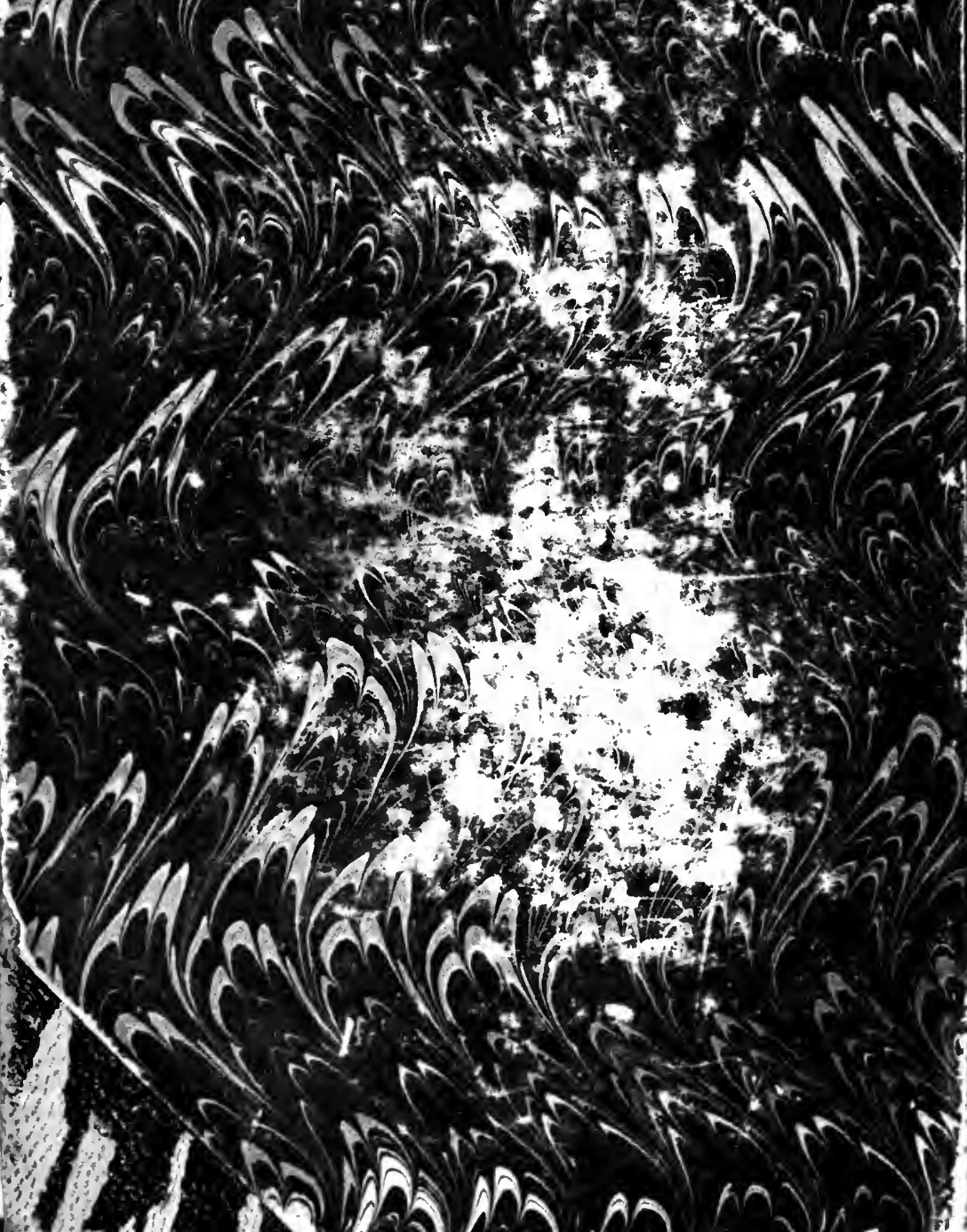
6. 